\title{
AN ENHANCED MODULATED \\ WAVEFORM MEASUREMENT SYSTEM
}

A thesis submitted to Cardiff University

In candidature for the degree of

Doctor of Philosophy

By

Muhammad Akmal, MS

Centre for High Frequency Engineering

Cardiff School of Engineering

Cardiff University

United Kingdom

November 2011 


\section{DECLARATION OF ORIGINALITY}

This work has not previously been accepted in substance for any degree and is not concurrently submitted in candidature for any degree.

Signed (candidate) Date

\section{STATEMENT 1}

This thesis is being submitted in partial fulfilment of the requirements for the degree of PhD.

Signed (candidate) Date

\section{STATEMENT 2}

This thesis is the result of my own independent work / investigation, except where otherwise stated. Other sources are acknowledged by explicit references.

Signed (candidate) Date

\section{STATEMENT 3}

I hereby give consent for my thesis, if accepted, to be available for photocopying and for inter-library loan, and for the title and summary to be made available to outside organisations.

Signed (candidate) Date

\section{STATEMENT 4}

I hereby give consent for my thesis, if accepted, to be available for photocopying and for inter-library loans after expiry of a bar on access approved by the Graduate Development Committee.

Signed (candidate) Date 


\section{ACKNOWLEDGMENTS}

In the name of Allah, the most merciful and most beneficent.

I would like to express heartfelt gratitude to Prof. Johannes Benedikt and Prof. Paul Tasker for giving me the opportunity to carry out this research work at the Centre for High Frequency Engineering, Cardiff University. I would like to thank Prof. Johannes Benedikt, who introduced me to the field of microwave engineering and nurtured me in becoming a researcher. His invaluable support and encouragement gave me confidence in overcoming difficult times. I would also like to express my profound thanks to Prof. Paul Tasker for his highly intellectual supervision. He guided me through the pursuit of this research work with his great academic wisdom, enthusiasm and infectious zest for the research. His expert advice has permanently been of great help, and incessant support with the sound knowledge in the field of RF and Microwave Engineering.

I would like to thank Engineering and Physical Sciences Research Council (EPSRC) for the financial support. This work would not have been possible without this support.

I have gained an invaluable knowledge in the microwave engineering field during my time at Cardiff University, due to the intellectually stimulating environment in the Centre for High Frequency Engineering. It has been a pleasure to be a part of an incredible team of scholars at Centre for High Frequency Engineering and I count it a privilege to have been part of this. I want to give a very special thanks to Dr. Jonathan Lees who has gone far beyond the call of duty to assist me and has taught me much of what I have learned about Microwave Engineering. His dedication and concern for this work has been largely responsible for its success. I wish to thank Dr. Simon Woodington for his patience and willingness to help me with the problems whenever I have had them. I would like to express my deepest appreciation to Vincenzo Carrubba, Zubaida Yusoff, James Bell, and Randeep Saini for their friendship and unreserved encouragement and strong support over the past several years.

I am thankful to my brothers and in particular to my sister who has served as my biggest fan and has always been available to provide good advice and to be a listening ear. Finally, I am grateful to my parents, for providing me the most important training I will ever receive: teaching me how to walk uprightly and developing honesty and integrity in my life. 


\section{ABSTRACT}

The microwave devices and circuits need to be characterized prior to being employed in the design of systems and components. Unfortunately the measurement systems required to characterize the microwave devices and circuits have not kept pace with the emerging telecommunication technologies demands. This has resulted into a situation where either the circuits being employed in the components are unoptimized or the yield and turn-around of optimized circuits are slow. One of the contributing factors of such situations is the limitations of the existing measurement systems to scale up in performance to fulfil the necessary requirements. This thesis presents an enhanced multi-tone, time domain waveform measurement and engineering system. The presented system allows for a more considered, and scientific process to be adopted in the characterisation and measurement of microwave power devices for modern day communications systems. The main contributions to the field of research come in two areas; firstly developments that allow for accurate time domain measurement of complex modulated signals using commercially available equipment; and secondly in the area of active impedance control, where significant developments were made allowing active control of impedance across a modulated bandwidth.

The first research area addressed is the fundamental difficulty in sampling multi-tone waveforms, where the main achievements have been the realisation of a high quality trigger clock for the sampling oscilloscope and a "Time Domain Partitioning" approach to measure and average multi-tone waveforms on-board. This approach allows the efficient collection of high quality vectoral information for all significant distortion terms, for all bands of interest.

The second area of research investigated suitable impedance control architectures to comprehensively investigate out-of-band impedance effects on the linearity performance of a device. The ultimate aim was to simultaneously present independent, baseband impedances to all the significant baseband (IF) frequency components and to $2^{\text {nd }}$ harmonic that result from a multi-tone excitation. The main achievement in this area was the ability of the enhanced measurement system to present the broadband impedance. At baseband this has been achieved in the time domain using a single arbitrary waveform generator (AWG) to synthesise the necessary waveforms to allow a specific IF impedance environment to be maintained across a wide IF bandwidth. To engineer the RF out-of-band load terminations at RF frequencies and to emulate specific power amplifier modes, a Tektronix AWG7000 Arbitrary Waveform Generator was used to deliver the desired impedances, practically fulfilling the wideband application requirements for reliable device characterisation under complex modulated excitations. 


\title{
LIST OF PUBLICATIONS
}

1. M. Akmal, J. Lees, Z. Yusoff, S. Bensmida, S. Woodington, V. Carrubba, S. Cripps, J. Benedikt, K. Morris, M. Beach, J. McGeehan, P. J. Tasker, "An Enhanced Modulated Waveform Measurement System for Robust Characterisation of Microwave Devices under Modulated Excitation", in Proc. of $41^{\text {st }}$ European Microwave Conference (EuMC), October 2011.

\begin{abstract}
This paper presents a refined modulated waveform measurement system for the robust characterization of nonlinear microwave devices when driven by broadband multi-tone stimuli. This enhanced system has the ability to present specific, constant impedances, not only to a large number of baseband (IF) components, but also to signals located around the carrier and significant harmonic frequencies. Achieving such comprehensive impedance control across wide modulation bandwidths is critical in allowing the 'emulation' of new power amplifier modes and architectures, and the subsequent waveform characterization of devices operating in these complex and often dynamic impedance environments. The enhanced system is demonstrated through a number of applications: firstly the experimental investigation and baseband optimization of a 10W GaN HEMT under nine-tone excitation, and secondly, the emulation of a modulated Class-J impedance environment that interestingly highlights the presence of separate optimum baseband impedance conditions necessary for the reduction of individual IM products.
\end{abstract}

2. M. Akmal, V. Carrubba, J. Lees, S. Bensmida, S. Woodington, J. Benedikt, K. Morris, M. Beach, J. McGeehan, P. J. Tasker, " Lineairty Enhancement of GaN HEMTs under Complex Modulated Excitation by Optimizing the Baseband Impedance Environment", in Proc. of IEEE MTT-S, International Microwave Symposium(IMS), June 2011.

Abstract - This paper demonstrates how the linearity performance of a $10 \mathrm{~W}$ GaN HEMT can be dramatically improved by actively engineering the baseband impedance environment around the device. An important refinement to existing active load-pull measurement capability is proposed that allows the precise and independent control of all significant baseband and RF components that result from the amplification of a complex 9carrier multi-sine modulation. The synthesis of constant, modulation frequency independent negative baseband impedances, resulting in specific baseband voltage waveforms has delivered a $24 \mathrm{~dB}$ improvement in ACPR compared to the classical baseband short case, even when the device is operating with RF components terminated into a non-optimal $50 \Omega$ RF environment. This linearization concept is further investigated 
through the broadband emulation of a class-J impedance environment around a single device. Using this enhanced system and a two-tone modulated excitation, optimum baseband loads are identified that result in a $18.5 \mathrm{~dB}$ and $24 \mathrm{~dB}$ improvement in $\mathrm{IM}_{3}$ and $\mathrm{IM}_{5}$ inter-modulation products respectively, again relative to the case of a traditional IF short circuit. The significance of this last observation is that unlike the $50 \Omega$ case, the optimum class-J IM and $\mathrm{IM}_{5}$ baseband impedances disperse, becoming reactive and moving away from the real axis.

3. M. Akmal, J. Lees, S. Bensmida, S. Woodington, J. Benedikt, K. Morris, M. Beach, J. McGeehan, P. J. Tasker, "Minimization of Baseband Electrical Memory Effects in GaN HEMTs Using IF Active Load-pull", in Proc. of Asia Pacific Microwave Conference (APMC), December 2010.

Abstract - This paper presents a rigorous way to quantify the role played by higher baseband impedances in determining baseband electrical memory effects observed in power transistors under two-carrier excitation. These effects typically appear not only as asymmetrical distortion terms in the frequency domain, but also more reliably as a recognizeable hysteresis or looping in the dynamic transfer characteristics extracted from measured input voltage and output current envelopes of a power device. Investigations have been carried out using a commercially available $10 \mathrm{~W}$ GaN HEMT device characterised at $2 \mathrm{GHz}$ within a high-power modulated wavefor measurement system. Active IF loadpull has been employed to present specific baseband impedance environments, allowing the sensitivity of IMD symmetry to baseband impedance variations to be investigated.

4. M. Akmal, J. Lees, S. Bensmida, S. Woodington, V. Carrubba, S. Cripps, J. Benedikt, K. Morris, M. Beach, J. McGeehan, P. J. Tasker, "The Effect of Baseband Impedance Termination on the Linearity of GaN HEMTs", in Proc. of $40^{\text {th }}$ European Microwave Conference (EuMC), September 2010, pages.

Abstract - This paper demonstrates the significant effect of baseband impedance termination on the linearity performance of a 10W GaN HEMT device driven to deliver a peak envelope power of approximately $40 \mathrm{dBm}$. The paper also proposes a further refinement to a state-of-art active IF load-pull measurement system to allow the precise independent control of all significant baseband components generated as a result of the multi-tone excitation used. The presentation of specific baseband impedances has delivered a $20 \mathrm{dBc}$ and $17 \mathrm{dBc}$ improvement in $\mathrm{IM}_{3}$ and $\mathrm{IM}_{5}$ inter-modulation products respectively, relative to the case of a classical, ideal short circuit. As expected for this device, this was achieved by emulating appropriate negative impedances lying outside of the Smith chart, and when this observation is 
considered alongside the Envelope Tracking PA architecture, this raises the interesting possibility of significantly improving PA linearity using the very mechanisms that are employed to improve PA efficiency.

5. M. Akmal, J. Lees, S. Bensmida, S. Woodington, J. Benedikt, K. Morris, M. Beach, J. McGeehan, P. J. Tasker, "The Impact of Baseband Electrical Memory Effects on the Dynamic Transfer Characteristics of Microwave Power Transistors", in Proc. of $4^{\text {th }}$ IEEE International Nonlinear Microwave Monolithic Integrated Circuit (INMMIC), April 2010.

Abstract - The inter-modulation distortion products can vary both in terms of amplitude and asymmetry due to the effects of baseband and 2nd harmonic impedance. This paper presents an investigation into the relationship between the IMD asymmetries caused by baseband impedance variation and the looping or hysteresis that can sometimes appear in the dynamic transfer characteristics of microwave power devices when subjected to modulated excitation. The investigation is carried out using a $2 \mathrm{~W}$ GaN HFET bare die device characterized at $2.1 \mathrm{GHz}$, and using IF active load-pull to clarify the role of baseband impedance on observed hysteresis in the dynamic transfer characteristics. Analysis is performed using the envelope domain in order to more effectively reveal the DUT's sensitivity to impedance environments and specifically electrical baseband memory effects. 


\section{JOINT PUBLICATIONS}

\& J. Lees, M. Akmal, S. Bensmida, S. Woodington, J. Benedikt, K. Morris, M. Beach, J. McGeehan, P. J. Tasker, "Waveform Engineering Applied to Linear-Efficient PA Design", in Proc. of $11^{\text {th }}$ IEEE Wireless and Microwave Technology Conference(WAMICON), April 2010.

* A. L. Clarke, M. Akmal, J. Benedikt, J. Lees, P. J. Tasker, "Investigation and Analysis into Device Optimization for Attaining Power Amplifiers with Efficiencies In-Excess of 90\%", in Proc. Of IEEE MTT-S, International Microwave Symposium (IMS), May 2010.

* Z. Yusoff, M. Akmal, V. Carrubba, J. Lees, J. Benedikt, P. J. Tasker, S.C. Cripps "The Benefit of GaN Characteristics over LDMOS for Linearity Improvement using Drain Modulation in Power Amplifier System", in Proc. $5^{\text {th }}$ International Nonlinear Microwave Monolithic Integrated Circuit(INMMIC), April 2011.

* S. Bensmida, K. Morris, M. Akmal, J. Lees, P. Wright, J. Benedikt, P. J. Tasker, J. McGeehan, M. Beach, "Generic Pre-destortion of a Class-J Power Apmlifier", in Proc. of $40^{\text {th }}$ European Microwave Conference(EuMC), September 2010.

* V. Carrubba, A. L. Clarke, M. Akmal, J. Lees, J. Benedikt, P. J. Tasker, S. C. Cripps, "The Continuous Class-F Mode Power Amplifier", in Proc. of $40^{\text {th }}$ European Microwave Conference(EuMC), September 2010.

V. Carrubba, A. L. Clarke, M. Akmal, J. Lees, J. Benedikt, P. J. Tasker, S. C. Cripps, "On The Extension of the Continuous Class- $F$ Mode Power Amplifier", IEEE Transaction on Microwave Theory and Techniques, Vol. 59, Issue 5, May 2011.

\& V. Carrubba, A. L. Clarke, M. Akmal, Z. Yusoff, J. Lees, J. Benedikt, S. Cripps, ,P. J. Tasker, "Exploring the Design Space for Broadband $P A s$ using the Novel Continuous Inverse Class-F Mode", in Proc. of $41^{\text {st }}$ European Microwave Conference, October 2011.

\& M. Paynter, S. Bensmida,K. Morris, M. Akmal, J. Lees, J. Benedikt, ,P. J. Tasker, M. Beach, J. McGeehan "Non-Linear Large Signal PA Modelling for Switching-Mode Operation (Class-F/Continuous Class$F)$ ", in Proc. of $41^{\text {st }}$ European Microwave Conference, October 2011.

* V. Carrubba, A. L. Clarke, S. P. Woodington, W. McGenn, M. Akmal, J. Lees, S. C. Cripps, P. J. Tasker, J. Benedikt, "High-Speed Device Characterization Using an Active Load-Pull System and Waveform Engineering Postulator", $77^{\text {th }}$ ARFTG Microwave Measurement Conference, June 2011. 


\section{LIST OF ACRONYMS}

AM - Amplitude Modulation

ACPR - Adjacent Channel Power Ratio

AET - Auxiliary Envelope Tracking

AWG - Arbitrary Waveform Generator

BER - Bit Error Rate

CCDF - Complementary Cumulative Distribution Function

CSA - Communication Signal Analyser

CDMA - Code Division Multiple Access

CW - Continuous Wave

DAC - Digital To Analogue Convertor

DUT - Device Under Test

DC - Direct Current

DPD - Digital Predistortion

ESG - Economy Signal Generator

EER - Envelope Elimination and Restoration

ET - Envelope Tracking

ESG - Economy Signal Generator

EVM - Error Vector Magnitude

FET - Field Effect Transistor

FDD - Frequency Division Duplex

FFT - Fast Fourier Transform

GaN - Gallium Nitride

GUI - Graphic User Interface

GaAs - Gallium Arsenide

GPIB - General Purpose Instrument Bus

HEMT - High Electron Mobility Transistor

HFET - Hetrojunction Field Effect Transistor

IFFT - Inverse Fast Fourier Transform

IF - Intermediate Frequency

IMD - Intermodulation Distortion

IMR - Intermodulation Distortion Ratio 
LSNA - Large Signal Network Analyzer

LTE - Long Term Evolution

LUT - Look Up Table

LDMOS - Laterally Diffused Metal Oxide Semiconductor

MTA - Microwave Transition Analyzer

NPR - Noise Power Ratio

NVNA - Nonlinear Vector Network Analyzer

OFDM - Orthogonal Frequency Division Multiplexing

PM - Phase Modulation

PA - Power Amplifier

PAR - Peak-to-Average Ratio

PEP - Peak Envelope Power

PSG - Performance Signal Generator

RF - Radio Frequency

RFC - Radio Frequency Chock

SNR - Signal to Noise Ratio

SCPI - Standard Commands for Programming Instructions

TRL - Thru Reflect Line

TRM - Thru Reflect Match

UMTS - Universal Mobile Telecommunication System

VSA - Vector Signal Analyzer

VNA - Vector Network Analyzer

WiMAX - Worldwide Interoperability for Microwave Access

3G - Third Generation

4G - Fourth Generation

3GPP - Third Generation Partnership Project 


\section{TABLE OF CONTENTS}

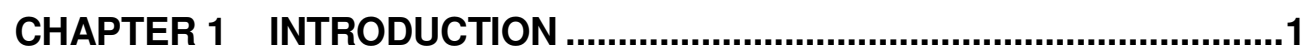

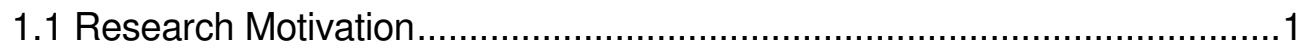

1.2 Power Amplifiers in Modern Wireless Applications ...................................

1.3 Challenges in Power Amplifier Design ................................................. 4

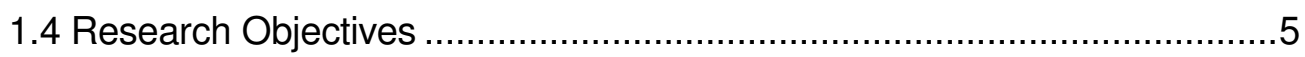

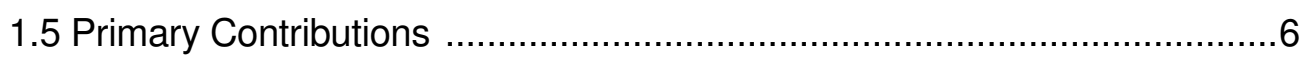

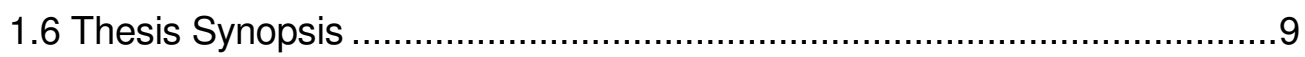

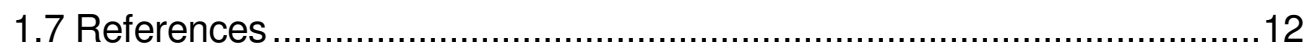

CHAPTER 2 MODULATED DOMAIN MEASUREMENT SYSTEMS..........14

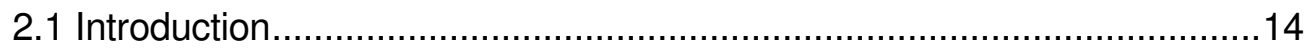

2.2 Memory Effects in Power Amplifier .................................................15

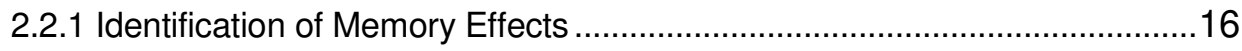

2.2.2 Sources of Memory Effects ............................................................... 17

2.2.3 Types of Memory Effects...................................................................... 18

2.2.3.1 Short Term Memory Effects ............................................................... 19

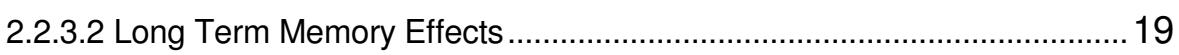

2.2.3.2.1 Thermal and Trapping Memory Effects ..................................................... 20

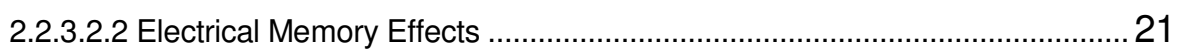

2.3 Modulated Domain Measurement Systems ........................................22

2.3.1 Industrial Measurement Solutions .......................................................28

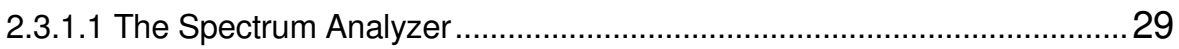

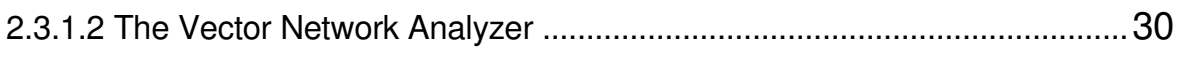

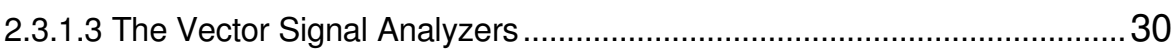

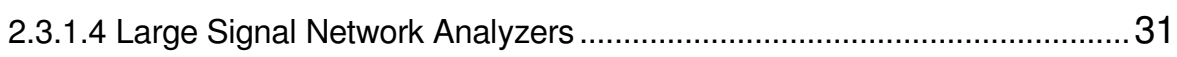

2.4 Previous Cardiff Modulated Measurement System .................................31

2.4.1 Previous Modulated Measurement System Limitations ...............................33

2.4.1.1 Triggering for Modulated Measurements ..................................................34

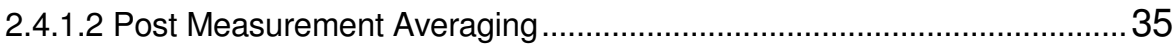

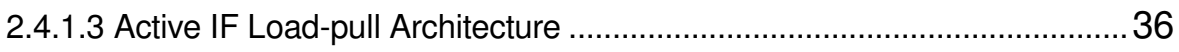

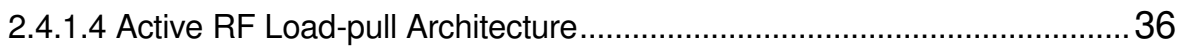

2.5 Enhanced Modulated Measurement System ...................................... 37

2.5.1 Necessary Measurement System Enhancements ...................................38

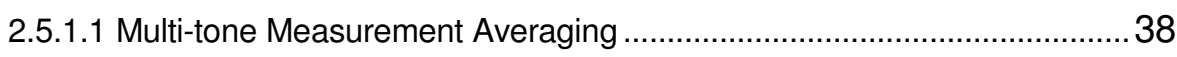


2.5.1.2 Multi-tone Measurement Software 40

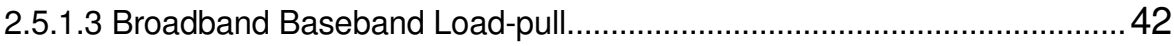

2.5.1.4 Why AWG for Modulated RF Load-pull? ............................................46

2.5.1.5 Broadband Modulated RF Load-pull ...................................................46

2.5.2 Capabilities of Enhanced Measurement System....................................48

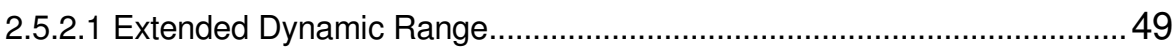

2.5.2.2 Extension of Measurement Bandwidth ................................................ 52

2.5.2.3 Possible Measurement Options ............................................................. 53

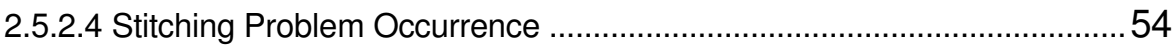

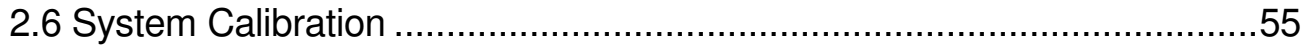

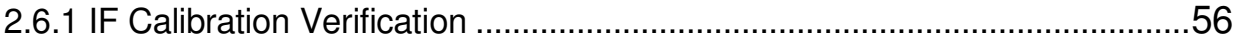

2.6.2 Measurement Accuracy Enhancement........................................................59

2.7 Demonstration of Multi-tone Measurement Capability ............................60

2.7.1 Output Power Spectrum Comparison ...........................................................63

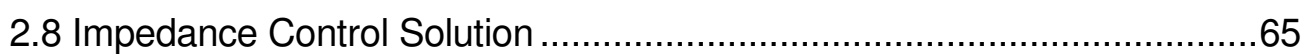

2.8.1 Demonstration of Baseband Load-pull Capability .......................................66

2.8.2 Demonstration of RF Load-pull Capability ....................................................

2.8.3 Why Out-of-Band Impedance Control is Necessary? .................................74

2.8.4 Comprehensive Out-of-Band Impedance Control ........................................75

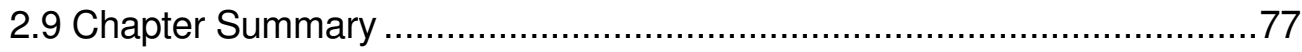

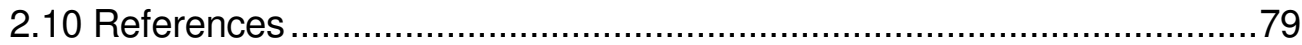

CHAPTER 3 BASEBAND ELECTRICAL MEMORY INVESTIGATIONS .83

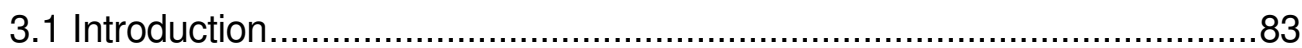

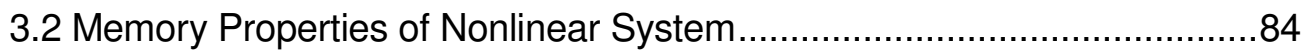

3.2.1 Characterisation of Memory Effects .........................................................89

3.2.1.1 Continuous Wave Characterisation ...................................................90

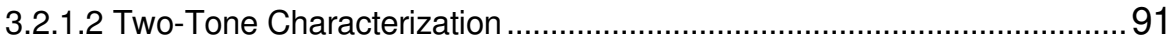

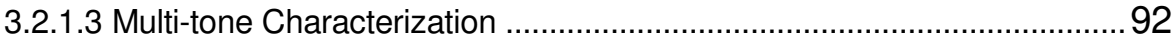

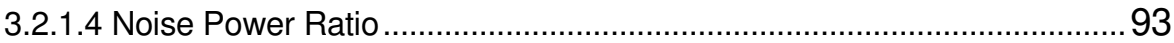

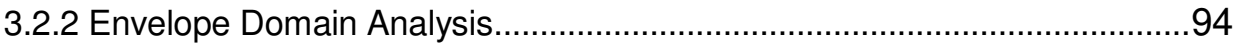

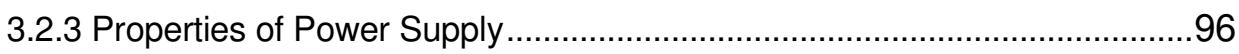

3.3 Memory Effects Measurements and Investigations...............................97

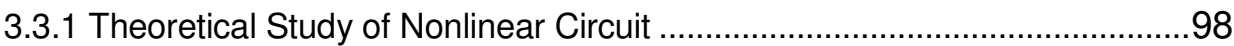

3.3.2 Effect of Drive Level on Output Envelopes Investigations ....................... 100

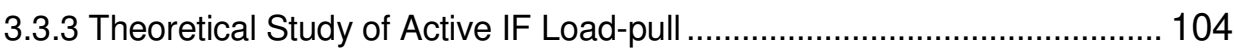

3.3.4 Application of Active IF Load-pull........................................................ 106 
3.3.5 Delay Adjustment Estimation 109

3.3.6 Effect of Baseband Impedance on $2^{\text {nd }}$ Harmonic 110

3.4 Broadband Baseband Impedance Termination....................................112

3.4.1 Dynamic Characteristics of Power Device............................................. 115

3.4.2 Minimization of Baseband Electrical Memory .......................................... 118

3.5 Chapter Summary ........................................................................120

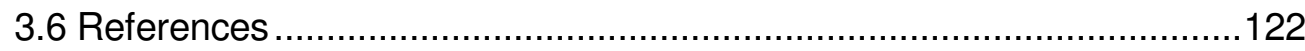

CHAPTER 4 ENVELOPE TRACKING FOCUSED INVESTIGATIONS ..124

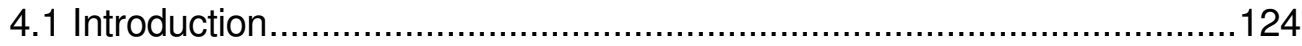

4.2 Efficiency Enhancement Techniques .............................................125

4.2.1 Peak to Average Ratio......................................................................... 126

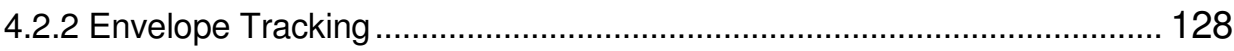

4.2.3 Envelope Tracking Power Supply .......................................................... 130

4.2.4 Auxiliary Envelope Tracking ............................................................... 131

4.3 Device Level Linearity of GaN HEMTs ............................................132

4.3.1 Continuous Wave Characterization of GaN HEMTs ............................. 132

4.3.2 Enhancing Linearity at Back-off Regime.............................................. 134

4.3.2.1 Baseband Signal Injection Technique..............................................135

4.3.2.2 Mathematical Analysis of Baseband Signal Injection ............................136

4.3.2.3 Linearity Investigations and Analysis ................................................ 137

4.3.2.4 Emulation of Auxiliary Envelope Tracking Environment.........................141

4.4 Enhancing Linearity at Compressed Drive Level ................................148

4.4.1 Optimized Device Linearity Performance ................................................ 155

4.4.2 Optimized Device Efficiency Performance .............................................. 156

4.4.3 Mathematical Analysis on $2^{\text {nd }}$ Harmonic Termination .............................. 158

4.4.4 Emulation of Class-J Mode of Power Amplifier ......................................... 159

4.4.5 Repeatability of Baseband and RF Load Emulation .............................. 161

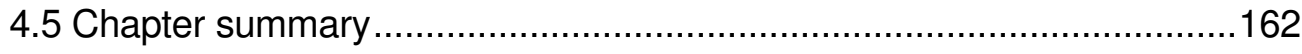

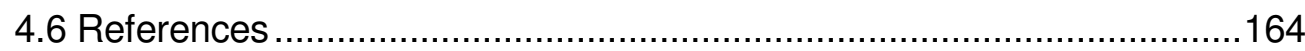

CHAPTER 5 MULTI-SINE MEASUREMENTS AND LINEARIZATION ...166

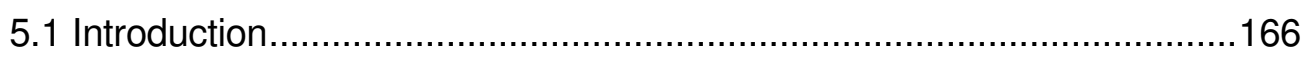

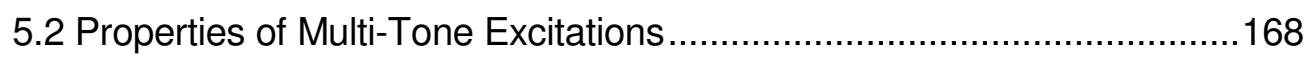

5.2.1 Correlation Between IMR and ACPR ….........................................169

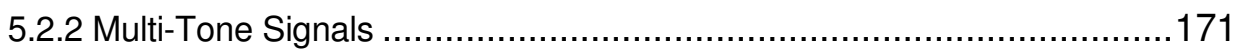

5.3 Multi-Tone Excitation and Characterisation........................................174 
5.3.1 Multi-Tone Investigations of Memory Effects .....................................176

5.4 Linearity Enhancements under Complex Excitations ............................182

5.4.1 Effect of Carrier Phase Distribution on Linearity .................................188

5.4.2 Optimum for IMR and ACPR Reduction ..........................................190

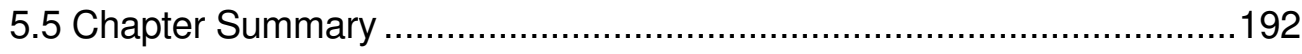

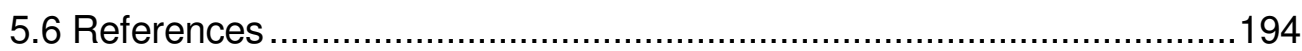

CHAPTER 6 CONCLUSIONS AND FUTURE WORK ...........................196

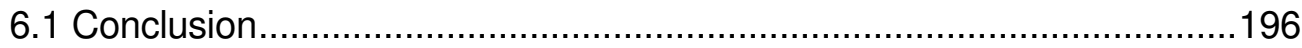

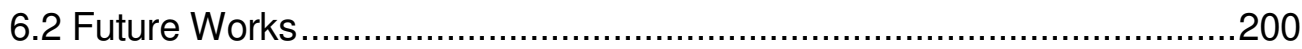

6.2.1 Improving Measurement Capability ................................................... 201

6.2.1.1 Automated Baseband Load-pull.........................................................201

6.2.1.2 Automated Modulated RF Load-pull ....................................................202

6.2.1.3 Load-pull Capability for Digitally Modulated Stimuli.............................202

6.2.2 Supplementary DUT Investigations ..................................................... 203

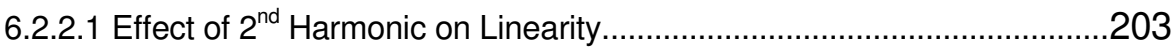

6.2.2.2 Combined LUT Predistortion and Baseband Injection ...........................204

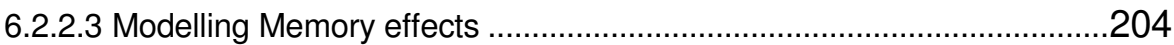

6.2.2.4 Envelope Square Injection for Linearity Enhancement ..........................205

6.2.2.5 Linearity Investigations of Continuous Modes .......................................206

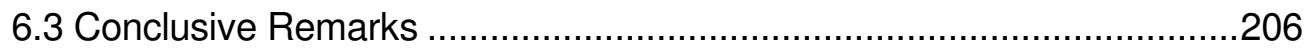

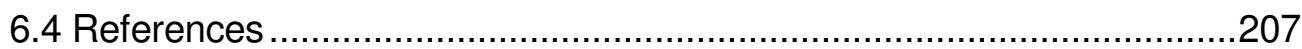

APPENDICES

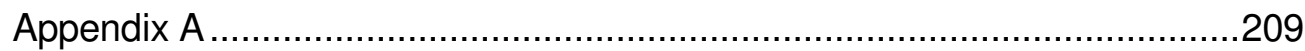

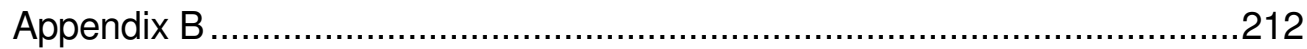

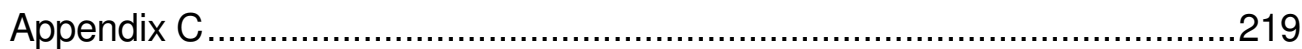

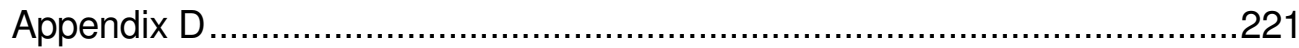

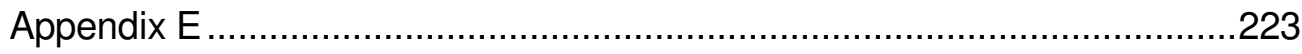

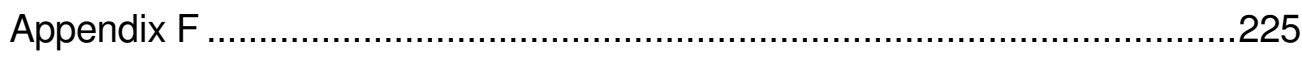

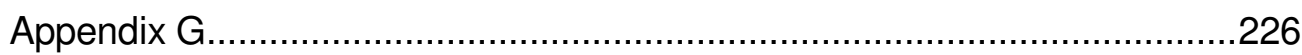

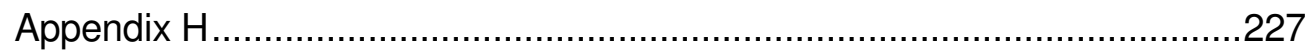

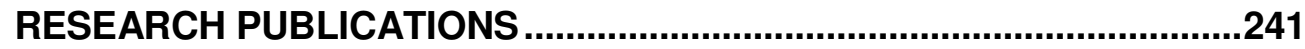




\section{Chapter}

\section{Introduction}

\subsection{Research Motivation}

The advent of fourth generation (4G) wireless systems, namely Long Term Evolution (LTE) and mobile WiMAX, has significantly increased the demand on Power Amplifier (PA) designers for multi-carrier capability; broadband for high data rate; high linearity to meet the adjacent channel power ratio (ACPR) requirements; multi-band; and multi-standards, so that the mobile systems can be used everywhere. The third generation (3G) comes under the umbrella of the International Mobile Telecommunications programme (IMT-2000) which employs wideband code division multiple access (W-CDMA), achieving a transmission rate of $2 \mathrm{Mbit} / \mathrm{s}$ with a $5-\mathrm{MHz}$ frequency bandwidth. The third generation of mobile communication systems is designed for applications such as Internet services, e-mail, database retrieval, video telephony, interactive video and sound. Despite the enhanced features of $3 G$ systems, they are still severely constrained by the bandwidth particularly when handling full-motion videos [1].

With the growing demand for higher data rates and ever increasing users on the $3 \mathrm{G}$ network linearity specifications have become acute in accomplishing the needed bandwidth. In this regard, the basestation power amplifier must be able to accommodate large peaks, which means that the power amplifier is usually operating well below its peak efficiency point. For instance, the 
Universal Mobile Telecommunication System (UMTS) power amplifier requirements according to $3 G$ partnership project (3GPP) specifications are to have frequency band (FDD, downlink) from $2.11 \mathrm{GHz}$ to $2.17 \mathrm{GHz}$, with $\mathrm{ACPR}$ of $-45 \mathrm{dBc}$ at $\pm 5 \mathrm{MHz}$ and $-50 \mathrm{dBc}$ at $\pm 10 \mathrm{MHz}$ carrier offset [1], [2]. Moreover, since the UMTS basestation power amplifiers are subjected to wideband code division multiple access (W-CDMA) signals, a peak-to-average ratio (PAR) ranging from $3 \mathrm{~dB}$ upto $12 \mathrm{~dB}$ must be sustained. The proposed $3 \mathrm{G}$ UMTS interface would employ orthogonal frequency division multiplexing (OFDM) for downlink with scalable bandwidths ranging from $1.25 \mathrm{MHz}$ to $20 \mathrm{MHz}$ [3]. This continuing developments in $3 G$ radio interface towards broadband $4^{\text {th }}$ generation capabilities targeting up to $100 \mathrm{Mb} / \mathrm{s}$ and $1 \mathrm{Gbit} / \mathrm{s}$ speeds is forcing rapid system enhancements.

The linear operation in conventional power amplifiers is achieved by reducing the RF input to a level sufficiently low to avoid saturation of the active devices [4]. When a high degree of linearity is required, back-off mode of operation inevitably degrades overall efficiency and output power. Consequently, as indicated in Figure 1.1, in modern RF power amplifier applications, fulfilling linearity for preserving fidelity of the signal together with power amplifier efficiency, impose two conflicting design requirements.

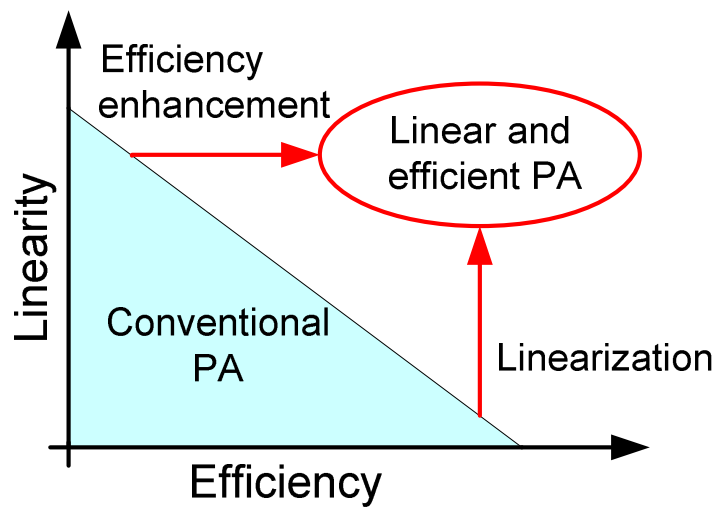

Figure 1.1: Linearity-efficiency trade-off in power amplifier design $[5,6]$

PA efficiency enhancement attempts to improve the efficiency of a linear but inefficient PA. Linearization takes a different approach by attempting to improve the linearity of a nonlinear but efficient PA. By resorting to highly efficient power amplifier architectures, however, memory effects can cause a bottleneck when implementing conventional linearization techniques. Memory 
effects manifest as an asymmetric behaviour between lower and upper intermodulation distortion (IMD) sidebands, which vary with the signal bandwidth. Such problematic effects can lead to significant difficulty in achieving PA performance that meets the required linearity specifications. Indeed, if the intermodulation distortion becomes strongly asymmetrical, then this can necessitate the need to employ different digital pre-distortion compensation requirements for lower and upper IMD sideband signals, which itself can be problematic. As a result, some common linearization techniques can be rendered ineffective because their success rely upon constant and equal intermodulation levels over the signal bandwidth [5]. Thus, it is of prime importance to understand the sources of this asymmetry. This thesis focuses on the role of baseband impedance variations with the aim being to improve the power amplifier linearity for future wireless communication systems.

\subsection{Power Amplifiers in Modern Wireless Applications}

In typical wireless transceivers, the PA is the last active building block in the transmitter chain. The foremost function of a PA is to amplify the signal to a level high enough that it can travel through the air to the intended receiver. Often, the PA dominates the power consumption of the entire transceiver; therefore, improving the PA's power efficiency, defined as the ratio of the output power to the DC power consumption, usually translates to longer battery life in the wireless device. In some wireless standards such as GSM, where the modulated signal has constant envelope, a nonlinear PA can be used. A nonlinear PA generally has higher efficiency than a linear PA, despite their higher efficiency; the data rates that these transceivers can transmit within a given bandwidth are low. In order to increase the data rate without increasing the bandwidth, a more spectrally efficient modulation scheme must be used. This makes the modulated signal's envelope nonconstant and therefore requires a linear PA to faithfully amplify the signal. Specifically this means that the design of a linear amplifier may not be achieved without compromising the efficiency. These requirements are interrelated which makes the design difficult to deal with independently. On the other hand the highest average efficiency is critical to maintain the low DC 
power consumption within the signal dynamic range accounting for PAR characteristics of the signal to be amplified. Hence, design solutions are under intensive investigation to improve the average efficiency as well as the linearity of the amplifier involved in the transmitter unit [7].

\subsection{Challenges in Power Amplifier Design}

To meet the requirements of the future generation of wireless communication systems, power amplifiers with high output power, high linearity, and broad bandwidth with high average efficiency are required. The basestation in the UMTS standard requires WCDMA modulation with $5 \mathrm{MHz}$ bandwidth and adjacent power ratio of $-45 \mathrm{dBc}$ at the offset frequency of $5 \mathrm{MHz}$ with a signal of PAR $7 \mathrm{~dB}$ to $10 \mathrm{~dB}$ [8]. The basestation PA needs to satisfy these stringent requirements as higher PAR values are expected for future standards like LTE and 4G [9].

The primary requirement in the basestation PA is to fulfil the linearity specifications. To satisfy the linearity requirements PA are typically operated at large back-off from the peak output power due to the high PAR of the signal, which leads to device operation at low average power and efficiency. The linearity of the amplifier can be improved through additional linearization but for the linearization method to be effective, the amplifier is also expected to have bandwidth independent linearity, for instance, minimum memory effects [9]. Consequently, the modern basestation PA should be designed to have high linearity, high output power, high average efficiency and a broad bandwidth. These multiple design challenges can be met with the following proposed solutions:

Basestation amplifiers with high output power (>100W) can be realized using new wide bandgap device technologies such as GaN HEMTs. The GaN HEMT devices provide architectural benefits and unique features to produce high output power [10], [11].

* The linearity at device level can be improved through the optimization of the baseband impedance environment. In this regard, the use of a two-tone test signal to analyze the device properties in terms of 
minimizing the intermodulation distortion is the pertinent possibility [12], [13].

* High peak efficiency can be achieved using single stage design architecture such as class B, harmonic tuning and switched mode amplifiers [14]. These power amplifiers typically have either low average efficiency, if operated at large back-off level, or high nonlinearity. The efficiency of these power amplifiers can be enhanced at back-off using enhancement techniques such as envelope elimination and restoration (EER), and envelope tracking (ET) which are still under intensive investigations [15].

The design of power amplifiers for future wireless communication systems should consider all these aspects to meet the requirements. A part of the problem will be embarked upon in this thesis whilst considering the high power device operating under the realistic modulation excitations to characterize the memory effects as well as the effects of envelope injection on the overall linearity of the device.

\subsection{Research Objectives}

The main objective of this research is to develop a modulated waveform measurement system that it is capable of measuring and engineering both the low frequency and RF signal components, hence is suitable for the characterisation and performance evaluation of high power microwave devices and power amplifier modes under multi-tone excitations. This research work can be divided into two significant parts. The first focuses on further refinement to a state-of-art active IF and RF load-pull measurement system to allow the precise independent control of all significant baseband components generated as a result of the multi-tone excitation used.

The previously developed Cardiff modulated waveform measurement system that incorporated an IF measurement capability was unfortunately only able to present the impedance to the two most significant baseband components (IF1 and IF2) generated as a result of 2-tone excitation and had no RF load-pull capability. The IF active load-pull was achieved by combining two, phase coherent arbitrary waveform generators (AWGs) whilst the device was driven 
at a relatively backed-off level, at $1 \mathrm{~dB}$ below the $1 \mathrm{~dB}$ compression point. However, when the device is driven more deeply into compression, significantly more mixing terms are generated, and in order to achieve a sufficiently broadband IF termination, significant modification of the baseband load-pull measurement system was required in order to accurately account for higher baseband harmonics. The RF architecture of the measurement system was modified to allow synthesise of RF loads for the robust characterisation of microwave devices in specific impedance environments. This was achieved in the time domain through dual channel Tektronix AWG7000 Arbitrary Waveform Generator to synthesize the excitation and constant, frequency independent RF loads around the fundamental and $2^{\text {nd }}$ harmonic tones.

The second part focuses on improving the triggering of Tektronix CSA8000, so that it could capture and average the more complex modulated waveforms directly. Through extensive testing it was found that the most robust solution to this problem was achieved by using a high quality trigger signal. This was provided through $120 \mathrm{MHz}$ Agilent arbitrary waveform generator (AWG). The measurement system was then demonstrated to capture consistent, repeatable, multi-tone waveforms without phase jitter, regardless of device behaviour.

\subsection{Primary Contributions}

The research work documented in this thesis is the result of the need to improve the linearity of RF power amplifiers in wireless communication systems, in order to increase power efficiency and reduce undesired emissions at the same time. This research makes available the following primary contributions, not found in the literature, to the field of modulated measurement capability, memory effect characterization and envelope injection linearization in power amplifiers.

To achieve a sufficiently broad baseband termination, significant modification of the baseband load-pull measurement system was required in order to accurately control the higher baseband components. The baseband active load-pull measurement system was 
thus modified to synthesise the necessary waveforms to allow a constant and specific baseband impedance environment to be maintained across a wide bandwidth. This was achieved in the time domain, using a single $80 \mathrm{MHz}$ arbitrary waveform generator (AWG).

* The role played by higher baseband impedances in determining baseband electrical memory effects observed in power transistors under two-carrier excitation was rigorously quantified. These effects typically appear not only as asymmetrical distortion terms in the frequency domain, but also more reliably as a recognizable hysteresis in the dynamic transfer characteristics extracted from measured input voltage and output current envelopes of a power device. The investigations were carried out thoroughly to identify the relationship between the IMD asymmetries caused by the baseband impedance variations and the hysteresis that appears in the dynamic transfer characteristics.

An additional feature of the modulated measurement system was identified and deemed usable in conjunction to improve the efficiency and linearity. In an envelope tracking (ET) architecture, the power amplifier supply voltage is dynamically adjusted to the most efficient level for the instantaneous output power level. This allows the power amplifier to operate near the maximum efficiency. The envelope

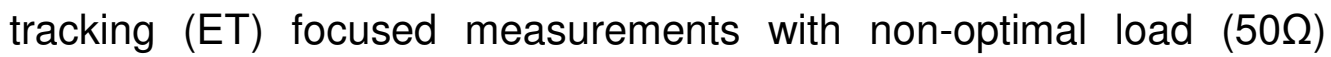
showed that efficiency as well as linearity can be improved at reduced drain supply voltages: for $\mathrm{V}_{\mathrm{dc}}=20 \mathrm{~V}$, the average drain efficiency was improved by approximately $5 \%$ together with an improvement of $10 \mathrm{dBc}$ in IM3 when compared to the static $V_{d c}$, where short circuit impedance was maintained for all four baseband components.

One noteworthy issue when employing the sampling-oscilloscope to average the multi-tone signals was an inability to 'freeze' the display of the sampling-oscilloscope. Consequently, software post-processing was mandatory to accurately time align the collected waveforms in 
order to allow averaging that is necessary to improve the overall quality of the measurements to an acceptable level. This slowed down the process of measurement due to the post measurement averaging and this technique was only applicable for two tone and three tone modulated measurements, as it was very difficult to align the vectoral information for more tones. Therefore, it was necessary to engineer an external clock to trigger measurements on the CSA 8000. The high quality trigger clock was successfully engineered thus allowing for onboard averaging of the oscilloscope.

\& New modulated waveform measurement software was developed in conjunction to improve the measurement speed as well as allowing the multi-tone measurements. The successive time windows were used to capture all of the relevant information and stitched together as the oscilloscope coherently sampled across the selected time window, when the end of the time window was reached the data was stored, processed and displayed. The number of time windows was computed based on the number of points which the oscilloscope can sample in one time window which means that consecutive time windows were required to capture one complete modulation cycle of waveforms in conjunction with the lower modulation frequencies $(\leq 1 \mathrm{MHz})$. Subsequently, this "Time Domain Partitioning" approach allowed measuring the broad bandwidth modulated signals very close to the noise floor with greater accuracy and enhanced the measurement speed in connection with the CW measurement in an example case when modulation frequencies are chosen such that total number of points to sample fit in one time window.

The RF architecture of the modulated waveform measurement system was modified to synthesize the RF loads. The Tektronix AWG7000 Arbitrary Waveform Generator was used as an RF synthesizer, and is used to synthesize both fundamental excitation and harmonic load-pull signals simultaneously, in the time domain. Using two independent yet coherent channels, this waveform measurement system is capable of 
maintaining independent and constant impedance control for each individual tone across the RF impedance environment, and over a wider modulation bandwidth. This modified architecture was then used to demonstrate the emulation of a modulated class-J power amplifier, through the application of modulated RF active load-pull.

The work described in this thesis has the potential to be used to characterise and reduce bandwidth dependent non-linear distortion in microwave devices under realistic stimulus relevant for future wireless communication systems. This is possible because it can now quantify, comprehensively, the out-ofband impedance on the performance of microwave devices and to emulate the specific PA modes under modulated excitations.

\subsection{Thesis Synopsis}

To accommodate the various aspects of enhanced modulated waveform system and its application to the device characterisation and measurements the thesis is sectioned into six chapters. Each chapter starts with the introduction and discusses the motivation and purpose of that particular chapter.

Chapter 2 gives an overview of the modulated domain measurement solutions used for device characterisation at microwave frequencies in order to address the contemporary problems in power amplifier design, most importantly memory effects. Most of the commonly known measurement solutions are discussed, with their pros and cons. The modulated measurement system developed at Cardiff University is then discussed in two sections; the first highlights the limitations of the previous measurement system in its load-pull capability and then introduces a refinement to state-ofart active IF and RF load-pull measurement system that allows the precise independent control of all significant baseband and RF components generated as a result of the multi-tone excitation used. The second section addresses the limitations of the previous measurement technique implemented for the device characterisation under modulated excitations and 
demonstrates the new measurement algorithms required to allow the capture of multi-tone information using a standard sampling oscilloscope. The final section of this chapter demonstrates the measurement system capabilities through the practical device measurements.

Chapter 3 explains an investigation into the relationship between the intermodulation distortion (IMD) asymmetries caused by the baseband impedance variations and the hysteresis or looping that sometimes appear in the dynamic transfer characteristics of microwave power devices when subjected to the modulated excitation using the enhanced modulated waveform measurement system. The role played by higher baseband impedances in determining baseband electrical memory effects observed in power transistors under two-carrier excitation is rigorously quantified through the application of an active IF load-pull to present specific baseband impedance environments, allowing the sensitivity of IMD symmetry to baseband impedance variations to be investigated. The baseband impedances are controlled over a significant bandwidth; in this case at least eight times the modulating frequency.

Chapter 4 reports an additional feature of the modulated waveform measurement system, which is emulating appropriate negative impedances lying outside of the Smith chart. When this feature is considered alongside the Envelope Tracking (ET) power amplifier (PA) architecture, this raises the interesting possibility of significantly improving PA linearity using the very mechanisms that are employed to improve PA efficiency. The architecture and detailed operation of linearity and efficiency enhancement are discussed. An investigation into the effect of variable supply voltage at the drain of the device under test (DUT) and the baseband impedance on the RF output performance, including output power, efficiency and linearity, is performed revealing an improvement in power efficiency and linearity when compared with using a fixed voltage power supply. Finally this chapter includes a comprehensive discussion on the evaluation and effectiveness of the envelope injection technique highlighting the advantages and short comings 
of this in applications, when applied to the realistic power amplifier architecture and modes.

Chapter 5 along with the fundamentals focuses on the multi-tone characterisation and linearization for the elimination of adjacent channel distortion products when the power device is subjected to multi-tone excitation. It describes the multi-tone characterization of baseband electrical memory effects and their reduction. It emphasizes on the envelope injection technique to reduce the non-linear distortion generated by the power device. The measurements performed evaluating the envelope injection technique, which is a promising candidate to achieve high linearity from the power device, realized through an external envelope signal that is simply generated by an arbitrary waveform generator (AWG), are discussed. The envelope signal contains all of the baseband frequency components generated by the power device and is then properly injected at the output of the power device where it actively modifies the impedance environment seen by the power device. The results highlight that this linearizing and efficiency enhancing technique could be extended to more complex multi-tone excitations.

Chapter 6 offers conclusions and the potential future directions for the developments of the modulated waveform measurement system and its application to further power device investigations. 


\subsection{References}

1. 3GPP Long Term Evolution specification, [Online]. Available: http://cp.literature.agilent.com/litweb/pdf/5989-8139EN.pdf

2. A.Toskala, H.H.a., "WCDMA for UMTS: Radio Access for Third generation Mobile Communications", West Sussex: john Wiley \& Sons, 2nd edition, 2002.

3. Towards Global Mobile Broadband, "Standardising the Future of Mobile Communications with LTE (Long Term Evolution)", white paper from UMTS Forum, Accessed in February 2011, www.umts-forum.org

4. P.B. Kenington, "High Linearity RF Amplifier Design", Norwood, MA: Artech House, 2000.

5. J. Vuolevi, J. Manninen, and T. Rahkonen, "Cancelling the memory effects in RF power amplifiers", in IEEE Int. Symp. Circuits and Systems, pp. 5760, 2001.

6. J. Vuolevi and Timo Rahkonen, "Distortion in RF Power Amplifiers", Norwood, MA: Artech House, 2003

7. F.H.Raab, P. Asbeck, S.C. Cripps, P.B. Kenington, Z. B. Popovic, N. Pothecary, J.F. Sevice, and N.O. Sokal "Power Amplifiers and Transmitters for RF and Microwave", IEEE Trans. on Microwave Theory and Tech, Vol.50, No.3, pp.814-826, 2002.

8. M. Feng, S. C. Shen, D. C. Caruth, and J. J. Huang, "Device Technologies for RF Front-End Circuits in Next Generation Wireless Communication", in Proc. of IEEE, Vol.92, No.2, pp.354-374, 2004.

9. A. Wright, "Multi-Carrier WCDMA Basestation Design Considerations Amplifier Linearization and Crest Factor Control", Technology White Paper, PMC-Sierra, Inc., Issue 1, 2002.

10. G. Fischer, "Architectural Benefits of Wide Bandgap RF Power Transistors for Frequency Agile Base Station", Proc. of wireless and Microwave Technology (WAMI) Conference, FD-1, 2004.

11.R. J. Trew, G. L. Bibro, W. Kuang, Y. Liu, and H Yin, "Microwave AlGaN/GaN HFETS", IEEE Microwave Magazine, Vol.6, No. 1, pp. 56-66, 2005.

12. Alghanim, A.; Lees, J.; Williams, T.; Benedikt, J.; Tasker, $\mathrm{P}$ "Using active IF load-pull to investigate electrical base-band induced memory effects in high-power LDMOS transistors", in Proc. Asia-Pacific Microwave Conference, 2007, 11-14 Dec. 2007 Page(s):1 - 4. 
13. Alghanim, A;Lees, J.; Williams, T.; Benedikt, J.; Tasker, P. "Reduction of electrical base-band memory effects in high-power LDMOS DevicesUsing Optimum Termination for IMD3 and IMD5 using active load-pull", in Proc., IEEE International Microwave Symposium, June 2008.

14.S. C. Cripps, "RF Power Amplifiers for Wireless Communications", Artech House Inc. Norwood, MA, 2006.

15. A. M. Saleh and D. C. Cox, "Improving the Power-Added Efficiency of FET Amplifers Operating with Varying Envelope Signals", IEEE Trans. On Microwave Theory and Tech., Vol. 31, No. 1, pp. 51-55, 1983. 


\section{Modulated Domain Measurement Systems}

\subsection{Introduction}

For wideband and higher power applications the dynamic behaviour of microwave devices, like memory effects can no longer be ignored. The first step to overcome this problem is to characterise the nonlinear microwave devices dynamically. Traditionally, high-frequency measurement systems employed continuous wave (CW) signals for the investigations of device characteristics. However, device characterisation at CW frequencies does not allow for the measurement and investigation of important device behaviour such as memory effects [1]. Therefore, a more dynamic measurement system capable of characterising a power device or a power amplifier is needed to fulfil the requirements of the $3 \mathrm{G}$ Universal Mobile Telecommunication System (UMTS) and 4G Long Term Evolution (LTE) spectral mask. The measurement system should also support multi-tone modulated signals in order to study the memory effects behaviour of the device under test (DUT) as nonlinear behaviour depends on the properties of the input signal. The introduction of two tone test signal revolutionized the perception of nonlinearity, setting up stringent rules on the measurement system requirements. A basic two tone test setup uses two discrete tones of equal power that fall within the passband of the DUT, which are applied as an excitation signal. The resulting baseband information and IMD components are then measured and cancelled [2]. This 
characterisation of power device is required to optimize the technology of the active device to eliminate or reduce the memory effects. It's a prerequisite in order to design a quasi memoryless broadband power amplifier, based on the robust characterisation of microwave device. Different design strategies for bias networks and matching networks can be taken into account in an accurate power amplifier design for future wireless communication systems.

This chapter reviews extensively the measurement techniques employed earlier to measure and quantify the memory effects with their disadvantages, advantages and limitations. This, then, makes a strong case for the development of high speed and robust modulated measurement system for memory effect analysis as well as envelope linearization of active power device and power amplifier. In this chapter the main focus will be on the newly developed oscilloscope based dynamic modulated waveform measurement system for active power device and power amplifier characterisation under modulated excitation.

\subsection{Memory Effects in Power Amplifiers}

Memory effects are recognised as a major obstacle in the design of highly linear power amplifiers for current and future wireless communication systems [1]. 'Memory' in this context is evident from variation in the magnitude and symmetry of intermodulation distortion (IMD) levels, as a function of signal bandwidth. These problematic effects can and do lead to significant difficulties and complexities in meeting the required power amplifier (PA) linearity requirements with standard linearization techniques often proving to be inadequate [1,2]. There are mainly two types of memory effects: electrical and thermal.

Electrical memory effects are generally caused by varying impedance across baseband (often significantly) as well as RF modulation bandwidths. In classical PA design, the dependency of IMD on the envelope frequency is minimized by engineering baseband impedances to be as small as possible, for example less than $1 \Omega$ over a baseband frequency range significantly larger than the operational bandwidth [3]. In reality, this is difficult to achieve over increasingly large baseband impedance ranges, and the result can be drain 
bias modulation effects that contribute significantly to the PAs overall spectral distortion with increased magnitude and asymmetry in measured IMD [4]. Nevertheless, smooth memory effects are not usually harmful to the linearity of the PA itself, a phase rotation of $10^{\circ}$ to $20^{\circ}$ or an amplitude change of less than $0.5 \mathrm{~dB}$, as a function of modulation frequency, has no dramatic effect on the linearity of the device $[1,2]$.

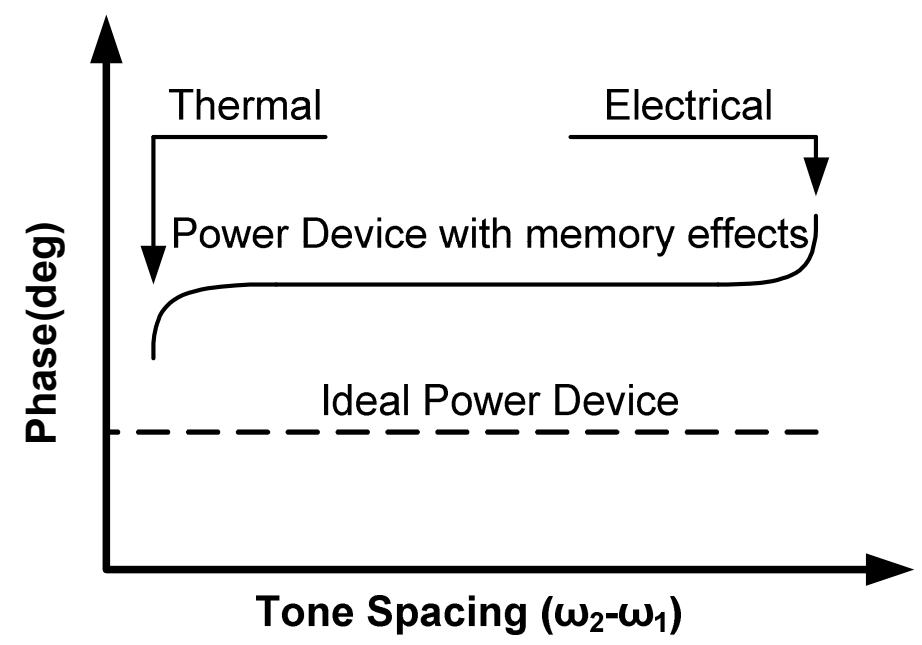

Figure 2.1: A representation of memory effects as a function of tone spacing.

Thermal memory effects are generated by the junction temperature, which is modulated by the applied signal. Thermal effects will be much more prominent in a slow sweep, or a stepped continuous wave test.

\subsubsection{Identification of Memory Effects}

A power device would be said to exhibit magnitude memory effect, if a tone spacing frequency sweep results in changes to the amplitude level of the inter-modulation distortion (IMD) as shown in Figure 2.2 [4]-[6]. These effects appear as a asymmetry between the signals at the lower and upper frequency components in the output spectrum. Generally these effects manifest themselves in the following observations;

- The upper and lower IMDs are a function of baseband frequency

- Lower and upper IMDs behave asymmetrically

- Lower and upper IMDs versus tone spacing vary in opposite direction with respect to the memory less case 
- Occurrence of some resonance effects in the lower and upper IMD Another way of describing memory effects is a time lag, defined as an existence of a hysteresis, between AM-AM and AM-PM response which creates uncertainty within the predictive model.

Fundamental

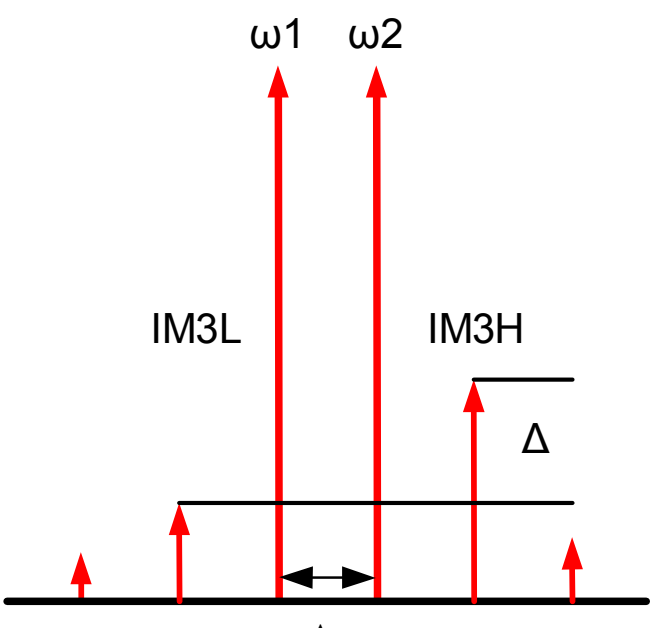

$\Delta \omega$

Figure 2.2: Memory effects definition and identification.

In other words, for a nonlinear device with memory, the output signal of the device is not going to be only a function of its present input, but also a function of previous inputs. Nevertheless, AM-AM and AM-PM are seldom used as precise characterisation technique for modelling devices with memory effect due to their inability to give comprehensive information on the device nonlinearity, such as in monitoring asymmetry in the IMD [6].

\subsubsection{Sources of Memory Effects}

Memory has a number of different origins, so are difficult to anticipate. The main sources of electrical memory effects are given by the bias network impedance variations. The self heating in the active device is the main source of thermal memory effects. Practically, there are many other additional sources, which contribute to the memory effect problem such as dispersion and trapping effects in a power device. All these effects are intensively analyzed in the literature for the different transistor technologies, indicating that at low frequency from $10 \mathrm{KHz}$ to several $\mathrm{MHz}$ the device internal components show dispersion and thermal effects which are low frequency 
dependent phenomena [6]-[9]. The most common sources of memory effect can be summarised as;

- Device reactive components

- RF Input and output matching network

- Biasing network

- Coupling and de-coupling capacitor

- Thermal and trapping memory effect

Figure 2.3 depicts the typical sources of memory in power device. The existence of all these effects result in the power device output signal to not only depends on the instantaneous input signal but also on previous output signal.

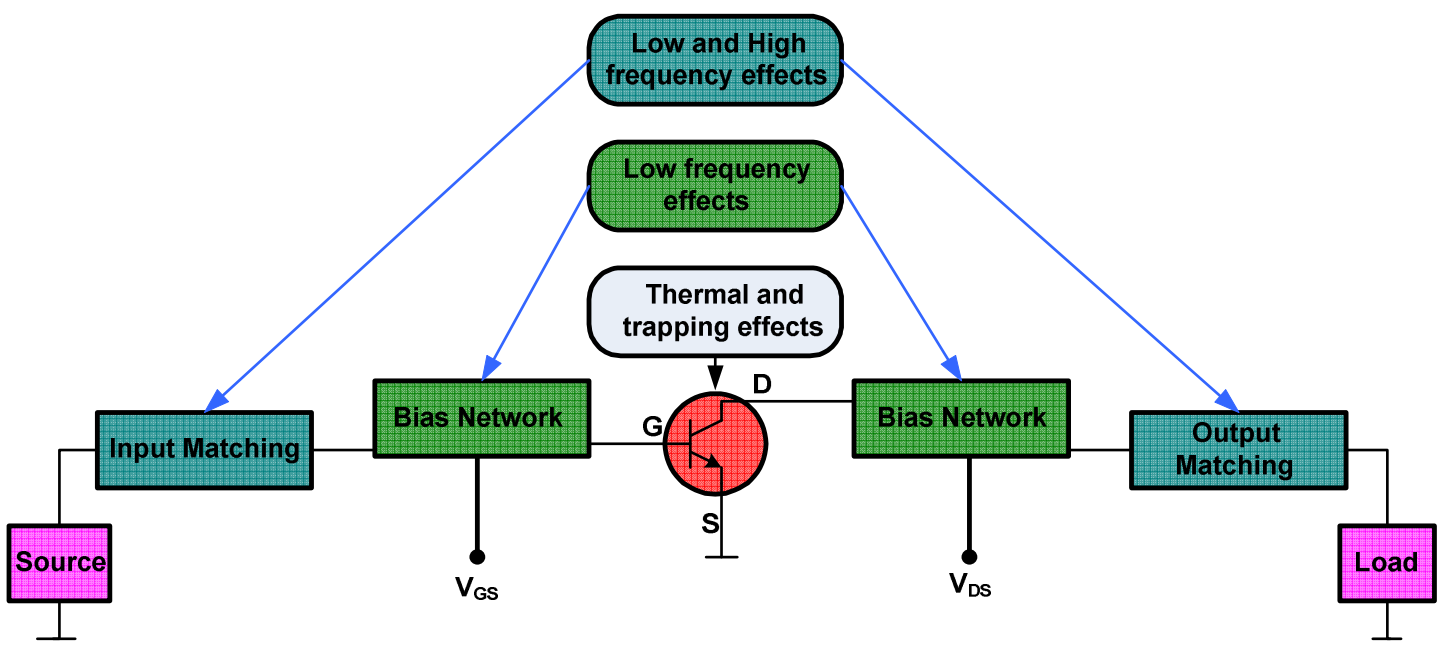

Figure 2.3: The location of memory effect in power amplifier [6].

\subsubsection{Types of Memory Effects}

All power amplifiers and power devices contain capacitive and inductive networks. The proportion of memory effects in the power amplifier are dependent on several dynamics, for instance power channelling capability, used transistor technology, stimulus signal statistics, envelope frequency bandwidth, design quality of bias networks and matching networks. Memory effects based on these dynamics are subdivided into two groups;

- Short Term Memory Effects

- Long Term Memory Effects 


\subsubsection{Short Term Memory Effects}

Short term memory effects are the easiest to deal with as they affect the RF signal. Short-term memory effects are those signals which have a higher frequency (shorter time scale) than the information signal in a range close to the carrier signal. This kind of electrical memory effect is mainly caused by both the reactive components of the device in addition to input and output matching networks [10],[11]. It is caused by the variation of either or both the fundamental and the harmonics impedances at different frequencies. However, designing a matching network with approximately constant impedance for the RF fundamental signal, in addition to its harmonic frequencies may not be considered a difficult task; therefore, by adjusting the matching network they can be minimized. Power amplifiers that have shortterm memory effects will usually have a static response for the information signal and may sometimes be treated as memory-less devices, since shortterm memory effect have a time constant much shorter than the time constant scale of the information signal [11].

\subsubsection{Long Term Memory Effects}

Long term memory effects, also called envelope memory, are the most disconcerting sources for the linearizer systems and are typically due to the biasing network. They appear as a long time delay in the impulse response of the system. A bias network needs to be constant and ideally of zero impedance over the envelope frequency range. Otherwise, $A C$ voltages may be generated and added to the power supply voltage, causing amplitude and/or phase modulation, and resulting in asymmetry in the IMD [12]. It is almost impossible to achieve a constant bias network impedance for many frequency decades. This highlights the shortcomings of the simple bias network and the complexity of bias network design [6].

Long term memory effects are responsible of various system impairments which increase the bit error rate (BER). The envelope memory effects can be categorized in two groups:

- Thermal and Trapping Memory Effects

- Electrical Memory Effects 


\subsection{Thermal and Trapping Memory Effects}

Thermal effects and trapping are inherent to the active device itself. They are caused by electrothermal couplings due to self heating, and these affect low modulation frequencies up to hundreds of $\mathrm{KHz}$, usually up to $1 \mathrm{MHz}$ [13]. In fact, any temperature variations caused by the dissipated power in the device are determined by thermal impedance which describes the heat flow from the device.

Due to the finite mass of the active device, thermal impedance is not purely resistive; instead it forms a distributed low-pass filter with a wide range of time constants. This means that the temperature variation caused by the dissipated power do not occur instantaneously, rather baseband frequencydependent phase shifts always exists. If any of the electrical parameters of the transistor are affected by temperature, thermal memory effects are unavoidable. Let us consider the dissipated power in a field effect transistor (FET) is given by the equation (2.1) [14].

$$
P_{D I S S}=V_{D S} \bullet I_{D S}
$$

Where $V_{D S}$ and $I_{D S}$ are the drain-source voltage and drain-source current respectively. The heat flow from the chip, packaging and the heat sink to the surrounding environment is depicted in figure 2.4.

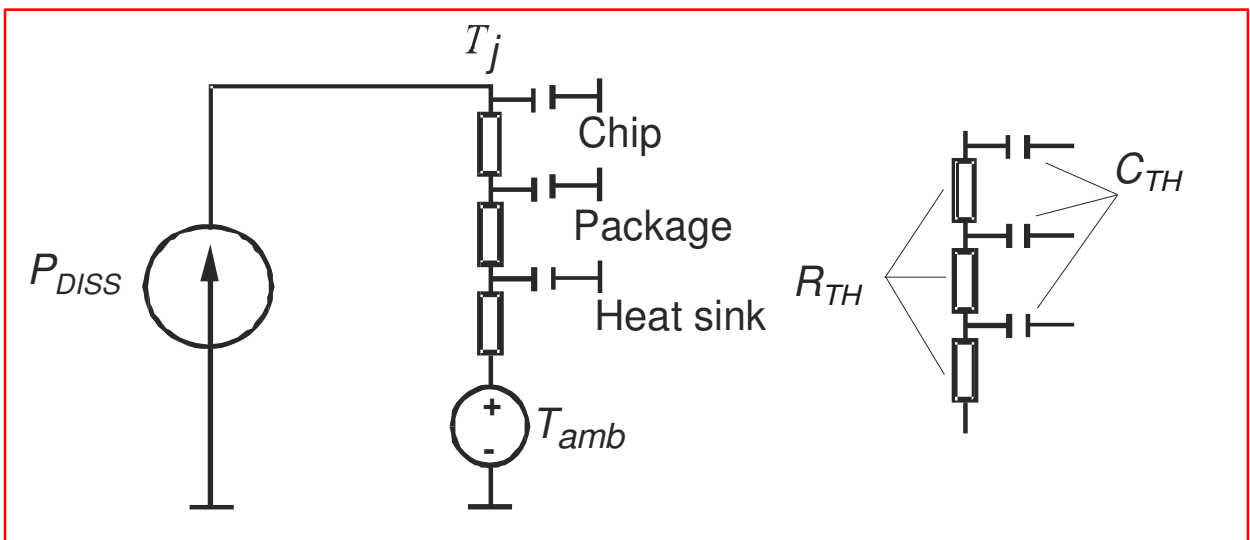

Figure 2.4: Electrical circuit model of the heat flow from power device

For a FET on silicon substrate, the silicon surface reacts to the dissipated power quickly. This results in chip temperature fluctuation up to several degrees over an input signal bandwidth of several hundreds of $\mathrm{kHz}$. Therefore, the chip temperature is low frequency dependent and it can be 
expressed by equation (2.2). Where $T_{a m b}$ is ambient temperature, $R_{T H}$ is thermal resistance resulting in the average temperature rise caused by self heating and $Z_{T H}$ is thermal impedance of the chip at envelope frequency. The $\mathrm{Z}_{\mathrm{TH}}$ is the main cause of the thermal memory effects in the active devices.

$$
T_{j}=T_{\text {amb }}+R_{T H} \bullet P_{D I S S}(d c)+Z_{T H}\left(\omega_{2}-\omega_{1}\right) \bullet P_{D I S S}\left(\omega_{2}-\omega_{1}\right)
$$

Some of the transistor parameters, such as drain-source current, output conductance and capacitance are temperature dependent; these dynamics of self-heating cause the distortions [14]. Theoretically all the power transistors exhibit thermal memory effects. Although, the dissipated power at high frequencies $(>1 \mathrm{MHz})$ is too fast to influence the instantaneous temperature of the silicon chip and the packaging, the thermal memory effects can be neglected in low power amplifiers [14,15]. But this is not the case for the power amplifiers that are used in the base stations for mobile communications.

\subsection{Electrical Memory Effects}

The bias networks are required to provide the supply voltage and current to an active device and keep them constant whilst isolating the RF signal from the bias supply and avoiding active device instability [16]. In traditional bias network design, the bias circuit is usually designed to provide relatively high impedance at $R F$ frequencies compared to the impedances of the input and output of the device and the matching circuits.

In the case of the input signal with non-constant envelope, the envelope frequency variations cause variation in the impedance of the bias network which causes memory effects as a function of envelope frequency [17]. The work presented in [18] demonstrated that the electrical memory effects can be changed by varying the values of the energy storing elements such as capacitances and inductances in the biasing networks of a power amplifier. This results in an improved quasi-memoryless power amplifier where the envelope frequency dependence of distortion has been reduced. The locations of these elements in a typical bias network used for bipolar and FET amplifiers are shown in figure 2.5. In an ideal case, to avoid envelope frequency dependent bias network, the impedance should be a short circuit 
at envelope frequencies and an open circuit at RF frequencies to isolate the RF signal from the bias supply [6],[15],[19]. Practically, it is difficult to realize such an impedance, which has two requirements that are conflicting with each other [19],[20].

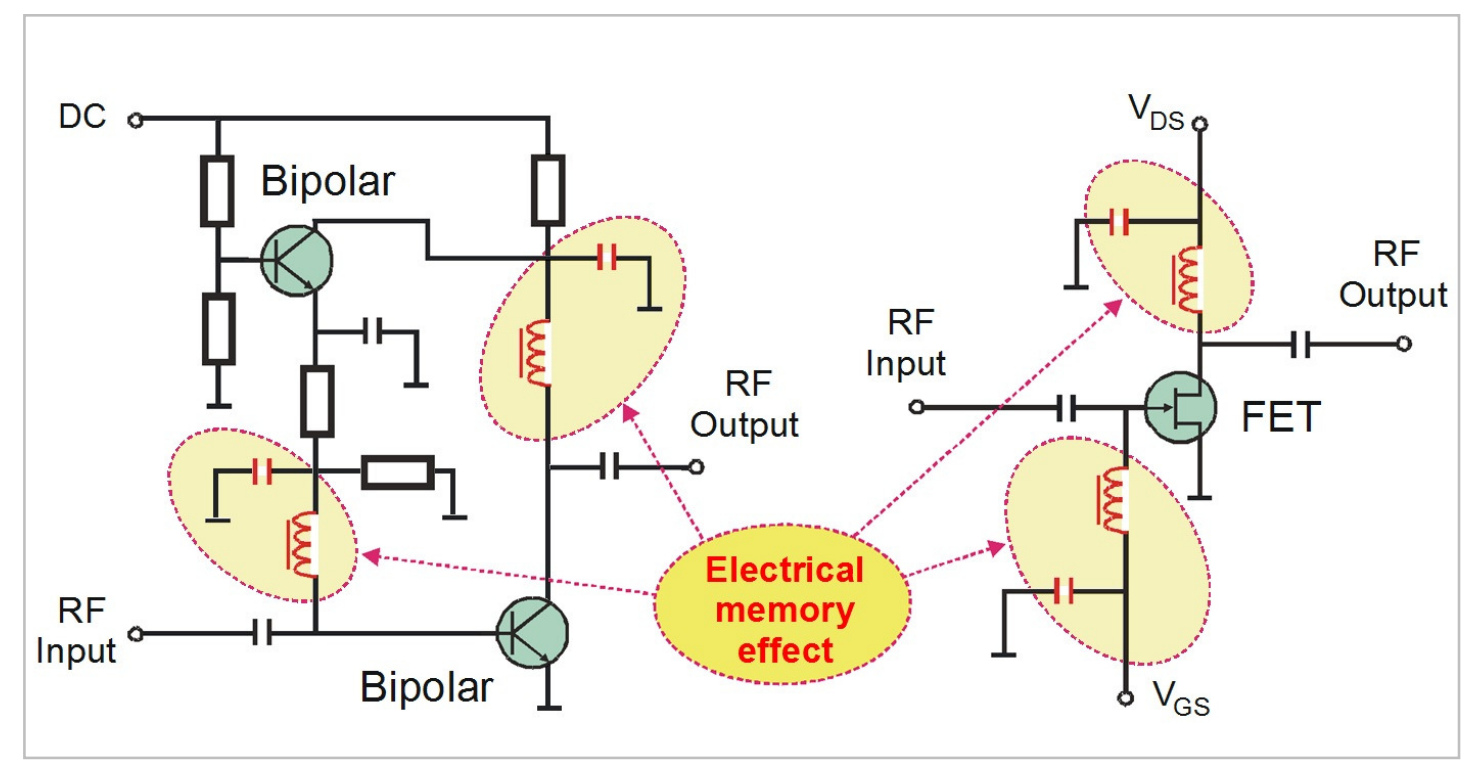

Figure 2.5: Memory effects in the bias networks of bipolar and FET power amplifiers [18].

Therefore, it is very difficult to realize short circuit impedance at broad baseband frequency bandwidth such as that required in broadband applications for multi-carrier operation.

\subsection{Modulated Domain Measurements Systems}

Electrical, thermal and trapping effects in the active power devices are of great concern in current and future wireless communication systems especially in wideband applications with advanced digital modulation formats. All of these effects are memory specific phenomena, which mean that, the output signal of the active device is not only a function of the instantaneous input signal but also a function of the previous input values.

In the last few years, these effects were intensively analysed in the literature and were called memory effects [21]-[24]. The baseband memory effects depend on baseband frequency ranging from $10 \mathrm{KHz}$ to several $\mathrm{MHz}$ and can be observed as amplitude variations up to $1 \mathrm{~dB}$ in the output signal at the 
fundamental frequency [14]. Generally, to measure and analyze memory effects in active devices as well as power amplifiers, the modulated measurement system should have the following capabilities [1],[18],[25],[26]:

- Envelope and radio frequency (RF) measurements to measure the signal in both frequency bands.

- Vector measurements to measure both amplitude and phase of these signals.

- High dynamic range to measure the higher order intermodulation products.

- Wide dynamic bandwidth to measure the broadband signals at the fundamental and at intermodulation frequencies.

- High power and frequency measurement accuracy to measure small amplitude variations $(<0.5 \mathrm{~dB})$.

A pioneering measurement setup to measure memory effects of power amplifiers is shown in Figure 2.6[18]. In this measurement setup, two signal generators are used to generate a two tone signal, which is synchronized by an external reference oscillator. The two tone input signal is swept for different power level and frequency spacings.

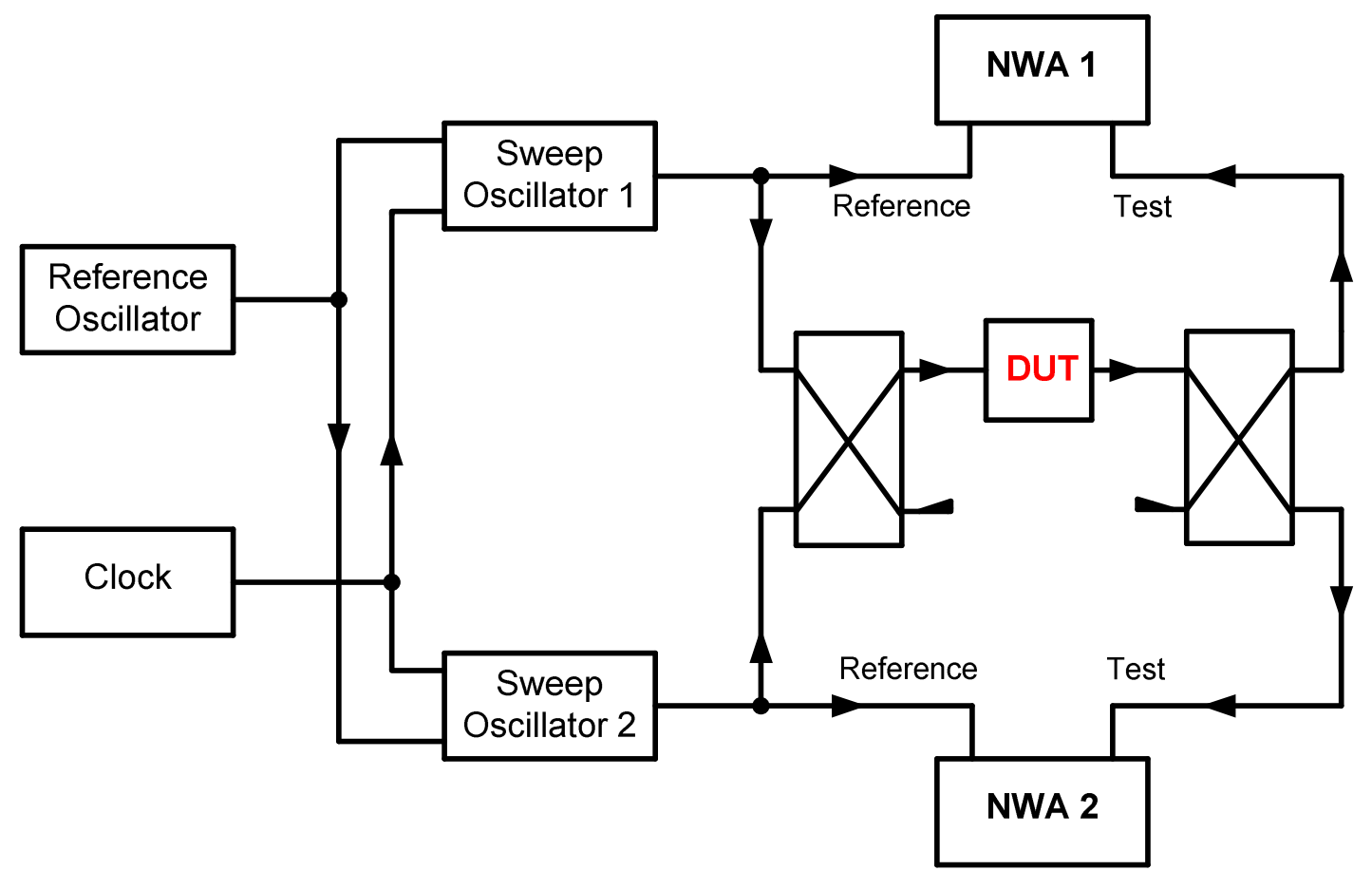

Figure 2.6: Measurement set-up for two tone dynamic power sweep measurements [18]. 
The complex input and output signals of the PA are measured using two separate network analyzers, which certainly increased the cost. Also a phase synchronized broadband two tone signal was difficult to generate due to the synchronization issues of two $\mathrm{CW}$ signal generators. The measurement setup presented in [26] was more suitable for the memory effects analysis in the power amplifiers. In this measurement setup, shown in Figure 2.7, the output signal of the power amplifier is demodulated to an intermediate frequency (IF) and the reference phase between the output signal and reference signal is measured.

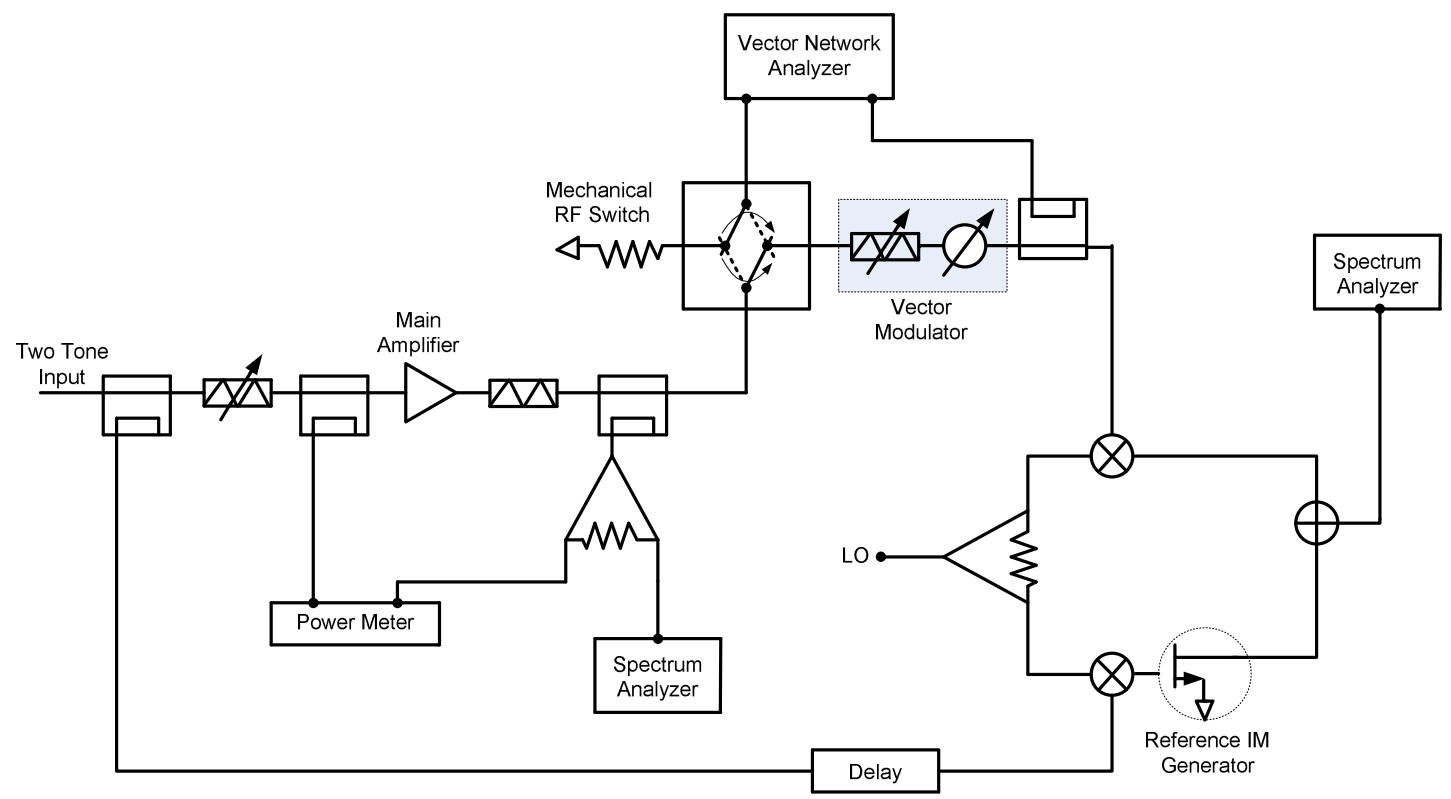

Figure 2.7: Measurement set-up for two tone transfer characteristics measurement [26].

The reference signal used as a nonlinearity reference is obtained through an external amplifier. In order to surmount the memory effects in the nonlinear reference component, it should be operated at low power and low frequency. The measurement setup requires many measurement instruments and long measurement time due to the time consumed by manually operating the amplitude and phase shifters during the sweeps. Another measurement setup was proposed in [1] to measure the memory effects. The key feature of this measurement setup is that the signal at the third order intermodulation distortion products (IMD) is applied together with a two tone input signal in 
order to cancel the third order IMD in the output spectrum of the power amplifier. The memory effects were measured by sweeping the input power and modulation frequency of two tone signal. This measurement does have many disadvantages such as it does not measure the IMD components at the output spectrum of the power amplifier and requires two signal generators as well as two spectrum analyzers. It also requires four signal generators to measure the memory effects at lower and upper third order intermodulation distortion products simultaneously.

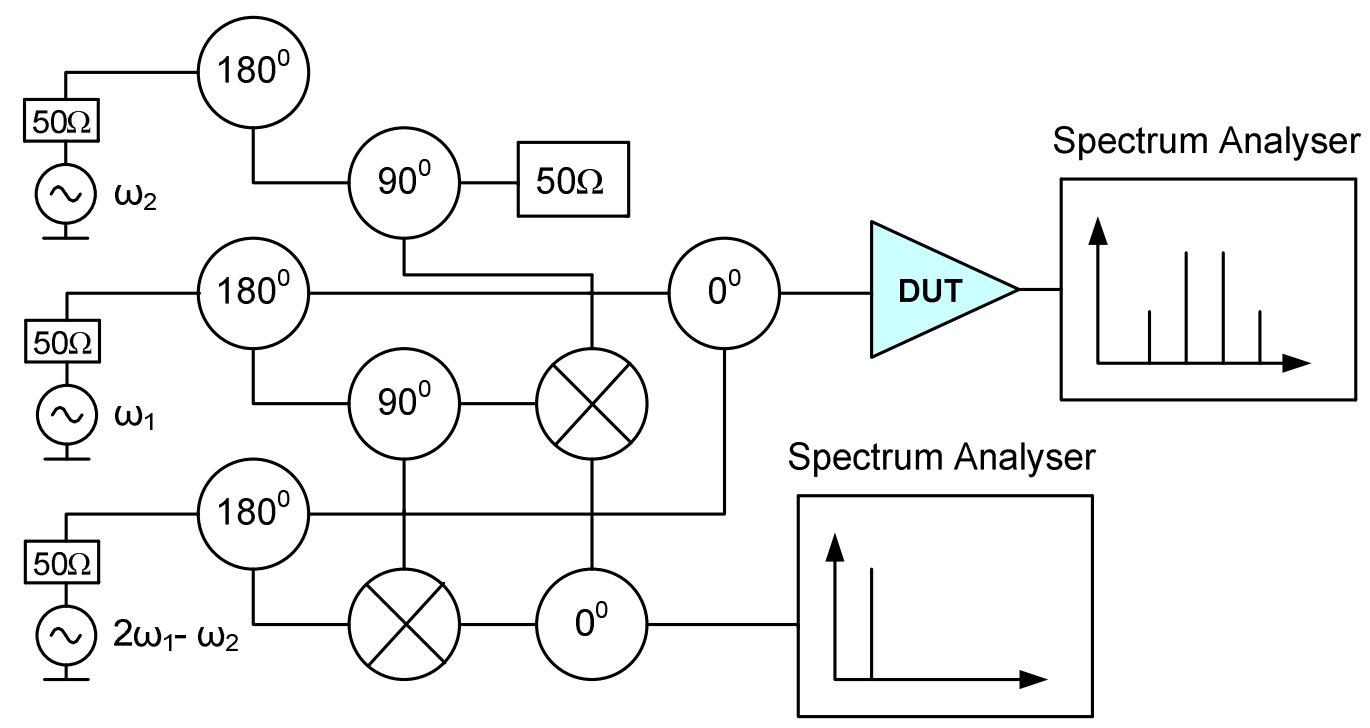

Figure 2.8: System for measuring memory effects proposed in [1], under two tone excitation using cancelling technique.

To measure the memory effects in the envelope time domain another method is detailed in [16] which is similar to the earlier discussed setup. In this measurement setup the power amplifier input and output signals after coupling are applied to the digital scope through amplitude and phase detectors, which enabled it to measure the complex data. All measurement setups discussed so far do not measure the output signal at baseband and RF harmonic frequencies.

The measurement system depicted in Figure 2.9 allows the characterization of memory effects in $50 \Omega$ environment. The RF signal from the multi-tone generator is first pre-amplified, using a linear power amplifier, and then passed through an isolator, bandpass filter and coupler to the input of the device under test (DUT). The isolator and bandpass filter are necessary to 
isolate the DUT from the signal generator to avoid impedance mismatching between the DUT and pre-amplifier. The RF input and output signals of the DUT after coupling and attenuation are measured using the vector signal analyzer (VSA). This system does not allow the engineering of out-of-band impedances but only allows the measurement of memory effects by passively terminating the baseband components to short circuit and RF impedances to $50 \Omega$.

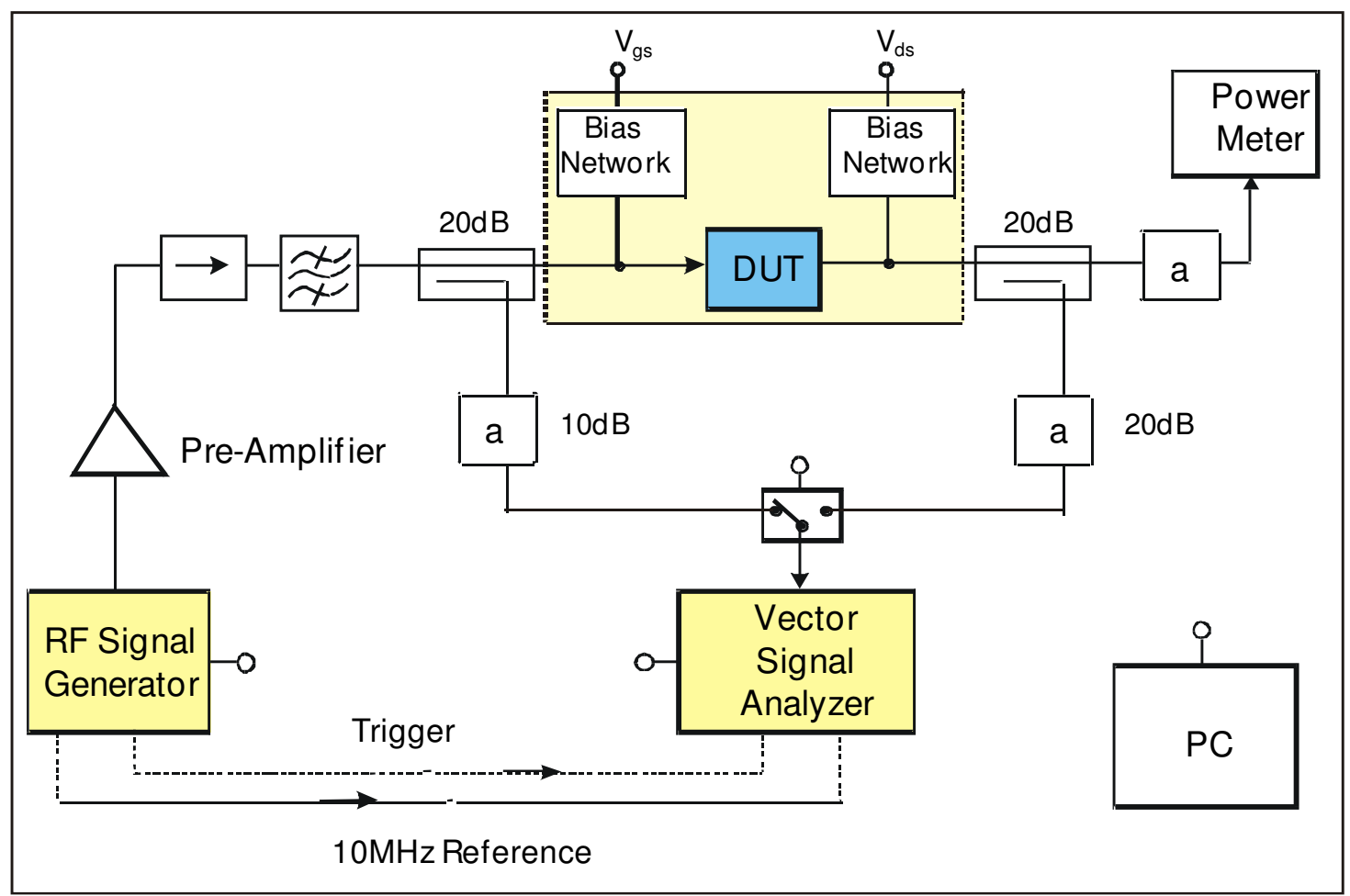

Figure 2.9: Schematic of the dynamic measurement setup for the analysis of memory effects [25].

The measurement system demonstrated in [27] allows the on wafer out-ofband device linearity optimization. It facilitates the independent control of the baseband, fundamental, and second-harmonic impedances at the device terminals, through a multiplexer board. This board decouples the frequency components of interest through the use of a multistub network, which employs $\lambda / 4$ lines at fundamental and $2^{\text {nd }}$ harmonic to obtain high isolation between the fundamental and second-harmonic signal path $(>30 \mathrm{~dB})$. It is important to mention that this network also presents low-reflection coefficients at the DUT 
for the frequency spectrum outside the controlled frequency bands at baseband, fundamental and second-harmonic impedances. The multiplexer board also facilitates the use of an external electronically controlled load or a modulated baseband signal. The baseband signals are sensed over the reference resistor. The value of this resistor can be set to 1,5 , and $50 \Omega$ to maximize the measurement accuracy of the baseband impedance. When the lowest baseband impedance is required, this reference resistor can also be short-circuited by a switch. Since the baseband impedance is controlled via the resistive switch bank, the resulting baseband impedances seen by the higher baseband components will be different. This makes it less effective for higher modulation frequencies $(>1 \mathrm{MHz})$ due to the dispersion in the baseband impedance presented to the baseband components. The precise control of baseband components, to quantify the baseband electrical memory effects, for at least a bandwidth of eight times that of the modulation frequency is required. This measurement system does not allow the characterization of high power microwave devices mounted on a fixture [27].

The modulated waveform measurement system reported in [20] is depicted in Figure 2.10 which combines two systems, a high frequency RF system and a low frequency baseband (IF) system. The high frequency measurement system is based on the HP-MTA (Microwave Transition Analyser), a two channel sampling scope, and the low frequency system which is identical in architecture and operation to the high frequency system, and is required to measure the IF1 $\left(\omega_{2}-\omega_{1}\right)$ component and its harmonics. A low frequency oscilloscope is used in this case to measure the incident and reflected voltage waves between $200 \mathrm{kHz}$ and $100 \mathrm{MHz}$. Unfortunately, this developed measurement system can only handle power levels of around $2 \mathrm{~W}$ thus making it unsuitable for characterising high power devices.

The waveform measurement systems based around real time digital oscilloscope that samples at $2 \mathrm{Ms} / \mathrm{s}$ was presented in [28]. An Arbitrary Waveform Generator (AWG) is used to generate modulated base-band signals, which are then fed into an I-Q modulator to produce the stimulus. Mixers are employed before the samplers to down-convert the complex time varying envelope of the fundamental carriers, both incident and reflected, at the input and output of the device. Significantly, the harmonics are filtered and 
are not taken into account, so the system does not capture all of the relevant information. The base-band information is also neglected, limiting the usefulness of the captured data in characterization of baseband memory effects in a power device as well as in power amplifier.

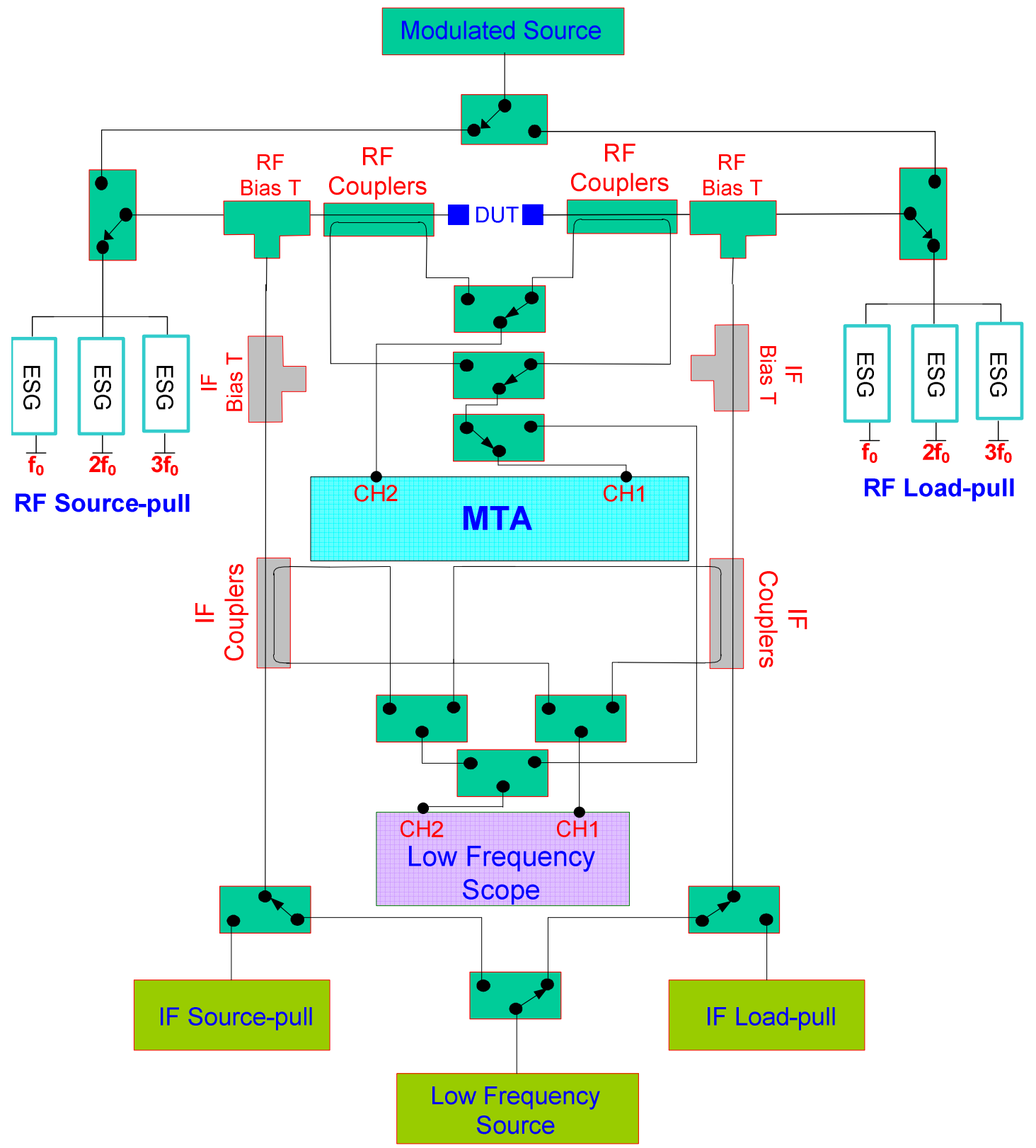

Figure 2.10: Two-tone waveform measurement system based around MTA [20].

\subsubsection{Industrial Measurement Solutions}

The industrially available high-frequency measurement solutions employ CW signals for the investigations of device characteristics. However, 
device characterisation at $\mathrm{CW}$ frequencies does not allow for the important measurement and investigation of devices under modulated excitation [1]. Unlike low frequency measurement it is rather difficult to measure power using voltage and current directly by using conventional voltmeter or ammeter at RF frequencies [6], [29], because they will interact and influence the accuracy of power measurements at RF and microwave frequencies. At low frequency the voltage can be directly measured using a high impedance probe and the current (through a node) measured using a series low impedance probe. This allows the admittance and impedance parameters to be computed. In terms of high frequency measurements, high and low impedances can only be maintained across a narrow bandwidth and could result in conditionally stable circuits becoming unstable within a test environment. Therefore, it emphasises the fact that the power, unlike current or voltage, is one of the few fundamental quantities that can be directly measured [30].

Due to the revolution in the wireless communications industry, microwave and $\mathrm{RF}$ frequencies usage is expanding; thus, developers of RF test equipments and measurement systems are driven to improve the performance of their measurement systems.

\subsubsection{The Spectrum Analyzer}

Spectrum analyzers are primarily a frequency domain measurement tool. They are considered to be a very powerful instrument for measuring signals with frequencies ranging from approximately $100 \mathrm{KHz}$ to approximately $110 \mathrm{GHz}$. The Spectrum Analyzer is a scalar instrument which measures a broad frequency spectrum in real time, whilst achieving very high dynamic range.

This allows the measurement of the magnitudes of the fundamental, harmonic and the intermodulation components in cases where modulated signals are used. However, the main limitation of such instruments is the inability to measure the phase information, thus limiting their suitability for use in device modelling applications. Despite this, the real time broadband behaviour of the instrument provides the capability for detecting and measuring spurious 
signals, which result from device instabilities and oscillations. This can be achieved without calibration or prior knowledge of the circuit behaviour or operation, making it an ideal instrument for evaluating the circuit and device performance.

\subsubsection{The Vector Network Analyzer}

Vector network analyzers are designed to measure both the magnitude and phase ratio of the voltage travelling waves. Their basic capability is to measure the s-parameters of an RF or microwave device and convert them to impedance and admittance using conversion matrices. Despite the valuable information gained from the s-parameter data, the measurement technique can only be applied when the superposition principle holds true [31]. This principle prevents energy being transferred from the stimulus frequency to other harmonic frequencies. This produces limitations when dealing with nonlinear networks containing active devices operating at high power levels, where the superposition principle cannot be applied. Non-linear behaviour produces additional harmonic frequency and intermodulation components. Thus, it would also be impossible to measure the non-linear behaviour of the microwave device.

\subsubsection{The Vector Signal Analyzer}

The vector signal analyzers employ digital signal processor to provide additional measurement capabilities and allow presenting the measured data in different forms compared to other comparable measurement systems. The vector signal analyzers can perform the same measurements as the spectrum analyzer while also providing the phase information of the signal. Their bandwidth is $40 \mathrm{MHz}$ which is not sufficient for full non-linear characterization of microwave power device under modulated excitation [32].

For instance, a device under two tone modulated excitation with a tonespacing of $10 \mathrm{MHz}$ will generate in-band and out-of-band distortion products. In order to study the performance of the in-band distortion up to only $5^{\text {th }}$ order will require a minimum bandwidth of $100 \mathrm{MHz}$, thus, making its application severely limited to power device characterization under modulated excitations. 
Although an extremely valuable instrument, it does not completely describe the full operating state of the device as it does not capture the out-of-band information that exists at both the base-band (IF) and around the harmonics of the carrier frequency $(R F)$.

\subsubsection{Large Signal Network Analyzers}

Large signal network analyzers are similar to vector network analyzer. They have a wide bandwidth. For example, Maury Microwave's MT4463A Large-Signal Network Analyzer operates from $600 \mathrm{MHz}$ to $20 \mathrm{GHz}$ with maximum power into test ports of $40 \mathrm{dBm}$ [33].

Further, they are capable of measuring the absolute values of the incident and the reflected travelling waves, which can be easily transformed to voltages and currents in the time-domain using an inverse Fourier transform. Even though they have large bandwidth their lower frequency is $600 \mathrm{MHz}$, which is relatively high. Therefore, they will not be suitable, for instance, to investigate low frequency memory effect of non-linear RF devices.

\subsection{Previous Cardiff Modulated Measurement System}

To bridge the gap between all of these commercially available solutions, the high power modulated measurement system developed at Cardiff University has been focused towards building a novel, fully functional modulated waveform time-domain measurement system. The basic RF architecture is an extension of the measurement system demonstrated in [34] and this measurement system is sometimes referred as Non-Linear Vector Network Analyzer (NLVNA), consisting of two directional couplers used to measure the incident and reflected waveforms with a bandwidth of $1 \mathrm{GHz}$ $12 \mathrm{GHz}$ and two bias tees of the same bandwidth and a maximum current and voltage handling of $10 \mathrm{~A}$ and $100 \mathrm{~V}$ respectively. The RF couplers and bias tees are capable of handling up to $100 \mathrm{~W}$ of $\mathrm{CW}$ RF power. The two triplexers are used to separate the fundamental, second, and third harmonic components to three ports, so that each frequency can be independently loadpulled using three ESGs to generate the desired terminating impedances (referred to as waveform engineering). The $\mathrm{CW}$ high power measurement 
system was further developed to measure the low frequency (IF) components and therefore study the effect of both IF and RF signals on device behaviour such as base-band memory effects. The RF test-set components have not been modified but specialised low frequency IF test-set components have been sourced to develop a complete modulated waveform measurement system.

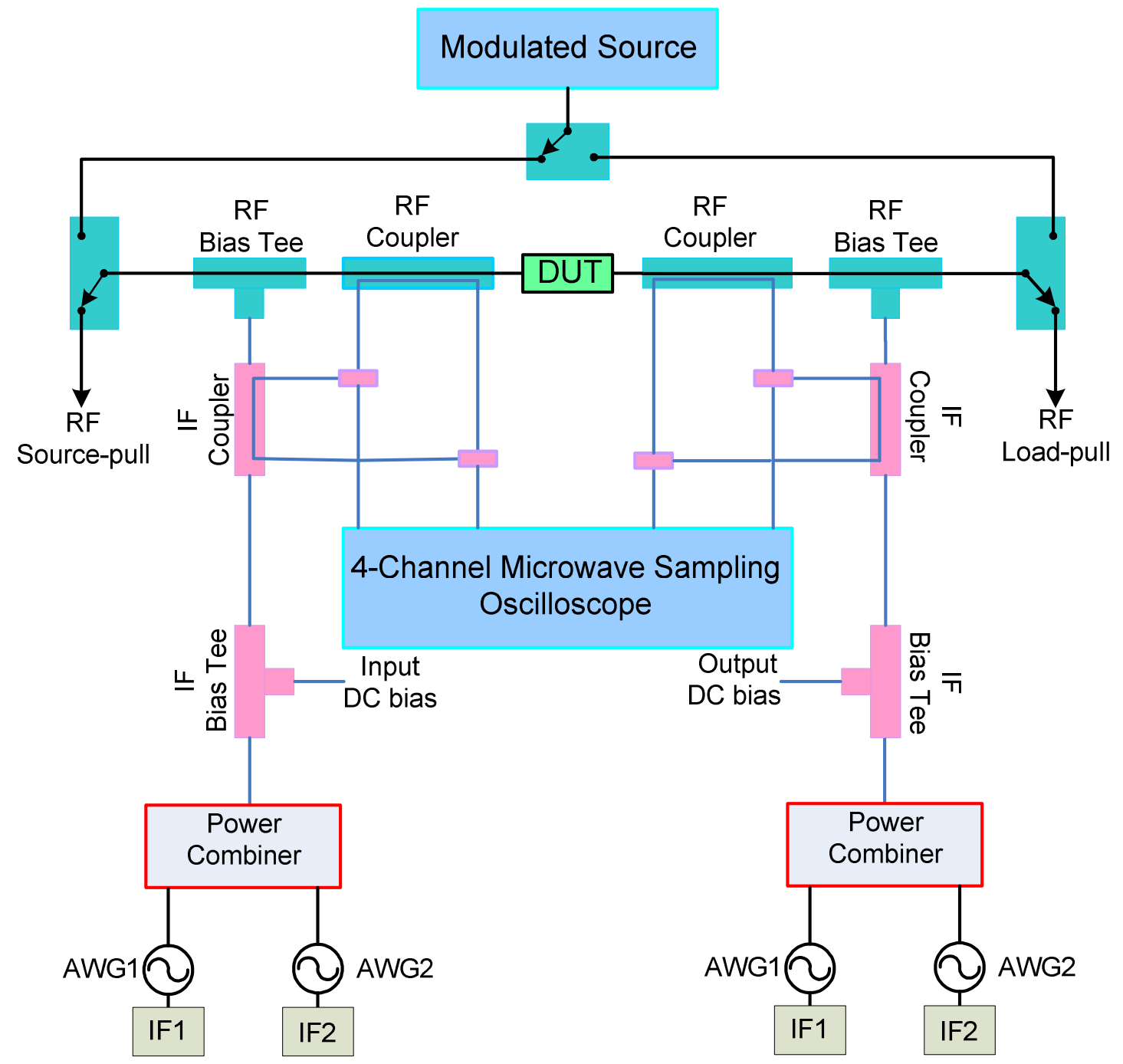

Figure 2.11: Modulated waveform measurement system based around oscilloscope [6].

The RF and IF signal components being combined using a diplexer prior to measurement is a key feature, and ensures phase coherence between measured IF and RF components. The four channel Tektronix sampling oscilloscope is used to measure the incident waveforms and the reflected 
waveform at the device input and output ports, which are vector error corrected before being converted to voltages and currents using the following fundamental equations.

$$
\begin{aligned}
& V_{n}=\sqrt{Z_{0}}\left(a_{n}+b_{n}\right) \\
& I_{n}=\frac{a_{n}-b_{n}}{\sqrt{Z_{0}}}
\end{aligned}
$$

Where $a_{n}, b_{n}$ are the travelling waves and $Z_{0}$ is the characteristic impedance of the system. The principle of operation and architecture of the IF test-set is an exact replica of RF test-set. The key feature of this modulated waveform measurement system is its ability to investigate the device performance in the time domain. This provides the full insight into the non-linear behaviour of the device under modulated two tone excitation. The resulting measurement system has the capability to handle RF power of more than $100 \mathrm{~W}$ and IF power of more than 50W along with current and voltage of more than $10 \mathrm{~A}$ and $100 \mathrm{~V}$ respectively. This measurement system can characterise devices for a bandwidth ranging from approximately $50 \mathrm{kHz}$ to approximately $12 \mathrm{GHz}$ and is depicted in Figure 2.11. This measurement system is, therefore, suitable for investigating high and low frequency memory effects [6].

\subsubsection{Previous Modulated Measurement System Limitations}

The previous modulated waveform measurement system is capable of characterising the high power devices in $50 \Omega$ RF environment under two-tone modulated excitation due to post averaging of successive modulated measurements. The "on scope" averaging was not possible due to the inability of the oscilloscope to freeze the time window for compressed sampled signals. Thus making the measurement system inapt for high power device characterisation under realistic communication signals and was considered to be a major obstacle in meeting the demands of the most modern communication systems, such as the base-station power amplifier market. Likewise, the existing low power IF test-set architecture is suitable for baseband (IF) load-pull over the bandwidth of four times the modulation frequency with two coherently synchronized arbitrary waveform generators (AWGs). The problem is further compounded due to the fact that more AWGs 
are required, in order to achieve a truly broadband baseband termination to load-pull all the baseband components when the device is driven into deep compression.

\subsubsection{Triggering for Modulated Measurements}

The oscilloscopes provide considerably more than simple views of waveforms. To capture the true waveforms the oscilloscope's trigger circuitry plays the very important role of telling the scope's other circuitry when to start drawing the trace. Since the scope display provides a graph of amplitude versus time, it is very important the scope starts drawing at the same point on the waveform each time it sweeps across the display. If the oscilloscope could not precisely trigger, it would be impossible to measure anything related to time. The sweep would start at a different point each time and therefore, the waveform would be moving to a different position on the display each time. When an external clock is used to trigger measurements on the CSA 8000 the user has the ability to select the hold off time of the trigger circuitry.

It is important to note that the hold off period occurs immediately after the completion of each sweep. This is the period during which the next sweep is inhibited. The length of the hold off period is controlled by the length of the next input clock pulse. This value is limited however by a minimum re-arm time of the trigger circuitry of the instrument due to an inherent variation or jitter in this re-arm time; therefore, it can lead to an unexpected trigger event. This variation however causes major issues when employing the frequency compressed sampling method described in [35] as a constant sampling frequency is required throughout the measurement. Formerly, the high frequency clock signal of $5 \mathrm{MHz}$ was used to trigger the measurements for all the modulation frequencies.

Though, it was possible to reduce the noise present in the measured signal and this also ensured that the sampling frequency remains constant throughout the measurement, the problem to capture the complex modulated signals persisted due to the lack of high quality trigger signal for complex modulated signals. The alternative solution to this problem is to use a 
modulation frequency adaptive higher quality clock source to trigger the multisine scope measurements.

\subsubsection{Post Measurement Averaging}

When the frequency compressed sampling technique was employed to sample the modulated multi-tone waveforms, it was found to be difficult to 'freeze' the display of the sampling-oscilloscope. The collection of each new multi-tone waveform is, in itself a valid measurement and captures all of the relevant information; however, due to triggering issues the consecutive measurements do not occur with a coherent time reference. This transpires because although the oscilloscope coherently samples across the selected time window, when the end of the time window is reached the data must be processed and displayed. This leads to an unknown and unpredictable number of clock edges being ignored, thus each new waveform collection begins at a different time-point in the repetitive waveform. This means that the on-board averaging of the oscilloscope cannot be employed. Therefore, software post-processing was required to accurately time align the collected waveforms in order to allow averaging that is necessary to improve the overall quality of the measurements to an acceptable level [35].

The required software processing is relatively trivial for a sampled continuous wave signal as each consecutive captured waveform is simply a time shifted version of its predecessor. In this case, averaging can be achieved by simply using the first waveform as a reference with all other waveforms time shifted to align. This approach allows for simple yet effective time-domain on board averaging. However, for modulated excitations, a simple time shift is no longer sufficient as the RF and Modulation naturally have different down-conversion ratios, which means that consecutive waveform collections are no longer just time shifted versions of each other. The envelope changes and RF changes occur on totally different time scales. Thus all subsequent waveform captures were aligned using the following sequence, first the modulation envelopes, then the RF cycles within the envelope. This post averaging technique decreased the measurement speed due to the download of data on the general purpose interface bus (GPIB) for each measurement. 


\subsubsection{Active IF Load-pull Architecture}

The active IF load pull measurements were successfully undertaken at different IF reflection coefficient, to determine the effects of the low frequency IF load impedances on the linear behaviour of the DUT, such as its effect on the distortion components when the device is driven into its non-linear region of operation. The active IF load pull was then later used to find the optimum IF load which might minimise the inter-modulation distortion terms.

Due to some limitations of the previous active load-pull implementation, the baseband impedance termination was limited to four times the modulation frequency. Therefore, this decreased the measurement system capability of load-pulling the IF components including the higher baseband components (IF3 which is three times of the modulation frequency and IF4 which is eight times of the modulation frequency).

\subsubsection{Active RF Load-pull Architecture}

Many design applications require the device to operate in a multicarrier environment, containing signals with multiple, closely spaced frequency components. Under these conditions the device will generate intermodulation distortion products about the carrier influenced by other design parameters, which cannot be accounted for using a single tone stimulus signal. In order to produce improved designs, the factors affecting these intermodulation products are required and are often used to provide an efficiency and linearity trade-off in the design.

Traditionally, the factors affecting linearity are obtained using a two-tone amplitude modulated (AM) measurement, where the figure of linearity is obtained from the in-band intermodulation (IM) distortion products. The measurement system has been demonstrated to fully characterise the device response, in a $50 \Omega$ environment, resulting from a two-tone modulated signal, and does not offer active source pull and load pull of harmonic and intermodulation components. This necessitates the development of the existing modulated waveform measurement system to characterize the device for future wireless communication system in non $50 \Omega$ RF impedance environments. 


\subsection{Enhanced Modulated Measurement System}

The enhancement of the measurement system has been performed at both baseband (IF) and RF impedance levels. The enhanced measurement system for characterization of memory effects can be used for measuring and analysing active power device as well as power amplifiers in a user defined specific RF impedance environment. This measurement set-up also allows investigation of broadband baseband load and source impedance effects on the linearity performance of a power device. Instead of $50 \Omega$ RF impedance environment, the RF architecture was modified to synthesize the specific RF loads for modulated excitation using the Tektronix AWG7000 at the fundamental and second harmonic.

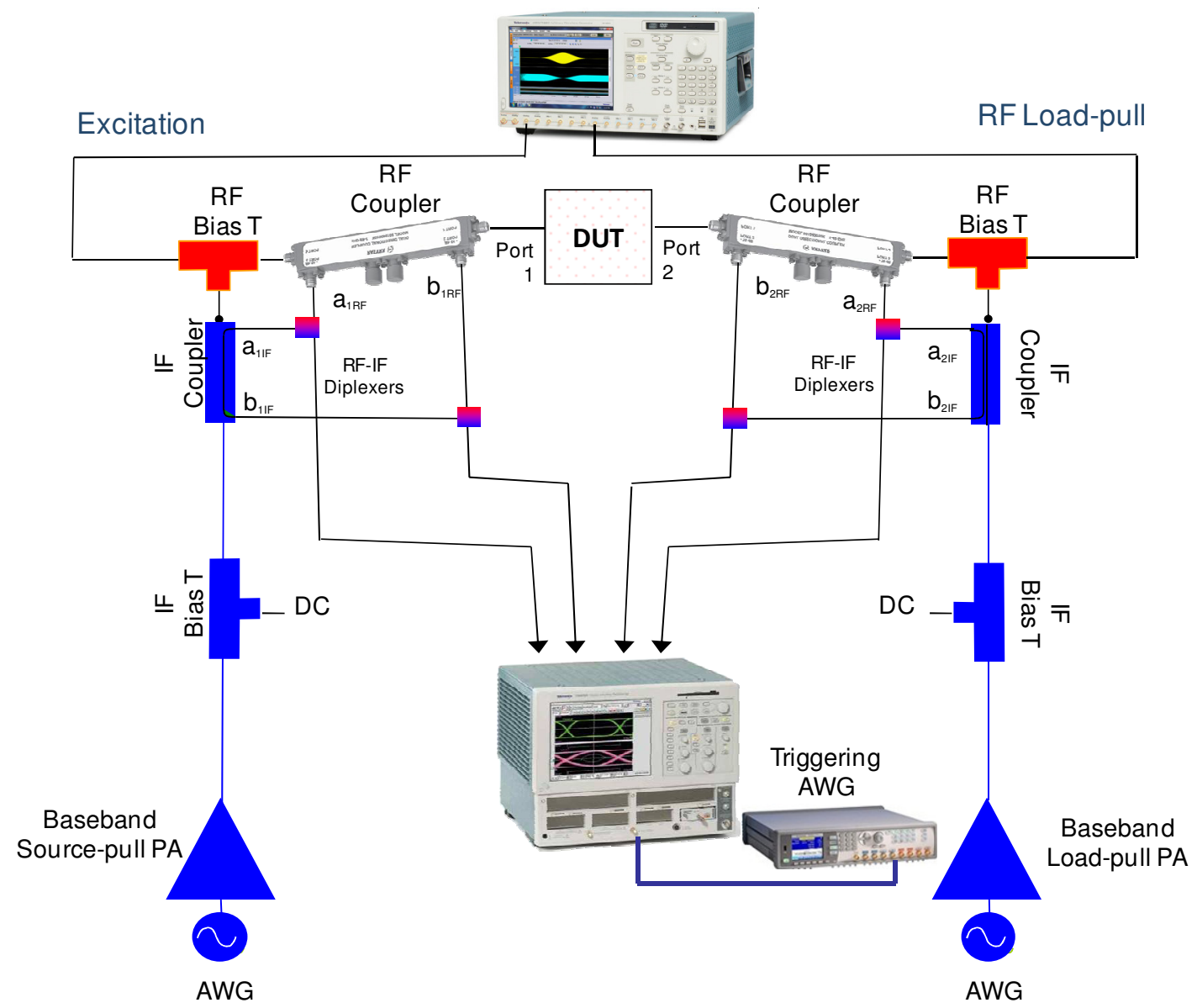

Figure 2.12: Enhanced modulated high power waveform measurement system based around an oscilloscope.

The enhanced architecture of the high power modulated waveform measurement system is depicted in Figure 2.12. In this measurement set-up 
the RF input multi-tone signal is generated from the arbitrary waveform generator channel-1 first pre amplified, using the linear driver power amplifier and then passed through an isolator, band pass filter and coupler to the input of the device under test (DUT). The isolator and band pass filter are necessary to isolate the DUT from the signal generator to avoid impedance mismatching between the DUT and the driver power amplifier. The modulated input and output signals of the DUT after coupling and attenuation are measured using the broadband receiver, Tektronix DSA8000. In order to synchronize, the $10 \mathrm{MHz}$ reference of the Signal Generator is used to adjust the clocks of all the measurement systems jointly.

The waveform measurement system is now capable of maintaining the independent and constant impedance control of each individual tone at baseband (IF) and RF. This requirement becomes very important to evaluate high power harmonic modulated active RF load pull characterisation of power devices when emulating of specific RF power amplifier modes. In the following sections the enhanced modulated waveform measurement system is described in more details.

\subsubsection{Necessary Measurement System Enhancements}

The modulated waveform measurement system needs to be made suitable for simultaneous RF and IF load-pull characterisations and measurements. It should, however, be remembered that what is said here is true for measurements and characterisation of RF active devices and power amplifiers on the whole. The emphasis has been to enhance the previously reported modulated waveform measurement system in order to decrease the time penalty for modulated measurements [6]. Here, the enhancements are classified into three sub-groups depending on the targeted performance parameters, multi-tone on-board averaging and measurements, broadband baseband load-pull, and modulated RF load-pull.

\subsubsection{Multi-tone Measurement Averaging}

Triggering from the input signal becomes impractical when we move away from CW stimulus and wish to sample multi-tone signals. The problem is 
further compounded for modulated signals, since each cycle of RF is no longer identical, thus the stimulus signal can no longer be used to trigger the measurement as the pattern no longer repeats on each rising edge. One imperative aspect of the enhanced modulated measurement system is that it allows the on-board averaging by engineering an appropriate trigger clock. Averaging is needed to improve the overall quality of the measurements to an acceptable level since it is most desirable due to high demand of high power devices characterisation under multi-tone and realistic communications signals.

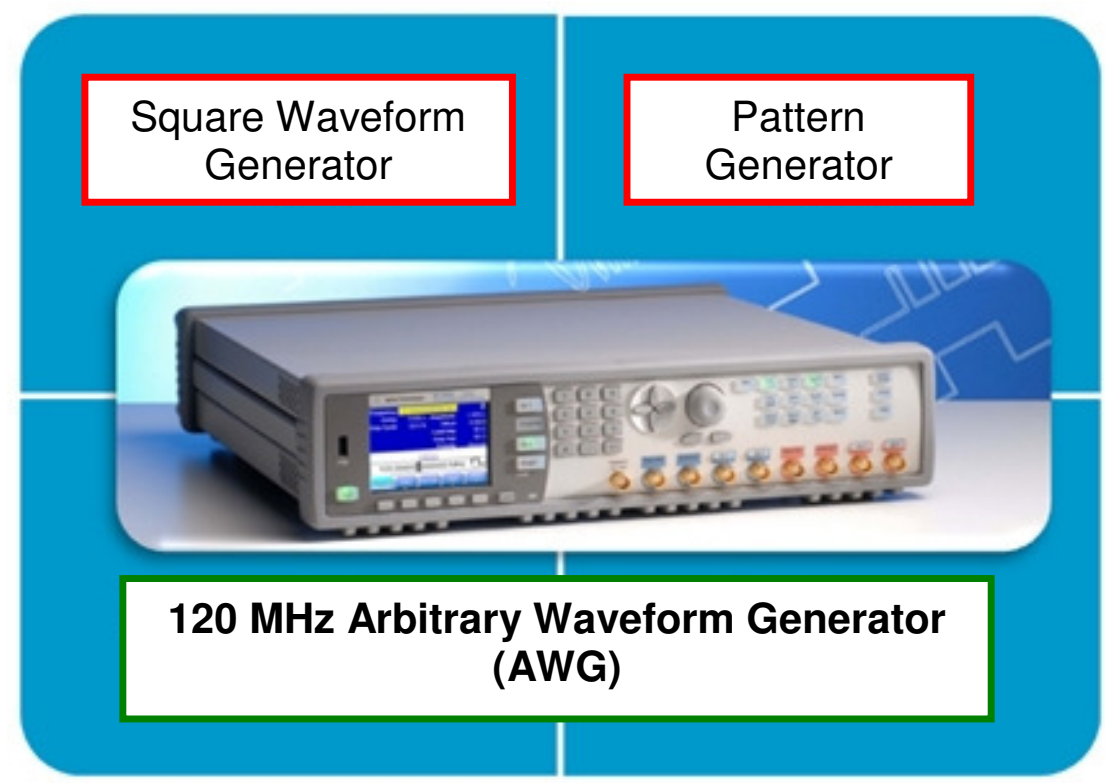

Figure 2.13: Agilent arbitrary waveform generator used for fine clock generation to trigger Tektronix oscilloscope for multi-tone measurements.

The triggering of the oscilloscope was achieved by using an Agilent $120 \mathrm{MHz}$ arbitrary waveform generator (AWG) which generates high quality repetitive square waveforms at the modulation frequency of interest. The trigger was set on square waveforms defined by amplitude, qualified by time (pulse width, slew rate, setup-and-hold, and time-out), and delineated by pattern. These resulting waveforms then triggered the oscilloscope for precise capture of signal across the time window. Actually, the oscilloscope's trigger function synchronizes the sampled horizontal sweep at the correct point of the signal, essential for clear signal characterization. Trigger control allows the stabilizing 
of repetitive waveforms and the capture of single-shot waveforms. The trigger made the repetitive waveforms appear static on the oscilloscope display by repeatedly displaying the same portion of the sampled signal. The oscilloscope's sample rate and record length was optimized to isolate a specific part of the sampled signal of interest on the horizontal trigger position. Thus, varying the horizontal trigger position, allows for the capture of the complete modulation cycle as it is depicted in Figure 2.14 for lower modulation frequencies $(\leq 1 \mathrm{MHz})$.

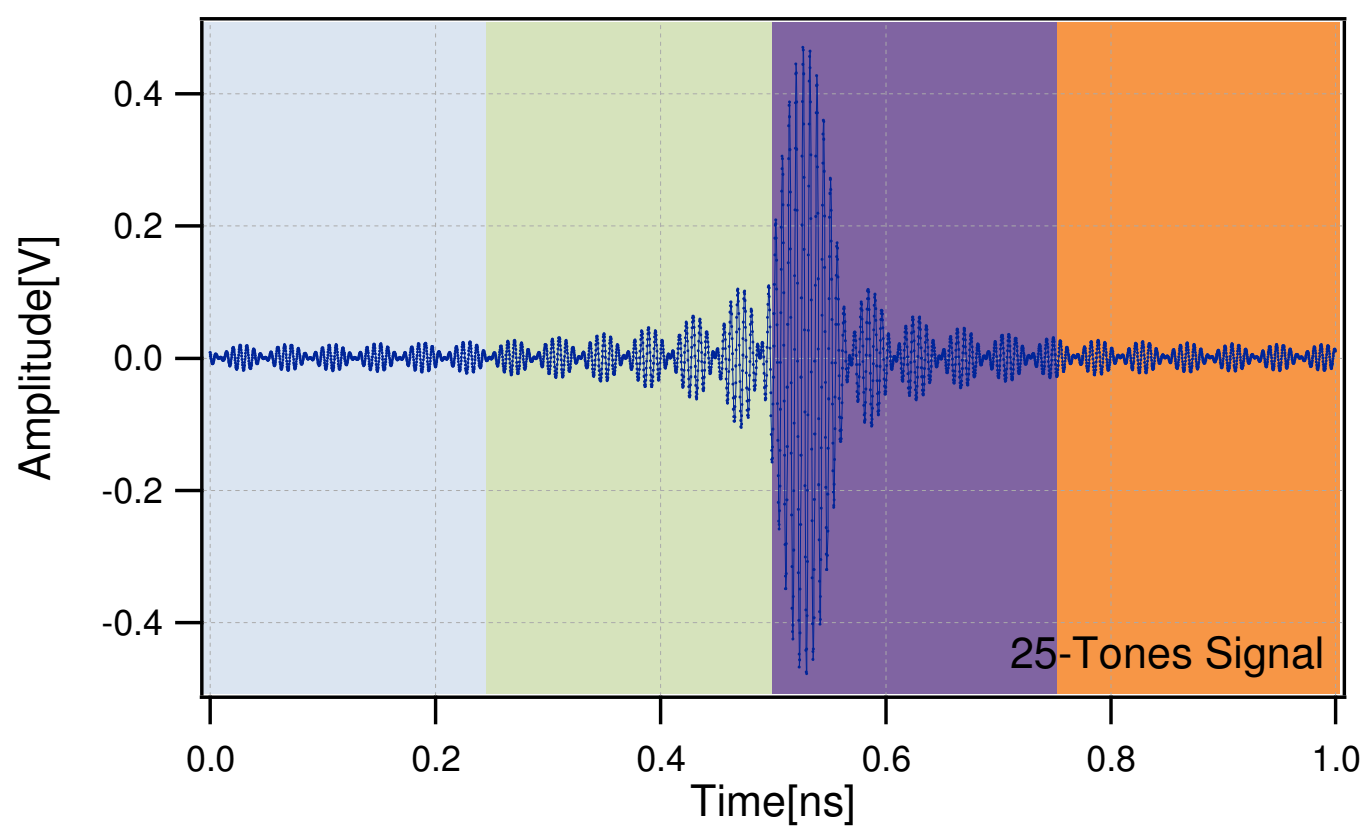

Figure 2.14: Four time widows stitched for one complete modulation cycle of on oscilloscope averaged 25- Tones modulated signal at carrier frequency of $2 \mathrm{GHz}$ with $500 \mathrm{KHz}$ modulation frequency [38].

\subsubsection{Multi-tone Measurement Software}

The function of the developed measurement software is to provide complex measurements automatically. For accurate measurements of device nonlinearities and memory effects, the device under test (DUT) measurement should be done for different power levels at both RF and IF bands which mean the number of measured sweep points increase and this could consume a significant amount of time. For full automatic control of the measurement set-up and acquisition of the measured data, new programmes 
have been developed using IGOR-pro software. Here are some details about the developed software;

* Performance Signal generator (PSG) is connected to the computer through a GBIP bus and has been automatically controlled using SCPI commands (standards commands for programming instructions). These commands have been used for remote control of the signal generator for setting the signal type (two-tone or multi-tone), power level, carrier frequency and modulation frequency.

\& To set up the measurement and collect the data, a broadband Tektronix oscilloscope (DSA8000) was used as a receiver and connected to the computer through GPIB. In order to capture the multitone signal using a standard sampling approach, a trigger was provided at the repetition rate of the modulated sequence. The DSA8000 has only 4000 measurement points available, allowed for collection of one complete modulation cycle making it impractical to capture all the relevant information at lower modulation frequencies with 4000 measurements points.

Therefore, a new technique was introduced that allows capture of one complete modulation cycle in a spectrally efficient way for lower modulation frequencies, referred to as "Time Domain Partitioning". The Tektronix DSA8000 swept across the display window for 4000 measurement points successively and data was downloaded to the computer, stored sequentially in the programme array for one complete modulation cycle. The formulation given in the following equations was used to capture the one complete modulation cycle for multi-tone signals.

$$
\begin{aligned}
& f_{\text {sampling }}=2 \bullet(\text { Harmonics }+1) f_{\text {Carrier }} \\
& T_{\text {Point } s}=\left(f_{\text {Sampling }} / f_{\text {Modulation }}\right) \\
& \text { Number of Point } s=\left(T_{\text {Point } s} / 4000\right)
\end{aligned}
$$

Where $f_{\text {sampling }}$ is the oscilloscope sampling frequency, $f_{\text {carrier }}$ is carrier frequency, $T_{\text {Points }}$ is total number of points. 
This technique has decreased modulated measurement time, for instance it now takes less than 1 minute to completely capture a device's non-linear response (including baseband and five harmonics) to a $1 \mathrm{MHz}$ modulated $2 \mathrm{GHz}$ carrier and it thereby allows capture of multi-sine signals effectively. Thus, the developed programme for the DSA8000 was used to automatically control the measurements and save the input and output signals of the DUT as a complex values such as;

$\checkmark$ Dynamic transfer characteristics, dynamic AM/AM and AM/PM characteristics at fundamental frequencies as a function of input power and input modulation frequency.

$\checkmark$ Distortion products IMD lower and upper in the case of two excitation, and ACPR upper and lower in case of multi-sine excitation as a function of input drive power and input signal modulation frequency.

* The switch driver controls the coaxial switch which is used for automatic selection of the input and output signals for the DUT. This switch driver is connected to the computer through GPIB bus and is automatically controlled.

* IGOR-pro software is used to save the measurement data, process it and display it in real time.

In brief, this measurement software development makes it possible to study the overall behaviour of the DUT in terms of its inherent nonlinearity and factors affecting its performance under multi-tone signals and realistic communication signals. Until now, it was only possible to study the DUT behaviour under two-tone signal due to the on-board averaging problem [39].

\subsubsection{Broadband Baseband Load-pull}

When the device is driven more deeply into compression, significantly more mixing terms are generated at baseband frequencies, and in order to achieve a sufficiently broadband IF termination, significant modification of the baseband load-pull measurement system was required in order to accurately 
account for higher baseband harmonics. This is now achieved in the time domain using a single arbitrary waveform generator (AWG) to synthesise the necessary waveforms to allow a constant and specific IF impedance environment to be maintained across a wide IF bandwidth.

The arbitrary waveform generates the baseband components which are the multiples of the baseband fundamental frequency. How the instruments are arranged to generate the arbitrary waveform is shown in Figure 2.15 and elaborates a little with respect to the triggering, what the trigger connects to, and where the $10 \mathrm{MHz}$ is used. A separate triggering AWG is used which is necessary because without external trigger, the ARB generator looses phase coherence between the modulation and the baseband when it is re-initiated to generate the arbitrary waveform. This then allows the ARB AWG to generate the precise waveform with the required amplitudes and phases.

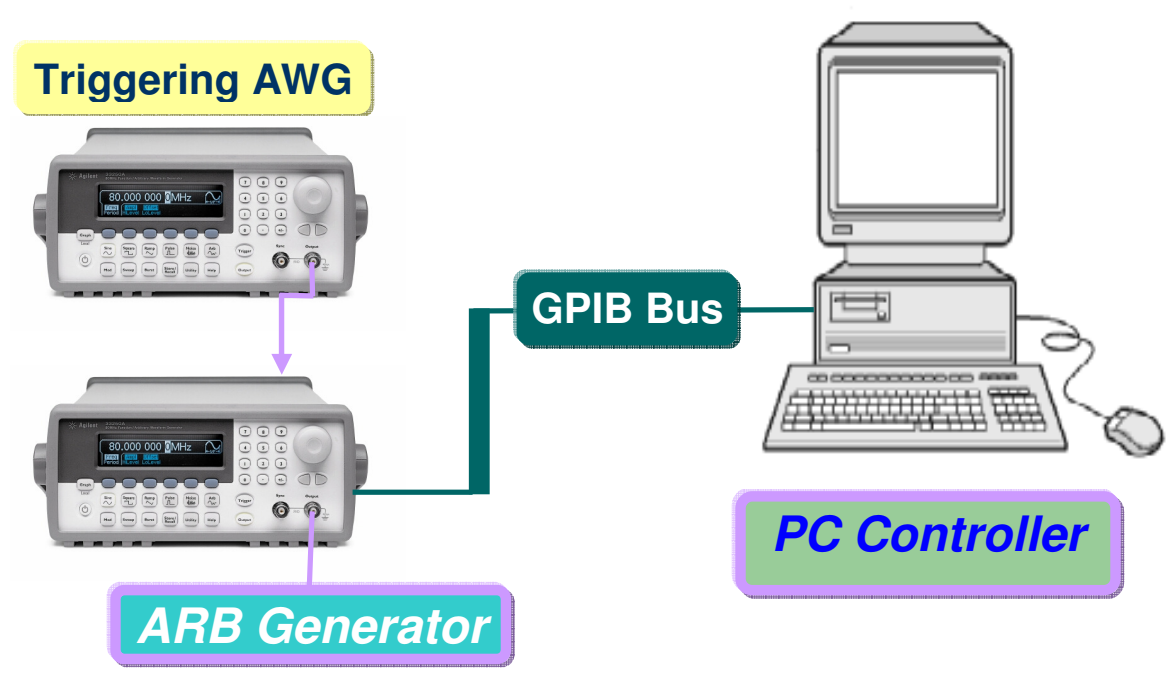

Figure 2.15: Arbitrary waveform generator arrangements to generate the baseband load-pull signal.

The mathematical equation (2.8) was used to generate the resultant arbitrary waveform containing all the frequency components at baseband frequencies.

$$
V(t)=A_{n} \sum_{n=1}^{\infty} \operatorname{Cos}\left(2 \pi f_{c} n t+n \varphi\right)
$$

Where $A$ is the amplitude in volts, $t$ is time in seconds (the horizontal axis), $V$ is the vertical axis in Volts, and $f$ is the frequency of cosine wave in $H Z, \varphi$ is 
the phase of the cosine wave (in the Figure 2.16, the waveform is shown with a phase of $180^{\circ}$ ). The arbitrary waveform generator used, uses a scale of -1 to 1 ; defining a waveform with values between 0 and 1 would only give half the amplitude resolution. Thus in order to create an arbitrary waveform it was ideal to use the entire vertical resolution for defining amplitude. The combined spectrum of the arbitrary waveform is shown in Figure 2.17 which was downloaded to the volatile memory of the AWG through the GPIB bus. After downloading the arbitrary waveform into the AWG, the AWG will always replicate the finite-length time record to produce a periodic version of the data in the waveform memory and play it continuously.

However, as a matter of fact, it is possible that the shape and phase of a signal may be such that a discontinuity may be introduced at the end of one cycle due to the inaccuracy of the AWG in replaying the waveform continuously. When the wave shape is repeated for all time, this end-point discontinuity will introduce leakage errors in the frequency domain because many spectral terms are required to describe the discontinuity. It is important to create arbitrary waveforms as a single period or as multiple periods and avoid a discontinuity.

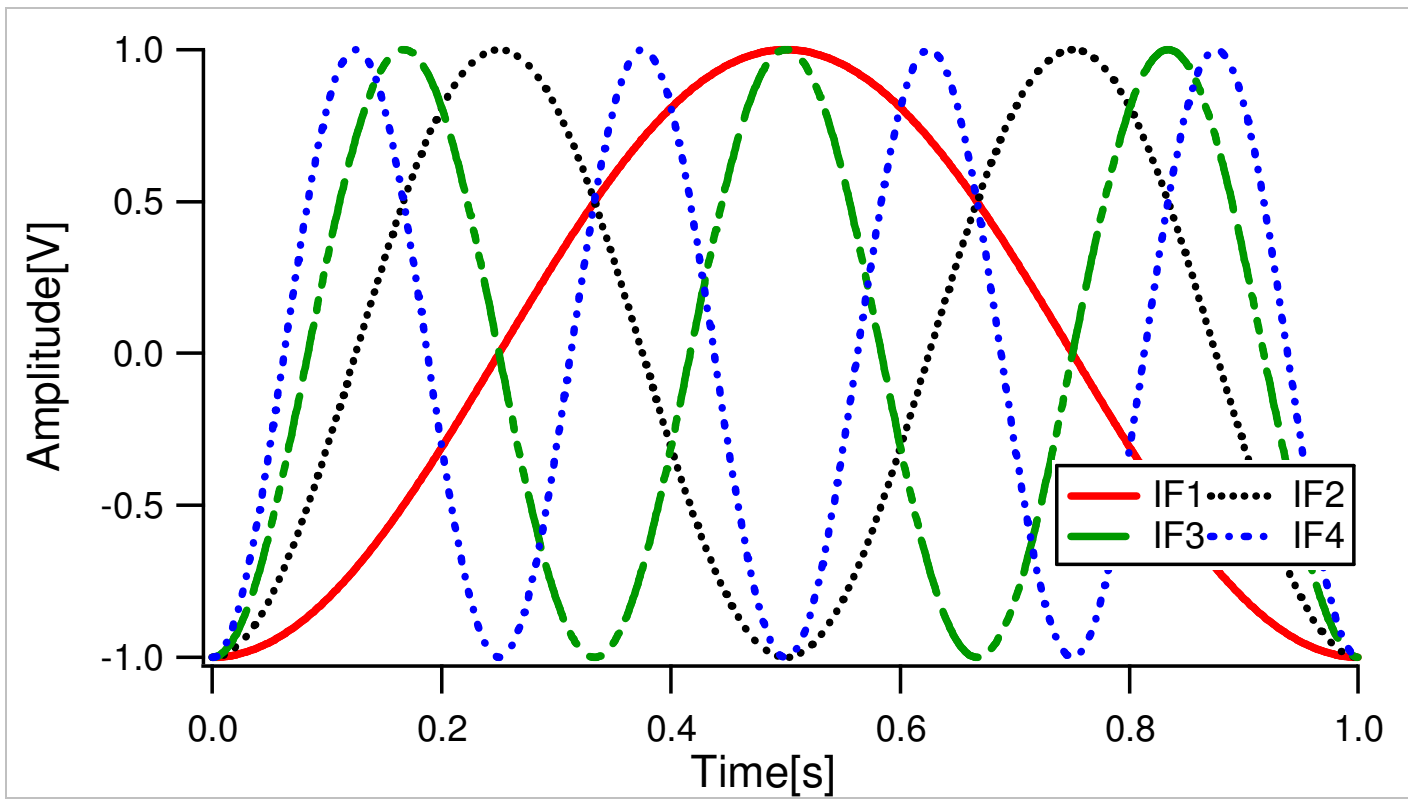

Figure 2.16: The sinusoidal representation of different waveforms for first four baseband components at amplitude of \pm 1 . 
The output frequency is typically limited by the bandwidth of the arbitrary waveform generator. The AWG outputs the entire arbitrary waveform at the specified rate. When an arbitrary waveform is defined as multiple cycles, the actual output frequency may be higher than the specified rate. For instance, if a waveform is defined as 10 cycles of a sine wave and is output at $1 \mathrm{MHz}$, the actual frequency will be $10 \mathrm{MHz}$. An anti-aliasing filter is placed at the output to smooth the voltage steps to create the final waveform. When using multiple cycles in an arbitrary waveform, especially at high frequencies, the final result may be attenuated. It could also be under-sampled. Therefore, to avoid this problem the final arbitrary waveform was scaled for one cycle.

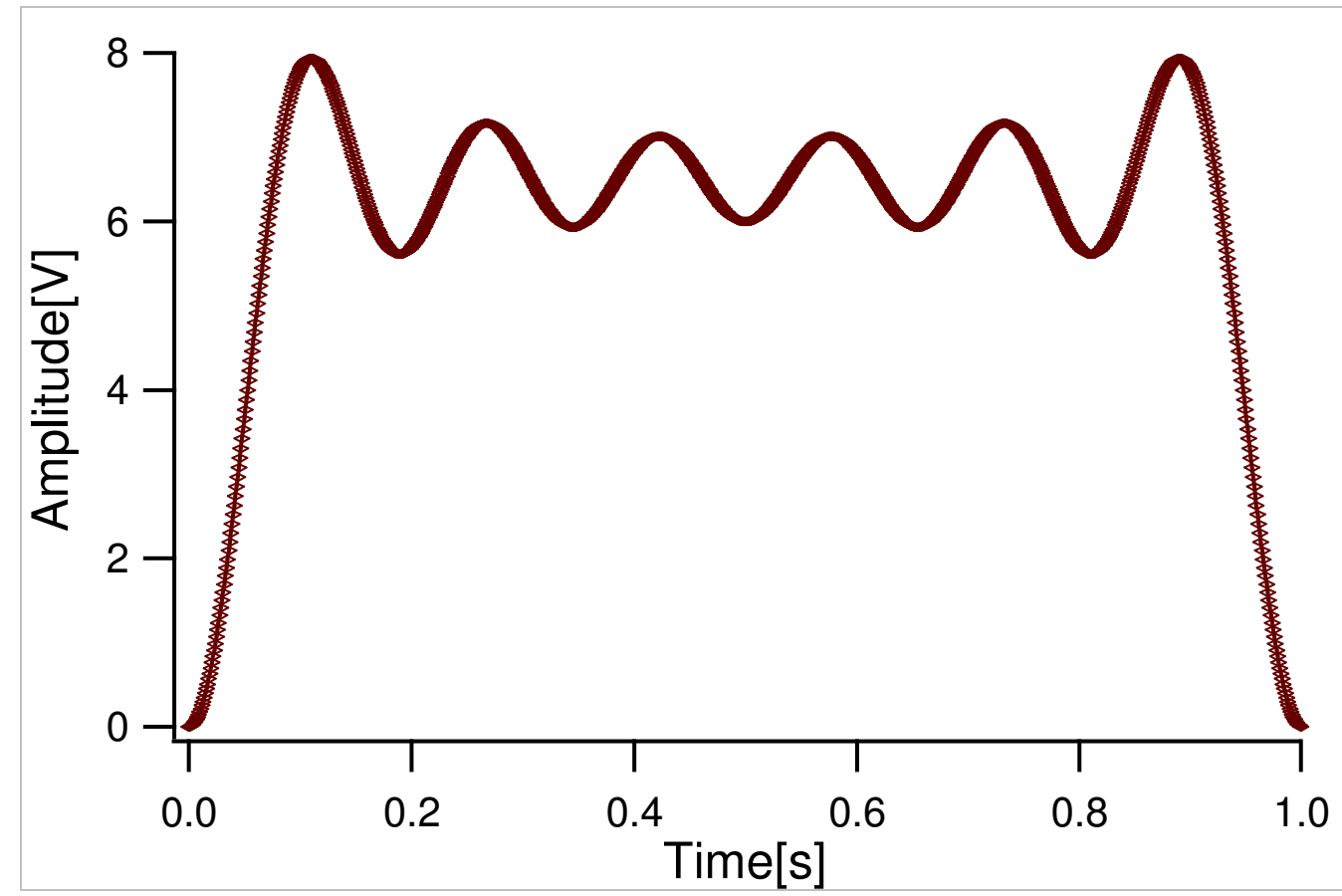

Figure 2.17: The combined spectrum of signal for the first four baseband components, for one cycle.

The advantage of using the IGOR programme to generate the waveform is that it is straightforward to modify the waveform and download it to the ARB generator. Combined with the baseband power amplifier that would allow an increase in the signal's amplitude in order to load-pull the baseband components when the device is driven into deep compression. This is done in order to quantify the affect of broadband baseband termination on the memory effects and device's overall linearity. 


\subsubsection{Why AWG for Modulated RF Load-pull?}

Active load pull of harmonic and intermodulation components was attempted but could not be achieved using the two PSG sources. To load pull the two fundamentals $\omega_{1}$ and $\omega_{2}$ and IM components when using a two-tone signal with a frequency separation of $1 \mathrm{MHz}$, the signal was generated using a PSG. The measurement software was used to determine the magnitude and phase of the signal generated by the PSG to achieve the desired load impedance. Once both the magnitude and phase for each tone of interest are altered, then the measurement software automatically updates the PSG waveform table and activated the waveform. However, the process of activating the table causes the ESG phase reference to relock to a new reference on the external coherent carrier signal. The problem is further compounded when the baseband generator fails to relock itself to an external pattern clock generator. This makes the newly activated phases obsolete and prevents the software from converging on the required load. A solution to this problem was investigated using the Tecktronix AWG 7000, dual channel synthesizer.

\subsubsection{Broadband Modulated RF Load-pull}

Today's digital wireless signals present an array of new challenges in synthesizing test signals. As the industry has shifted to digital wireless technology, it has brought a vast increase in modulation waveform complexity. Fortunately the steadily rising AWG performance now enables flexible, efficient generation of very complex test stimulus. The concept behind the arbitrary waveform generator is using a variable frequency clock to step through a pre-stored digital representation of an arbitrary waveform, which is then converted into an analogue signal.

Generating baseband IQ signals with the AWG offers some advantages beyond just having two outputs to drive a modulator. The wide AWG bandwidth relative to most modulation bandwidths usually allows a significant level of over-sampling hence lowers the noise floor. Furthermore, the DAC output of the arbitrary waveform generator produces some level of quantization noise but arbitrary waveform generators like the Tektronix 
AWG7000 that uses 14 bits to represent each signal sample at baseband, sufficiently to prevent the noise floor from becoming a problem. The AWG7000 offer up to 10 bits of linear digitization with 12 GS/s sample rate has also provided high DAC linearity which is essential to maximize the Dynamic Range. Therefore, the traditional limitations of AWG in dynamic range, bandwidth and frequency are now minimised with this AWG 7000. The AWG 7000, thus, was used to uncover large-signal RF performance, focused around modulated characterisation; it is therefore not surprising that in the impedance control architecture documented in this thesis that it has been applied to modulated signals for RF impedance emulation. In addition, the use of modulation during the characterisation process introduces a new band of components generated at base-band frequencies, also around the carrier and harmonics. The control of the impedances presented to these components forms an integral part of the PA optimisation process as well as emulation of different PA modes. To accomplish RF impedance control, two simultaneous stimulus channels of the AWG7000 were used, which guarantee signal RF phase coherence, to create continuous signals by repeating the available time window over and over. With the use of the AWG7000, the use of many signal generators and signal synchronization problems are avoided.

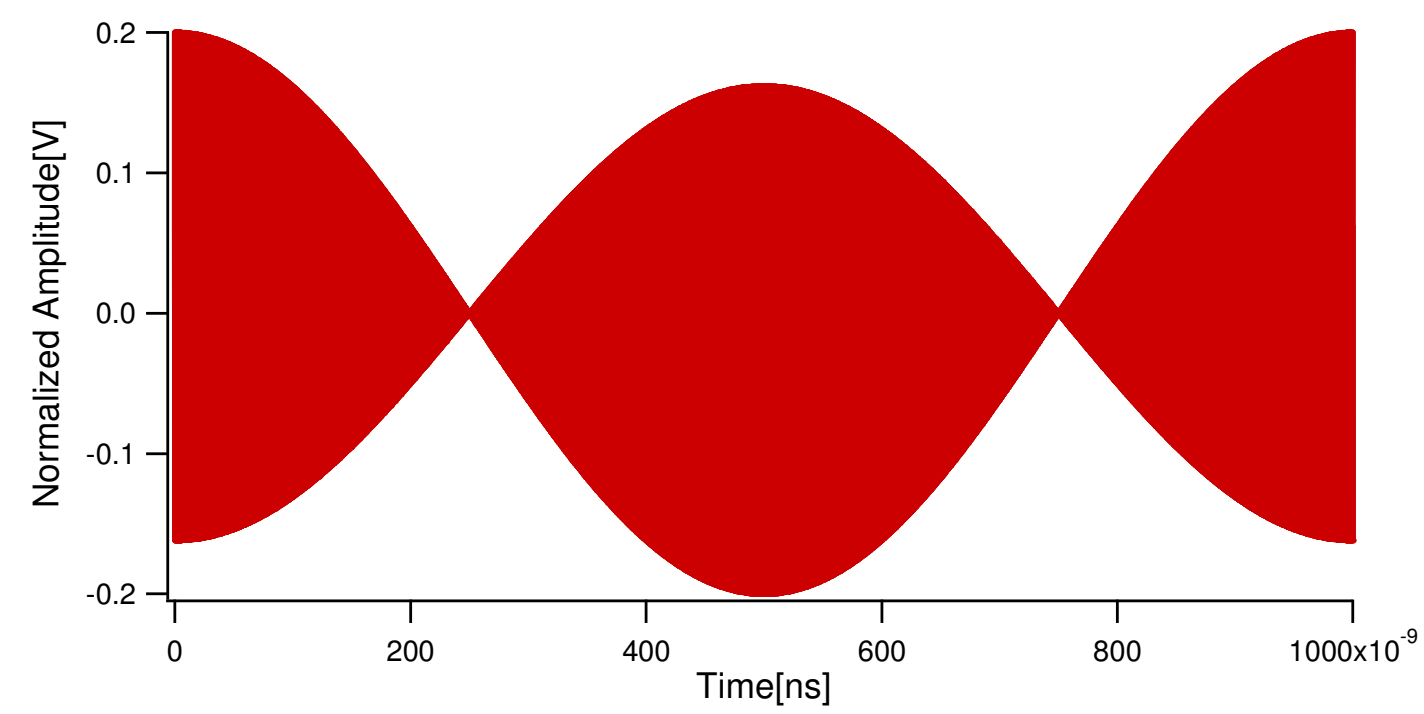

Figure 2.18: The 2-tone modulated detailed RF time domain waveform generated by using the channel-1 of the AWG to excite the device.

Figure 2.18 depicts the two-tone signal with $2 \mathrm{MHz}$ frequency spacing, for the required power level, and phase distribution, generated by using channel-1 of 
the AWG7000. The program for AWG control, integration and signal generation was written in $\mathrm{C \#}$ and it allows the phase distribution of the tones to be either random, uniform, or fixed by the user which can be accessed using GPIB command. A variation in the magnitude and phase of each tone is achieved relative to the $\mathrm{RF}$ output power and carrier phase of the fundamental tones. Time domain representation of waveform containing the fundamental and $2^{\text {nd }}$ harmonic components, generated by the channel-2 of the AWG7000 is shown in Figure 2.19, with absolute amplitude and phase relationship between frequencies.

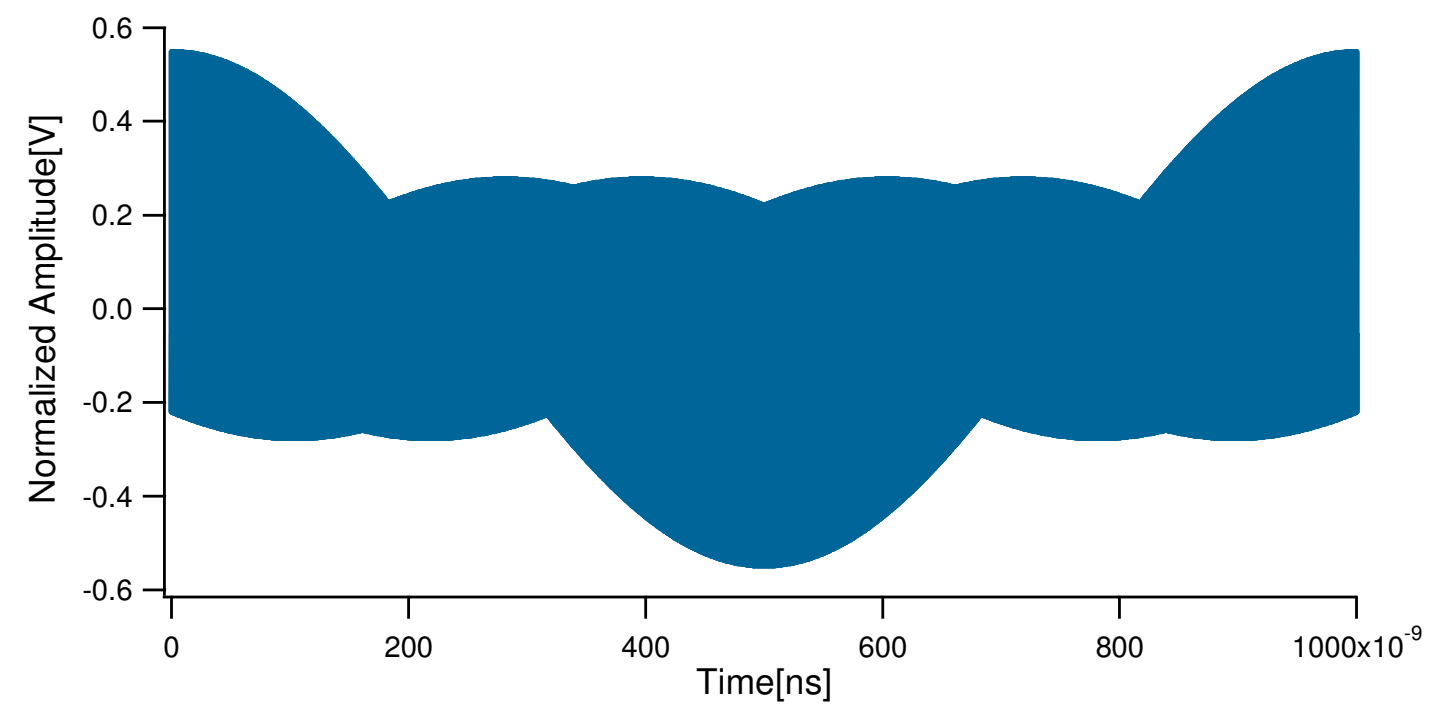

Figure 2.19: The combined time domain waveform for fundamental and $2^{\text {nd }}$ harmonic.

\subsubsection{Capabilities of Enhanced Measurement System}

The enhanced measurement system is used to measure the multi-sine signals in a wide bandwidth and to evaluate resulting output signals from the device under test (DUT) very close to the noise floor. For instance, in evaluating a power amplifier and power device this measurement system was used to identify the spurs, harmonics, and intermodulation distortion products and evaluate them with very narrow resolution bandwidths. It was also used to quantify, analyse and measure the relevant information with magnitude, phase and time domain capabilities and change between the frequency domain and time domain representation. For vector measurements, the developed measurement software uses the fast Fourier transform (FFT) to 
convert the signal from time domain to frequency domain and provides the following measurement capabilities.

- The spectral components at IF and RF

- Baseband frequency dependent modulated AM/AM and AM/PM measurements under multi-tone excitations

- Influence of drain bias on the output signals at fundamental and IMD frequency components

Furthermore, due to the ability of this measurement system to analyse the signal in different domains, different characterisation techniques can be deployed, starting from very traditional two-tone to multi-sine signals and digital modulated signals.

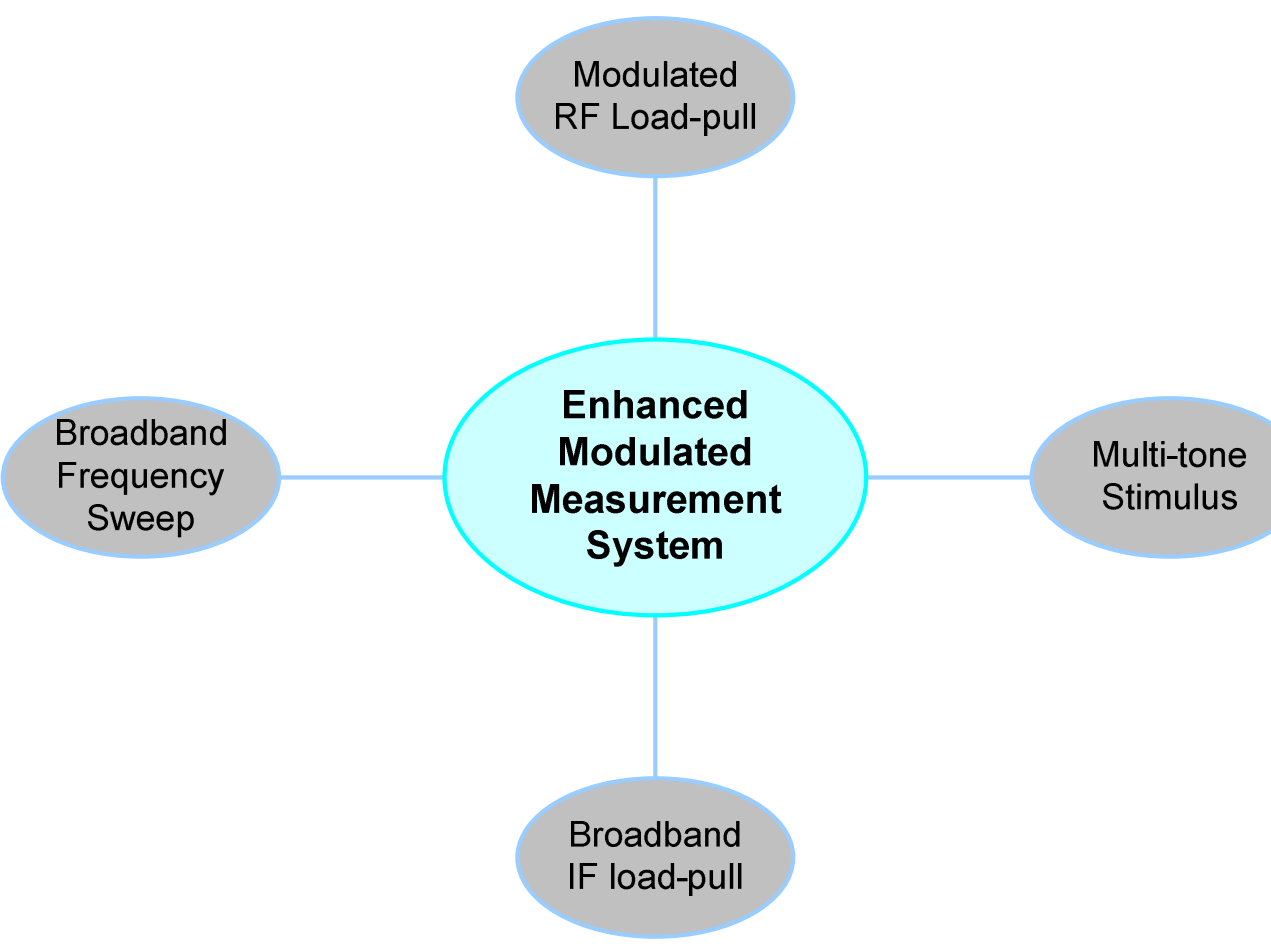

Figure 2.20: Measurement Capabilities of the enhanced modulated high power waveform measurement system.

\subsubsection{Extended Dynamic Range}

Distortion products generally have lower power levels than the fundamentals in power transistors and power amplifiers. This is especially the case for power amplifiers operating in a weakly nonlinear region. Therefore, it is important to ensure that any characterisation or measurement systems has 
enough dynamic range to be able to measure low distortion levels when there are high fundamental levels present. Factors that reduce dynamic range are thermal noise, quantisation noise, and nonlinearity in the characterisation system and excitation sources. Investigations documented in this thesis propose a rule of thumb, that the measurement dynamic range of the measurement system should be at least $50 \mathrm{~dB}$ to measure the third-order IMD levels below the fundamentals. The dynamic range of the measurement system will need to be sufficient to see the smallest components that are to be measured. The experience of developing this thesis has shown that a dynamic range of around $60 \mathrm{~dB}$ is sufficient to accurately characterize thirdorder nonlinearity.

To demonstrate the improved dynamic range of the enhanced measurement system, a thru measurement was conducted using a 9-tone stimulus with a notch created by progressively suppressing the central tone. For this case, and as can be seen from Figure 2.21, the measurement system can measure this tone to approximately $50 \mathrm{dBc}$. To be meaningful here, the system measured the actual response of the signal generator, as what is measured is the leakage from the modulator. From the same plot, the out-of-band dynamic range is closer to $60 \mathrm{~dB}$, and this is perfectly sufficient to be able to measure the relatively low distortion levels of interest here.

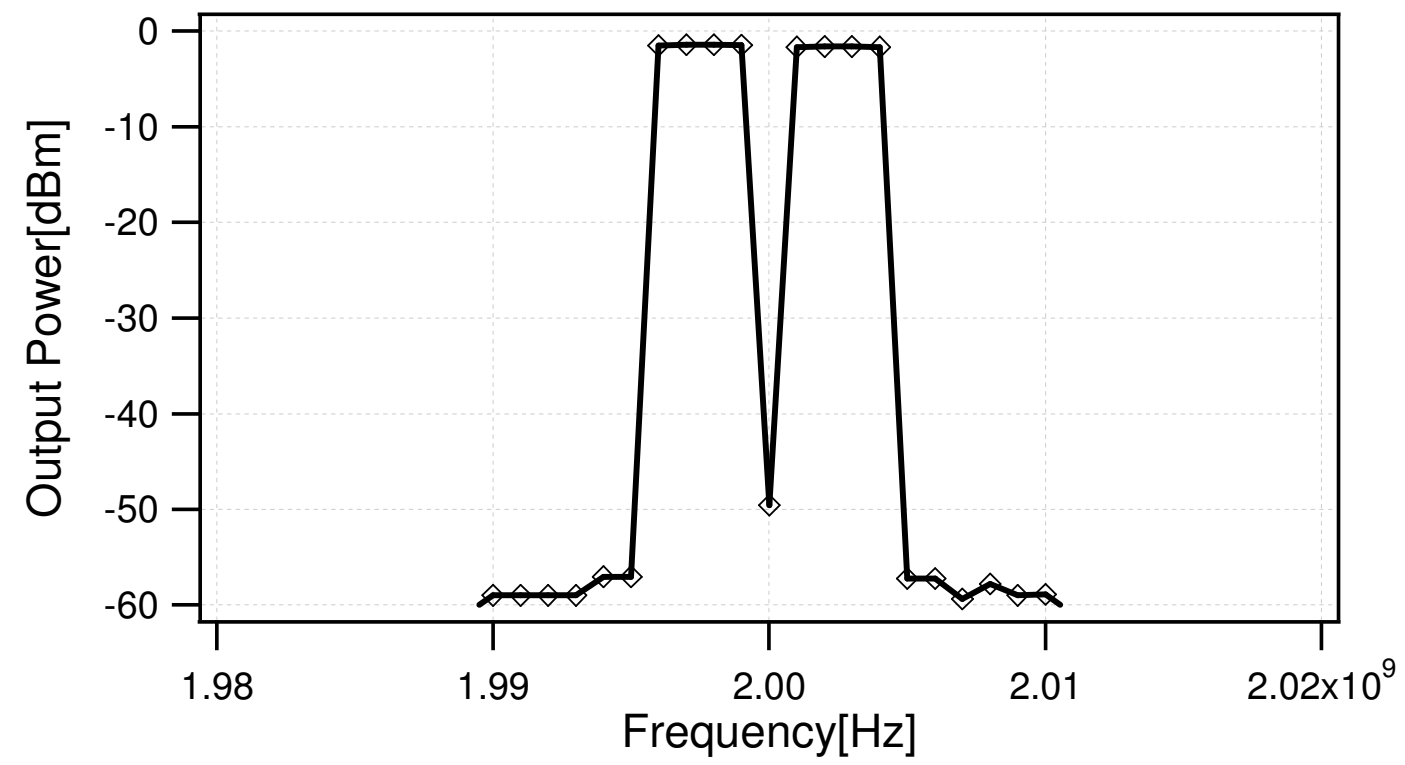

Figure 2.21: Measured output spectrum for thru standard using 64 averages with the lowest spectral information [39]. 
A closer analysis of measurement system dynamic range for modulations using different numbers of tones, and using 64 averages is shown in Table2.1. Increasing averaging beyond this has little effect on the in-band dynamic range as this is eventually limited by the leakage distortion properties of the modulated source. One important observation to note is that when the phases of the tones used in the excitation are randomised, the dynamic range of measurement system is slightly reduced. For all measurements, the tone amplitudes were adjusted to ensure a constant peak envelope power. The effect of averaging on the dynamic range of the enhanced measurement system has been quantified using 25-tone stimulus.

TABLE 2.1: Dynamic range of enhanced modulated measurement system for different multi-tone excitations [39].

\begin{tabular}{|l|c|c|c|c|}
\hline Fixed Amplitude & $\begin{array}{c}\text { 2-Tone } \\
\text { [dB] }\end{array}$ & $\begin{array}{c}\text { 5-Tone } \\
\text { [dB] }\end{array}$ & $\begin{array}{c}\text { 9-Tone } \\
\text { [dB] }\end{array}$ & $\begin{array}{c}\text { 25-Tone } \\
\text { [dB] }\end{array}$ \\
\hline Fixed Phase & 64 & 62 & 60 & 45 \\
\hline Random Phase & 61 & 59 & 56 & 39 \\
\hline
\end{tabular}

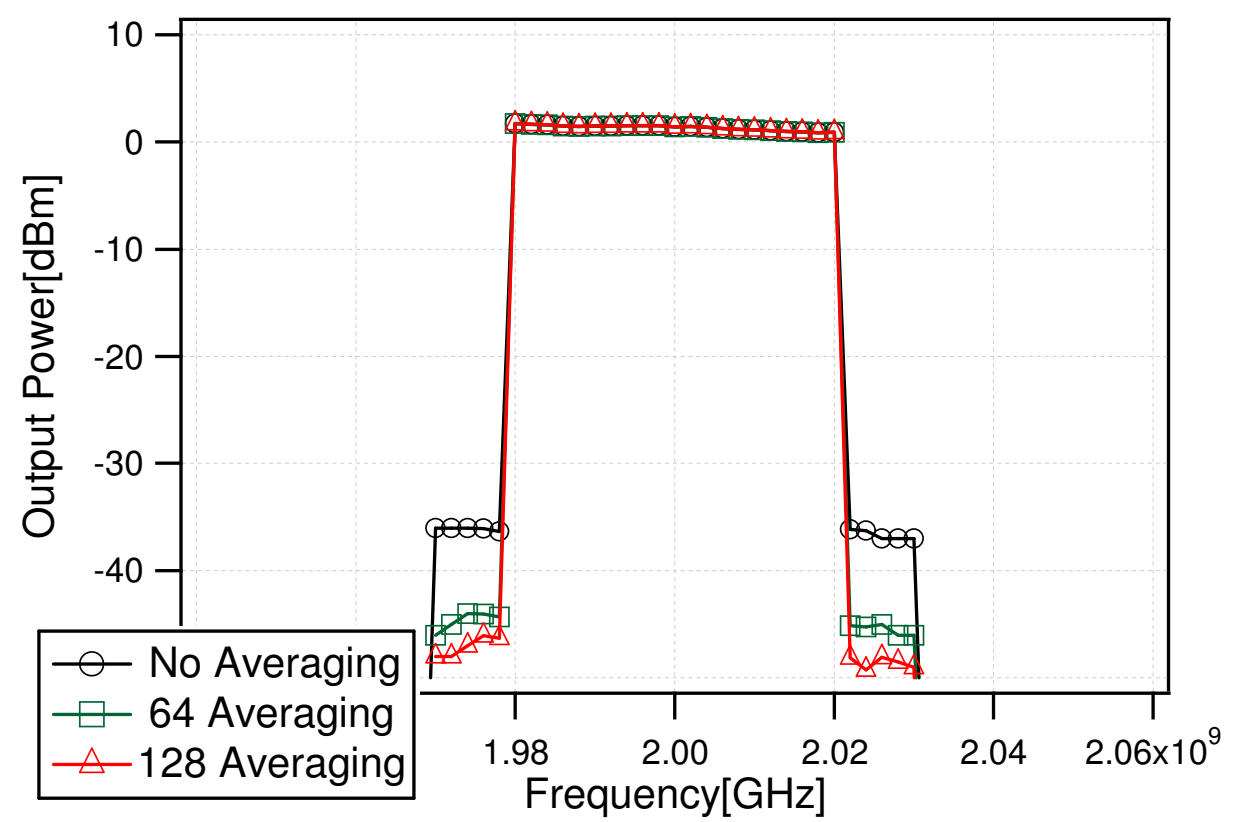

Figure 2.22: The effects of averaging on a typical thru measured modulated signal.

The raw performance of the measurement system with no averaging is depicted in Figure 2.22 along with the averaging. The averaging has reduced 
the noise floor without affecting the fundamental tones. With averaging, effective dynamic range is increased by some $8 \mathrm{~dB}$ by using 64 averages and $10 \mathrm{~dB}$ using 128 averages. Further increased averages did not improve the dynamic range due to limitation imposed by the distortion in the signal sources.

\subsubsection{Extension of Measurement Bandwidth}

The bandwidth of wireless communication systems has increased over the last decade and it has been of great interest to jointly amplify signals from different systems and bands in one PA. Thus, if efficiently and cheaply done, there is basically no limit to the bandwidth requirements of the PA. In addition, and maybe more important from a measurement system view, the output signal of a nonlinear PA, due to the nonlinearities, has a considerably larger bandwidth than the input signal. A typical example could be that the input signal has a bandwidth of $20 \mathrm{MHz}$, while the bandwidth of the output signal will increase to $100 \mathrm{MHz}$ considering up to IM5. The bandwidth is here somewhat loosely defined but is essentially the frequency range for which the distortion raises above the noise floor. The enhanced measurement system allows capturing of all the relevant spectral information at baseband, around the carrier and harmonics which are sufficient to understand and study the nonlinear behaviour of the device across these bands. It is, therefore, desired to measure the nonlinear behaviour of power device over a frequency range over which the electrical memory effects are significant. It would be useful if the measurement system could excite and measure down to at least $1 \mathrm{MHz}$. This frequency limit is selected as an approximate goal for a number of reasons:

* Firstly, it is difficult to make bandwidth dependent IMD measurements with a spectrum analyser at tone spacings greater than $1 \mathrm{MHz}$ and less than $20 \mathrm{MHz}$.

* Typical measurement systems do not have significant capability to load-pull the baseband frequencies of several $\mathrm{MHz}$.

* The $1 \mathrm{MHz}$ is the corner frequency of baseband electrical memory effects in most devices. Memory characteristics of any power device 
with strong inherent nonlinear effects typically vary above that frequency anyway.

Of course, some devices will exhibit frequency dependent dynamic behaviour at lower frequencies than this, but this limit will be sufficient for the devices used in this thesis. Such a low frequency limit also includes the possibility of using the AWG to load-pull all the baseband components and the bandwidth of baseband load-pull is limited by the bandwidth of AWG which is currently $80 \mathrm{MHz}$.

\subsubsection{Possible Measurement Options}

Many kinds of multitude measurements can be performed with the presented system. This flexibility is obtained by varying some independent parameters of the system. The parameters that can be varied are:

$\checkmark$ Input power

$\checkmark \quad$ Number of tones

$\checkmark$ Carriers phase distribution

$\checkmark$ Load impedance

$\checkmark$ Biasing conditions

The measurement system measures the in-band and out-of-band distortion products resulting from the DUT under multi-tone excitations.

$\checkmark \quad$ IMR measurements (in-band/out-band)

$\checkmark$ ACPR measurements(in-band/out-band)

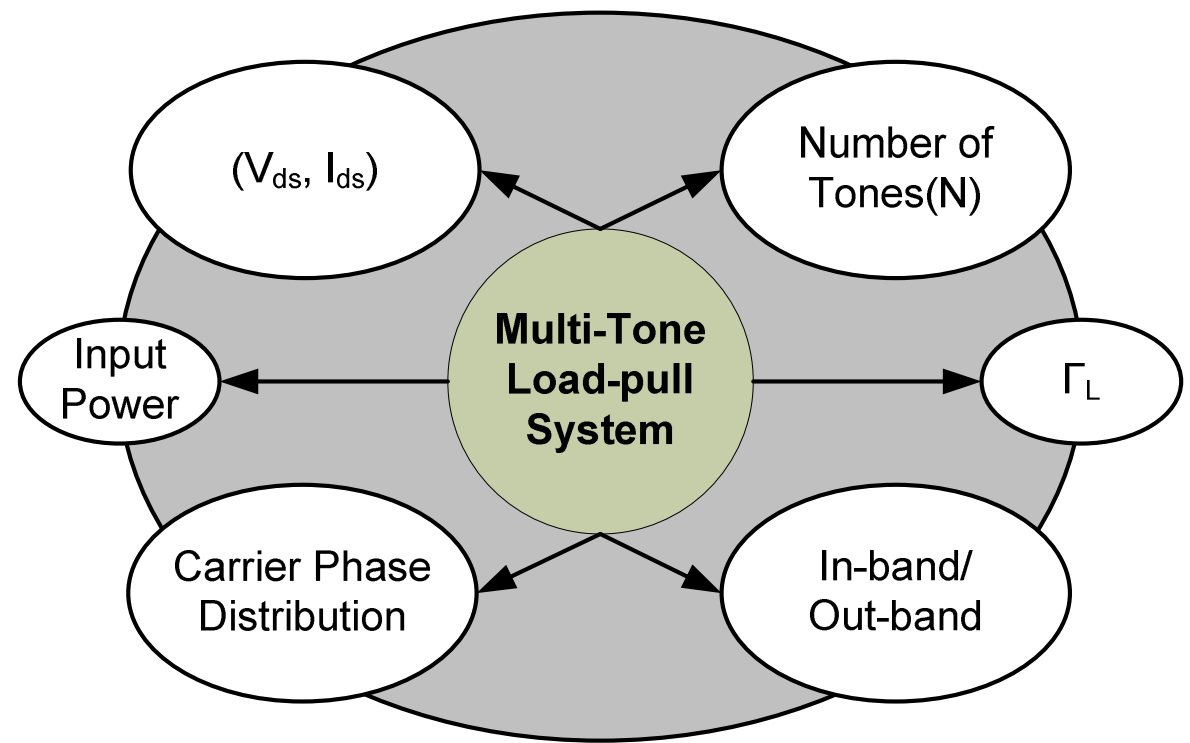

Figure 2.23: Parameter variation options in the measurement system. 
All the possible variation options of these parameters are shown in Figure 2.23. Therefore, performing measurements by varying these independent parameters will generate a complete database which would be useful in the development of different plots for a given transistor with given nonlinear characteristics.

\subsubsection{Stitching Problem Occurrence}

The stitching of measured data from multiple measurements is required, to obtain the amplitude and phase of the various tones of the multiharmonic wide-band periodically modulated signal. The frequency of the tones used to generate the modulation need to be considered carefully however to avoid waveform 'stitching' problems and subsequent spectral re-growth. The correct stitching, then, enables one to step through the modulated waveform, and accurately capture one complete modulation cycle in sufficient detail and accuracy for meaningful analysis.

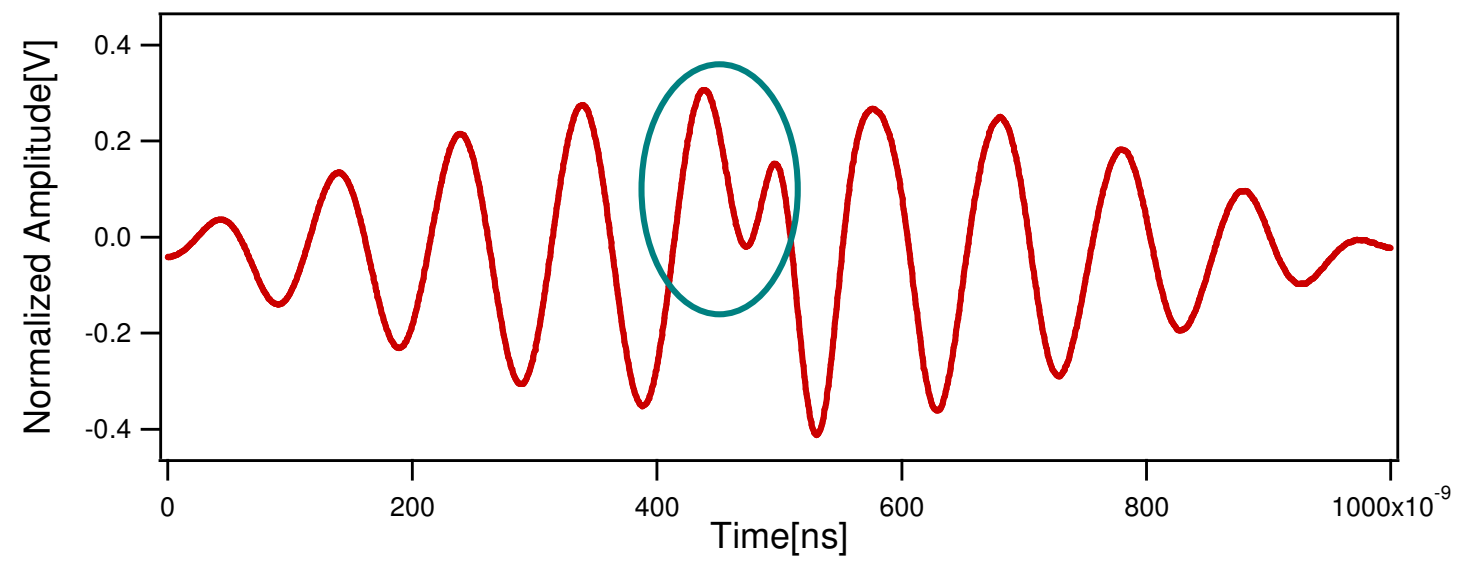

Figure 2.24: Zoomed in RF waveform to demonstrate the stitching problem.

Figure 2.24 identifies the stitching problem which is due to the fact that the prime number division results in infinite fractional points for modulation frequency of $3 \mathrm{MHz}$. As frequency is related to time by:

\section{Time=1/Frequency}

The fractional points thus make it impractical to employ time partitioning approach without fine tuning of the modulation frequencies. It can be seen from the table 2.2 that the number of windows is effectively defined by the total number of sampling points, so the modulation frequency can be altered for most investigations. The stitching problem at certain modulation 
frequencies has been overcome through the precise rounding off at a particular modulation frequency, to offer the measurement flexibility.

Table 2.2: Number of windows required to capture different modulation frequencies with stitching issues.

\begin{tabular}{|c|c|c|c|c|}
\hline \multicolumn{5}{|c|}{ Carrier frequency 2GHz, Harmonics 3, Multi-tone Stimulus } \\
\hline $\begin{array}{c}\text { Modulation } \\
\text { Frequency[MHz] }\end{array}$ & $\begin{array}{c}\text { Sampling } \\
\text { Frequency[GHz] }\end{array}$ & $\begin{array}{c}\text { Sampling } \\
\text { Points }\end{array}$ & $\begin{array}{c}\text { Number of } \\
\text { Windows }\end{array}$ & $\begin{array}{c}\text { Stitching } \\
\text { Problem }\end{array}$ \\
\hline 1 & 16 & 16000 & 8 & No \\
\hline 2 & 16 & 8000 & 4 & No \\
\hline 3 & 16 & 5333.33 & 1.333 & Yes \\
\hline 4 & 16 & 4000 & 1 & No \\
\hline 5 & 16 & 3200 & 0.8 & No \\
\hline 6 & 16 & 2666.67 & 0.667 & Yes \\
\hline 7 & 16 & 2285.71 & 0.571 & Yes \\
\hline 8 & 16 & 2000 & 0.5 & No \\
\hline 9 & 16 & 1777.78 & 0.444 & Yes \\
\hline 10 & 16 & 1600 & 0.4 & No \\
\hline
\end{tabular}

\subsection{System Calibration}

To de-embed the measured raw data taken though the Tektronix DSA to the reference measurement planes of the transistor, the TRM (thru-reflectmatch) technique was used. For power corrections, the measuring ports have been calibrated with an independent power meter. Other calibration techniques (e.g., thru-reflect line (TRL)) can be used to improve the system flexibility and precision, and to extend the system to perform on-fixture measurements. A calibration procedure is of paramount importance to account for any errors introduced due to losses, mismatches and imperfect directivities in the system. This allows for the measurement of the complete multi-tone voltage and current waveforms that exist at the DUT plane. The calibration employed uses the well-established calibration procedure presented in detail in [37], [38]. This calibration procedure has been 
successfully implemented and utilised on various previous iterations of measurement systems developed at Cardiff University and further additions and alterations made to allow calibrations on a multi-tone grid were presented in [35]. In order to calibrate for a multi-tone measurement, it is necessary to apply the calibration procedure outlined in [37], [38] both at base-band and at $\mathrm{RF}$ frequencies covering fundamental and significant harmonic bands. The baseband and RF calibration results can then be combined to create a single calibration file containing all necessary error coefficients for application to raw waveform modulated measurements. Using the standard sampling technique of the DSA8000 and capturing one complete window for modulated measurements, all expected frequency components are known. Thus a calibration grid was defined that covers all of the frequency components in the spectrum, ensuring that only relevant components are calibrated. The ability to vary the modulation frequency during a measurement is a common requirement when performing multi-tone measurements. For example, it is desirable in order to quantify the effect of the different modulation rates on transistor performance in order to investigate various memory phenomena. If the calibration grid is defined in the same way as the measurement grid then a new calibration must be completed for each change in modulation frequency, which makes measurements rather time consuming. To surmount this potential problem an interpolated calibration procedure has been utilised. An interpolation process is only viable if the error coefficients are reasonably well behaved across the entire measurement bandwidth. This is shown to be the case in Appendix-A, where the error coefficients are plotted and the measurements indicate that the error coefficients are well behaved in each of the measured bands and as such can be easily interpolated. The error coefficients for each band are then combined to form a calibration file for use in the waveform measurement software.

\subsubsection{IF Calibration Verification}

The commercially available microwave power devices utilized for baseband impedance variation investigation purposes in this thesis are mounted in a custom microstrip $50 \Omega$ fixture and therefore the need of IF 
calibration arises so that the measurement reference plane can be shifted from sampler to the device package plane. This calibration will account for the systematic errors occurring due to imperfections in the analyzer, test set, couplers, switches etc. This calibration will not correct for the random errors introduced due to repeatability problems in the instrument and the drift errors which are dependent on environmental factors such as variation in temperature. Therefore to achieve accurate measurements of the device, error coefficients must be determined which is of prime importance to minimize any error which a system can possibly introduce. The calibration procedure used for the small signal CAL was TRM (Thru - Reflect - Match) and for the large signal Cal, extend two ports indirectly via the source-pull port was used. The IF test set was connected with the RF test set as shown in Figure 2.25 and the arbitrary waveform generator (AWG) was used as the IF source and was connected to IF Cal Switch.

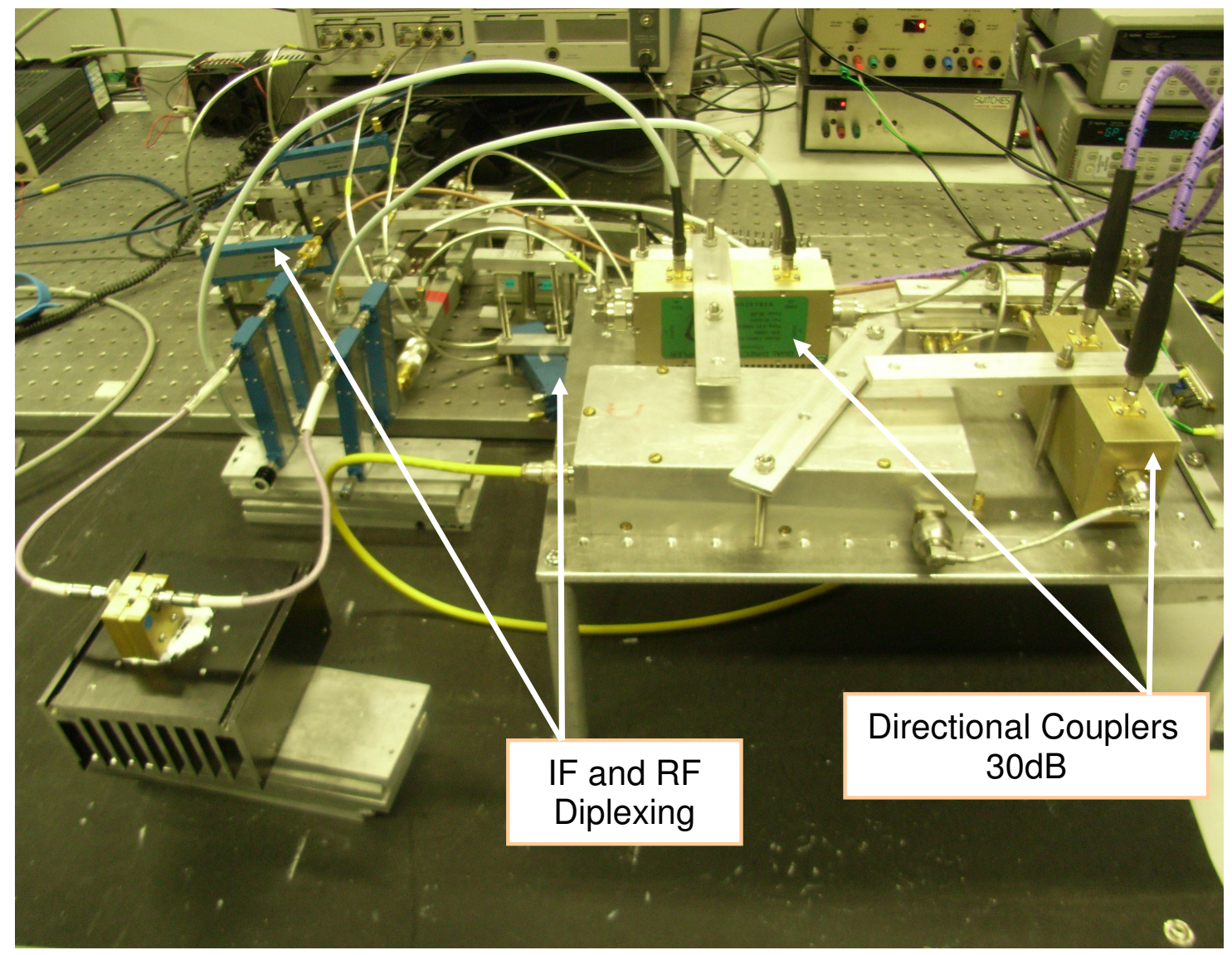

Figure 2.25: Integration of IF test-set into the RF test-set for calibration.

The complete IF and RF calibration test set-up is shown in Figure 2.25. In this complete configuration, there is a need to diplex the RF and IF travelling wave 
components before measurement and it is achieved by blue Krytar back-toback couplers. The calibration (small signal and large signal) was done by using a $3.5 \mathrm{~mm}$ standard cal kit at the end of piece of the co-axial cable. The comprehensive calibration verification is listed in Appendix-B for different standards. However, for simplicity only the validation of the IF Calibration is presented through the measurement of small piece of cable. A cable of $4.94 \mathrm{nsec}$ delay (delay was measured through the 8753 VNA for a frequency range of $1-20 \mathrm{MHz}$ ) was used to perform an off-set IF calibration verification. The measured $\mathrm{S} 21$ for the cable is listed in Table 2.3 and the delay is calculated by using 2.10 and compared with the obtained result through the 8753 VNA.

$$
\text { Phase Delay }=\Phi / 360^{*} \text { Frequency }
$$

Measured delay through the enhanced measurement system was $5 \mathrm{~ns}$ for all frequency ranges and measured delay on 8753 VNA was 4.94ns which shows an excellent match between both measured delays.

Table 2.3: Measured S21 for cable

\begin{tabular}{|c|c|c|}
\hline \multicolumn{3}{|c|}{ Measured S21 } \\
\hline Frequency(MHz) & Magnitude & Phase(Degrees) \\
\hline 1 & 0.999089 & 1.827038 \\
\hline 2 & 0.998033 & 3.633365 \\
\hline 3 & 0.997932 & 5.456631 \\
\hline 4 & 0.997736 & 7.264744 \\
\hline 5 & 0.997135 & 9.06323 \\
\hline 6 & 0.996747 & 10.846911 \\
\hline 7 & 0.996808 & 12.655551 \\
\hline 8 & 0.996419 & 14.441444 \\
\hline 9 & 0.996441 & 16.236152 \\
\hline 10 & 0.999089 & 18.031014 \\
\hline
\end{tabular}

Finally, the calibration was loaded into the measurement software and thru measurements performed. A $0 \mathrm{dBm}$ signal was injected at $1 \mathrm{MHz}$ and simple $50 \Omega$ line was used to calculate the power at the DUT plane (centre of the line was used as a reference). Also, an oscilloscope was used to measure the voltage for all the frequency spacings and then converted to the power using the formula giving in equation 2.11.

$$
\text { Power }=\left(V_{p-p} / 2\right)^{2} / 2 * R
$$


The voltage measured at the centre of the line with the oscilloscope was $571 \mathrm{mV}$ and then converted to average power.

$$
\text { Power }=\left(573^{*} 10^{-3} / 2\right) / 2 * 50=0.82 \mathrm{~mW}
$$

This power was then converted to $\mathrm{dBm}$ which is $-0.84 \mathrm{dBm}$, as can be seen from the below panel the input is $-0.82 \mathrm{dBm}$ which is close enough to the calculated power at the device reference plane. Since a $50 \Omega$ line was measured, the power at the input was transferred to the output. The calibration reference plan for RF and IF was established at device package plan for accurate power device measurements. The IF calibration verification signifies that the baseband memory effects and linearity measurements and investigations documented in this thesis in the subsequent chapters are true, accurate and legitimate due to the accurate IF calibration of the IF test-set.

\begin{tabular}{|c|c|c|c|c|c|}
\hline \multicolumn{6}{|c|}{ Enhanced Measurement System Measured Input Parameters } \\
\hline & Input & Reflected & Load $\mathrm{F}$ & I. Coef & Impedance \\
\hline IF & 0.827511 & -26.1404 & 0.0542446 & -78.6937 & 51.3617 \\
\hline Lower Tone & 4.93758 & -17.3737 & 0.0766361 & -160.199 & 43.3377 \\
\hline Fundamental & -42.4071 & -55.8863 & 0.211857 & -101.402 & 50.3143 \\
\hline Upper Tone & 4.81422 & -17.2663 & 0.0786995 & -162.228 & 43.0821 \\
\hline
\end{tabular}

Figure 2.26: Measured Input parameters under two tone modulated excitation at $1 \mathrm{MHz}$ modulation frequency using the enhanced measurement system.

\subsubsection{Measurement Accuracy Enhancement}

During large signal power device characterization, it is desirable to obtain a reliable data. The following aspects have been observed and hence implemented to enhance the time domain measurement accuracy. The DSA 8000 has a limited dynamic range, if overdriven there is a risk that the DSA 8000 provides invalid measurements. In order to ensure the linear and safe operation of DSA 8000 the attenuators placed before the sampling heads of channel are set corresponding to the amount of signal power measured. Note the attenuators values must be set high enough such that the desired distortion products to be measured are driven below the noise floor. By setting the relevant and appropriate trigger events corresponding to the type of test signal being characterized, it is possible to measure signal components 
having a stable amplitude and phase at a predefined frequency point. When considering dynamic range improvement when measuring intermodulation distortion products in case of multi-tone excitation, it is very important to use distortion free RF signal source to avoid any unwanted noise at the output. It is highly recommended to operate the RF signal source in its linear region of power generation and then driver amplifier can be used to maintain an adjustable power level at the DUT. This will keep the IMD generated by the RF source at the lowest power level which will then consequently increase the quality of IMD and memory effects characterization of the measurement setup. By time averaging, which is simply a process of integration; a significant improvement in the dynamic range can be achieved for precise measurement of spectral components close to the receiver's detectable noise level. Averaging increases the measurement quality to the acceptable level whilst using the PSG as an RF signal source ensures sufficient dynamic range. However, challenges exist at the instrument level in improving the dynamic range if it is replaced with the Tektronix AWG 7000 as a signal source. However, after certain time averages it is not possible to sufficiently increase the measured output signal quality. A further refinement can be achieved by decreasing the resolution bandwidth which, however, increases in sweep time during the measurements.

\subsection{Demonstration of Multi-tone Measurement Capability}

In order to demonstrate the multi-tone measurement capability of the enhanced measurement system, the multi-tone measurement was conducted on the novel continuous class- $F$ power amplifier. The Continuous Class- $F$ mode [40] is a new PA mode which allows the design and realisation of broadband power amplifiers [41]. This new theory has demonstrated that by presenting a new formulation on the voltage waveform, a new family of voltage waveforms is yielded whilst maintaining the constant half-wave rectified sinusoidal current waveform. The new, different waveforms compared with the standard class-F case will reveal the same constant output power and drain efficiency compared with the conventional class- $F$ case. This is very important when designing broadband power amplifiers, as now by 
presenting simultaneously the appropriate fundamental and second harmonic impedances in accordance with the continuous class- $F$ theory (while maintaining an open-circuit third harmonic load) constant output performance is achieved for a wide design space [40]. The enhanced measurement system was calibrated over a relatively wide baseband bandwidth $50 \mathrm{MHz}$, and over $100 \mathrm{MHz}$ RF bandwidths centred around fundamental, second and third harmonics to characterise and investigate the linearity performance of this newly designed continuous class-F power amplifier under multi-tone signal. The continuous class- $F$ power amplifier was excited using a 9-tone stimulus signal with peak-to-average ratio of $9.54 \mathrm{~dB}$ depicted in Figure 2.27 at the presumed optimum bias point.

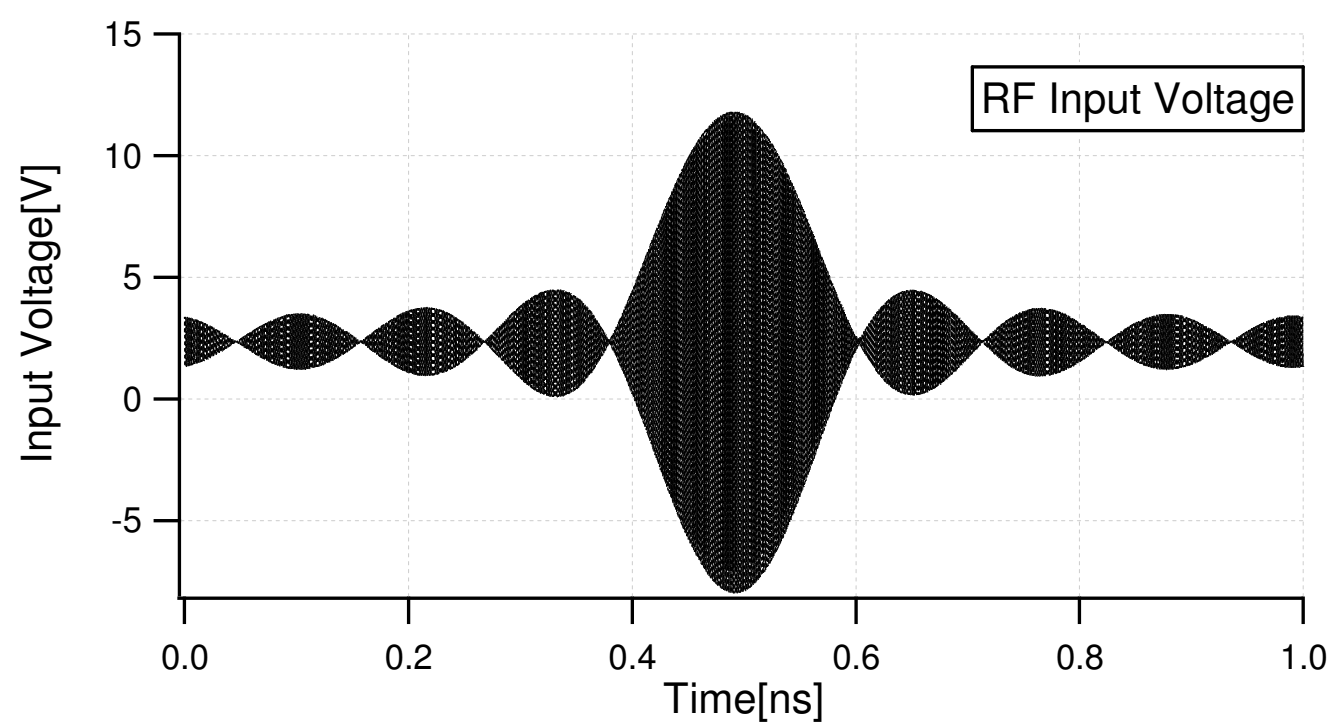

Figure 2.27: The Input 9-tone stimulus to continuous class-F power amplifier.

Figure 2.28 shows the peak envelope output power and adjacent channel power (ACPR $R_{L}$ and $A C P R_{U}$ ) behaviour. It can be seen from this measurement that the class-F amplifier has about $-33 \mathrm{dBc}$ linearity in ACPR at $8.5 \mathrm{~dB}$ back-off. It maintains, however, low asymmetry in the upper and lower ACPRs $(<2 \mathrm{~dB})$ with $-34.5 \mathrm{dBc}$ of $\mathrm{ACPR}$ at $8.5 \mathrm{~dB}$ back-off as shown in Figure 2.29. It can be seen that the continuous class- $F$ amplifier provides a slightly improved performance in peak envelope efficiency(PEE) by $2.1 \%$ at $\mathrm{P}_{1 \mathrm{~dB}}$ for two-tone excitation as compared with nine-tone excitation with fixed phases at $8 \mathrm{~dB}$ back- off. On the other hand, the continuous class-F involves complicated circuitry and vast effort in matching network design 
procedure. Summary of the performance of the designed continuous class- $F$ amplifiers is given in table 2.4 .

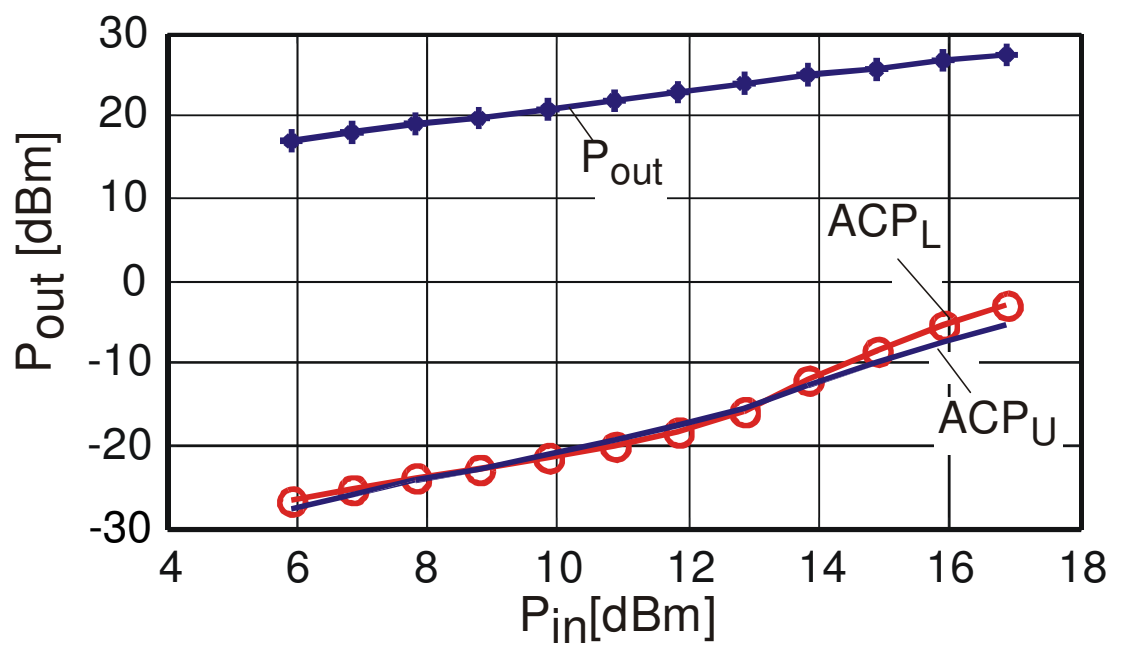

Figure 2.28: Measured peak envelope output power, upper and lower ACPs under nine-tone excitation for $10 \mathrm{~W}$ continuous class- $\mathrm{F}$ amplifier at bias point: $\mathrm{Vds}=28 \mathrm{~V}, \mathrm{Vgs}=-4.6 \mathrm{~V}, \mathrm{f}_{0}=1 \mathrm{GHz}$

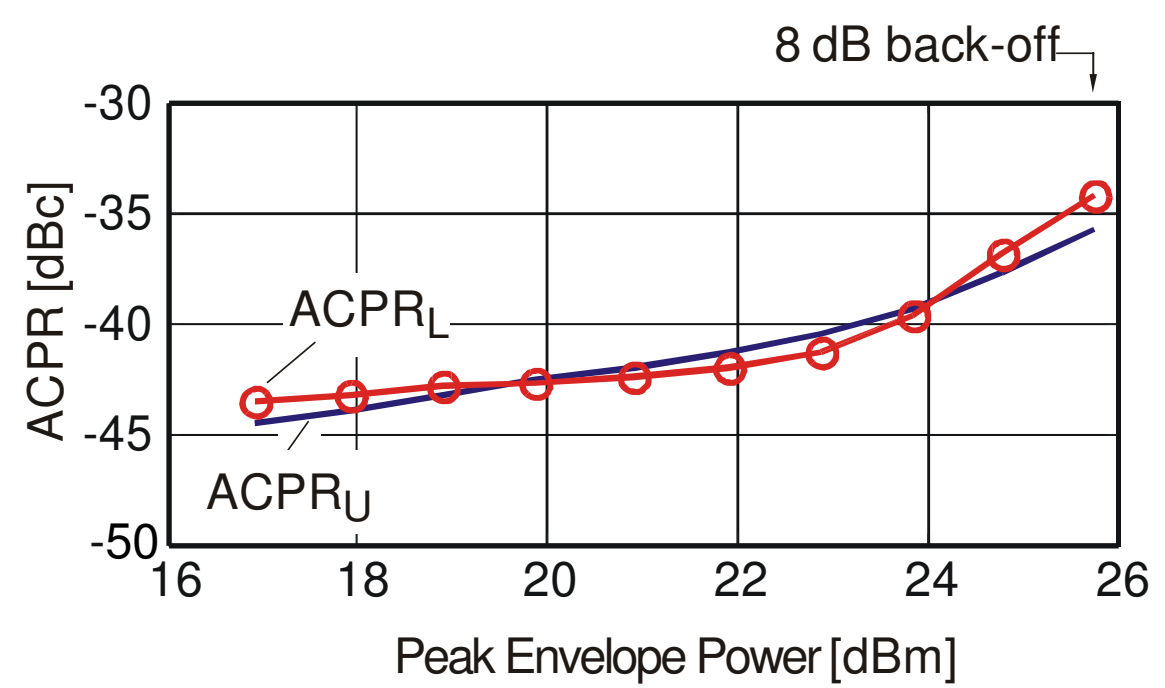

Figure 2.29: Lower and upper ACPs measured up to the 8dB back-offs at bias point: $\mathrm{Vds}=28 \mathrm{~V}, \mathrm{~V}_{\mathrm{gs}}=-4.6 \mathrm{~V}, \mathrm{f}_{0}=1 \mathrm{GHz}$.

Table 2.4: Summary of the performance of the 10W GaN HEMT continuous class-F power amplifiers.

\begin{tabular}{|l|l|}
\hline \multicolumn{2}{|c|}{ Novel Continuous Class-F Power Amplifier } \\
\hline \multicolumn{2}{|c|}{ Two-Tone Stimulus } \\
\hline Gain & $11 \mathrm{~dB}$ \\
\hline Output power at $\mathrm{P} 1 \mathrm{~dB}$ & $32.6 \mathrm{dBm}$ \\
\hline
\end{tabular}




\begin{tabular}{|c|c|}
\hline Maximum IMD3 asymmetry & $7.5 \mathrm{~dB}$ \\
\hline PEE $\eta$ (at 8.5dB back-off) & $19.1 \%$ \\
\hline \multicolumn{2}{|c|}{ Nine-Tone Stimulus } \\
\hline Gain & $13 \mathrm{~dB}$ \\
\hline PEP (at $8.5 \mathrm{~dB}$ back-off) & $26 \mathrm{dBm}$ \\
\hline PEE $\eta$ (at $8.5 \mathrm{~dB}$ back-off) & $15 \%$ \\
\hline ACPR (at $8.5 \mathrm{~dB}$ back-off) & $-33 \mathrm{dBc}$ \\
\hline
\end{tabular}

\subsubsection{Output Power Spectrum Comparison}

For a high power amplifier, such as the continuous class- $F$ power amplifier used in this measurement excited with two-tone, the IF components generated are large. This is especially true of IF1 (twice the modulation frequency) and IF2 (four times the modulation frequency), the most dominant base-band components and higher baseband components IF3 (six times the modulation frequency and IF4 (eight times the modulation frequency). The magnitude of the output power of IF1 was approximately comparable to the magnitude of $\omega_{1}$ and $\omega_{2}$, this signal (IF1) could not be load pulled directly using only an arbitrary waveform generator (AWG). A high power baseband ENI $240 \mathrm{~L} 20 \mathrm{kHz}$ to $10 \mathrm{MHz}$, 50W linear power amplifier was used to amplify the signal from the arbitrary wave generators (AWG), in order to actively loadpull significant baseband components e.g. IF1 and IF2.

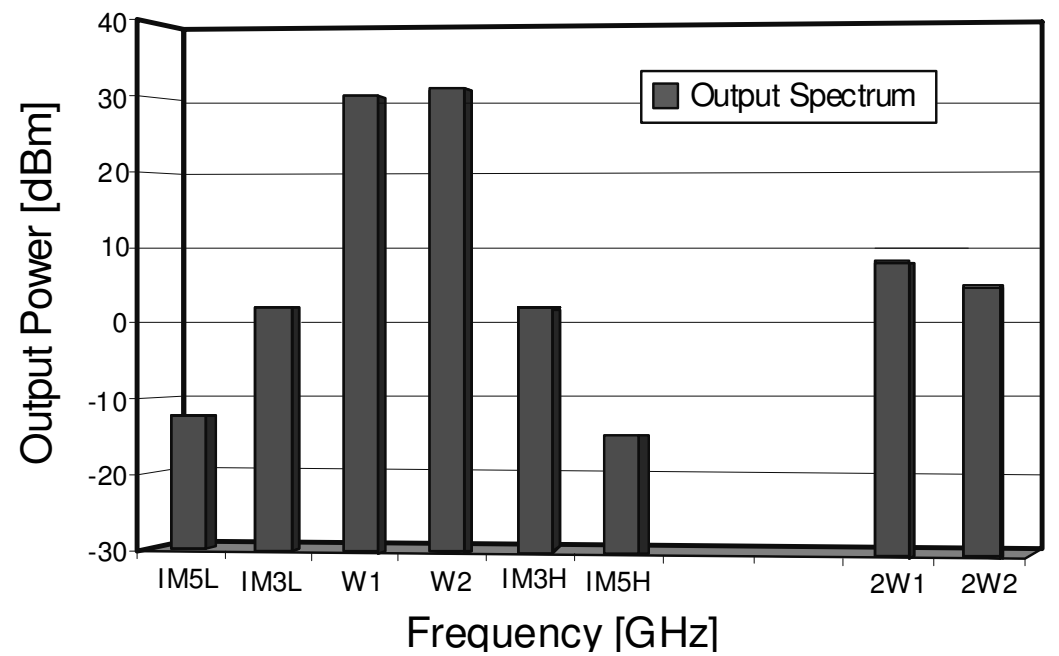

Figure 2.30: Measured simplified two-tone spectrum with enhanced measurement system for a continuous class- $\mathrm{F}$ power amplifier at carrier frequency of $1 \mathrm{GHz}$ with $500 \mathrm{KHz}$ modulation frequency when biased at $\mathrm{Vds}=28 \mathrm{~V}, \mathrm{Vgs}=-4.6 \mathrm{~V}$. 
Figure 2.30 shows the output spectrum of the continuous class-F power amplifier, measured when driven $1 \mathrm{~dB}$ into compression whilst presenting short circuit impedance to all the significant baseband components. The results indicate that the asymmetries in IMD products have greatly been minimized and a reduction of $8 \mathrm{dBc}$ is achieved in IMD3 products. The decisive comparison of the measurement system performance is the measurement of output power spectrum of the DUT. To validate the measured performance of the power amplifier the output spectrum of power amplifier was measured using a spectrum analyzer to measure output power precisely at the fundamental frequency in the frequency domain whilst keeping the measurement conditions strictly the same.

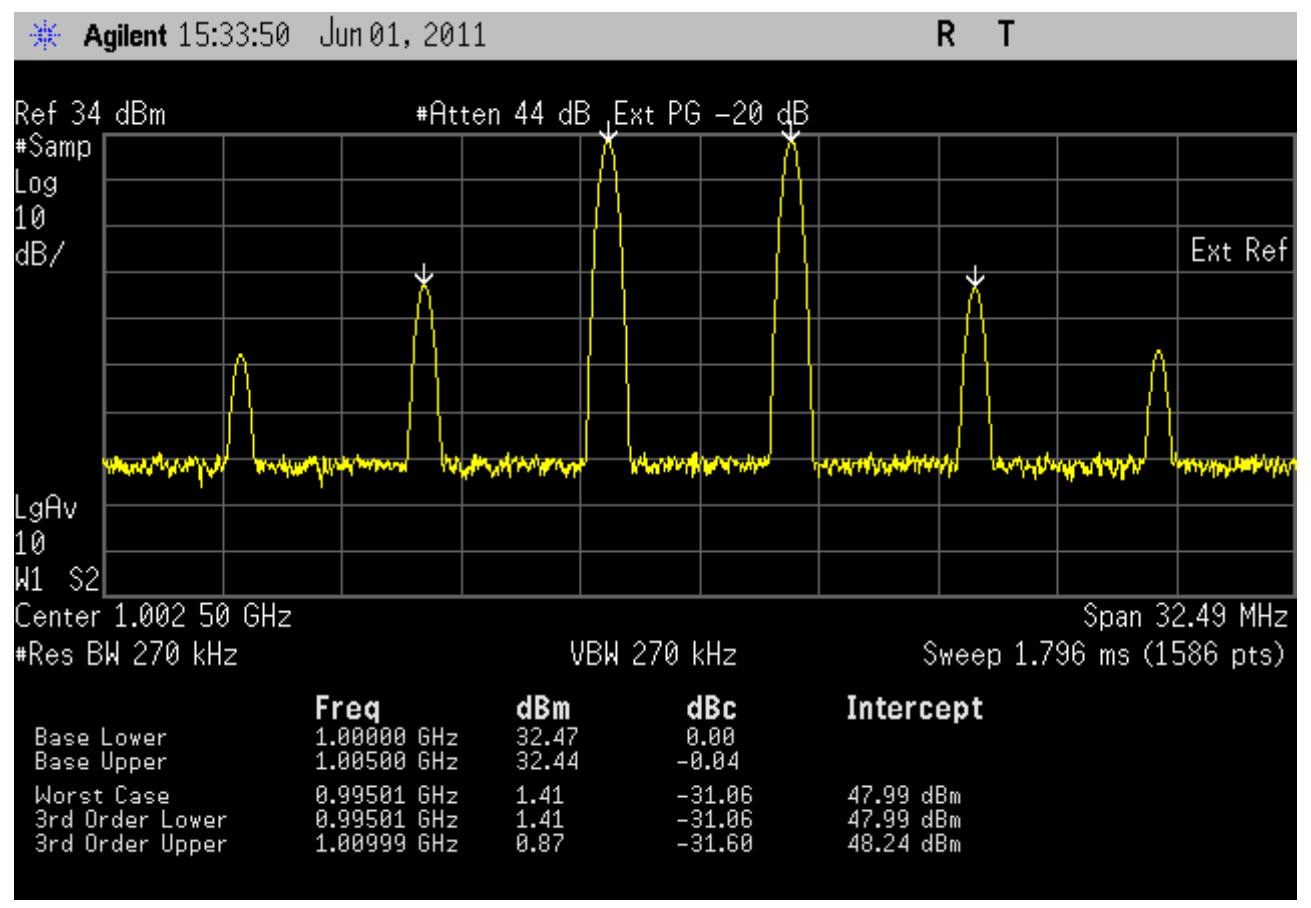

Figure 2.31: Measured simplified two-tone spectrum of continuous class-F power amplifier with Agilent spectrum analyzer for carrier frequency of $1 \mathrm{GHz}$ at $500 \mathrm{KHz}$ modulation frequency when biased at $\mathrm{Vds}=28 \mathrm{~V}, \mathrm{Vgs}=-4.6 \mathrm{~V}$.

The Agilent spectrum analyzer was connected to the load side of the enhanced measurement system to measure the distortion products of the continuous class-F amplifier accurately, in order to perform the comparison with the measured output power spectrum by modulated measurement software. It can be seen from Figure 2.30 and Figure 2.31 that the 
measured output power performance of the continuous class- $F$ power amplifier is almost identical. There exists an excellent agreement between both measured output spectrums with a slight variation of $\leq 1 \mathrm{~dB}$ in intermodulation products.

\subsection{Impedance Control Solution}

Tuning the impedance environment around a device can in turn result in improved device performance. For example gain, power, efficiency and linearity are all reliant on the design of the correct impedance matching network. In order to analyse the effect of impedance matching, it is necessary therefore to vary the impedance either at the input (source-pull) or at the output (load-pull) of the device, and to measure the resulting performance. For full optimisation, it is necessary to simultaneously control the impedance at the baseband, fundamental and significant harmonics, both at the input and at the output. Such systems are termed harmonic source and load-pull. The load reflection coefficient $\left(\Gamma_{\mathrm{L}}\right)$ can be described in terms of incident and reflected travelling waves. Analysis is often considered in a similar way to sparameters, using the analogous names $a_{1}, b_{1}, a_{2}$ and $b_{2}$ for the forward and reverse travelling waves, where $a_{1}$ is the incident travelling wave, $b_{1}$ is the travelling wave reflected at the device input, $b_{2}$ is the output of the device and $\mathrm{a}_{2}$ is the travelling wave reflected off the load. From this, it is clear that the load and source reflection coefficients are given by the ratios shown in equation 2.13 and 2.14 respectively.

$$
\begin{aligned}
& \Gamma_{L}=\frac{b_{2}}{a_{2}} \\
& \Gamma_{S}=\frac{b_{1}}{a_{1}}
\end{aligned}
$$

Using this fundamental theorem, measurement systems have evolved to emulate the impedance seen by the device by changing the reflected power injected into the device output port. This can be achieved either passively or actively. The intensive literature review of existing load-pull capabilities is documented in [42] which emphasize that there is need for a new concept to allow control and engineering of frequency components generated when a 
device or circuit is driven with a multi-tone stimulus. The impedance control required for multi-tone measurements can be broken down into two distinct sections: frequency components generated at RF frequencies and frequency components generated at baseband frequencies. The test set architecture was designed in such a way that baseband and RF components were separate, with the base-band and RF signal components recombined for measurement by the sampling oscilloscope. This architecture also ensures that there are separate load networks for base-band and RF frequencies, allowing different approaches to be adopted for each. The following sections will detail the capability of measurement system to maintain constant and frequency impedance broadband loads at IF and RF frequencies.

\subsubsection{Demonstration of Baseband Load-pull Capability}

The base-band impedance control architecture employed builds upon an active open-loop baseband load-pull architecture presented in [6]. This architecture takes little effort to control generated higher baseband components IF3 (six times the modulation frequency) and IF4 (eight times the modulation frequency). The effectiveness of IF active load-pull in maintaining constant and frequency independent impedance for the significant baseband components IF1 (twice the modulation frequency) and IF2 (four times the modulation frequency), and higher baseband components (IF3 and IF4) is demonstrated for 10W GaN HEMT, with deep class AB bias. The fundamental frequency for the modulated two-tone measurement was $2 \mathrm{GHz}$ with separation frequency of $2 \mathrm{MHz}$. The fundamentals and harmonic impedances were nominally terminated into a $50 \Omega$ environment. Figure 2.32 illustrates a measurement where the phase of the significant baseband components (IF1 and IF2) and higher baseband components (IF3 and IF4) were varied simultaneously, in steps of $18^{\circ}$ around the perimeter of the Smith chart, whilst keeping the magnitude of IF reflection co-efficient at unity. The RF output voltage and output current envelopes are shown in Figure 2.33 for the baseband reflection coefficient $\Gamma_{\mathrm{IF}}=1 \angle 180^{\circ}$. The computation of magnitude of the voltage and current envelope components at the fundamental frequency 
provides the possibility of directly observing the linearity performance of the device [43].

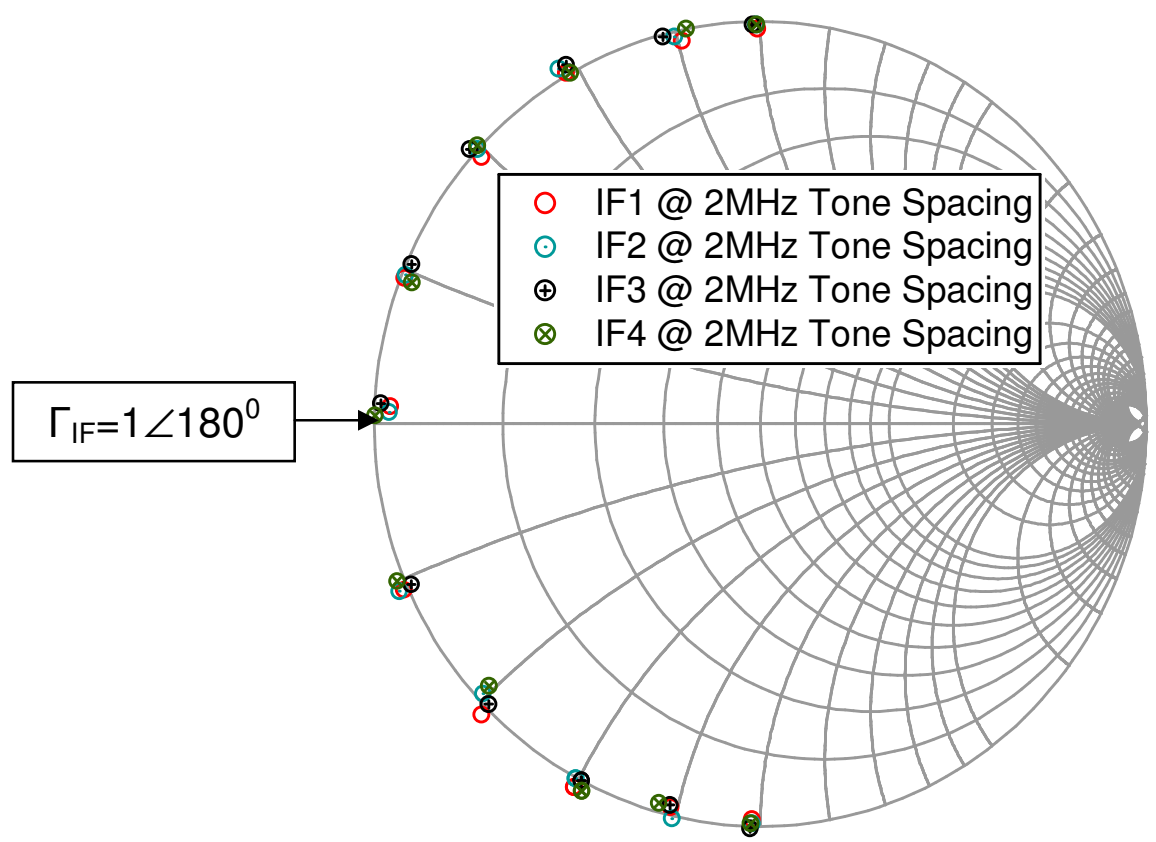

Figure 2.32: Measured IF impedances at $2 \mathrm{MHz}$ tone spacing with active IF load-pull.

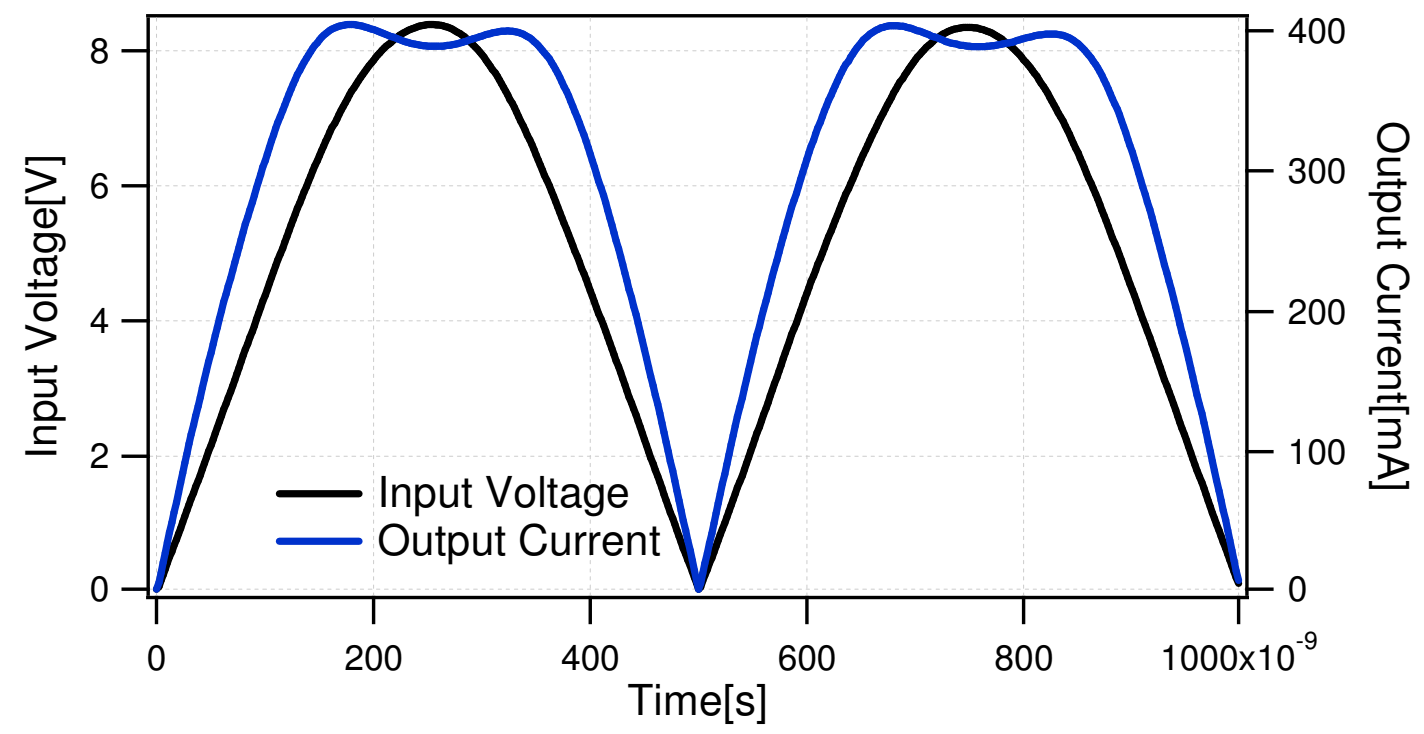

Figure 2.33: Measured input voltage and output current envelopes for baseband reflection coefficient $\left(\Gamma_{\mathrm{IF}}=1 \angle 180^{\circ}\right)$

Figure 2.34 shows the dynamic IF and RF load lines. In the case of baseband short circuit termination ( $\Gamma_{\mathrm{IF}}=1 \angle 180^{\circ}$ ), the IF load-line (achieved by plotting baseband current vs baseband voltage) remains completely vertical with no looping observed The analysis of measured results with respect to the 
dynamic load lines shows that the baseband impedance influences the RF dynamic load lines interaction with the knee region. This is due to the output voltage generated by the IF component which causes the measured output current and voltage dynamic load lines to interact with the knee region. In the case when a short circuit baseband impedance is presented to the device, it minimizes the knee interaction with transistor's I-V characteristic, producing a symmetrical knee interaction.

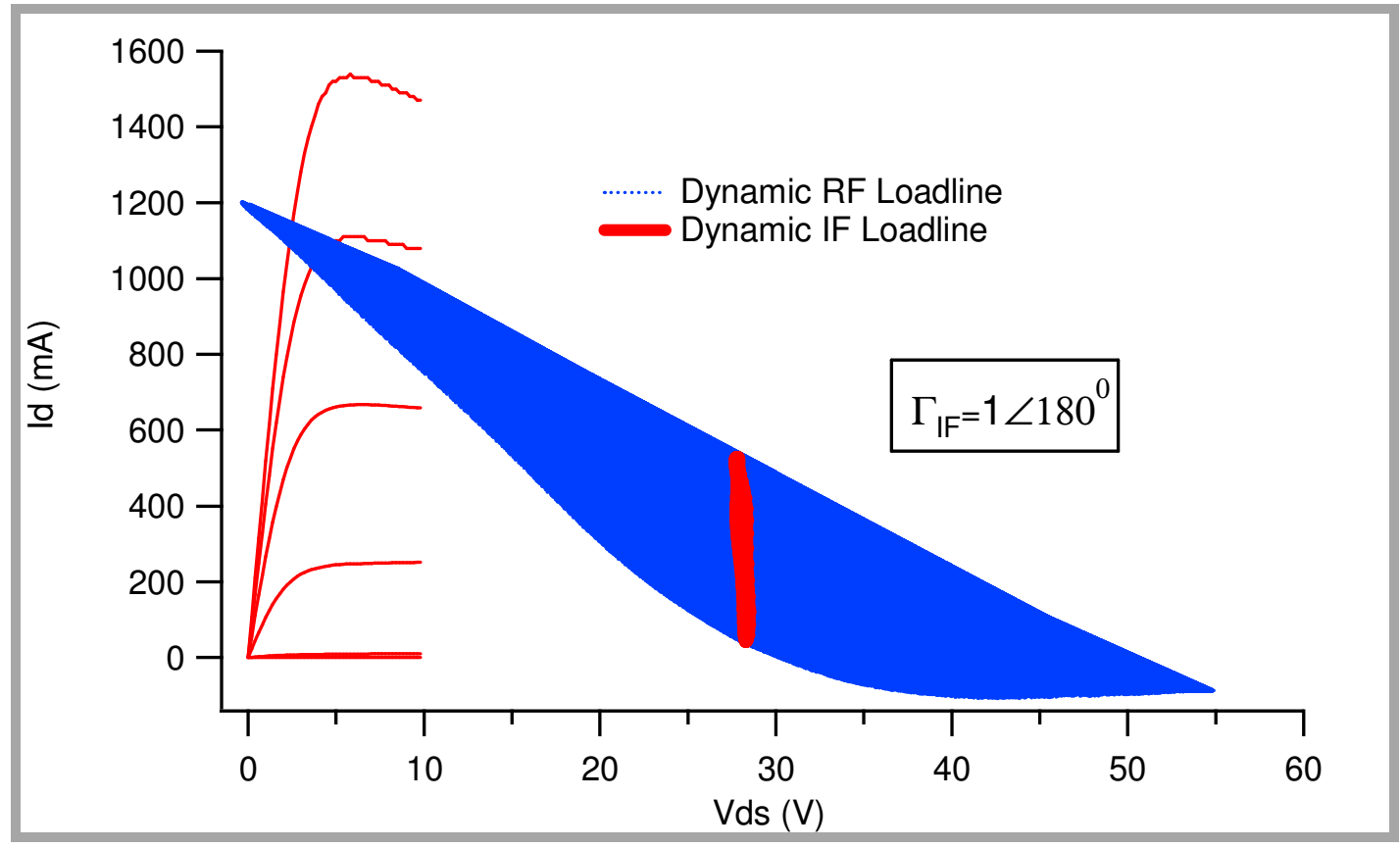

Figure 2.34: The measured dynamic RF and IF load-lines for baseband reflection coefficients $\left(\Gamma_{\mathrm{IF}}=1 \angle 180^{\circ}\right)$

Additionally, it is sometimes possible in the design process to use bias modulation to actually improve device performance. For example, a growing number of companies and universities are investigating the development of Envelope Tracking power amplifiers. These architectures use a variable voltage power supply, sometimes called a DC-DC converter or Modulator, which is dynamically adjusted to track the envelope of the applied modulation. In this way a designer can ensure that the amplifier always operates close to compression, even when operating at reduced output power level, therefore greatly improving the average efficiency. The presented enhanced system with the improved IF load-pull would allow a clear measurement of device performance under such a mode of operation and is therefore seen as a key 
tool in any such investigations, allowing a direct measurement and manipulation of the entire base-band spectrum, combined with the ability to view and analyze the resulting effect on RF performance. Active IF load-pull however has an important advantage in that it is able to seamlessly synthesise both positive impedances within the Smith chart, as well as negative impedances outside the Smith chart. This offers an alternate linearization approach which allows exploring further the optimum baseband impedances for best linearity conditions, the broadband IF impedance was swept over a measurement grid, including the short circuit condition, and extending some way outside the Smith chart.

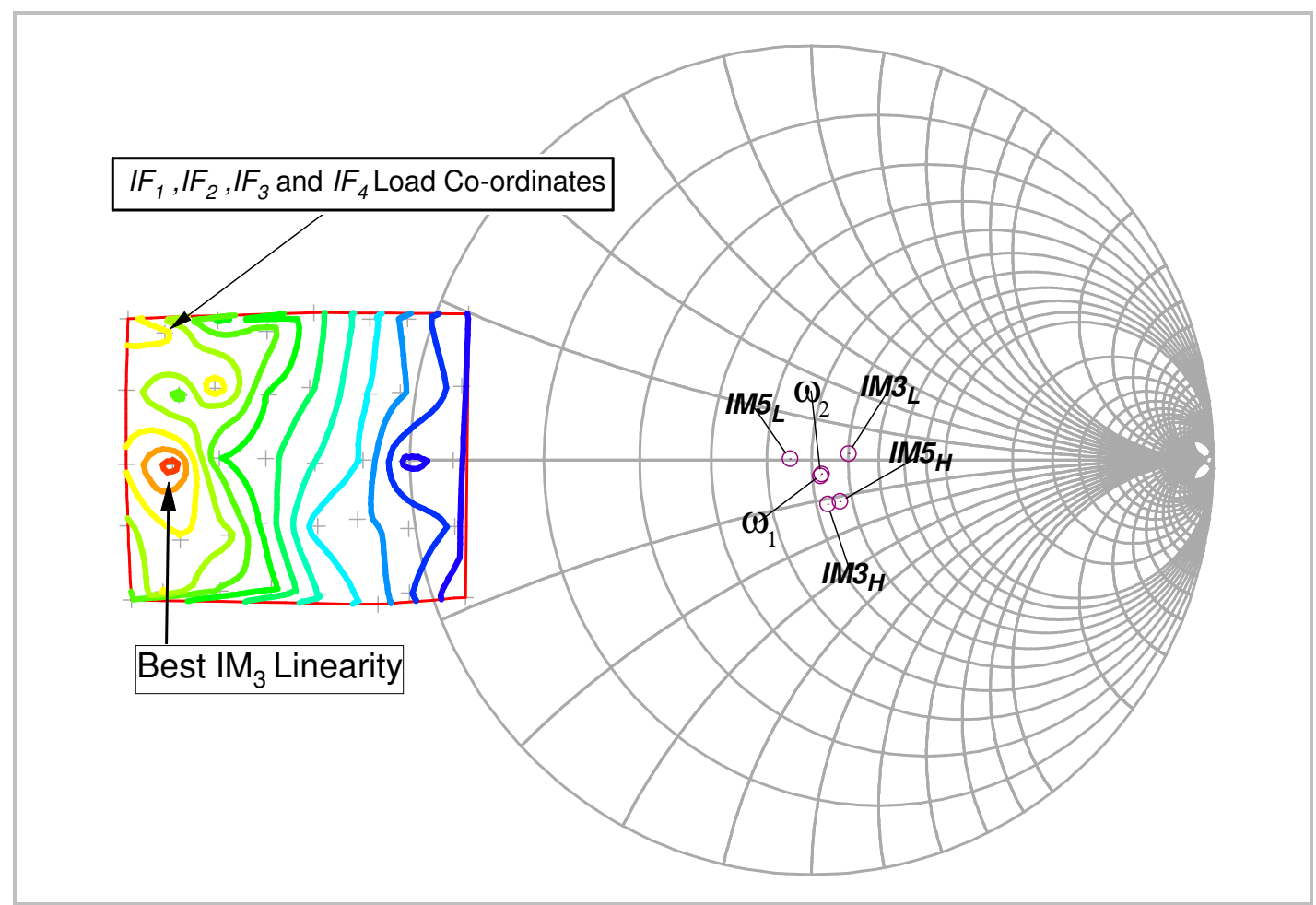

Figure 2.35: Measured $\mathrm{IM}_{3}$ linearity contours as a function of IF1, IF2, IF3 and IF4 loads.

Analysis of the measured results with respect to the dynamic load lines depicted in Figure 2.36 shows that the load IF impedance influences the linearity once the dynamic load lines interact with the knee region. In this case an optimum IM3, IF load impedance minimises the interaction with the transistor's I-V characteristic in the knee region, producing a condition with the most linear performance. In contrast to this, the response to varying the 
baseband impedance is quite evident from the contour plots. When the dynamic load lines interact with the knee region, significant improvements can be achieved by presenting the real negative baseband impedance, while significant variations in the distortion are achieved with reactive baseband impedance.

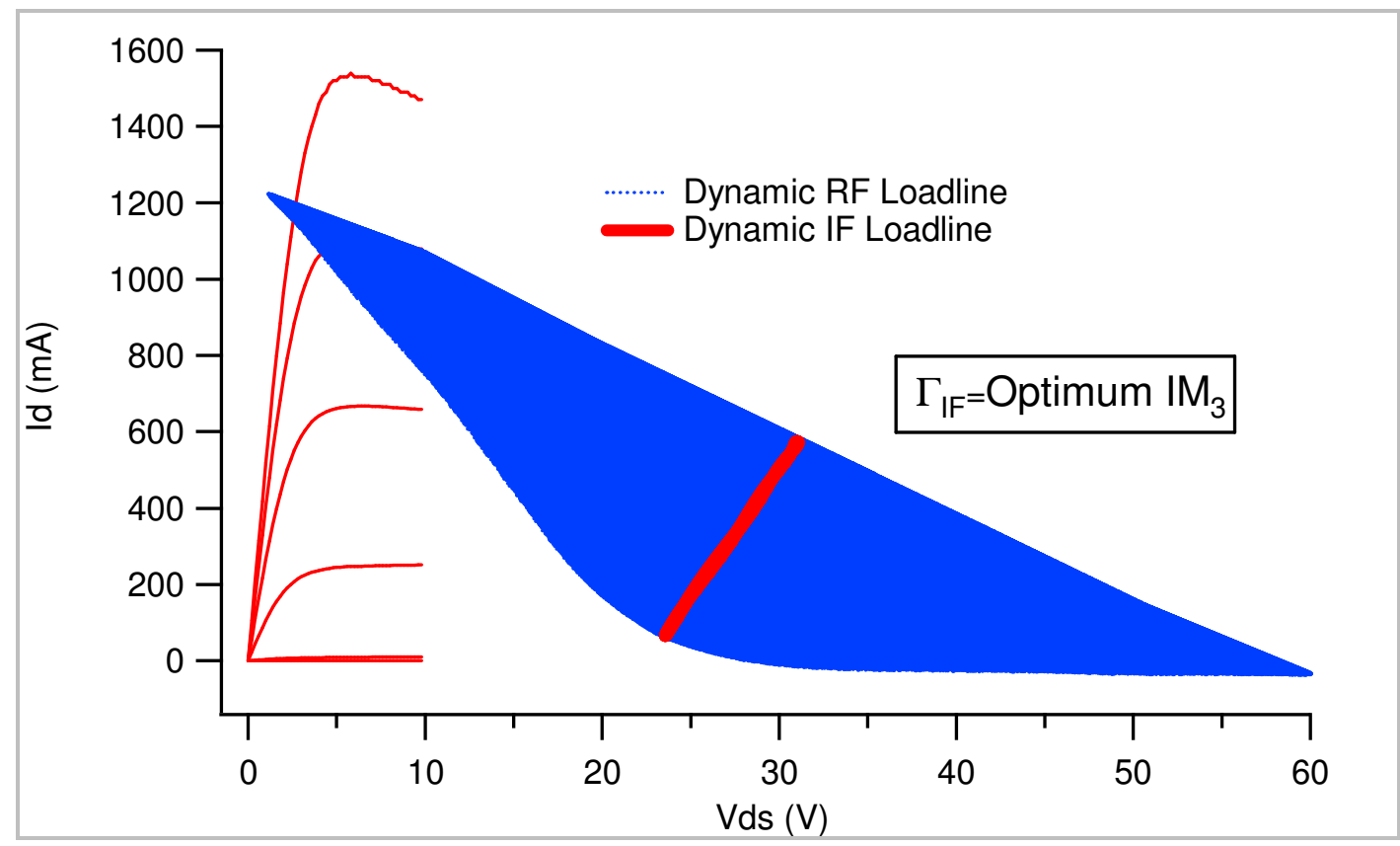

Figure 2.36: Dynamic RF and IF load-lines with static DCIV inset for best IM3 linearity.

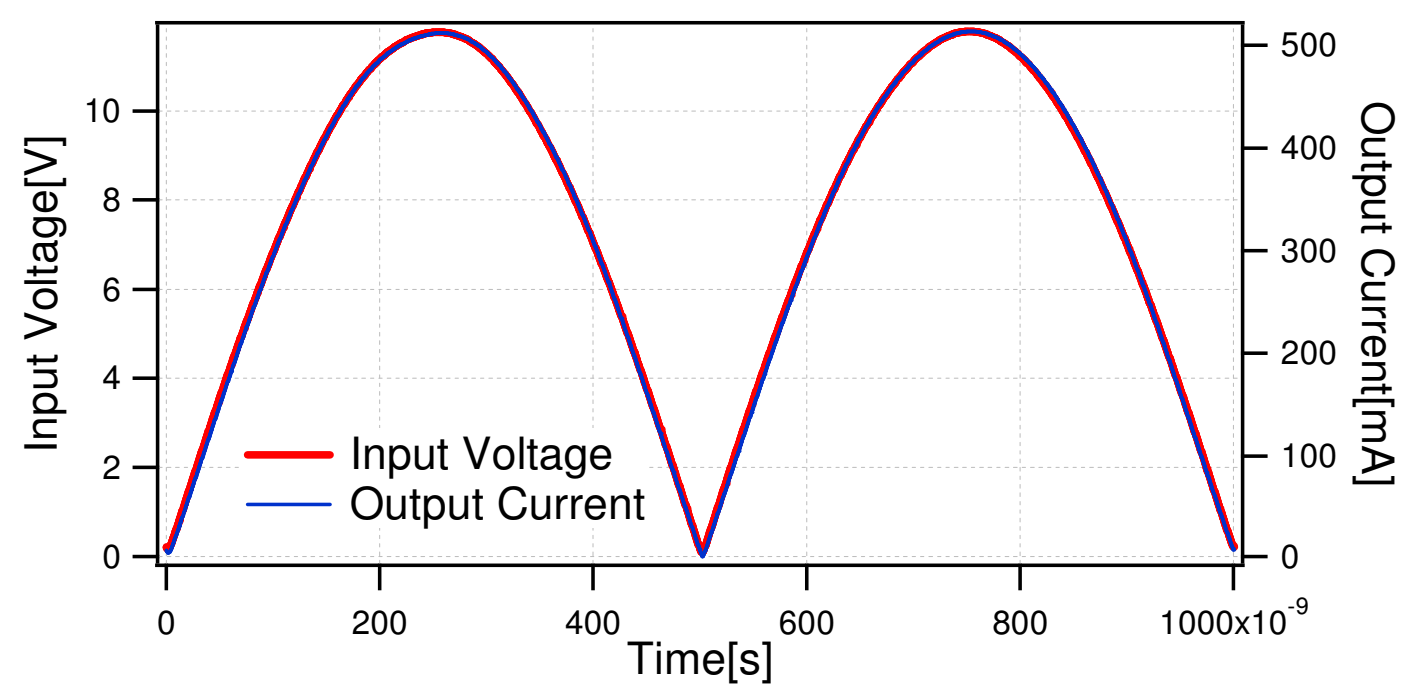

Figure 2.37: Measured input voltage and output current RF envelopes for best IM3 linearity. 
The improved linearity can be better explained by considering the output current and input voltage envelopes. The linearity performance of the device is quite evident from the magnitude of the voltage and current envelope components at the fundamental frequency. It can be seen from Figure 2.37 that the measured output current envelope from the non-linear behaviour of the transistor is quite symmetrical due to the termination of baseband components for optimum $\mathrm{IM}_{3}$ linearity.

The results presented here will be revisited in chapter 3 and chapter 4 of the thesis where applications of the enhanced measurement system will be discussed in greater details.

\subsubsection{Demonstration of RF Load-pull Capability}

Synthesis of active RF loads has been achieved through an open loop configuration. The main advantage is the high speed with which the load can be changed and the possibility to reach a reflection coefficient $>1$ and hence compensate for losses in the system making full matching possible at the DUT interface. The main drawback is the need for high power amplifier to increase load-pull power. An open loop system is shown in Figure 2.38.

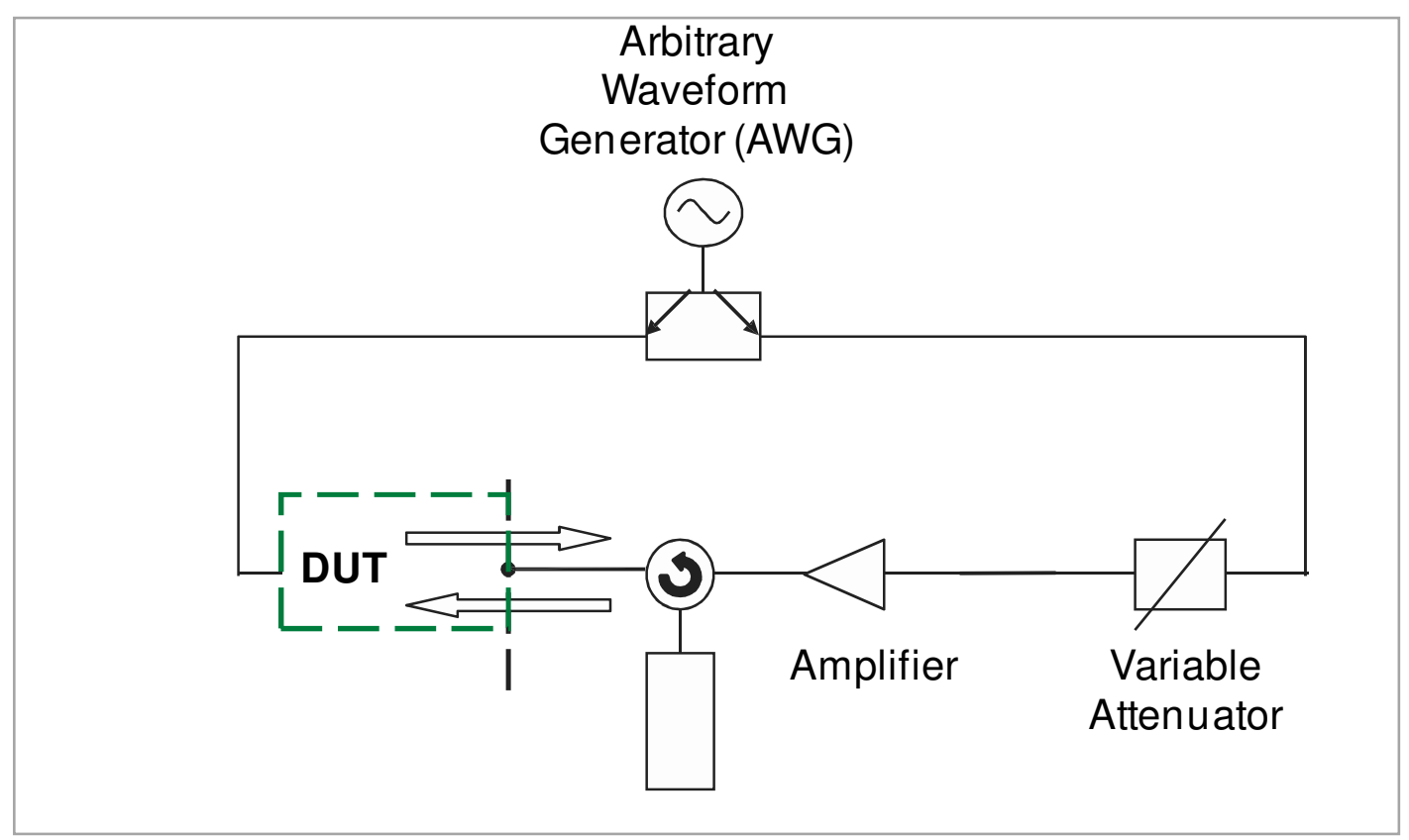

Figure 2.38: Two-tone spectrum up to third-order component polynomial transfer characteristic. 
In the open-loop active load-pull architecture of the modulated waveform measurement system, the channel-1 of the AWG was used as an excitation signal and channle-2 was used to load-pull the RF output components around the fundamental and $2^{\text {nd }}$ harmonic of the device under test, DUT. By changing the phase and magnitude of the load-pull signal the impedance seen by the DUT was changed manually. Active load-pull has been used for both for RF impedance tuning and IF impedance tuning at baseband frequencies. The power handling capability is limited mainly by the amplifier but is normally in the range of a few watts. Repeatability and speed can be questioned due to manual control but is not a major problem since active loads are used with insitu impedance measurements (not pre- calibrated). The ability to control RF impedances is vital in the full large signal characterisation of a device or circuit. As soon as we move away from linear measurements, it is no longer possible to predict the performance of a device or circuit purely from measurements conducted in a $50 \Omega$ environment. Load-pull, therefore, is an integral component in any measurement system designed to characterise and investigate non-linear performance.

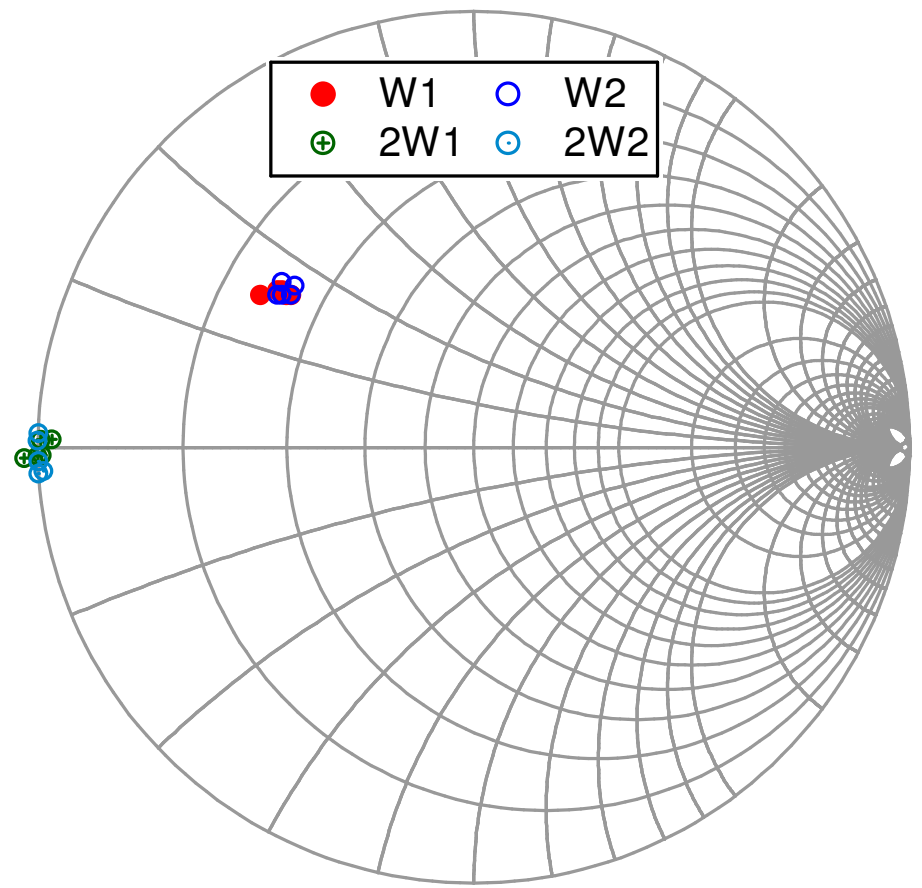

Figure 2.39: Emulation of class-B impedance environment for tone spacing ranging from $1 \mathrm{MHz}$ to $5 \mathrm{MHz}$ for $10 \mathrm{~W}$ GaN HEMTs, under two-tone excitation. 
Figure 2.39 shows the measured fundamental and $2^{\text {nd }}$ harmonic load impedances, independent of the drive level, when the device was driven into compression under two-tone excitation. The RF impedance plot suggests that different impedances can be synthesized other than $50 \Omega$, which is an essential case for emulation of different power amplifier modes. It can be seen that the dispersion in RF loads is minimal and RF fundamental load-pull closer to the optimum impedance was maintained as the tone spacing was swept from $1 \mathrm{MHz}$ to $5 \mathrm{MHz}$ to achieve a more appropriate characterisation capability. Furthermore, the ability to hold the load constant under complex modulated excitation is imperative to characterize the devices under realistic measurement conditions.

It has been effectively demonstrated that the precise load impedances can be maintained, under 9-tone excitation as can be seen from the Figure 2.40, using the modulated load-pull architecture elaborated earlier in this chapter and that the system can maintain constant impedance for varying drive conditions, this measurement also determines the scalable bandwidth of the system.

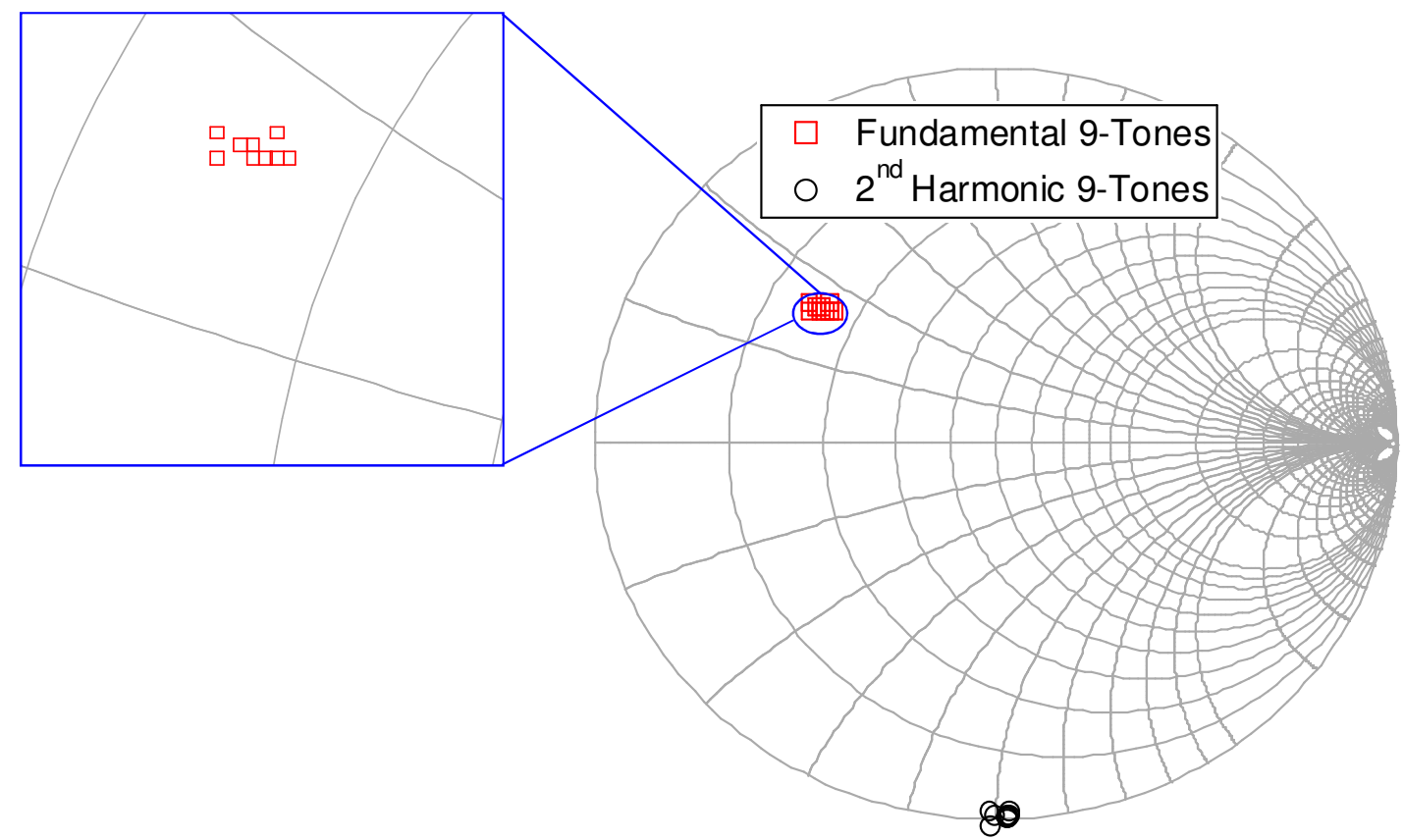

Figure 2.40: Emulation of class-J impedance environment, when 10W GaN HEMTs was subjected to nine-tone excitation at $2 \mathrm{GHz}$ with $1 \mathrm{MHz}$ modulation frequency. 
This quantifies the performance of the microwave device that can be expected when used with complex multi-tone signals rather that the two-tone modulated signal.

\subsubsection{Why Out-of-Band Impedance Control is Necessary?}

The out of-band components are usually utilized to control the in-band distortion, because of their distance from the fundamental signals, which occurs in close proximity to the requisite fundamental signal and is consequently quite difficult to directly filter without compromising the desired signal. Subsequently, out-of-band distortion components are considered to be a very important factor in terms of their effects on the behaviour of in-band distortion. Figure 2.41 shows the output signals $\omega_{1}$ and $\omega_{2}$, in addition to the unwanted distortion generated by the non-linear behaviour of the RF power device up to the third-order. The second-order out-of-band spectra consists of two frequency bands, namely the base-band (DC and IF band) as well as the $2^{\text {nd }}$ harmonic band (falling to the right of the in-band region) as can be seen in Figure 2.41. The baseband (including DC) consists of the base-band components caused by those components of an even-number order such as second-order IF1 $\left(\omega_{2}-\omega_{1}\right)$; fourth-order IF2 $\left(2 \omega_{2}-2 \omega_{1}\right)$; sixth-order IF3 $\left(3 \omega_{2}-\right.$ $\left.3 \omega_{1}\right)$ and so forth, together with the harmonic band generated by second and higher-order components. The baseband components generated due to device nonlinear behaviour are controlled and engineered in the work documented so far. The second-order products $2 \omega_{1}, 2 \omega_{2}$ and $\omega_{1}+\omega_{2}$ in the second harmonic zone need to be engineered or controlled to quantify their effect on the linearity of the microwave device.

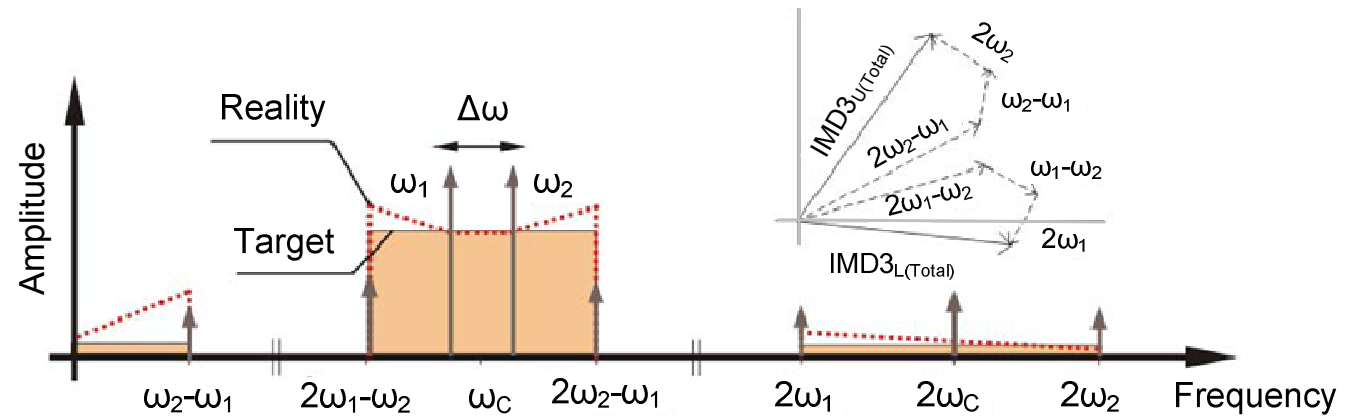

Figure 2.41: Two-tone spectrum up to third-order component polynomial transfer characteristic. 
Figure 2.41 illustrates a typical spectrum up to third-order nonlinearity. The transfer characteristic with third-order terms is as follows:

$$
i(t)=a_{1} * v(t)+a_{2} * v^{2}(t)+a_{3} * v^{3}(t)
$$

For third-order nonlinearity of the microwave device the mathematical analysis yields:

$$
\begin{aligned}
& y_{3}(t)=a_{3} * V^{3}(t)=a_{3}\left(V_{1} \cos \left(\omega_{1} t\right)+V_{2} \cos \left(\omega_{2} t\right)\right)^{3} \\
& =\cos \left(\omega_{1} t\right)\left(\frac{3}{4} a_{3} V_{1}^{3}+\frac{3}{2} a_{3} V_{1} V_{2}^{2}\right)+\cos \left(\omega_{2} t\right)\left(\frac{3}{4} a_{3} V_{2}^{3}+\frac{3}{2} a_{3} V_{1}^{2} V_{2}\right) \\
& +\frac{3}{2} a_{3} V_{1}^{2} V_{2} \cos \left(\left(2 \omega_{1}-\omega_{2}\right) t\right)+\frac{3}{2} a_{3} V_{1} V_{2}^{2} \cos \left(\left(2 \omega_{2}-\omega_{1}\right) t\right) \\
& +\frac{3}{2} a_{3} V_{1}^{2} V_{2} \cos \left(\left(2 \omega_{1}+\omega_{2}\right) t\right)+\frac{3}{2} a_{3} V_{1} V_{2}^{2} \cos \left(\left(2 \omega_{2}+\omega_{1}\right) t\right) \\
& +\frac{1}{4} a_{3} V_{1}^{3} \cos \left(3 \omega_{1} t\right)+\frac{1}{4} a_{3} V_{2}^{3} \cos \left(3 \omega_{2} t\right)
\end{aligned}
$$

Third-order terms at frequencies $2 \omega_{1}-\omega_{2}$ and $2 \omega_{2}-\omega_{1}$ are the most interesting inter-modulation products since they fall within the channel bandwidth. Generally speaking, odd-order terms generate in-band distortion. Interestingly, with respect to IMD behaviour, as illustrated, the enhanced modulated waveform measurement system provides constant load impedance at the relevant frequency bands and this is demonstrated in the following section.

In general, a precise control of impedances at baseband and $2^{\text {nd }}$ harmonic frequencies is indeed demanded, with the latter more specifically for enhancing overall efficiency, which highlights that the comprehensive control of the out-of-band impedance can be achieved which is essential to emulate the different PA modes through the application of active load-pull. However, for wideband applications, it becomes imperative to practically fulfil all these requirements simultaneously, leading to the reliable characterization of the device behaviour.

\subsubsection{Comprehensive Out-of-Band Impedance Control}

The ability of the system to maintain baseband, fundamental and second harmonic loads is critical over a wider bandwidth. This capability is evident in Figure 2.42 where the second harmonic and fundamental loads are 
kept constant with a slight spreading in the emulated load reflection coefficients takes place. The emulation of desired load reflection coefficients at the baseband causes the spreading to occur at the respective tones around the fundamental and $2^{\text {nd }}$ harmonic impedances. The load emulation around the frequency bands of interest is very tricky and time consuming under complex tones (i.e. more than two and three tones), however, every effort was made to minimize the spread to demonstrate the system capability in emulating the impedances when the device was subjected to modulated excitation.

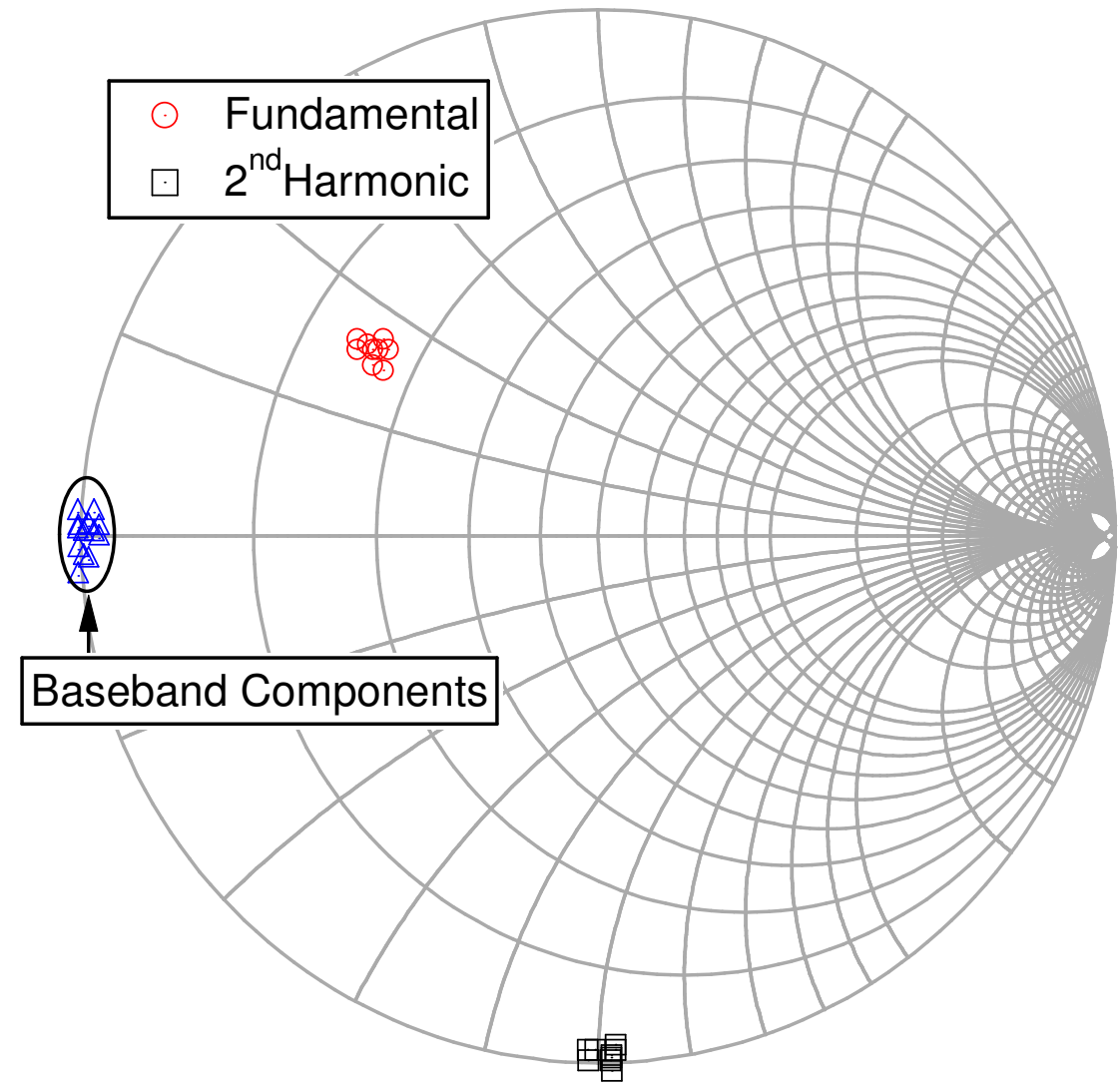

Figure 2.42: Measured baseband loads $\left(\Gamma_{\mathrm{IF}}\right)$ under 2-tone excitation, for $10 \mathrm{~W}$ GaN HEMTs biased in class $A B$, at for different modulation frequencies for a class-J emulated RF impedance environment.

In other words, the system is able to emulate the desired loads at the baseband, carrier and $2^{\text {nd }}$ harmonic without any trouble. Figure 2.42 shows that system can maintain constant and frequency independent impedances at the baseband and RF simultaneously. 


\subsection{Chapter Summary}

This chapter presented an extensive overview of memory effects in microwave device along with the measurement systems and approaches that have been used to characterize them. From this review it is evident that the existing measurement systems although able to characterize microwave devices, suffer from certain limitations.

these systems are either time consuming, not capable for extensive out-of-band impedance characterization or are not able to capture the relevant information simultaneously at baseband, around the carrier and harmonics

\& they are not suitable for characterizing devices operating under multitone stimuli

For holistic characterization of microwave devices, it is important that a rapid, robust and effective measurement approach is developed which can tackle both these issues, mentioned above, prevalent in the existing approaches. The chapter detailed the required necessary enhancement carried out to improve the measurement system accurately describing a non-linear device's response to a multi-tone signal, including all of the significant distortion terms. Firstly, a suitable trigger clock was engineered to allow on-board averaging on the scope to increase the quality of the measurements which was not possible previously due to inability of the sampling oscilloscope to freeze the multi-tone signals. Then a new measurement technique referred as "Time Domain Partitioning" was formulated that allowed for spectrally efficient capture of repetitive multi-tone signals.

Measurement speed and dynamic range have both been dramatically improved through the adoption of the new technique thus employing the full capabilities of the sampling oscilloscope used. To quantify the persistent effect of baseband impedance on the microwave power device, the baseband load-pull architecture was modified to account for higher baseband components which are multiples of the baseband fundamental frequency. The modification of the RF architecture to provide for active synthesis of loads thorough the use of a Tektronix AWG7000 Arbitrary Waveform Generator, and its two independent yet coherent channels over a wide modulation 
bandwidth was presented. As a result, the waveform measurement system is able to maintain independent and constant impedance control for each individual tone present across both the IF and the RF impedance environment. A section here detailed the calibration interpolation software and the verification of the IF calibration that allowed for efficient characterisation of low frequency components for multi-tone measurements. The final section of the chapter has discussed and described the full functionality of the enhanced measurement system to measure the power amplifiers under multi-tone excitations. The impedance control for robust characterization of the device is demonstrated experimentally across a wide bandwidth at both baseband and RF frequencies simultaneously to ensure control of all significant components. Applications of this new measurement technique and developed system are discussed and explained in depth in the subsequent chapters. 


\subsection{References}

1. J. Vuolevi, J. Manninen, and T. Rahkonen, "Measurement technique for characterising memory effects in RF power amplifiers", IEEE Transactions on Microwave Theory and Techniques, 2001, p. 1383-1389.

2. J. Vuolevi, J. Manninen, and T. Rahkonen, "Cancelling the memory effects in RF power amplifiers", in IEEE Int. Symp. Circuits and Systems, pp. 5760, 2001.

3. N. Le Gallou, et. al, "Analysis of Low Frequency Memory and Influence on Solid State HPA Intermodulation Characteristics," IEEE MTT-S Int. Microwave Sym. Dig., pp. 979-982, May 2001.

4. N. B. Carvalho and J. C. Pedro, "A Comprehensive Explanation of Distortion Sideband Asymmetries," IEEE Trans. on Microwave Theory and Tech., Vol. MTT-50, September 2002, pp. $2090-2101$.

5. H. Ku and J. S. Kenney, "Behavioural Modelling of RF power Amplifiers Considering IMD and Spectral Regrowth Asymmetries," IEEE MTT-S Int. Microwave Symp. Dig., June 2003, pp. $799-802$.

6. A. A Alghanim," High Power Waveform Measurement System Enabling Characterisation of High Power Devices Including Memory Effects" Ph.D. thesis, Cardiff University, March 2008.

7. S. Nuttink,E. Gebara, J. Laskar, B. Wnager, M. Harris. "RF Performance and Thermal Analysis of AlGaN/GaN power HEMTs in Presence of Self Heating Effects," IEEE MTT-S Int. Microwave Symp. Dig., June 2002, pp. $921-924$.

8. S. Augaudy, R. Quere, J. P. Teyssier, M. A. Di Forte-Poisson, s. Cassette, B. Dessertenne, S. L. Delage "Pulse Characterization of Trapping and Thermal Effects of Microwave GaN Power FETs" ," IEEE MTT-S Int. Microwave Symp. Dig., May 2001, pp. 427 - 430.

9. J. Brinkhoff and A. E. Parker "Effect of Baseband Impedance on FET Intermodulation", IEEE Trans. on Microwave Theory and Tech., vol.51,no.3, March 2003, pp.1045-1051.

10. Martins, J.P., N.B. Carvalho, and J.C. Pedro, "A Figure of Merit for the Evaluation of Long Term Memory Effects in RF Power Amplifiers", in 2006. IEEE MTT-S International, Microwave Symposium Digest, June 2006, pp. 1109-1112.

11. José Carlos Pedro, Nuno Borges Carvalho, "Intemodulation Distortion in Microwave and Wireless Circuits", Artech House, 2003.

12. M. Franco, A. Guida, A. Katz, P. Herczfeld, "Minimization of Bias-Induced Memory Effects in UHF Radio Frequency High Power Amplifiers with 
Broadband Signals", in Proc of IEEE Radio and Wireless Symp. 2007, pp.369-372

13. J. Vuolevi and T. Rahkonen, "Third-order intermodulation distortion caused by thermal power feedback," in Proc. Norchip'99, Oslo, Norway, pp. 121125.

14.J. Vuolevi and Timo Rahkonen, "Distortion in RF Power Amplifiers", Norwood, MA: Artech House, 2003.

15.L. Gallou, J. M. Nebus, E. Ngoya, and H. Buret, "Analysis of Low Frequency Memory and Influence on Solid State HPA Intermodulation characteristics", IEEE MTT-S Int. Microwave Symp. Dig., Phoenix, Arizona, May 2001.

16.S. C. Cripps, "RF power amplifiers for wireless communications", $2^{\text {nd }}$ edition. Norwood, MA: Artech House, 2006.

17.P. B. Kenington, "High Linearity RF Amplifier Design", Norwood, MA: Artech House, 2000.

18. W. Bösch and G. Gatti, "Measurement and Simulation of Memory Effects in Predistortion Linearizer", IEEE Transactions on Microwave Theory and Tech, Vol.37, December 1989, pp.1885-1890.

19.A. Khanifar, N. Maslennikov, and B. Vassilakis, "Bias Circuit Topologies for Minimization of RF Amplifier Memory Effects", 33rd European Microwave Conference, Germany, October 2003, pp. 13491352.

20.D. J. Williams, J. Leckey, and P. Tasker, "A Study of the Effect of Envelope Impedance on Intermodulation Asymmetry Using a Twotone Time Domain Measurement System", IEEE MTT-S Int. Microwave Symp. Dig., Seattle, WA, June 2002, pp. 1841-1844.

21.J. Vuolevi and T. Rahkonen, "Analysis of Amplitude Dependent Memory Effects in RF Power Amplifiers", Proc. Of ECCTD, Helsinki University of Technology, Vol. II, Finland, August 2001, pp.37-40.

22.H.Ku, M. D. Mckinley, and J. S. Kenney, "Quantifying Memory Effects in RFPower Amplifiers", IEEE Trans. on Microwave Theory and Tech, Vol.50, No. 12, December 2002, pp.2843-2849.

23. A. E. Parker and J. G. Rathmell, "Novel Techniques for Determining Bias, Temperature and Frequency Dependence of FET Characteristics", IEEE MTT-s Internationsl Microwave Symp. Dig., Seattle, WA, June 2002, pp.993-996. 
24. N. B. De Carvalho and J. C. Pedro, "A Comprhensive Expalantion of Distortion Sideband Asymmetries", IEEE Trans. on Microwave Theory and Tech, Vol.50, No. 9, September 2002, pp.2090-2101.

25. A. Ahmad, E. R. Srinidhi, and G. Kompa, "Efficient PA modelling Using Neural Network and Measurement Set-up for Memory Effect Characterization in the Power Device", IEEE MTT-S International Microwave Symp. Dig., Long Beach, USA, June 2005.

26. Y. Yang, J. Yi, J. Nam, B. Kim and M. Park, "Measurement of Two Tone Transfer Characteristics of High Power Amplifier", IEEE Transactions on Microwave Theory and Tech, Vol.49, no. 3, March 2001, pp.568-571.

27.Spirito, M. , Pelk, M.J. , van Rijs, F. , Theeuwen, S.J.C.H., Hartskeerl, D. , de Vreede, L.C.N. , "Active Harmonic Load-Pull for On-Wafer Out-of-Band Device Linearity Optimization", IEEE Transaction on Microwave Theory \& Techniques., vol. 54,no. 12, pp. 4225-4236, Dec. 2006.

28. Macraigne, F.; Reveyrand, T.; Neveux, G.; Barataud, D.; Nebus, J.-M.; Soury, A.; NGoya, E. "Time-domain Envelope Measurements for Characterization and Behavioral Modeling of Nonlinear devices with Memory" IEEE Transactions on Microwave Theory and Techniques, Volume 54, Issue 8, Aug. 2006 Page(s):3219 -3226.

29. J. M. Golio, "The RF and Microwave Handbook", Boca Raton, CRC Press, London, 2008.

30. David M. Pozar "Microwave and RF Design of Wireless Systems", Second Edition, John Wiley \& Sons 2001.

31.S.A. Maas, "Nonlinear Microwave Circuits", Second Edition, Artech House Microwave Library, 2003.

32. "Vector Signal Analyser (VSA )," Accessed the user documentations, in January 2011 at http://www.agilent.com

33. "Large Signal Network Analyser Technology," Accessed the preliminary product Overview, in January 2011 at http:// www.maurymw.com

34. M. Demmler, P.J. Tasker, and M. Schlechtweg, "A Vector Corrected High Power On-Wafer Measurement System with a Frequency Range for the Higher Harmonics up to $40 \mathrm{GHz}$, in 24th IEEE European Microwave Conference,1994, pp.1367-1372.

35. Tudor V. Williams, "A Large Signal Multi-tone Time Domain Waveform Measurement System With Broadband Active Load Impedance Control', Ph.D. thesis, Cardiff University, December 2007.

36. Williams D. J., "Non-linear Measurement System and Techniques for RF Power Amplifier Design", PhD thesis, Cardiff University,2003. 
37.Benedikt, J.; Gaddi, R.; Tasker, P.J.; Goss, M. "High-power time-domain measurement system with active harmonic load-pull for high-efficiency base-station amplifier design" IEEE Transactions on Microwave Theory and Techniques, Volume 48, Issue 12, Dec. 2000 Page(s):2617 - 2624.

38. R. Gaddi, "Characterisation and Modelling of Silicon RF LDMOS Transistors" Ph.D. thesis, University of Wales Cardiff, January 2002.

39. M. Akmal, J. Lees, Z. Yusoff, S. Bensmida, S. Woodington, V. Carrubba, S. Cripps, J. Benedikt, K. Morris, M. Beach, J. McGeehan, P. J. Tasker, "An Enhanced Modulated Waveform Measurement System for Robust Characterisation of Microwave under Modulated Excitation", in Proc. of $41^{\text {st }}$ European Microwave Conference, October 2011.

40. V. Carrubba, A. L. Clarke, M. Akmal, J. Lees, J. Benedikt, P. J. Tasker, S. C. Cripps, "The Continuous Class-F Mode Power Amplifier", in Proc. $40^{\text {th }}$ European Microwave Conference, October 2010, Page(s): 1674 - 1677.

41. V. Carrubba, J. Lees, J. Benedikt, P. J. Tasker, S. C. Cripps, "A Novel Highly Efficient Broadband Continuous Class-F RFPA Delivering 74\% Average Efficiency for an Octave Bandwidth," IEEE MTT-S International Microwave Symp. Dig., Baltimore, US, June 2011, Page(s): 1 - 4.

42. M.S. Hashmi, "Analysis, Realization and Evaluation of Envelope Load-pull System for Both CW and Multi-tone Applications," PhD thesis, Cardiff University, February 2009.

43.J. Lees, T. Williams, T.; S. Woodington,P. McGovern, S. Cripps, J. Benedikt, P. Tasker, "Demystifying Device related Memory Effects using Waveform Engineering and Envelope Domain Analysis" in Proc. $38^{\text {th }}$ European Microwave Conference, October 2008, Page(s): 753 - 756. 


\section{Baseband Electrical Memory Investigations}

\subsection{Introduction}

High power amplifiers, used in cellular basestations, commonly employ digital predistortion to improve their linearity and efficiency. Terms such as look up tables and memory polynomials are used to describe the types of digital predistortion. From an RF perspective these terms are significantly different from the typical specifications like linearity, efficiency and even peak to average ratio (PAR). Predistortion is not a new concept to power amplifier design. It is, in essence, creating a signal that has "anti-phase" spectral information which is then used to eliminate the spectral errors at the output of the amplifier. Features of this predistorted signal include an increased bandwidth and an increased PAR of the input signal. In an RF sense, having a wider bandwidth requires the designer to increase the amplifier capabilities over a wider bandwidth to eliminate roll off at the edge of the band when the correction is applied. Also the higher peaks of the input signal can drive the amplifier deeper into saturation as the PAR expands. This can push an amplifier into unknown states closer to breakdown. It has been shown that different algorithms achieve different levels of performance increase for different signals and amplifiers [1]. Examining the information from these predistorters can provide some insight to the limiting factors that exist for each. For instance, looking at a simple memory polynomial predistorter for an 
amplifier at different back off locations can show that the memory coefficients change. Examining these coefficients shows that more computational memory is needed as the amplifier is driven deeper into compression. However, it is more likely that the algorithm is just having difficulty in capturing the true compression characteristics and not an actual increase in the memory of the power amplifier. Especially, when considering the memory dispersion of modulated $\mathrm{AM} / \mathrm{AM}$ at an average power level there is a very small difference between each power level. Examining the predistorter and the signal to the power amplifier, show the limitations to the power amplifier and the algorithms being used to improve the linearity of basestation power amplifiers.

Hence, deterioration of the distortion compensation becomes a problem. Thus, it is imperative to understand the memory effects in order to be able to reduce or compensate for them. This is because these dynamic effects impact signal distortions (linearity) as well as power efficiency performances. This chapter aims to provide a demonstration through measurements, as it is unfortunately quite usual that the simulation models fail to simulate such high level nonlinear phenomena as memory effects, that electrical memory effects can influence intermodulation. Only baseband electrical memory effects are considered, because they are believed to be the dominant cause of memory effects in microwave power transistors. The drive levels are set to ensure weakly and strong nonlinear behaviour from microwave power device under two-tone which is a simplistic excitation and helps understanding multiple mixing, in order to quantify the device's inherent nonlinearity. Once investigated and examined, it is clear that the measured hysteresis on the dynamic transfer characteristics and inter-modulation product asymmetry, can be very sensitive to variations in baseband impedance, and this reinforces the importance of optimization of broad baseband impedance termination at the device output for reduction in baseband electrical memory.

\subsection{Memory Properties of Nonlinear System}

An intensive research carried out into designing a predistortion linearizer for IMD cancellation, with AM-AM and AM-PM characteristics inverse to that of power amplifiers to be linearized [2]. This approach was 
acceptable under the condition that predistortion circuits could reproduce the same characteristics for any signal envelope frequencies, which unfortunately could not be fulfilled. As a result, there is significant degradation in IMD cancellation performance followed by a more sophisticated implementation of linearization techniques. At this point, it becomes rather interesting to raise the subject of nonlinear system classification with respect to the inherent memory property. Accordingly, nonlinear systems can be classified into memoryless system, quasi-memoryless system, and systems with memory.

However, it is important to understand the type of excitation signal essential for characterizing a system's memory behaviour. From the application point of view, a static single-tone power sweep measurement is generally favoured during power amplifier characterization, additionally aiding the design of matching topologies. However, it is well known that a CW characterization does not provide sufficient information on the system's memory. Moreover a statically linearized power amplifier reported in [2], under single-tone stimuli, fails to show any improvement in IMD characteristics compared to a nonlinearized power amplifier under dynamic two-tone excitation. This is due to the presence of non-negligible memory, which forced the system response to vary with the baseband frequencies. As a result, such effects can be characterized only under a modulated test signal environment.

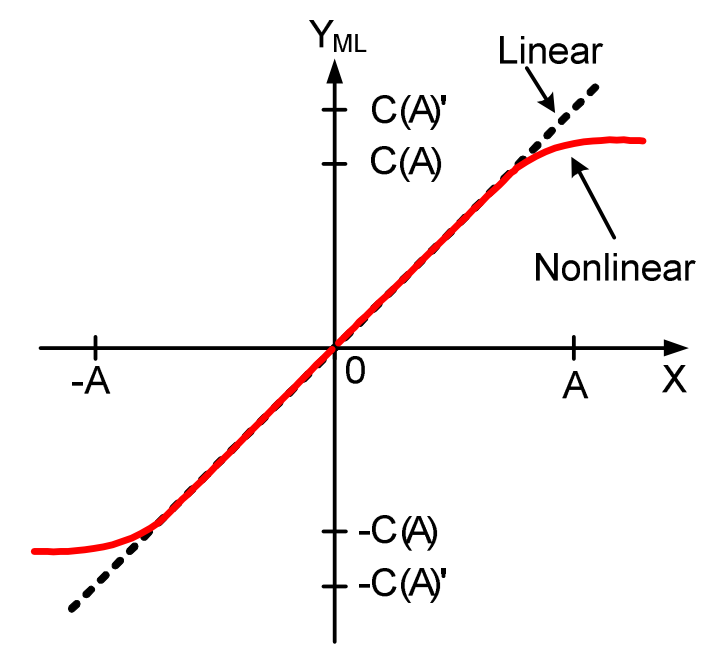

Figure 3.1: Nonlinear input-output characteristics of a memoryless system [3].

Consider a sinusoidal input signal $\mathrm{x}(\mathrm{t})$ with an angular frequency of $\omega_{\mathrm{c}}$, which is modulated by amplitude $A(t)$ and phase $\varphi(t)$

$$
x(t)=A(t) \cdot \cos \left[\omega_{c} t+\varphi(t)\right]
$$


In order to simplify the analysis, in case of small-signal excitation, the linear system response of a memoryless system $\mathrm{y}_{\mathrm{ML}}(\mathrm{t})$ can be expressed as

$$
y_{M L}(t)=C(A) \cdot \cos \left(\omega_{c} t+\varphi\right)
$$

Where $C(A)$ is the amplitude conversion curve, commonly referred to as AMAM curves, which is always defined at a specific amplitude or power level.
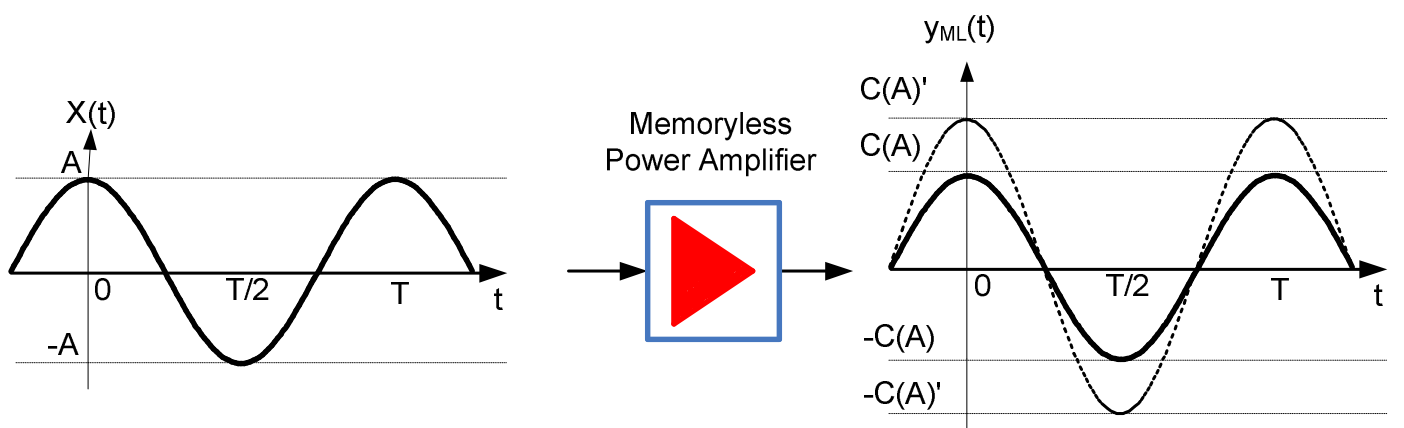

Figure 3.2: Time domain waveforms of a memoryless system [3].

The input-output response characteristics of memoryless nonlinear systems are shown in Figure 3.1 as a single curve. Figure 3.2 clearly shows the absence of phase shift between the input and the output waveform. For a quasi-memoryless system the output response $\mathrm{y}_{\mathrm{QM}}(\mathrm{t})$ takes the following form;

$$
y_{Q M}(t)=C(A) \cdot \cos \left[\omega_{c} t+\varphi-\Phi(A)\right]
$$

Where $\Phi(A)$ is the input amplitude dependent phase distortion additionally induced in a quasi-memoryless system due to AM-PM conversion. Thus, if phase distortion is present, the system must posses certain amount of memory.

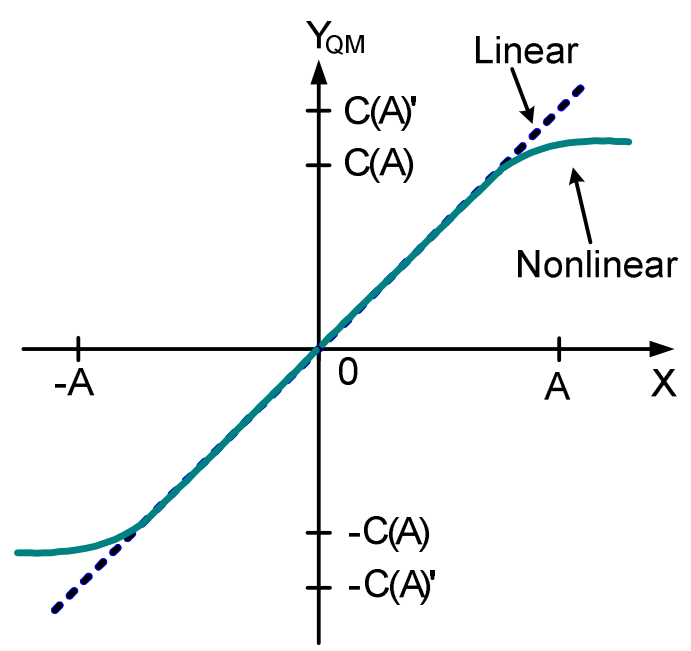

Figure 3.3: Depiction of nonlinear input-output characteristics of quasimemoryless system [3]. 
At any given time instant, the response amplitude $C(A)$ and the phase $\Phi(A)$ are functions of only instantaneous input amplitude $A$ as shown in Figure 3.3 and Figure 3.4, respectively, with the assumption that $A$ is time invariant. In case of a modulated single carrier, the system time constant $T \ll 1 / f_{\text {env }}$, where $f_{e n v}$ is the maximum baseband frequency indicating negligible memorizing time. In such systems, symptoms of memory effect manifest mainly in terms of phase distortion. A nonlinear system with memory constitutes a more realistic scenario, a consequence of which is that the output of the system will no longer respond instantaneously. As shown in Figure 3.5, which depicts the nonlinear time domain input-output characteristics, a phenomenon of hysteresis emerges due to the system's memorizing effect preventing the overlap of input-output trajectory with the increase in signal swing.
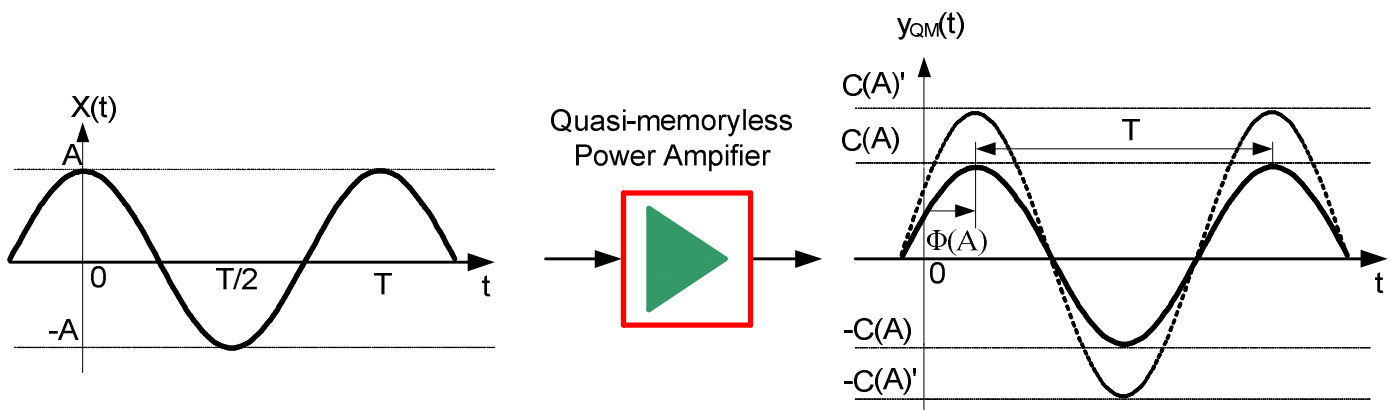

Figure 3.4: Time-domain waveforms of a quasi-memoryless power amplifier system [3].

In other words, as indicated in Figure 3.5, the rising curves from s' to $s$ fail to maintain the same trajectory when the amplitude is increased from $A^{\prime}$ to $A$ [3].

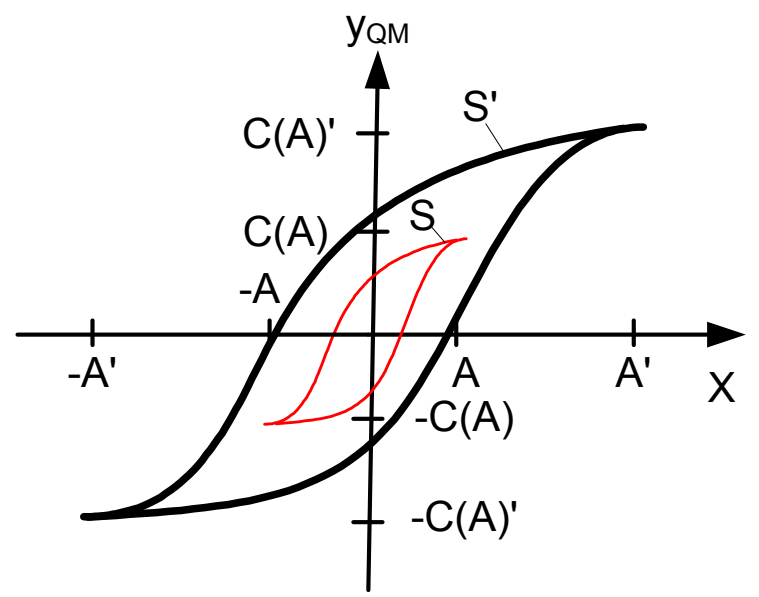

Figure 3.5: Nonlinear input-output characteristics for a quasi-memoryless system with memory representing the hysteresis behaviour [3]. 
The hysteresis behaviour, hence, tends to show a strong dependence of memory-effect with input amplitude. In frequency-domain, the AM-AM and AM-PM characteristics become a function of the carrier frequency. In the case of a modulated carrier, for instance, the system time constant $T$ will be comparable to $1 / f_{\text {env }}$ indicating a significant amount of memorizing time. However when the device is driven over the drive level ' $A$ ' then the impedance seen by the baseband components can in fact have a dramatic effect on the overall measured waveforms. If a generated base-band current component is presented with high impedance then it will naturally generate a voltage. This generated voltage manifests itself as a modulation on the bias lines of the device. This modulation is a common cause of electrical memory, which is probably the most common memory effect exhibited by the device. Any modulation on the drain voltage supplied also results in a dynamic characteristic interacting with the device's boundary conditions, causing additional distortion.

An illustration of the difficulties in correctly characterising nonlinear power amplifiers with memory effects is given in Figure 3.6 where measurement noise and linear propagation delay of the system or device can be seen as broadening similar to that caused by memory effects, however, broadening of the peak after $A$ and $-A$ is typically due to memory effects only. The broadening in the trailing part of the input-output characteristics is demystified in [4] and hysteresis on the upper part is demystified as part of this work through the precise and independent control of baseband impedance.

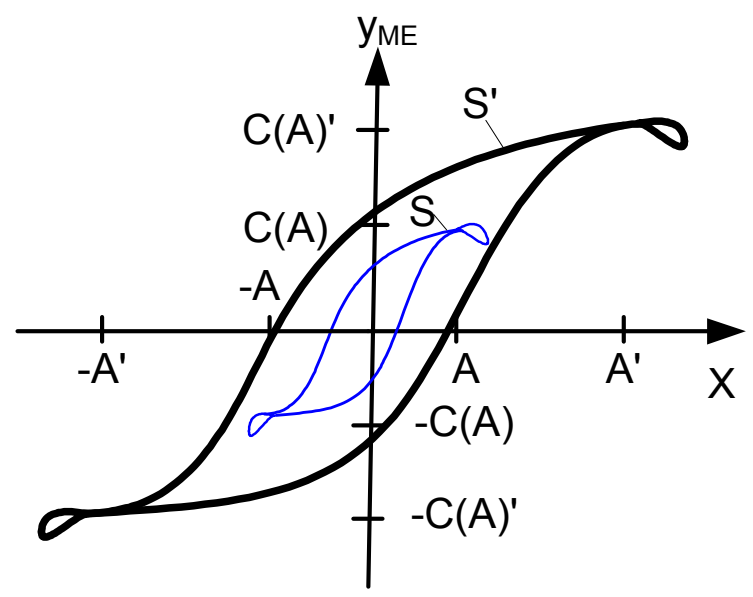

Figure 3.6: Nonlinear input-output characteristics for a system with memory representing the hysteresis behaviour [3]. 


\subsubsection{Characterization of Memory Effects}

When the power device is relatively backed-off, its performance is linear and operation is in the linear zone. On the other hand, when the power device is driven into compression, the device is operating in its nonlinear zone. There are two main nonlinear effects that are produced by the device under modulated excitations, "in-band effects" and "out-of-band effects". The first one, the "in-band effects", produces a constellation distortion and consequently, a worse BER (Bit Error Rate) value. These are not easy to remove due to their proximity to the signal. The second group, the "out-ofband effects", produces a spectrum widening and so, a higher ACPR (Adjacent Channel Power Ratio) value. Thus, it highlights the importance to characterise these anomalous nonlinear effects in order to understand and correct them.

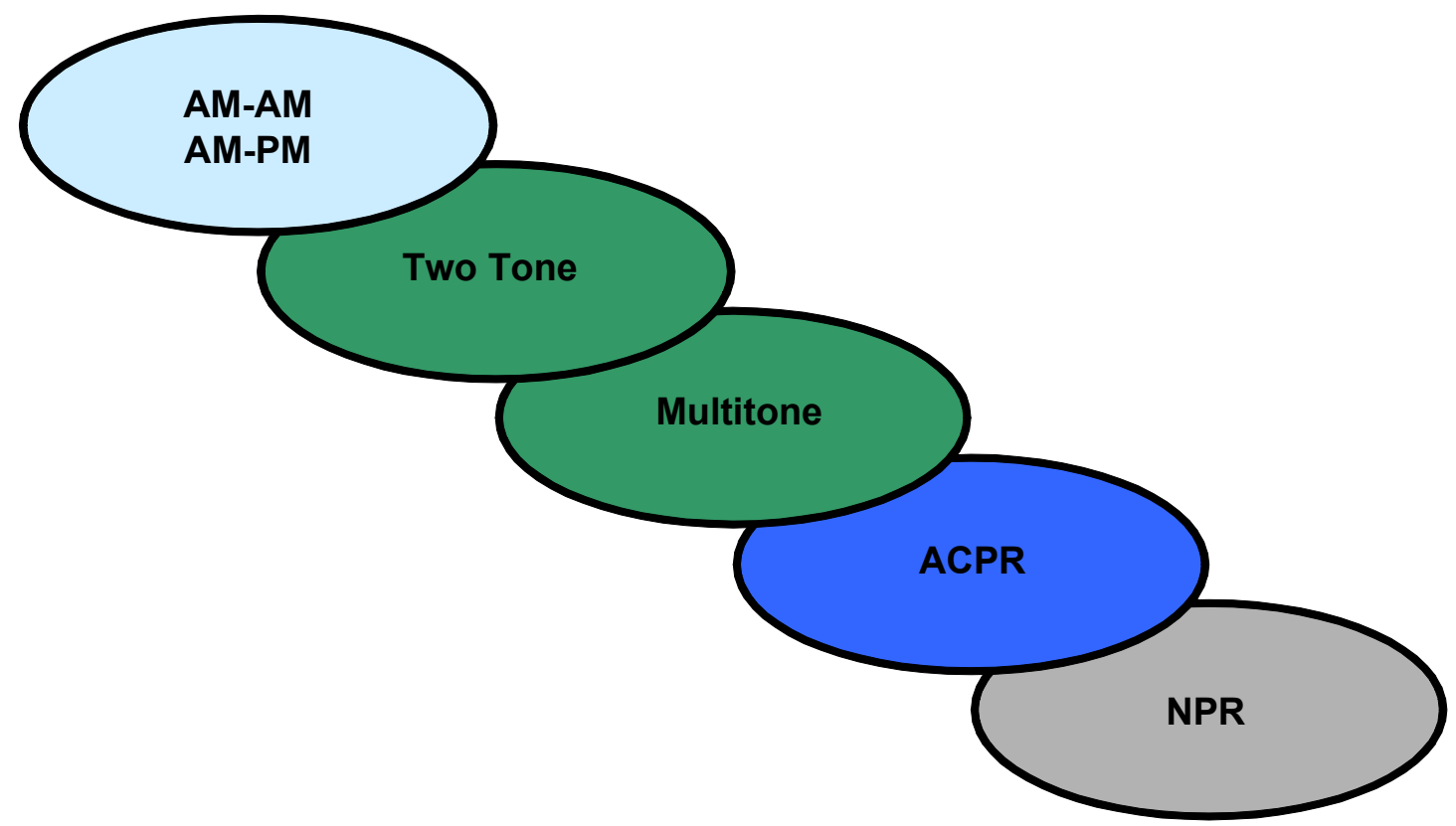

Figure 3.7: Different device inherent nonlinearity characterization methods.

There are many methods that can be used to characterize nonlinear behaviour of power device as can be seen from Figure 3.7. Common methods include two-tone, multi-tone, and noise power ratio measurements. These conventional test methods provide a means of comparing figures of merit (like third-order intercept) from different devices. Indeed it is meaningful and straight forward to test the nonlinear device under a realistic signal environment. However, since these signals are unpredictable in nature, it is 
rather difficult to interpret the device behaviour from conventional figure of merits such as the third-order intercept and the $1 \mathrm{~dB}$ compression point. Therefore, more complex dynamic characterization methods are needed in order to analyse and extract more information about nonlinearity in broadband and high power devices.

\subsubsection{Continuous Wave Characterization}

A nonlinear system when subjected to a sinusoidal input signal produces output spectral components harmonically related to the input fundamental frequency. The nonlinearity of the system can be estimated from the fundamental tone only, by sweeping the input amplitude and measuring the output response of the fundamental tone. Any deviation from the linear response is known as amplitude dependent amplitude distortion AM/AM. Furthermore, nonlinear systems always exhibit some amount of memory and thus the phase of the output response will also deviate as a function of input amplitude, which is known as amplitude dependent phase distortion AM/PM.

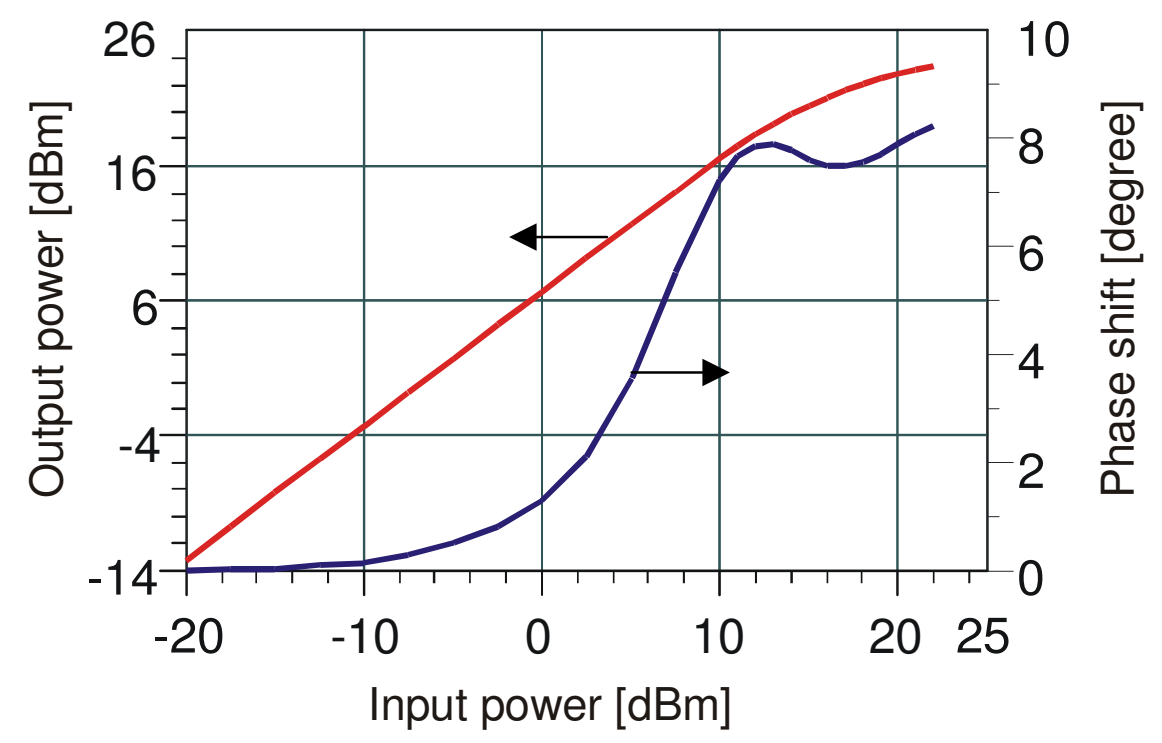

Figure 3.8: AM/AM and AM/PM conversion of a power device [5].

Usually, $\mathrm{AM} / \mathrm{AM}$ and $\mathrm{AM} / \mathrm{PM}$ conversions are measured by sweeping the power of a single-tone sine wave as the input signal of the amplifier. The AM/AM characteristics enable the evaluation of an important figure of merit called the 1-dB compression point. It has been noted that $A M / A M$ and 
AM/PM characteristics are only a function of the input signal level and independent of its envelope frequency which can be seen from Figure 3.8.

These characteristics are generated from quasi-memoryless power device, which means that the device output signal is only a function of the instantaneous input signal. These characteristics will cause a symmetrical output spectrum when the amplifier input signal is multi-tone [5], [6], [7]. Similarly, we get a symmetrical spectral regrowth in the output spectrum when input to the amplifier is a digitally modulated signal.

\subsubsection{Two-Tone Characterization}

The power device's spectral regrowth behaviour can not be observed under static continuous wave (CW) stimuli. It can only be revealed by considering modulated signals which are rather more complex than the $\mathrm{CW}$ signal. A more simplistic representation of a telecommunication signal is a two-tone, which has indeed, proved to an efficient solution for evaluating the linearity properties of a nonlinear microwave device.

A simple two tone excitation signal with tones having equal amplitude and zero phase offset can be expressed by equation (3.4);

$$
\begin{aligned}
& x(t)=A *\left[\cos \left(\omega_{1} t\right)+\cos \left(\omega_{2} t\right)\right] \\
& x(t)=2 A * \cos \left(\frac{\omega_{1}-\omega_{2}}{2} t\right) * \cos \left(\frac{\omega_{1}+\omega_{2}}{2} t\right) \\
& x(t)=2 A * \cos \left(\omega_{m} t\right) * \cos \left(\omega_{c} t\right)
\end{aligned}
$$

Where $\omega_{m}=\left(\omega_{1}-\omega_{2}\right) / 2$ and $\omega_{c}=\left(\omega_{1}+\omega_{2}\right) / 2$ are the modulation and carrier frequencies. The corresponding nonlinear output signal would be given by equation (3.5).

$$
y(t)=\sum_{p}^{\infty} A_{0 p} * \cos \left(\omega_{p} t+\varphi_{0 p}\right)
$$

Where $\omega_{p}=m \omega_{1}+n \omega_{2}$ with $m, n \in Z$, which shows that the output signal will be composed of many mixing spectral terms involving all possible combinations of $\pm \omega_{1}$ and $\pm \omega_{2}$ as shown in Figure 3.9. The output signal of the device will have unwanted frequency components generated at frequencies $2 \omega_{1}-\omega_{2}$ and $2 \omega_{2^{-}} \omega_{1}$ which are termed as lower and upper $3^{\text {rd }}$ order intermodulation distortion (IMD3) products respectively. Also the lower and 
upper $5^{\text {th }}$ order intermodulation products (IMD5) products can be seen at frequencies $3 \omega_{1}-2 \omega_{2}$ and $3 \omega_{2}-2 \omega_{1}$ respectively which become very significant when the device is driven near the compression.

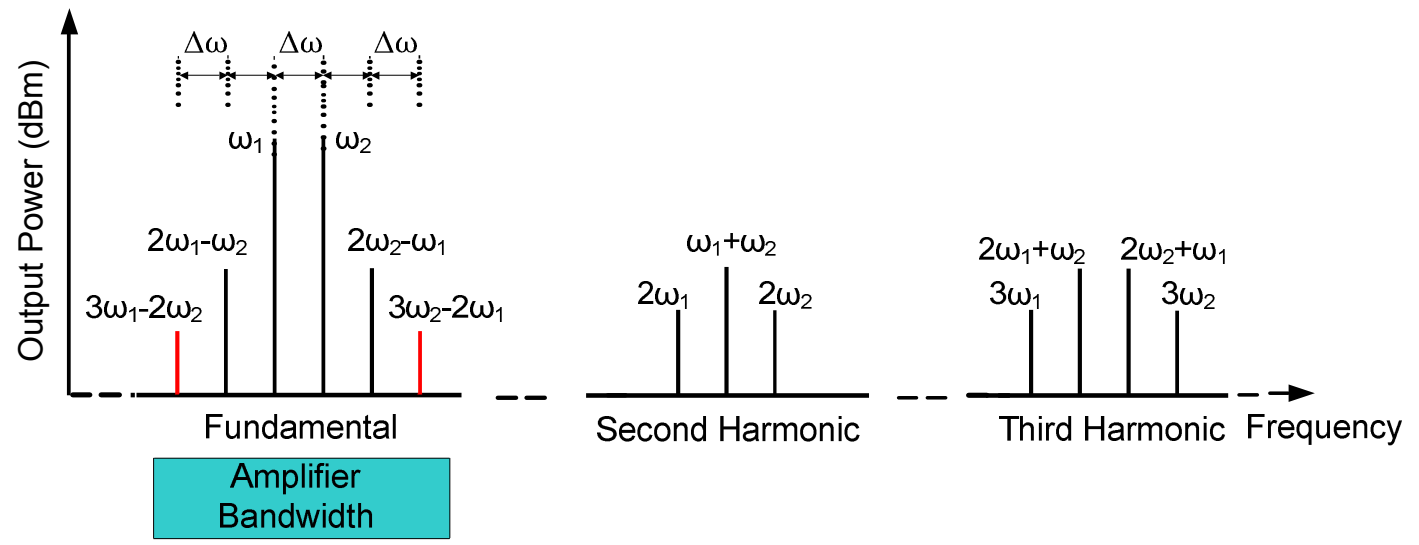

Figure 3.9: Two-tone output spectral components in frequency domain $[6,8]$.

The IMD products appearing at $2 \omega_{1}-\omega_{2}$ and $2 \omega_{2}-\omega_{1}$ are of great concern as they appear in-band and will thus distort the desired shape of the fundamental signal components. The in-band IMD cannot be easily filtered out unlike the other IMD products, which are located far away from the fundamental frequency components (out of band) and can be easily filtered out. The most commonly used measure of IMD is the ratio of the largest IMD, almost always third order IMD, to the one of the two-tone [6]. To obtain valuable information on the device's IM distortion behaviour, the two-tone characterization has been used for the work presented in this chapter, in order to investigate the device related memory effects due to baseband impedance variations.

\subsubsection{Multi-Tone Characterization}

Multi-tone characterization technique is another option for characterizing device nonlinearity with more than two tones. This has gained importance in recent years as a substitute for the actual telecommunication signal. Interpretation of power device nonlinearity in terms of the time domain waveforms of a digitally modulated signal is rather complicated. Nevertheless, amplifier linearity under a digitally modulated test signal is characterized by measuring the degree of output spectrum regeneration across the lower and 
upper sidebands. In all cellular systems it is necessary for the system design to impose specifications on the maximum amount of distortion that can be introduced in adjacent channels. Adjacent channel main power ratio (ACPR) is defined as the measured power in an adjacent communication channel relative to the power in the main channel of specified bandwidth as depicted in Figure 3.10. ACPR is often used as a measure of linearity in nonlinear system when complex digital modulated signal is applied as an input signal. It can be measured using a spectrum analyzer.

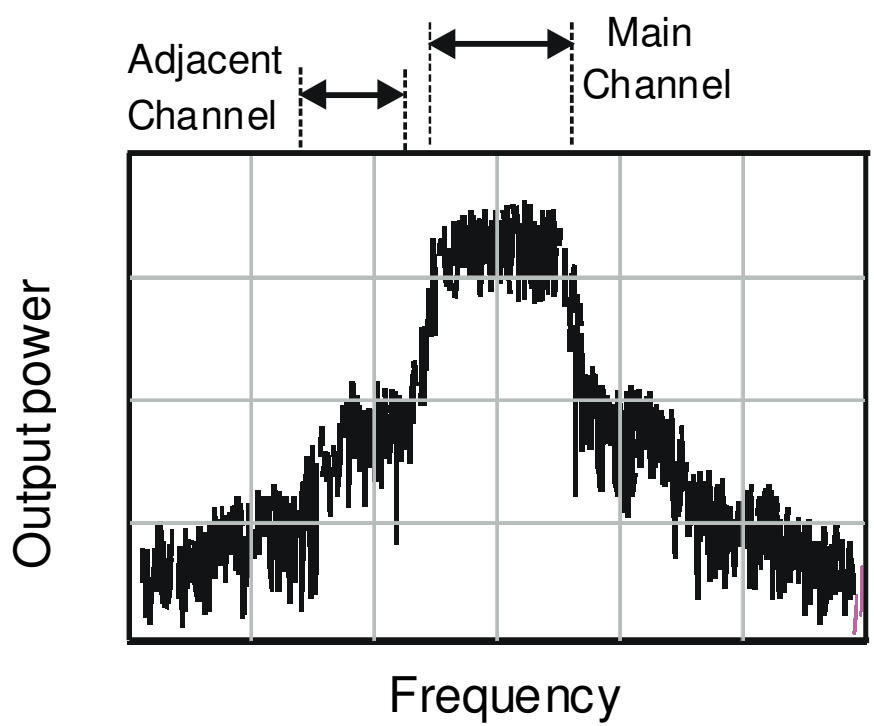

Figure 3.10: Adjacent channel leakage power in the output spectrum of the amplifier.

Today, in most advanced communication systems which use multi- carrier signals with digital modulation formats [6], [9], the ACPR has become one of the important power amplifier linearity requirements. For example, the maximum ACPR requirement given in third generation partnership project should be $\geq 45 \mathrm{dBc}$ for bandwidth up to $5 \mathrm{MHz}$ and $\geq 50 \mathrm{dBc}$ for $10 \mathrm{MHz}$ bandwidth [8],[10].

\subsubsection{Noise Power Ratio}

Noise power ratio (NPR) is a measure of the unwanted in-channel distortion power caused by the nonlinearity of the power amplifier. This can be measured by extracting a portion of the input signal, using a notch filter, and examining the level of distortion 'filling in' the space within resulting gap. NPR 
is defined as the ratio between the noise power spectral density of a white noise signal passing through the amplifier, measured at the notch of the output signal, to the noise power spectral density without the notch filter. The amplifier is driven at the same power level in each case. Further details of this test and concept are illustrated in Figure 3.11.

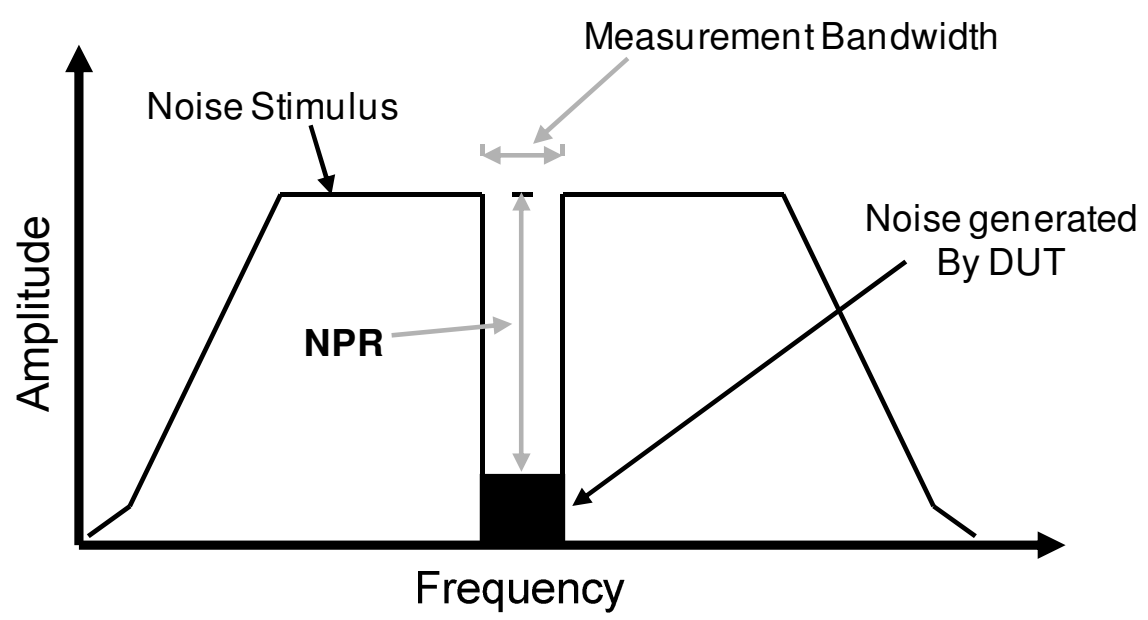

Figure 3.11 Conceptual representation of Noise Power Ratio (NPR).

\subsubsection{Envelope Domain Analysis}

The large signal measurement system measures the full spectral content of the voltage and current waveforms and provides both the magnitude and phase behaviour of the device. Analysis of these waveforms in the time domain becomes difficult due to the frequency difference between the baseband IF and the RF carrier, resulting in a large number of zero crossing points within the envelope of the signal. This makes the overlaying of time domain waveforms, produced by an increase in the input power, difficult to interpret.

Alternatively, the analysis of the spectral contents can be achieved in the frequency domain using the magnitude and phase information of each generated component as a function of the input power. Again this analysis will become more complicated as the number of input frequency components is increased. This is as a result of, each additional frequency term resulting in the generation of additional in-band frequency components. An alternative approach to both problems is the combined use of time domain and frequency domain techniques to perform an envelope domain analysis of the measured 
data. In envelope domain analysis, the modulated signal is described as a complex time varying envelope containing both the magnitude and phase information for each Fourier coefficient. The distortion in the output spectrum of the device is quantified in terms of the generated 'envelope harmonic components' around the carrier and its harmonics [11].

This process involves extracting time varying $I$ and $Q$, or magnitude and phase components that are associated with the fundamental and all harmonic bands. Computation of the complex envelope is achieved by considering a modulated signal described by equation (3.4), where an RF carrier is amplitude modulated as a function of time by equation (3.5).

$$
\begin{aligned}
& \left.v(t)=A(t) \cos \left(\omega_{R F} t-\varphi_{R F}\right)\right) \\
& \left.A(t)=A \cos \left(\omega_{I F} t-\varphi_{I F}\right)\right)
\end{aligned}
$$

Using standard trigonometric identities for a double angle, equation (3.4) can be represented as;

$$
\left.v(t)=A(t)\left[\cos \left(\omega_{R F} t\right) \cos \left(\varphi_{R F}\right)+\sin \left(\omega_{R F} t\right) \sin \left(\varphi_{I F}\right)\right)\right]
$$

From this, the in phase $\mathrm{I}(\mathrm{t})$ and quadrature phase $\mathrm{Q}(\mathrm{t})$ modulation components can be expressed by equations (3.7) and (3.8).

$$
\begin{aligned}
& I(t)=A(t) \cos \left(\varphi_{R F}\right) \\
& Q(t)=A(t) \sin \left(\varphi_{R F}\right)
\end{aligned}
$$

Thus further application of trigonometric identities and the rearranging of the Fourier coefficients create a new spectral representation that contains only the spectral information related to that particular band, where the centre of the band is made to lie around DC.

The In-phase (I) and quadrate (Q) time domain signals are then extracted from the spectrum, achieved by summing the magnitude and phase of each of the spectral location using equations (3.9) and (3.10).

$$
\begin{aligned}
& I_{n}(t)=\sum_{i=- \text { order }}^{\text {order }} \rho_{n+1} \cos \left(i \omega_{I F} t-\varphi_{n+1}\right) \\
& Q_{n}(t)=\sum_{i=- \text { order }}^{\text {order }}-\rho_{n+1} \sin \left(i \omega_{I F} t-\varphi_{n+1}\right)
\end{aligned}
$$

The envelope modulation components are computed between -order and order about the centre point of carrier or harmonic frequency component $n$ [12]. The computed $\mathrm{I}(\mathrm{t})$ and $\mathrm{Q}(\mathrm{t})$ components generated about each harmonic, 
equations (3.11) and (3.12), can be used to compute the magnitude and phase of the time varying envelopes.

$$
\begin{aligned}
& A_{n}(t)=\sqrt{I_{n}(t)^{2}+Q_{n}(t)^{2}} \\
& \phi_{n}(t)=\tan ^{-1}\left(\frac{Q_{n}(t)}{I_{n}(t)}\right)
\end{aligned}
$$

The representation of the data in the envelope domain allows for the direct observation of the device response to an input stimulus signal. Analysis of time varying behaviour in the envelope domain can often provide significant insight into a number of dynamic non-linear processes including the presence of device related 'memory' [13].

\subsubsection{Properties of Power Supply}

In general, the amplifier power supplies are applied to the gate and the drain sides in order to provide the necessary dc voltage or current for active device operation. Particularly, in the case of the input signal with a non-constant envelope, a current variation flowing from the dc power supply causes dc voltage variations. These voltage variations cause an additional amplitude modulation of the RF signal (re-modulation) as illustrated in $\mathrm{F}$ igure 3.12. Moreover, when the impedance of the bias network is reactive, not a short circuit, the additional amplitude modulation will be out of phase compared to the original $\mathrm{RF}$ signal resulting in memory effects or envelope frequency dependent and asymmetrical of the output signal. In an ideal case, the amplifier power supply should provide bias current to the active device with minimal voltage variation at varying envelope frequency. To reduce the power supply variation effect in the amplifier, the bias networks should be carefully designed in order to isolate the current variations drawn from the dc power supply.

Imperfections in the design can increase the variation in the dc voltage and consequently the memory effects will appear [6], [7]. In the last few years, many bias networks design strategies have been intensively investigated and analyzed [8], [17]. Theoretically, they conclude that an ideal bias network should have short circuit impedance at baseband frequency and an open circuit at fundamental frequency. However it is very difficult to realize such 
impedances, which has two requirements that tend to conflict with each other. Therefore, actually in practice, the bias network design strategies are still under development and more research work has to be done.

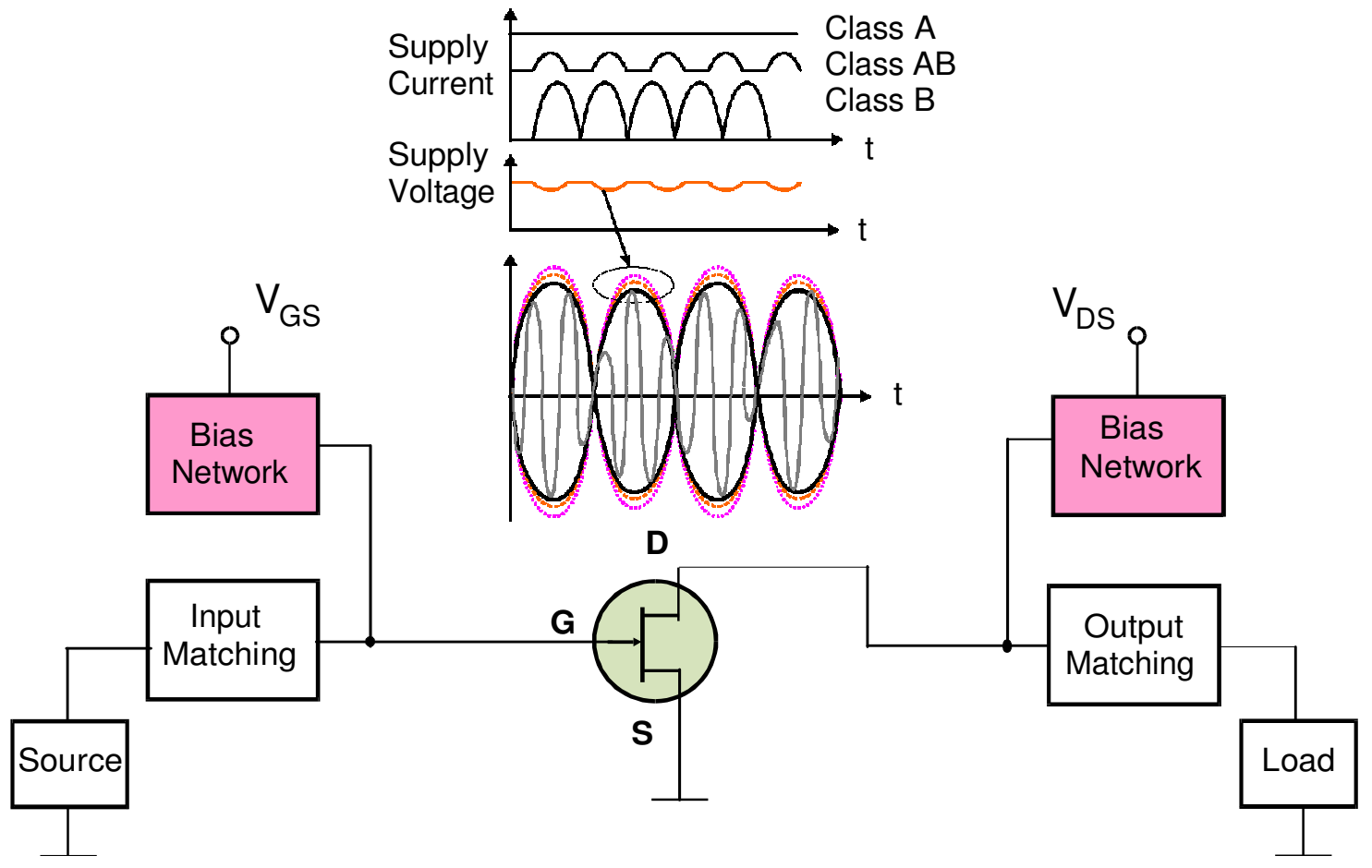

Figure 3.12: Memory effects due to power supply variations [8].

\subsection{Memory Effects Measurements and Investigations}

To support the successful control of the out-of-band frequency components, particularly at baseband, a dedicated measurement setup has been developed and demonstrated in [14], [19] to investigate the device related baseband electrical memory under modulated excitations. In earlier work [13], [15] only the impedance presented to the two most significant baseband components (IF1 and IF2) generated as a result of 2-tone excitation was controlled. This was achieved by combining two, phase coherent arbitrary waveform generators (AWGs) whilst the device was driven at a relatively backed-off level at $1 \mathrm{~dB}$ below the $1 \mathrm{~dB}$ compression point.

However, when the device is driven more deeply into compression, significantly more distortion components are generated, and in order to achieve a sufficiently broad baseband termination, significant modification of the baseband load-pull measurement system was required in order to 
accurately control the higher baseband components. The integrated measurement architecture is shown in Figure 3.13 and provides the ability to present independent impedances to all the baseband components that result from a multi-tone excitation.

Enhancements performed elaborated in chapter-2 as part of this research work allow this to now be achieved in the time domain, using a single $80 \mathrm{MHz}$ arbitrary waveform generator (AWG) to synthesise the necessary waveforms to allow a constant and specific baseband impedance environment to be maintained across a wide bandwidth.

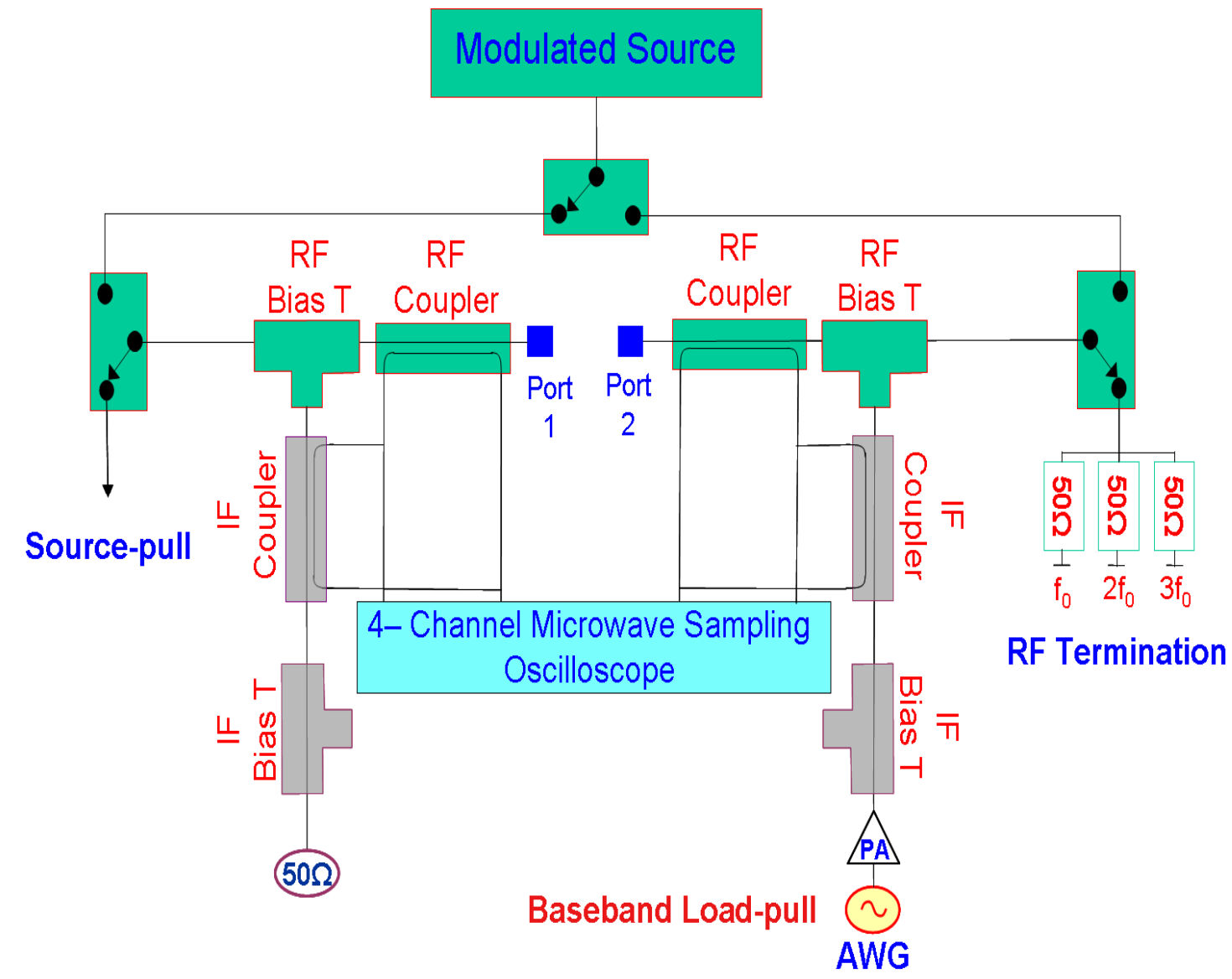

Figure 3.13: Modulated waveform measurement system with broadband active IF load-pull.

\subsubsection{Theoretical Study of Nonlinear Circuit}

An example of an output power amplifier equivalent circuit model which is used for theoretical study is presented in Figure 3.14. It comprises of a nonideal bias-T ( $L_{B}$ is a RFC inductor and $C_{B}$ is a $D C$ blocking capacitor) 
connected to a linear dynamic matching network. For the sake of simplicity, it is assumed that this matching network presents a short circuit to all the baseband components and has a wider bandwidth than the signals processed. For analysis an input RF signal composed by a carrier at $\omega_{c}$, modulated by a complex envelope is assumed.

$$
v_{\text {in }}(t, \tau)=\operatorname{Re}\left[r(\tau) \bullet e^{j \phi(\tau)} \bullet e^{j \omega_{c} t}\right]
$$

Where $r(\tau)$ and $\Phi(\tau)$ are the modulating complex envelope amplitude and phase respectively. Similarly, the output will be given by a sum of all the harmonic components of the envelope and the carrier.

$$
v_{D S}(t, \tau)=\sum_{k_{1}=-k_{1}}^{k_{1}} \sum_{k_{2}=-k_{2}}^{k_{2}} \operatorname{Re}\left[r_{k_{1} k_{2}}(\tau) \cdot e^{j \phi_{k_{1} k_{2}}(\tau)} \cdot e^{j k_{2} \omega_{c} t}\right]
$$

Looking into Figure 3.14 and performing a simple circuit analysis, it is possible to derive a set of differential equations that governs the $V_{D S}(t)$ and $I_{D S}(t)$ envelope dynamics, $\mathrm{V}_{\mathrm{DS}}(\tau)$ and $\mathrm{I}_{\mathrm{DS}}(\tau)$.

$$
\begin{aligned}
& V_{D S}(\tau)=V_{D D}-L_{B} \frac{d i_{L_{B}}(\tau)}{d t}=V_{D D}-\frac{1}{C_{B}} \int_{0}^{\tau} i_{C_{B}}(\tau) d \tau \\
& I_{D S}(\tau)=i_{L_{B}}(\tau)+i_{C_{B}}(\tau)
\end{aligned}
$$

This analysis indicates that the PA dynamic behaviour will be strongly affected by the baseband impedance presented to the transistor [5], which will be shown by the transfer characteristics plots.

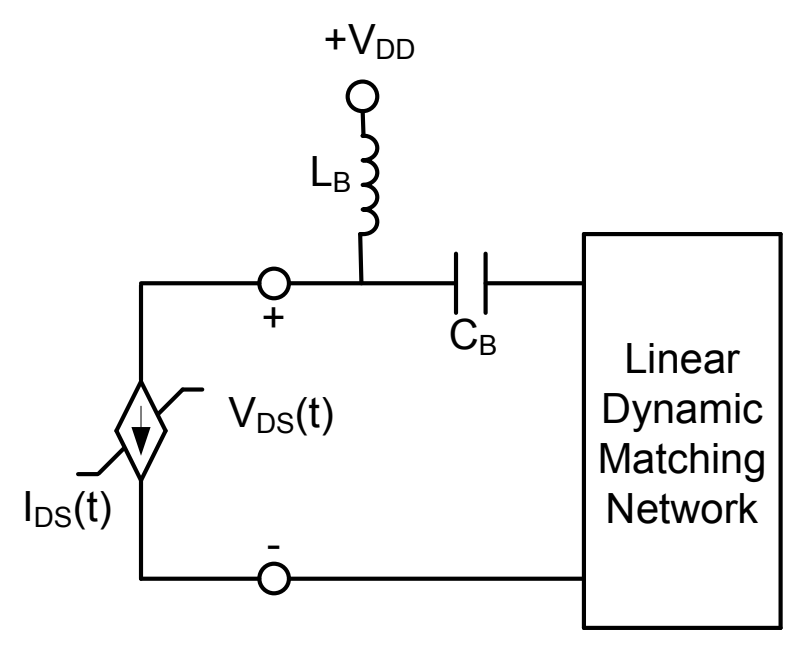

Figure 3.14: Simplified power amplifier output circuit [5]

Indeed, as it will be shown in the later part of this chapter, if the output baseband signal frequency range coincides with a zone where the output 
baseband impedance, as seen by the transistor, is resistive, inductive or capacitive, the dynamic transfer characteristics will reflect these different types of induced long term memory effects.

\subsubsection{Effect of Drive Level on Output Envelopes Investigations}

Power transistor operating in high efficient modes produce distorted transmitted signals containing both inter-modulation distortion products and harmonics. It is the in-band intermodulation distortion around the fundamental RF carrier that directly influences the linearity of the communication systems, as harmonic distortion can easily be filtered out. However, the transistor distortion generated around the fundamental RF carrier is influenced by many external parameters; DC bias, temperature, base-band impedance, RF fundamental impedance and its variation over the modulated bandwidth, RF harmonic impedances, to name just a few. Thus to fully characterize the power transistor linearity performance, ideally all these parameters should be varied systematically and independently. The basic baseband power transistor linearity measurements and investigations involve stimulating the device with a suppressed carrier amplitude modulated (AM) signal, two-tone stimulus, and then measuring and characterizing the distortion products, $\mathrm{IMD}_{3}$ and $\mathrm{IMD}_{5}$, generated. The measurements presented here were carried out on a 2 W GaN HFET bare die supplied by CREE. For baseband electrical memory investigations, a two-tone stimulus signal given in equation (3.17) with a centre frequency of $2.1 \mathrm{GHz}$ was used and the tone-spacing was varied between 1 and $10 \mathrm{MHz}$. The device was biased at approximately $20 \%$ IDSS in Class-AB at a quiescent current of $130 \mathrm{~mA}$, and all significant RF signals were passively terminated into $50 \Omega$. The characteristics were measured dynamically and found to be strongly dependent on the separation frequency. The characteristics were measured dynamically and found strongly dependent on the separation frequency for the device considered.

$$
\begin{aligned}
& A\left(\cos \omega_{1} t+\cos \omega_{2} t\right) \\
& =2 A \cos \left[\left(\frac{\omega_{1}-\omega_{2}}{2}\right) * t\right] \cos \left[\left(\frac{\omega_{1}+\omega_{2}}{2}\right) * t\right]
\end{aligned}
$$


Where the modulation frequency, $\omega_{\mathrm{m}}$, is given by

$$
\omega_{m}=\left(\omega_{1}-\omega_{2}\right) / 2
$$

To observe the dynamic behaviour of the device whilst operating within a realistic operational environment, and in response to an applied modulated input stimulus signal, envelope domain analysis is used.

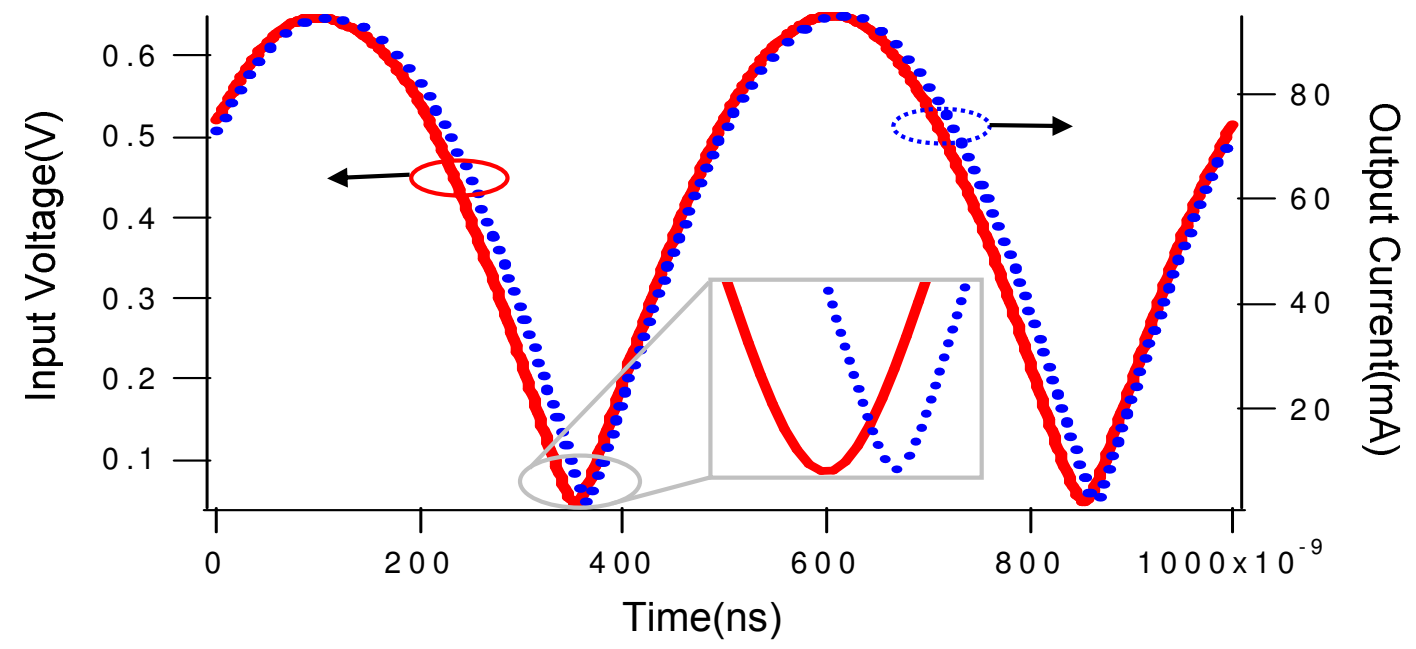

Figure 3.15: Input voltage and output current envelopes at $5 \mathrm{MHz}$ tone spacing

Figure 3.15 shows the fundamental output current and input voltage modulation envelopes for a $\mathrm{P}_{1 \mathrm{~dB}}-3 \mathrm{~dB}$ backed-off drive level, and clearly show that there is a significant phase delay between the input and output of the device at a tone spacing of $5 \mathrm{MHz}$, whereas at $1 \mathrm{MHz}$ tone spacing, negligible phase delay was observed as shown in Figure 3.16.

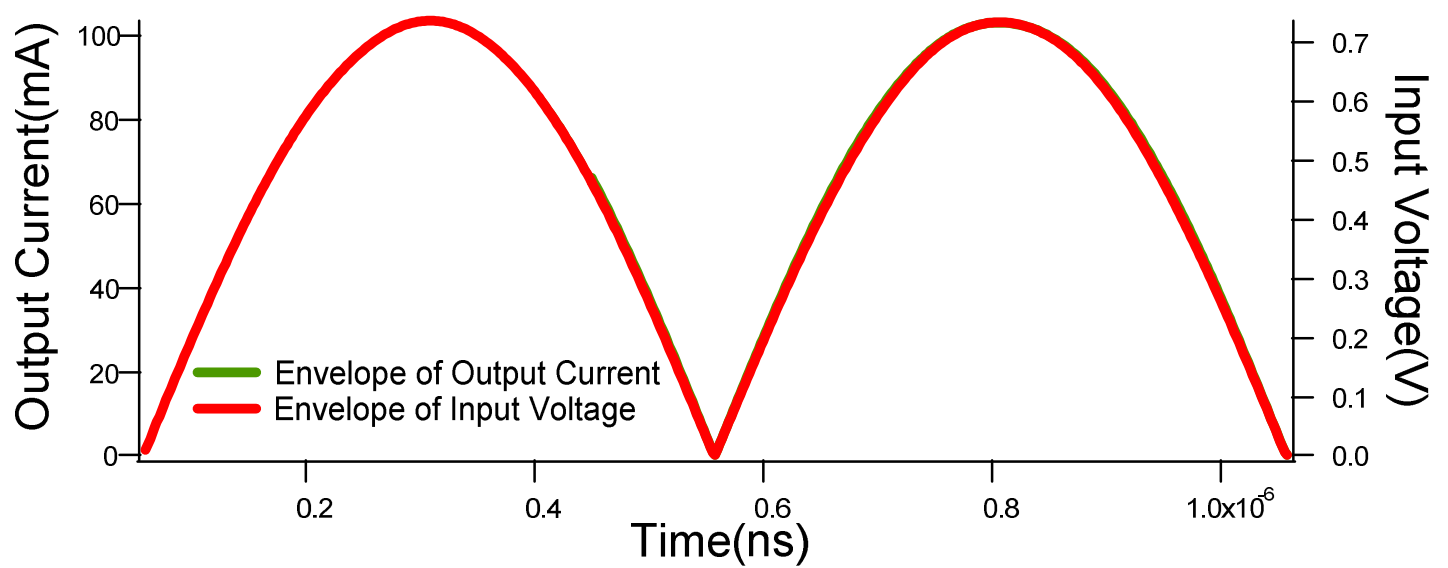

Figure 3.16: Input voltage and output current envelopes at $1 \mathrm{MHz}$ tone spacing. 
At this backed-off drive level, the output power spectrum illustrated the lowlevel of baseband and near absence of $2^{\text {nd }}$ harmonic distortion; a condition which unsurprisingly resulted in symmetrical $\mathrm{IMD}_{3}$ behaviour. The input voltage is plotted against the output current, plotted this way it reveals the looping in the device dynamic transfer characteristics, as both envelops are symmetrical and have no phase shift consequently no broadening appears in the upper part of the dynamic transfer characteristics.

Figure 3.17 shows a broadening in the dynamic transfer characteristic due to linear device delay as reported in [4]. It can be seen however that when the device is driven $1 \mathrm{~dB}$ into compression, the observed nonlinearities are much more significant and appear as a soft, asymmetrical clipping of the output current envelope, which significantly increases the $2^{\text {nd }}$ harmonic signal level and causes a significant baseband signal components to develop.

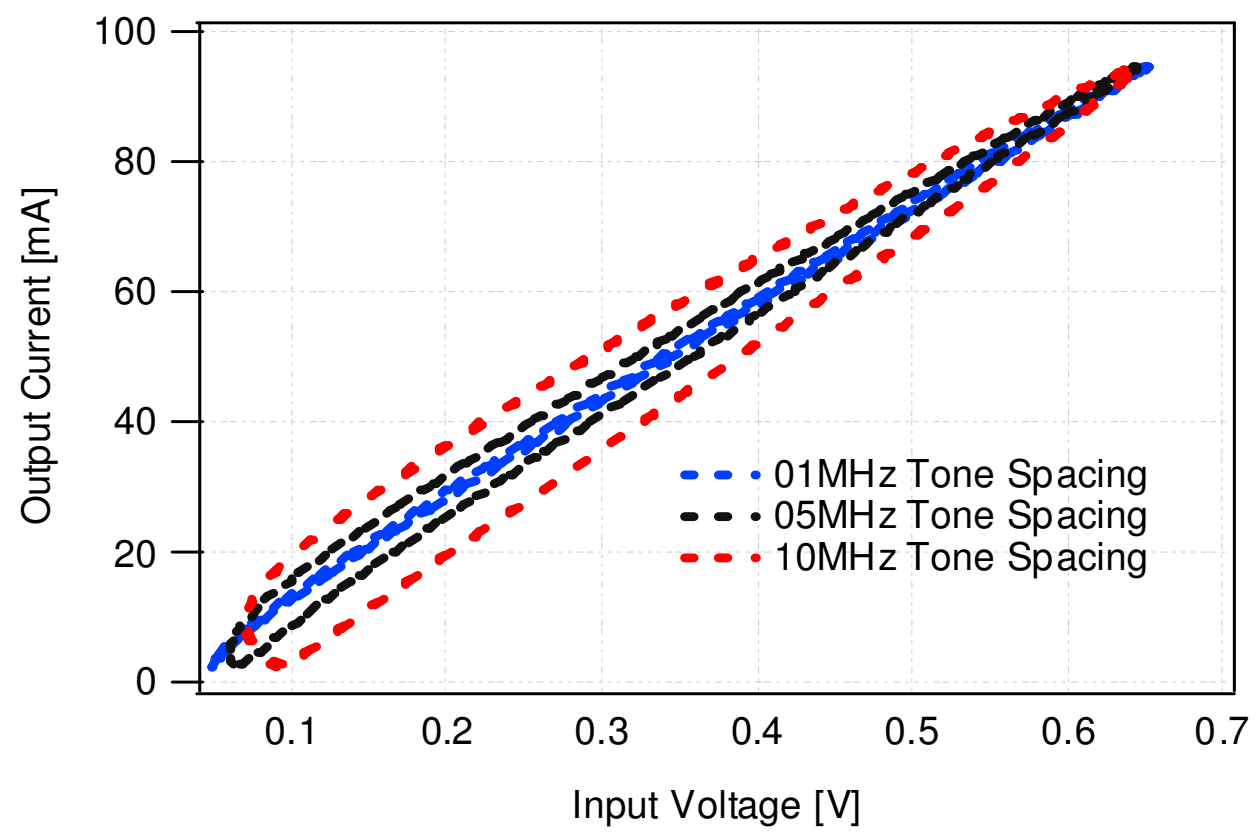

Figure 3.17: Dynamic transfer characteristics at back-off drive level for different tone spacings.

The asymmetrical envelops observed are shown in Figure 3.18 which are an explicit manifestation of presence of nonlinear memory effects at higher drive levels (when device is driven into deep compression). The baseband signal generated from the device also includes higher order terms ( IF2 which is four times of the modulation frequency and IF3 which is six times of the modulation frequency), and it is the asymmetric envelope shape that 
determines which $\operatorname{IMD}_{3}$ product $\left(2 \omega_{2}-\omega_{1}\right)$ or $\left(2 \omega_{1}-\omega_{2}\right)$ will have the greater magnitude.

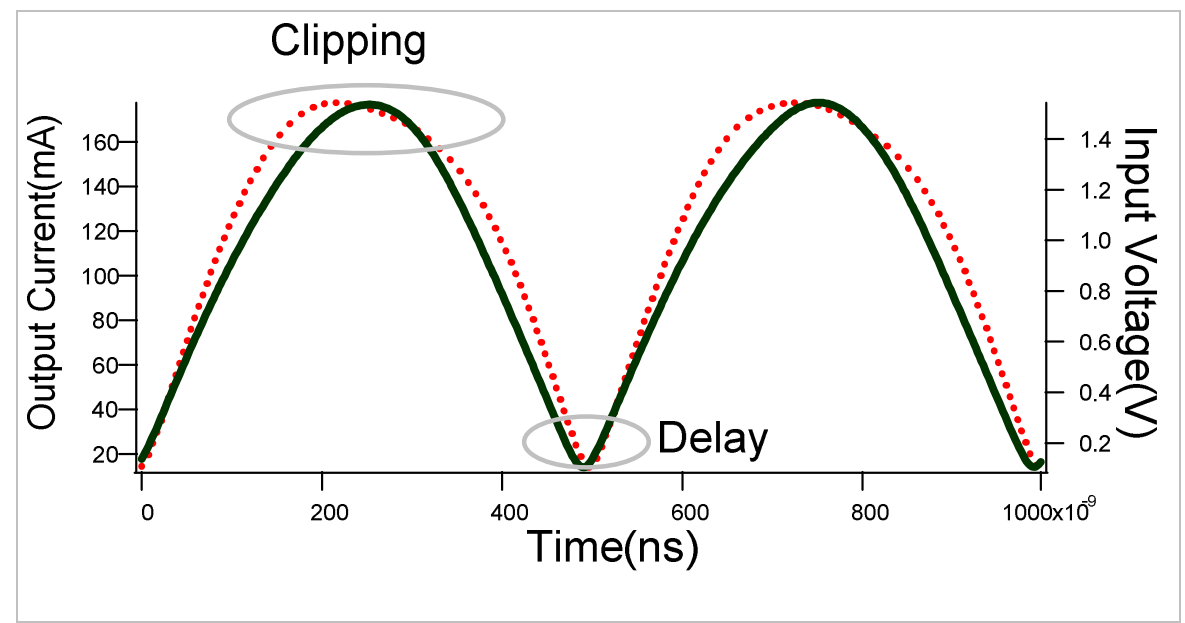

Figure 3.18: Input voltage and output current envelops at $5 \mathrm{MHz}$ tone-spacing.

The presence of these asymmetrical spectral current components manifest themselves as recognizable electrical memory effects and can be clearly seen in the envelope domain as significant looping at the top-end of the input voltage / output current transfer characteristic. The hysteresis in the dynamic transfer characteristics worsen with the increasing tone spacing and can be seen explicitly from Figure 3.19.

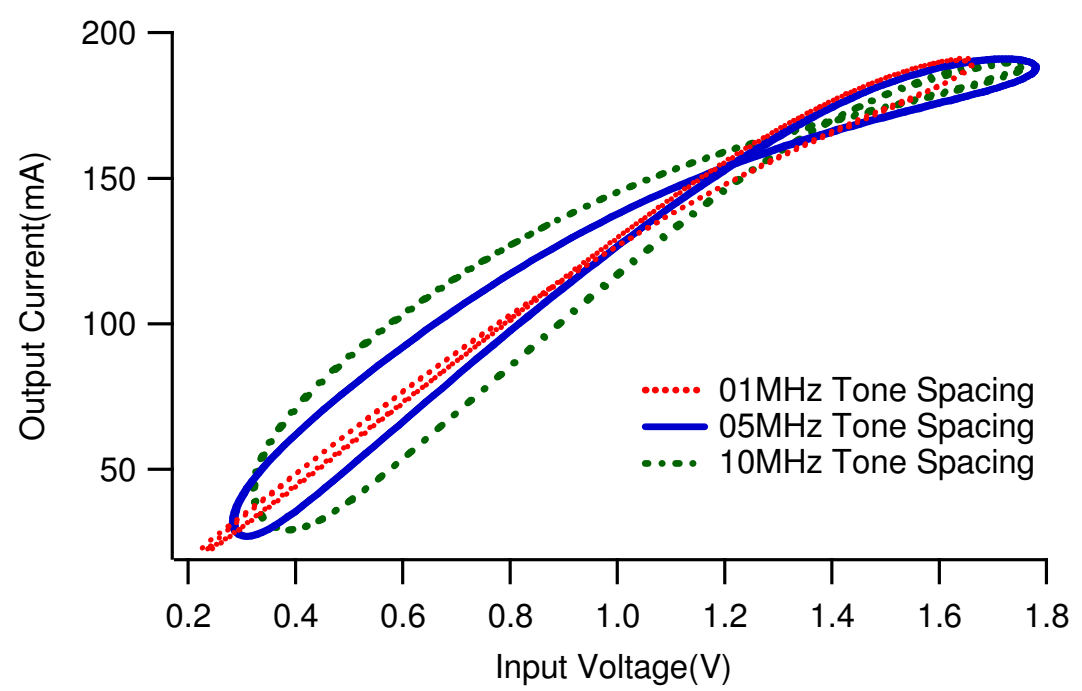

Figure 3.19: Dynamic transfer characteristics at different tone spacings for compressed drive level.

In this case, the significant baseband frequency components are to be expected and are due to the fact that the significant baseband current 
components are terminated into relatively high broadband impedance of $50 \Omega$. These are clearly the most significant contributor towards the observed electrical memory effects.

\subsubsection{Theoretical Study of Active IF Load-pull}

The active load-pull principle is the most practical way to optimize the out-of-band impedances without affecting the fundamental matching, as fundamental impedance mixes with the baseband and $2^{\text {nd }}$ harmonic impedances to generate IM products. The impedance seen by the baseband components can be affected by adding an external low frequency signal source at the same frequency. Now the apparent impedance can easily be engineered by adjusting the amplitude and phase of the signal source. The Figure 3.20 demonstrates the active load-pull principle. The circuit consists of just input impedance $Z_{I N}$ and the impedance $Z_{P I}$, the total baseband impedance $Z_{\mathrm{BB}}$ can be seen as a parallel connection of these two. The Norton's equivalent of the circuit is presented in Figure 3.20(b) and a circuit that also includes the nonlinear current source is shown in Figure 3.20(c).

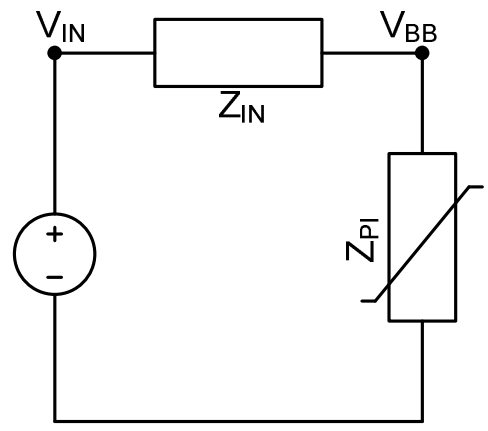

(a)

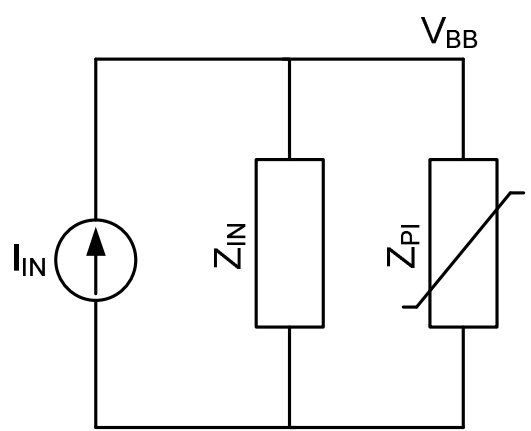

(b) $\quad Z_{B B}=Z_{I N} \| Z_{P I}$

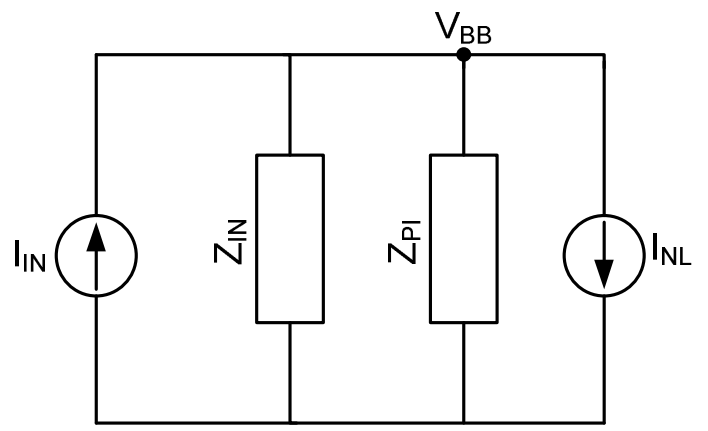

(c)

Figure 3.20: (a) A simple nonlinear circuit, (b) its Norton equivalent, and (c) the circuit consisting of the nonlinear current source. 
By applying the modulated two tone signal to the input, the envelope voltage $\mathrm{V}_{\mathrm{BB}}$ at the drain can be written as;

$$
V_{B B}\left(\omega_{2}-\omega_{1}\right)=\left(I_{I N}\left(\omega_{2}-\omega_{1}\right)-I_{N L}\left(\omega_{2}-\omega_{1}\right)\right) \bullet Z_{B B}\left(\omega_{2}-\omega_{1}\right)
$$

The nonlinear current can be further written as;

$$
I_{N L}\left(\omega_{2}-\omega_{1}\right)=K_{2} \bullet V_{B B}\left(\omega_{2}\right) \bullet V_{B B}\left(-\omega_{1}\right)
$$

However, it is not necessary to calculate the value of the nonlinear current source, it can be observed from the measurements by tuning the $I_{\mathrm{NL}}\left(\omega_{2}-\omega_{1}\right)$ and monitoring the $\mathrm{V}_{\mathrm{BB}}\left(\omega_{2}-\omega_{1}\right)$. Once the $\mathrm{V}_{\mathrm{BB}}\left(\omega_{2}-\omega_{1}\right)$ is forced to zero, the following requirement is fulfilled.

$$
I_{\text {IN }}\left(\omega_{2}-\omega_{1}\right)=I_{N L}\left(\omega_{2}-\omega_{1}\right)
$$

Since the impedance is defined as a ratio between the node voltage and the current, the impedance seen by the nonlinear current source can be modified and written as;

$$
Z_{B B e f f}=\left(\frac{I_{I N}\left(\omega_{2}-\omega_{1}\right)}{I_{N L}\left(\omega_{2}-\omega_{1}\right)}-1\right) \cdot Z_{B B}\left(\omega_{2}-\omega_{1}\right)
$$

Equation 3.22 is explained in more detail in Figure 3.21 where $Z_{\mathrm{BB}}$ represents the original node impedance of the base. Once $I_{\mathbb{N}}$ is applied according to equation 3.21, no baseband voltage waveform is seen at the base, which means that $Z_{B B e f f}$ is virtually driven to zero at the baseband frequency. Next $I_{\mathbb{N}}\left(\omega_{2}-\omega_{1}\right)$ is adjusted by $180^{\circ}$ which means $I_{\mathbb{N}}$ and $I_{N L}$ are summed up with the same phase.

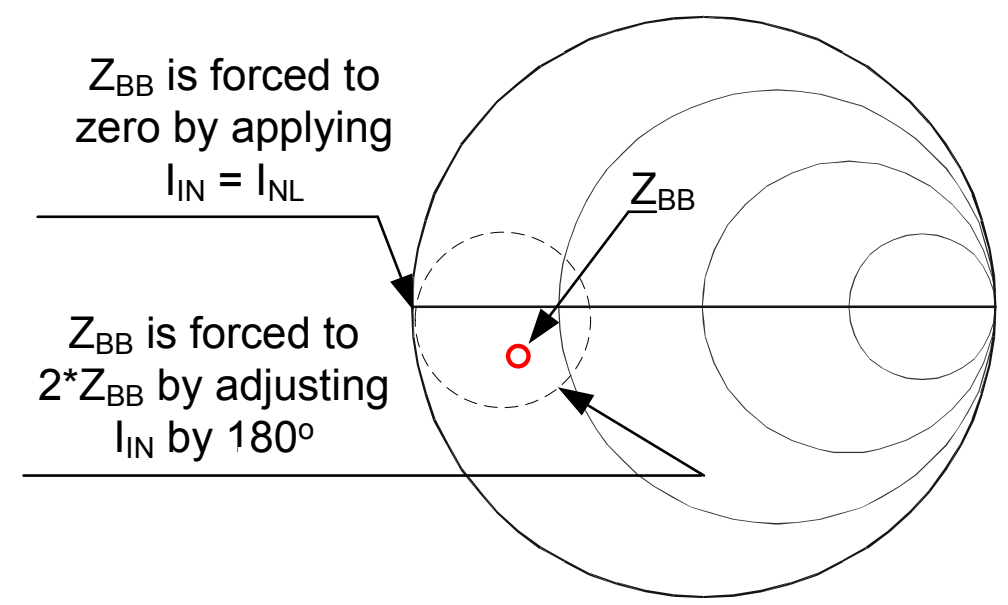

Figure 3.21: The effectiveness of IF active load-pull in generating the baseband impedance $Z_{\mathrm{BB}}$ 
By studying equation 3.22, it can be seen that the effective $Z_{\text {BBeff }}$ is now twice the actual base impedance $Z_{\mathrm{BB}}$. This is also demonstrated in Figure 3.21. By adjusting the amplitude and phase of $I_{1 N}\left(\omega_{2}-\omega_{1}\right)$ all impedances can be generated.

Albeit the circuit used in this study is greatly simplified, the same principle also holds to more complicated systems. Actually, it does not matter how many base baseband frequency components are generated, which are just produced by exciting the device with modulated stimulus. The tuned $Z_{B B}$ is optimal from the overall baseband memory reduction point of view and highlights the significance of active load-pull at baseband frequencies to minimize the baseband electrical memory.

\subsubsection{Application of Active IF Load-pull}

In a practical PA design, the first instinctive step is to nullify or cancel these unwanted baseband voltage components, preventing any contamination or 're-modulation' of the output bias supply. Therefore, the termination of these frequency components into short circuits is usually desirable and the normal course of action. For the measurements presented here, active IF load-pull has been used as means of controlling the contribution of the significant baseband components (IF1 and IF2). The validity of this approach in maintaining a constant low-frequency impedance environment is reported in [16] where IF1, which is twice of the modulation frequency and IF2, which is four times the modulation frequency were actively load-pulled. In the work presented here, frequency independent IF1 and IF2 short circuit impedances were maintained for different tone separation and at a single drive level. The observed variation in impedance as a function of tone spacing is very small and can be seen from Figure 3.22 to be less than 0.005 in magnitude and approximately 0.1 degrees in phase over the entire IF bandwidth, and are actually not harmful to the linearity of the device itself.

The behaviour of the carrier tones $\left(\omega_{1}\right.$ and $\left.\omega_{2}\right)$ is clearly independent of the tone spacing as shown in Figure 3.23. It is also evident that a reduction in $\mathrm{IMD}_{3}$ components appears to have been possible by providing the IF short circuit impedance for frequencies ranging from $1 \mathrm{MHz}$ to $10 \mathrm{MHz}$. This is due to 
the fact that the non-negligible modulation amplitude dependent baseband electrical memory effects are greatly reduced.
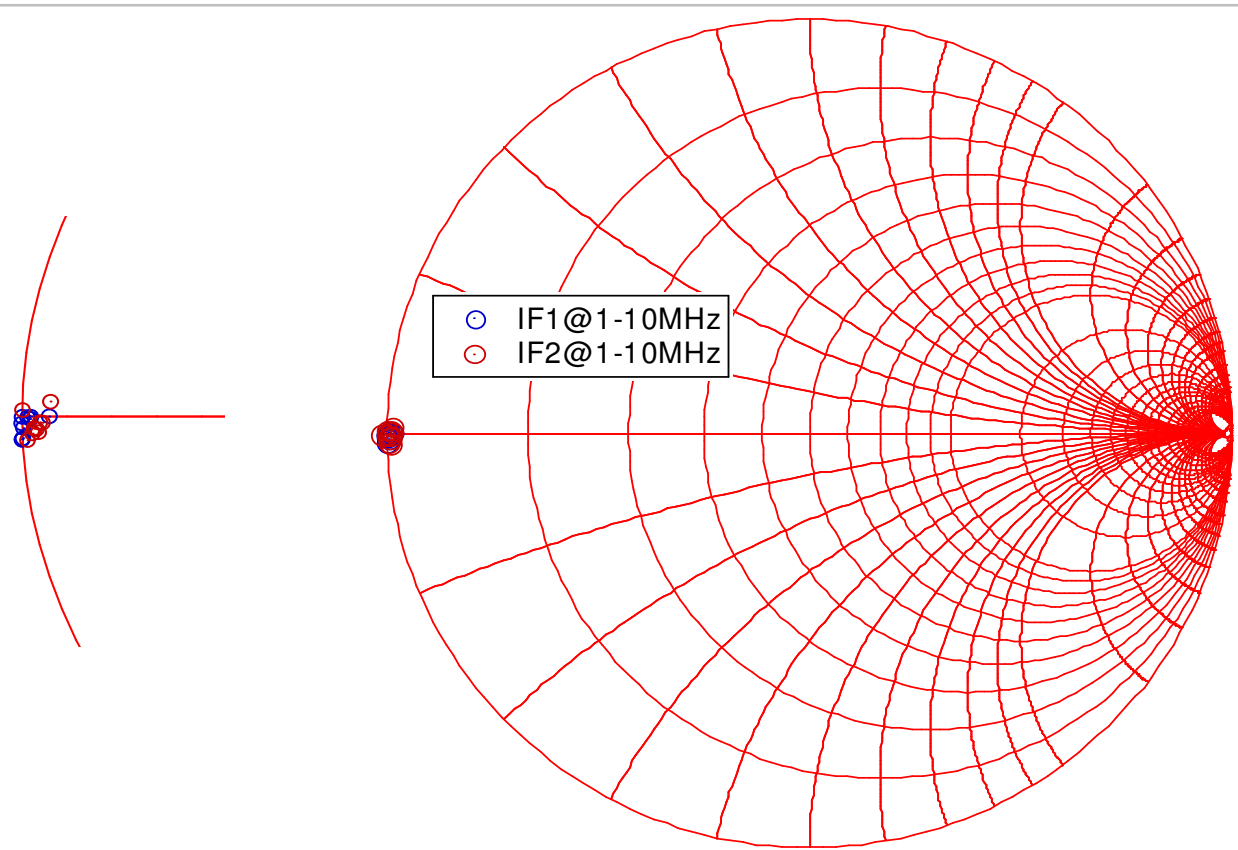

Figure 3.22: Measured $\mathrm{IF}_{1}$ and $\mathrm{IF}_{2}$ impedances on a Smith chart at different tone-spacing.

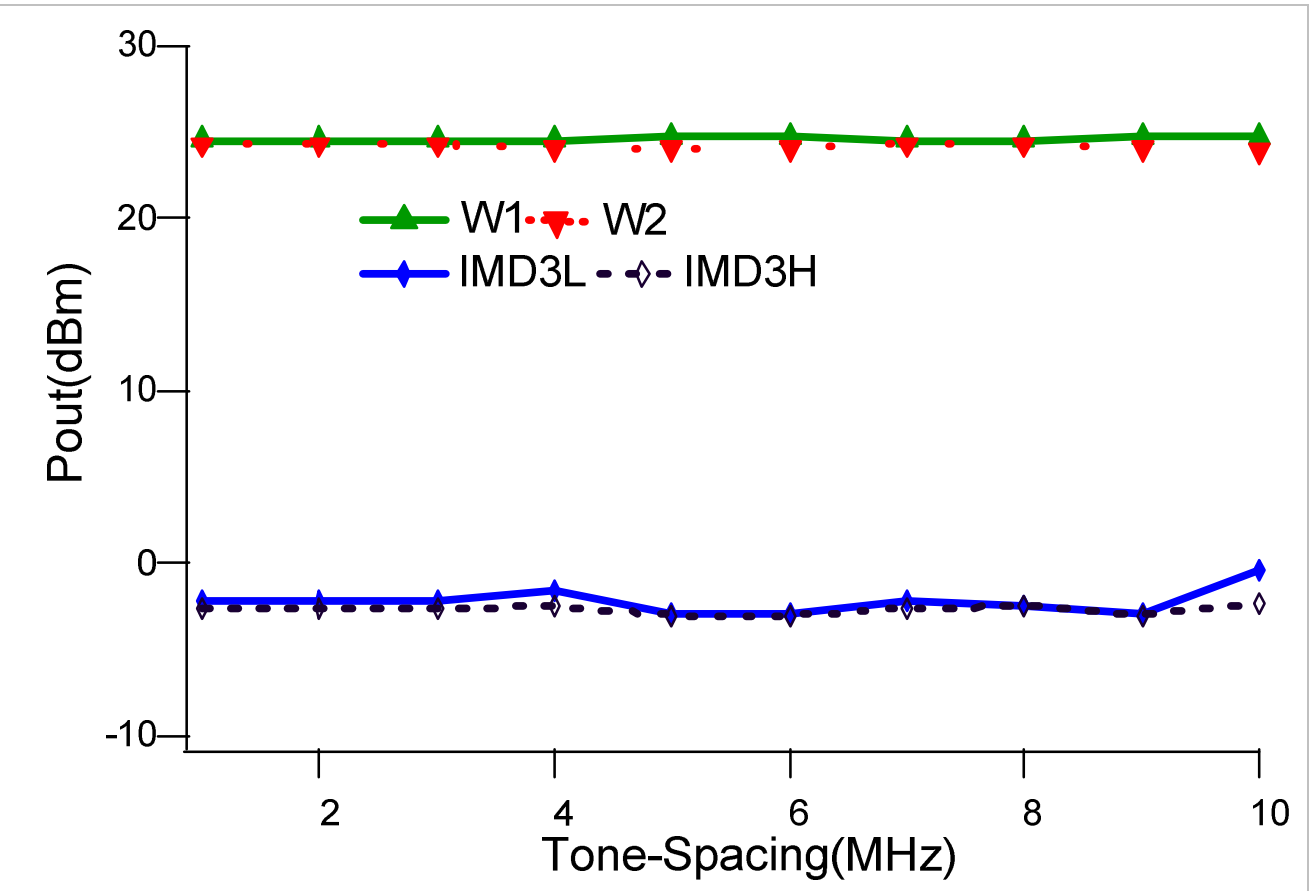

Figure 3.23: Measured fundamental and $\mathrm{IMD}_{3}$ power for different tonespacing at constant drive level of $1 \mathrm{~dB}$ compression point. 
Since the fundamental and second harmonic impedances play a minor role, memory effects are for the most part produced by the baseband impedances. Therefore, it can be concluded that by the careful design of bias network the memory effects due to baseband impedance can greatly be reduced. Measurements under such conditions show a notable reduction in $\mathrm{IMD}_{3}$ asymmetry and confirm the validity of IF active load-pull in maintaining a constant baseband impedance for the two significant baseband components IF1 and IF2 over the bandwidth of at least four times the modulation frequency.

The baseband voltage components are greatly suppressed whilst maintaining the short-circuit baseband impedance for significant baseband components (IF1 which is twice of the modulation frequency and IF2 which is four times of the modulation frequency).

Under such conditions, the IF load-line was almost completely vertical with only slight current variation present as can be seen from the Figure 3.24. This condition resulted in negligible looping in IF load-lines, hence the RF load-line follows the same trajectory in and out of the boundary conditions; consequently this reduced the hysteresis in the dynamic transfer characteristics at the carrier frequency.

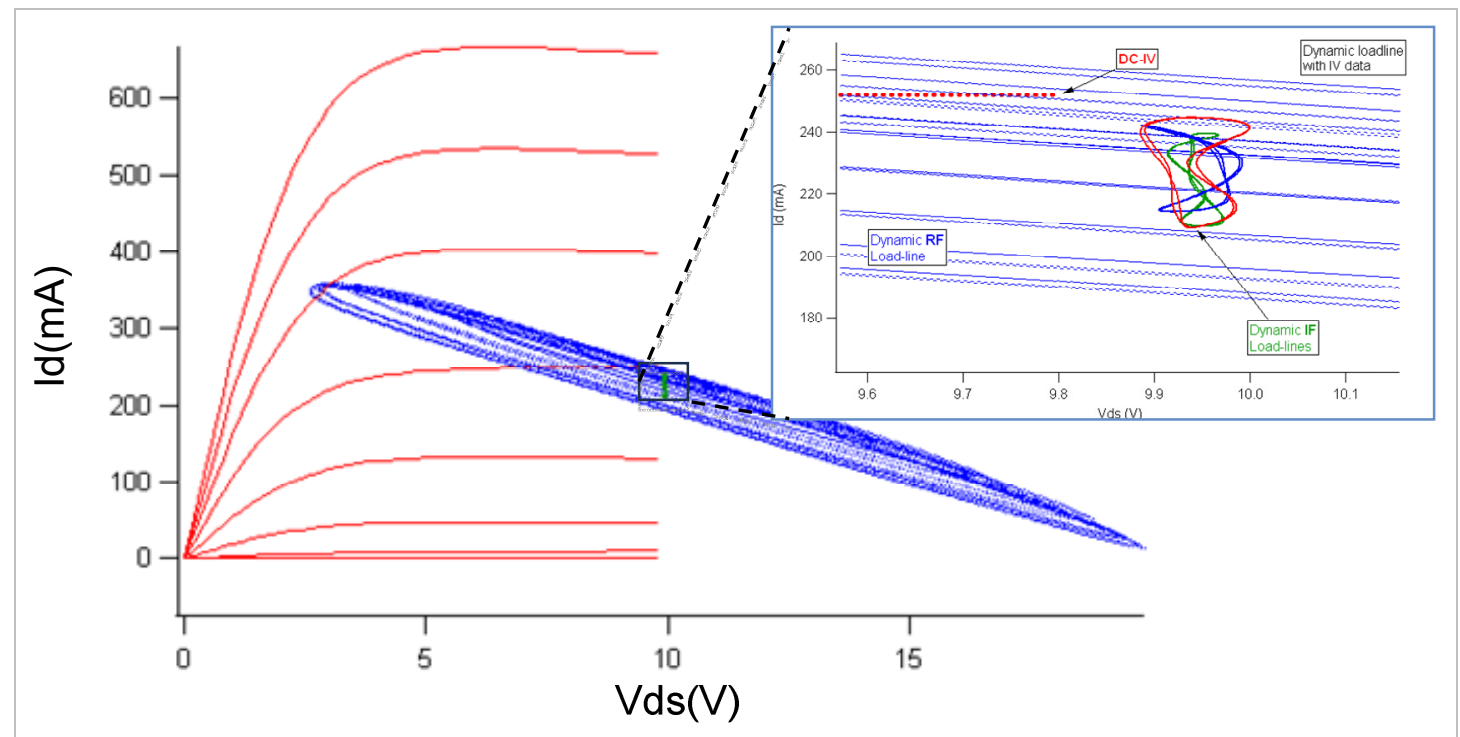

Figure 3.24: Dynamic RF load-line with the IF load-line inset at different tone spacings. 


\subsubsection{Delay Adjustment Estimation}

The device linear propagation delay results in the output signal envelope being of later time interval then the input signal envelope. This delay has to be accurately computed so that the output envelope can be time aligned compared to input envelope. The device's linear propagation delay has to be accurately computed so that the input and output envelopes can be time aligned. The delay is computed using the equation 3.23 and applied to the output envelope.

$$
\begin{aligned}
& \cos \left(\omega_{1} t\right)+\cos \left(\omega_{2} t+\phi\right) \\
& =2 A \cos \left[\left(\frac{\omega_{1}-\omega_{2}-\phi}{2}\right) * t\right] \cos \left[\left(\frac{\omega_{1}+\omega_{2}+\phi}{2}\right) * t\right]
\end{aligned}
$$

Whereas the envelope delay is given by

$$
\cos \left[\left(\omega_{1}-\omega_{2}-\varphi\right)^{*} \mathrm{t}\right]
$$

Figure 3.25 shows that the envelopes are perfectly aligned at their minima. However there appears to be non-quasi static envelope distortion even after terminating the significant baseband components into short circuits.

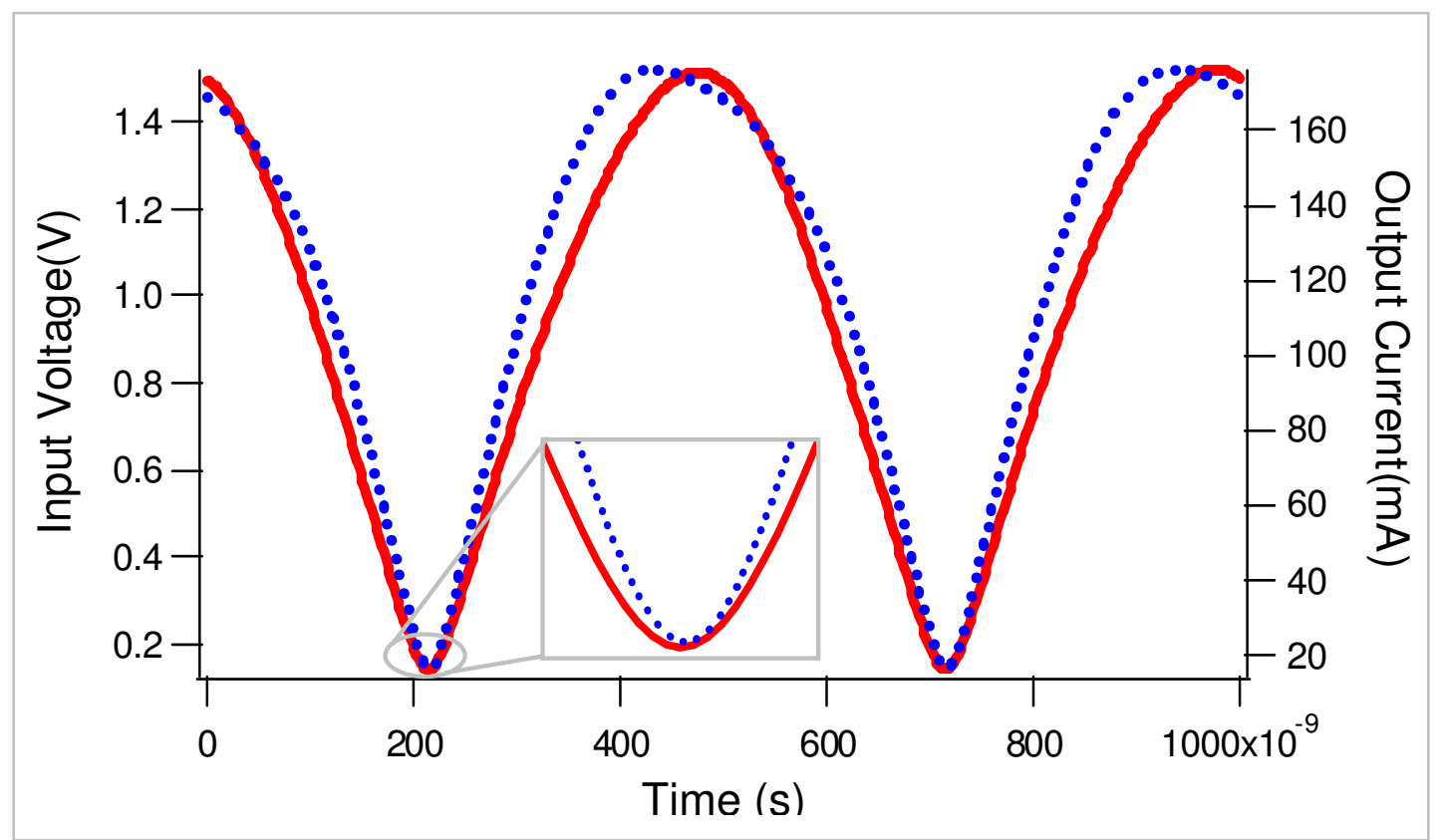

Figure 3.25: Delay compensated output current envelope at $5 \mathrm{MHz}$ tone spacing.

This alignment changes the shape of the transfer characteristics such that the broadening at the bottom is diminished whilst the looping in the upper part of 
the characteristic remains as shown in Figure 3.26. Interestingly however, in the case of IF short circuit termination, it was observed that the approach of shorting these low-frequency voltage components is effective in influencing the IMD asymmetry, suggesting that the hysteresis in the dynamic transfer characteristics is also be minimized by terminating the significant baseband components IF1 and IF2 into short circuits.

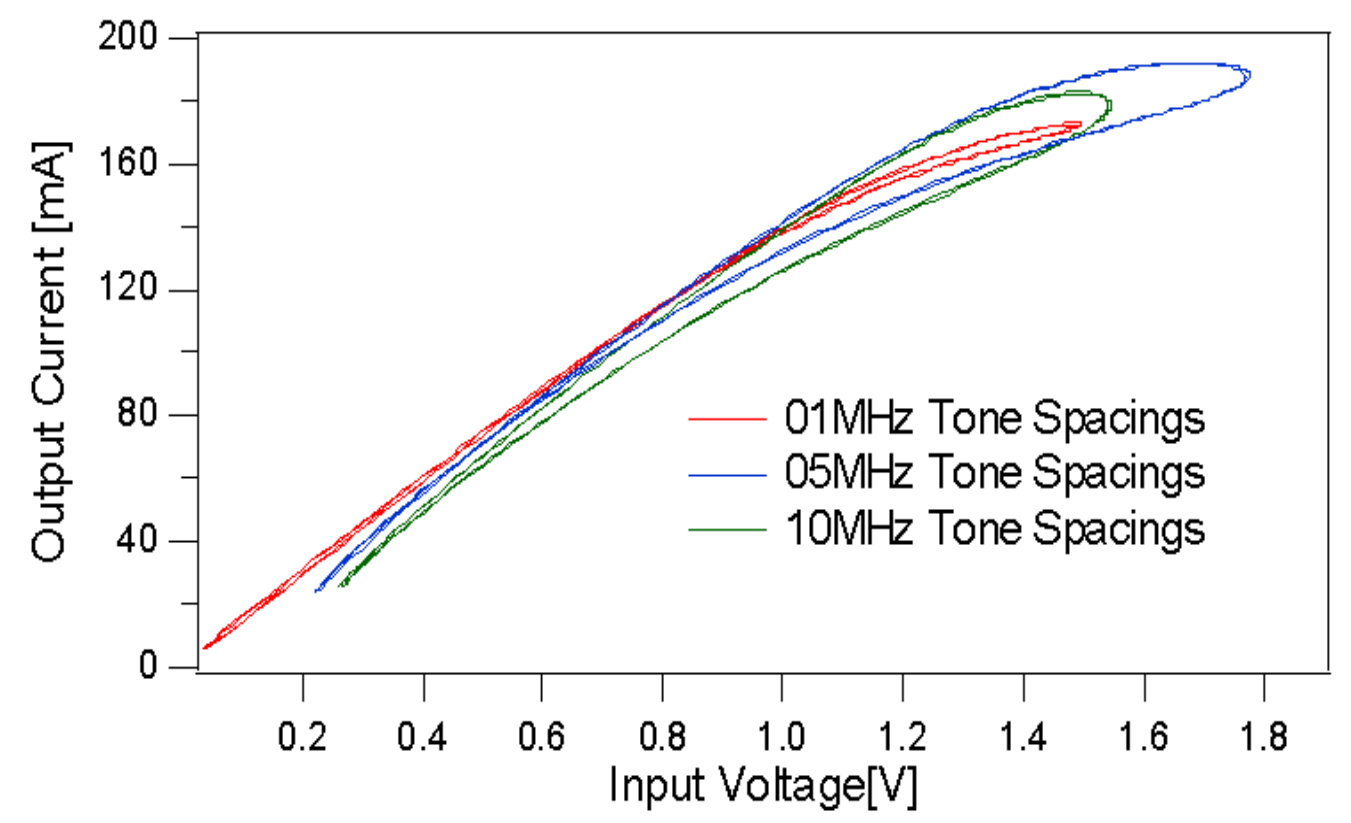

Figure 3.26: Delay compensated dynamic transfer characteristics at different tone spacings for slightly different drive levels at $5 \mathrm{MHz}$ tone spacing.

The residual spread remains in the dynamic transfer characteristics as can be seen from Figure 3.26 for certain bias conditions and tone spacings, and is attributable to non-linear device delay or other sources of memory, specifically thermal effects, $2^{\text {nd }}$ harmonic impedance [17][18], since in this case, is not due to electrical baseband memory.

\subsubsection{Effect of Baseband Impedance on $2^{\text {nd }}$ Harmonic}

Terminating the baseband components with short impedance to suppress intermodulation distortion is desirable. Experimental measurements using a 2W GaN HFET bare die device characterised at $2.1 \mathrm{GHz}$ for varying tone spacing and biased as class $A B$ using a two-tone stimulus have been explained in the preceding section. Here measurements for two different tone 
spacings are considered to compare the output power spectrum, in order to demystify the residual looping in the dynamic transfer characteristics for higher tone spacings. The measured RF two-tone output spectral power for the two-tone spacings of $1 \mathrm{MHz}$ and $10 \mathrm{MHz}$ is shown in Figure 3.27 for when short circuit impedance was presented to IF1 and IF2. The tone spacing of $1 \mathrm{MHz}$ is considered as a reference case due to the fact that at this frequency there appears negligible $\mathrm{IMD}_{3}$ asymmetry and looping in the dynamic transfer characteristics. It was observed that for a tone spacing of $10 \mathrm{MHz}$, the in-band distortions are suppressed while it results in an increase in the out-of-band second harmonic distortions, in contrast to tone spacing of $1 \mathrm{MHz}$. Clearly it can be seen from Figure 3.27 that there is an asymmetry of approximately $4 \mathrm{~dB}$ between the lower and upper IMD3 products. These results, therefore, indicates that there is a very strong link between the injected baseband signal and the second harmonic components.

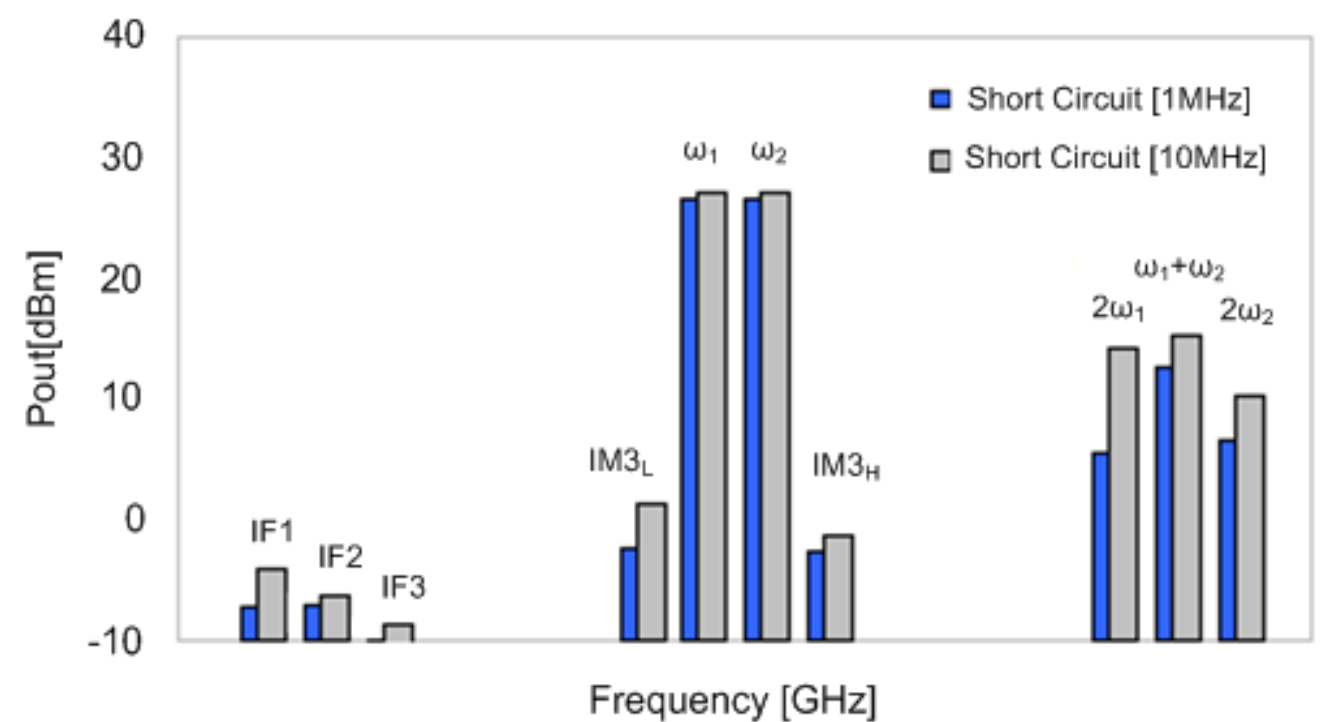

Figure 3.27: Measured output power spectrum for Tone spacing of $1 \mathrm{MHz}$ and $10 \mathrm{MHZ}$ when short circuit was presented to IF1 and IF2.

It could therefore be concluded on the basis of this result that this behaviour is due to the mixing process between output components. To be more precise, when the output fundamental signals mix with IF1 signals, there is a possibility that they generate products which are out of phase with $\mathrm{IMD}_{3}$ (reduction in $\mathrm{IMD}_{3}$ ) and in phase with second harmonic components (increment in $2 \omega_{1}, 2 \omega_{2}$ and $\left.\omega_{1}+\omega_{2}\right)$. Consequentially, base-band impedance modifies not only the 
level of in-band distortion components but also modifies the level of out-ofband distortion components especially those around the second harmonic of the carrier. The results confirm that some of residual spread in the dynamic transfer characteristics is associated with the $2^{\text {nd }}$ harmonic components, not due to baseband electrical memory effects.

\subsection{Broadband Baseband Impedance Termination}

To explicitly investigate the effect of broadband baseband impedance on the output envelopes of the device and reduction of electrical baseband memory, the system was calibrated using a custom $50 \Omega$ test fixture over a bandwidth of $50 \mathrm{MHz}$ at baseband, and over $180 \mathrm{MHz}$ fundamental RF bandwidths. This fully vector error corrected system can account for any errors introduced due to losses, mismatches and imperfect directivities in the system and allows for the measurement of the complete modulated voltage and current waveforms that exist at the DUT plane. In this set of measurements, RF fundamental and associated harmonics were terminated into a nominal impedance of $50 \Omega$, whilst the IF components were actively load-pulled. The investigations were performed on a 10W GaN device biased in class- $A B$ and characterized at a fundamental frequency of $2 \mathrm{GHz}$, for different values of tone spacing. Respective drain and gate bias voltages of $28 \mathrm{~V}$ and $-2.05 \mathrm{~V}$ resulted in a quiescent drain current of $250 \mathrm{~mA}$ ( $\mathrm{I}_{\mathrm{DSQ}} \cong 5 \%$ $\left.\mathrm{I}_{\mathrm{DSS}}\right)$. The device was driven to approximately $1.5 \mathrm{~dB}$ of compression resulting in $39.5 \mathrm{dBm}$ peak envelope power (PEP). To understand the effects of baseband impedance, particularly the significant baseband components IF1 (twice the modulation frequency) and IF2 (four times the modulation frequency), the higher baseband components IF3 (six times the modulation frequency) and IF4 (eight times the modulation frequency) on memory effects and dynamic transfer characteristics, the baseband impedance environment was optimized. Generally, terminating baseband components into nominal $50 \Omega$ impedance will cause a ripple to appear on the DC drain voltage present at the device plane, which effectively results in additional modulation or remodulation of the RF signal. Therefore, the IF active load-pull capability was employed as a means to control all of the baseband components. The 
magnitude of the synthesized reflection coefficient for all baseband components was set to unity whilst the phase was varied in the area of the short circuit at the edge of the Smith chart. Figure 3.28 shows the variation of the four most significant baseband load impedances $\left(\Gamma_{\mathrm{IF}}\right)$ that result for a tone separation frequency of $2 \mathrm{MHz}$.

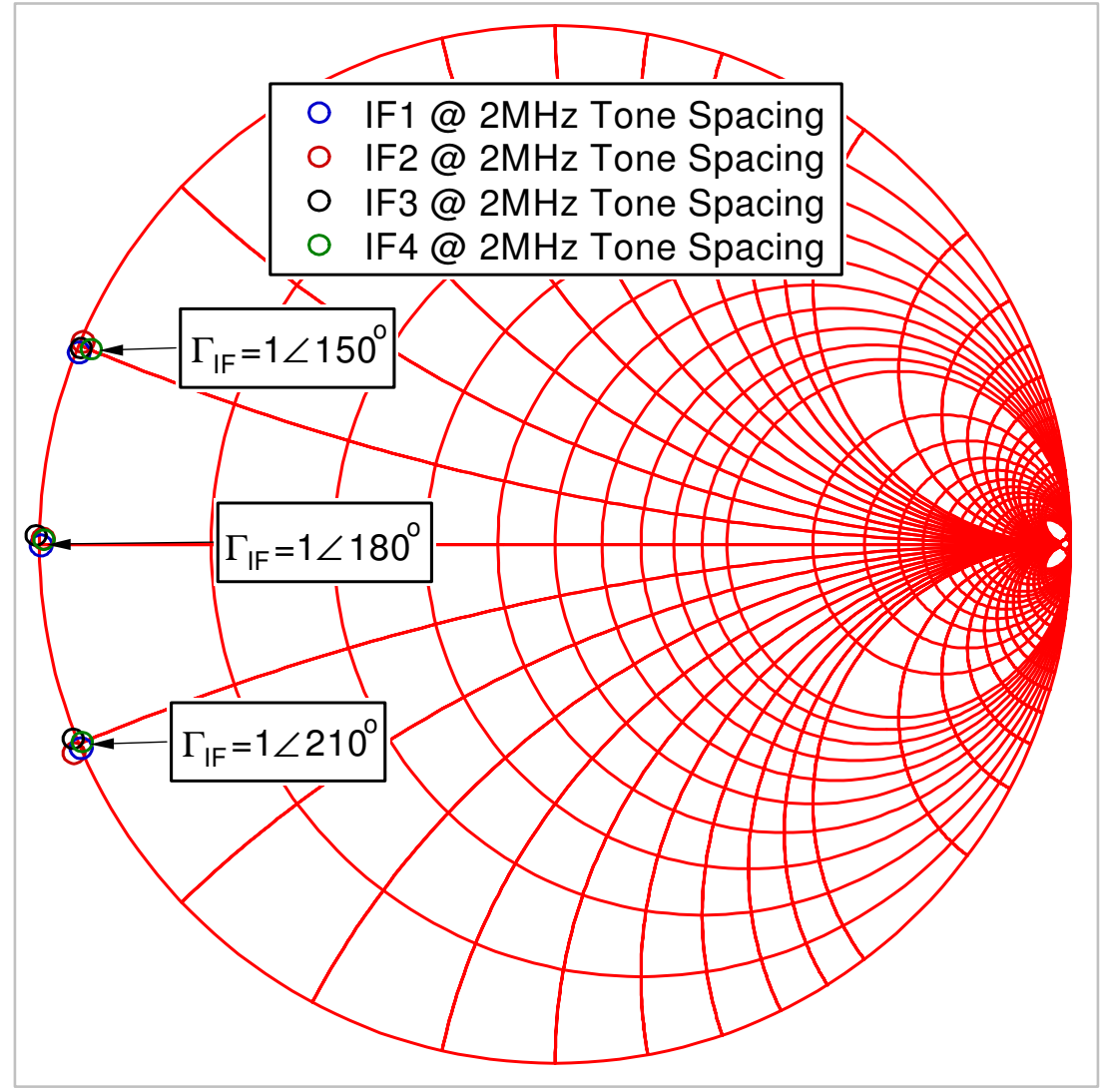

Figure 3.28: Measured baseband impedances at $2 \mathrm{MHz}$ tone spacing for different baseband reflection coefficient $\left(\Gamma_{\mathrm{IF}}\right)$.

It can be seen that $\Gamma_{\mathrm{IF}}=1 \angle 150^{\circ}$ clearly corresponds to an inductive baseband termination and $\Gamma_{\mathrm{IF}}=1 \angle 210^{\circ}$ corresponds to capacitive baseband termination. Working in the envelope domain, and using the measured RF voltage and current envelopes extracted from the measured magnitude and phase of all significant tones around the carrier, it is possible to begin to investigate the causes of envelope asymmetry. Figures 3.29, 3.30 and 3.31 show the input voltage and output current envelopes for each of the three cases of IF load shown in Figure 3.28, and illustrate how terminating the baseband components with reactive impedances dramatically changes the shape of the output current envelopes and result in the different types of induced memory 
effects, observable in the dynamic transfer characteristics; it is this asymmetrical envelope shape that determines which $I \mathrm{IMD}_{3}$ product $\left(2 \omega_{2}-\omega_{1}\right)$ or $\left(2 \omega_{1}-\omega_{2}\right)$ will have the greater magnitude.

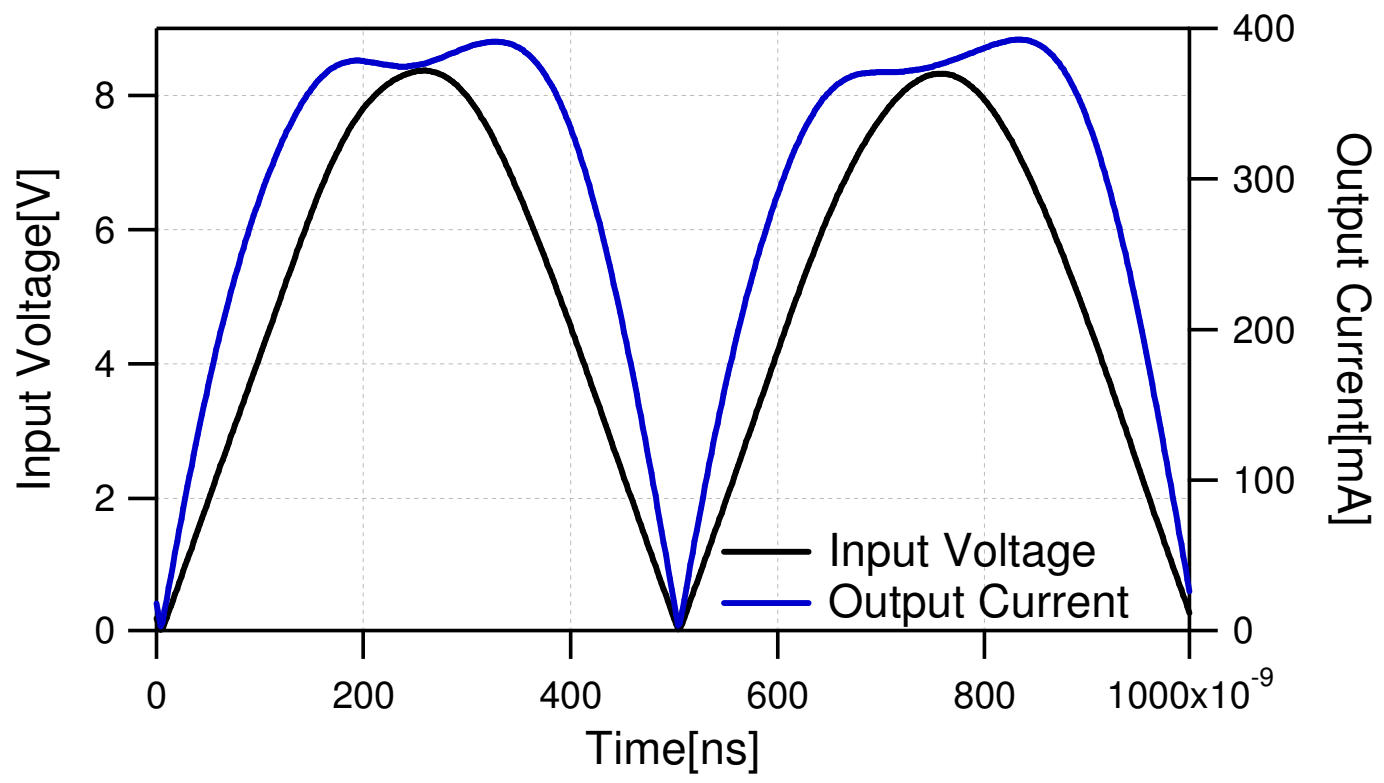

Figure 3.29: Measured dynamic input voltage and output current envelopes for $2 \mathrm{MHz}$ tone spacing at $\Gamma_{\mathrm{IF}}=1 \angle 150^{\circ}$

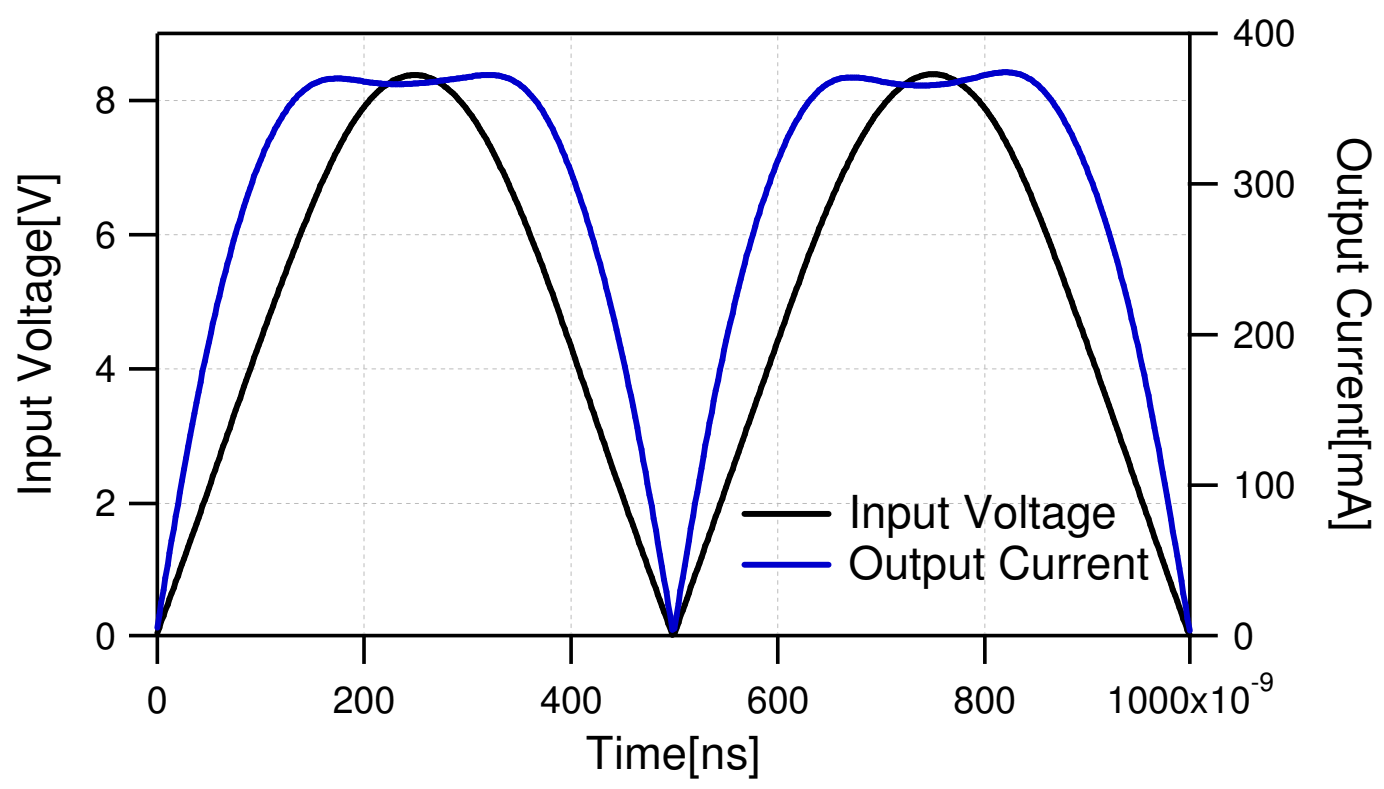

Figure 3.30: Measured dynamic input voltage and output current envelopes for $2 \mathrm{MHz}$ tone spacing at $\Gamma_{\mathrm{IF}}=1 \angle 180^{\circ}$

Indeed, the response of the active power device is often different whereas the same instantaneous power level is applied to the power device if the impedance seen by the device is different at baseband frequencies. 
Therefore, the key outcome of this study reveals that the asymmetrical output envelopes of an active power device profoundly depend on the baseband impedance terminations.

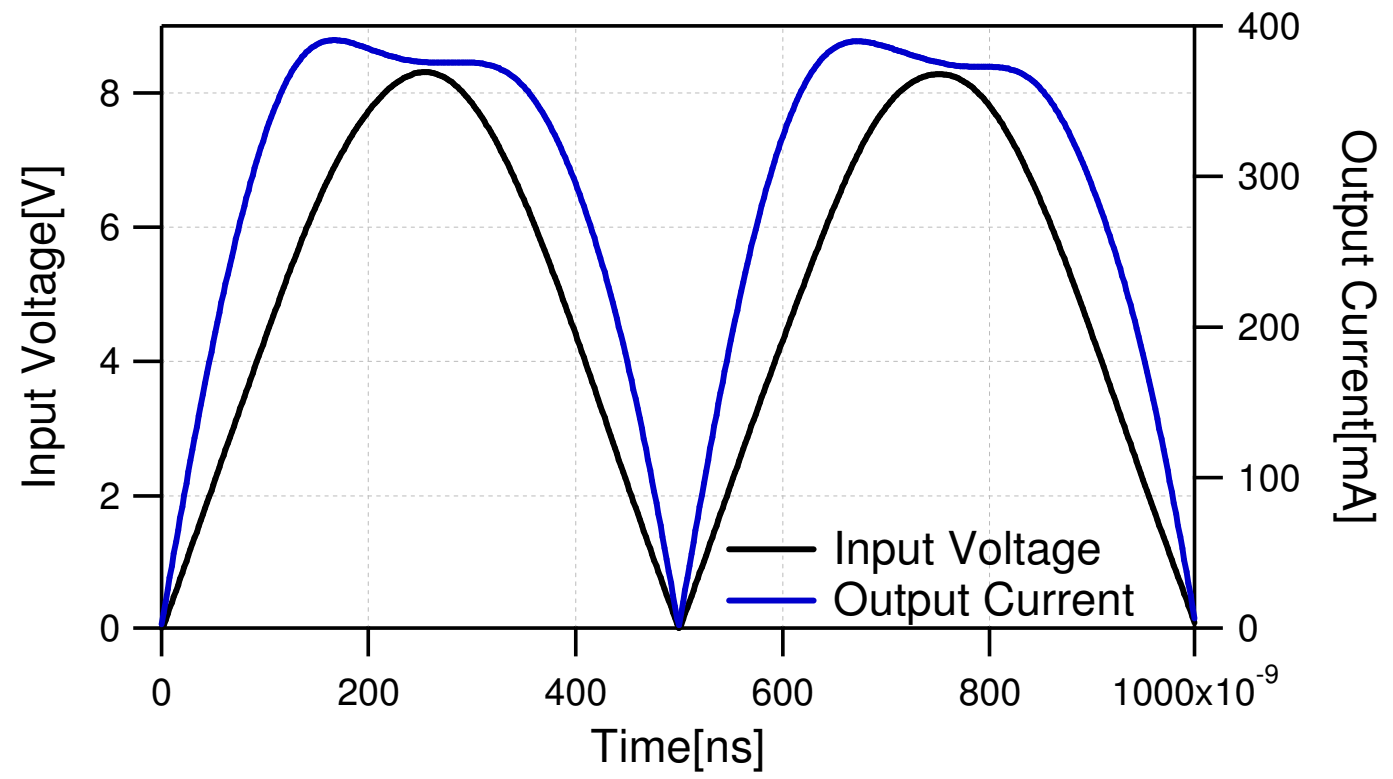

Figure 3.31: Measured dynamic input voltage and output current envelopes for $2 \mathrm{MHz}$ tone spacing at $\Gamma_{\mathrm{IF}}=1 \angle 210^{\circ}$.

\subsubsection{Dynamic Characteristics of Power Device}

Envelope domain representation of device dynamic transfer characteristics gives clear understanding of memory effects and reveals the presence of memory effects. Thus the dynamic transfer characteristics obtained for the same three cases of $\Gamma_{\mathrm{IF}}$ are depicted in Figure 3.32. In the cases where the baseband impedance presented to the transistor's output is no longer a short circuit, the presence of the inductive or capacitive reactance causes the baseband current and voltage waveforms to become phase shifted and as a consequence the output RF current envelope to become asymmetrical, appearing as a phenomenon of hysteresis in the $\mathrm{V}_{\text {in }} \mathrm{I}_{\text {out }}$ dynamic transfer characteristic. However, when short circuit impedance $\left(\Gamma_{\mathrm{IF}}=1 \angle 180^{\circ}\right)$ was maintained, then negligible hysteresis was observed. The asymmetrical current envelopes and hence hysteresis in the transfer characteristic is explained by the fact that at higher drive levels, the RF dynamic load line begins to interact with the knee boundary region, and the degree and nature of the interaction becomes a strong function of the baseband impedance environment. In the case of baseband short circuit 
termination $\left(\Gamma_{\mathrm{IF}}=1 \angle 180^{\circ}\right)$, the IF load-line (achieved by plotting baseband current vs baseband voltage) remains completely vertical with no looping observed, as shown in Figure 3.32.

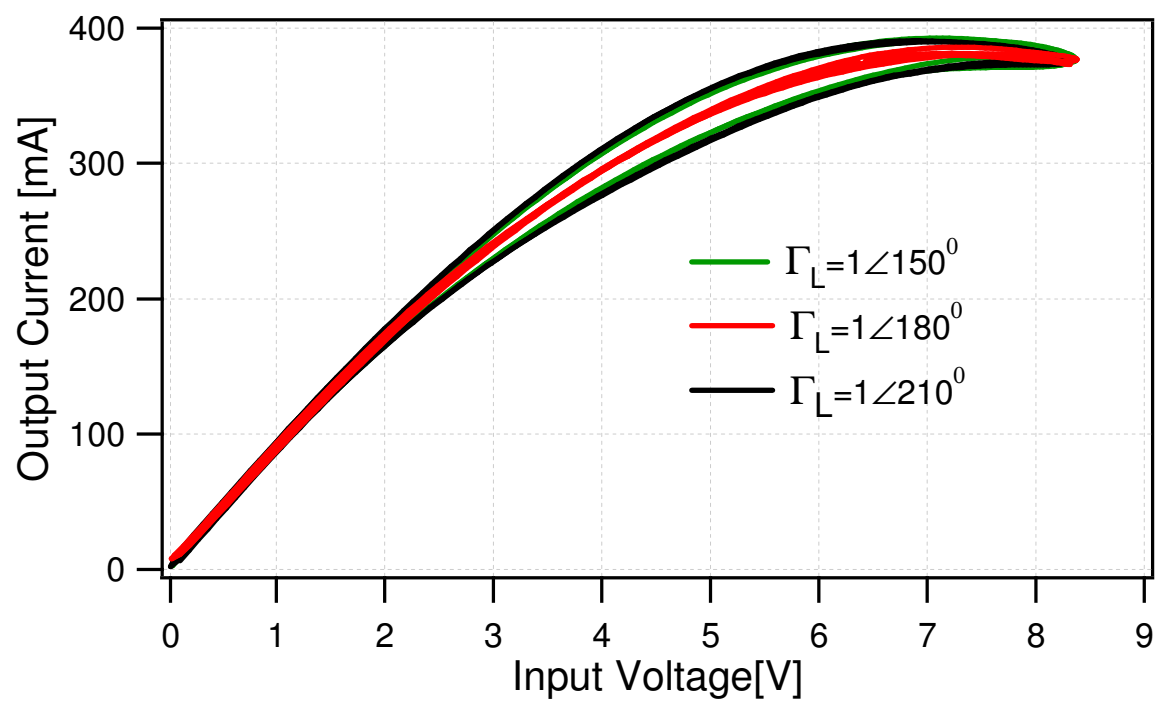

Figure 3.32: Dynamic transfer characteristics at different baseband reflection coefficients for $2 \mathrm{MHz}$ tone spacing.

This particular behaviour of the IF load-line ensures that the RF load-line expands and contracts along the same trajectory, both in the linear region and when it interacts with the knee boundary. As a result, no hysteresis is observed in the dynamic transfer characteristics.

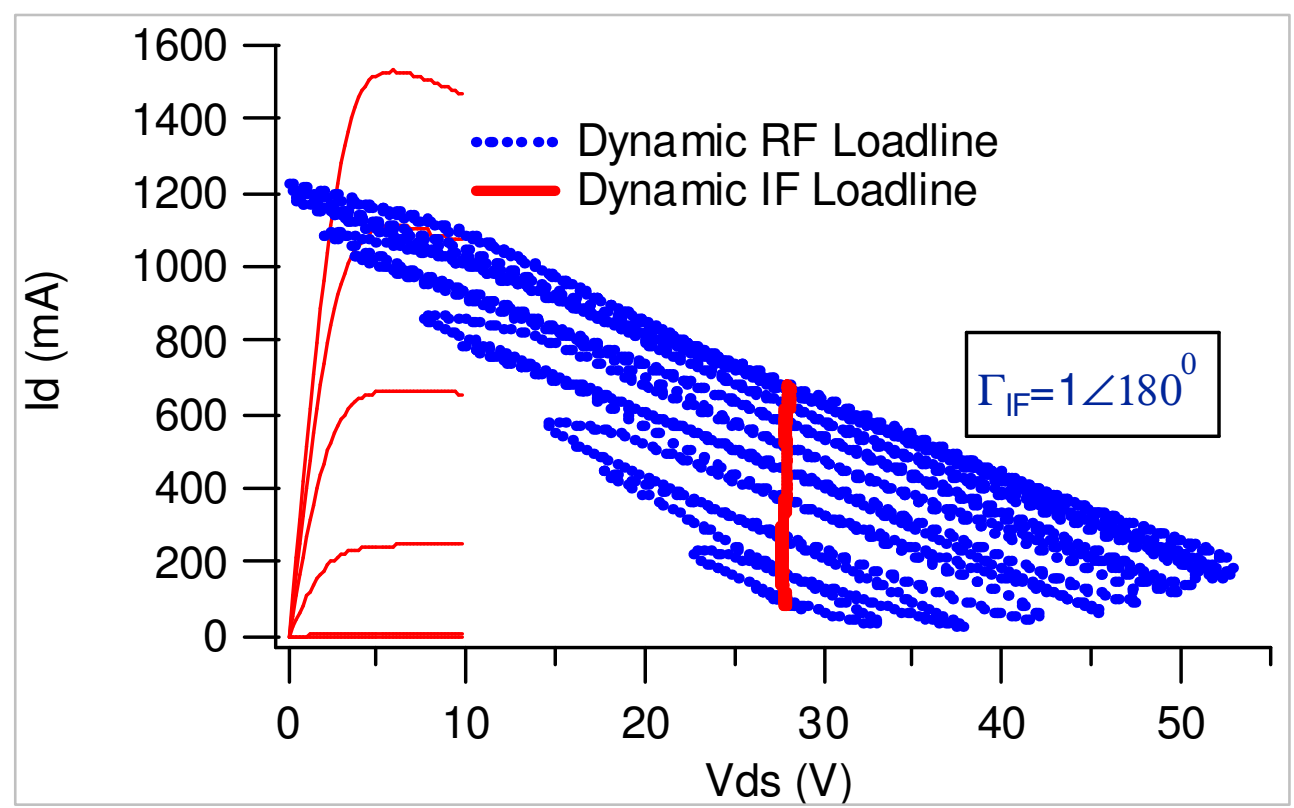

Figure 3.32: Dynamic RF load-line and IF load-lines at baseband reflection coefficients $\Gamma_{\mathrm{IF}}=1 \angle 180^{\circ}$. 
For the induced reactive baseband impedances conditions, there is a significant looping in the IF load-lines, both of which will cause an asymmetrical interaction with the knee boundary condition, as shown in Figure 3.33 and Figure 3.34. The impact of this interaction is clearly visible on either the rising edge or falling edge of each half of the modulation cycle. This behaviour causes the RF load-line to follow a different path in-to and then outof the knee region; as a result asymmetrical output current envelopes emerge.

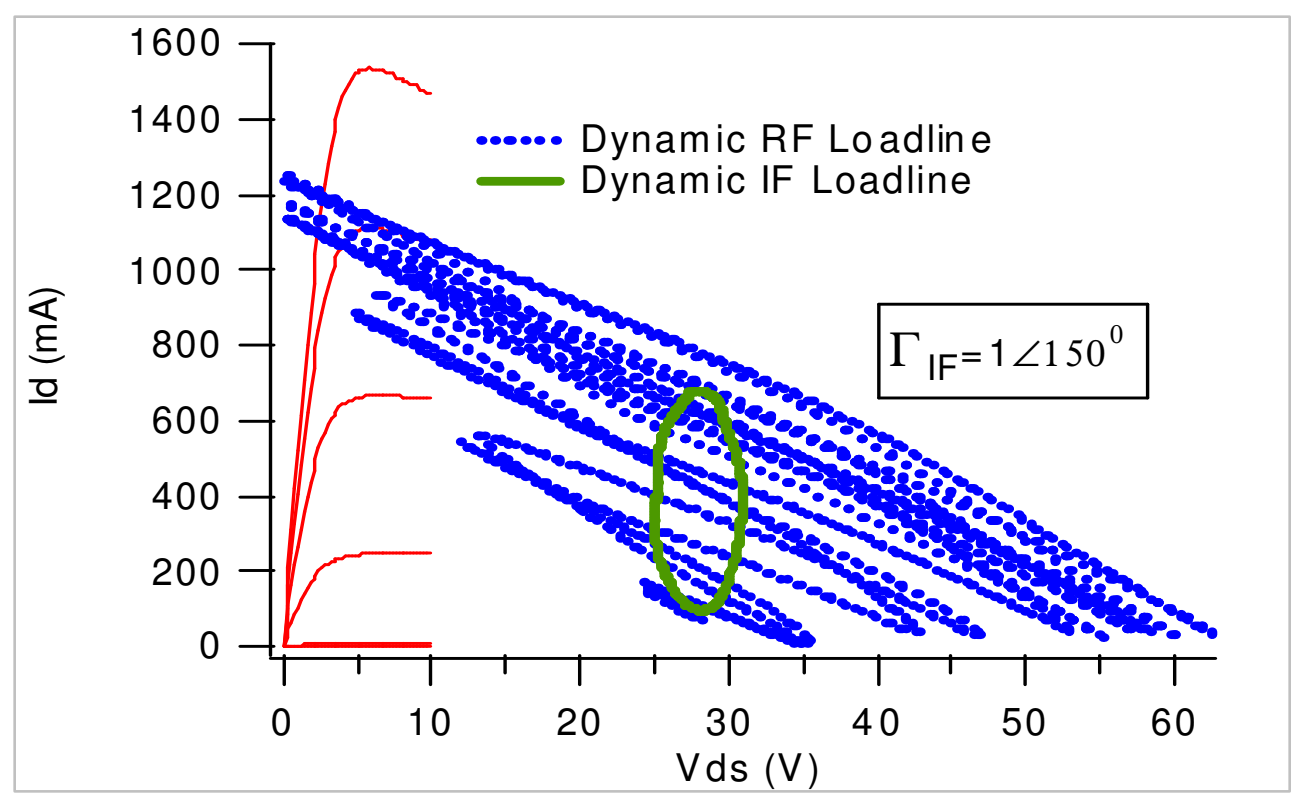

Figure 3.33: Dynamic RF load-line and IF load-lines at baseband reflection coefficients $\Gamma_{\mathrm{IF}}=1 \angle 150^{\circ}$.

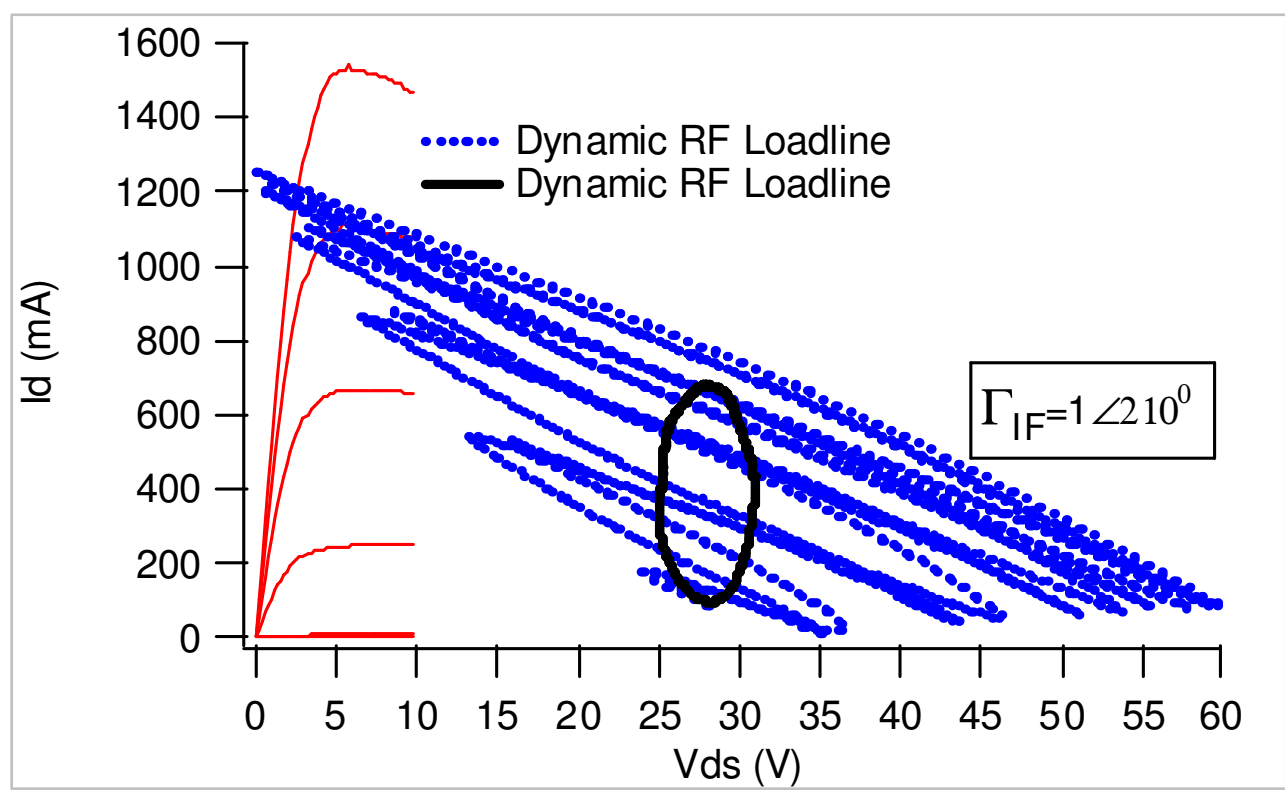

Figure 3.34: Dynamic RF load-line and IF load-lines at baseband reflection coefficients $\Gamma_{\mathrm{IF}}=1 \angle 210^{\circ}$. 


\subsubsection{Minimization of Baseband Electrical Memory}

The electrical memory-effect originating from the baseband is not only a function of IF1 $\left(\omega_{2}-\omega_{1}\right)$, IF2 $\left(2 \omega_{2}-2 \omega_{1}\right)$ but also the higher baseband components (IF3 and IF4). In terms of suppression of baseband electrical memory effects, it is clear that all significant baseband frequencies need to be terminated with near short circuit impedance [6] - a common practice for envelope termination in order to achieve symmetrical IMD terms. This is indeed critical under realistic test signals which may have baseband components that extend from zero up to $100 \mathrm{MHz}$.

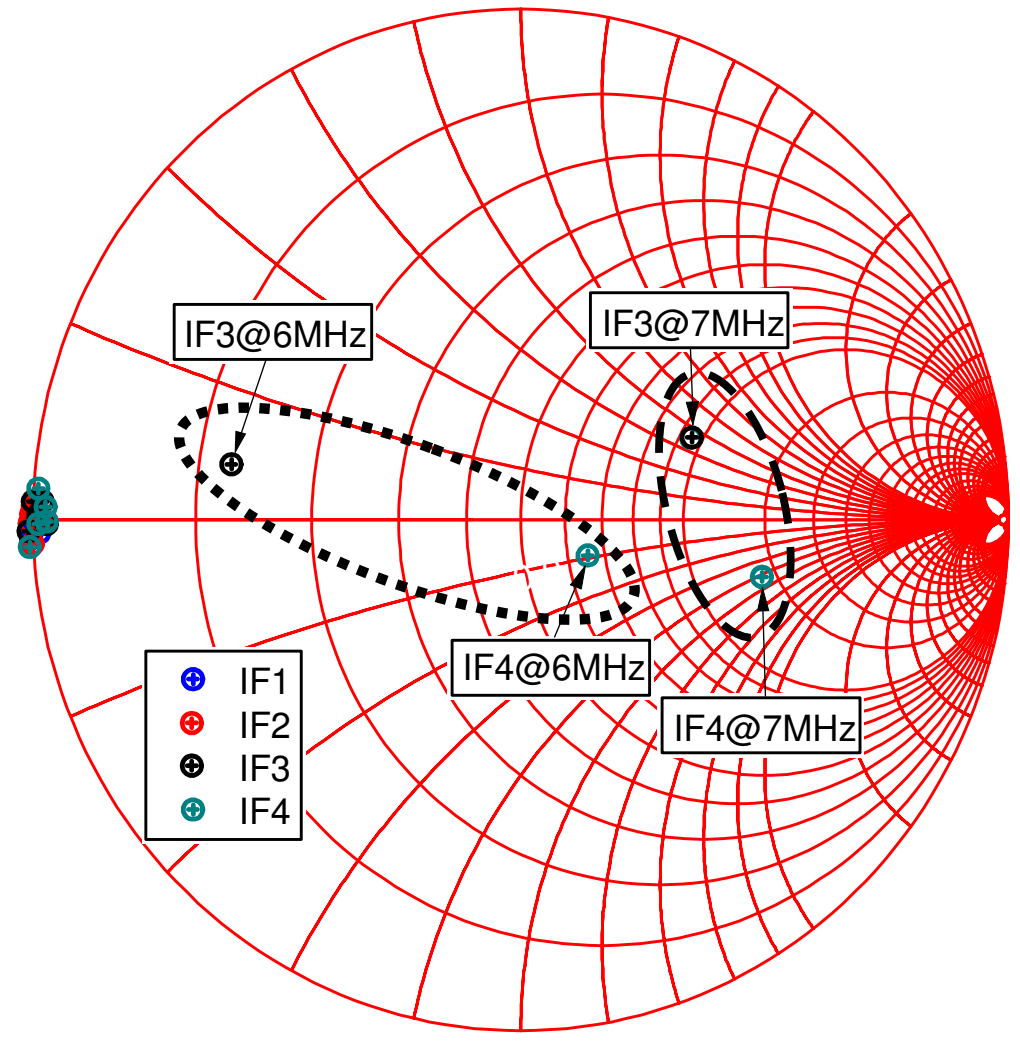

Figure 3.35: Measured baseband impedances at different tone spacing after IF active load-pull.

The overall suppression of baseband components using active IF load-pull, emphasize the importance of broadband termination of the measurement setup when performing linearity analysis of an active device. The bandwidth of the active IF load-pull was limited to approximately $20 \mathrm{MHz}$ by the baseband power amplifier used to amplify the signal generated by the arbitrary waveform generator (AWG). This means that it becomes impossible to 
actively control the higher baseband components (IF3 and IF4) for tone separations above $5 \mathrm{MHz}$. This can be seen by the scattering of these components in the Smith chart plot of Figure 3.35.

The $\mathrm{IMD}_{3}$ and $\mathrm{IMD}_{5}$ behaviour for different tone-spacing values ranging between $1 \mathrm{MHz}$ and $7 \mathrm{MHz}$, at a constant drive level is depicted in Figure 3.36. The behaviour of the two output tones $\omega_{1}$ and $\omega_{2}$ is clearly observed to be almost independent of the tone-separation frequency. The reduction in $\mathrm{IMD}_{3}$ and $\mathrm{IMD}_{5}$ asymmetry confirms that if a frequency independent constant short circuit baseband impedance termination is utilized in the power amplifier drain bias network, no modulation frequency sensitivity in IMD responses should be observed. The $\mathrm{IMD}_{3}$ and $I M D_{5}$ responses are found to be modulation frequency independent only from $1 \mathrm{MHz}$ to $5 \mathrm{MHz}$.

It is important to note that the variations in the magnitude of $I M D_{3}$ and $I M D_{5}$ components above $5 \mathrm{MHz}$ tone spacing are related to variation in base-band impedance, since it was difficult to present the short circuit impedance to the higher baseband components (IF3 and IF4) due to the bandwidth limitation of active IF load-pull power amplifier.

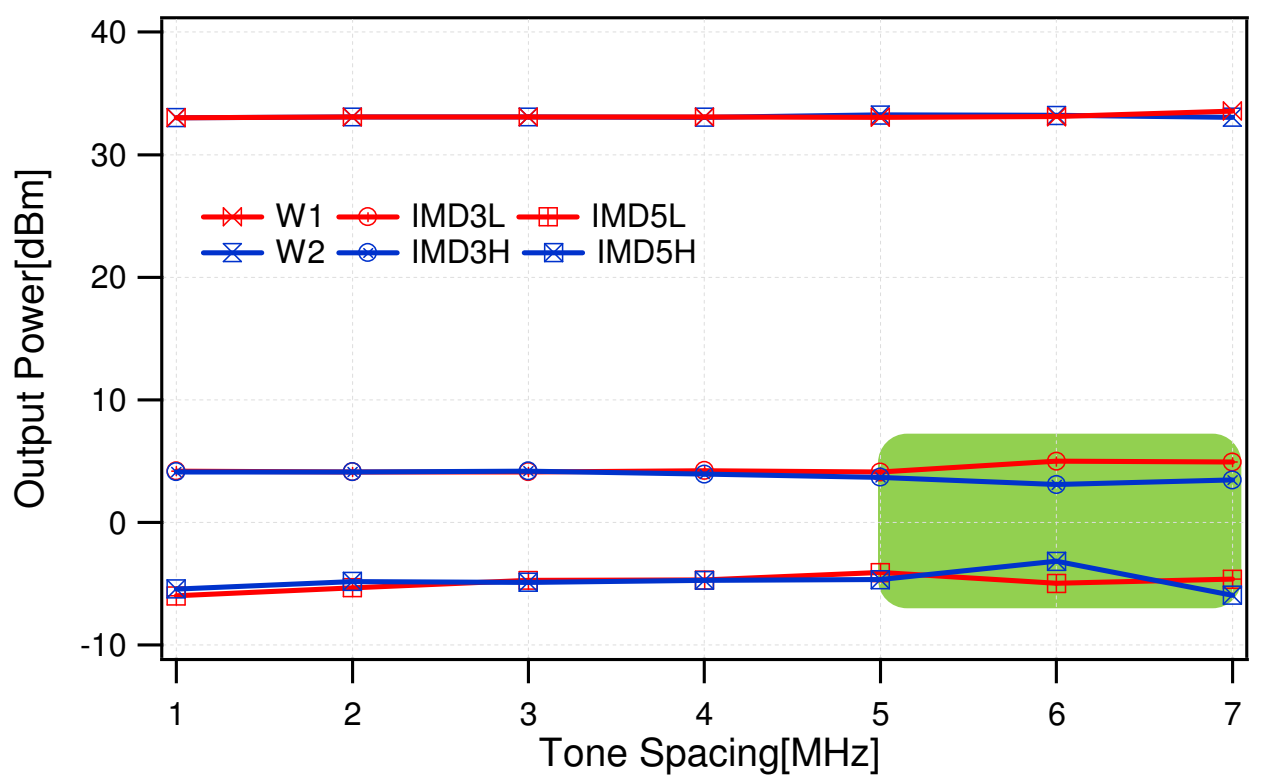

Figure 3.36: Measured fundamental and IMD power for different values of tone spacing at a constant drive level of $1.5 \mathrm{~dB}$ compression point.

These results summarized that the suppression of electrical memory has been achieved by the synthesis and presentation of frequency invariant IF 
impedances to significant baseband components through active IF load-pull. The measurements also demonstrated and confirmed that hysteresis behaviour in the dynamic transfer characteristics has significantly been improved by providing short circuit impedances to all significant baseband components.

The results also suggest however that IMD behaviour in 10W GaN HEMT is not only dependent on the most significant IF component (IF1 and IF2), but is also sensitive to higher order IF components. In order to obtain the frequency independent response, the bandwidth over which the base-band impedances must be engineered should be extended to at least eight times the modulated bandwidth.

\subsection{Chapter Summary}

In this chapter an enhanced modulated waveform measurement system has been demonstrated to confirm and establish the basic understanding of passive baseband termination by synthesising the positive baseband impedances within the Smith chart. It has been shown how important it is to investigate the device related memory behaviour of microwave power transistors in order to mitigate the memory related effects. The investigations were carried out on two different devices using the twotone modulated excitation which provided a more realistic understanding of the factors affecting the performance of a power amplifier operating within a real communication system. The investigation documented in this chapter has mainly focused on the sensitivity of the power device to the terminating baseband impedances at the transistor output. The analysis of the measured voltage and current waveforms in the envelope domain and the resulting dynamic load lines have provided a detailed insight into the causes of asymmetry in the intermodulation distortion products.

Firstly investigations have been carried out on the 2W GaN HFET and envelope domain analysis has revealed the effect of drive levels on envelope distortion and on the measured dynamic transfer characteristic. It is highlighted that at a backed-off drive level the device operates linearly; consequently symmetrical modulation envelopes are observed. When driven 
into compression, these envelopes become asymmetrical however. Using IF active load-pull to control the behaviour of the significant baseband components, $\mathrm{IMD}_{3}$ asymmetry was significantly improved; an effect due to the suppression of baseband electrical memory. The causes of this 'electrical' effect was understood by analysis of the IF dynamic load-lines - explaining that any significant looping in the IF load-line will result in different trajectories of the RF load-line through the static IV characteristic, for different parts of the modulation cycle. The suppressed baseband voltages translated to greatly reduced hysteresis for lower tone spacing, significant hysteresis remained for higher tone spacing in case of $2 \mathrm{~W}$ GaN HFET [19] which is attributable to $2^{\text {nd }}$ harmonic impedance variations.

Secondly, in the case of the 10W GaN HEMT, the suppression of electrical memory has been achieved by the synthesis and presentation of frequency invariant IF impedances to significant baseband components through active IF load-pull. The measurements showed that hysteresis behaviour in the dynamic transfer characteristics was significantly improved by providing short circuit impedances to all significant baseband components. The results also suggested however that IMD behaviour in 10W GaN HEMT was not only dependent on the most significant IF component (IF1 and IF2), but also sensitive to higher order IF components. In order to obtain the frequency independent response, the bandwidth over which the base-band impedances must be engineered should be extended to at least eight times the modulated bandwidth as the modulation bandwidth rapidly increases, 4G systems (LTE and mobile WiMAX) have up to a $20 \mathrm{MHz}$ modulation bandwidth, in order to perform baseband memory effects measurement would thus necessitate a constant, or engineered impedance across a $100 \mathrm{MHz}$ bandwidth considering up to $\mathrm{IMD}_{5}[20]$. 


\subsection{References}

1. O. Hammi, S. Boumaiza, F. M. Ghannouchi, "On the Robustness of Digital Predistortion Function Synthesis and Average Power Tracking for Highly Nonlinear Power Amplifiers", IEEE Transactions on Microwave Theory and Techniques,June 2007, vol. 55, no. 6, pp 1382-1389

2. W. Bösch and G. Gatti, "Measurement and Simulation of Memory Effects in Predistortion Linearizer", IEEE Transactions on Microwave Theory and Tech, Vol.37, December 1989, pp.1885-1890.

3. V. Meghdadi, J. P. Cances, F. R. Chevallier, and J. M. Dumas, "Method for the Simulation of a Nonlinear Amplifier with Envelope Memory Effect", USA Patent, 6.216.100, April 2001.

4. J Lees, T Williams, S Woodington, P McGovern, S Cripps, J Benedikt, and P Tasker, "Demystifying Device Related Memory Effects Using Waveform Engineering and Envelope Domain Analysis", in Proc. $38^{\text {th }}$ European Microwave Conference, October 2008.

5. J. C. Pedro and N. B. Carvalho, "Intermodulation Distortion in Microwave and Wireless Circuits", Norwood, MA: Artech House, 2003.

6. P. B. Kenington, "High Linearity RF Amplifier Design", Norwood, MA: Artech House, 2000.

7. S. C. Cripps, "RF power amplifiers for wireless communications", $2^{\text {nd }}$ edition. Norwood, MA: Artech House, 2006.

8. A. A Alghanim, "High Power Waveform Measurement System Enabling Characterisation of High Power Devices Including Memory Effects", Ph.D. thesis, Cardiff University, March 2008.

9. B. Sklar, "Digital Communications Fundamentals and Applications", Prentice Hall, 1987.

10. José Carlos Pedro, Nuno Borges Carvalho, "Intemodulation Distortion in Microwave and Wireless Circuits", Artech House, 2003.

11.S.A. Maas "Nonlinear Microwave Circuits", Second Edition, Artech House Microwave Library 2003

12.Tudor V. Williams, "A Large-Signal Multi-Tone Time Domain Waveform Measurement System With Broadband Active Impedance Control' Ph.D. thesis, Cardiff University, December 2007.

13. J. Lees, M. Akmal, S. Bensmida, S. Woodington, J. Benedikt, K. Morris, M. Beach, J. McGeehan, P. J. Tasker, "Waveform Engineering Applied to Linear-Efficient PA Design", in Proc. of $11^{\text {th }}$ IEEE Wireless and Microwave Technology Conference(WAMICON), pages:1- 5, April 2010. 
14. Alghanim, A.; Benedikt, J.; Tasker, P., "A measurement test-set for characterisation of high power LDMOS transistors including memory effects", High Frequency Postgraduate Student Colloquium, 2005 , vol., no.pp. 29- 32, 5-6 Sept. 2005.

15. A. Alghanim , J. Lees, T. Williams, J. Benedikt, and P. J. Tasker, "Reduction of Electrical Baseband Memory Effect in High-Power LDMOS Devices using Optimum Termination for IMD3 and IMD5 using Active Load-Pull", IEEE MTT-S International Microwave Symposium Digest, 2008 , pp. 415-418,June 2008.

16. A. Alghanim, J. Lees, T. Williams, J. Benedikt, P. J. Tasker, "Using active IF load-pull to investigate electrical base-band induced memory effects in high-power LDMOS transistors", in Proc. Asia-Pacific Microwave Conference, Page(s):1 - 4, 11-14 Dec. 2007.

17.N. Le Gallou, J.M. Nebus, E. Ngoya, H. Buret, "Analysis of low frequency memory and influence on solid state HPA intermodulation characteristics", IEEE MTT-S Digest, Phoenix, vol.2, pp.979-982, May 2001.

18.Z. Ouarch, J.M. Collantes,J.P. Teyssier, R. Quere, "Measurement based nonlinear electrothermal modeling of GaAs FETwith dynamical trapping effects", IEEE MTT-S Digest, Baltimore, vol.2, pp.599-602, Jun 1998.

19. M. Akmal, J. Lees, S. Bensmida, S. Woodington, J. Benedikt, K. Morris, M. Beach, J. McGeehan, P. J. Tasker, "The Impact of Baseband Electrical Memory Effects on the Dynamic Transfer Characteristics of Microwave Power Transistors", in Proc. of $4^{\text {th }}$ International Nonlinear Microwave Monolithic Integrated Circuit(INMMIC), pages: 148 - 151, , April 2010.

20. M. Akmal, J. Lees, S. Bensmida, S. Woodington, J. Benedikt, K. Morris, M. Beach, J. McGeehan, P. J. Tasker, "Minimization of Baseband Electrical Memory Effects in GaN HEMTs Using IF Active Load-pull", in Proc. Of Asia Pacific Microwave Conference (APMC), pages: 97-100, December 2010. 


\section{Chapter 4}

\section{Envelope Tracking Focused Investigations}

\subsection{Introduction}

High efficiency radio frequency (RF) power amplifiers (PAs) are critical in portable battery operated wireless communication systems because they can dominate the power consumption. Linear power amplifiers are required for applications using non-constant envelope modulated signals to minimize distortions. Traditionally, linear power amplifiers are implemented by "backingoff" the output power of PAs for a spectrally efficient high peak-to-average (PAR) signal, the average PA efficiency is much lower than the peak efficiency, which is undesirable. This poses conflicting design requirements, in particular, to maintain both high efficiency and meet the spectral requirements in modern wireless communication systems. Thus, in order to achieve truly broadband, highly efficient linearization of increasingly complex PA architectures, baseband as well as RF effects must be carefully considered. This can be of particular importance when working with architectures such as the Doherty PA, where successful design relies upon the precise and highly optimised interaction between two or more active devices. These devices themselves may be operating in efficient or efficient-linear modes of operation such as inverse class-F or class-J, as well as using different types or versions of device. Such designs become challenging when steps are made to improve efficiency by the application of 'envelope' based approaches such as 
Envelope Elimination and Restoration (EER) and Envelope Tracking (ET) [1]. The envelope tracking has widely gained acceptance in improving the PA efficiency but does present some problems, most notably the need for a highly efficient baseband tracking amplifier and a great dependency on the linearization techniques to achieve the acceptable level of linearity. This chapter builds upon the work documented in chapter 3 which focused primarily upon emulating passive baseband impedances inside the Smith chart, such as those that can be presented by an actual bias insertion network.

The aim of this chapter is to explore in more detail the impact of broadband baseband impedance termination upon linearity, and efficiency through the synthesis of negative broadband baseband impedances that allows for example the emulation of Envelope Tracking (ET) environments around a particular device. It outlines an important variation of the envelope tracking technique, termed as auxiliary envelope tracking (AET), in power management to reduce the DC average power consumption of power amplifier which tends to operate at less than maximum power. It is shown that efficient and rapid adjustment of the supply voltage for the power amplifier can provide up to an order of magnitude higher average efficiency and major linearity improvements through the use of an injected baseband signal. This also highlights the fact that the PA linearity can be improved using the very mechanisms that are employed to improve PA efficiency whilst ensuring that the power content of the injected AET signal remains very low percentage of the DC supply to the power amplifier.

\subsection{Efficiency Enhancement Techniques}

Power amplifiers universally suffer from reduced efficiency when operated below the maximum output power as limited by the battery voltage, a condition known as power back-off [2]. This problem is compounded by the use of high data-rate standards. Higher data rates tend to require larger amounts of amplitude modulation to increase the number of bits per symbol [2]. However, the signals used in modern wireless communication systems have high PAR, which requires the amplifier to operate at higher back-off. 
This is needed to avoid excessive clipping of the signal and, hence, to operate at higher linearity (minimum spectral spreading). These two requirements in the amplifier, i.e. linearity and efficiency as emphasized before, are the two contrasting parameters in amplifier design. Hence, to satisfy the efficiency-linearity trade-off, one of the following methods is generally followed:

Efficiency enhancements of the power amplifier at higher back-off so that it operates over the entire signal dynamic range with high efficiency. This requires the use of an efficiency enhancement technique like Envelope Tracking Doherty structure [3], Doherty [4], and envelope elimination and restoration [5].

4 Linearization of the power amplifier and operation near its maximum output power. This method makes use of linearization techniques like digital pre-distortion, feed-forward and feed-back. Although the linearity requirements are fulfilled, this method, however, may reduce the overall efficiency due to the additional circuitry involved in the linearization method.

* Application of efficiency enhancement techniques and linearization techniques at the same time. Methods like the ET perform both functions but with an important variation on the existing ET implementation. In ET, the baseband injected signal tracks the RF envelope which potentially results in high efficiency and at the same time high linearity.

In this chapter, the last approach will be followed based on the Envelope Tracking technique, which is one of the most common techniques for improving efficiency at back-off output power level as well as at the maximum output power.

\subsubsection{Peak to Average Ratio}

The peak to average power ratio (PAR) is the ratio between the maximum value of the instantaneous power and the average power of the 
signal [6]. Power amplifiers have their specific peak-to-average ratio, which is very important for amplifier nonlinearity characterization. When the input signal peak level becomes greater than the linear amplification capability of the amplifier, then the signal compression occurs. This causes distortion in the output signal in the form of intermodulation distortion or spectral regrowth [6]. Normally, the amplifiers are designed such that the signal peak is within their peak power capability. The total peak power of the multi-carrier signal can be calculated as [7]:

$$
P_{\text {peak }}=\sum^{N} \frac{V_{r m s}^{2}}{R}=\frac{N \bullet V_{\max }^{2}}{R}=N \bullet P_{\max }
$$

Where $N$ is the number of signal carriers, $R$ is the channel resistance in ohms, $V_{\max }$ is the maximum signal voltage and $P_{\max }$ is the maximum signal output power. It is important to note that, in case of CDMA signal $N$ codes, the peak power is similar to the peak power of $N$ carriers [7]. The total envelope average of multicarrier signal can be expressed as a sum of the voltage square, that is:

$$
P_{\text {average }}=\sum^{N} \frac{V_{r m s}^{2}}{R}=\frac{N \bullet V_{r m s}^{2}}{R}=N \bullet P_{r m s}
$$

Where $V_{r m s}$ and $P_{r m s}$ are the envelope average or root mean square value of the voltage and power respectively. The envelope voltage $V_{r m s}$ is equal to the instantaneous average value over one cycle $(T)$ and can be calculated by [6]:

$$
V_{r m s}=\sqrt{\frac{1}{T} \cdot \int_{0}^{T} V^{2}} d t
$$

Where $T$ is the period of the signal. Consequently, the envelope peak to average power ratio (PAR) in $\mathrm{dB}$ can be expressed as:

$$
P A R=10 \log _{10}\left(\frac{P_{\text {peak }}}{P_{\text {average }}}\right)
$$

In the case of a multi-tone signal, in which each carrier is represented as unmodulated sinewave signal with constant envelope, the envelope PAR for $N$ carriers can be calculated in $\mathrm{dB}$ as:

$$
P A R=10 \log _{10}(N)
$$

The maximum power added efficiency of class A amplifier is equal to $50 \%$ at maximum output power. Moreover, if the input signal has $10-\mathrm{dB}$ peak to 
average power ratio, the efficiency will be equal to $5 \%$, which means, to transmit a 1 Watt average power, the amplifier should be able to pass 10 Watt peak power [7]. Therefore, efficiency and linearity of all amplifier classes are dependent on PAR.

In general, by using digital modulation signals, the efficiency of the signal bandwidth is improved. However, the peak power level changes continuously and randomly with the digital data transmitted. In table 4.1, some examples are given to compare the PAR for different signal types [8]. The different PAR are provided due to change in signal peak power which is dependent on different factors such as modulation scheme type, baseband filtering and number of carriers[6], [7].

Table 4.1: Different PAR values for different signal types [8].

\begin{tabular}{|c|c|c|}
\hline Signal Type & Modulation Method & PAR[dB] \\
\hline Single Tone & FM & 3 \\
\hline Two Tone & AM & 3.2 \\
\hline Three Tone & AM & 9.5 \\
\hline Nine Tone & AM & 1.5 \\
\hline GSM & GMSK & 3.5 \\
\hline TDMA & T/4 DQPSK & $10-12$ \\
\hline CDMA & QPSK/DSSS & $8-9$ \\
\hline WCDMA & QPSK/DSSS & 0 \\
\hline
\end{tabular}

\subsubsection{Envelope Tracking}

Modulated RF input signal is split in two paths with a power splitter, one part going directly to the PA and the other part being fed to an envelope detector. Detected envelope signal is amplified with a DC-DC converter which modulates the PA supply voltage. Thus the envelope tracking method enhances the operation of a conventional linear PA by modulating the supply voltage in accordance to the input signal envelope. As the amplifier is linear, the supply variations do not need to follow the amplitude exactly. In an 
envelope tracking system shown Figure 4.1, the PA supply voltage is dynamically adjusted to the most efficient level for the instantaneous output power level. This allows the PA to operate near maximum efficiency even while in power back-off. To increase the efficiency of the RFPA, the power supply must be adjusted efficiently and rapidly and this requires a fast switching regulator power supply. However, this implementation has a stringent requirement that the properties of a linear PA when used in envelope tracking system are anything but static. Several of a transistor's properties are closely tied to its gate and drain voltages such as input and output impedances, gain, phase shift and thermal properties. Therefore the designer of an envelope tracking amplifier is bound to have to make compromises between linearity, power gain, efficiency and bandwidth.

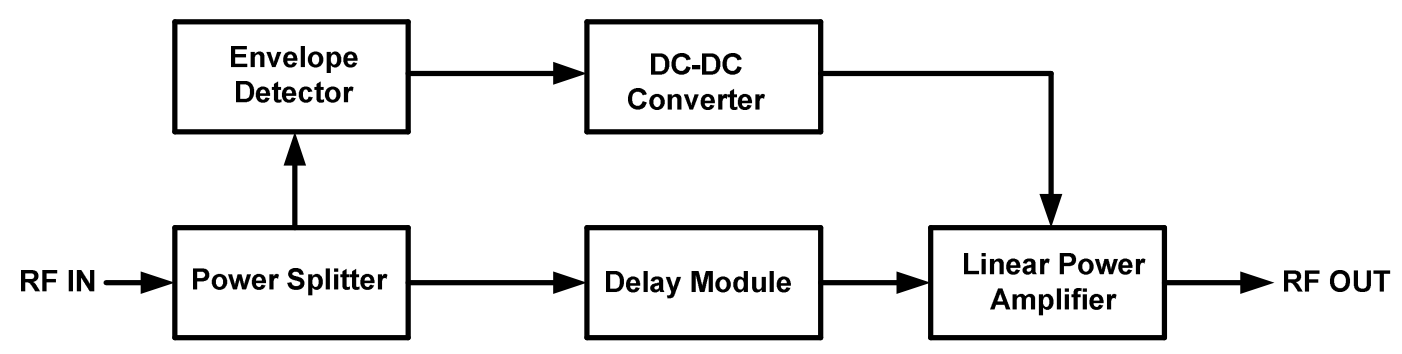

Figure 4.1: Basic diagram of an envelope tracking system [2]

The problem that surfaces because of envelope tracking is the difficulty of power supply filtering due to requirement for broadband power supply modulation. This rules out any excessive capacitor and inductor based filtering. The majority of distortion in envelope tracking system comes from transistor's gain and phase dependence on the supply voltage. Gain and phase dependence give rise to the $A M / A M$ and $A M / P M$ distortion, respectively.

Another source of distortion is the ripple in the drain power supply, which is an effect caused by insufficient power supply filtering. This mechanism may cause asymmetrical distortion to amplified signal, which can be observed as an asymmetric intermodulation distortion (IMD) spectrum [3]. Lastly the DCDC converter adds to the distortion with imperfect amplitude and phase responses. As the signal is split into two separate paths, precise synchronization has to be done before the final PA. This makes the system a 
bit more complicated as a delay module should be added to the design. Some implementations leave it out and instead use the RMS value of the signal to modulate supply voltage. This unfortunately degrades the efficiency of the system [4].

\subsubsection{Envelope Tracking Power Supply}

The purpose of a dynamic supply is to create a time varying supply voltage for the power amplifier and it is instructive to consider a simple case where two supply voltages are available as shown in Figure 4.2. The supply switch, in principle, can be realized using low cost and readily available semiconductors, which for envelope speeds in the $\mathrm{MHz}$ range do not have to be exceptionally fast, by modern standards. The drive signal to the switch would conventionally be derived by using an envelope detector and a threshold circuit which sets the level at which the switch was activated. A modern implementation would probably use a drive signal generated by the system digital signal processor (DSP), which could conveniently be time synchronized with appropriate pre-distortion of the input RF signal. This will include necessary compensation for the small changes in gain and phase in the RFPA as the supply is switched, is clearly an important design choice. This choice will have an optimum value, dependent on the statistics of the signal environment. As with selection of breakpoint in a PA, the intuitive choice would be to set the lower voltage to switch in at the mean power level, the higher supply being used up to the peak power[9].

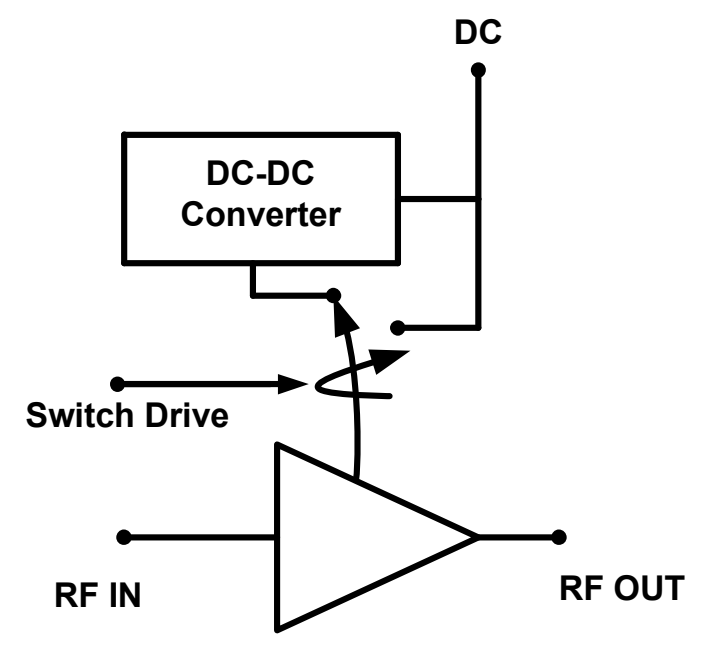

Figure 4.2: Envelope tracking system using two level switched supply. 
The efficiency characteristics shown in [9] assumes that the PA follows the ideal class $B$ power back off (PBO) efficiency characteristics for each supply voltage setting, reaching a maximum value of $70 \%$ at the onset of envelope clipping in each case. In order to evaluate the impact of such a characteristic in a specific signal environment, it is necessary to generate a representative signal burst and evaluate the average efficiency. This process can be repeated for various breakpoint and peak envelope power (PEP) back-off settings.

\subsubsection{Auxiliary Envelope Tracking}

The two carrier and three carrier signals are used in Auxiliary Envelope Tracking (AET) techniques and significant enhancement in efficiency and linearity is achieved. The three carrier input signal with $100 \% \mathrm{AM}$ modulation was amplified by a driver amplifier before being applied to 10W GaN HEMT. In the system described in figure 4.3, the $2 \mathrm{MHz}$ AET signal was generated by the arbitrary waveform generator (AWG). The magnitude and phase of the signal was adjusted appropriately, so that the optimum IMD suppression was achieved. This signal was amplified by an external baseband amplifier and was fed to the output of device through purposely built IF test-set [10]. This injected signal then modulates the supply and starts tracking input signal. This technique then not only improves the efficiency but also improves the linearity. The key point in AET is that the power content of the injected AET signal typically remains a very low percentage $(<10 \%)$ of the DC supply to the power device.

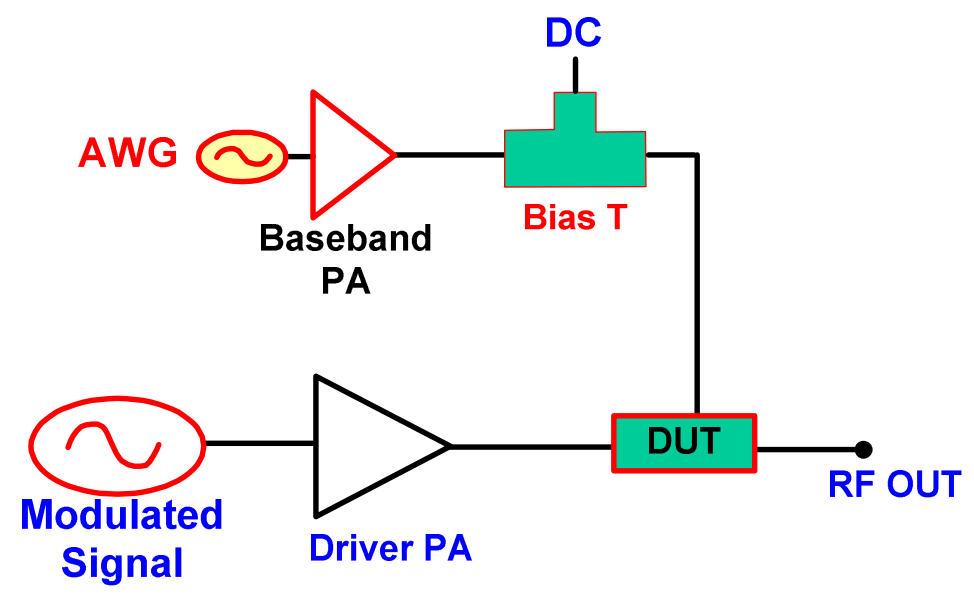

Figure 4.3: Auxiliary envelope tracking system block representation. 


\subsection{Device Level Linearity of GaN HEMTS}

In recent years, regarding linearity of $\mathrm{RF}$ devices and power amplifiers, research has been mainly focussed on characterising power devices in terms of linearity and external linearization of RF power amplifiers (PAs). However, only very few initiatives have been taken so far to analyze or to improve the linearity at device level. According to the previous findings documented in chapter 3 , it is clear that the baseband impedance should be kept constant and as small as possible over the bandwidth of eight times the modulation frequency in order to achieve the modulation frequency independent IM3 and IM5 performance. In this chapter, investigations of improving GaN HEMT linearity are presented based on the aforementioned findings. It is shown that baseband impedance variations have significant influence on device linearity, which can be optimized in order to improve device level linearity. An idea to make an ultra-linear device by the application of additional baseband voltage is also presented.

\subsubsection{Continuous Wave Characterization of GaN HEMTs}

Single tone $(\mathrm{CW})$ measurement provides a basic method of evaluating the linearity characterisation of RF devices. The CW power sweep, for example, is a helpful tool in showing the most common parameter describes the linearity performance extracted under single tone conditions, AM-AM and AM-PM distortion along with quantifying the device $1 \mathrm{~dB}$ compression point. Single tone measurements have been performed on Cree 10W GaN HEMTs characterised at $2 \mathrm{GHz}$ biased in deep class $\mathrm{AB}$.

In the $\mathrm{CW}$ measurement at $2 \mathrm{GHz}$, the RF power was swept at different drain bias voltage from $8 \mathrm{~V}$ to $28 \mathrm{~V}$. Figure 4.4 shows the $\mathrm{P}_{\mathrm{in}} / \mathrm{P}_{\text {out }}$ characteristics of the device obtained by sweeping the input power at fundamental frequency. It can be clearly seen that the output power varies significantly with the drain supply voltage. The AM/AM and AM/PM characteristics of the device when the drain bias voltage is varied are shown in Figure 4.5 and Figure 4.6. The AM/PM characteristics show that the phase changes for a given input power at maximum of about 10 degrees. The measurement showed that the gain varied significantly from $12 \mathrm{~dB}$ to $18 \mathrm{~dB}$ as shown in Figure 4.5. At drain voltage 
of $28 \mathrm{~V}$, the gain measured was around $18 \mathrm{~dB}$ but compresses as the peak input drive level was reached. The behaviour of gain at different drain bias voltage was almost similar but at each higher drain bias voltage, the gain increased by about $0.5 \mathrm{~dB}$.

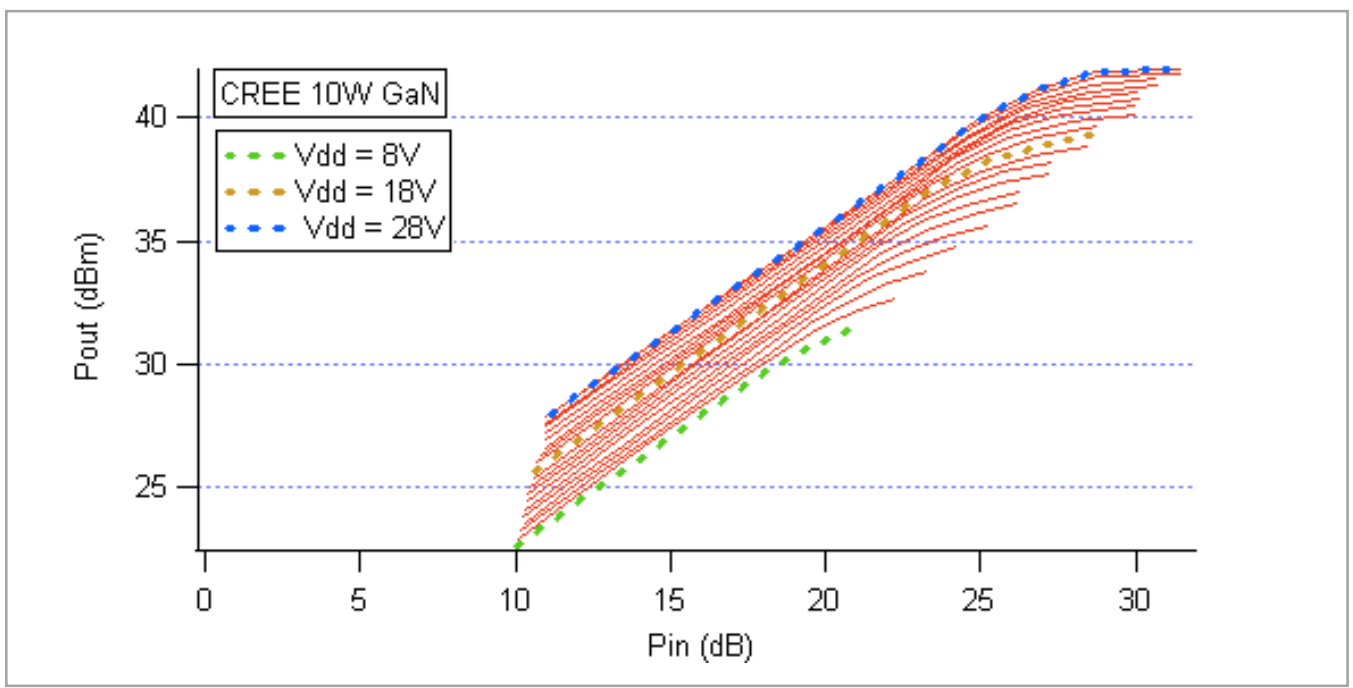

Figure 4.4: Measured single tone power sweeps at $2 \mathrm{GHz}$ for varying drain supply voltage.

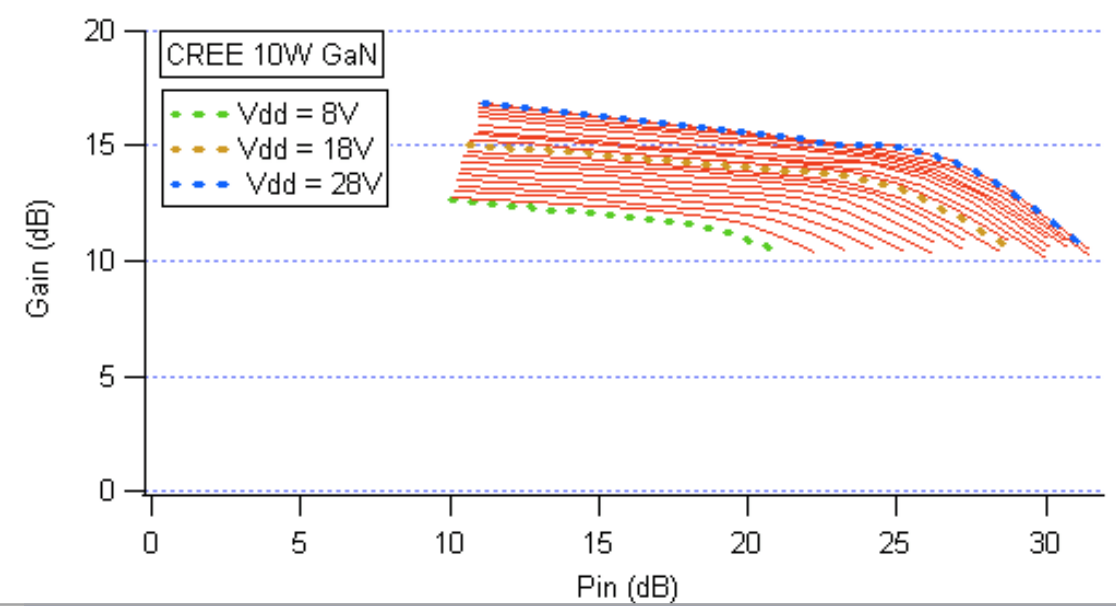

Figure 4.5: Measured AM/AM characteristics of Cree $10 \mathrm{~W}$ GaN for varying drain supply voltage.

The measurement results above show that the GaN 10W device has an expansive gain characteristic with increasing drain voltage. Theoretically, when the device reaches the peak power level the device compressive behaviour can be linearized by the expansive gain characteristic if the drain 
bias voltage is suitably increased. Investigations have been carried out to enhance the linearity of the device through the suitable drain bias voltage when the device is driven into compression to deliver the peak output power.

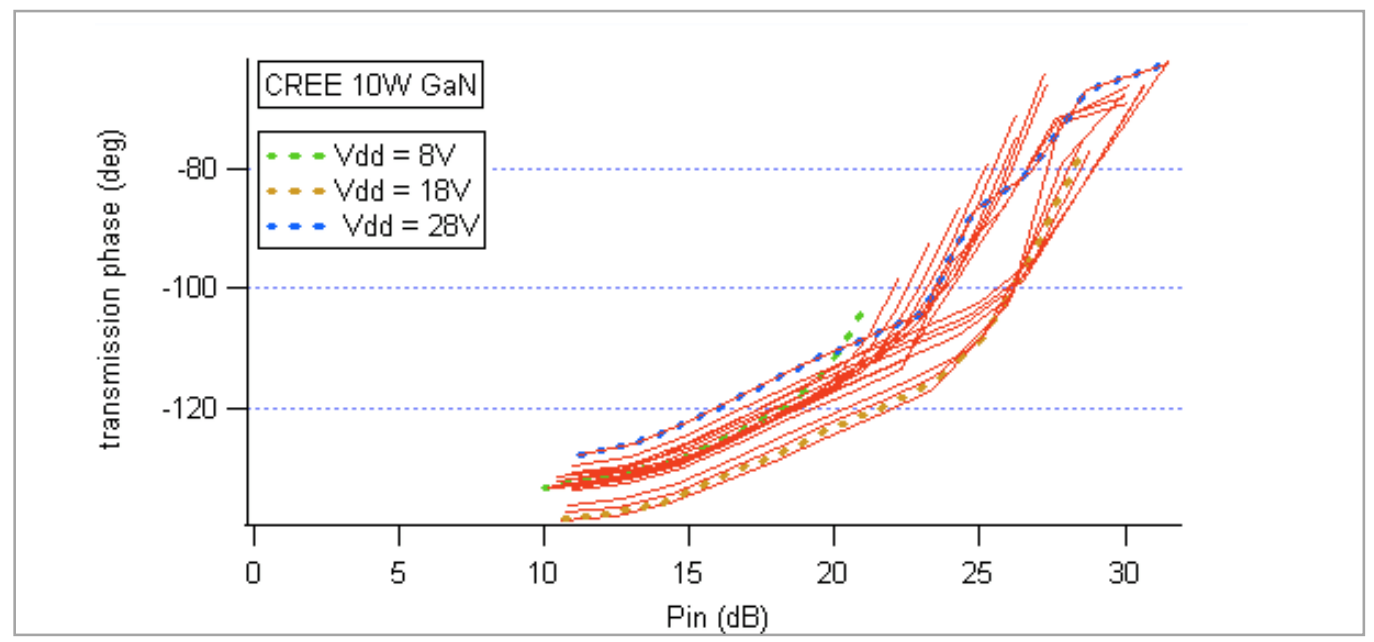

Figure 4.6: Measured AM/PM characteristics of Cree 10W GaN for varying drain supply voltage.

As the linearity of the device improves, a linear and flat gain at peak output power will be observed as well at back-off and compressed drive level. Such drain voltage adjustment has been fed to power amplifier system to improve the linearity, inasmuch as the additional supply voltage is primarily intended to linearize the device, rather than improve its efficiency [11].

\subsubsection{Enhancing Linearity at Back-off Regime}

Wideband digital modulated signals usually have very high peak to average ratio. Therefore, amplifiers are operated at high back-off to meet the linearity specification at peak power. Therefore linearity near saturation is a very important issue to operate a PA efficiently. On the other hand, astonishingly, modern mobile phone basestations have an overall efficiency of $5-10 \%$ that is very bad in terms of energy cost and environment. The unfortunate dichotomy between, linearity and efficiency offers relatively little compromise for an RF circuit designer. ClassA amplifiers are known to be very linear but inefficient as the amplifier is always on and consumes DC power even when there is no RF signal at the input. Higher efficiency classes usually bias the device near or below 
pinch-off so that no DC power is consumed during idle time. Linearity of these classes is usually bad due to clipping effects. It has been shown that, class-B can be linear if the gate closes sharply during pinch-off and the device can be precisely biased [9].

\subsubsection{Baseband Signal Injection Technique}

The influences of second-order components of fundamentals to the third- order intermodulation products in microwave power devices have been investigated. The second order components termination control is a special strategy for the amplifier linearization to eliminate the second-order components such that the interaction of the second order components with the carrier frequencies through the second-order nonlinearities of the amplifier can be greatly suppressed. However, second-order components can be injected into the output of device, to impede the formation of the overall IMD3, to eliminate them. The second order injection technique has been practically considered in this work, and showed a superior performance and the advantage of using the baseband signal injection to maintain and enhance the device linearity performance.

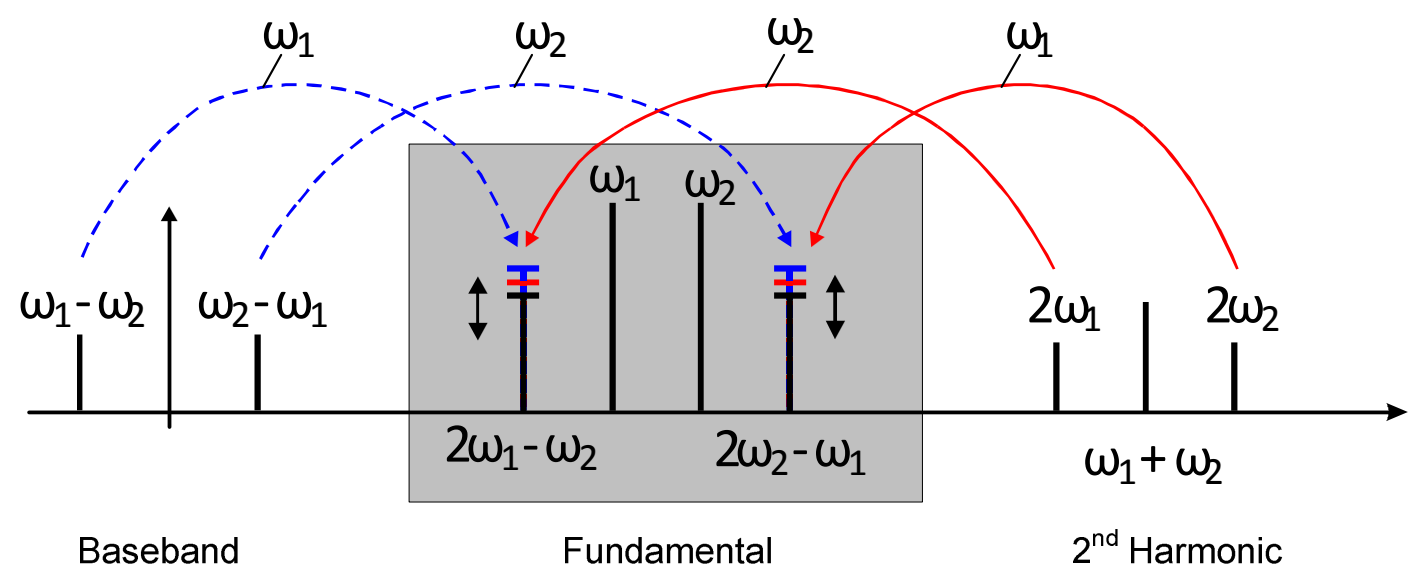

Figure 4.7: Spectrum composition under two tone modulated excitation.

The microwave device generates a set of $3^{\text {rd }}$ order intermodulation distortion (IMD3) products which have the magnitude and phase but to realize the IMD3 cancellation, the signal injection technique at the baseband modulation frequency with the same magnitude but out-of-phase to the original one has to be used. Figure 4.7 shows the spectral representation for the output signal 
of a nonlinear power device under the two tone excitation. The baseband injection satisfies the reduction of intermodulation products without sacrificing the fundamental signal power levels due to the indirect mechanism of reducing the distortion products.

Compared to other approaches, this technique tends to be simpler and can achieve a good linearity improvement as well as efficiency. A recent experimental study [1], [10] has demonstrated the effectiveness of this technique through an excellent suppression on IMD3 levels by using both baseband signal injection techniques. Since, baseband signal utilizes the baseband frequency even order components only to indirectly generate a signal with the same frequency as the distortion products. This controlled frequency is relatively low and out of the carrier frequency band. Moreover, gain variation at fundamental frequency incurred by this method would be lower. In the following section, a brief explanation of the baseband signal injection technique is first introduced. The operation principle is derived from the assumption of regarding the transconductance as the only nonlinearity in the amplifier, an assumption used to analyze the baseband injection linearization technique. The analytical expressions of the distortion terms modified by the additional baseband injection signal are derived and the theoretical condition for complete cancellation of IMD3 has been listed in the following section.

\subsubsection{Mathematical Analysis of Baseband Signal Injection}

For the baseband injection technique, the power series provides the qualitative information about the injection effect on the intermodulation distortion products. In the following, the output expression of the IMD3 is deduced from the drain current in order to illustrate the basic principle of baseband injection operation. Equation 4.6 relates the drain current to the input voltage as follow;

$$
I_{d}=g m_{1} V_{i n}^{1}+g m_{2} V_{i n}^{2}+g m_{3} V_{i n}^{3}
$$

Consider two tone signal input with baseband signal at frequency multiple of $\omega_{2}-\omega_{1}$, with amplitudes $A_{1}$ and $A_{2}$ at frequencies of $\omega_{1}$ and $\omega_{2}$ respectively, which is represented by: 


$$
V_{\text {in }}=A_{1} \cos \omega_{1} t+A_{2} \cos \omega_{2} t+A_{\text {env }} \cos \left(\left(\omega_{2}-\omega_{1}\right) t+\varphi_{\text {env }}\right)
$$

The substitution of 4.7 into 4.6 , the analysis yields the IMD3 as follow;

$$
\frac{3}{4} A_{1} A_{2}^{2} g m_{3} \cos \left(\left(2 \omega_{2}-\omega_{1}\right) t\right)+A_{1} A_{\text {env }} g m_{2} \cos \left(2 \omega_{2} t-\omega_{1} t+\varphi_{\text {env }}\right)
$$

The perfect cancellation of IMD3 can be obtained when,

$$
A_{\text {env }}=-\frac{3}{4} \frac{A_{2}^{2} g m_{2}}{g m_{2}} \text { and } \varphi_{\text {env }}=180^{\circ}
$$

It can be seen from these equations that the complete cancellation principle of IMD3 relies on the separate treatment of IMD3 products generated directly by third order nonlinearities, and IMD3 products that are generated directly by mixing of first and $2^{\text {nd }}$ order components through $2^{\text {nd }}$ order nonlinearities.

Therefore, one of the mixing components, baseband signal, is injected and its magnitude is controlled. Through the proper selection of the amplitude and phase of the injected baseband signal, the IMD products can be ideally cancelled in principle when newly generated IMD products have equal amplitude and are out of phase to the original IMD products of power transistor.

\subsubsection{Linearity Investigations and Analysis}

The specific focus of this measurement activity was to investigate the impact of active baseband impedance conditions upon device linearity. For this reason, the fundamental and all harmonic frequency components were terminated into $50 \mathrm{Ohms}$ and IF load-pull architecture was used to provide an active impedance control of the most significant IF components; IF1 and IF2. This was achieved using an Agilent 33502A 80 MHz AWG in combination with a high-power (200W), low-frequency power amplifier. All measurements were performed using a CREE CGH40010 10W GaN device within a custom 50 $\mathrm{Ohm}$ test fixture. Calibrated reference planes were established the package plane using error terms generated using a TRM coaxial calibration, embedded with the extracted s-parameters of each half of the test fixture. In earlier work [12]-[14], only the impedance presented to the two most significant baseband components (IF1 and IF2) generated as a result of 2-tone excitation were controlled, which specifically looked at the performance of ideal, passive baseband terminations, and if these can be optimised. The work conducted in 
[13],[14] showed that the linearity of a measured LDMOS device could be improved by presenting specific, positive complex impedances to the IF1 and IF2 components when the device was driven at a relatively backed-off level at $1 \mathrm{~dB}$ below the $1 \mathrm{~dB}$ compression point.

The application of active load-pull to both RF and baseband frequency components has some important advantages however: the approach is not limited to synthesising positive impedances within the Smith chart, and can just as easily synthesise negative impedances outside of the Smith chart. Synthesising negative baseband impedances allows, for example, the emulation of Envelope Tracking (ET) environments around a particular device, whilst at the same time being able to investigate the efficiency, linearity and most importantly the 'predistortability' of PAs that employ and depend upon such approaches. It is possible for example to directly investigate some of the key problems associated with ET such as 'drain-lag', where non-optimal tracking voltage envelopes lead to degraded performance due to unintentional RF load-line / device boundary interaction.

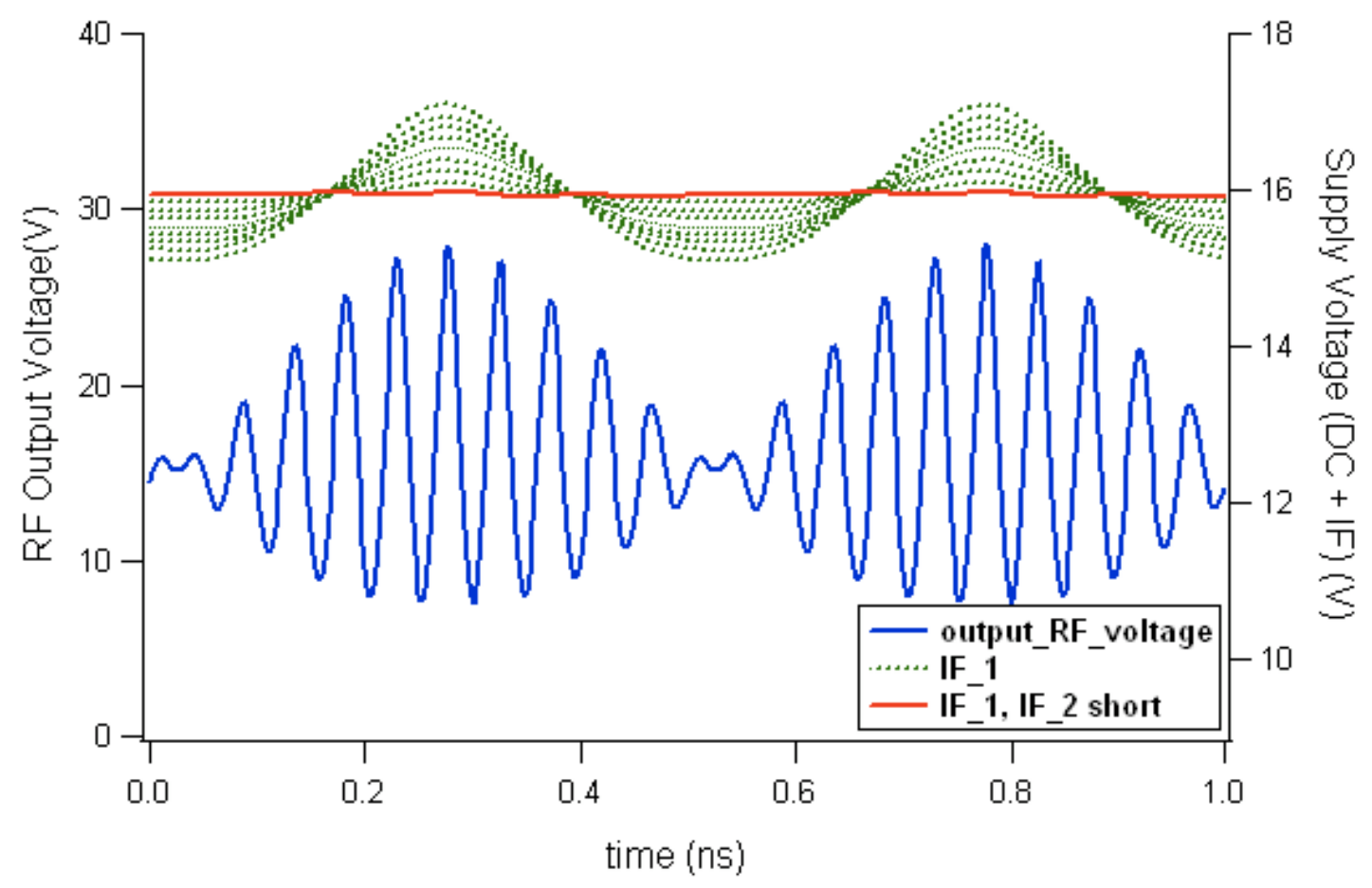

Figure 4.8: Measured modulated output voltage waveform and supply voltage.

The first investigation involved biasing a CREE CGH40010 10W GaN device in class $A B\left(I_{D Q}=250 \mathrm{~mA}\right)$, using a drain bias voltage of $16 \mathrm{~V}$ and exciting with a symmetrical 2-tone modulation. $A$ reduced value of $V_{d d}$ was used to 
represent a typical ET application and the magnitude of the excitation limited to emulate a backed-off drive condition. In this way, the device remained largely linear with no significant load-line / knee boundary interaction occurring. Figure 4.8 shows the measured voltage waveform present at the device plane, where the backed-off and linear operating point is evident from the limited voltage peak-to-peak swing between $8 \mathrm{~V}$ and $28 \mathrm{~V}$ - well away from the device's knee voltage at approx 4V[1]. The baseband loads IF1 and IF2 were then actively synthesised, held together and swept over the measurement grid shown in Figure 4.9 and Figure 4.10. The supply voltages comprising DC+IF that correspond to the cases of IF1 and IF2 load along the real axis are shown together in Figure 4.8. These are plotted relative to the right-hand axis, and are not to scale. The reference case is shown as a central, solid red line where both IF1 and IF2 are terminated into short circuits.

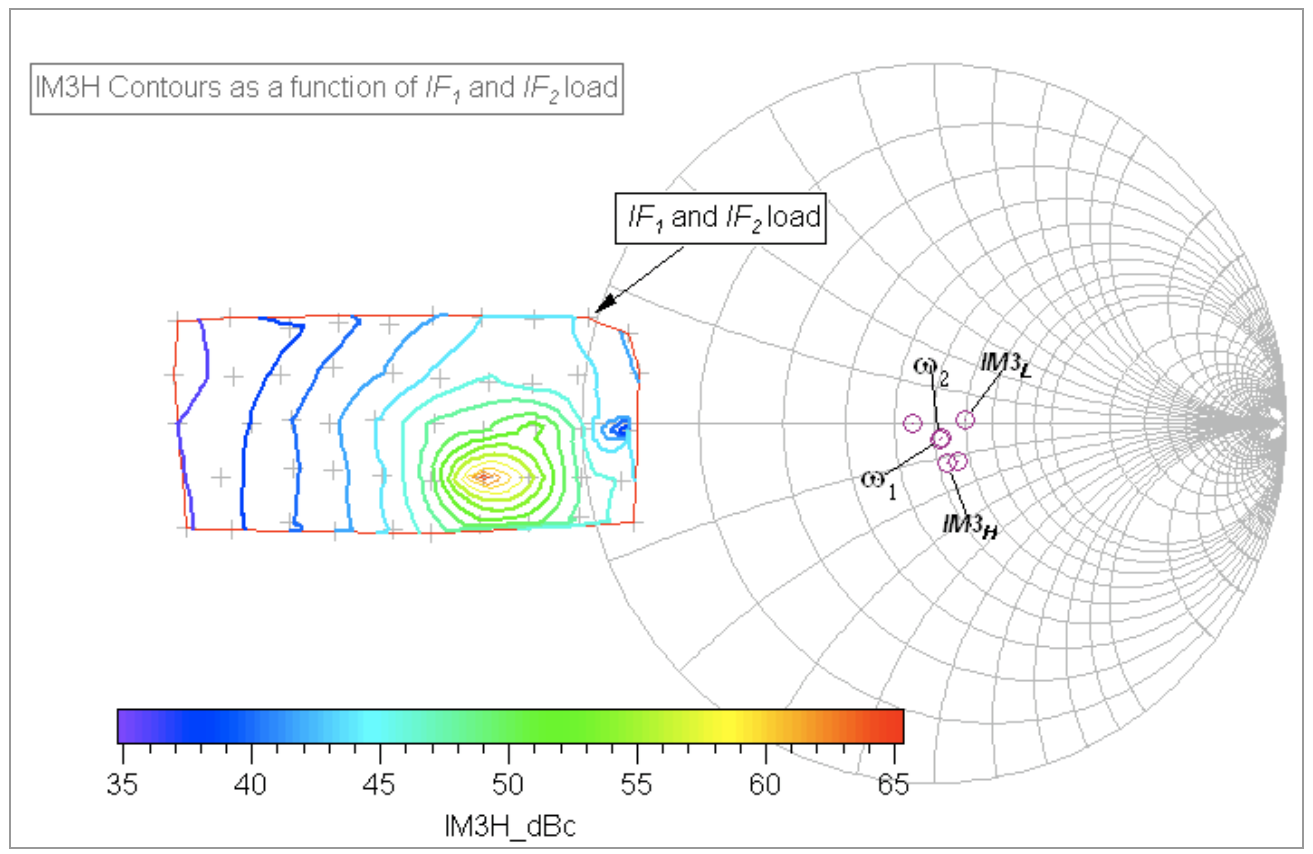

Figure 4.9: IM3-high contours as a function of IF1 and IF2 load [1].

The most negative IF1 load point results in approximately a $2 \mathrm{~V} p-p$ IF waveform established on a $16 \mathrm{~V}$ supply rail. For each of the measurement points, the bias and drive level was maintained constant, and the IM3 behaviour measured. Due to the linear operation of the device and the dynamic range limitations of the system $(60 \mathrm{~dB})$, it was not possible to accurately measure the IM5 behaviour. The contours of IM3 low and high are 
shown in Figure 4.9 and Figure 4.10, and show that interestingly, separate local optimum IF loads for linearity exists slightly outside the Smith chart, and that for this device, a 15-20 dB improvement in IM3-high magnitude exists for this load in comparison to the reference case where IF1 and IF2 are terminated into short circuits, as may be the case for example when using a conventional video bypass bias network arrangement. A slightly smaller improvement and different optimum load is observed for IM3-low.

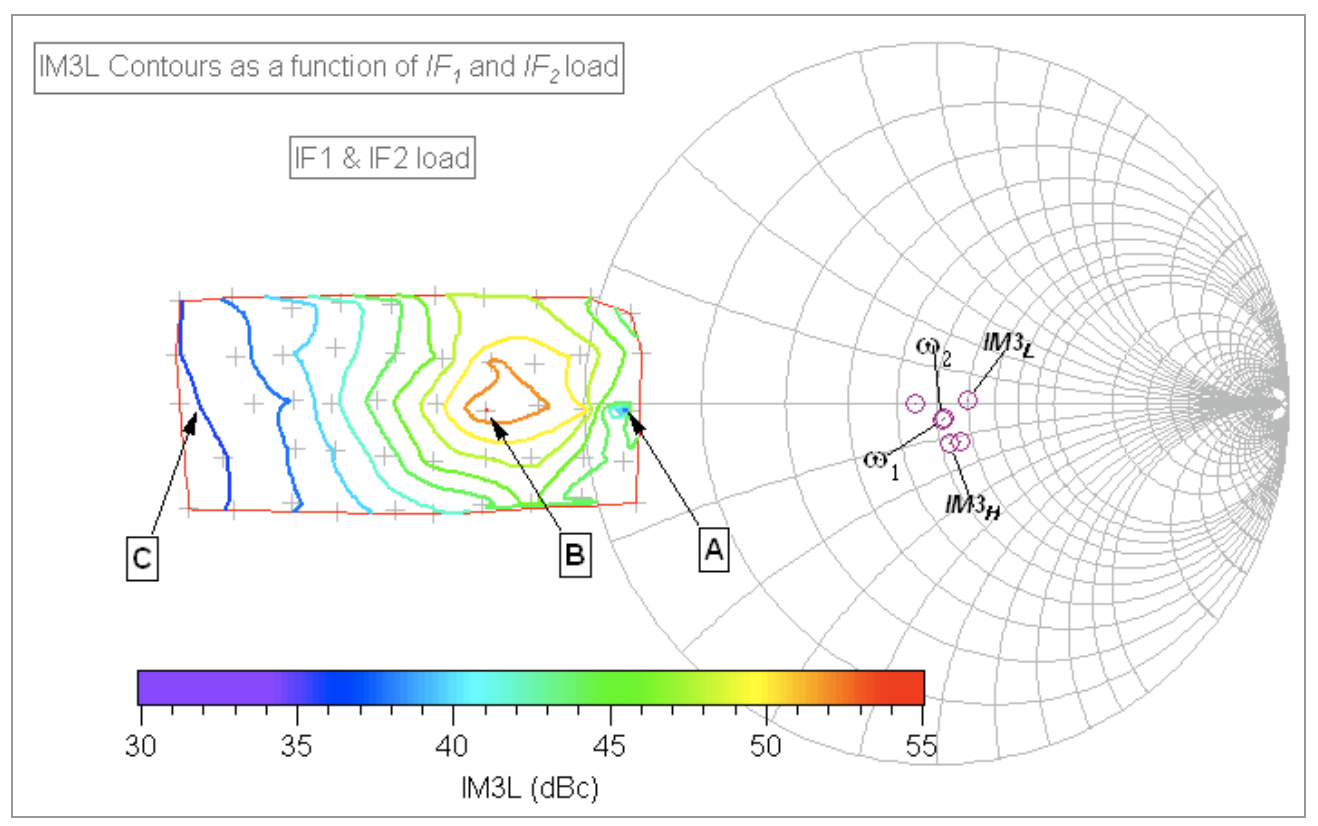

Figure 4.10: IM3-low contours as a function of IF1 and IF2 load.

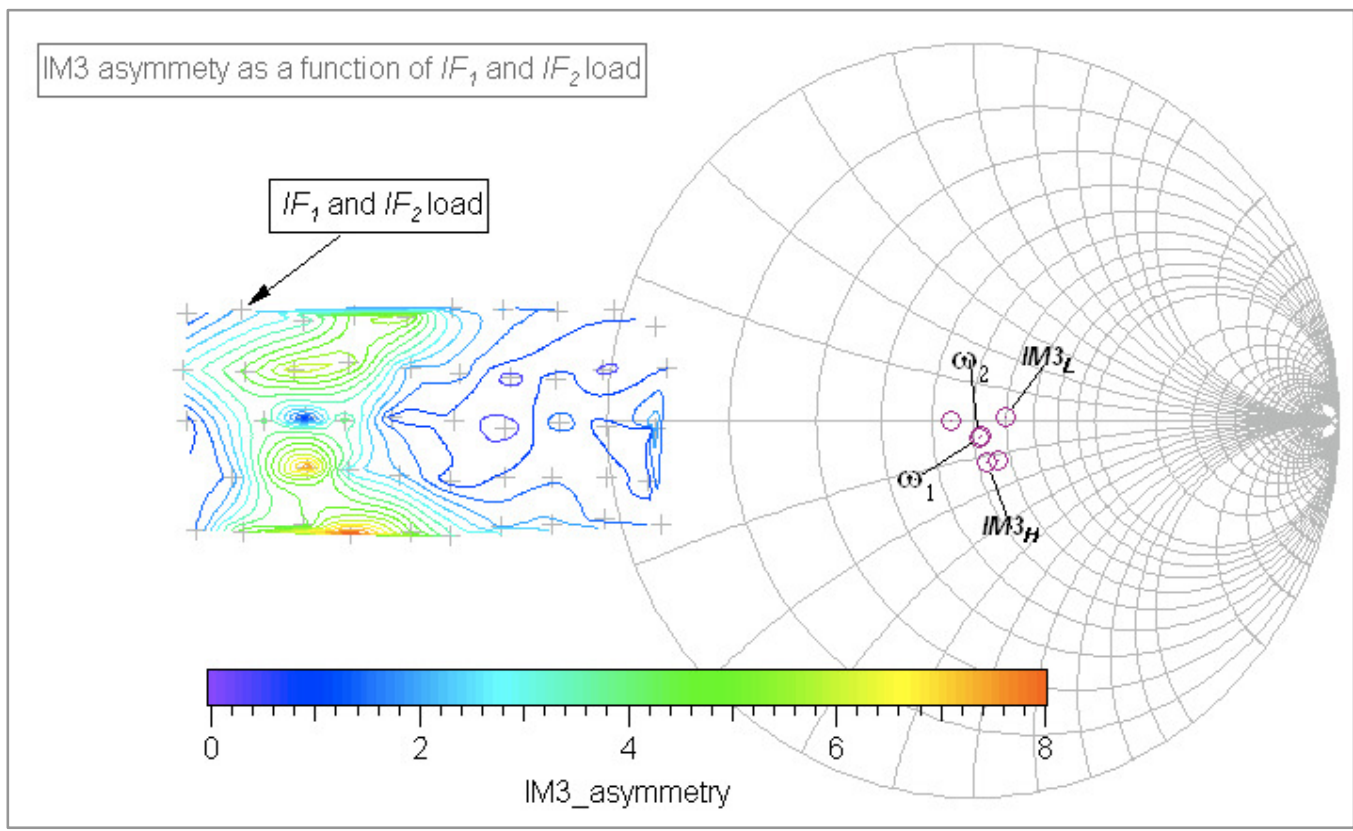

Figure 4.11: IM3-low asymmetry contours as a function of IF1 and IF2 load [1]. 
The IM3 asymmetry representation is shown in figure 4.11 which reflects that the asymmetries are minimal when purely negative resistance was maintained for significant IF components. Access to the envelope domain allows the dynamic analysis of other device parameters such as gain; drain efficiency, etc as a function of the applied modulation envelope. Figure 4.12 shows for example how the gain changes throughout the cycle of modulation, in this case for three cases of IF1 \& IF2 load identified in Figure 4.10 as A - near IF short circuit, B - identified optimum and C - significant ET-like IF component. It is clear from this graph that the positive real load at point-A results in the higher gain, yet the negative load at point-B offer the improved linearity, at least in terms of IM3 behaviour. A load at Point-C causes significant distortion in the dynamic gain characteristic, which is reflected in the poor linearity performance [1].

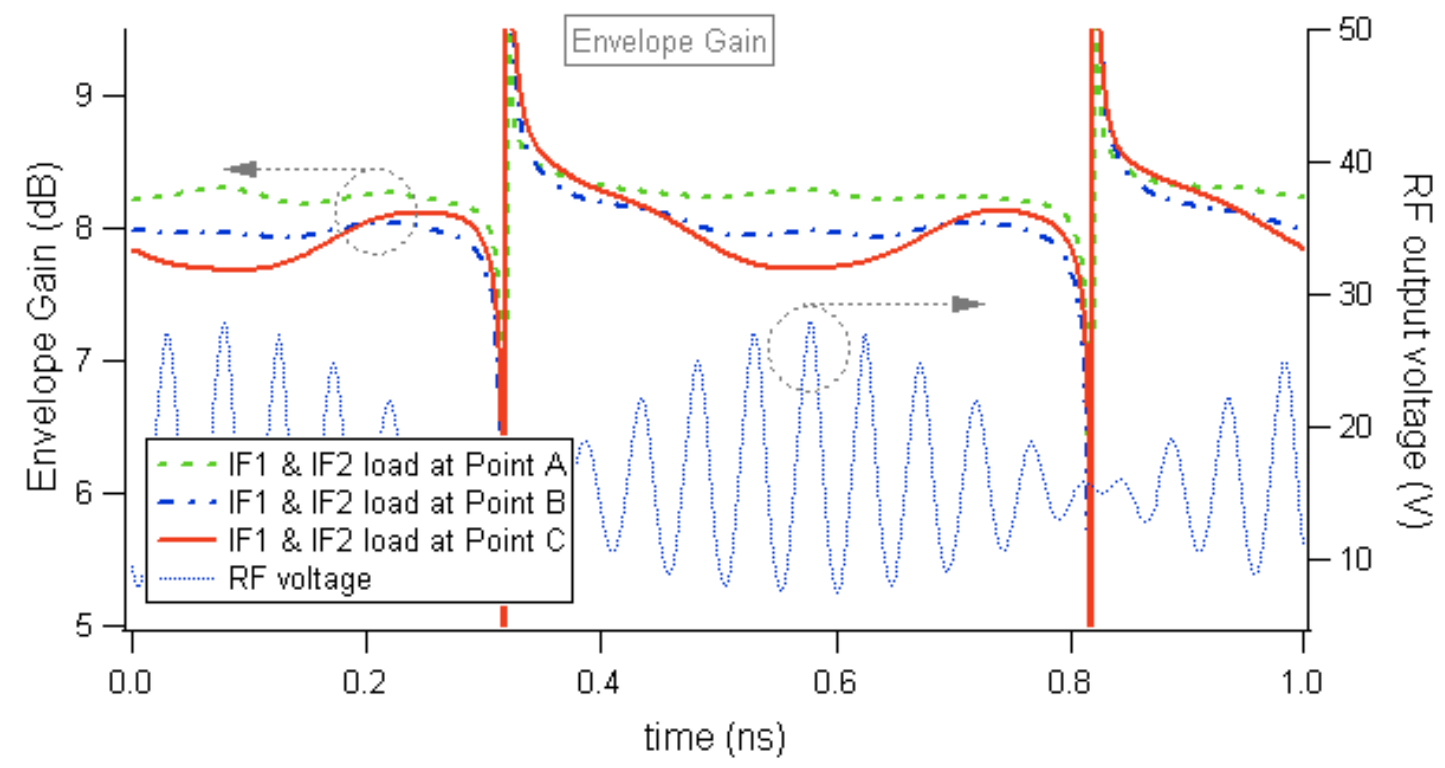

Figure 4.12: Envelope gain profiles for IF1 and IF2 loads in identified positions $\mathrm{A}, \mathrm{B}$ and $\mathrm{C}$.

\subsubsection{Emulation of Auxiliary Envelope Tracking Environment}

The above analysis is interesting from a linearity perspective, yet it is limited in that it explores performance only in a backed-off condition. The synthesise of negative IF impedances does allow to linearize the device with additional IF power. This measurement approach can be extended from simple linearity investigations to for example full ET emulation. In this next 
measurement sequence, the same device has been excited using a symmetrical 3-tone signal centred at $2.1 \mathrm{GHz}$, resulting in $100 \% \mathrm{AM}$ modulation with modulation frequency of $1 \mathrm{MHz}$. The Device was driven into approximately $2.5 \mathrm{~dB}$ of compression with fundamental and harmonic components terminated into a passive $50 \Omega$ load. At this frequency, the device has an optimum output impedance of approximately $25+j 10$ Ohms, and although the device is not operating into the optimal fundamental load impedance, it is sufficiently representative for this analysis.

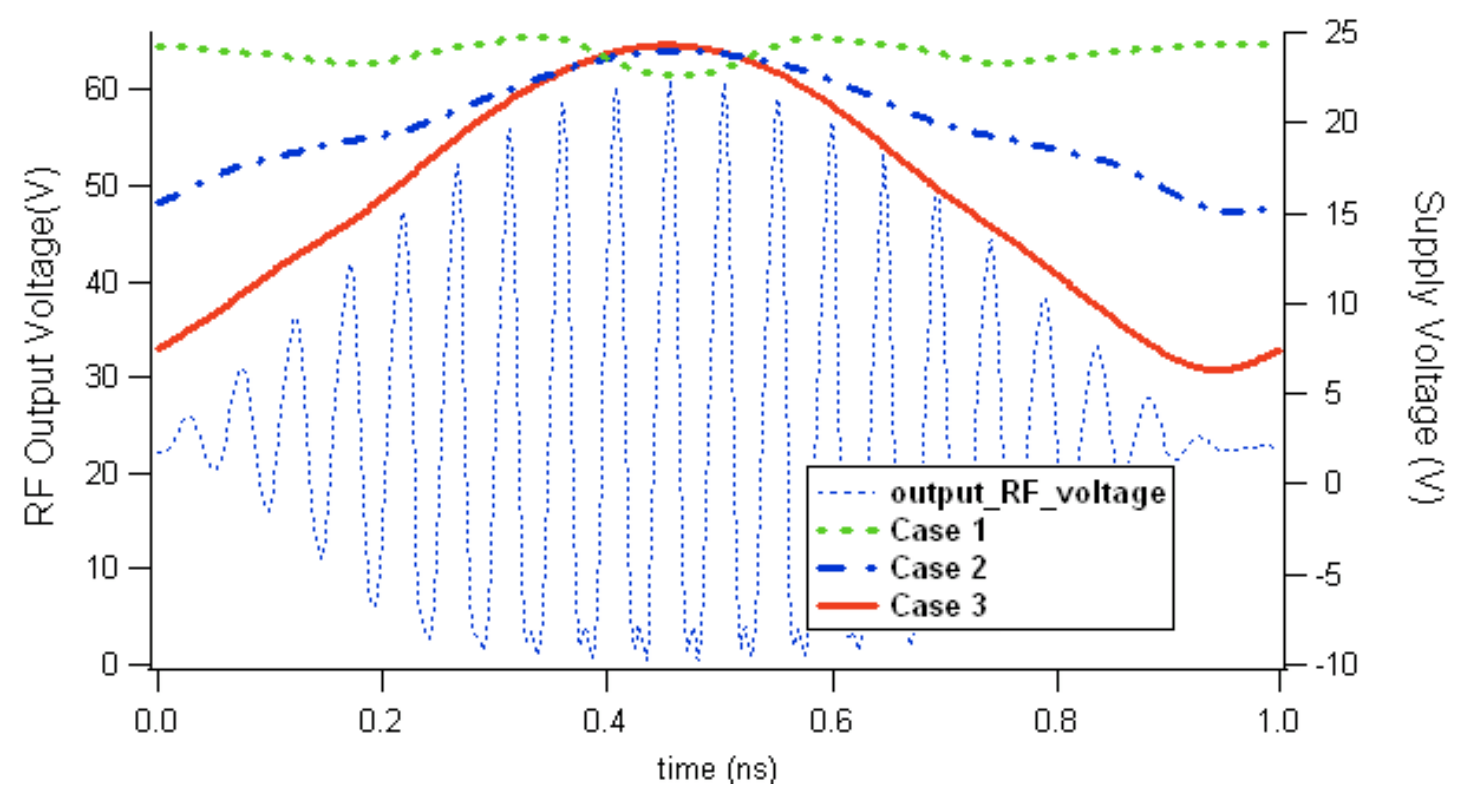

Figure 4.13: Synthesised IF voltages for the three cases of IF load, together with RF output voltage waveform - note that different scales are used.

Three separate measurement cases were considered; case-1 (reference case): $V_{d}=24 \mathrm{~V}$ and IF1 and IF2 components actively terminated into short circuits, case-2: $V_{d}=20 \mathrm{~V}$, injected $I F 1=8 V_{p-p}$ and IF2 terminated into a short circuit, and finally case $3: V_{d}=16 V$, injected $I F 1=16 V_{p-p}$ and IF2 again terminated into a short circuit. These combinations of static DC supply and injected IF1 component ensured that the peak supply voltage remained as close as possible to $24 \mathrm{~V}$, as shown in the representative graph in Figure 4.13, allowing meaningful comparison between the three cases [1].

Plotting the measured (RF, IF \& DC) time-domain voltage vs. time-domain current yields a complete dynamic load-line which can be used to describe the trajectory of the modulated envelope relative to the device's DC characteristic. 
This intuitive view shows the actual voltage and current behaviour at the calibrated reference plane, and importantly, the nature of any interaction between the envelope and device boundary conditions. This is a familiar plot in CW waveform engineering yet adds an extra dimension to modulated analysis as it can show interesting effects such as self-bias, and in the case here of an emulated ET environment, the proximity of the load-line to the knee region. Plotting the measured (RF, IF \& $D C$ ) time-domain voltage vs. timedomain current yields a complete dynamic load-line which can be used to describe the trajectory of the modulated envelope relative to the device's DC characteristic. This intuitive view shows the actual voltage and current behaviour at the calibrated reference plane, and importantly, the nature of any interaction between the envelope and device boundary conditions. This is a familiar plot in CW waveform engineering yet adds an extra dimension to modulated analysis as it can show interesting effects such as self-bias, and in the case here of an emulated ET environment, the proximity of the load-line to the knee region.

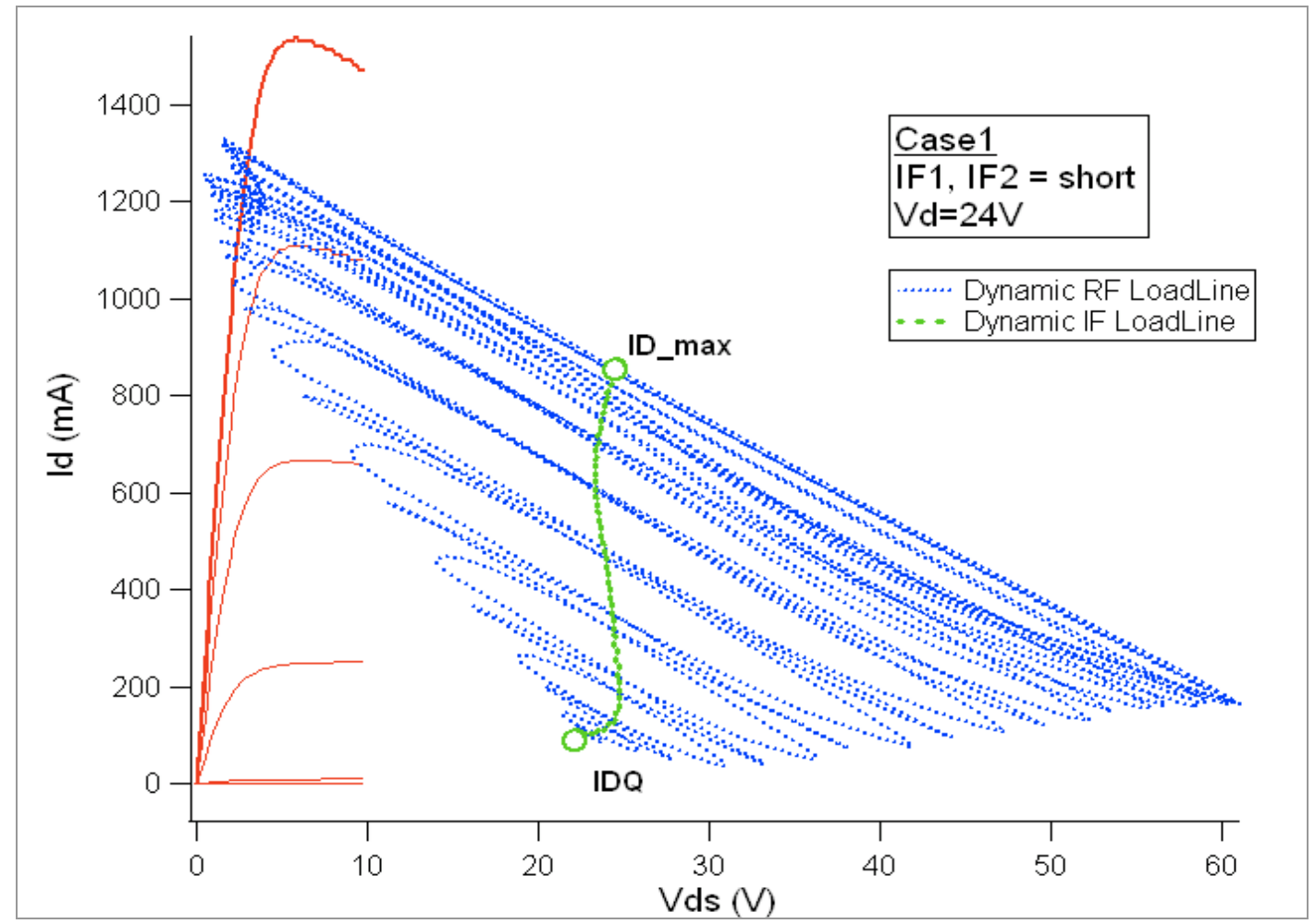

Figure 4.14: RF and IF dynamic load-lines overlaid on DCIV characteristic for reference Case-1. 
Figure 4.14, 4.15 and 4.16 show the full dynamic load-lines for the three cases considered, along with the IF load-line trajectories, which have been added by plotting and overlaying the baseband current vs. the baseband voltage waveforms. These clearly show the degree of baseband voltage (horizontal) and current (vertical) variation throughout the modulation cycle. The IF load-line itself can be considered as the component that 'pushes' or 'pulls' the dynamic RF load-line, in the case of electrical memory, asymmetrically into the knee region, and in the case of ET, into more efficient parts of the characteristic [1].

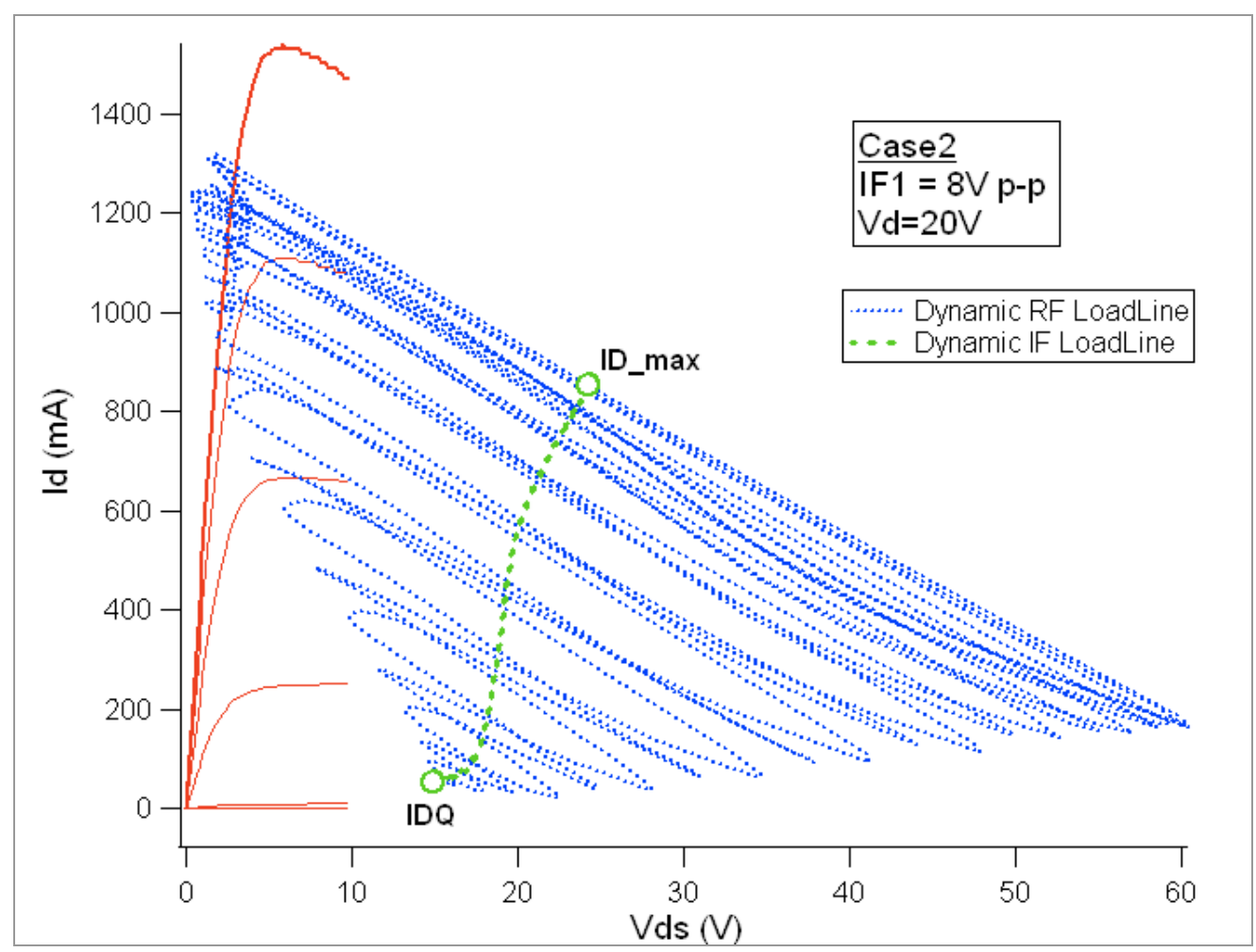

Figure 4.15: RF and IF dynamic load-lines overlaid on DCIV characteristic for reference Case-2.

A Perfect IF short would result in a straight, vertical IF load-line, and the shape of the IF load-line allows scope for baseband waveform engineering and optimisation for example of the proximity of dynamic RF load-line to knee boundary region. In case of negative impedance termination the IF load-lines show the developed voltage at the drain of power device whilst reducing the knee interaction. Figure 4.17 shows the extracted gain envelopes for the three cases, and how at the point in the envelope where the DC supply voltage is 
the same at $24 \mathrm{~V}$, the gain is the same, albeit compressed. This plot also shows however how for other parts of the envelope, the gain is very different for the three cases.

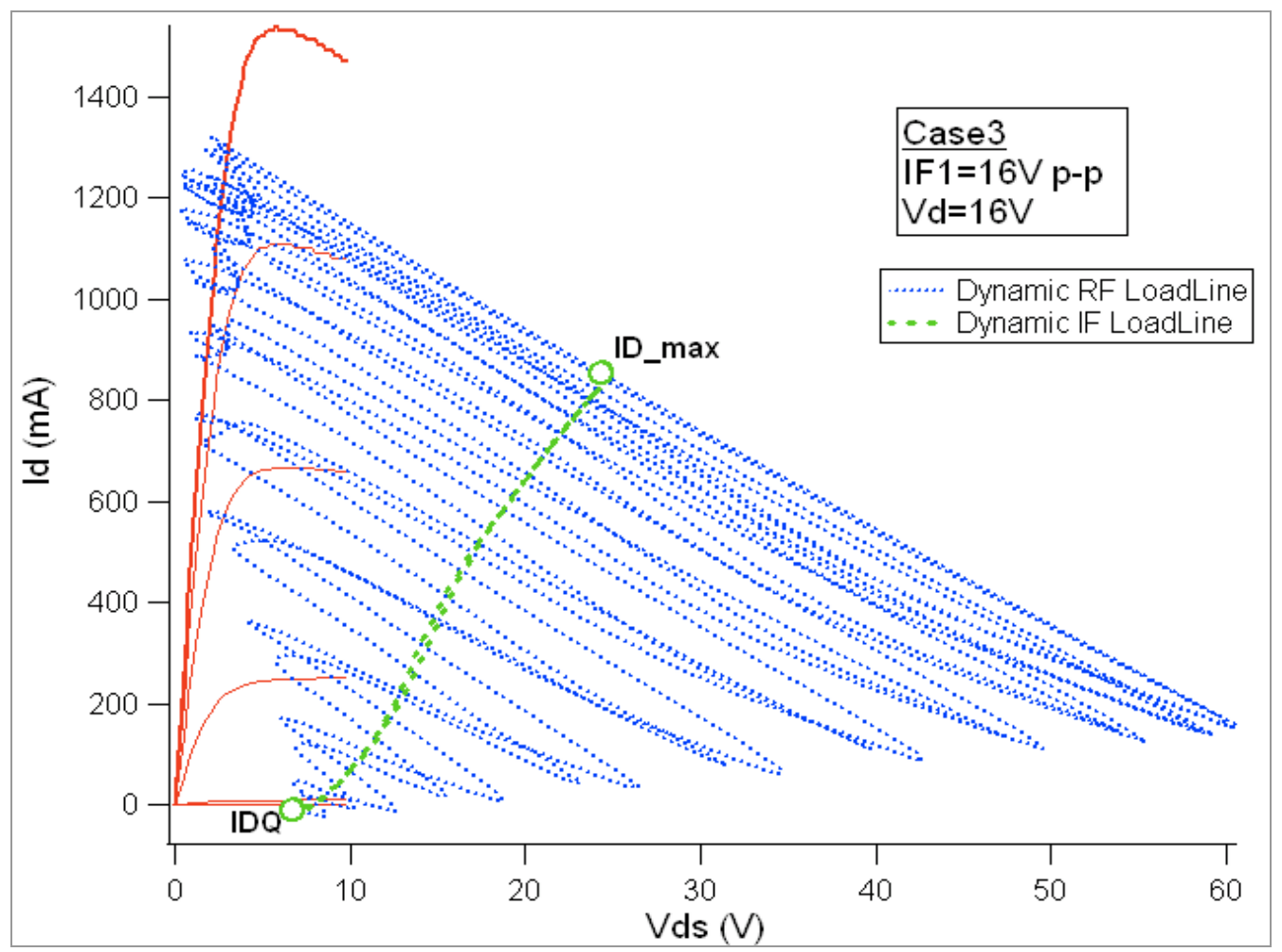

Figure 4.16: RF and IF dynamic load-lines overlaid on DCIV characteristic for reference Case-3.

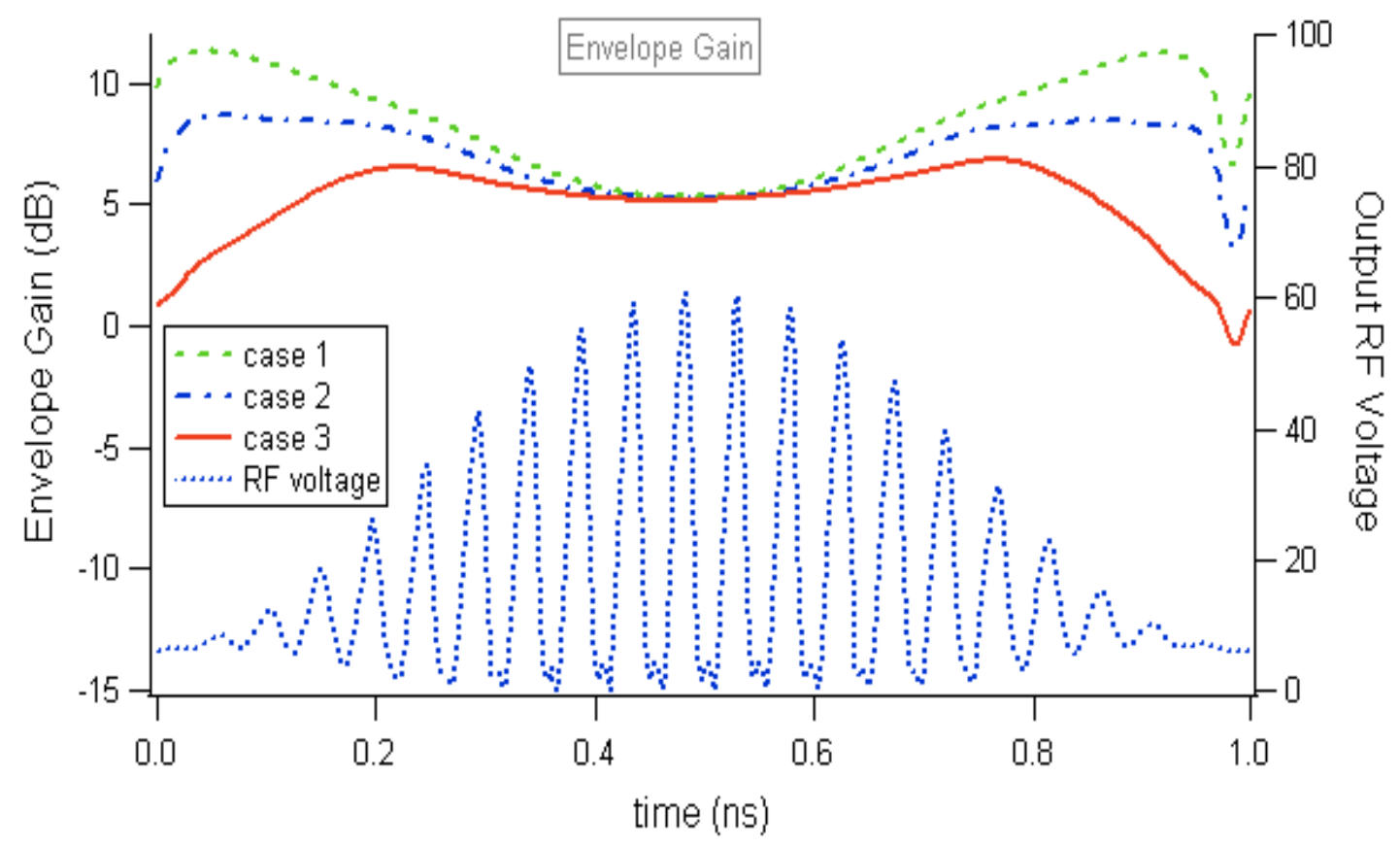

Figure 4.17: The comparison of gain envelopes for the three cases. 
The output RF power envelopes are depicted in Figure 4.18 which show that the peak output envelope power for all three cases is almost similar but the rise in peak envelope power of the device is apparent in the 'skirts' of output peak power envelopes. Similarly, there exists an increase in the DC power dissipation around the 'skirts' of the envelopes as can be seen from the figure 4.19.

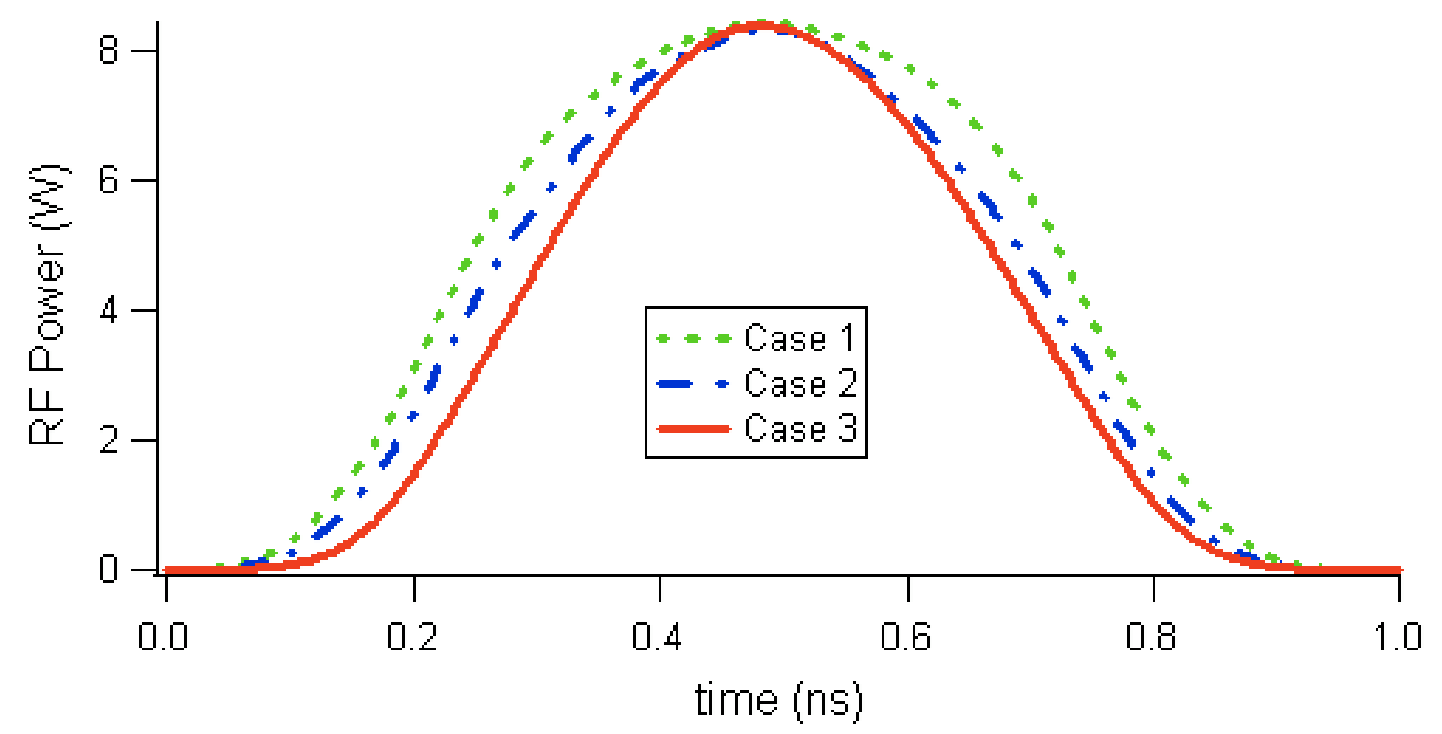

Figure 4.18: RF Power envelopes for the three cases

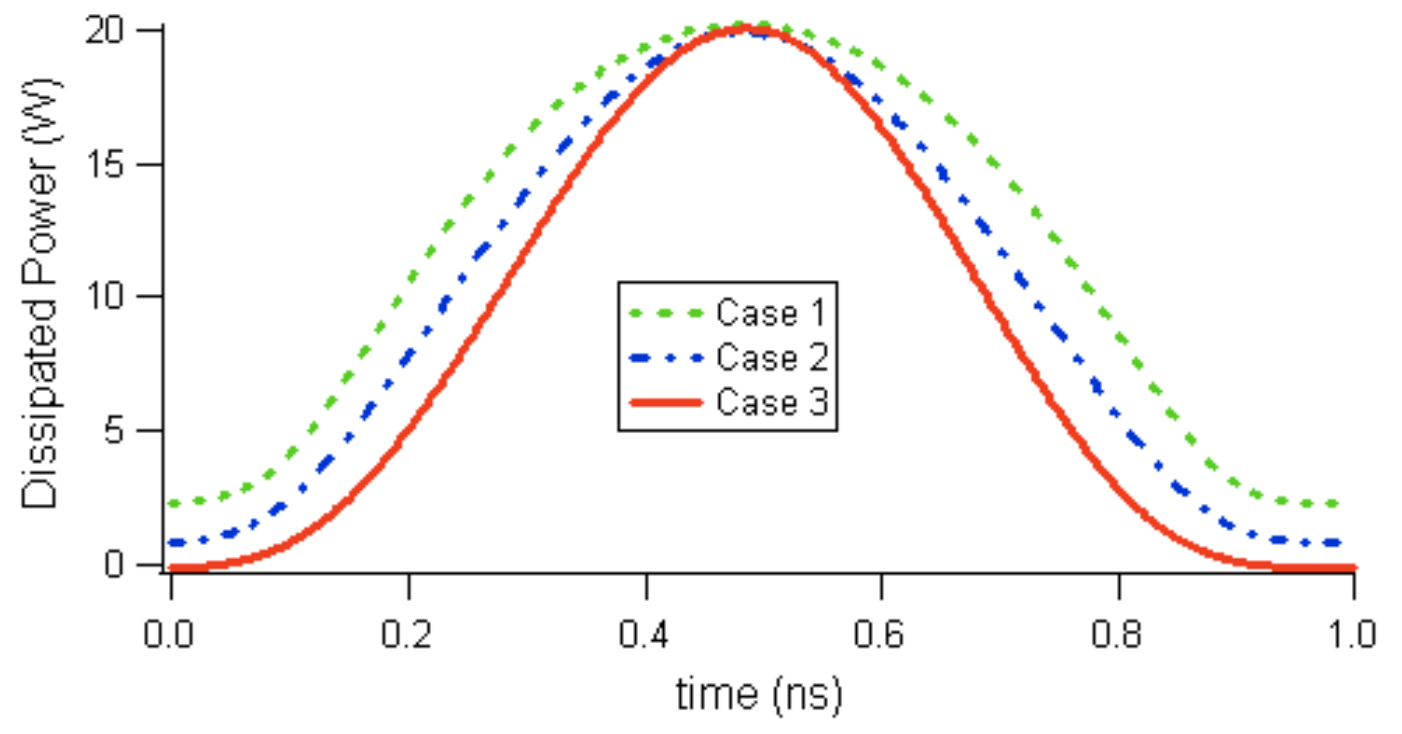

Figure 4.19: Dissipated DC Power envelopes for the three cases

As with gain, it is possible to show the efficiency as a function of the envelope, and Figure 4.20 shows a comparison of the three cases. Although it is clear from this graph that the applied ET does improve the average efficiency, it is also clear that the improvement is surprisingly small. In trying to explain this, it 
must be remembered that in each case, the device is driven fairly hard into compression, and no improvement can be expected in this region of the envelope. Although improvement is apparent around the envelope 'skirts', as mentioned, it maybe isn't as large as one would expect. This is again probably explained by the fact that for this device, the gain, output RF power and hence efficiency reduces with reducing drain voltage [1].

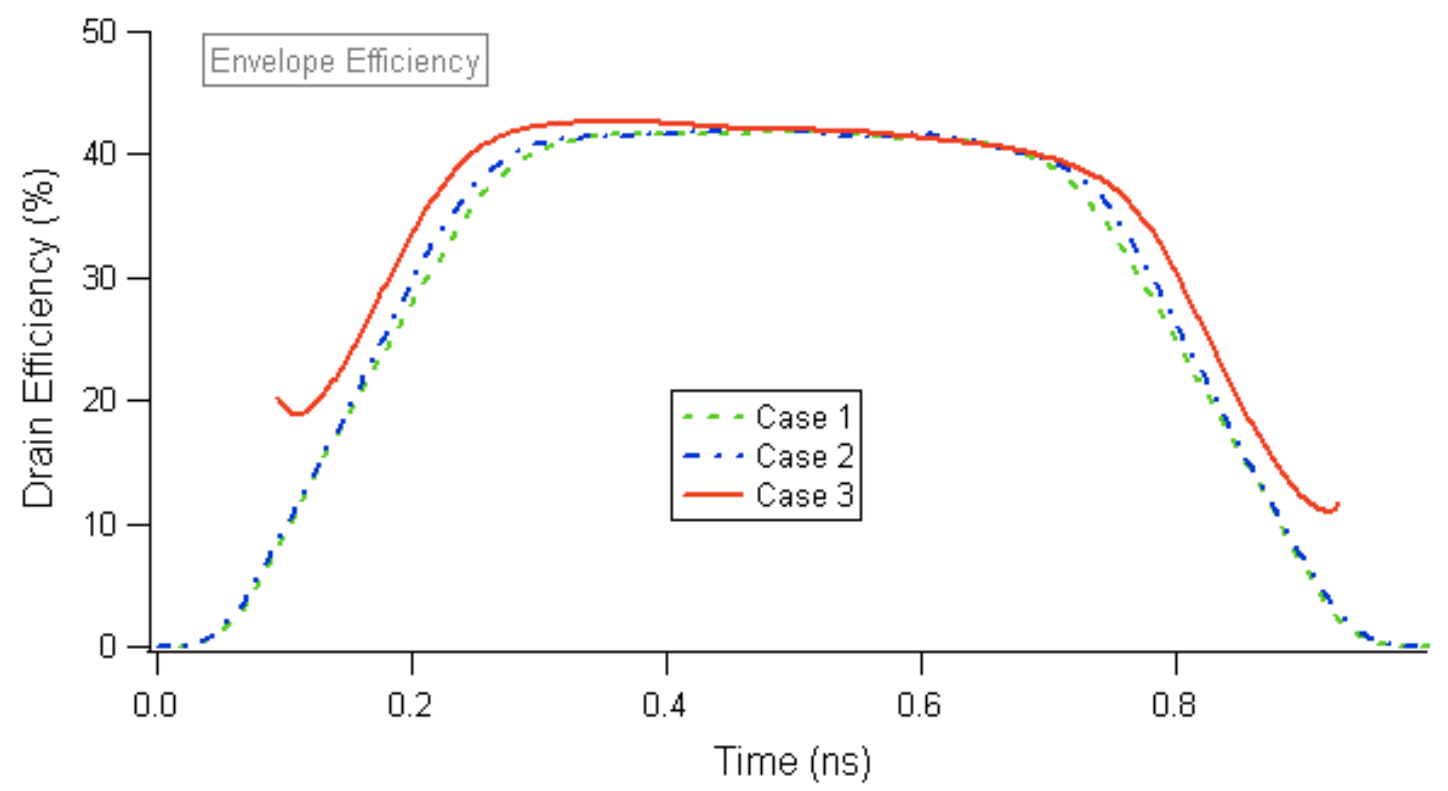

Figure 4.20: The comparison of drain efficiency envelopes for the three cases [1].

Table 4.2: Linearity for the three cases [1].

\begin{tabular}{|l|c|c|c|c|c|c|c|c|}
\hline Case & $\begin{array}{c}\text { Supply } \\
\text { Voltage(V) }\end{array}$ & $\begin{array}{c}\text { IM5L } \\
\mathrm{dBc}\end{array}$ & $\begin{array}{c}\text { IM3L } \\
\mathrm{dBc}\end{array}$ & $\begin{array}{c}\text { W1 } \\
\mathrm{dBm}\end{array}$ & $\begin{array}{c}\text { Wc } \\
\mathrm{dBm}\end{array}$ & $\begin{array}{c}\text { W2 } \\
\mathrm{dBm}\end{array}$ & $\begin{array}{c}\text { IM3H } \\
\mathrm{dBc}\end{array}$ & $\begin{array}{c}\text { IM5H } \\
\mathrm{dBc}\end{array}$ \\
\hline Case-1 & 24 & $\mathbf{4 1 . 5 7}$ & $\mathbf{1 2 . 7 7}$ & 27.04 & 34.95 & 27.10 & $\mathbf{1 2 . 9 4}$ & $\mathbf{4 1 . 0 2}$ \\
\hline Case-2 & 20 & $\mathbf{4 7 . 4 6}$ & $\mathbf{1 6 . 3 2}$ & 27.13 & 34.29 & 27.22 & $\mathbf{- 1 6 . 5 3}$ & $\mathbf{4 3 . 5 7}$ \\
\hline Case-3 & 16 & $\mathbf{3 1 . 9 3}$ & $\mathbf{2 3 . 6 2}$ & 27.42 & 33.57 & 27.46 & $\mathbf{2 4 . 4 3}$ & $\mathbf{3 1 . 2 3}$ \\
\hline \multicolumn{8}{|c|}{ All IM values are in dBc, rative to W1 } \\
\hline
\end{tabular}

The linearity in terms of measured IM3 and IM5 mixing terms is shown in Table 4.2, which indicates that there is an approximate $10 \mathrm{~dB}$ improvement in IM3 for case 1 compared to the reference IF1=IF2=short case, and only a slight improvement in IM5 for case 2. 


\subsection{Enhancing Linearity at Compressed Drive Level}

A real power device has essentially nonlinear behaviour. A nonlinear power device has an amplified output but distorted version of the input signal. For modern wireless communications this generated distortion should be minimized or suppressed. In addition, power device nonlinearity might be frequency dependent if it is excited by a signal with broad envelope bandwidth. In this case, the power device is not only nonlinear but might also suffer from memory effects.

This, in particular, is a crucial phenomenon to be dealt with and the results depicted in the following section, firstly, confirm and demonstrate that baseband electrical memory effects can be greatly reduced by terminating the baseband impedance into ideal short circuits; an impedance environment that would result from conventional design and the use of video bypass capacitors. Then secondly considers 'if' and 'how' this situation can be improved by considering alternative baseband impedance conditions. As expected, for the GaN device considered, and for this degree of compression, the measured linearity significantly improves when negative baseband impedances are presented. Although such impedances are non-realiseable using conventional, passive designs, this is not the case when active, baseband injection architectures such as Envelope Tracking (ET) are employed. In a former section, only the impedance presented to the two most significant baseband components (IF1 and IF2) generated as a result of 2-tone excitation were controlled. This was achieved by combining two, phase coherent arbitrary waveform generators (AWGs) whilst the device was driven at a relatively backed-off level. However, when the device is driven more deeply into compression, significantly more mixing terms are generated, and in order to achieve a sufficiently broadband IF termination, baseband active load-pull was employed to accurately account for higher baseband harmonics. The measurements presented in this section are for a CREE CGH40010 discrete 10W GaN HEMT device, characterised at the centre frequency of $2 \mathrm{GHz}$, within a custom $50 \Omega$ test fixture. This fixture was calibrated over a relatively wide $50 \mathrm{MHz}$ baseband bandwidth, and over $100 \mathrm{MHz}$ RF bandwidths centred around fundamental, second and third harmonics, with both baseband and RF 
calibrated reference planes established at the device's package plane. This allowed the accurate and absolute measurement of all the significant voltage and current spectra generated at the input and output of the device. Two-tone measurements were performed using a $2 \mathrm{MHz}$ tone spacing, with the device class-AB biased. Respective drain and gate bias voltages of $28 \mathrm{~V}$ and $-2.05 \mathrm{~V}$ resulted in a quiescent drain current of $250 \mathrm{~mA}\left(\mathrm{I}_{\mathrm{DSQ}} \cong 5 \% \mathrm{I}_{\mathrm{DSS}}\right)$.

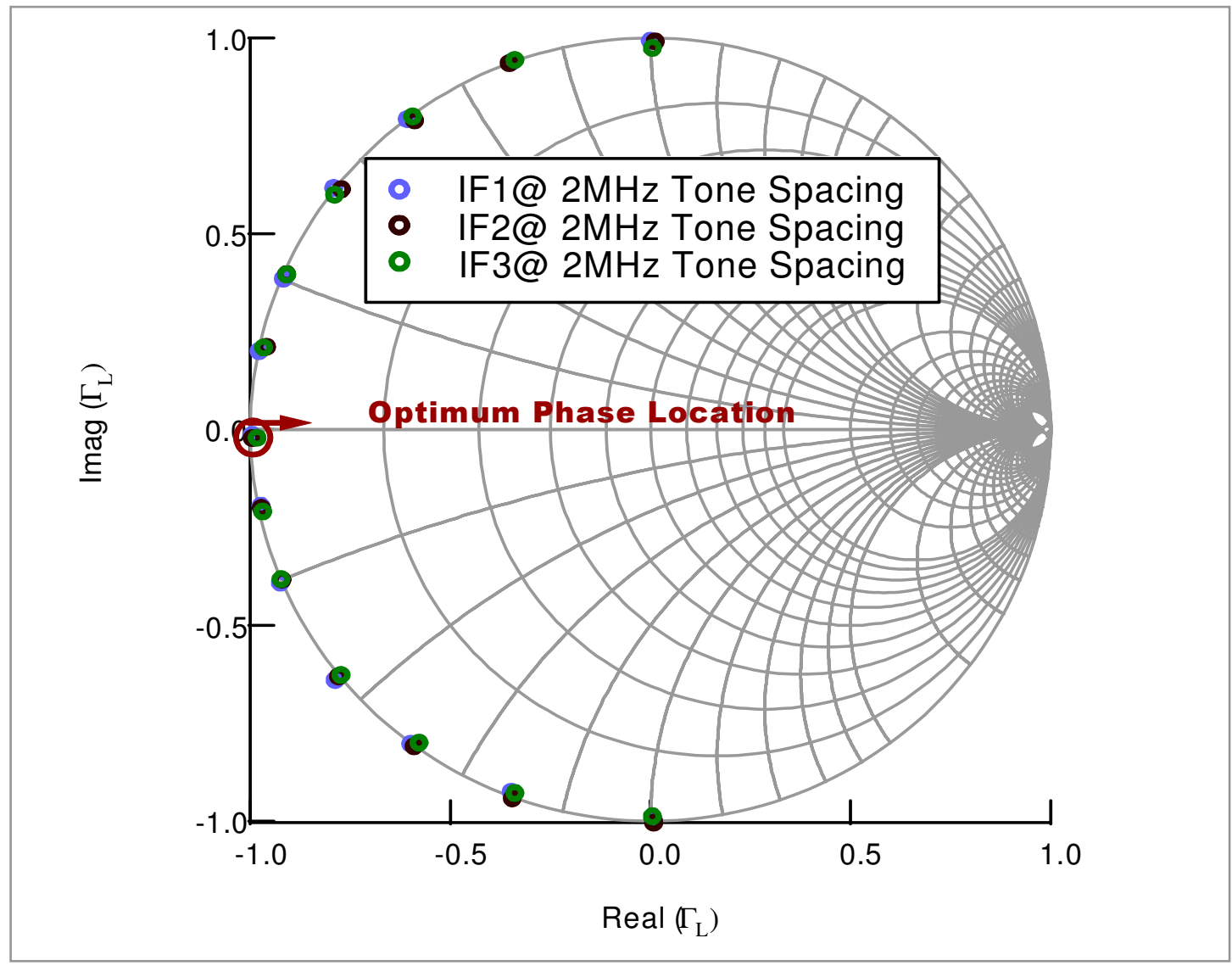

Figure 4.21: Measured IF impedances at $2 \mathrm{MHz}$ tone spacing using IF active load-pull.

The device was driven into approximately $1.5 \mathrm{~dB}$ of compression whilst delivering $39.5 \mathrm{dBm}$ output peak envelope power (PEP) with fundamental and harmonic components terminated into a nominal impedance of $50 \Omega$, at both the input and the output. It should be noted that broadband $50 \Omega$ load is used to simplify the required measurement architecture. Although this is nonoptimal, but is considered sufficiently representative for the linearity analysis presented here. Active IF load-pull was then used to synthesise a range of IF reflection coefficients in order to quantify the effects of the low frequency 
broad-band IF load impedance termination on the non-linear behaviour of the DUT. Figure 4.21 illustrates a measurement where the phase of the IF1, IF2 and IF3 loads were varied simultaneously, in steps of $15^{\circ}$ around the perimeter of the Smith chart, whilst keeping the magnitude of IF reflection coefficient at unity. The results depicted in Figure 4.22 clearly show that, as expected, there exists a strong dependence of IM3 and IM5 magnitude on the phase of the baseband impedance. The results explicitly identify an expected optimum phase in the region of $180^{\circ}$ for IF1, IF2 and IF3 loads, where IM3 and IM5 distortion products are minimised. The measured inter-modulation distortion products presented in Figure 4.22 show that when a perfect short impedance $\left(\Gamma_{\mathrm{IF}}=1 \angle 180^{\circ}\right)$ is presented to the significant baseband components, the measured IM3 and IM5 magnitudes can be seen to be $24 \mathrm{dBC}$ and $-38 \mathrm{dBC}$ respectively.

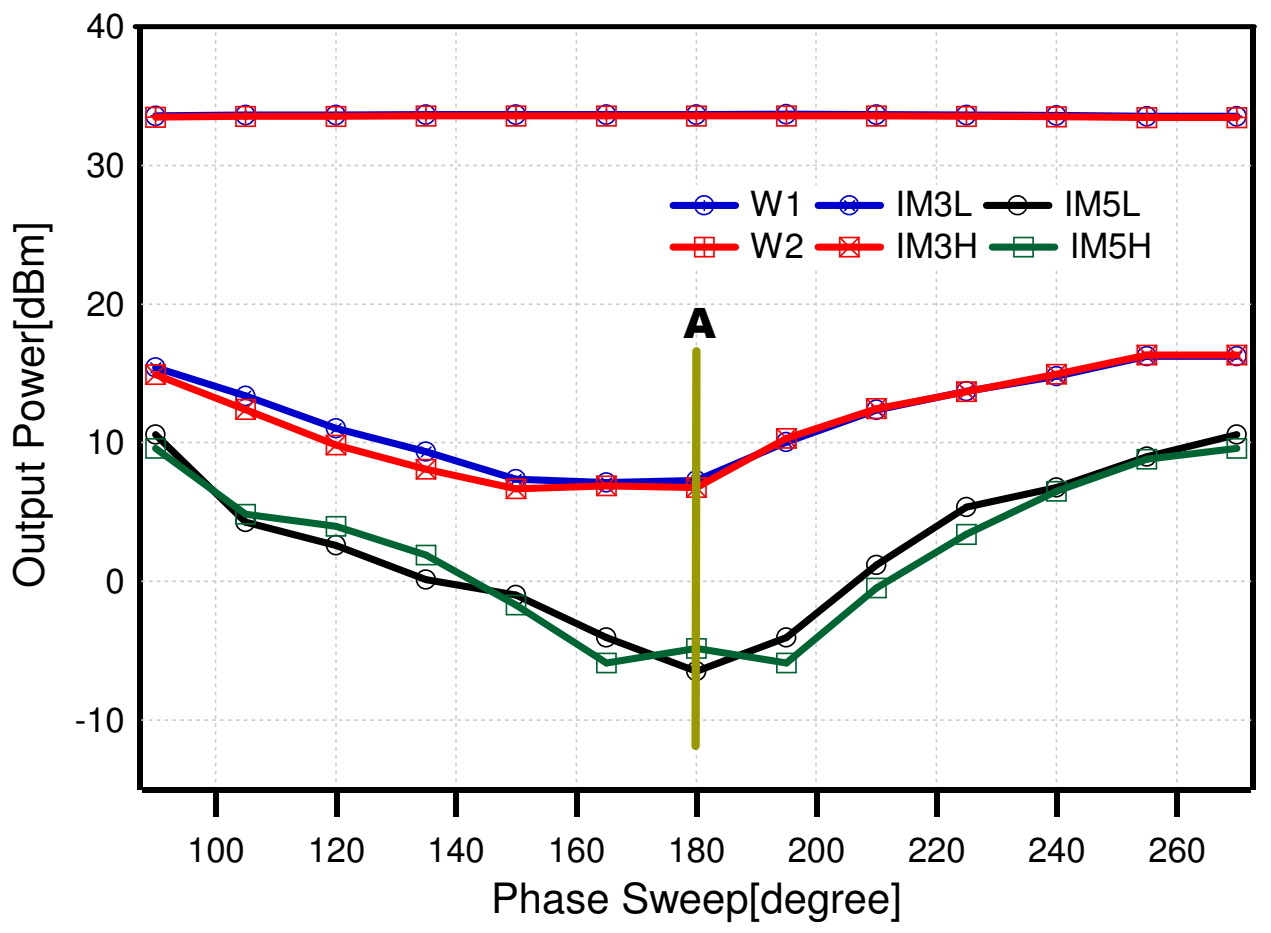

Figure 4.22: Measured fundamental and IMD magnitudes at $2 \mathrm{MHz}$ Tone Spacing as a function of IF1, IF2 and IF3 phase.

Active load-pull however has an important advantage in that it is able to seamlessly synthesise both positive impedances within the Smith chart, as well as negative impedances outside the Smith chart. Thus, in order to explore further the optimum baseband impedances for best linearity 
conditions, the broadband IF impedance was swept over a measurement grid that included the short circuit condition and extended some way outside the Smith chart. $\mathrm{IM} 3_{\mathrm{L}}$ and $\mathrm{IM} 5_{\mathrm{L}}$ contours were then plotted and are shown in Figure 4.23 and Figure 4.24 respectively, and in both cases show purely resistive negative optimum impedance. The optimum IM3 $3_{\mathrm{L}}$ performance (point $\mathrm{B}$ ) is found to be $-43.5 \mathrm{dBc}$, and is approximately $19.5 \mathrm{dBc}$ better than the case where usual short circuit is provided to all the significant baseband components (point $\mathrm{A}$ ). With regard to the $\mathrm{IM} 5_{\mathrm{L}}$ and $\mathrm{IM} 5_{\mathrm{H}}$, an improvement of $17 \mathrm{dBc}$ was achieved at an optimum termination (point $\mathrm{C}$ ) as compared to the short circuit case (point $A$ ). As the contours for $I M 3_{L}$ and $I M 3_{H}$ were found to be almost identical, as was the case for $I M 5_{\mathrm{L}}$ and $I M 5_{\mathrm{H}}$, only $\mathrm{IM} 3_{\mathrm{L}}$ and $\mathrm{IM} 5_{\mathrm{L}}$ contours are presented here.

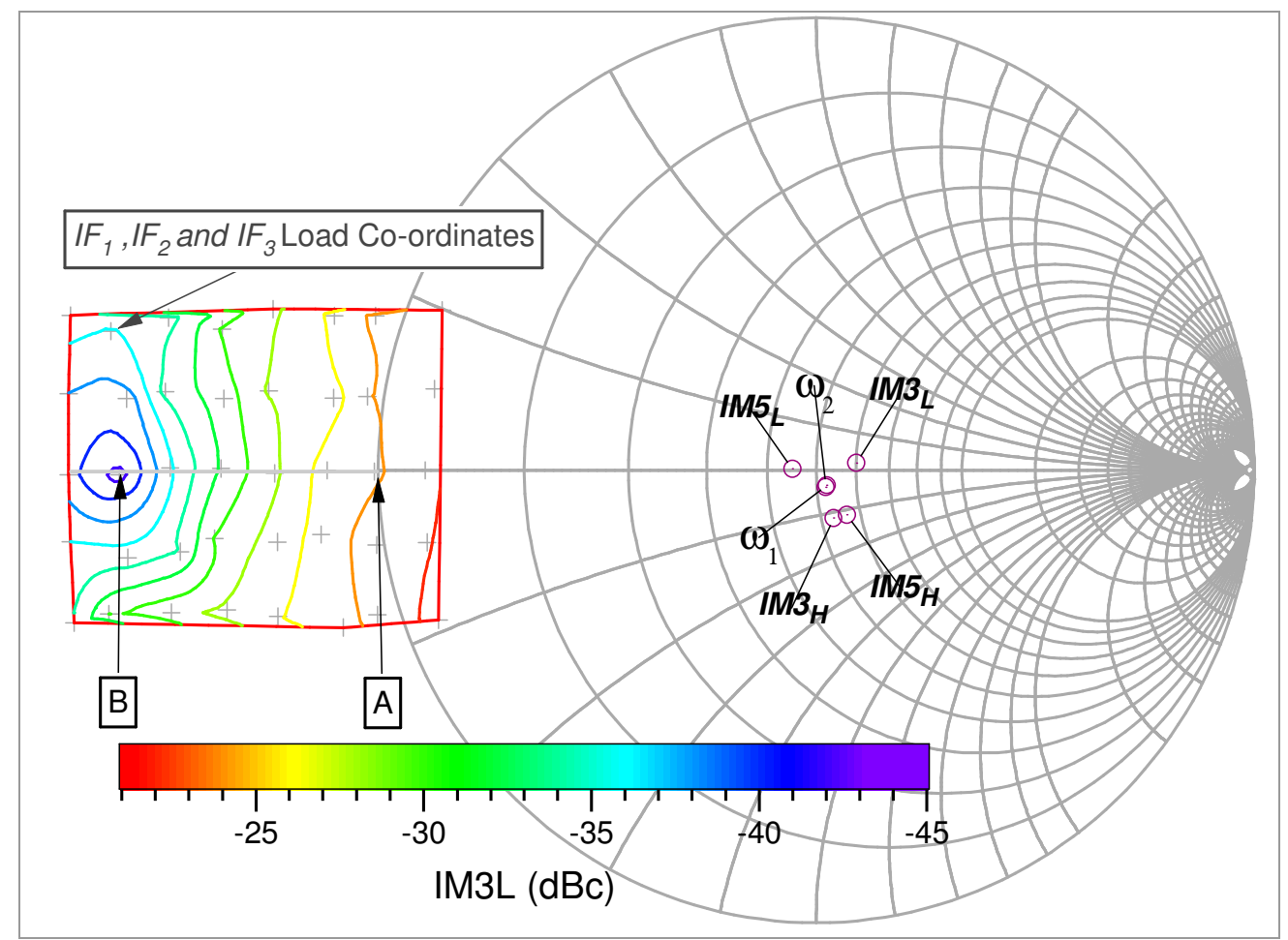

Figure 4.23: Measured IM3L linearity contours as a function of IF1, IF2 and IF3 loads.

If we consider the behaviour of IM3 and IM5 components for the cases of IF loads only located along the real axis, it can be seen that with regard to Figure 4.25, the real baseband impedances required to minimize IM3 and IM5 are different, and located at points $\mathrm{B}$ and $\mathrm{C}$ respectively. Establishing the 
broadband IF load at point $B$ leads to an approximate $11 \mathrm{~dB}$ degradation in the established IM5 optimum.

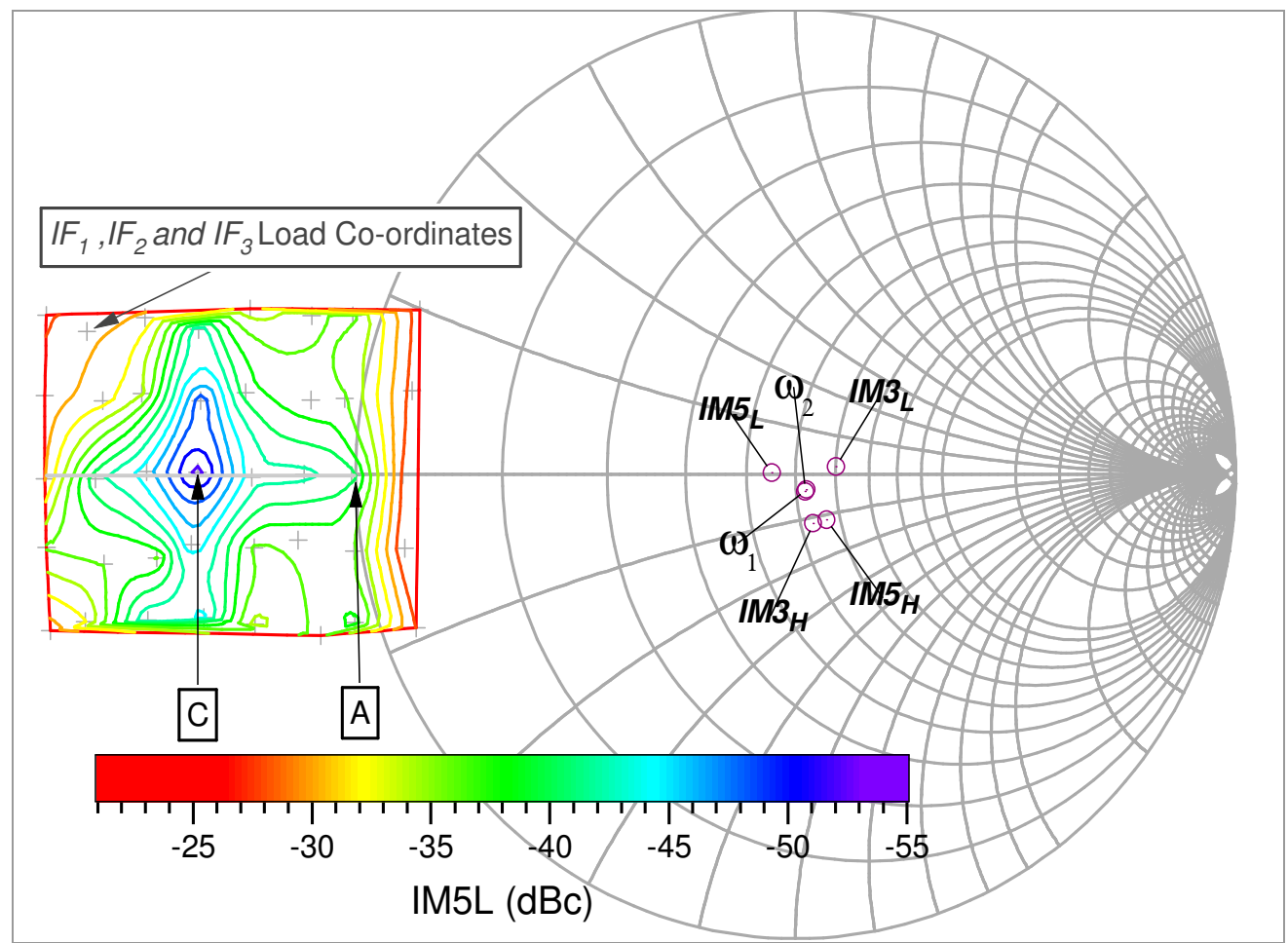

Figure 4.24: Measured IM5L linearity contours as a function of IF1, IF2 and IF3 loads.

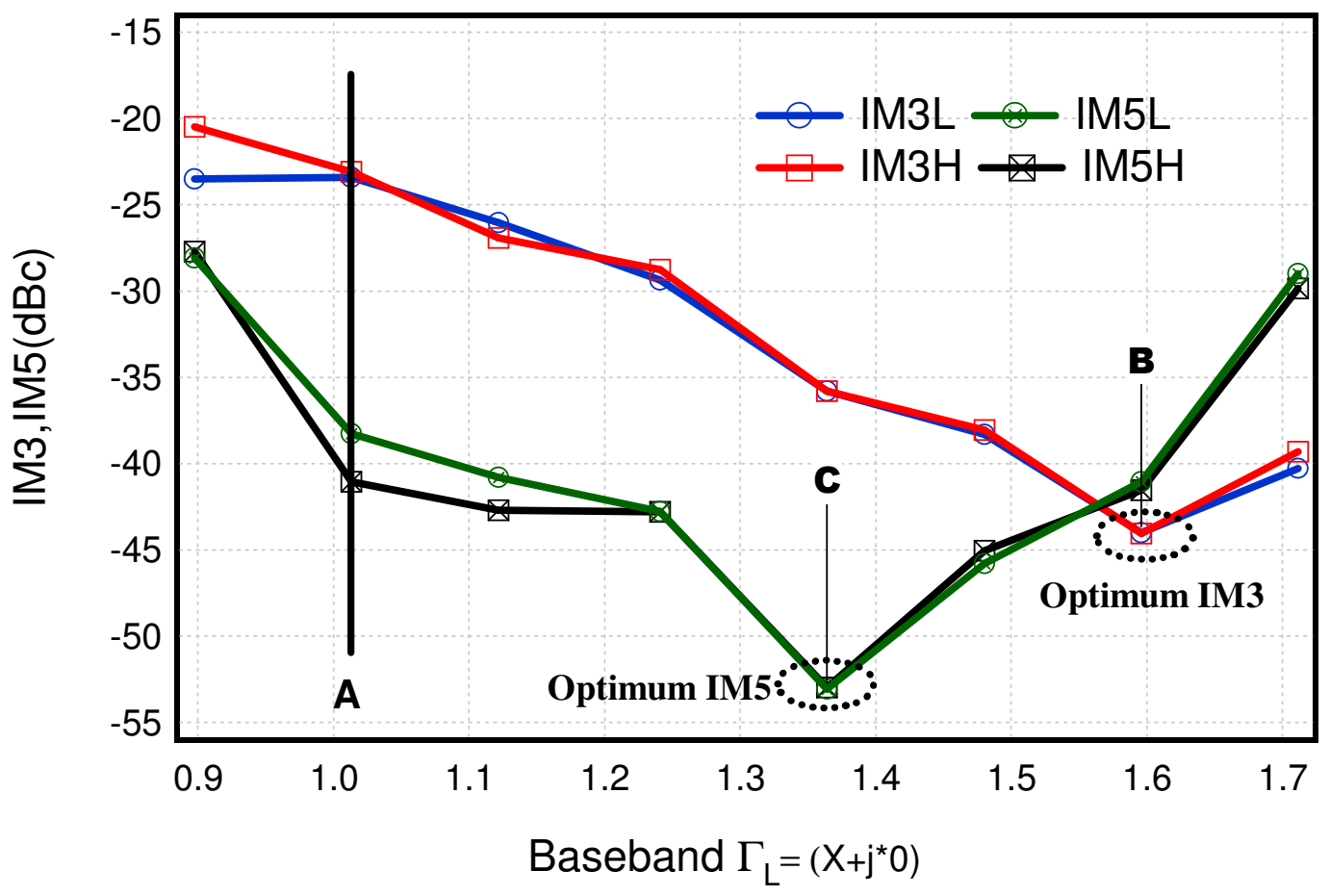

Figure 4.25: Measured IM3 and IM5 linearity as a function of baseband reflection coefficient $\left(\Gamma_{\mathrm{L}}\right)$. 
Conversely, fixing the IF load at point $C$ results in an approximate $7 \mathrm{~dB}$ degradation from the established IM3 optimum. Having said this, it can be seen that adopting a broadband IF load impedance between points $B$ and $C$ offers a significant improvement in linearity when compared to the usual short circuit termination located at point-A.

Figure 4.26 shows the baseband voltage waveforms that result when the IF impedances for point $A, B$ and $C$ are presented to the device. The waveform engineering through the application of active IF load pull demonstrates the importance of providing the suitable baseband impedance to the device under test in order to achieve better linearity performance. It is shown later how effective this technique is for minimising inter-modulation distortion components and envelope distortion by maintaining the low impedance $(\leq 1 \Omega)$ for all the baseband components.

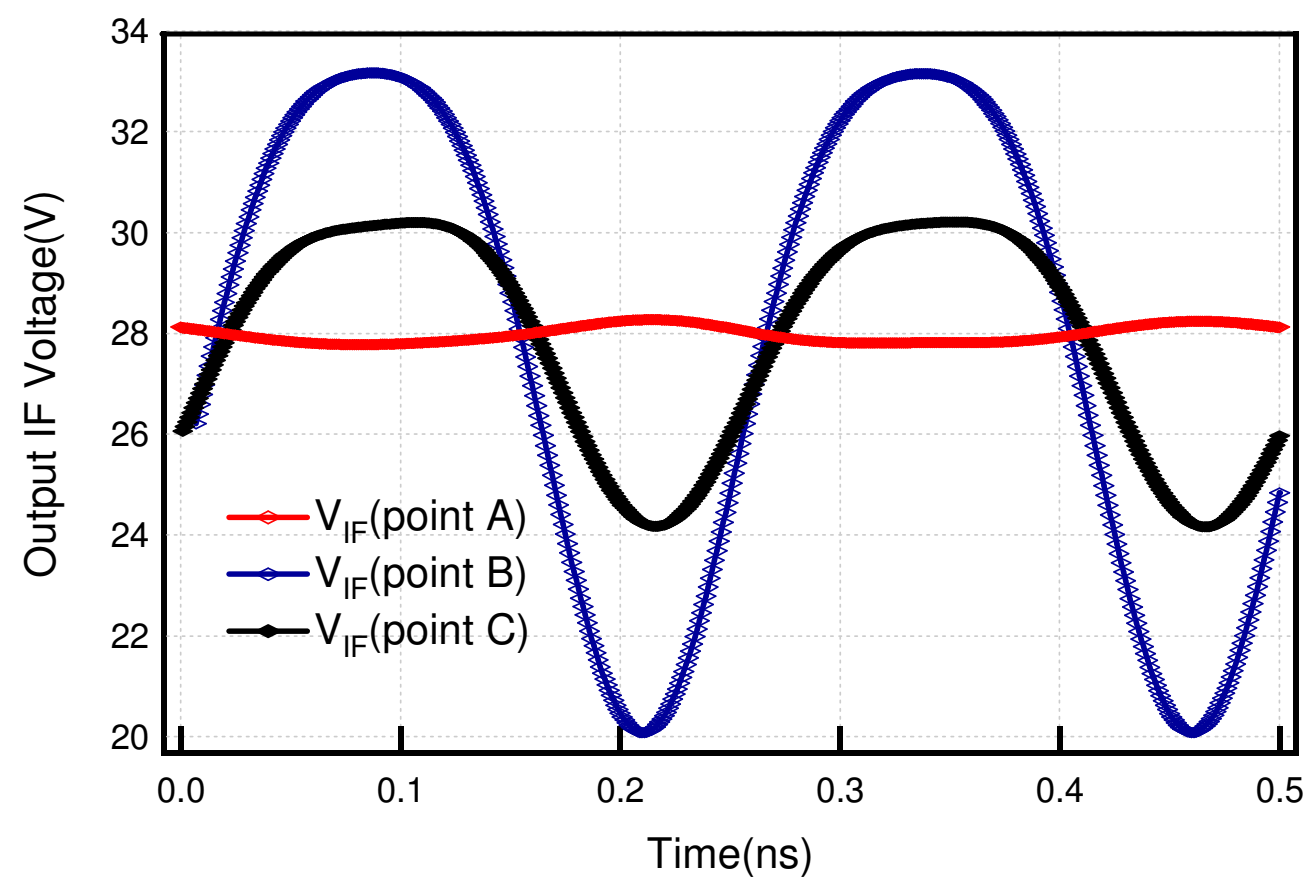

Figure 4.26: Baseband voltage that resulted for the two cases of IF load impedances for minimum IM3 and IM5.

The measured RF and IF output voltage waveforms corresponding to the purely negative resistive load condition at point-B are depicted in figure 4.27. The incident $b_{2}$ wave generated by the device, was phase shifted and its magnitude was varied by shifting the phase and varying the magnitude of 
actively injected baseband signal which leads to modifying the reflected wave $\mathrm{a}_{2}$ from device at the baseband frequency level.

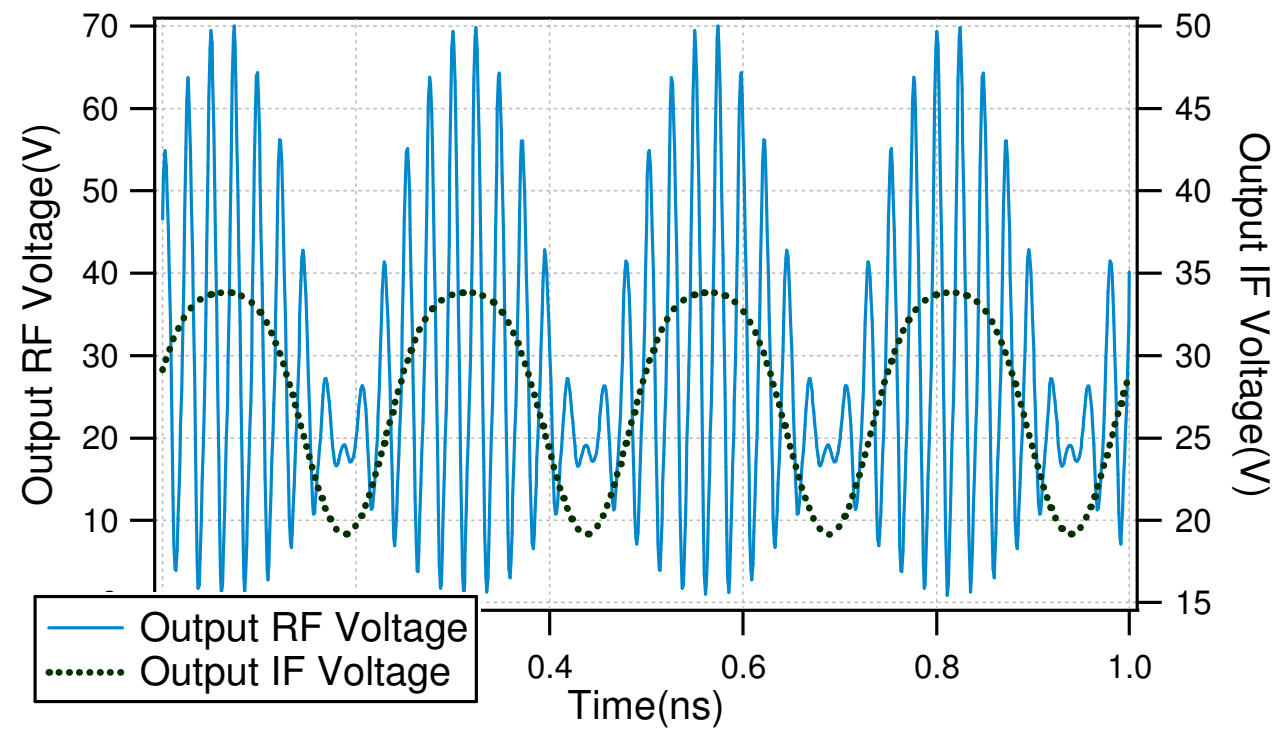

Figure 4.27: The output RF and IF voltage waveforms at point-B for optimum linearity of IM3.

At this point the output RF voltage obtained for the best IM3 linearity is dramatically shaped by the presence of substantial baseband voltage at the drain of the device. The subtraction of baseband voltage from the output RF voltage waveform results in a linearized and symmetrical output RF voltage waveform with the complete absence of distortion as can be seen from the Figure 4.28.

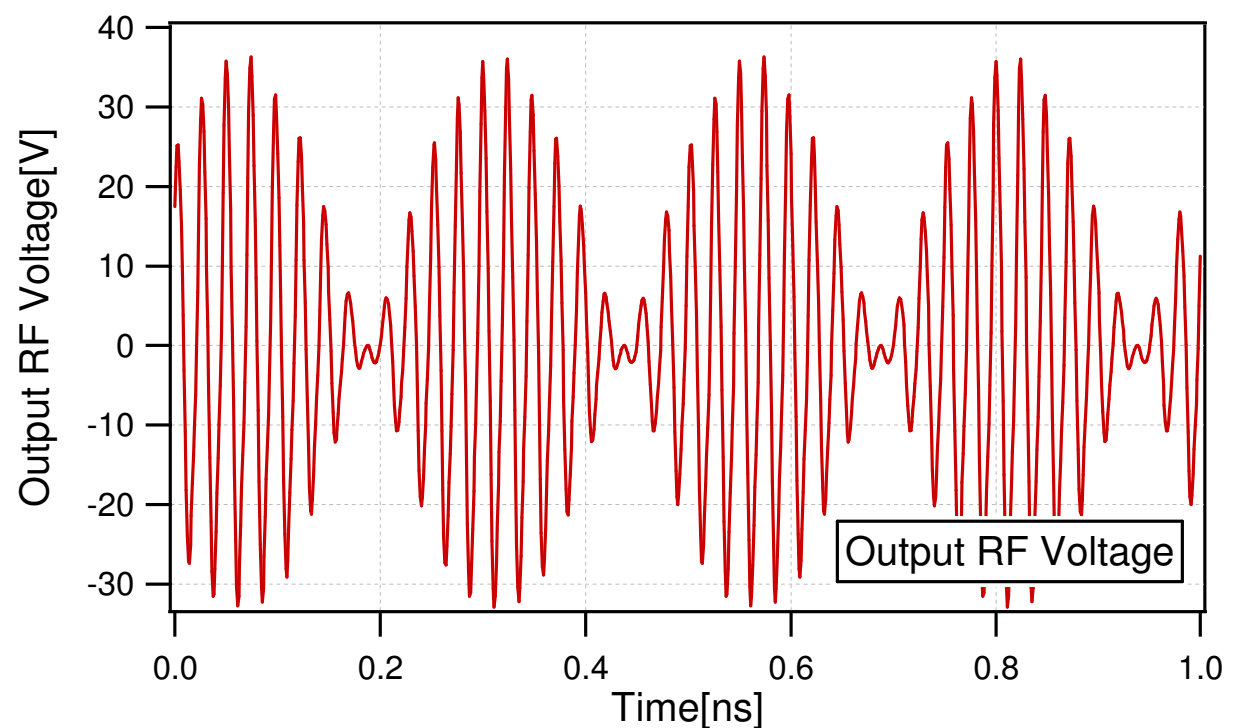

Figure 4.28: The linearized output RF voltage waveforms at point-B for optimum linearity of IM3. 


\subsubsection{Optimized Device Linearity Performance}

From the previous two-tone measurements it was clear that, for this device, the optimum baseband impedance for linearity resides outside the Smith chart. To investigate this observation further and specifically, to explore the possibility of linearization through the direct application of an ET voltage waveform, additional measurements were carried out for three different cases of $\mathrm{DC}$ drain voltage $\left(\mathrm{V}_{\mathrm{dc}}\right): 28 \mathrm{~V}, 24 \mathrm{~V}$ and $16 \mathrm{~V}$.

The device was deep class-AB biased, and driven approximately $2.5 \mathrm{~dB}$ into compression with fundamental and harmonic components again terminated into a passive $50 \Omega$ load. Using a symmetrical 3-tone, 100\% AM excitation signal centred at $2 \mathrm{GHz}$ with an envelope frequency of $2 \mathrm{MHz}$, a different IF load condition was used for each case of $V_{d c}$.

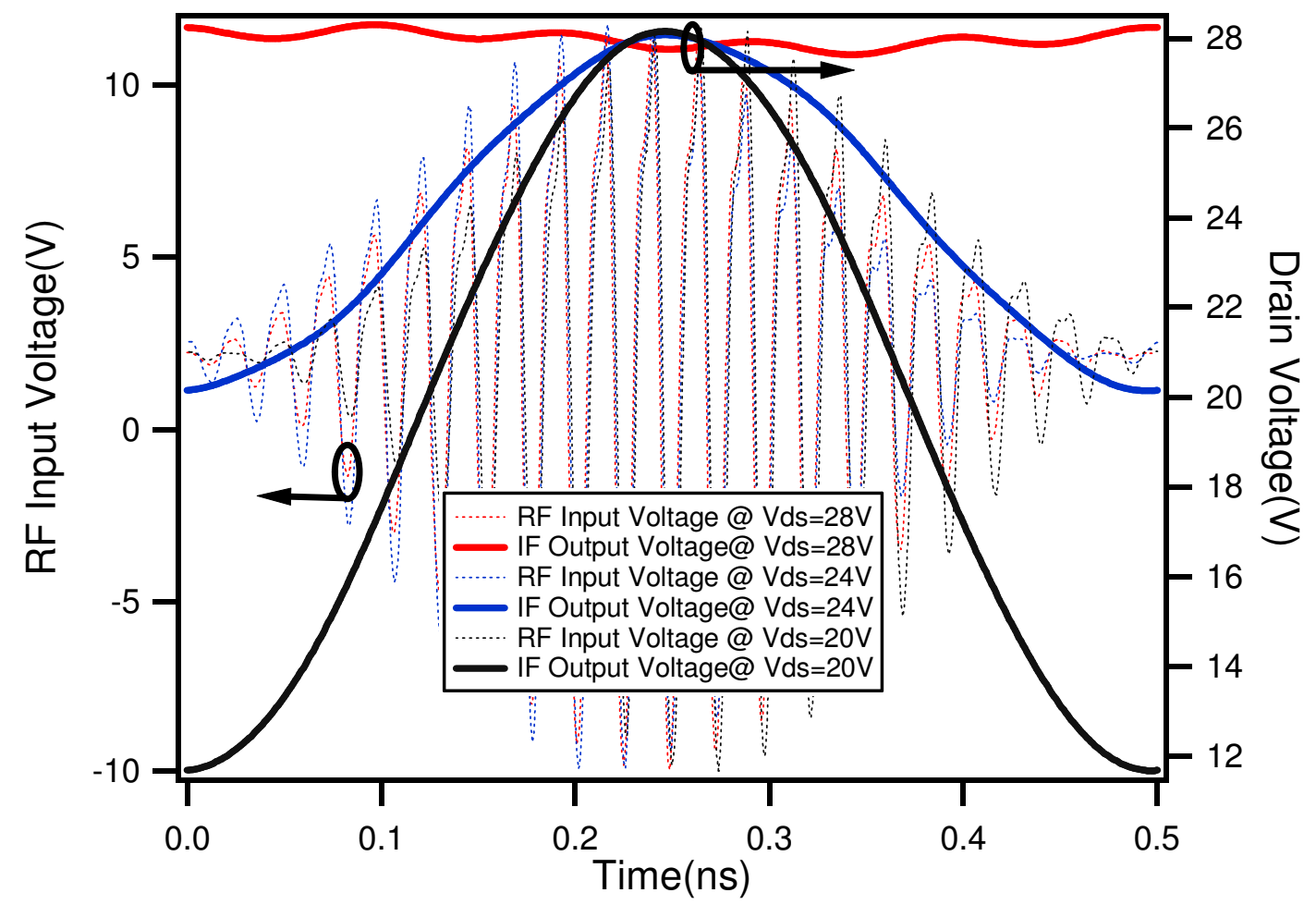

Figure 4.29: Measured dynamic IF voltage envelops in-phase with input RF voltages.

For the case of $\mathrm{V}_{\mathrm{dc}}=28 \mathrm{~V}$, an ideal short circuit termination was synthesised for each of the four significant baseband components, resulting in a near static supply rail. The slight 'ripple' that remained was associated with IF5, but this was very small and in the order of $200 \mathrm{mV}_{\mathrm{p}-\mathrm{p}}$, so was considered not to be of 
concern. For the second case of $\mathrm{V}_{\mathrm{dc}}=24 \mathrm{~V}$, a sufficiently negative resistance was presented to all four IF components to result in an $8 \mathrm{~V}_{\mathrm{p}-\mathrm{p}}$ baseband voltage being developed. For the third case of $\mathrm{V}_{\mathrm{dc}}=20 \mathrm{~V}$, the IF load was again adjusted such that a $16 \mathrm{~V}_{\mathrm{p}-\mathrm{p}}$ baseband voltage waveform was developed. Figure 4.29 shows how in each case, the injected baseband signals track the RF input signal envelope and provide an 'ET-like' variable supply voltage to the device. It is also clear that for the three cases, the peak baseband voltage is the same at $28 \mathrm{~V}$, thus allowing meaningful comparison.

For the case of $\mathrm{V}_{\mathrm{dc}}=28 \mathrm{~V}$, an average drain efficiency of $38.1 \%$ was achieved at a peak envelope power (PEP) of $39.72 \mathrm{dBm}$. For the case of $\mathrm{V}_{\mathrm{dc}}=24 \mathrm{~V}$, the average drain efficiency was $41.5 \%$ at a PEP of $40.3 \mathrm{dBm}$. A slightly better performance in terms of efficiency and output power was achieved with $\mathrm{V}_{\mathrm{dc}}=20 \mathrm{~V}$. Therefore, applying a dynamic drain voltage has increased the achievable peak output power, and has thus enabled a higher efficiency than was possible with a fixed supply voltage of $28 \mathrm{~V}$.

Linearity performance is summarized in Table 4.3, where it is shown that for the case of $\mathrm{V}_{\mathrm{dc}}=20 \mathrm{~V}$, IM3 and IM5 distortions are suppressed by $10 \mathrm{dBc}$ and $3 \mathrm{dBc}$ respectively compared to the static $\mathrm{V}_{\mathrm{dc}}=28 \mathrm{~V}$ case where a short circuit impedance was maintained for all four IF components. For the case where $\mathrm{V}_{\mathrm{dc}}=24 \mathrm{~V}$, only a small improvement in IM3 distortions was observed along with an $8 \mathrm{dBc}$ improvement in IM5 distortion.

Table 4.3: Three tones measured linearity results for three distinct drain voltages

\begin{tabular}{|c|c|c|c|c|c|c|c|}
\hline $\begin{array}{c}\text { Supply } \\
\text { Voltage }(\mathrm{V})\end{array}$ & $\begin{array}{c}\text { IM5L } \\
\mathrm{dBc}\end{array}$ & $\begin{array}{c}\text { IM3L } \\
\mathrm{dBc}\end{array}$ & $\begin{array}{c}\text { W1 } \\
\mathrm{dBm}\end{array}$ & $\begin{array}{c}\text { Wc } \\
\mathrm{dBm}\end{array}$ & $\begin{array}{c}\text { W2 } \\
\mathrm{dBm}\end{array}$ & $\begin{array}{c}\text { IM3H } \\
\mathrm{dBc}\end{array}$ & $\begin{array}{c}\text { IM5H } \\
\mathrm{dBc}\end{array}$ \\
\hline 28 & $\mathbf{- 3 5 . 6 8}$ & $-\mathbf{- 1 2 . 8 3}$ & 27.85 & 35.69 & 28.02 & $-\mathbf{- 1 3 . 1 2}$ & $\mathbf{- 3 6 . 4 7}$ \\
\hline 24 & $-\mathbf{4 3 . 0 5}$ & $-\mathbf{- 1 5 . 8 6}$ & 28.08 & 35.18 & 28.15 & $\mathbf{- 1 6 . 3 7}$ & $\mathbf{- 4 3 . 2 6}$ \\
\hline 20 & $\mathbf{- 3 8 . 9 5}$ & $-\mathbf{- 2 2 . 8 1}$ & 28.39 & 34.39 & 28.49 & $\mathbf{- 2 2 . 5 7}$ & $\mathbf{- 3 9 . 4 1}$ \\
\hline
\end{tabular}

\subsubsection{Optimized Device Efficiency Performance}

Modern complex modulation schemes have the disadvantage of requiring linear power amplification characteristics, which compromises 
overall system efficiency because RF power amplifiers (RFPA) are much less efficient when operating in the linear region than they are at maximum power. The power amplifier and its associated components consume up to $40 \%$ of the overall power in a cellular basestation and account for a similar proportion of operating costs. Conventional designs for improving PA efficiency are inherently narrowband, requiring multiple amplifiers to be used in order to span all the required bands. The efficiency of the PA is dependent on the 'crest factor' or Peak-to-Average Ratio (PAR) of the signal being amplified. In a WCDMA system, the PA is usually operating far below peak power, as the signal crest factor is typically 6.5 - 7.0dB. OFDM systems such as 3GPP LTE and WiMAX use even higher crest factors typically 8.5 - 9.5dB for improved spectral efficiency but this results in traditional PA designs having even lower efficiency. It is possible, however, to achieve a significant improvement in PA linearity and the efficiency of the entire network, along with the increase in efficiency, using Auxiliary Envelope Tracking (AET) reported in [10][11].

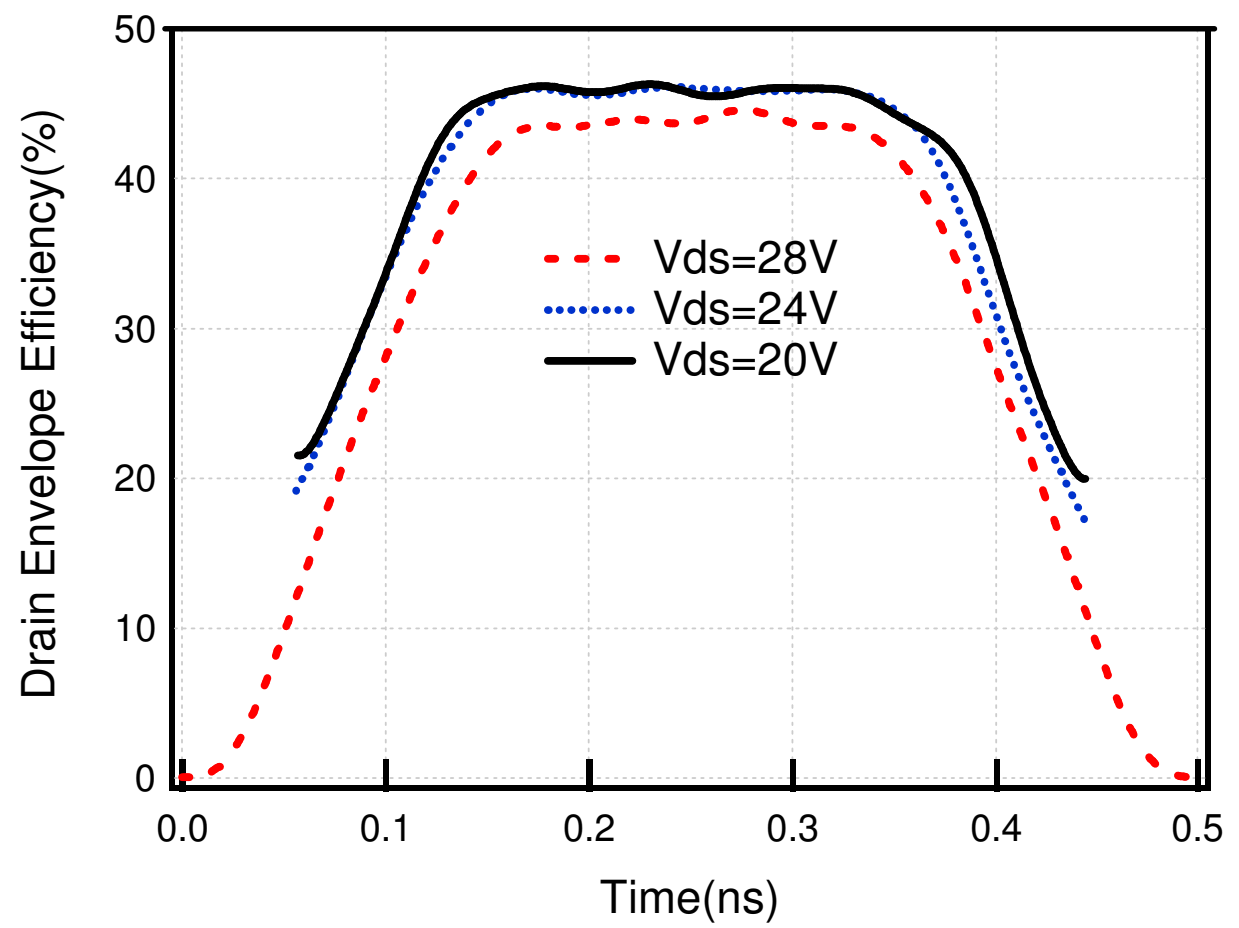

Figure 4.30: Drain envelope efficiencies for three different cases at constant input drive and fixed bias level $\mathrm{V}_{\text {bias }}=-2.05 \mathrm{~V}$.

The results depicted in Figure 4.30 are achieved through a technique based on envelope tracking that has been proven to offer a significant improvement 
in efficiency, from typically $1 \%$ to over $10 \%$, while offering improved linearity performance. The true essence of AET is that, the power supply tracks the RF input signal envelope and provides a variable supply voltage Vds to the power device. Both Vds and output power vary together, greatly increasing power efficiency (a comparison of efficiencies can be seen from figure 4.30 for three different drain bias, that is an envelope domain representation of efficiencies [1]) compared with using fixed voltage power supply. It can be seen that there exists an increase of envelope efficiencies for variable drain voltage in comparison with the fixed drain bias $\left(V_{d c}=28 \mathrm{~V}\right)$ which was resulted when short circuit impedance was provided to all the baseband components. The increase in efficiency is quite evident at the skirts of the envelopes.

\subsubsection{Mathematical Analysis on $2^{\text {nd }}$ Harmonic Termination}

In order to have more insight on the effect of the $2^{\text {nd }}$ harmonic termination, a transistor's nonlinearity can be approximated by three term power series expansion for the drain current as:

$$
\mathrm{I}_{\mathrm{d}}=\mathrm{gm}_{1} \mathrm{~V}_{\text {in }}^{1}+\mathrm{gm}_{2} \mathrm{~V}_{\text {in }}^{2}+\mathrm{gm}_{3} \mathrm{~V}_{\text {in }}^{3}
$$

Considering a two-tone fundamental signal fed to the power device with second harmonic in which $\varphi_{1}$ and $\varphi_{2}$ are the phases of second harmonic:

$$
V_{\text {in }}=A_{1} \cos \omega_{1} t+A_{2} \cos \omega_{2} t+A_{11} \cos \left(2 \omega_{1} t+\varphi_{1}\right)+A_{22} \cos \left(2 \omega_{2} t+\varphi_{2}\right)
$$

By substituting (4.11) in (4.10) gives all the relevant components and for brevity the third order $1 \mathrm{M} 3_{\mathrm{H}}$ components are given in equation (4.12).

$$
\begin{aligned}
I_{d}\left(2 \omega_{2}-\omega_{1}\right) & =\frac{3}{4} A_{1} A_{2}^{2} g m_{3} \cos \left(2 \omega_{2}-\omega_{1}\right) t \\
& +A_{1} A_{22} g m_{2} \cos \left(2 \omega_{2} t-\omega_{1} t+\varphi_{2}\right) \\
& +\frac{3}{2} A_{11} A_{22} g m_{3} \cos \left(2 \omega_{2} t-\omega_{1} t+\varphi_{2}-\varphi_{1}\right)
\end{aligned}
$$

The third term in the equation (4.13) is small in comparison to the two other terms and can possibly be ignored. In order to cancel out the IM3 completely following condition must be satisfied.

$$
A_{\text {env }}=-\frac{3}{4} \frac{A_{2}^{2} g m_{3}}{g m_{2}} \text { and } \varphi_{2}=180^{\circ}
$$

This shows that if the first two terms in equation are not out-of-phase between each other, suppression of IM3 is difficult to realize because the 
intermodulation products at frequencies $2 \omega_{1}-\omega_{2}$ and $2 \omega_{2}-\omega_{1}$ generated from the second harmonic termination other than the $0^{\circ}$ and $180^{\circ}$ would possess different phase with respect to each other. Consequently the newly generated IM terms will have different magnitude and phase. It is possible to attain a complete suppression of one of the IM products whilst giving rise to other when the phase of the second harmonic is varied and the reference phase is defined by the intrinsic parameters of the device which was found to be $0^{0}$ for optimum linearity performance of this device in case when RF components were terminated with nominal $50 \Omega$ impedance.

\subsubsection{Emulation of Class-J Mode of Power Amplifier}

In order to further investigate the suitability of this efficiency enhancing and linearizing technique under a realistic PA mode of operation, a class-J RF impedance environment was emulated. The device was biased in deep class- $\mathrm{AB}$, driven into approximately $2 \mathrm{~dB}$ of compression and excited using a $4 \mathrm{MHz}$ spaced two-tone modulated excitation centred at $2 \mathrm{GHz}$. With knowledge of the device's intrinsic and extrinsic parasitics, the required class$\mathrm{J}$ impedances were emulated and presented to the package plane [15]. This involved maintaining constant, specific impedances for both fundamental tones as well as the tones $\left(2 \omega_{1}, 2 \omega_{2}\right)$ located around the $2^{\text {nd }}$ harmonic. The third harmonic components were terminated arbitrarily.

The measurement results documented in the previous section clearly indicated that significant linearity benefit can be gained by optimizing the baseband impedance environment, as opposed for example to simply presenting a short circuit. For this class-J investigation, the IF impedance presented to all significant baseband tones was swept over a similar measurement grid, again including the short circuit condition, and again extending outside the Smith chart. The IMD contours are plotted in Figure 4.31, and similarly show that the optimum IF impedance for reduction of IMD resides outside the Smith chart. Importantly in this case however, the measurement shows that optimum baseband impedances are different for $\mathrm{IM} 3_{\mathrm{L}}, \mathrm{IM} 3_{\mathrm{H}}, \mathrm{IM} 5_{\mathrm{L}}$ and $\mathrm{IM} 5_{\mathrm{H}}$ and these are not, as was the case in the previous $50 \Omega$ measurement, co-located on the real axis. The optimum $1 \mathrm{M} 3_{\mathrm{L}}$ and $I \mathrm{IM} 3_{\mathrm{H}}$ baseband termination is found to be $-43.2 \mathrm{dBc}$ and $-45.5 \mathrm{dBc}$ respectively, and 
is approximately $18.5 \mathrm{dBc}$ better than the case where usual short circuit is provided to all the significant baseband components. But from an IM5 perspective, the optimum $\mathrm{IM} 5_{\mathrm{L}}$ and $\mathrm{IM} 5_{\mathrm{H}}$ showed the minimum distortion products which were found to be $-59.3 \mathrm{dBc}$ and $-61.1 \mathrm{dBc}$ respectively.

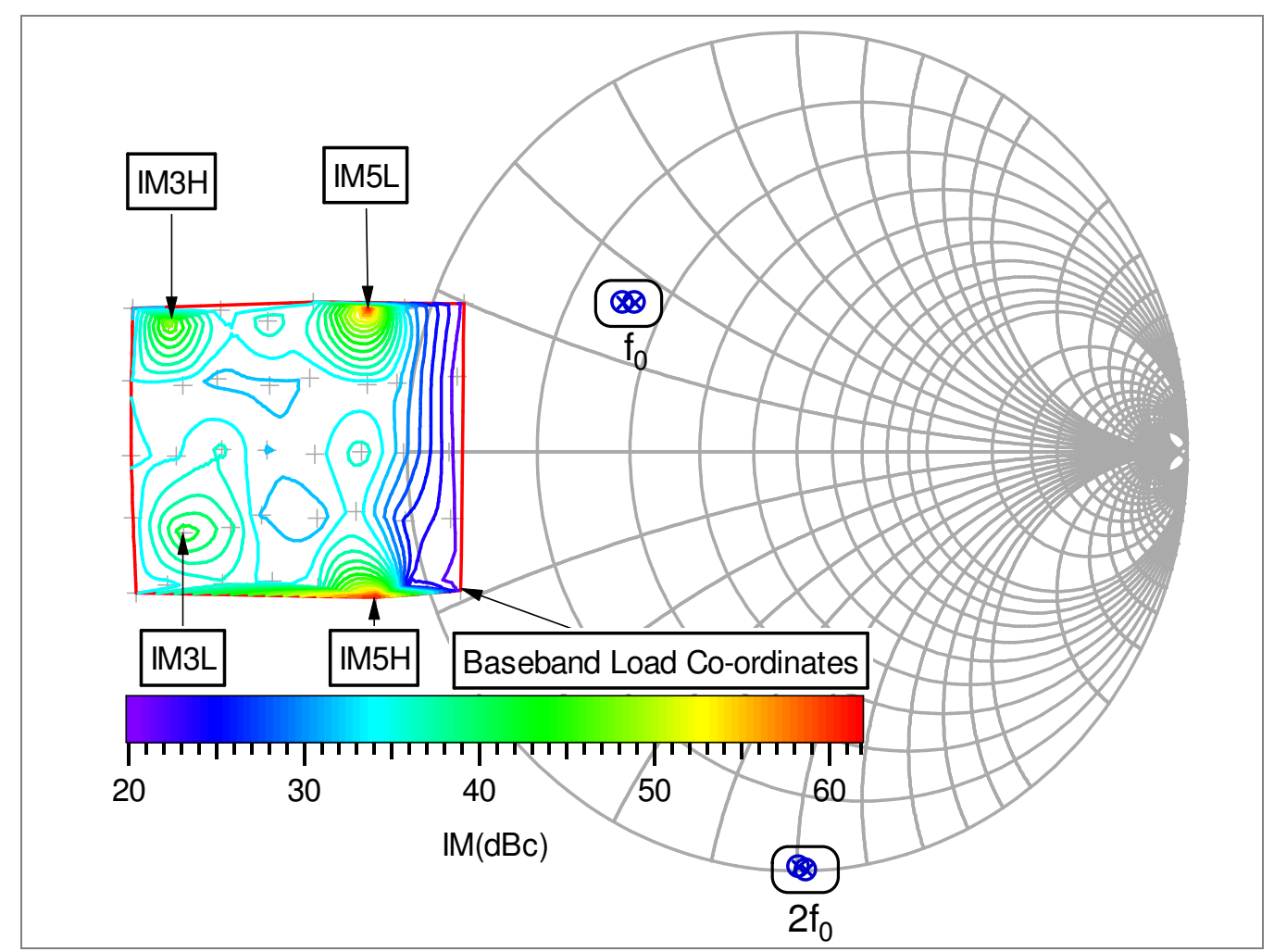

Figure 4.31: Measured IM linearity contours as a function of IF reflection coefficient $\left(\Gamma_{\mathrm{L}}\right)$.

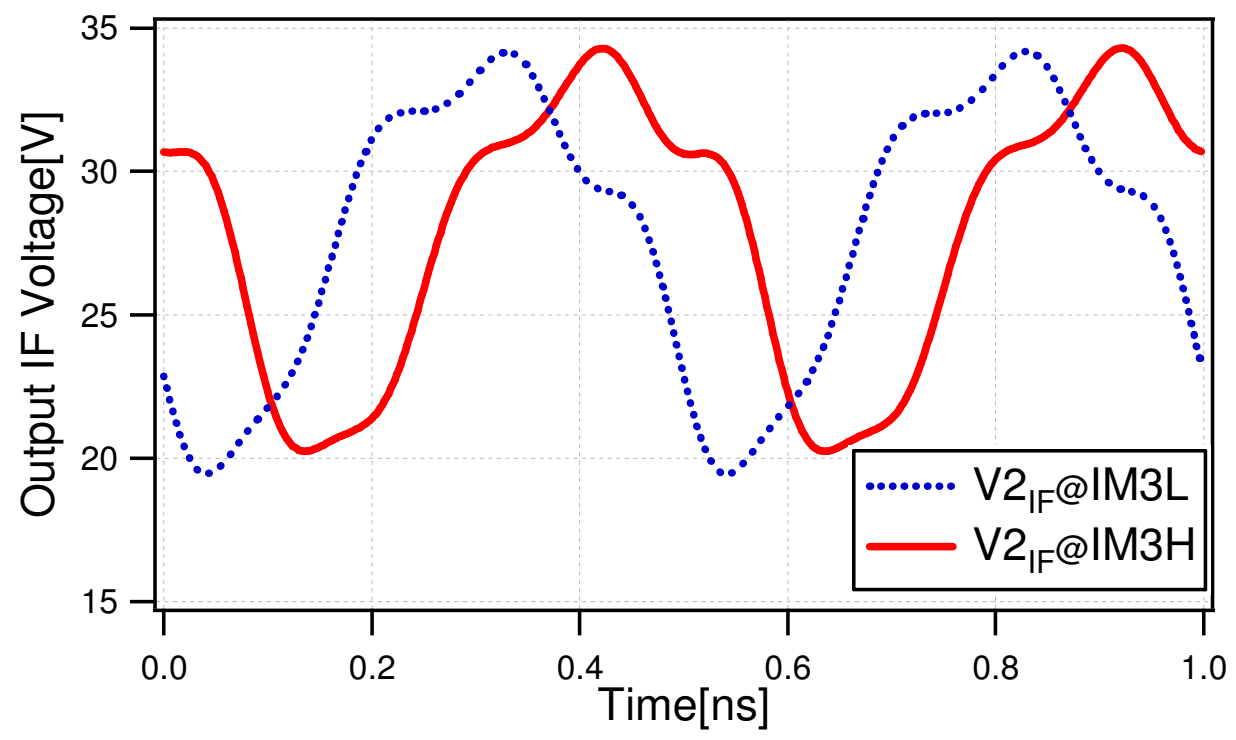

Figure 4.32: Baseband voltages that resulted for the best IM3 linearity performance. 
Figure 4.32 and Figure 4.33 show the baseband voltage waveforms that resulted when the IF impedances for best IM low and high linearity were presented to the device. Although the magnitude and shape of both waveforms is similar, the phase is clearly different.

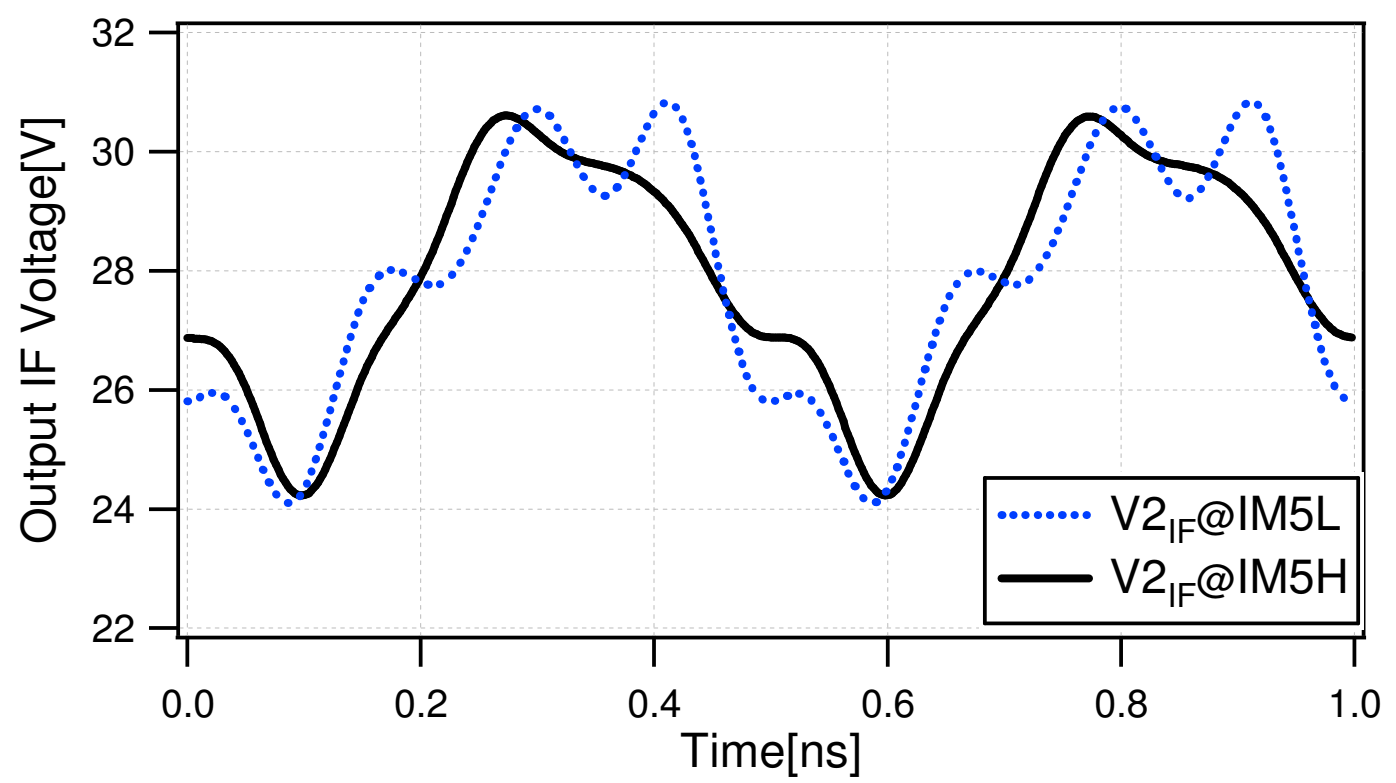

Figure 4.32: Baseband voltages that resulted for the best IM5 linearity performance.

\subsubsection{Repeatability of Baseband and RF Load Emulation}

The ability of the system to maintain broadband baseband, fundamental and second harmonic loads was thus utilized. This capability is evident in Figure 4.33 where the second and fundamental loads for all 40 baseband points are overlaid on the same smith chart. The drive level of device was kept constant for all these 40 measurements.

The variation and dispersion in the fundamental and $2^{\text {nd }}$ harmonic loads can be seen to be minimal and in terms of normalised Cartesian coordinates, this was measured to be in the region of $0.6 \%$ (1SD) for both fundamental and second harmonic loads.

The very low standard deviation in the oscilloscope measurements for 40 repeated measurements for different numbers of targets at baseband demonstrates the capability of the measurement system in maintaining the constant and frequency independent RF loads whilst providing different impedance to baseband components 


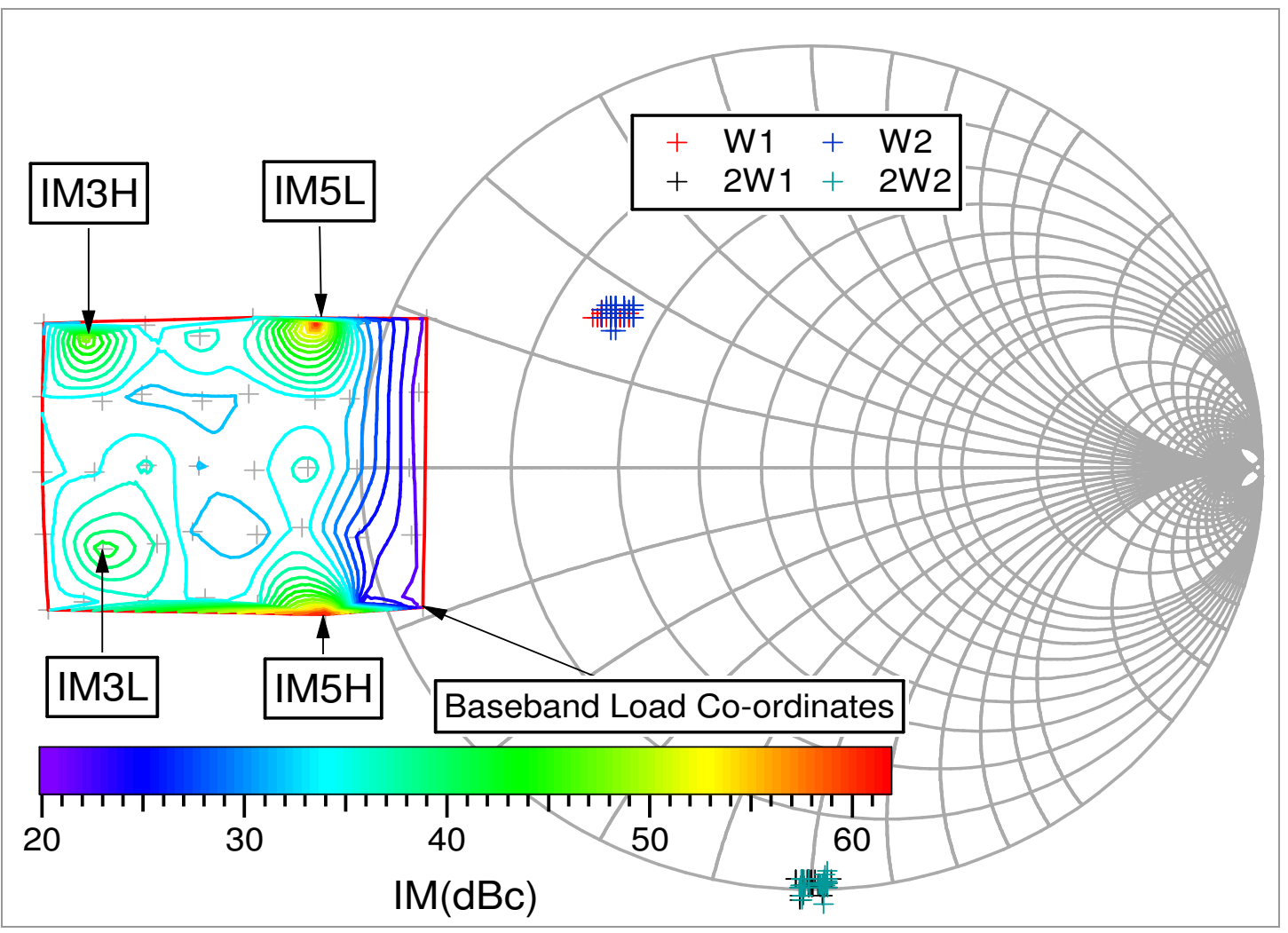

Figure 4.33: The measured 40-points baseband grid for a class-J emulated RF impedance environment.

\subsection{Chapter Summary}

An important application of the enhanced measurement system is discussed where both positive (inside the Smith chart) and negative (outside the Smith chart) baseband impedances were presented to all baseband (IF) components. This chapter has detailed the impact of baseband impedance termination on the linearity of GaN HEMTs in both backed-off and compressed drive level regimes. In the case of a 'backed-off' device, contours of IM3-high and IM3-low indicate separate local optimum IF loads that lie some way outside the Smith chart, and for this particular device, a 15-20 dB improvement in IM3-high magnitude is observed in comparison to the reference case, where IF1 and IF2 are terminated into short circuits. Whereas dynamic RF load-lines are a familiar plot in CW waveform engineering, the inclusion of baseband voltage and current to these plots adds an extra dimension that shows interesting and useful modulated behaviour, such as self-bias, and in this case of an emulated ET environment, the proximity of the 
load-line to the knee region, which may of course be optimised. For the ET analysis, where a significant IF component is injected, it is shown how plotting the envelope efficiency and gain is useful in understanding the device behaviour [1]. In this case, it is clear from these time domain plots that there is an overall reduction in gain and hence output power with the application of an envelope tracking voltage, an effect that is largely explained by the fact that, for this device, the gain reduces with reducing drain bias voltage as has been described through CW investigations for varying drain supply voltage.

Furthermore, this chapter sheds light on the optimized linearity investigations of the 10W GaN HEMT under modulated excitations when driven strongly into compression. This has been shown that for the device considered, the optimum impedance for best linearity lies outside the Smith chart as was the case for the backed-off drive level. Interestingly, the results also suggest that there exists separate optimum impedances for suppression of IM3 and IM5 distortion products. Additionally, auxiliary envelope tracking measurements

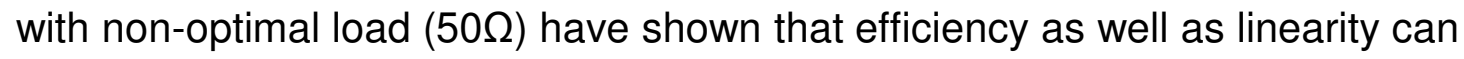
be improved at reduced drain supply voltages: for $\mathrm{Vdc}=20 \mathrm{~V}$, the average drain efficiency is improved by approximately $5 \%$ together with an improvement of $10 \mathrm{dBc}$ in IM3 when compared to the static Vdc, where short circuit impedance was maintained for all four baseband components [10].

It was shown that that enhanced modulated waveform measurement system allows the emulation of novel PA modes and architectures, including for example class-J and envelope tracking respectively. Direct observation of the effects of baseband impedance variations is now possible over wide bandwidths, and under complex multi-sine excitations. The true benefit of this enhanced system becomes apparent through the broadband emulation of a class-J mode of operation. The results also suggest that there exist separate optimum impedances for suppression of IM distortion products when emulating a class-J mode of operation for power amplifiers. This imperative observation has large implications for modern PA linearization techniques; emphasize the fact that the linearity of power amplifiers can greatly be enhanced by using the very mechanisms that are employed to improve the efficiency. 


\subsection{References}

1. J. Lees, M. Akmal, S. Bensmida, S. Woodington, J. Benedikt, K. Morris, M. Beach, J. McGeehan, P. J. Tasker, "Waveform Engineering Applied to Linear-Efficient PA Design", in Proc. of $11^{\text {th }}$ IEEE Wireless and Microwave Technology Conference(WAMICON), pages:1- 5, April 2010.

2 T. H. Lee, "The design of CMOS radio-frequency integrated circuits", 2nd edition, Cambridge University Press, 2004.

3. M. D. Weiss, F. H. Raab, Z. Popovic "Linearity of X-Band Class-F Power Amplifiers in High-Efficiency Transmitters", IEEE Transactions on Microwave Theory and Techniques, VOL. 49, NO. 6, JUNE 2001.

4. W. H. Doherty, "A New High Efficiency Power Amplifier for Modulated Waves", Proc. of the IRE, Vol. 24, No. 9, pp. 1163 -1182, 1936.

5. L. R. Khan, "Single Sideband Transmission by Envelope Elimination and Restoration", Proc. of the IRE, Vol. 40, No. 10, pp. 803 806, 1952.

6. P. B. Kenington, "High Linearity RF Amplifier Design", Norwood, MA: Artech House, 2000.

7. N. Pothecary, "Feedforward Linear Power Amplifiers", Norwood, MA: Artech House, 1999.

8. Powerwave Technologies Application Note, "Multi-Carrier Power Amplifiers for W-CDMA Wireless Systems", June 1998, http:// www.powerwave.com.

9. S. C. Cripps, "RF power amplifiers for wireless communications", $2^{\text {nd }}$ edition. Norwood, MA: Artech House, 2006.

10. M. Akmal, J. Lees, S. Bensmida, S. Woodington, V. Carrubba, S. Cripps, J. Benedikt, K. Morris, M. Beach, J. McGeehan, P. J. Tasker, "The Effect of Baseband Impedance Termination on the Linearity of GaN HEMTs", in Proc. of $40^{\text {th }}$ European Microwave Conference(EuMC), September 2010, pages: $1046-1049$

11.Z. Yusoff, J. Lees, J. Benedikt, P. J. Tasker, S.C. Cripps, "Linearity Improvement in RF Power Amplifier System using Integrated Auxiliary Envelope Tracking System", in Proc., IEEE International Microwave Symposium, June 2011, Page(s):5- 8.

12. A. Alghanim, J. Lees, T. Williams, J. Benedikt,P. Tasker, "Using active IF load-pull to investigate electrical base-band induced memory effects in high-power LDMOS transistors," in Proc. Asia-Pacific Microwave Conference, 2007, 11-14 Dec. 2007 Page(s):1 - 4. 
13. A. Alghanim, J. Less, T. Williams, J. Benedikt, P. Tasker, "Reduction of electrical base-band memory effects in high-power LDMOS DevicesUsing Optimum Termination for IMD3 and IMD5 using active load-pull', in Proc., IEEE International Microwave Symposium, June 2008.

14. J. Lees, T. Williams, S. Woodington, P. McGovern, S. Cripps, J. Benedikt, and P. Tasker, "Demystifying Device Related Memory Effects Using Waveform Engineering and Envelope Domain Analysis", in Proc. $38^{\text {th }}$ European Microwave Conference, October 2008, pages: 753-756.

15.P. Wright, J. Lees, P. J. Tasker, J. Benedikt, S.C. Cripps, "An efficient, linear, broadband class-J-mode $P A$ realised using $R F$ waveform engineering", in Proc. of IEEE MTT-S International, June 2009, pages: 653 $-656$. 
Chapter 5

\section{Multi-Sine Measurements and Linearization}

\subsection{Introduction}

The continuing evolution of wireless standards such as WiMAX and Long Term Evolution (LTE) are driving the need for power amplifiers (PAs) to multiple modulation schemes at multiple frequencies, whilst also demanding spectral efficiency to meet the ever-increasing linearity requirements. These standards invariably impose higher peak-to-average ratio (PAR), which typically require increasing degrees of back-off to maintain good linearity, thus reducing the efficiency of the power amplifier considerably. As an example, the PAs used in LTE systems, employing orthogonal frequency division multiplexing (OFDM) need to accommodate scalable bandwidths ranging between 1.4 MHz and $20 \mathrm{MHz}$, with high PAR [1].

The goal of a communication system may be broadly defined as: to transfer information across a given medium with a minimum of information loss and minimal interference to other users. The fact is that communication channels add unwanted signals to the desired information thus making it difficult to achieve the desired goal. These undesirable signals include random noise and distortion. Random noise is not correlated with the information, whereas distortion generation is a strong function of the information signal [1]. Distortion arising from the inherent nonlinearity of microwave devices is the topic of interest in this thesis. One impact of device inherent nonlinearity on 
communication systems is the corruption of information and spectral regrowth. Today's mobile communication systems usually use a wideband digitally modulated signal. Such a signal can be imagined as densely spaced multiple tones. If the device is excited with a multi-sine signal then a broad spectrum of the distortion product can be seen. This gives a more clear idea how a device would behave under wide-band excitations. Figure 5.1 shows the mechanism of growing sidebands from multi-tone excitation. If the channels are defined, then one can measure the ratio of total power in the signal and the power in the side band. This measurement is called adjacent channel power ratio (ACPR).

Signal

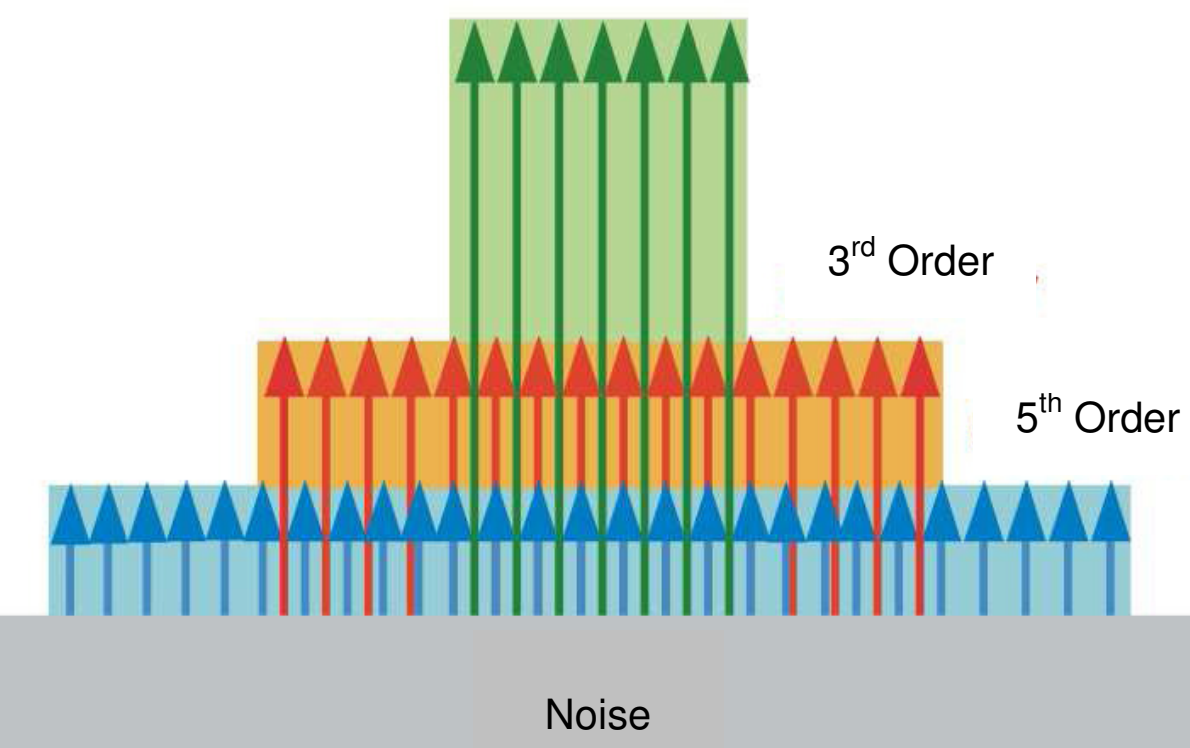

Figure 5.1: The output spectrum when power device is excited with the multitone signal

ACPR can not generally be reduced by filtering because of it is close frequency proximity to the desired signal. This problem is serious because ACPR from one user can fall inside the bandwidth allocated to another user in the already crowded spectrum. Regulatory authorities impose strict regulations on spectral regrowth, which must be met for a product to be approved. Once again, because of the complexity of modern modulation schemes, and especially the use of multi-carrier techniques [2], designing amplifiers that meet such specifications is becoming increasingly difficult. 
Linearization refers to a group of methods that aim to increase the linearity of a system without significantly decreasing its efficiency. For example, predistortion linearization is one widely used method. It works by distorting the input signal before it reaches the nonlinear system, in such a way that it will counteract the distortion introduced by the system.

Linearization has led to large gains in the linearity and efficiency trade-off in many cases. With the increasing bandwidth and dynamic-range demands of recent communication systems, it is frequently observed that linearization does not perform as well as expected. Linearization may be effective for one particular amplifier, while yielding almost no improvement for another amplifier. In addition, the performance of linearization is often seen to be dependent on the signal bandwidth and when PAs are driven into compression, predistortion algorithms have difficulty in capturing the true compression characteristics of the PA instead of increase in memory effects [3]. This has lead to explore the possibility of linearization through the precise control of the out-of-band impedance environment, in particular at baseband frequencies under more complex modulation excitations. To verify the observation made under two-tone and three-tone excitation in chapter 4 , the complex modulated measurements were conducted to further investigate the effect of baseband impedance termination on the linearity of $10 \mathrm{~W}$ GaN HEMT. This chapter confirms and demonstrates that substantial linearity improvement can be achieved under a complex nine-tone excitation, when specific negative baseband impedances are presented to the device.

\subsection{Properties of Multi-Tone Excitations}

The peak-to-average (PAR) of multi-tone excitations significantly affects the ACPR and efficiency performance. Often, lowering a PAR is considered to yield a better linearity performance from the microwave power amplifier (PA). The justification is that high peaks overload the PA, producing unwanted nonlinear effects and strongly impact the ACPR capabilities of a PA system. It is true that the nonlinear effects, caused by high power peaks, can degrade the ACPR value $[1,4]$. However, the correlation between PAR and ACPR is not as straightforward as it seems. Peak power and PAR are time 
domain notions, while ACPR is a frequency domain notion. Increasing PAR values indicate that the peak power is being shifted further in excess of the average power which in turn largely affects the ACPR. Therefore, the PAR describes a form of power back-off that is required to meet the spectral specification of microwave devices. The efficiency of the PA is dependent on the PAR of the signal being amplified. In a W-CDMA system, the PA is usually operating far below peak power, as the signal PAR is typically $6.5-7.0 \mathrm{~dB}$. The OFDM systems such as 3GPP LTE and WiMAX use even higher PAR, typically 8.5 - 9.5dB for improved spectral efficiency but this result in traditional PA designs having even lower efficiency. It is difficult to achieve flexible, high efficiency, highly linear PA designs by means of 'RF only' efficiency enhancement techniques, such as Doherty power amplifier.

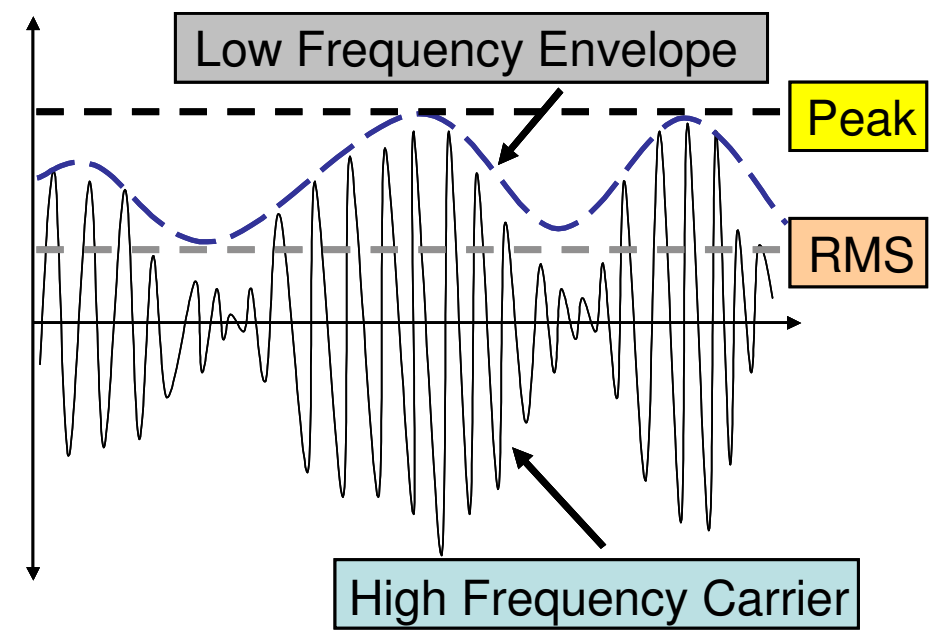

Figure 5.2: Typical time domain definition of peak and average voltages.

A large number of variants are required to cover all the stringent requirements, such as high efficiency and ACPR linearity specifications. It also takes considerable development time to achieve high production yields, and it is difficult to maintain the efficiency as systems evolve to higher PAR values.

\subsubsection{Correlation Between IMR and ACPR}

Two-tone characterization has been traditionally used to characterize nonlinearity of both active and passive RF components, its correlation with the currently implemented complex digitally modulated test signal are highly 
desirable. This is well described in [5] providing compact formulas relating the distortion figures of merit encountered under modern test signal and well established two-tone stimulus. For assessing the nonlinear distortion performance of a FET device operating under a real telecommunication test signal, each combination of two sinusoidal components of the continuous spectrum may be interpreted as two-tone excitation. Further, these multitones can be categorized, for convenience, into multiple two-tone signals with variable carrier spacing up to $3.84 \mathrm{MHz}$, focusing on the Universal Mobile Telecommunication System (UMTS) application as an example. Taking into account the $3.84 \mathrm{MHz}$ adjacent channel bandwidth, which is used to compute adjacent channel power (ACP), typically both third-order and fifth-order mixing products (also higher order) impact ACPR characterization [6], [7].

This is illustrated in Figure 5.3, which considers two cases of two-tone excited nonlinear output spectrum with carrier spacing $\Delta \omega_{1}$ (dotted) and $\Delta \omega_{2}$ (solid) depicting the superposition of IMD3 and IMD5 products onto the W-CDMA spectral mask. Table 5.1 gives the boundary conditions of the carrier spacing which produce the third-order and fifth-order products occurring simultaneously within the sidebands under consideration. As a result the fundamental property of IMD3 observed under two-tone excitation no longer prevails under digitally modulated test signals.

Table 5.1: IMD3 and IMD5 mixing products falling into the adjacent channel bandwidth of $3.84 \mathrm{MHz}$

\begin{tabular}{c|c|c}
\hline Stimuli & $3^{\text {rd }}$ Order (IMD3) & $5^{\text {th }}$ Order (IMD5) \\
\hline 2-Tones & $2.053 \mathrm{MHz} \leq \Delta \mathrm{f} \leq 4.613 \mathrm{MHz}$ & $1.232 \mathrm{MHz} \leq \Delta \mathrm{f} \leq 2.768 \mathrm{MHz}$ \\
\hline
\end{tabular}

Considering the analogy of a multi-tone excited nonlinear output spectrum, each distortion product in the sideband is dominated by its lowest order component, for instance, third-order mixing product for typical $2 \omega_{2}-\omega_{1}$ case. This is true at the small-signal excitation regime; however, when the excitation level is increased, higher order contributions start to dominate over the lower order components. Moreover, each of the sidebands at the output is a result of the vectorial sum of many mixing products of order $\geq 2 n+1$, where $n$ is the 
total number of input tones or carriers [7]. Depending on the phase of individual mixing products occurring at a given frequency, the voltage vectors may interfere in a constructive or destructive manner. Consequently, above certain power levels the IMD3 behaviour under two-tone stimuli can no longer be correlated to the sidebands of multi-tone/digitally modulated signals. Moreover, from the perspective of the nonlinear output spectrum of digitally modulated signals, wherein the input spectral components typically have randomised phase (equivalent to having uncorrelated tones), and integrated distortion power in the sidebands will have different null conditions [7]. In most cases it is a device technology dependent, devices with weak fifth-order nonlinearities may exhibit reasonable correlation between IMR and ACPR characteristics.

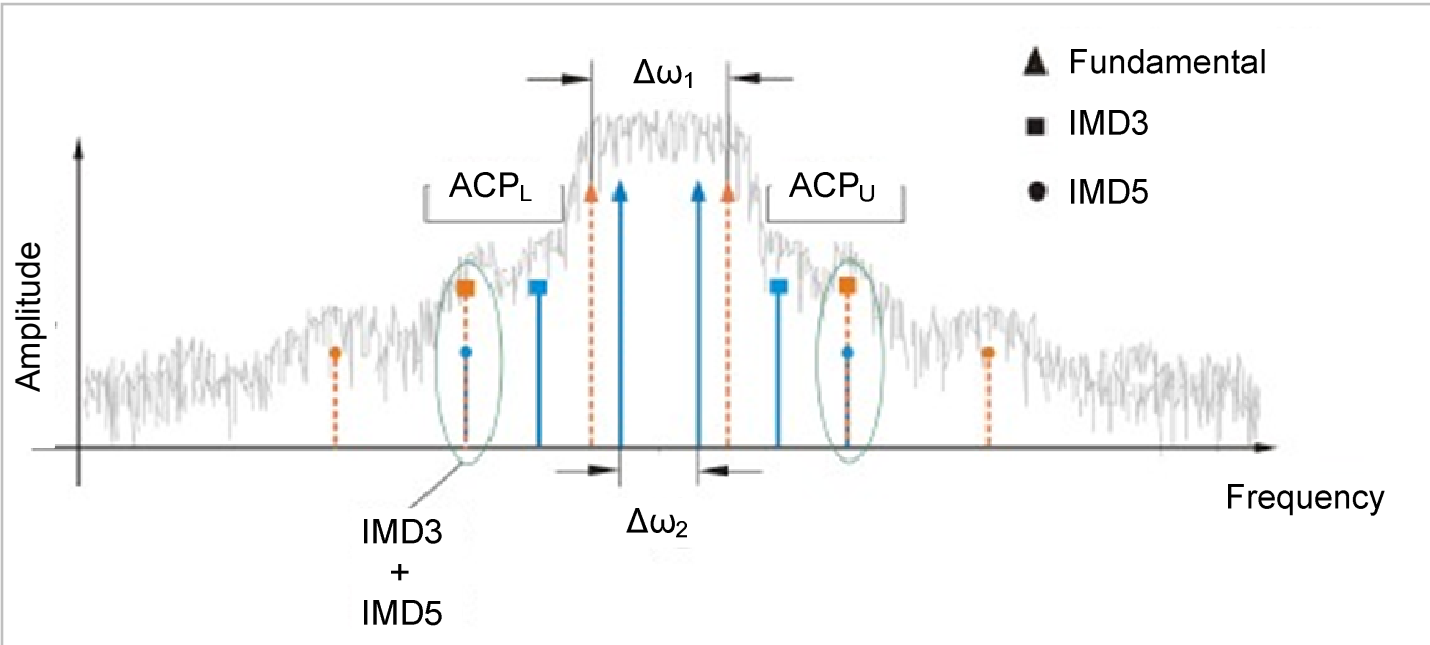

Figure 5.3: Two-tone excited discrete nonlinear output spectrum superimposed onto a WCDMA excited nonlinear output spectrum.

\subsubsection{Multi-Tone Signals}

Multi-Tone signals are useful for measurements in the laboratory where periodic well characterized signals can be readily synthesized to approximate complex modulated radio frequency (RF) signals. Indeed multi-tones can be synthesized using Agilent PSG which provides reliable test signals that have representative statistics to various types of digitally modulated signals such as code division multiple access (CDMA), orthogonal frequency division multiplexing (OFDM) and Long Term Evolution (LTE). The results of the analyses in the preceding chapters were used to reduce bandwidth- 
dependent distortion in simple ways, two-tone stimulus signals were considered sufficient to characterize the nonlinearities in power amplifiers due to the small amplitude variations and narrow-band characteristics of the signals the microwave device were to amplify. In this sense, measurements of the power device for two-tone excitations have provided a good and reliable method for investigating nonlinearities in microwave devices. Moreover, nonlinear characteristics, including intermodulation distortion (IMD), memory effects and gain compression, could be fairly well explained by two-tone experiments [9],10]. However, the growing demand for wireless communication services has brought about signals with wider bandwidth and higher PAR characteristics. Modern wireless signals can no longer be accurately characterized by simple two-tone test signals due to the inherent memory effects in the microwave devices.

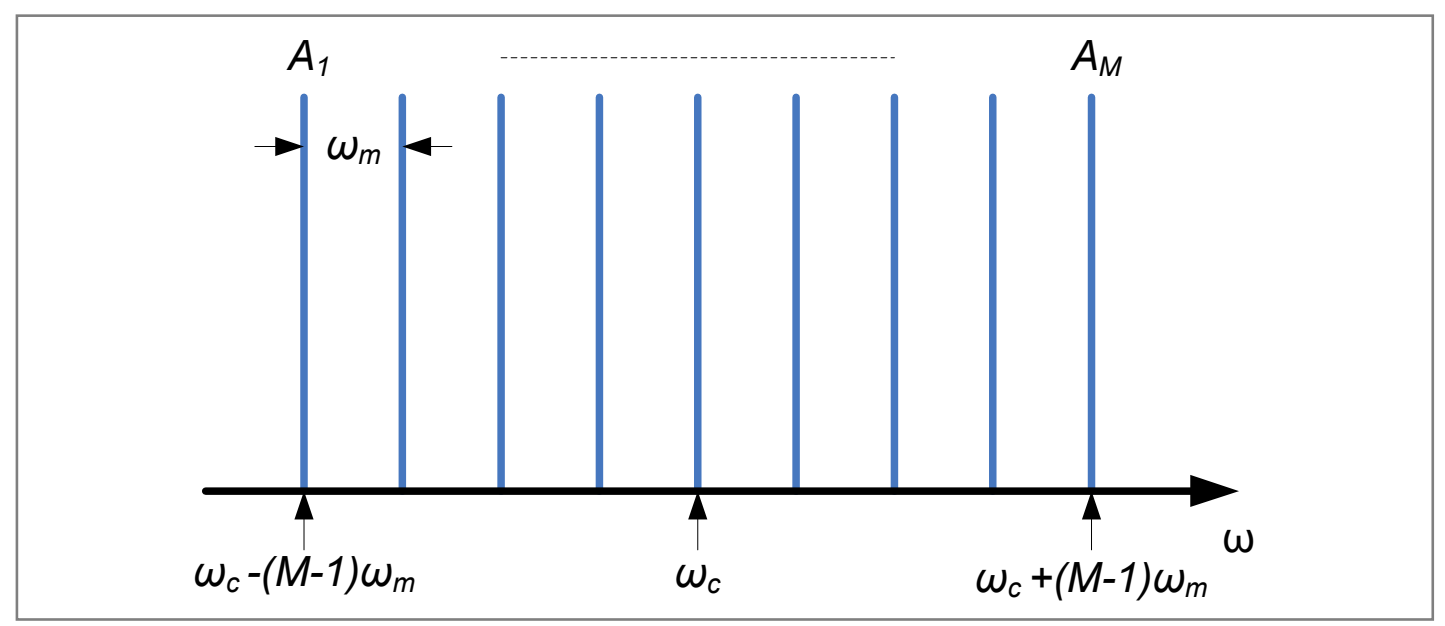

Figure 5.4: Multi-tone stimulus signal at centre frequency $\omega_{c}$ with modulation frequency $\omega_{\mathrm{m}}$.

Today digitally modulated signals are wide band and contain significant amplitude variation as a byproduct of implementing high-spectral-efficiency modulation schemes in order to maximize the amount of data transmitted in a fixed signal bandwidth. The suitable signals in laboratory experiments should have approximately similar statistics to characterise the inherent nonlinearity of microwave devices, generally equally spaced multi-tone signal with $\mathrm{M}$ tones, centred around fundamental frequency $\left(\omega_{c}\right)$, with modulation frequency $\left(\omega_{\mathrm{m}}\right)$ as shown in Figure 5.4 has been used. A complex envelope of the multitone signal depicted in Figure 5.4 can be described as: 


$$
x_{1}(t)=\sum_{i=1}^{M} c_{i} e^{j(2 i-M-1) \omega_{m} t}
$$

Where, $c_{i}=A_{i} e^{j \theta_{i}}$ and $\omega_{\mathrm{m}}\left(2 \pi f_{m}\right)$ is the modulation frequency of the multi-tone signal. The Fourier transform of $\mathrm{x}_{1}(\mathrm{t})$ is:

$$
X_{1}(\omega)=\sum_{i=1}^{M} \frac{c_{i}}{2} \delta\left(\omega-(2 i-M-1) \omega_{m}\right)
$$

A multi-tone signal has a much different peak-to-average ratio (PAR) and signal statistics depending on the phase of each term even though the magnitude of each term is same [Appendix-F]. For example, the characteristics of a 9-tone complex envelope signal with the same magnitudes for each tone are investigated. Two cases are considered in Table 5.2. Case 1 is when the phase of each term is fixed and gives the maximum PAR. Case 2 is when the terms have a random phase difference and give the reduced PAR. The phase information and PAR are shown in Table 5.2 for two distinct cases of nine tone signal.

\begin{tabular}{|c|c|c|}
\hline Case & Phase Schemes & PAR \\
\hline 1 & 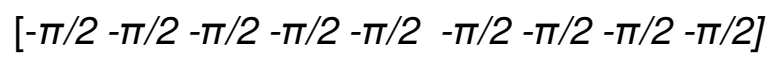 & 12.54 \\
\hline 2 & {$\left[\begin{array}{llllllll}0 & 0 & 1.365 & 0.716 & 5.436 & 2.959 & 4.751 & 1.625\end{array}\right]$} & 6.86 \\
\hline
\end{tabular}

Table 5.2: Phase and peak to average ratio for a 9-tone signal

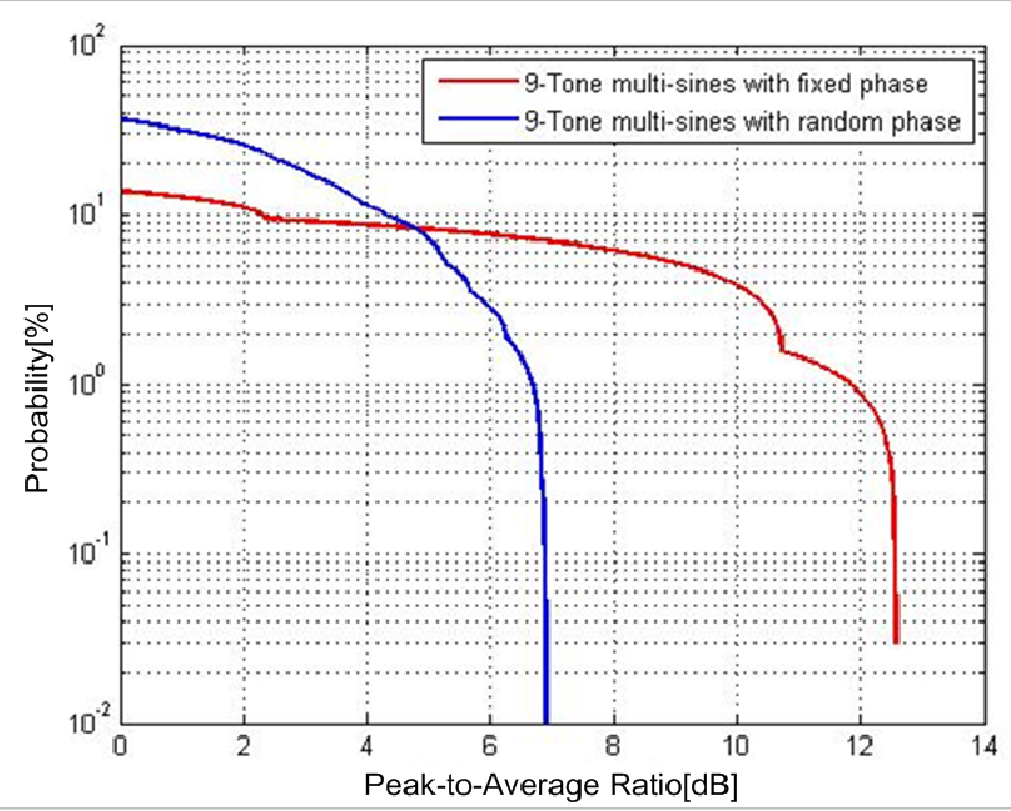

Figure 5.5: Nine-tone signal complementary cumulative distribution function (CCDF) function for different phase schemes. 
The complementary cumulative distribution functions (CCDF) for these cases are plotted in Figure 5.5. The minimum peak to average ratio is about $6.86 \mathrm{~dB}$ and maximum crest factor is about $12.54 \mathrm{~dB}$. The signal characteristics show a significant difference depending on the phases of the nine tone signal. For the simple case when all the magnitudes and phases of the multi-tones have the same values, the CCDF functions of multi-tone signals are plotted in Figure 5.6. In this case the crest factor is $10 \log (M)$. Thus, a nonlinear device can be characterized using a multi-tone signal for $2(M-1) \omega_{m}$ bandwidth and up to $10 \log (M)$ PAR power ranges.

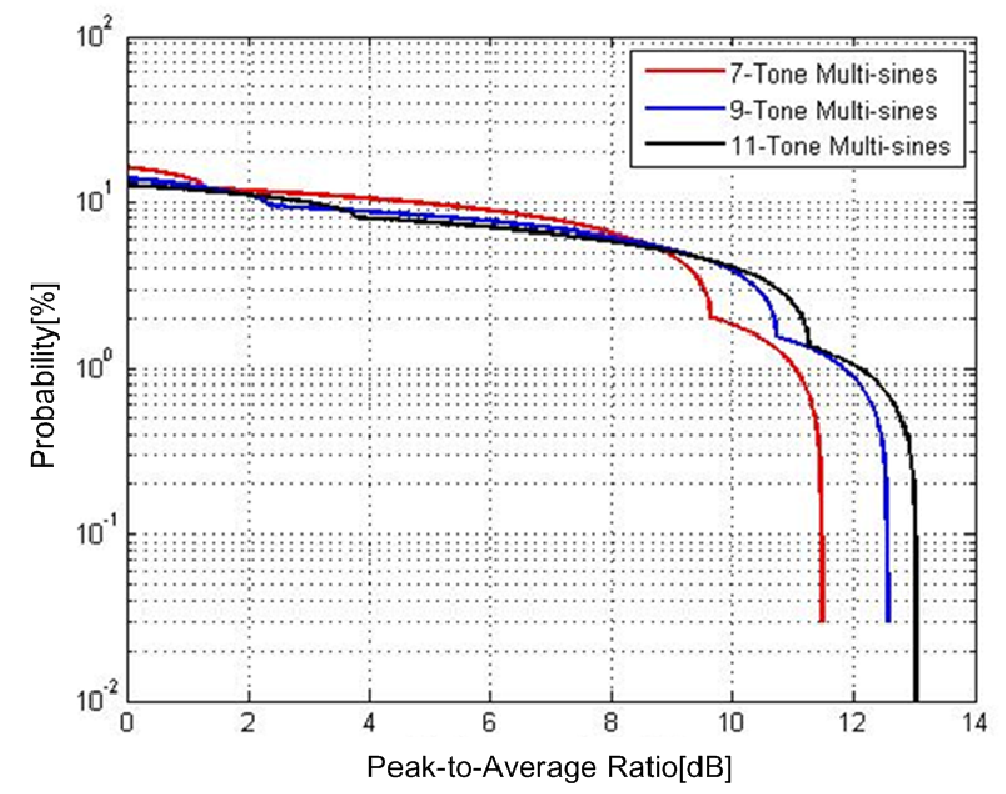

Figure 5.6: Multi-tone signal peak-to-average ratio for various numbers of tones, when the phases are fixed.

\subsection{Multi-Tone Excitation and Characterisation}

The multi-tone characterisation technique is a robust technique for characterising the device nonlinearity when excited with more than two tones, which has gained importance in recent years as a substitute for the practical communication signals. The measured time domain input voltage waveform of a nine tone signal with equal amplitude and fixed phases is depicted in Figure 5.7 along with the fundamental input and output spectral components. In the output spectrum it can be seen that the magnitude of the IMD products diminish with the order, exhibiting the spectral regrowth behaviour. It also highlights the important details of the spectral mask on an input-output power 
spectral density. Interpretation of the microwave device's nonlinearity in terms of the time domain waveforms of a multi-tone signal is rather easy and simple.

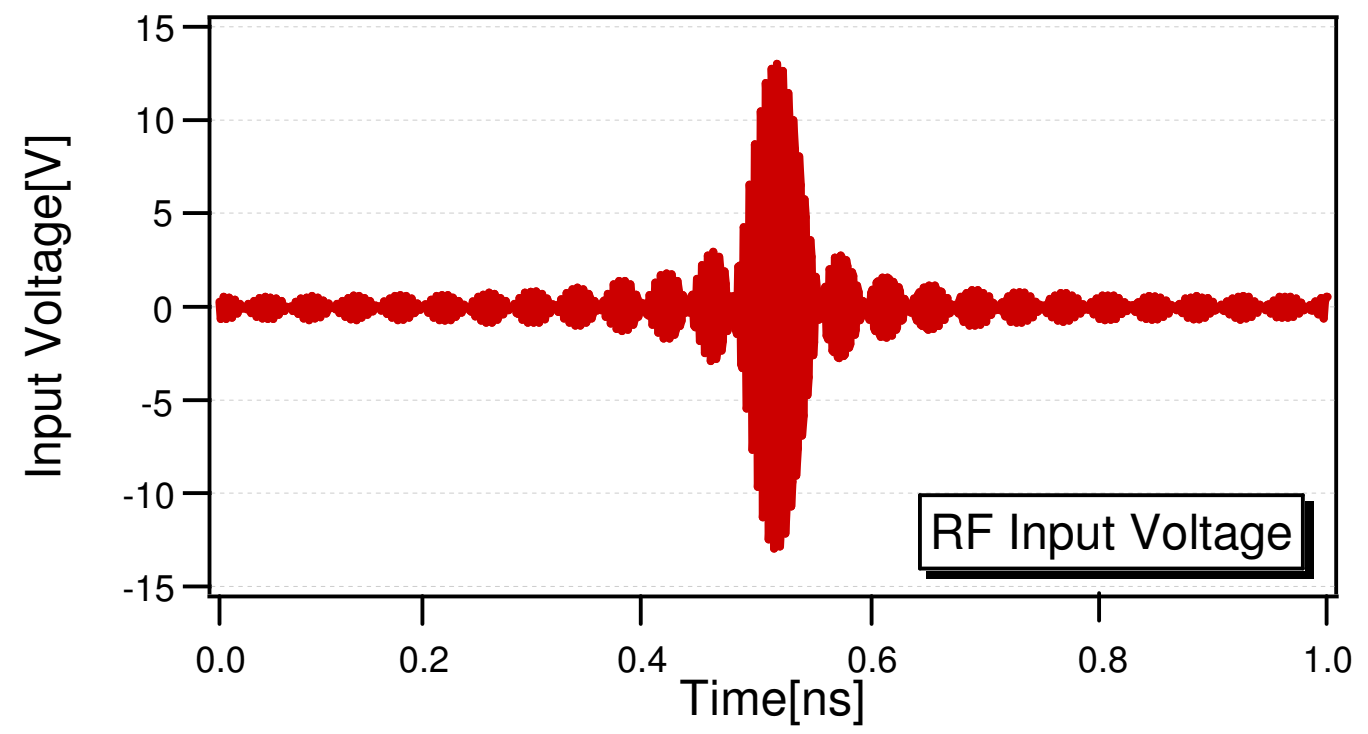

(a)

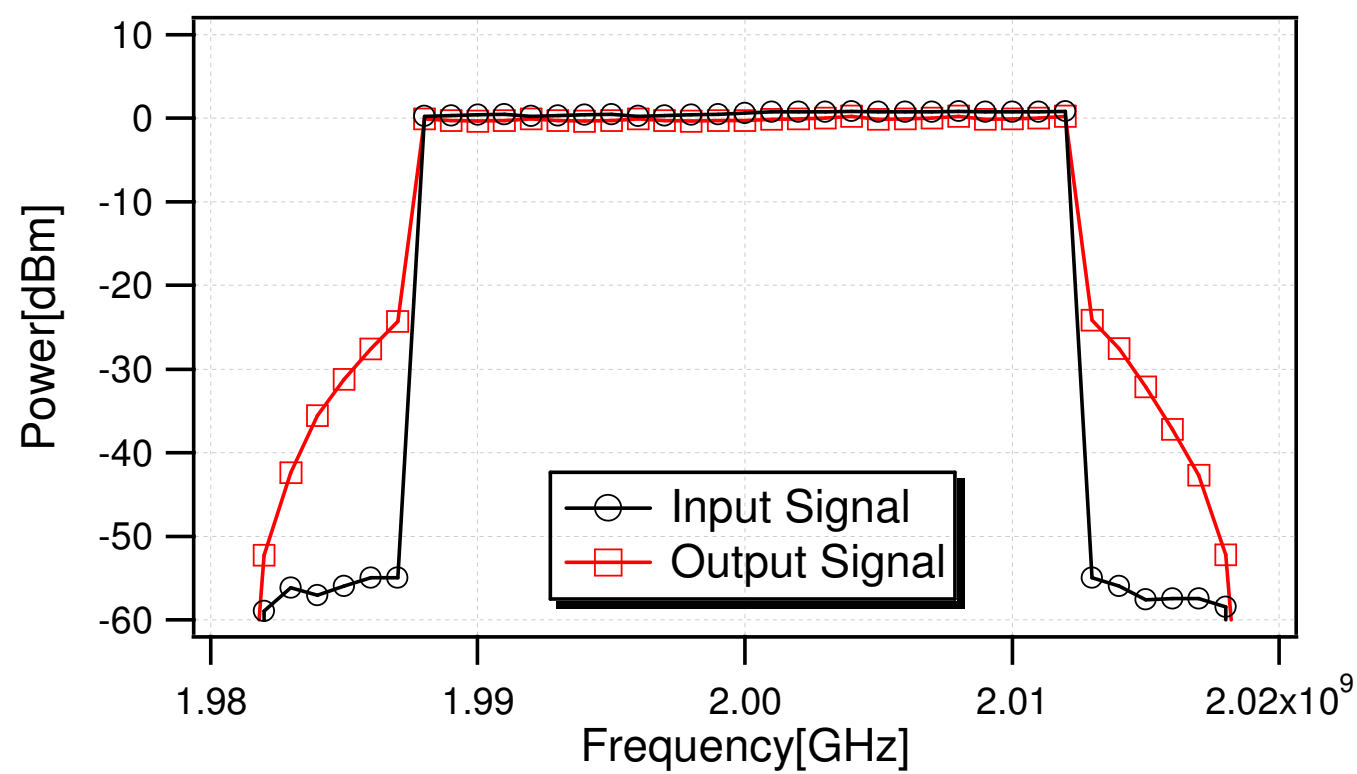

(b)

Figure 5.7: Measured (a) 25-tones time domain RF input voltage waveform deriving a 10W GaN HEMT device into nonlinear operation, biased at deep class-AB and (b) measured fundamental input and output spectrum using real time oscilloscope.

Nevertheless, device linearity under multi-tone test excitation is characterised by measuring the degree of output spectrum regeneration across the lower and upper sidebands, computed by using equation (5.9). In all the cellular 
systems it is necessary for the system designer to impose specifications on the maximum amount of distortion that can be introduced in adjacent channels.

$$
A C P R=\frac{\text { Total power of in }- \text { band } 25-\text { tones }}{\text { Total power of adjacent band } 25-\text { tones }} \quad[\mathrm{dBm}]
$$

However, since it is impossible to practically design for absolute distortion levels, relative levels of power in the adjacent channel to power radiated in the main channel, is made obligatory, defining the most commonly known figure of merit called adjacent channel power ratio (ACPR).

\subsubsection{Multi-Tone Investigations of Memory Effects}

Under multi-tone stimulus, based on the complex vector error-corrected travelling waves measured using the enhanced modulated measurement documented in details in Chapter 2, the memory effect behaviour of a $10 \mathrm{~W}$ GaN HEMT was further investigated under class-AB operation in a custom $50 \Omega$ fixture. The calibrated system was thus utilised to acquire the modulated RF signals in multiple measurements. The stitching of measured data from multiple measurements is therefore required to obtain the amplitude and phase of the various tones of the multi-harmonic wide-band periodically modulated signals. The triggering frequency to trigger the DSA8000 was set alternatively to the modulation frequencies used in the nine-tones broadband input stimulus. This trigger then assures that the phase relationship between each tones remains the same in successive measurements. As mentioned in the preceding chapters, the excitation used for the measurements plays an important role to characterize microwave power transistors for modern wireless communication systems including WiMAX and LTE. Therefore, a 9tone multi-sine signal was considered as an approximation of realistic complex digitally modulated signals. A pre-driver amplifier was used after the signal generator to keep the input stimulus clean. An isolator was also used before the input port which effectively reduced the second harmonic spectra getting at the input of device. The measurements were conducted to experimentally validate and confirm that the same optimum $\left(\Gamma_{\mathrm{IF}}=1 \angle 180^{\circ}\right)$ 
exists for reduction of memory effects under nine tone stimulus as was the case for two-tone modulated stimulus investigations documented in Chapter 3 for the same device.

The device delivered $39.5 \mathrm{dBm}$ peak envelope power (PEP) when driven approximately $1.5 \mathrm{~dB}$ into compression and active IF load-pull was used to present the constant baseband impedance to most significant components IF1 and IF2, the higher baseband components IF3 and IF4. Figure 5.8 depicts the contours of $A C P R_{L}$ when a measurement grid was swept to find the optimum for reduction of memory effects, as it is reported in [11] that the linearizability of a memory-less device is much better than the devices with memory.

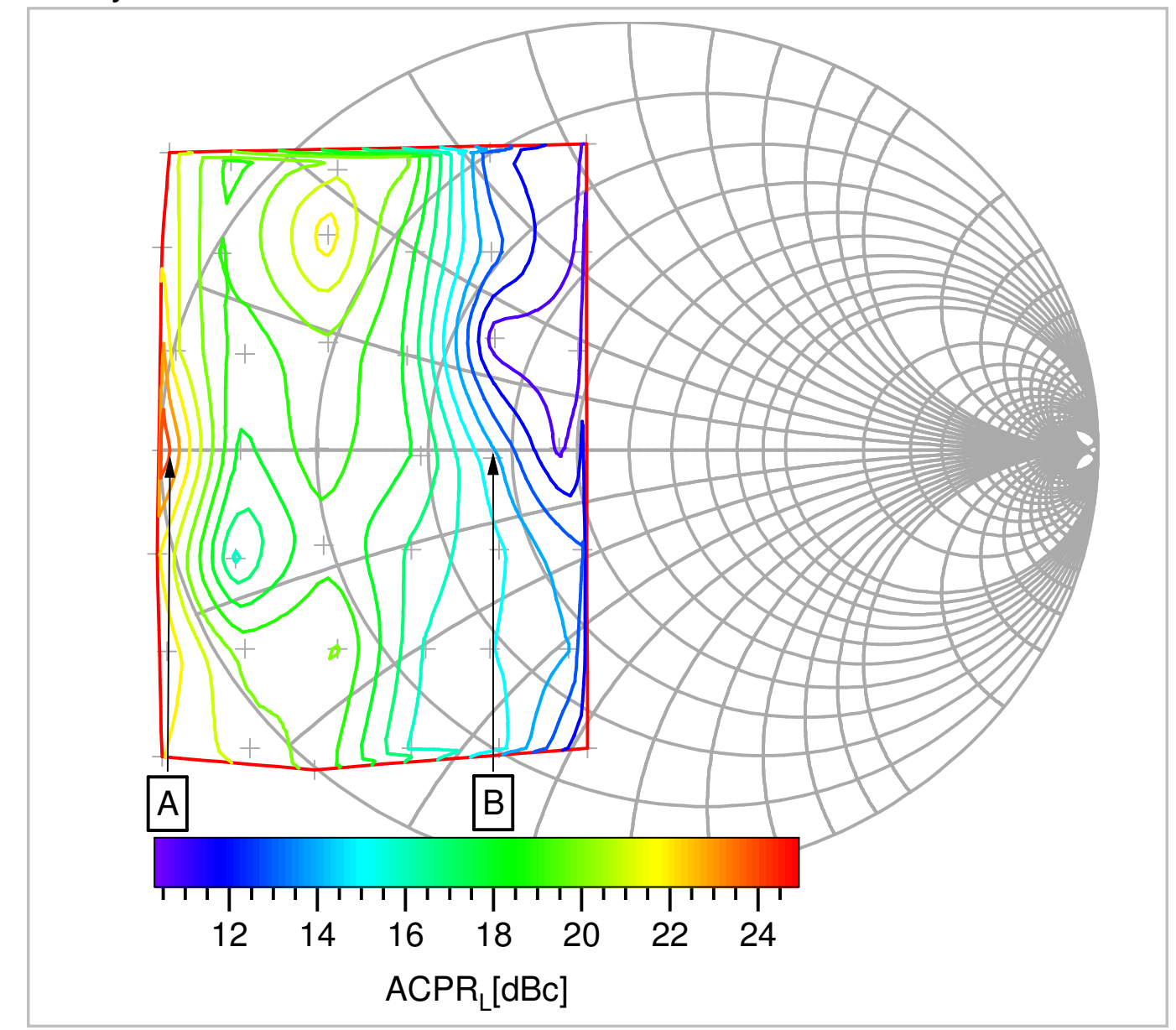

Figure 5.8: Baseband load-pull characterization of 10W GaN HEMT under 9tone stimulus for optimum $A C P R_{L}$ as a function of baseband impedance.

The significant observation to note is that there are pronounced ACPR variations and asymmetry around the measurement grid. These 
measurements suggested that the optimum baseband load for minimum asymmetries is indeed located at point- $A$ and then terminating the baseband components at an experimentally determined optimum point- $A$, a reduction of approximately $10 \mathrm{~dB}$ in ACPR was observed in comparison with point-B and it highlights the strong dependence of ACPR on the baseband impedance termination.

The output spectra for two cases when a different baseband termination is maintained for the baseband components are depicted in Figure 5.9. Terminating the baseband components at point-B caused a ripple to appear on the DC drain voltage present at the device output plane, which effectively resulted in additional modulation of the $\mathrm{RF}$ signal. This, then, leads to the change in the ACPR levels and it can be seen that significant memory effects exist with more than 6dB ACPR asymmetry between upper and lower ACPR products. For the case when the optimum impedance is maintained to the baseband components at a modulation frequency of $1 \mathrm{MHz}$ there are no baseband electrical memory effects, so the spectral regrowth shows no asymmetry for the optimum baseband impedance termination.

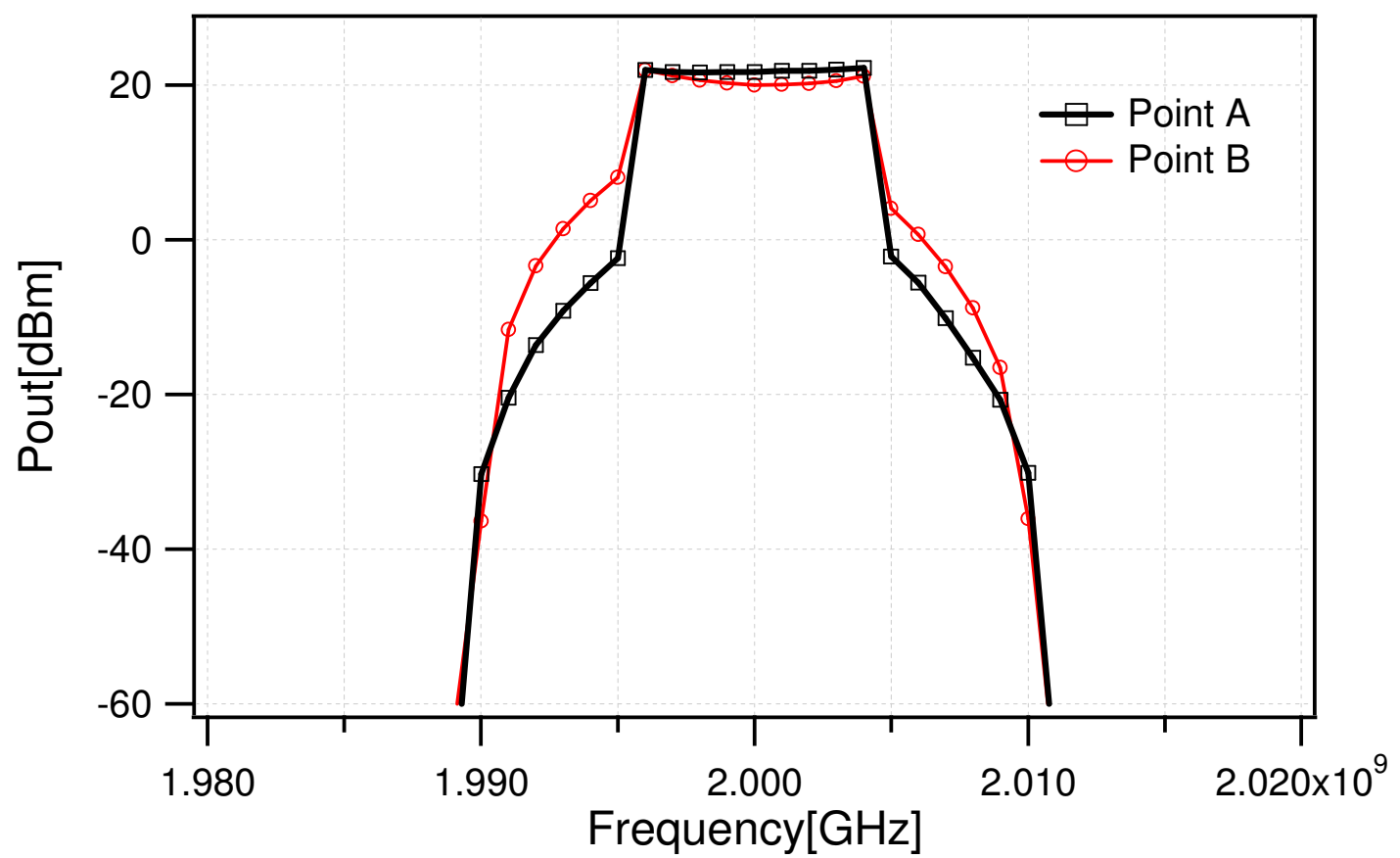

Figure 5.9: Measured output spectrum at modulation frequency of $1 \mathrm{MHz}$, when different impedances were provided to baseband components with nominal $50 \Omega$ RF termination. 
The measured output RF voltage envelopes obtained for the two baseband impedance conditions, are shown in Figure 5.10. In the case of the baseband impedance at point- $B$, an asymmetrical envelope is clearly observed that determines which ACPR product will have the greater magnitude. Whilst terminating the baseband components at point- $A$ which has been demonstrated to be the optimum load termination, an undistorted output envelope is produced.

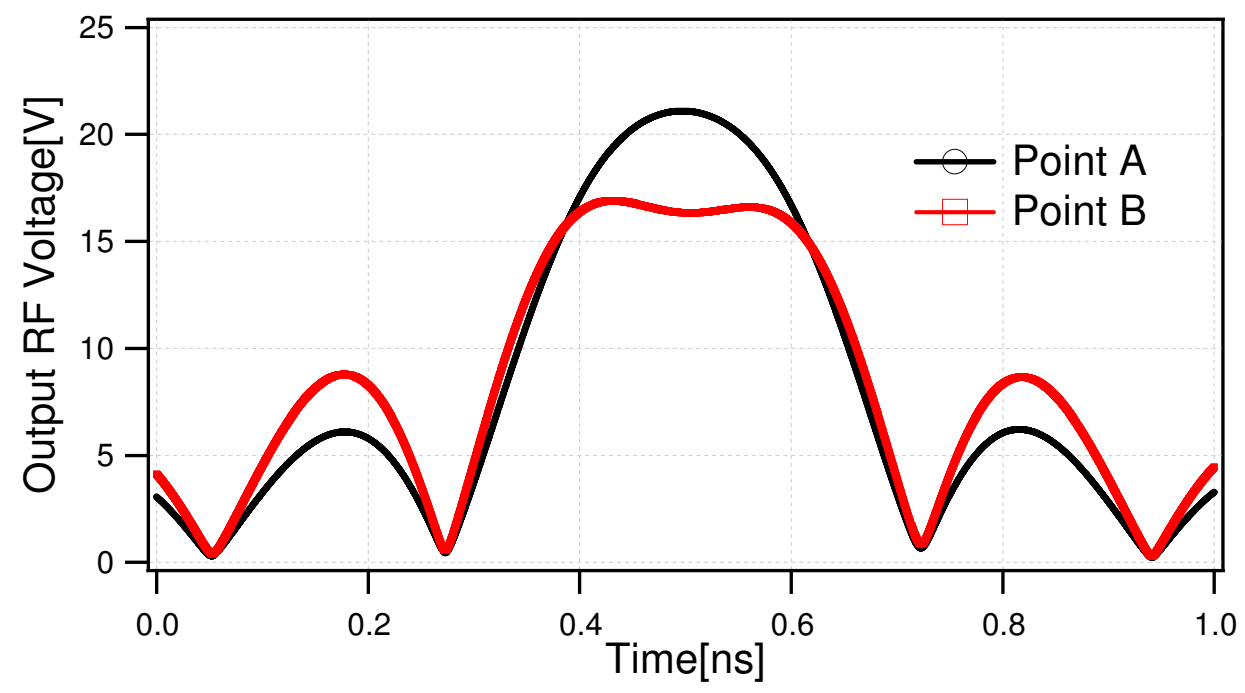

Figure 5.10: Measured RF output voltage envelopes for different baseband impedance environment at a modulation frequency of $2 \mathrm{MHz}$.

The broadband baseband short circuit $\left(\Gamma_{\mathrm{IF}}=1 \angle 180^{\circ}\right)$ impedance termination was utilized for modulation frequencies ranging from $0.5 \mathrm{MHz}$ to $7 \mathrm{MHz}$, in order to achieve the symmetrical ACPR response. The magnitude of the IF reflection coefficient could not be brought precisely to a short after $5 \mathrm{MHz}$ because of the bandwidth limitation of the baseband load-pull PA, as can be seen from the scattering of baseband components after modulation frequency of $5 \mathrm{MHz}$ in Figure 5.11.

The fundamental output power of nine tones remains constant and independent of the modulation frequency. With regards to the ACPR distortion $\mathrm{ACPR}_{\mathrm{L}}$ and $\mathrm{ACPR}_{\mathrm{H}}$ depicted in Figure 5.12, it is clear that the symmetry level of ACPR is degraded at higher modulation frequencies after $5 \mathrm{MHz}$ which is due to impedance variations in higher baseband components (IF3 and IF4). It is important to note that the observed variations in $A C P R_{L}$ and $A C P R_{H}$ 
magnitude observed below $1 \mathrm{MHz}$ are not related to variations in baseband impedance and attributable to other sources of memory.

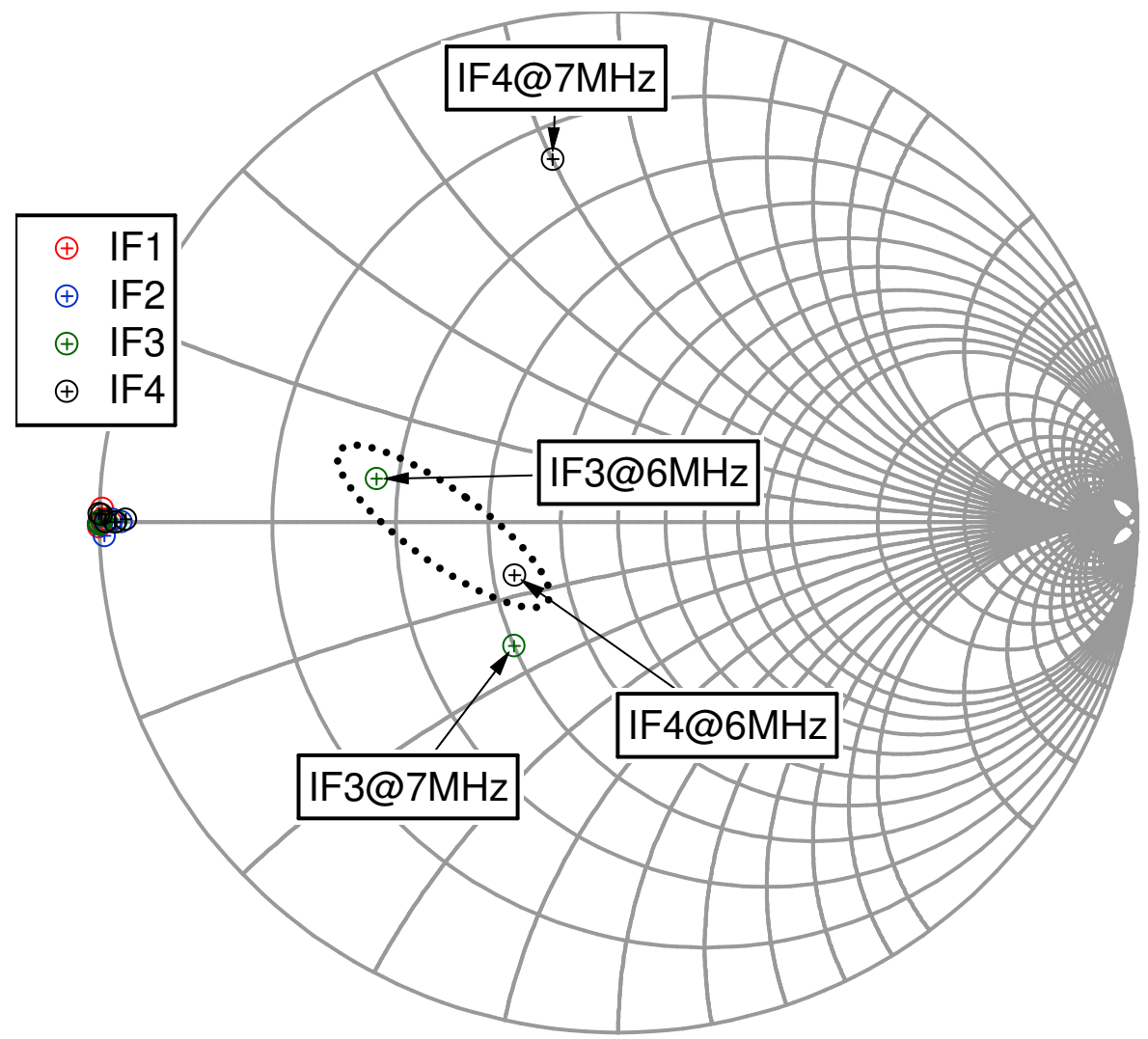

Figure 5.11: Measured IF1, IF2, IF3 and IF4 for modulation frequencies ranging from $0.5 \mathrm{MHz}$ to $7 \mathrm{MHz}$.

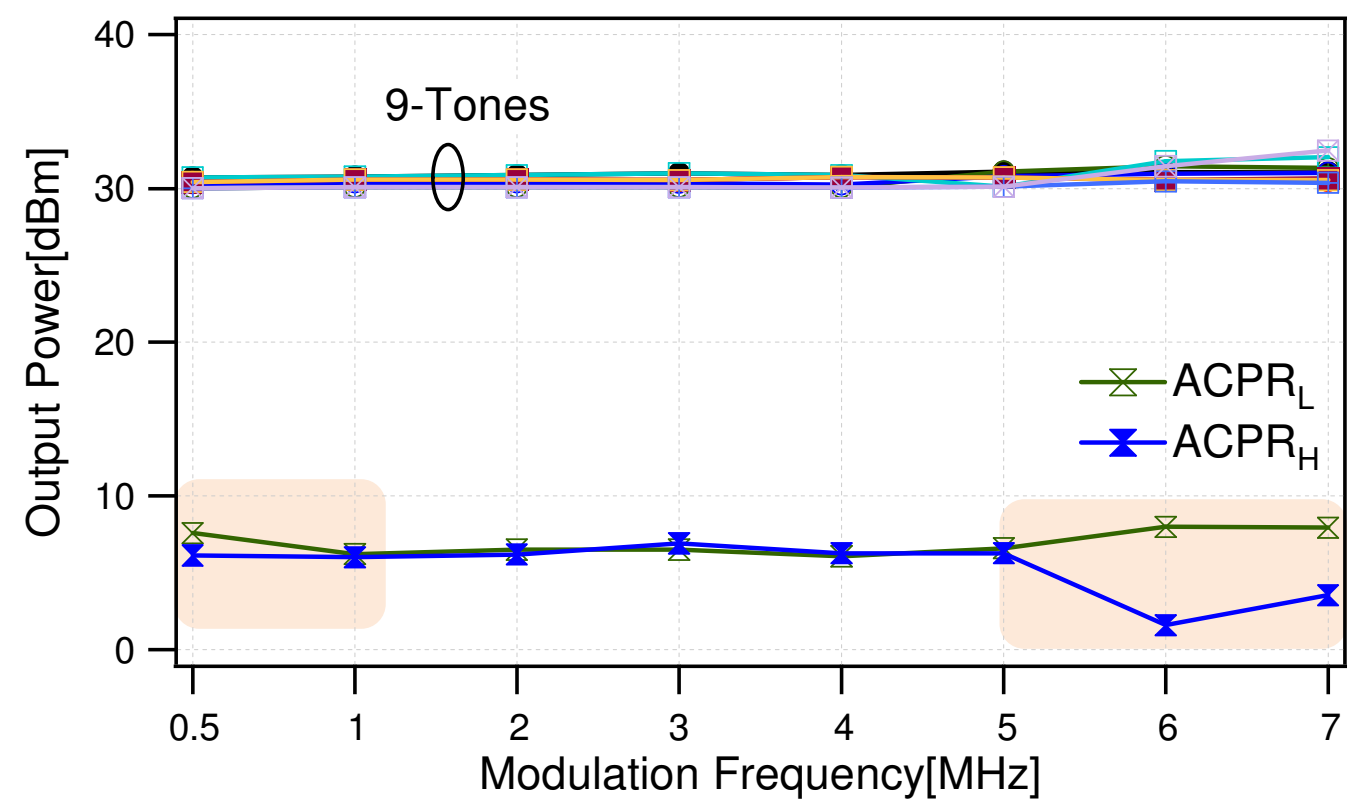

Figure 5.12: Measured fundamental nine-tones and ACPR power for different modulation frequencies. 
The dynamic transfer characteristics depicted in Figure 5.13 correspond to the case when short circuit impedance was presented to baseband components, thus, there is an absence of hysteresis in the dynamic transfer characteristics which emphasize the absence of baseband electrical memory effects. The analysis of measured results with respect to the dynamic load line shows that the baseband impedance influences the RF dynamic load line interaction with the knee region. This is due to the output voltage generated by the IF component which causes the measured output current and voltage dynamic load lines to interact with the knee region. Consequently, this causes the looping in the IF load-line which results in an asymmetrical knee interaction of $\mathrm{RF}$ load-line with the knee region. The asymmetrical knee interaction of RF load-line with the knee region manifests itself as a hysteresis on the dynamic transfer characteristics. In the case when short circuit baseband impedance is presented to the device, it minimizes the knee interaction with transistor's I-V characteristic, producing a symmetrical knee interaction.

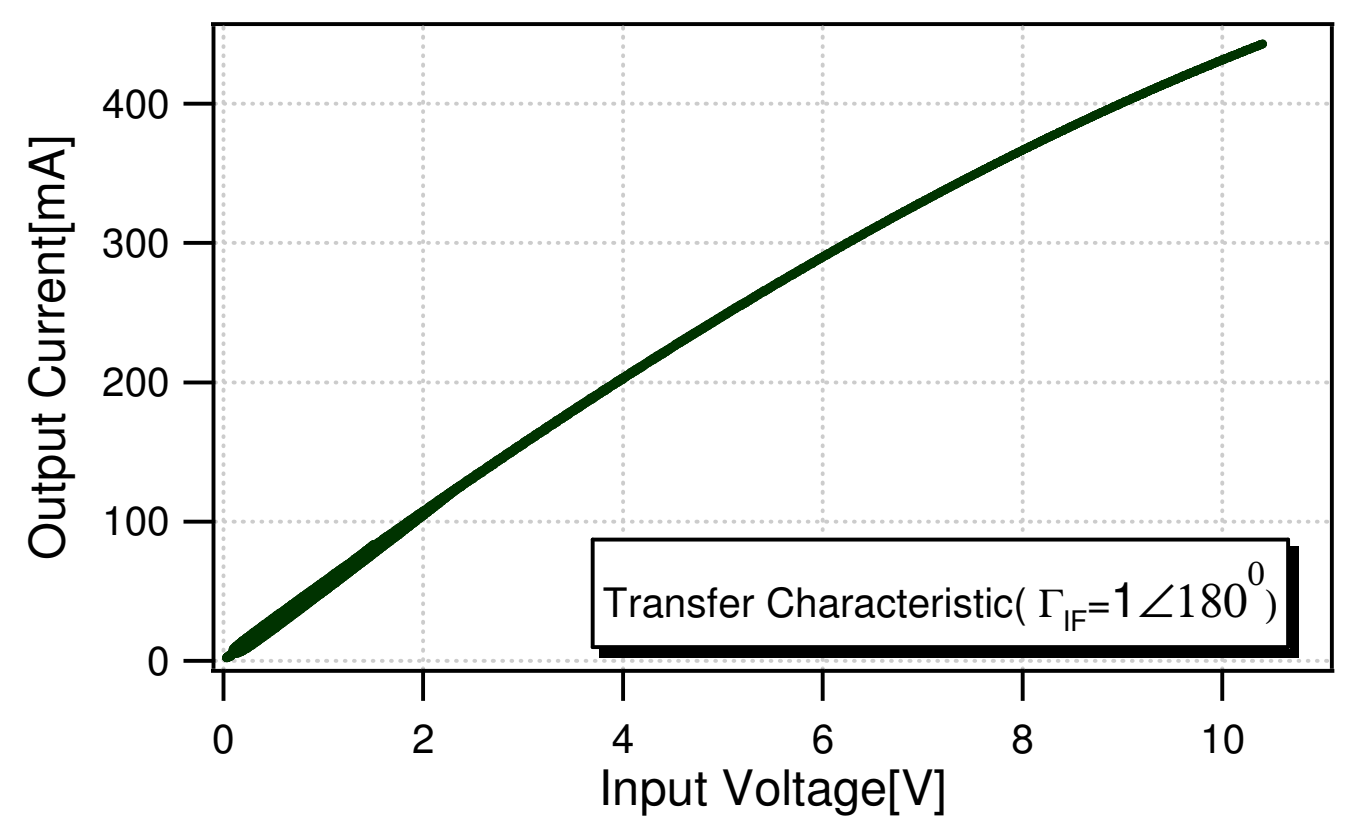

Figure 5.13: Measured dynamic transfer characteristic for nine-tone stimulus when short circuit impedance was presented to all the baseband components for a modulation frequency of $1 \mathrm{MHz}$.

Figure 5.14 shows the dynamic RF and IF load-lines for short circuit baseband impedance. Terminating the baseband components with short 
circuit impedance resulted in static IF voltage, consequently, no looping is observed in the dynamic transfer characteristics depicted in Figure 5.12.

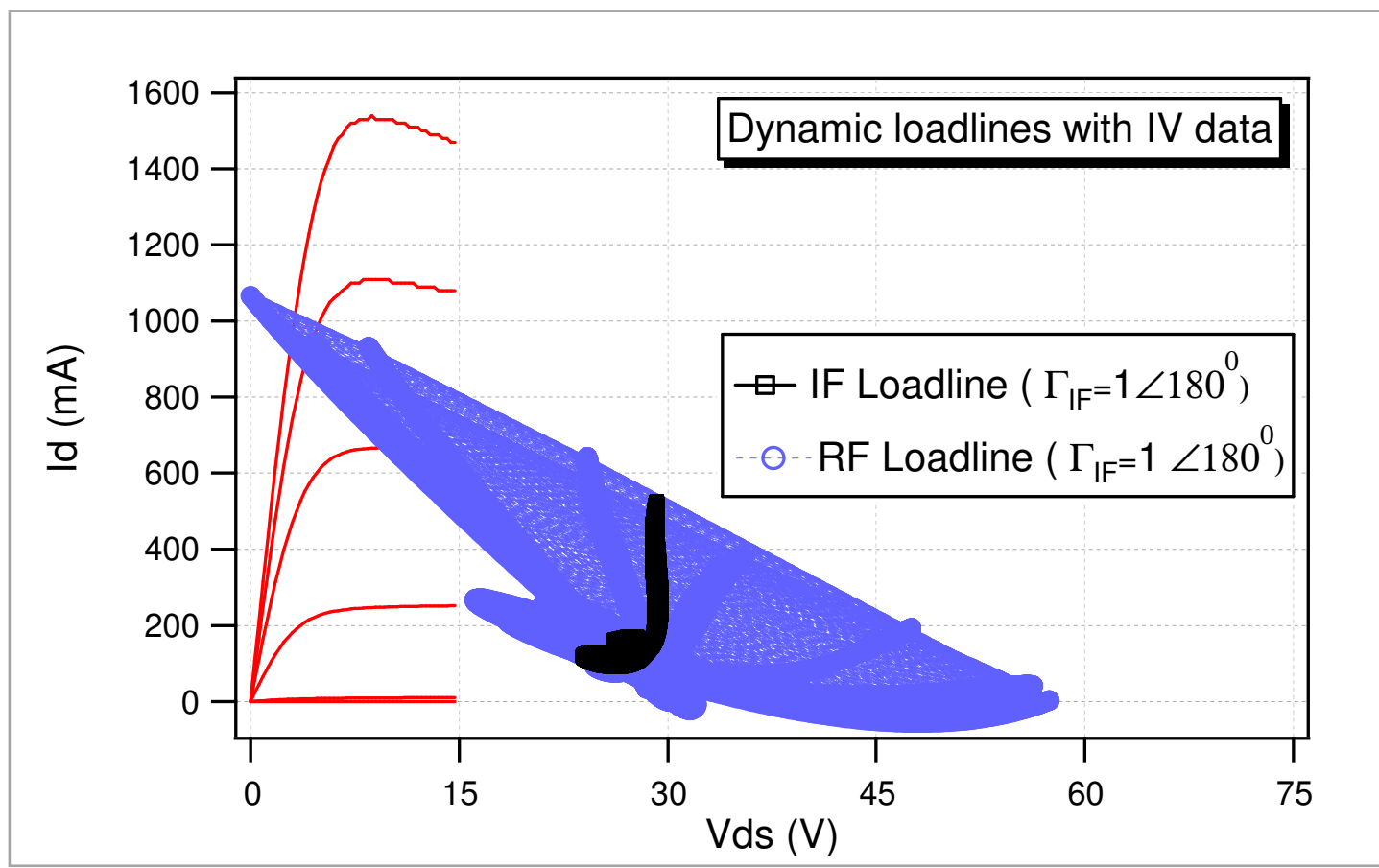

Figure 5.14: The measured dynamic RF and IF load-lines for baseband reflection coefficients $\left(\Gamma_{\mathrm{IF}}=1 \angle 180^{\circ}\right)$

\subsection{Linearity Enhancements under Complex Excitation}

This section confirms and demonstrates that substantial linearity improvement can be achieved using this approach, when using a complex 9tone excitation, as has already been shown for the two-tone excitation in chapter-4. As expected, for the GaN device considered, and for this degree of compression, the measured linearity significantly improves when specific negative baseband impedances are presented. All the measurements presented in this section are for a CREE CGH40010 discrete 10W GaN HEMT device, characterized at the centre frequency of $2 \mathrm{GHz}$, within a custom built $50 \Omega$ test fixture. This fixture was calibrated over a relatively wide $50 \mathrm{MHz}$ baseband bandwidth, and over $100 \mathrm{MHz}$ RF bandwidths centred around fundamental, second and third harmonics, with both baseband and RF calibrated reference planes established at the device's package plane. This allowed the accurate and absolute measurement of all the significant voltage and current spectra generated at the input and output of the device. 
Modulated measurements under nine-tone stimulus with PAR of $9.54 \mathrm{~dB}$ were performed using a $1 \mathrm{MHz}$ modulation frequency, with the device class- $\mathrm{AB}$ biased. Respective drain and gate bias voltages of $28 \mathrm{~V}$ and $-2.05 \mathrm{~V}$ resulted in a quiescent drain current of $250 \mathrm{~mA}$ ( $\left.\mathrm{I}_{\mathrm{DSQ}} \cong 5 \% \mathrm{I}_{\mathrm{DSS}}\right)$. The device was driven into approximately $1.5 \mathrm{~dB}$ of compression whilst delivering a peak envelope power (PEP) of $41 \mathrm{dBm}$ with fundamental and harmonic components terminated into a nominal impedance of $50 \Omega$, at both input and the output. It should be noted that the braodband $50 \Omega$ load was used in this case to simplify the required measurement architecture. Although this is a non-optimal load condition, it was considered sufficiently representative for the linearity analysis presented here. Active IF load-pull was then used to synthesize a range of IF reflection coefficients in order to quantify the effects of the low frequency, broadband baseband load impedance environment on the non-linear behaviour of the DUT.

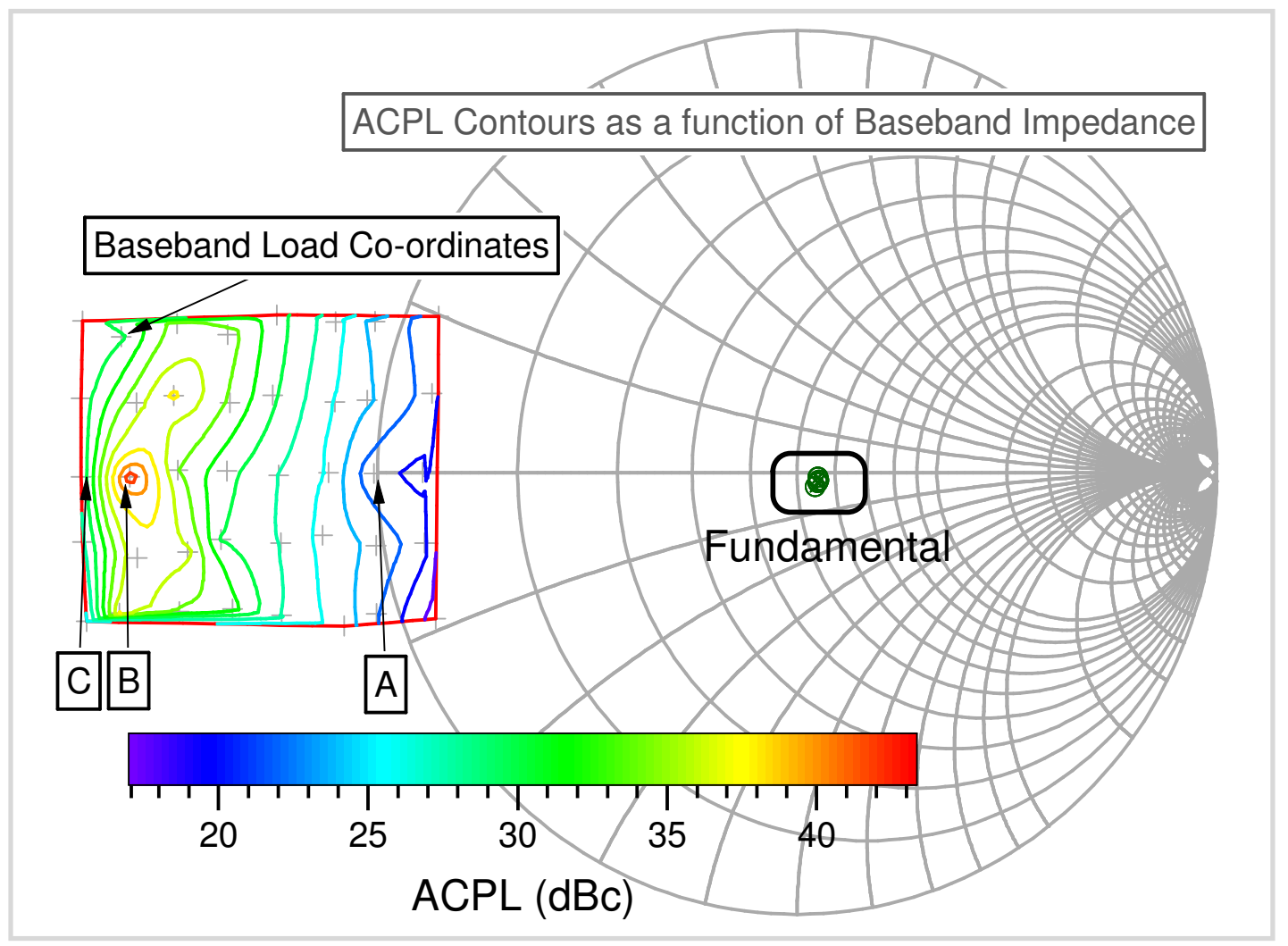

Figure 5.15: Measured lower adjacent channel power linearity contours as a function of IF reflection coefficient $\left(\Gamma_{\mathrm{L}}\right)$.

The optimum IF impedance for the best linearity as has been identified in [12],[13], lies outside the smith chart and Figure 5.15 illustrates a 
measurement where the broadband IF impedance was held constant for all baseband tones and swept over a measurement grid, including the short circuit condition, and extending some way outside the Smith chart. For each of the measurement points, the bias and drive level was maintained constant. The optimum loads for $A C P R_{L}$ and $A_{C P R_{H}}$ lie on the real axis and are almost identical in terms of their position and the degree of linearization they offer. The contours of $A C P R_{L}$ are plotted in Figure 5.15 and show that at point $B$, the performance is found to be $-43 \mathrm{dBc}$, which is approximately a $25 \mathrm{~dB}$ improvement over the classical short circuit case (point A). Figure 5.16 shows the baseband voltage waveforms that resulted when the baseband impedances for point $A, B$ and $C$ were presented to the device. When short circuit impedance was provided to all the baseband components a static DC supply was resulted with negligible 'ripple' that remained.

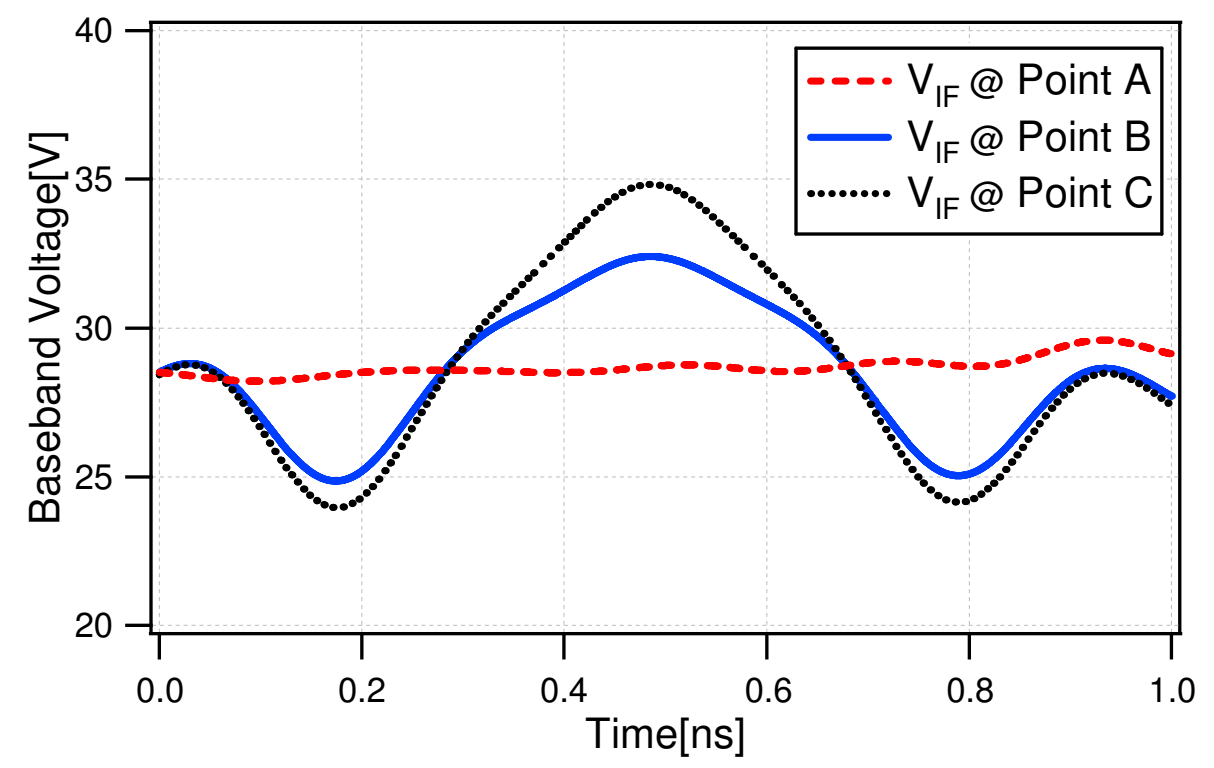

Figure 5.16: Baseband voltages that resulted for the three cases of baseband (IF) load impedances identified at point $A, B$ and $C$.

From the output power spectrum depicted in Figure 5.17, it can be clearly seen that terminating all the significant IF components at point-B drastically improves the device's linearity over a $10 \mathrm{MHz}$ bandwidth, to a level of approximately $43 \mathrm{dBc}$. Thus, it can also be seen that spectral regrowth for multi-tone signals depends on all the significant baseband components, in that it affects the whole spectral regrowth curve, from close in to the extremity. This confirms that this method of baseband linearization is not only applicable 
to two-tone excitations, but can also apply to far more complex, multi-sine signals with high degrees of PAR.

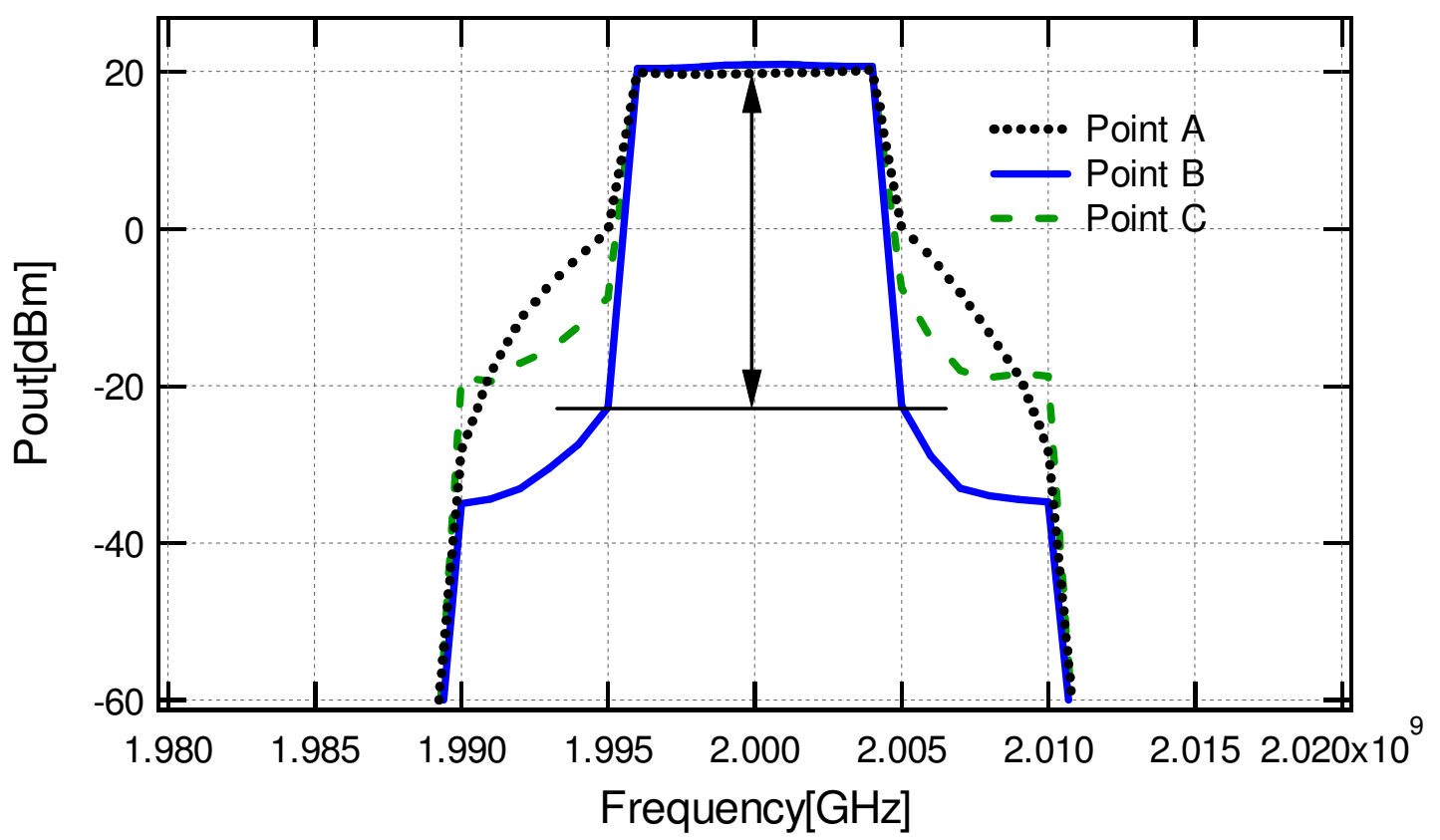

Figure 5.17: Measured output power spectrum as a function of IF reflection coefficient $\left(\Gamma_{\mathrm{L}}\right)$ when device was subjected to 9-tone stimulus at $2 \mathrm{GHz}$ carrier frequency with $1 \mathrm{MHz}$ modulation frequency.

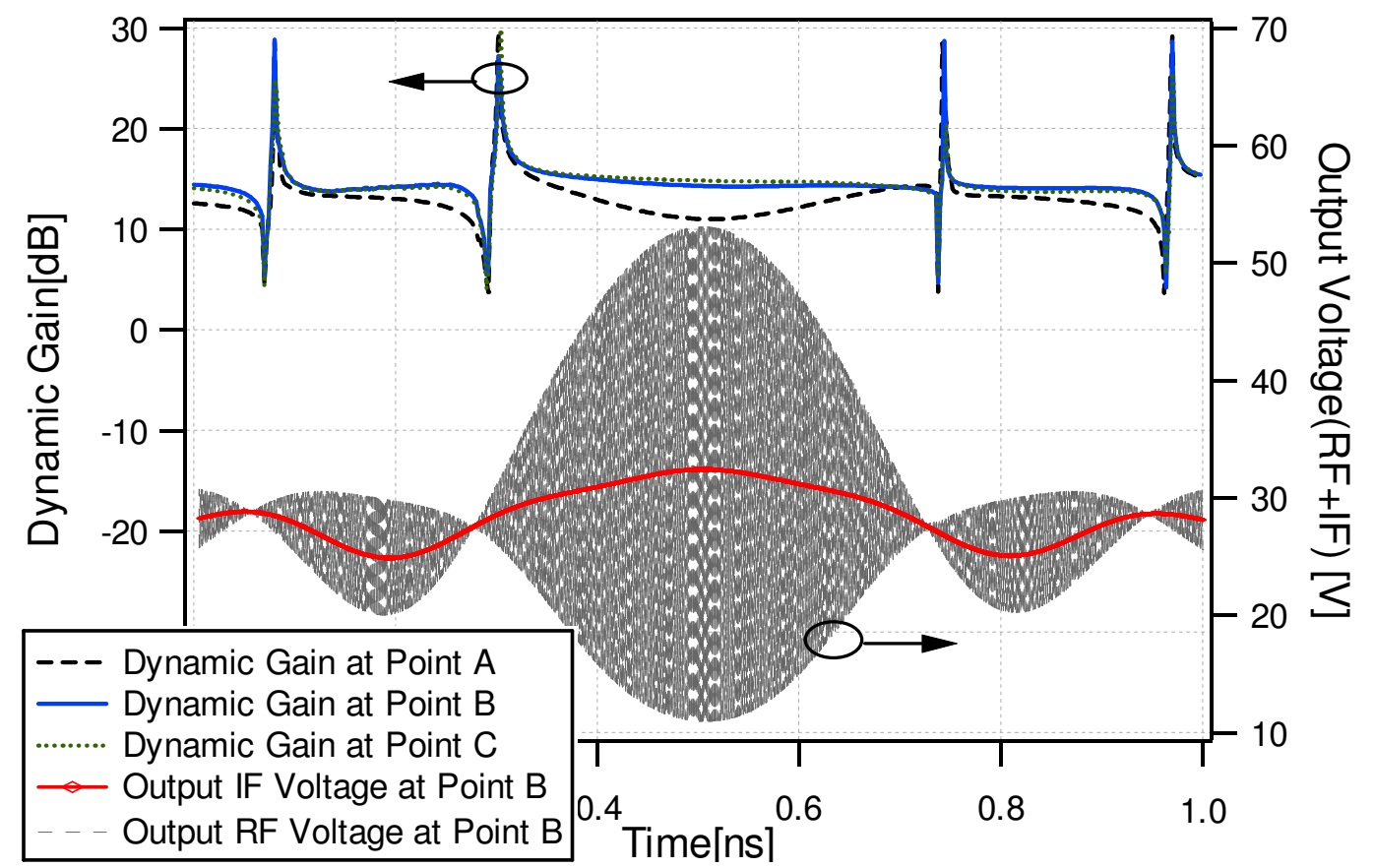

Figure 5.18: Envelope domain [14] representation of gain as a function of IF reflection coefficient $\left(\Gamma_{\mathrm{L}}\right)$ at the indentified points, alongside RF and baseband voltage waveforms. 
Figure 5.18 shows the dynamic changes in gain over the modulation cycle for the three cases of baseband load reflection co-efficient, identified by points $A$, $B$ and $C$ in Figure 5.15. It is clear from this graph that the negative IF impedance presented at point-B results in an increased and almost constant gain and improved linearity. The effect of the baseband voltage at point- $B$ in shaping and 'positioning' the output voltage waveform can clearly be seen. Setting the baseband load at a short circuit (Point-A) results in a significant depression in the dynamic gain envelope. Whilst point- $C$ delivers almost the same envelope gain as point $B$, the linearity is reduced by approximately $13 \mathrm{~dB}$. The phase shown in Figure 5.19 is defined here as the difference between measured $a_{1}$ and $b_{2}$ modulation envelopes, and its dependency on negative baseband impedance is shown in Figure 5.19. It should be noted that the discontinuities observed in both the envelope gain and phase traces are measurement artifacts that occur due to the effect of linear delay as the magnitude of the modulation envelope approaching zero. Interestingly, there is a phase variation of approximately $18^{\circ}$ between point- $A$ and point- $B$, and this remains relatively constant over the entire period of the modulation cycle which is due to inherent linear RF propagation delay of the device.

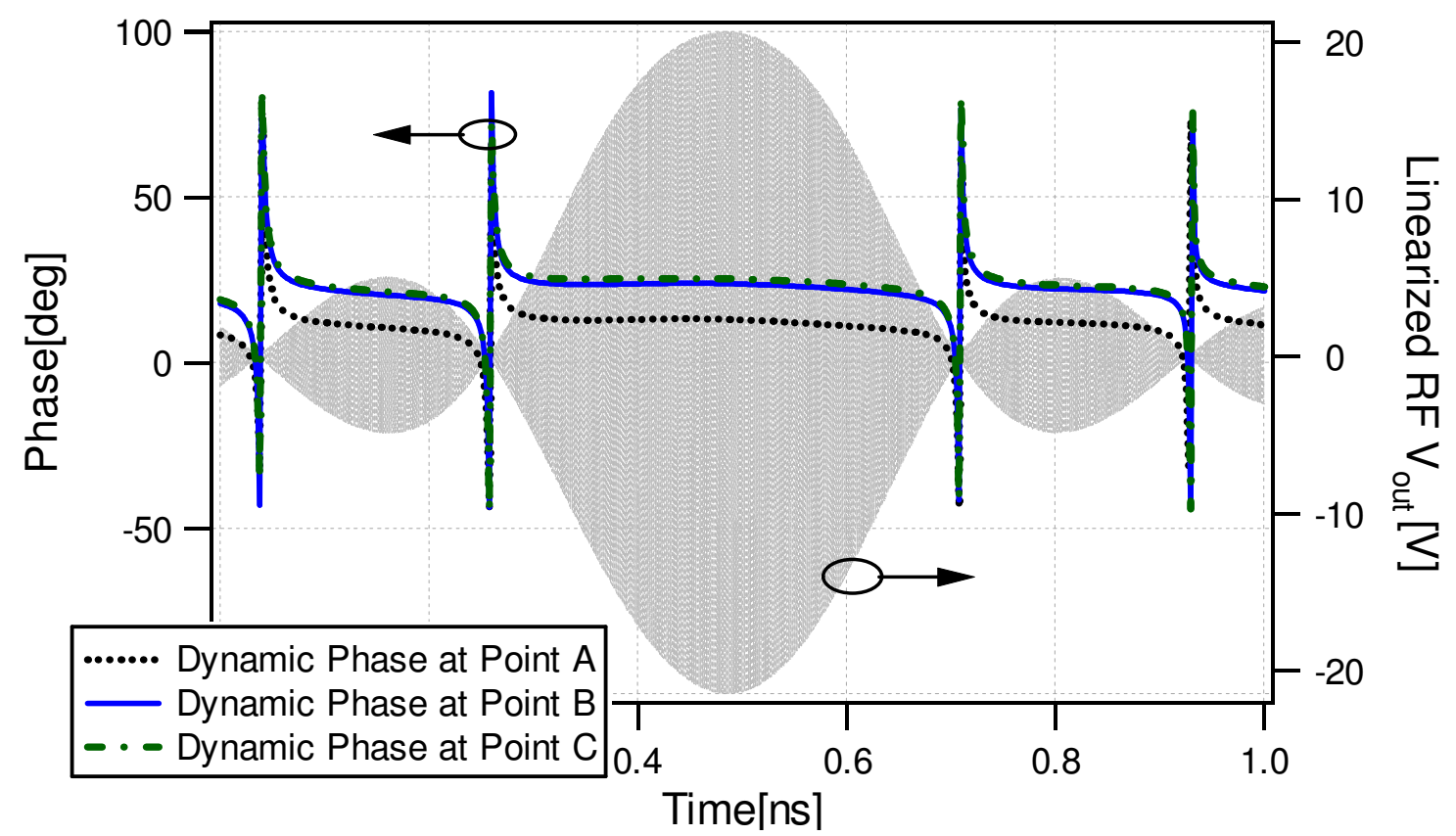

Figure 5.19: Measured envelope phase $(\angle \mathrm{b} 2-\angle \mathrm{a} 1)$ as a function of IF reflection coefficient $\left(\Gamma_{\mathrm{L}}\right)$ at the indentified points with linearized output $R F$ voltage at point-B inset. 
Figure 5.20 depicts the baseband voltage and current waveforms required to present the device with the IF impedance of point B in Figure 5.15. These relatively complex linearizing waveforms are clearly in-phase due to the purely real negative nature of the baseband termination and contain five baseband harmonics.

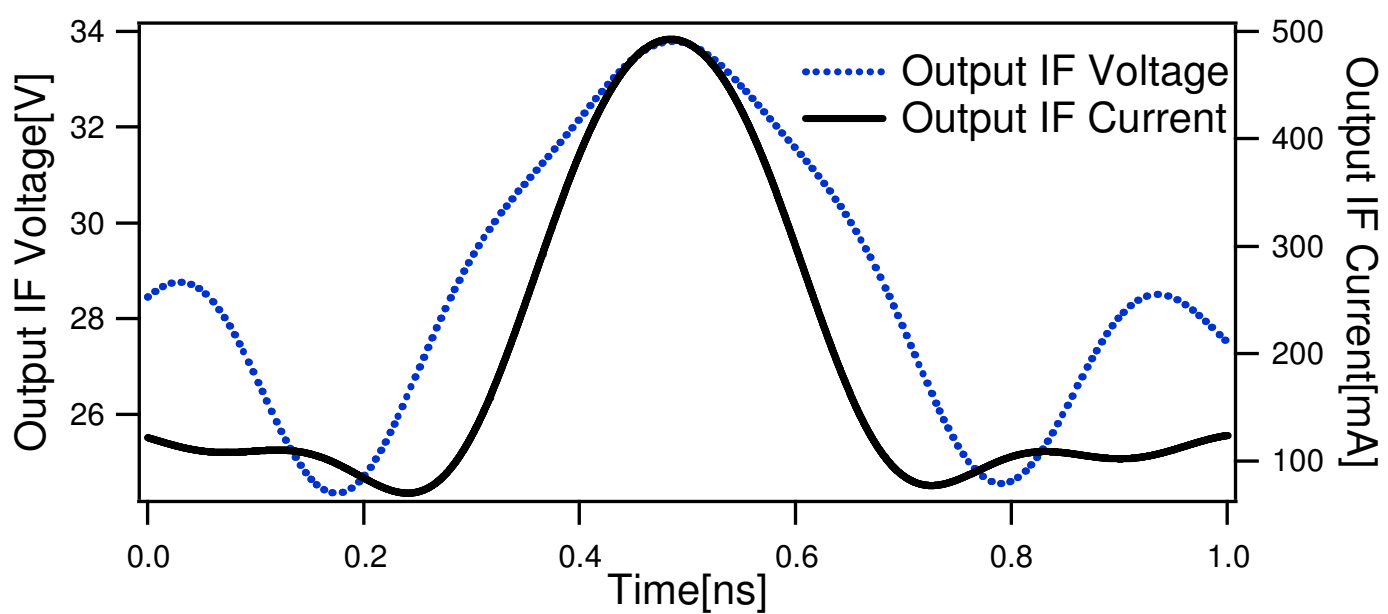

Figure 5.20: Measured output baseband voltage and current for best linearity point $-B$.

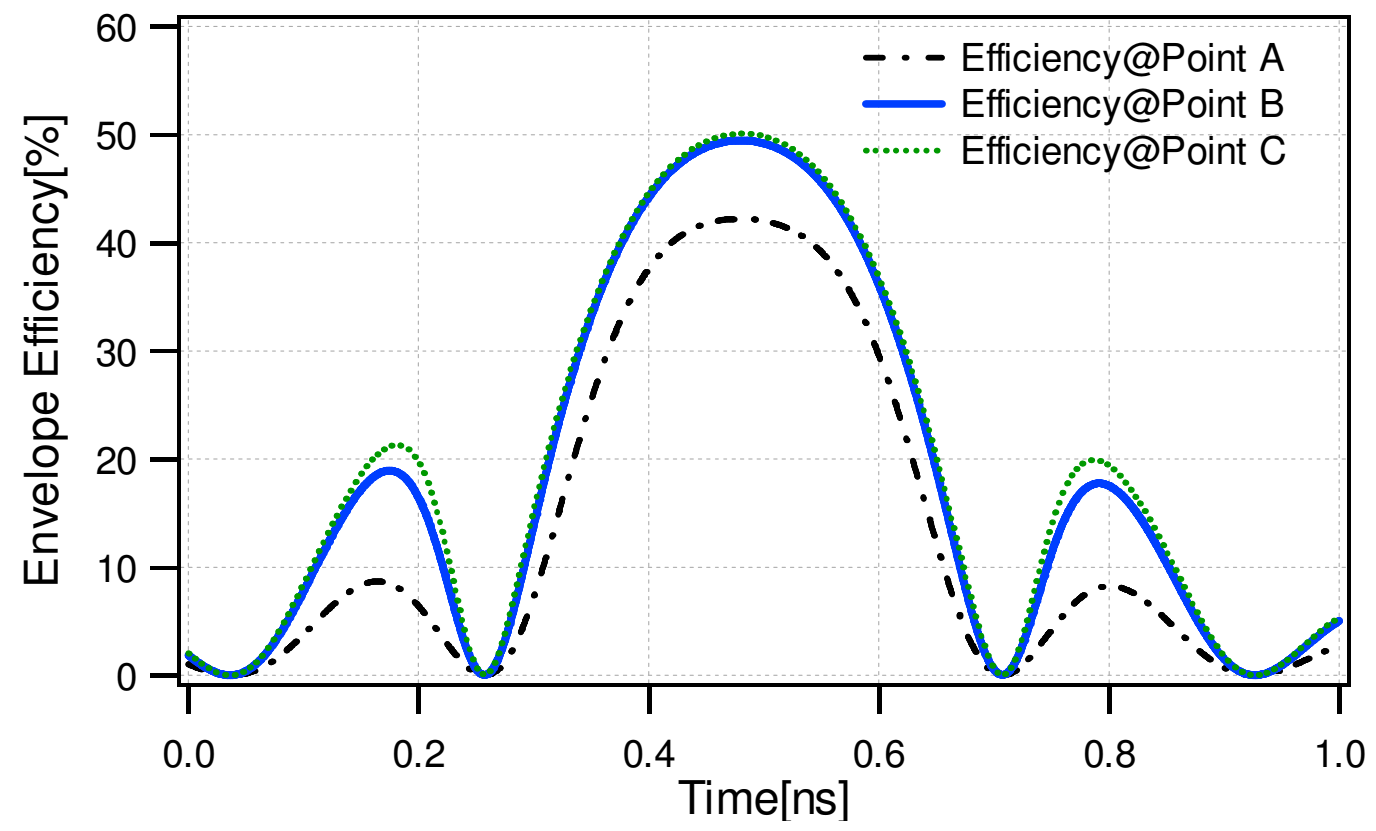

Figure 5.21: Dynamic peak efficiency envelopes at an identified baseband loads.

The peak envelope efficiency is depicted in Figure 5.21, and shows a maximum improvement of $8.1 \%$ for point-B compared to the short circuit case. 
This observation shows that presenting negative impedances at point- $B$ increases the achievable peak output power and has thus enabled a higher efficiency than is possible at point-A.

\subsubsection{Effect of Carrier Phase Distribution on Linearity}

The measurements documented in the previous section were carried out on a $10 \mathrm{~W}$ GaN HEMT when biased in deep class-AB at $2 \mathrm{GHz}$ again under 9-tone complex stimulus with fixed phases at modulation frequency of $1 \mathrm{MHz}$. The nonlinear nature of microwave devices determines a close relation between the usefulness of a multi-tone characterization technique and the similarity of the excitation signal with the real world's excitation. Thus, to characterize and measure the device under more realistic stimulus the 9-tone stimulus was generated in a way that all the tones are related in phase but randomly. Since all the tones are correlated in phase, there may be time spots where they all attain their highest maximum, and other time spots where they may cancel each other and generate a peak power to average power ratio of $6.86 \mathrm{~dB}$. This is due to the fact that the peak-to-average characteristics of the signal vary depending upon the phase relationships of the tones.

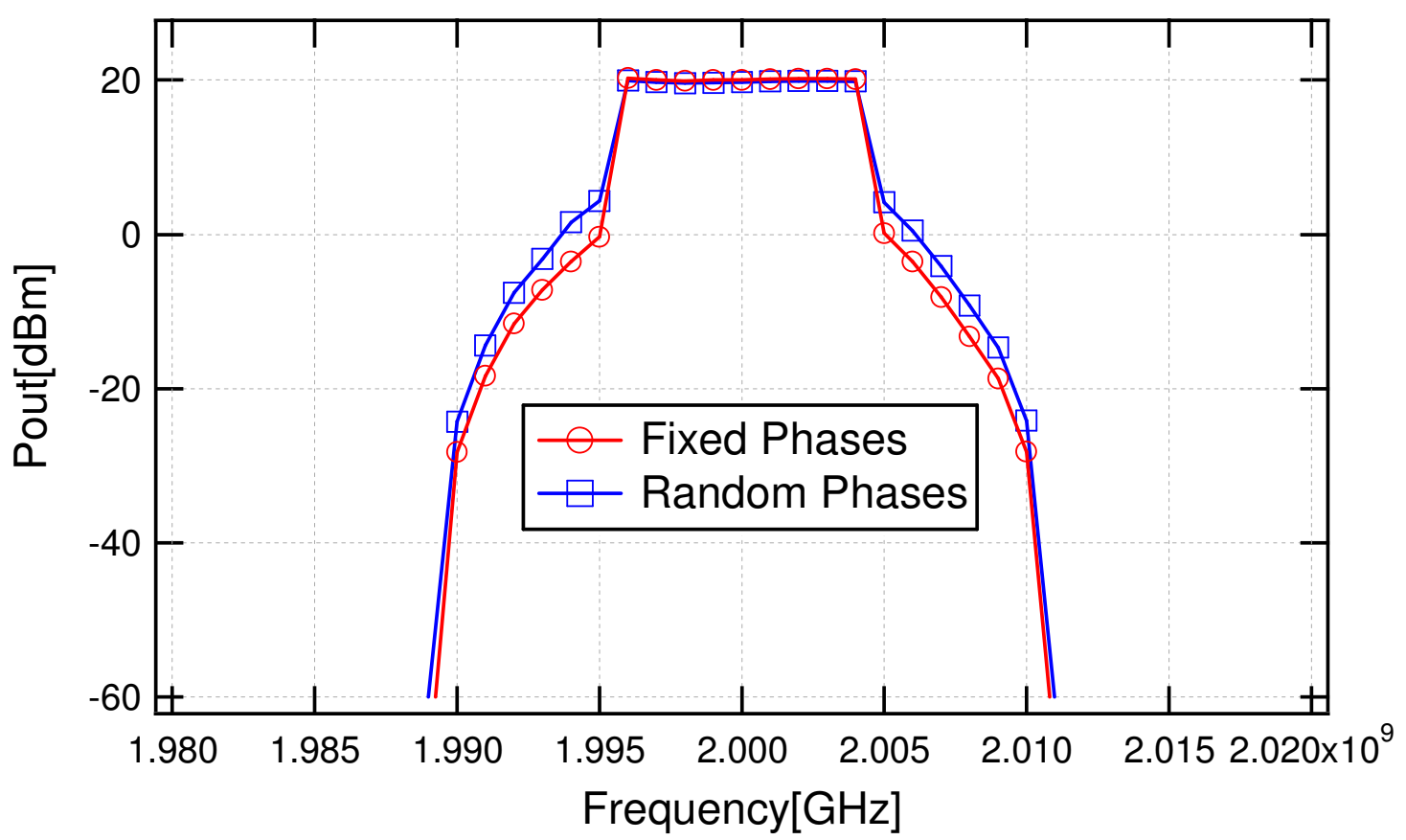

Figure 5.22: The measured output power spectrum for two different phase distributions of 9-tone stimulus when a short circuit was presented to all the significant baseband components. 
Remember, in the time domain, the composite waveform is the summation of each sinusoid (i.e. tone). The difference in peak-to-average ratio would be even bigger if the number of tones considered were different. Baseband loadpull measurements were performed by keeping the output power constant and terminating the RF components with nominal $50 \Omega$. For comparison purposes, for two types of excitation signal the average output power was adjusted such that the peak envelope output power for all 9-tones was $40 \mathrm{dBm}$. It can be concluded from the output spectrum depicted in Figure 5.22, that the phase distribution of the carriers has a strong influence on the transistor adjacent intermodulation products. It can also be seen that there is a difference up to $5 \mathrm{dBc}$ between the adjacent intermodulation products degradation values of the measured output spectrum for random carrier phase distribution.

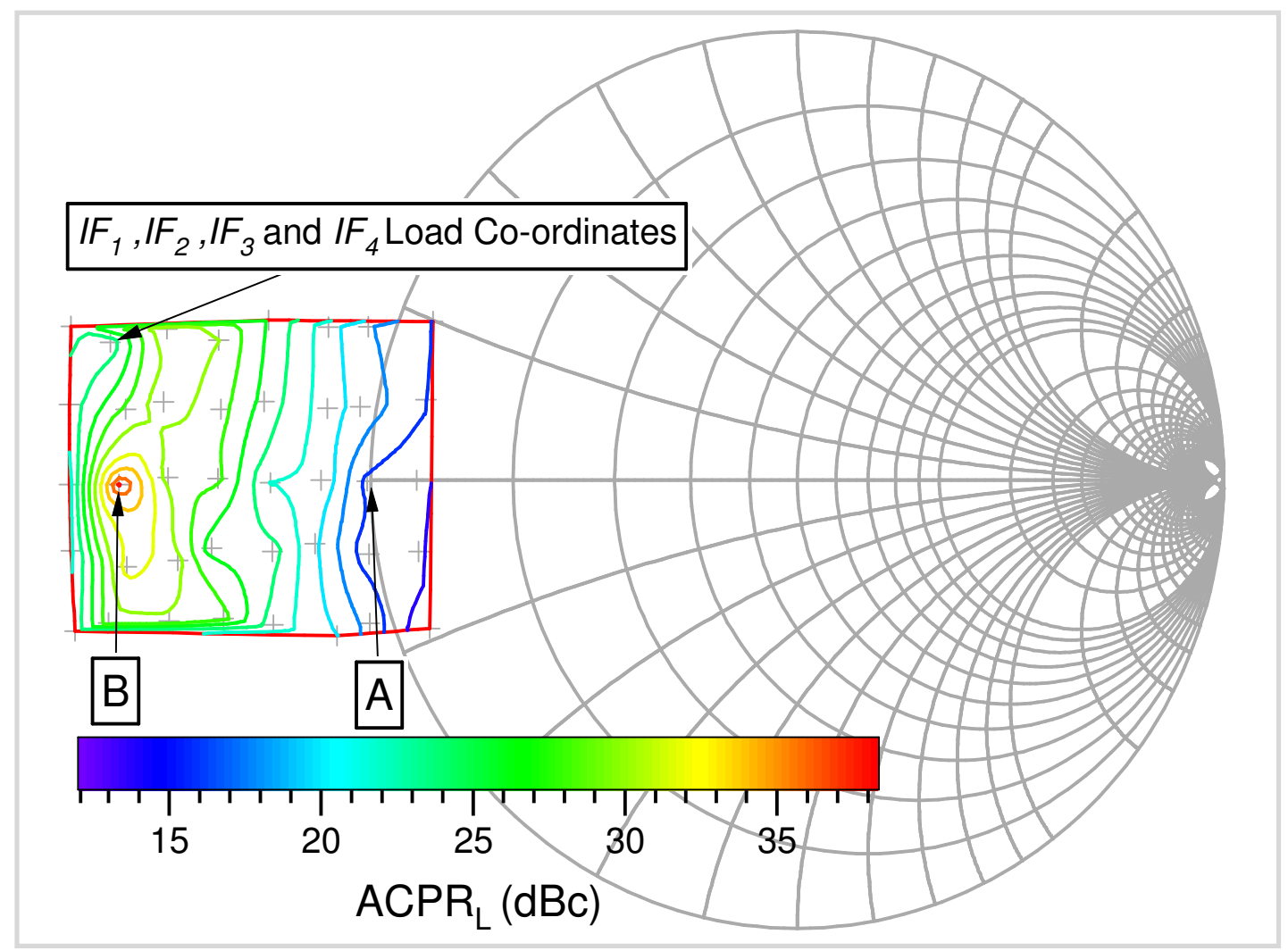

Figure 5.23: Measured lower adjacent channel power linearity contours as a function of IF reflection coefficient $\left(\Gamma_{\mathrm{L}}\right)$ for random phase distribution of the 9-tone stimulus.

The output peak envelope power was measured to be $40 \mathrm{dBm}$ approximately for all the points shown in the Figure 5.23. Again the optimum impedance 
point is located outside the smith chart for the optimum $A C P R_{L}$ performance under 9-tone excitation with random phase distribution. It can be seen from the $A C P R_{L}$ contours depicted in Figure 5.23 that the location of the optimum on the smith chart for random phase distribution of fundamental tones matched those using a fixed phase distribution of fundamental tones with a different PAR value. The baseband load-pull measurements show that the $A C P R_{L}$ results are predominately dependant on PAR of the signal used as an excitation and it is observed that there is a degradation of approximately $5 \mathrm{dBc}$ in ACPRL in comparison with the nine-tone stimulus with fixed phases. The baseband voltage and current for the best linearity "point-B" is depicted in Figure 5.24. It clearly shows that both baseband voltage and current are inphase due to the purely negative resistive baseband termination. The linearity improvement observed by emulating the auxiliary envelope tracking environment around the single device is obtained with significant increase in efficiency, through the additional $7 \mathrm{~V}_{\mathrm{p}-\mathrm{p}}$ variation on the $\mathrm{DC}$ drain supply as can be seen from the Figure 5.24.

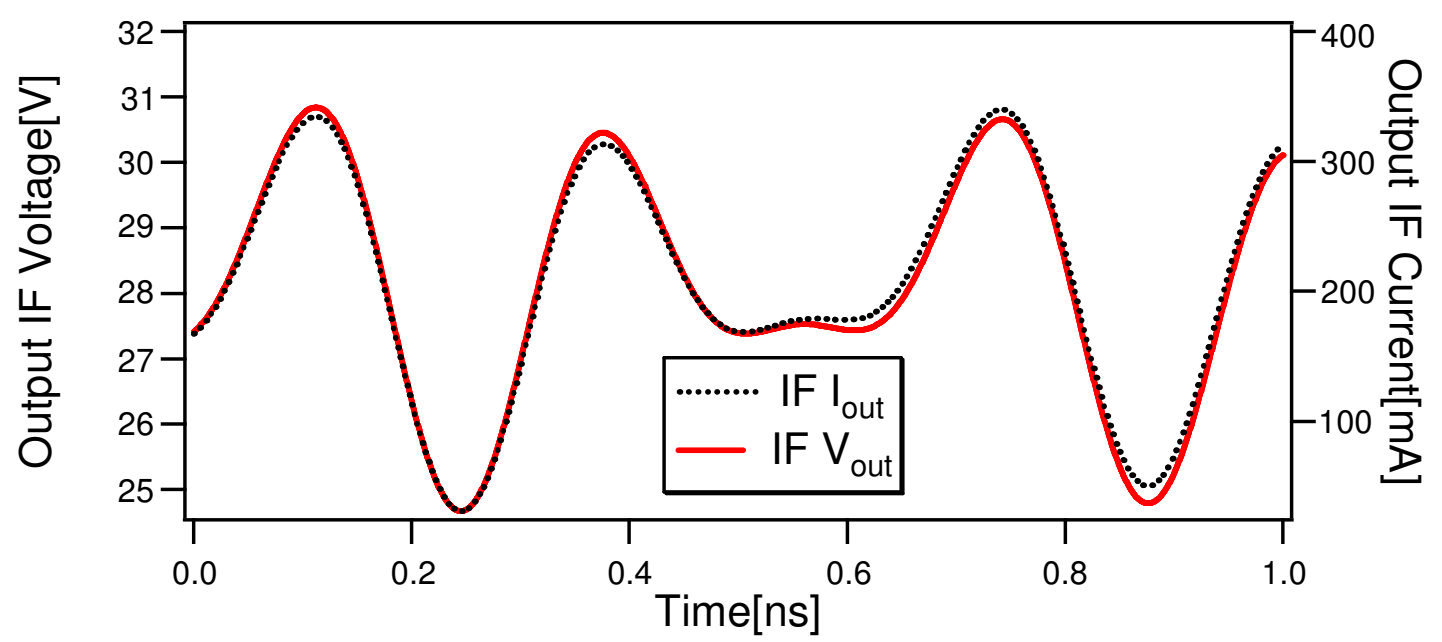

Figure 5.24: Measured output baseband voltage and current at for best linearity point $-B$ when the device was subjected to nine-tone stimulus with random carrier phase distribution.

\subsubsection{Optimum for IMR and ACPR Reduction}

In [15] [16], the authors pointed out that ACPR can be related to the two-tone IM3 performance. This relationship holds for RF devices whose performance is dominated by $3^{\text {rd }}$ order distortion products. Contours for $I M 3_{L}$ 
and $A C P R_{L}$ as a function of baseband reflection coefficient are shown in Figure 5.25 and 5.26 respectively.

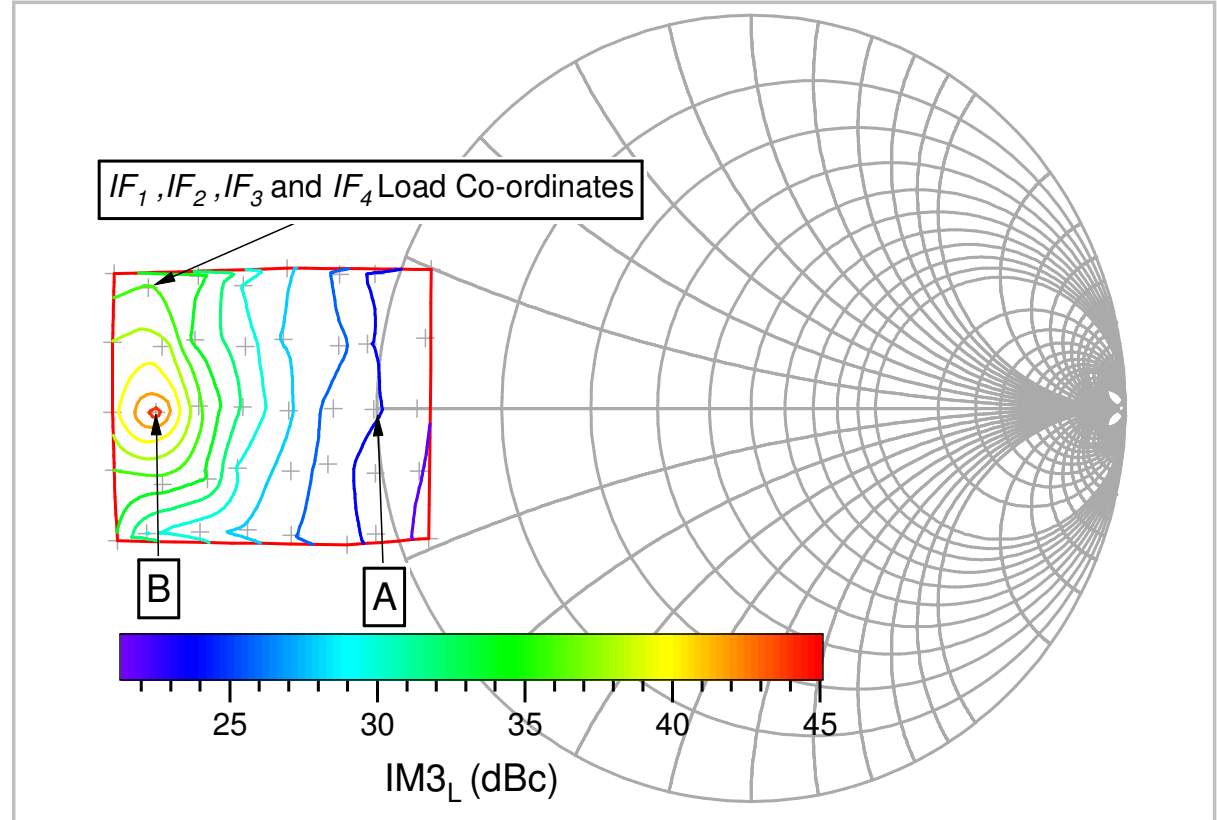

Figure 5.25: Measured output IM3 ${ }_{L}$ linearity contours for two tone excitation when the device was biased in class-AB and driven to deliver $40 \mathrm{dBm}$ peak envelope output power (PEP).

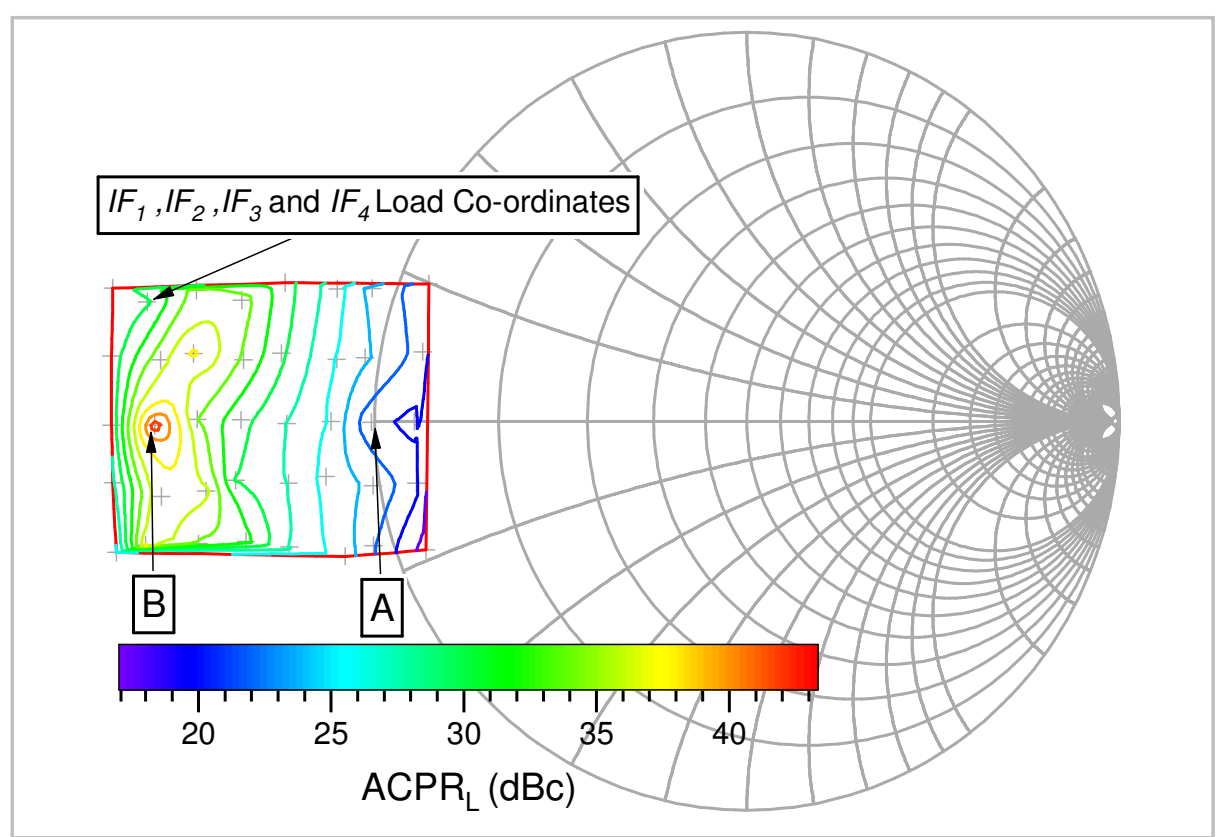

Figure 5.26: Measured output $A C P R_{L}$ linearity contours for 9- tone excitation with fixed phase distribution, when the device was biased in class-AB and driven to deliver $40 \mathrm{dBm}$ peak envelope output power (PEP).

It is seen that $I M 3_{L}$ and $A C P R_{L}$ curves have a similar shape indicating a correlation between them. There is about $20 \mathrm{dBc}$ improvement in $\mathrm{IM} 3_{\mathrm{L}}$ and 
$A C P R_{L}$ at point-B in comparison to point- $A$ where the short circuit impedance was maintained for all baseband components. The results suggest that there is a common optimum point for $I M 3_{L}$ and $A C P R_{L}$ reduction. $A$ very significant conclusion can be drawn being that the device related $3^{\text {rd }}$ nonlinearity should be minimized to achieve a good correlation between the measured IM ${ }_{\mathrm{L}}$ and $A C P R_{L}$ for the device considered in these investigations.

\subsection{Chapter Summary}

The chapter has presented the application of new multi-tone measurement technique in characterization and measurement of microwave device under complex modulated excitations. The memory effects investigations 10W GaN HEMT under 9-tone excitations have confirmed and validated that the baseband electrical memory effects can be greatly reduced by suppressing the significant baseband components IF1, IF2 and higher baseband components IF3, IF4. These measurements clearly demonstrate how non-ideal, frequency dependent, low frequency baseband impedances will induce significant memory effects in high power GaN power amplifiers. The results show that the bandwidth over which the base-band impedances need to be controlled must be extended to at least four times the modulated bandwidth under 9-tone stimulus. This important observation has significant implications for modern PA linearization techniques, as well as requiring careful consideration when designing PA bias networks. For applications utilizing wide modulation bandwidths this will become a serious design constraint.

The linearity investigations and analysis of $10 \mathrm{~W}$ GaN HEMT under 9-tone excitations have shown that for the device considered the optimum impedance for best linearity lies outside the Smith chart. The results showed that under 9-tone stimulus with fixed phases, the presentation of specific baseband loads with non-optimal load $(50 \Omega)$ at point-B has delivered $24 \mathrm{dBc}$ and $8.1 \%$ improvement in linearity and peak envelope efficiency respectively, in comparison with the point-A where short circuit impedance was maintained for all the significant baseband components. This highlights that this important observation holds true for more complex excitation in $50 \Omega$ RF impedance 
environment, as was shown for case of two-tone and three-tone modulated excitation in chapter 4 . To present the fair comparison between the fixed phase and random phase distribution of 9-tone stimulus the measurement condition were kept constant. It was particularly observed that for a 9-tone stimulus with random phase distribution, the optimum for the best linearity was purely negative resistive load lying outside the Smith chart. It was shown that the carriers' randomized phase distribution conditions affect the transistor performance in terms of linearity but resulted in identical linearity optimums as was the case for 9-tone stimulus with fixed phase carrier distribution.

These results suggest that this method of emulating the negative baseband impedance is not only applicable to simplest two-tone signal, but can also be extended to multi-tone signals with variable PAR where the peak signal power is much higher than the average power. Towards the end of the chapter the comparison of measured two-tone and nine-tone results has been performed in terms of location of optimum and the degree of linearity achieved by emulating the specific baseband impedance lying outside the Smith chart. 


\subsection{References}

1. José Carlos Pedro, Nuno Borges Carvalho, "Intemodulation Distortion in Microwave and Wireless Circuits", Artech House, 2003.

2. J. Feigin, "Don't let linearity squeeze your WLAN performance”, Communication Systems Design, Page(s):12-16, October 2003.

3. M. D. LeFevre, D. W. Runton, C. T. Burns, M. K. Mellor, "Digital Predistortion from an RF Perspective”, 2010 IEEE Topical Symposium on Power Amplifiers for Wireless Communications, September 2010.

4. P. B. Kenington, "High Linearity RF Amplifier Design", Norwood, MA: Artech House, 2000.

5. N. B. De Carvalho and J. C. Pedro, "Compact Formulas to Relate ACPR and NPR to Two-Tone IMR and IP3" Microwave Journal, Vol. 42,No. 12, pp.70-84, December 1999.

6. J. F. Sevice, G. R. Simpson and M. B. Steer, "Automated Large Signal Load-pull Characterization of Adjacent Channel Power Ration for Digital Wireless Communication Systems" , Application Note 5C-047, Maury Microwave Corporation, September 2000.

7. N. B. De Carvalho and J. C. Pedro, "Inferring Nonlinear Distortion Performance of Power Amplifiers Subject to Telecommunication Signals from Two-Tone Measurments", IEEE MTT-S International Microwave Symp. Dig., Long Beach, USA, June 2005, Page(s):817 -820.

8. B. Aleiner, "Correlation Between P1dB and ACP in TDMA Power Amplifiers," Applied Microwave and Wireless, No.3, March 1999.

9. J. Vuolevi and Timo Rahkonen, "Distortion in RF Power Amplifiers", Norwood, MA: Artech House, 2003.

10. N. B. De Carvalho and J. C. Pedro, "A Comprhensive Expalantion of Distortion Sideband Asymmetries", IEEE Trans. on Microwave Theory and Tech, Vol.50, No. 9, September 2002, Page(s):2090-2101.

11. J. P. Martins, P. M. Cabral, N. B. Carvalho,J. C. Pedro, "A Metric for the Quantification of Memory Effects in Power Amplifiers", Microwave Theory and Techniques, IEEE Transactions on Microwave Theory and Techniques, December 2006, vol.54, pages: 4432-4439

12. M. Akmal, J. Lees, S. Bensmida, S. Woodington, V. Carrubba, S. Cripps, J. Benedikt, K. Morris, M. Beach, J. McGeehan, P. J. Tasker, "The Effect of Baseband Impedance Termination on the Linearity of GaN HEMTs", in Proc. of $40^{\text {th }}$ European Microwave Conference, September 2010, pages: $1046-1049$. 
13. M. Akmal, V. Carrubba, J. Lees, S. Bensmida, S. Woodington, J. Benedikt, K. Morris, M. Beach, J. McGeehan, P. J. Tasker, "Lineairty Enhancement of GaN HEMTs under Complex Modulated Excitation by Optimizing the Baseband Impedance Environment", in Proc. of IEEE MTT-S, International Microwave Symposium(IMS), June 2011, Page:1 - 4.

14.J. Lees, T. Williams, T.; S. Woodington,P. McGovern, S. Cripps, J. Benedikt, P. Tasker, "Demystifying Device related Memory Effects using Waveform Engineering and Envelope Domain Analysis" in Proc. $38^{\text {th }}$ European Microwave Conference, October 2008, Page(s): 753 - 756.

15. Michael Leffel, "Intermodulation Distortion in a Multi-signal Environment," RF Design Magazine, June 1995, pp. 78-84.

16. Nuno Borges Carvalho and Jose Carlos Pedro, "Compact Formulas to Relate ACPR and NPR to Two-Tone IMR and IPE," Microwave Journal, December 1999, pp. 70-84. 


\section{Chapter 6}

\section{Conclusions and Future Work}

\subsection{Conclusions}

The strategies to explore the possibilities of improving the linearity of power amplifiers at higher output power are still under development. The intensive research activities have been initiated worldwide to understand and reduce the distortions in power devices. In the present development phase, it is very important to characterize and analyze the device properties and performance systematically. As GaN HEMTs are targeted to be employed in the wireless communication circuits, linearity performance is one of the most important issues. In particular, in-band intermodulation distortions of wideband signals, which cannot be eliminated by using filters, must be characterized and minimized.

Besides the benefits of high efficiency offered by the existing GaN devices, due to rapidly developing broadband multi-standard air interface with multicarrier solutions, the situation has become even more challenging to meet the 3GPP linearity specifications. With the advent of 4G UMTS-LTE, which incorporates OFDM for downlink having scalable bandwidths ranging from $1.25 \mathrm{MHz}$ to $20 \mathrm{MHz}$, the complexity of satisfying telecommunication linearity requirements would rapidly escalate. In this thesis a new multi-tone measurement technique has been documented to analyze, identify and minimize the distortion sources in GaN devices. It was identified that a key 
advancement of the measurement system over the previous one documented in [1] was the requirement to be able to characterize the microwave devices under realistic stimuli with the ability to measure voltage and current waveforms at all relevant frequencies.

Thus, modulated waveform measurement system architecture was modified to allow characterisation of a power device or a power amplifier to fulfil the requirements of 3G Universal Mobile Telecommunication System (UMTS) and 4G Long Term Evolution (LTE) spectral masks. This enhanced system has the ability to present specific, constant broadband impedances, not only at baseband (IF) frequency, but also at RF frequencies, particularly, around the carrier and significant harmonics. Achieving such comprehensive impedance control across wide modulation bandwidths is critical in allowing the 'emulation' of new power amplifier modes and architectures, and the subsequent waveform characterization of devices operating in these complex and often dynamic impedance environments. The capability of the enhanced measurement system is experimentally demonstrated through the number of applications.

Firstly the experimental investigations to quantify the role of higher baseband harmonics IF3 and IF4 under two-tone suppress carrier modulated excitation on the baseband electrical memory, which appears as a recognizeable hysteresis on dynamic transfer characteristics of power device, preventing the overlap of input-output trajectory with the increased signal swing. The memory effects contributed by the baseband impedance variations impact the device performance under modulated excitation and reduction of baseband electrical memory by terminating the baseband impedances into a short circuit provides the better insight into the effects of variation of baseband impedances on the overall device performance.

The introduction of two tone test signal revolutionized the perception of nonlinearity, setting up stringent rules on the measurement system requirements. A basic two tone test setup uses two discrete tones of equal power that fall within the passband of the DUT, are applied as excitation signals. The resulting baseband information and IMD components are then measured and can be cancelled by engineering the appropriate baseband impedance. To observe the dynamic behaviour of the device whilst operating 
within a realistic operational environment, and in response to an applied modulated input stimulus signal, envelope domain analysis was used. This feature provides a clear representation and inspection of the cause of nonlinearity and memory effect. Under two tone modulated excitation, maximum of $15 \mathrm{dBc}$ and $18 \mathrm{dBc}$ suppression in $\mathrm{IMD}_{3}$ and $\mathrm{IMD}_{5}$ together with the minimization of IMD asymmetry were achieved in 10W GaN HEMT using IF active load-pull. Measurements and investigations show that hysteresis behaviour in the dynamic transfer characteristics as well as IMD behaviour are not only dependent on the most significant IF component (IF1, IF2), but is also very sensitive to higher order IF components (IF3 and IF4). The results show that in order to obtain the frequency independent response the bandwidth over which the baseband impedances must be controlled should be extended to at least eight times the modulated bandwidth for two tone excitation [2], [3].

The measurement system is also used for nine-tone modulated signals to investigate the memory effects of the device under test (DUT) as nonlinear behaviour depends on the properties of the input signal significantly.

For wideband and higher power applications the dynamic behaviour of nonlinear power transistor, like memory effects can no longer be ignored. For illustration, it has been shown that it is possible to suppress the electrical baseband memory effects and hysteresis which sometimes appear on the dynamic transfer characteristics by providing the short circuit impedance to all the significant baseband components generated as result of inherent nonlinearity of power device under more complex multi-tone excitations. These measurements clearly demonstrated a constant spectral symmetry over a wide modulation bandwidth. The origin of memory effects is secondorder distortion, simultaneous minimisation of second-order and third-order distortion allows linearization that is not dependent on bandwidth.

Secondly the enhanced measurement system is used to study the effect of baseband impedance termination on the linearity of power transistor which explains the need to engineer the baseband components in excess of five times that of the modulation frequency to obtain the modulation independent device behaviour [3]. In addition, the capability of load- pulling baseband frequency components for high power device through the application of active 
IF load-pull provides an insight into the effect of the baseband impedances on the overall device performance. The linearization performance is often degraded by bandwidth-dependent distortion; the baseband impedances that reduce bandwidth-dependent distortion were outlined under tow-tone modulated excitation [4].

The successful linearization over much larger bandwidths was achieved through the presentation of negative impedances to all the substantial baseband components, which is referred as baseband signal injection linearization. The presentation of negative baseband impedances has delivered a $20 \mathrm{dBc}$ and $17 \mathrm{dBc}$ improvement in IMD3 and IMD5 intermodulation products respectively, relative to the case of a classical, ideal short circuit. Similar improvement in IMD3 and IMD5 has been reported in [5] on PA system level using the signal injection technique. Additionally, auxiliary envelope tracking $(A E T)$, an important variation on the existing envelope tracking (ET) techniques, focused measurements with nonoptimal RF load $(50 \Omega)$ have shown that efficiency as well as linearity can be improved at reduced drain supply voltages: for $\mathrm{Vdc}=20 \mathrm{~V}$, the average drain efficiency is improved by approximately $5 \%$ together with an improvement of $10 \mathrm{dBc}$ in IM3 when compared to the static Vdc, where short circuit impedance was maintained for all four baseband components. The significance of AET is that the power content of the injected signal is a very low percentage of the DC supply to the microwave device.

Furthermore, the baseband injection linearization technique was exploited to investigate the suitability of this baseband linearizing approach to real-world PA architectures. The enhanced measurement system was used to emulate a modulated class-J impedance environment, through the application of modulated RF active load-pull. This has been done to investigate the effectiveness of using this baseband linearizing approach in improving PA linearity when the fundamental and reactive harmonic loads presented to the device become highly reactive.

The results suggested that although linearity is significantly improved, the optimum impedances become dispersed and move away from the real axis. This important observation has large implications for the linearization of 
emerging, broadband PA architectures, especially when these involve efficiency enhancing techniques such as envelope tracking [6], [7].

Finally enhanced measurement system is demonstrated through the investigation into the effectiveness of this linearity and efficiency enhancement technique under more complex modulated excitations. Linearity investigations and analysis of $10 \mathrm{~W}$ GaN HEMT under complex multi-tone excitations have shown that the optimum impedance for best linearity lies some way outside the Smith chart. The device linearity performance improved in a substantial leap and is summarized in Table 6.1 as has been shown for the two-tone case. The results suggest that for a nine tone stimulus, the presentation of specific baseband loads has delivered a linearity improvement of $24 \mathrm{~dB}$ and an efficiency improvement of $8.1 \%$, relative to the baseband short-circuit case.

Table 6.1: Linearity performance under complex modulated excitation [6], [7].

\begin{tabular}{|l|cccc|}
\hline & Pout $[\mathrm{dBm}]$ & ACPR $_{\mathrm{L}}[\mathrm{dBc}]$ & $\mathrm{ACPR}_{\mathrm{H}}[\mathrm{dBc}]$ & $\eta_{\mathrm{D}}[\%]$ \\
\hline Point-A & 39.12 & -20.27 & -20.12 & 42.1 \\
Point-B & 40.78 & -43.59 & -43.07 & 49.9 \\
Point-C & 40.93 & -28.63 & -27.21 & 50.7 \\
\hline
\end{tabular}

\subsection{Future Work}

For reliable distortion characterization of future WiMAX and LTE systems, device level linearity issues resulting from out-of-band terminations should be carefully analyzed. This thesis has predominantly been concerned with the enhancement of modulated measurement system and accurate characterization of broadband distortion and linearization in GaN HEMT devices through the optimization of out-of-band impedance environment, particularly at baseband frequencies.

Furthermore, the work outlined in this thesis stresses how linearity optimum results under $50 \Omega$ RF impedance environment change when measured as the RF impedance environment is changed to emulate realistic PA modes. The enhanced measurement system is capable of maintaining independent and 
constant impedance control for each individual tone across both the IF and RF impedance environment simultaneously, and over a wide modulation bandwidth.

This has provided a significant step forward in enabling high power robust device characterization under complex modulated excitations and introduced the considerations required to improve this system. This section suggests some future work necessary in moving forward the work undertaken in this thesis, which will make modulated measurement system more proficient, an automated and effective in the characterization of microwave devices for future wireless communication standards.

\subsubsection{Improving Measurement Capability}

The "Time Domain Partitioning" approach for modulated measurements has demonstrated great potential in investigating the dynamic behaviour of microwave devices and measurement of power amplifiers in the $50 \Omega$ environment as well as under a specific power amplifier mode. But currently the rapidity is limited by the relatively manual load-pull measurements which are especially problematic when harmonic load-pull is desired; such a problem is further compounded when simultaneously load-pull is desired at baseband and RF to investigate the device nonlinear behaviour under a specific PA mode. This necessitates the further developments of the load-pull capabilities of measurement system.

\subsubsection{Automated Baseband Load-pull}

An application of enhanced active baseband load-pull has been demonstrated though the control of significant baseband components over a significant bandwidth; in this thesis at least ten times of modulating frequency. The magnitude and phase of each IF component was adjusted manually to present the specific impedance. This, in principle, is very time consuming in terminating and load-pulling the IF components together.

In fact this shortcoming can be overcome by a programme routine that allows the baseband components to be held, moved and terminated together with appropriate baseband impedance termination. This improvement will allow the 
baseband load-pull system to operate with full automated functionality. In a nutshell, fully automated control can be used to either eliminate the variation on the baseband components for better accuracy or improving the baseband load-pull capability by emulating real world bias network impedance through the precise control of baseband components individually and together.

\subsubsection{Automated Modulated RF Load-pull}

The RF load-pull is a technique based on the search for the optimum impedance seen by the device in order to investigate its performance in terms of efficiency, linearity, gain, and output power. This is important especially for the nonlinear device in which the performance with different load cannot be predicted using small signal s parameters.

The major development of the RF modulated load-pull system through this work has been achieved with the aim of characterising the microwave devices under realistic PA mode RF impedances. A limited linearity investigation has been performed on 10W GaN HEMT transistors with some success in emulating the class-J mode of operation, with the implementation of the open loop architecture using the Tektronix AWG7000. To further this investigation and to enable the performance of different devices to be investigated, the modulated RF active load pull system must be automated to avoid the excessive time penalty in controlling the individual tones around the carrier and harmonics. A development of the measurement software is, therefore, required to enable load pull to be performed on the output spectrum of a modulated signal at both the fundamental and harmonic frequencies; this would provide the ability to directly measure the behaviour of the device operating in each mode of operation, thus providing further validation of the behaviour models ability to predict the effect of the fundamental load and harmonic terminations on the distortion products.

\subsubsection{Load-pull Capability for Digitally Modulated Stimuli}

Further enhancements of measurement system can be carried out towards a large-signal fully automated load-pull system for characterization of wireless communication systems dependant on digitally modulated signals. The traditional load-pull measurements have been limited to the utilization of 
single-tone and two-tone signals for optimizing output power and third order intermodulation products performance of transistors. However, most of today's wireless communication systems rely exclusively on digital modulation.

Load-pull measurements to determine optimum load impedances for power gain and adjacent-channel power ratio (ACPR) in microwave devices stimulated with wideband code division multiple access and orthogonal frequency-division multiplexing signals of various peak-to-average ratios is of utmost importance. The obtained results can then be compared to load-pull measurements of gain and third-order intermodulation products (IMD3), based on a two-tone source signal to establish that the optimum match impedance changes depending on whether a two-tone or a digitally modulated source is used as a stimulus or not.

\subsubsection{Supplementary DUT Investigations}

The push for wider bandwidths and higher distortion reduction will continue, driven by emerging wireless technologies. It seems that memory effects are probably the most important issue. These phenomena are so complex and depend on so many interactive variables that a simple solution to the problem does not exist. This doctoral thesis attempted to quantify them and reduce some of the more relevant memory effects, but further reduction is certainly possible. This emphasizes that RF electrical memory effect can be investigated in addition to baseband frequencies. It would be interesting to use the enhanced measurement system to find a figure of merit for both the optimum baseband and second harmonic components at the same time, however, memory effect can also be modelled effectively and it would also be possible to use the mix of linearization techniques to enhance the linearity performance of microwave devices.

\subsubsection{Effect of $2^{\text {nd }}$ Harmonic on Linearity}

The apparent future work is also to quantify the effect of $2^{\text {nd }}$ harmonic impedance on the linearity performance of a power device. Equation 6.1 indicates that it would be possible to linearize the power device through the 
precise engineering of second harmonic impedance environment using $R F$ load-pull.

$$
i(t)=a_{1} * v(t)+a_{2} * v^{2}(t)+a_{3} * v^{3}(t)
$$

It is not yet clear how much the variation in in-band distortion is related to the variation in the second harmonic impedance, not engineered precisely in this work but terminated to a reactive for emulation of class-J mode of PA operation. It would be important to investigate in sufficient detail the impact of second harmonic termination on in-band distortion and possibly find a figure of merit for the one that has the greater impact on it. Is it base-band or second harmonic impedance?

\subsubsection{Combined LUT Predistortion and Baseband Injection}

Look-Up-Table (LUT) based predistortion, and baseband injection can be considered simultaneously for improved linearity performance, mainly in terms of adjacent channel power ratio (ACPR) reduction, error vector magnitude (EVM) improvement. The LUT predistortion plus injection advantages can be quantified on their comparisons with baseband injection only, and can be verified its abilities by applying to a PA system having both nonlinearities and memory effects. The LUT predistortion plus baseband injection has the potential to be controlled using artificial intelligence techniques.

The advantage of this would be that the coefficients of LUT and baseband injection can be calculated automatically rather than manual measurement and calculation. The logical reason for applying LUT predistortion first then baseband injection, rather than baseband injection first then LUT, is that the LUT predistorter will first reduce the majority of the PA's nonlinearities and hence the new intermodulation products generated from the interaction of injection and pre-linearized signal will be much less significant and will almost suppress the intermodulation products completely.

\subsubsection{Modelling Memory Effects}

The memory effects are problematic effects in power amplifier design. These represent a source of nonlinearity which is difficult to formulate in the 
modelling approach. Since memory effects would contribute to the distortion characteristics of a power amplifier, neglecting memory effects in modelling may reduce optimal performance of power amplifiers. So it's worth considering a modelling approach somehow by accounting for the memory effects. There are basically three factors which contribute to memory effects. The thermal effects cause memory effects due to the heat generated during the operation of transistor devices which results changes of temperature and consequently dispersion of device characteristics. It's possible to model this bit once the thermal measurement is available. The temperature can be a model parameter which allows the model to respond differently in different thermal states. The major factor which causes memory effects is the baseband modulation. Due to the imperfect nature of the DC biasing circuit, the impedance variations across the base-band seen by the device causes asymmetrical IM sidebands when the device is excited by a wideband or multi-tone signals.

The asymmetry can be a serious problem for common PA linearization schemes such as pre-distortion. Since these kinds of effects are mainly caused by the DC biasing network, it's possible to model the effects using practical DC biasing network in simulation. There are various avenues to be explored in relation to bandwidth-dependent memory effects modelling. Many modelling techniques could be considered, particularly those more suitable to model baseband memory effects, generally speaking it's difficult to model such effects, to reduce second-order distortion, and hence increase linearization possibility of power amplifiers.

\subsubsection{Envelope Square Injection for Linearity Enhancement}

A comprehensive work has been documented in this thesis on envelope injection which was used to compensate for memory effects of the microwave transistor and it is essentially referred to as a real-time version of the load-pull technique [3]. Generally, the main causes of memory effects are the existence of envelope impedances. It is important to remember that most memory effects arise as mixing products at the envelope frequency. While the envelope signal virtually generates the desired envelope impedances over a 
range of modulation frequencies, which are used to compensate the original envelope impedances. Interestingly this method was then used to achieve better power transistor linearity by nullifying the $2^{\text {nd }}$ order nonlinearity of the device as has been demonstrated in [4], [6], [7].

However, the investigations on an envelope square injection signal can be conducted to explore its effectiveness to correct the AM/AM nonlinearity of power transistor regardless of the excitation signal statistics. Intuitively speaking, this should suppress the distortion side bands over a broad dynamic output range and for a wide modulation frequency variation; however, this requires investigations to be validated.

\subsubsection{Linearity Investigations of Continuous Modes}

The effectiveness of the linearization documented in this thesis needs to be investigated for continuous mode of operation of power amplifier [8], [9]. The imperative observation made under class-J mode of operation has large implications for modern PA linearization techniques emphasizing the fact that the linearity enhancement of continuous mode of operation would be rather challenging by using the very mechanisms that are employed to improve the efficiency.

\subsection{Conclusive Remarks}

For what small measure of knowledge this work may contain, and perhaps might impart to others, there is yet a vast frontier waiting to be explored. The advent of $4^{\text {th }}$ generation wireless communication systems has truly given rise to a new era of un-paralleled speed and versatility in electronic communication. Literally every day, new directions are being wrought out. Though the letter of this work now comes to a close, it is hoped with great earnest that, in the mind of the reader, the spirit of this work will continue onward. 


\subsection{References}

1. A. A Alghanim," High Power Waveform Measurement System Enabling Characterisation of High Power Devices Including Memory Effects" Ph.D. thesis, Cardiff University, March 2008.

2. M. Akmal, J. Lees, S. Bensmida, S. Woodington, J. Benedikt, K. Morris, M. Beach, J. McGeehan, P. J. Tasker, "The Impact of Baseband Electrical Memory Effects on the Dynamic Transfer Characteristics of Microwave Power Transistors", in Proc. of 4th International Nonlinear Microwave Monolithic Integrated Circuit(INMMIC), April 2010, pages: 148 - 151.

3. M. Akmal, J. Lees, S. Bensmida, S. Woodington, J. Benedikt, K. Morris, M. Beach, J. McGeehan, P. J. Tasker, "Minimization of Baseband Electrical Memory Effects in GaN HEMTs Using IF Active Load-pull", in Proc. Of Asia Pacific Microwave Conference (APMC), December 2010, pages: 5-8.

4. M. Akmal, J. Lees, S. Bensmida, S. Woodington, V. Carrubba, S. Cripps, J. Benedikt, K. Morris, M. Beach, J. McGeehan, P. J. Tasker, "The Effect of Baseband Impedance Termination on the Linearity of GaN HEMTs", in Proc. of $40^{\text {th }}$ European Microwave Conference(EuMC), September 2010, pages: 1046-1049.

5. Z. Yusoff, J. Lees, J. Benedikt, P. J. Tasker, S.C. Cripps, "Linearity Improvement in RF Power Amplifier System using Integrated Auxiliary Envelope Tracking System", in Proc., IEEE International Microwave Symposium, June 2011, Page(s):5- 8.

6. M. Akmal, V. Carrubba, J. Lees, S. Bensmida, S. Woodington, J. Benedikt, K. Morris, M. Beach, J. McGeehan, P. J. Tasker, "Lineairty Enhancement of GaN HEMTs under Complex Modulated Excitation by Optimizing the Baseband Impedance Environment", in Proc. of IEEE MTT-S, International Microwave Symposium(IMS), June 2011, Page:1 - 4.

7. M. Akmal, J. Lees, Z. Yusoff, S. Bensmida, S. Woodington, V. Carrubba, S. Cripps, J. Benedikt, K. Morris, M. Beach, J. McGeehan, P. J. Tasker, "An Enhanced Modulated Waveform Measurement System for Robust Characterisation of Microwave under Modulated Excitation", in Proc. of $41^{\text {st }}$ European Microwave Conference, October 2011, Page:1-4.

8. V. Carrubba, A. L. Clarke, M. Akmal, J. Lees, J. Benedikt, P. J. Tasker, S. C. Cripps, "The Continuous Class-F Mode Power Amplifier", in Proc. $40^{\text {th }}$ European Microwave Conference, October 2010, Page(s): 1674 - 1677.

9. V. Carrubba, J. Lees, J. Benedikt, P. J. Tasker, S. C. Cripps, "A Novel Highly Efficient Broadband Continuous Class-F RFPA Delivering 74\% Average Efficiency for an Octave Bandwidth," IEEE MTT-S International Microwave Symp. Dig., Baltimore, US, June 2011, Page(s): 1 - 4. 


\section{APPENDICES}




\section{APPENDIX-A}

\section{Calibration Interpolation}

This section provides an explanation of the calibration interpolation panel and features available in the interpolation software implemented in IGOR. All controls are accessed using the Main Panel depicted in Figure A-1

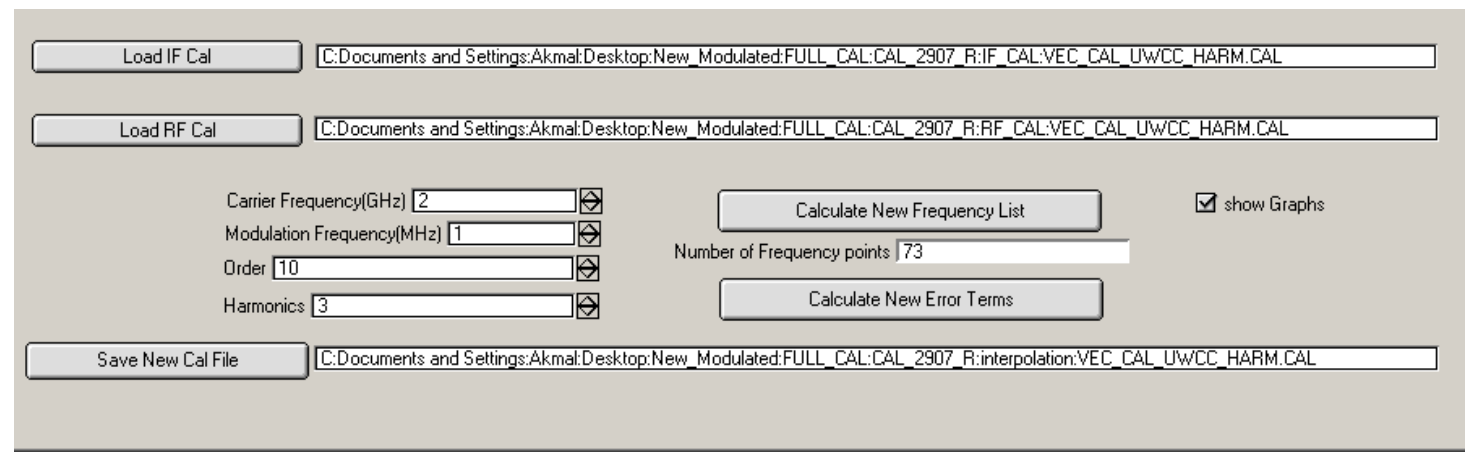

Figure A-1: The main user panel for calibration interpolation

As shown in Figure A-1, there are four sections of functions that can be controlled via the interface. They are "Load IF Cal", "Load RF Cal", "Calculate New Frequency List", "Calculate New Error Terms", "Save New Cal File", and Show Graphs.

Load IF Cal allows to select the relevant directory, loads the IF calibration file and store the error coefficients and the IF frequency list of the system in a programme array.

Load RF Cal allows selecting the relevant directory to load the RF calibration file and stores the error coefficients and the RF frequency list of the system in a programme array.

Calculate New Frequency List this computes the interpolated frequency list to define a precise measurement grid

Calculate New Error Terms this computes the interpolated error terms of the integrated IF and RF measurement system. Before calculating the new error terms, the information about the calibration data set, for instance, Carrier Frequency, Modulation Frequency, Order and Harmonics are needed to be defined.

Save New Cal File allows saving the new interpolated calibration file by specifying the path Show Graphs displays the interpolated error terms graphs. The following graphs show the calculated error coefficients of the measurement system, the system is calibrated at baseband, fundamental band and harmonic-bands up to the $3^{\text {rd }}$ harmonic. The system has been calibrated over a $45 \mathrm{MHz}$ bandwidth at the base-band, and over a $60 \mathrm{MHz}$ band at RF. The presented graphs of the error coefficients confirm that the system does not vary widely over the calibrated bandwidth, making it possible to easily interpolate the error coefficients. 

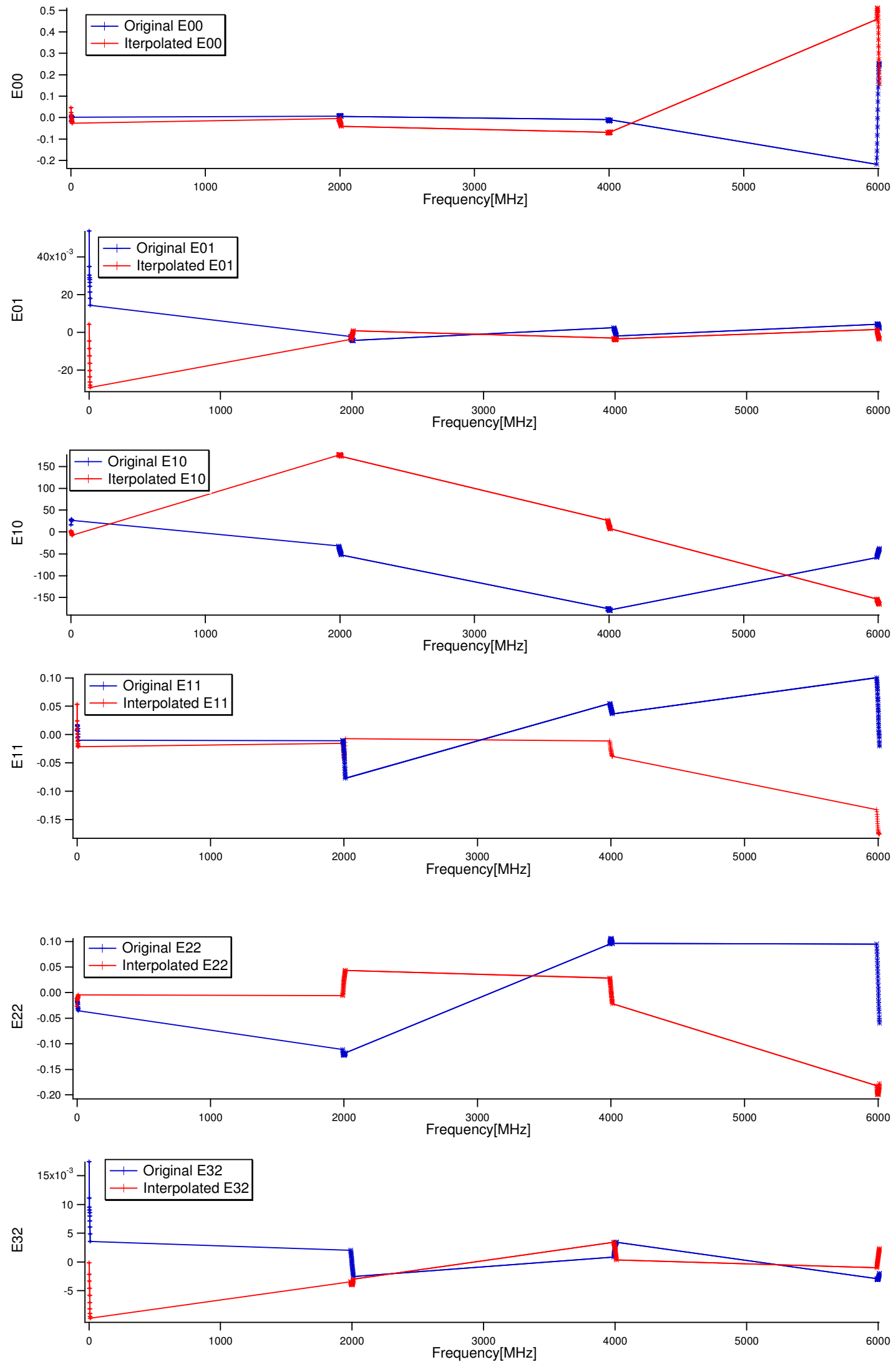

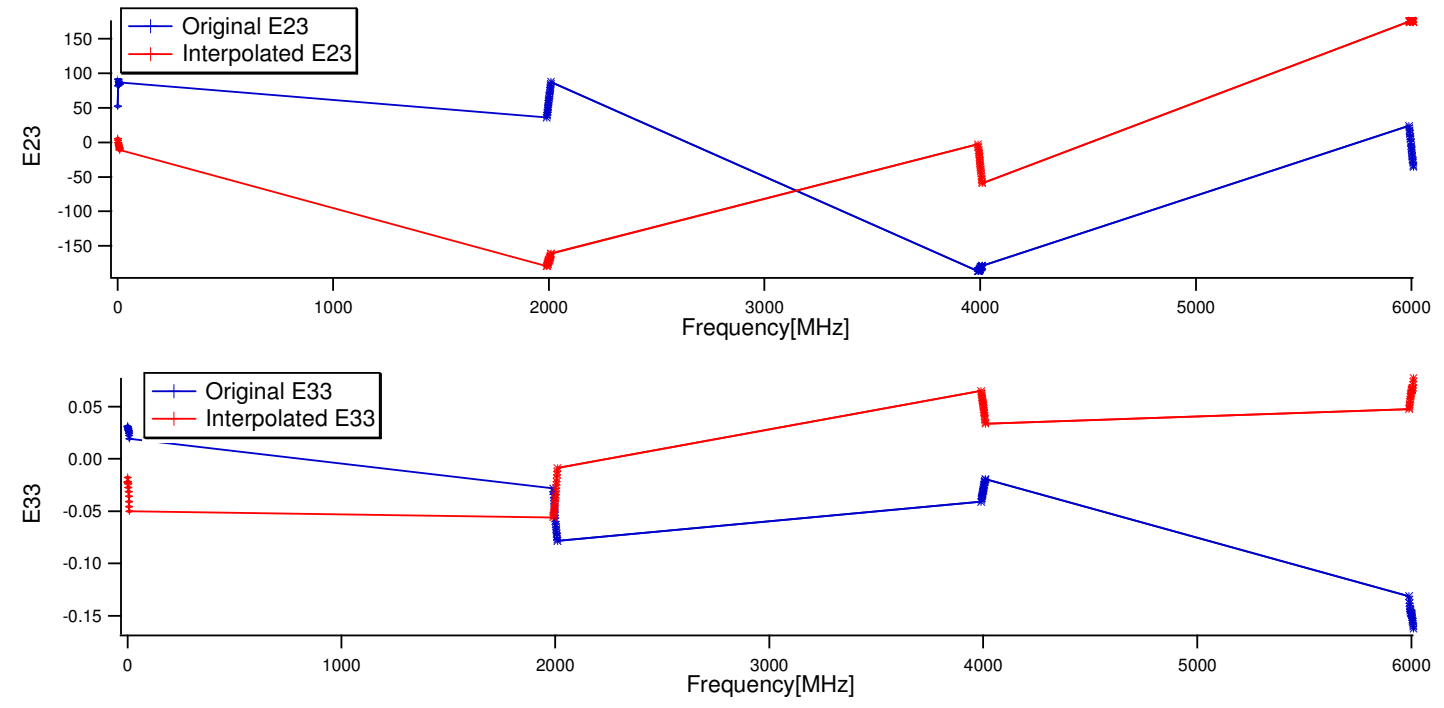

Figure A-2: The calibrated original and interpolated error terms 


\section{APPENDIX-B}

\section{IF Test-Set Calibration and Validation}

In order to measure a device it is of prime importance to minimize any error which a system can possibly introduce. Therefore to achieve accurate measurements of the device, error coefficients must be determined. The calibration procedure used for the small signal CAL is TRM (True - Reflect - Match) and for large signal Cal, extend two ports indirectly via sourcepull port is used. The IF test set is connected as shown in Figure1. AWG is used as the IF source and it was connected to IF Cal Switch.

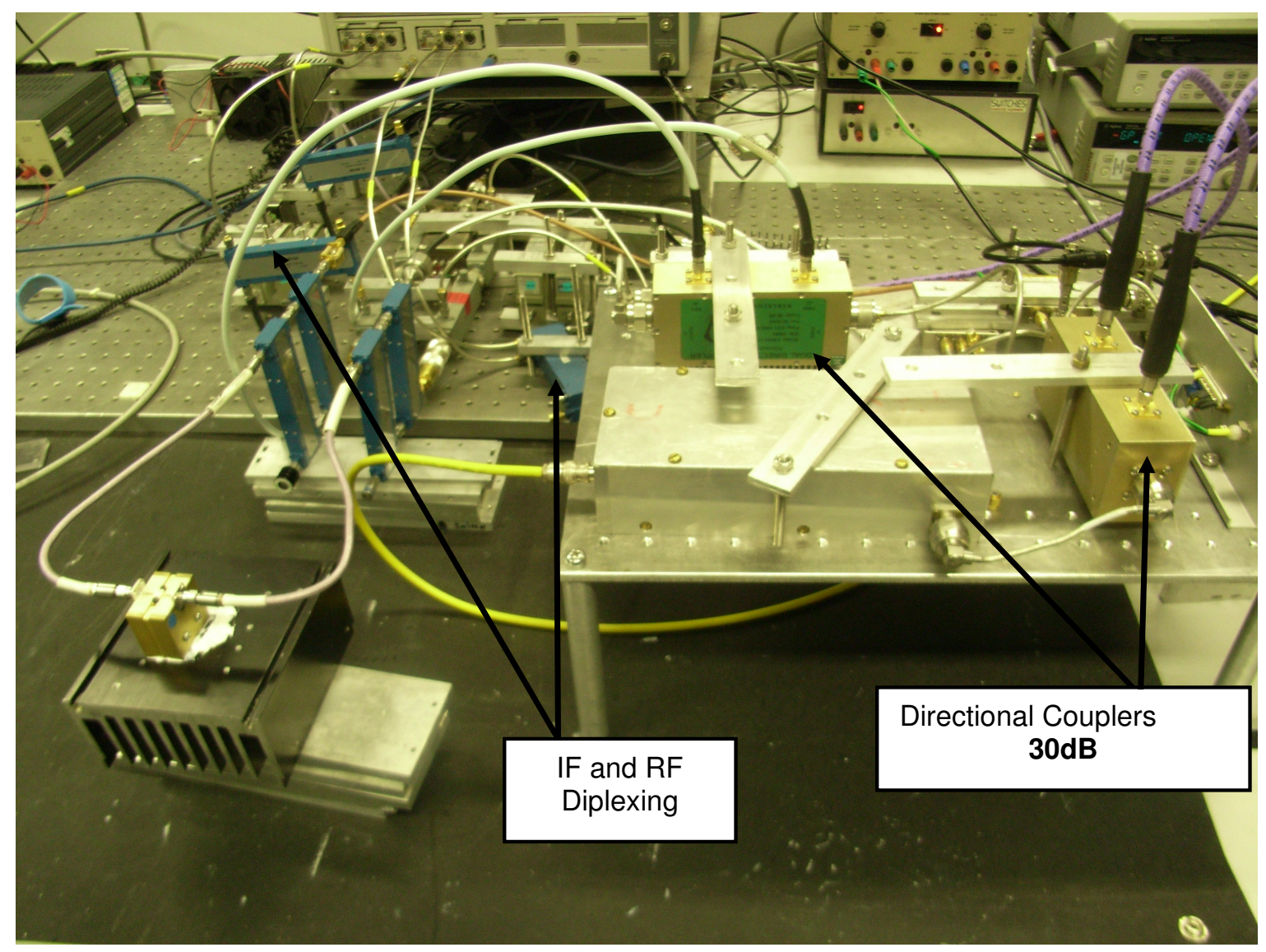

Figure B-1: Integration of IF test-set into the RF test-set

As can be seen from the figure the complete IF and RF calibration test set-up. In this complete configuration, there is a need to diplex the RF and IF travelling wave components before measurement and it is achieved by blue Krytar back-to-back couplers. 


\section{IF Full Calibration}

After the full calibration the Thru measurements with delay of $-16.584 p s e c$ are depicted in figure B-2.
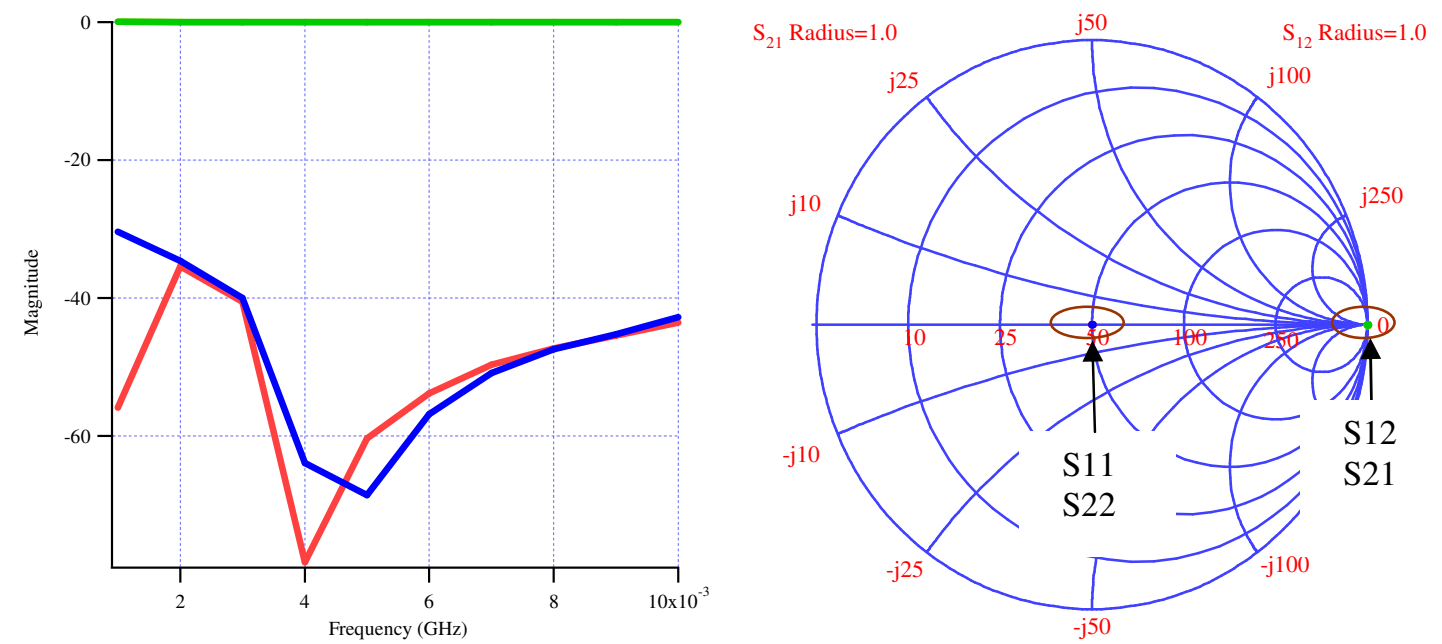

Figure B-2: Thru measurement for S-parameters

\section{Verification of IF Calibration}

\section{Step 1:}

To validate the IF Cal a short standard measurement is performed and S11, S22 can be seen perfectly on short location on smith chart pointed by circles.
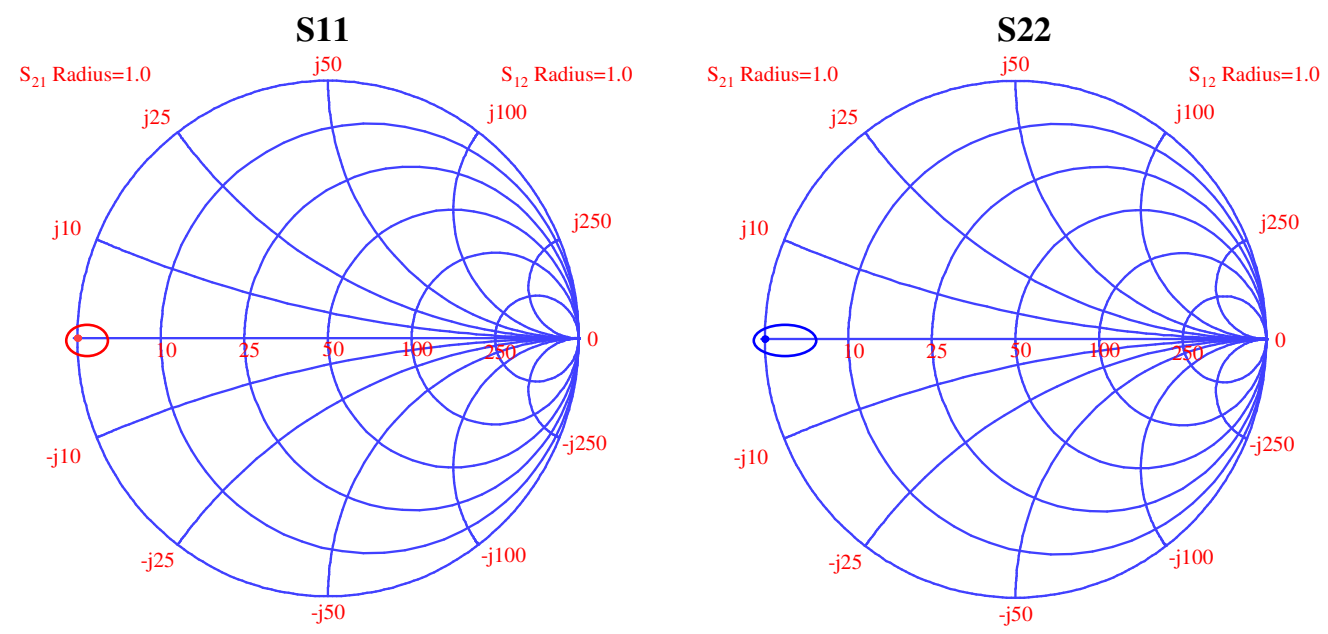

Similarly open standard measurement is performed in order to validate the IF full Calibration. 

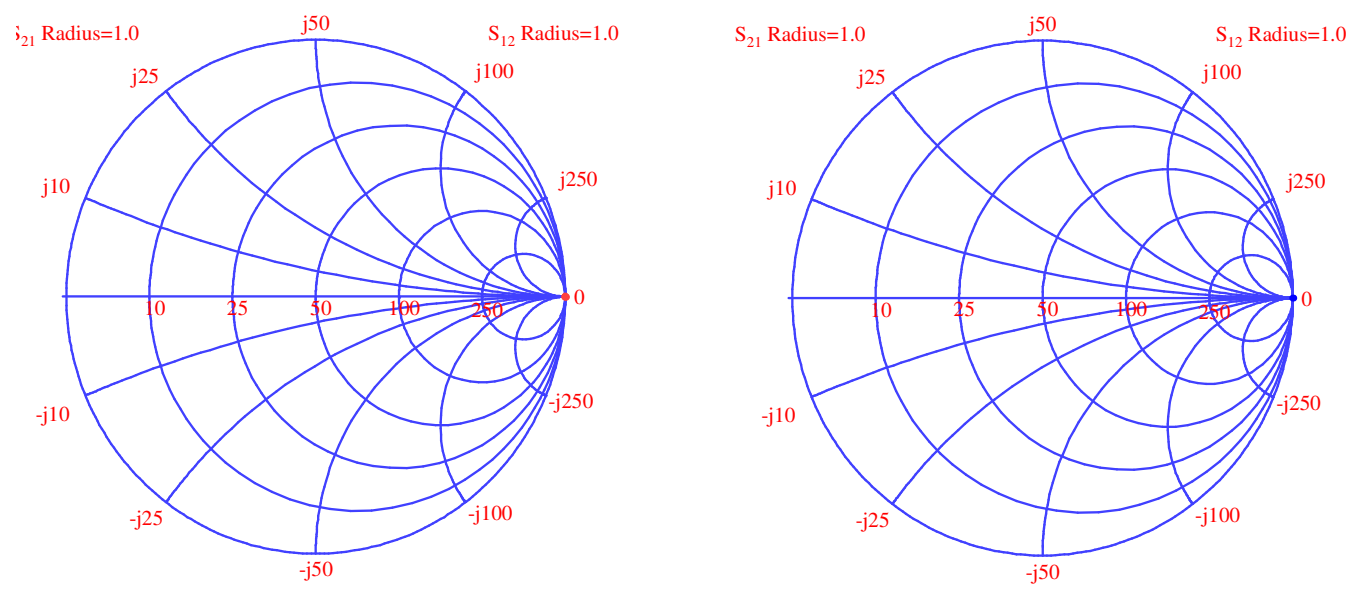

Match Measurement (S11, S22)

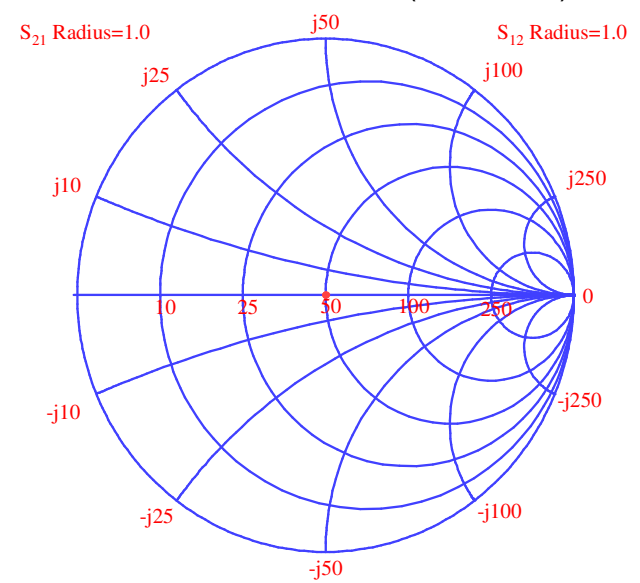

\section{Step 2:}

A cable of $4.94 n s e c$ delay (delay is measured through VNA 8753 for frequency ranging from $1-20 \mathrm{MHz}$ ) is used to perform off-set IF calibration verification.
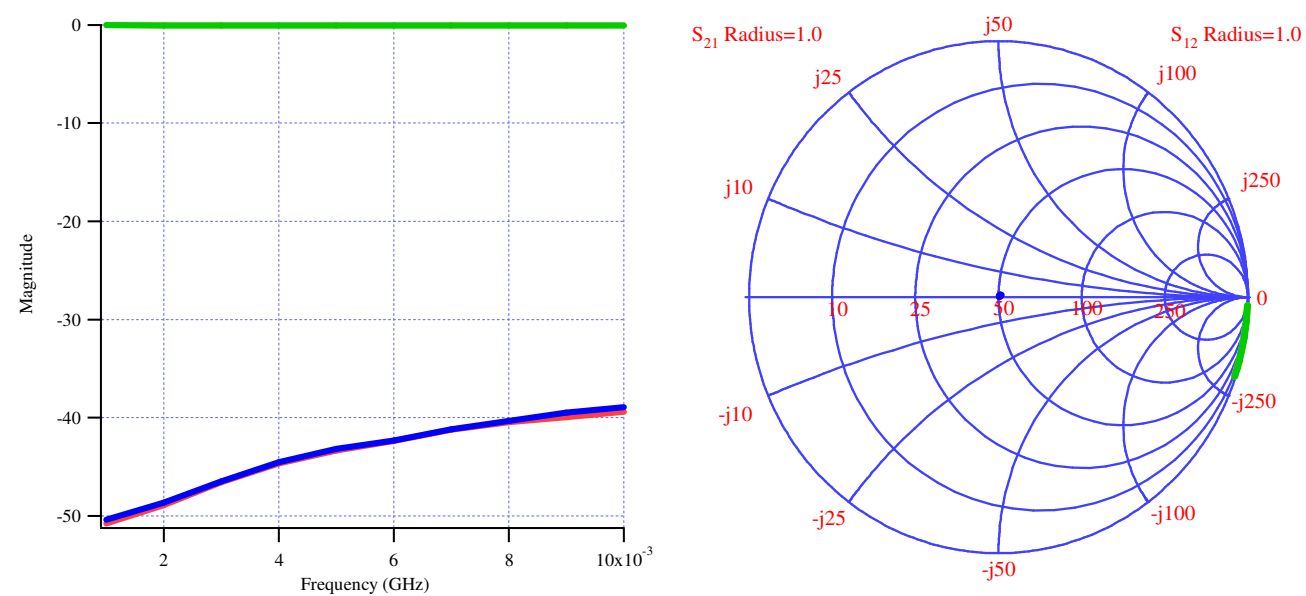

The measured S21 for thru is listed in Table B-1 and the delay is calculated by using B-1 and compared with the obtained through VNA 8753.

Phase Delay $=\Phi / 360^{*}$ Frequency

\begin{tabular}{|c|c|}
\hline Magnitude & Phase(Degrees) \\
\hline 0.999089 & 1.827038 \\
\hline
\end{tabular}




\begin{tabular}{|c|c|}
\hline 0.998033 & 3.633365 \\
\hline 0.997932 & 5.456631 \\
\hline 0.997736 & 7.264744 \\
\hline 0.997135 & 9.06323 \\
\hline 0.996747 & 10.846911 \\
\hline 0.996808 & 12.655551 \\
\hline 0.996419 & 14.441444 \\
\hline 0.996441 & 16.236152 \\
\hline 0.996035 & 18.031014 \\
\hline \multicolumn{2}{|l|}{ Table B-1: Measured S21 for Thru } \\
\hline
\end{tabular}

Measured delay through measurement system $=5 \mathrm{~ns}$ for all frequency ranges and measured delay on VNA $8753=4.94$ ns which shows an excellent match between both measured delays.

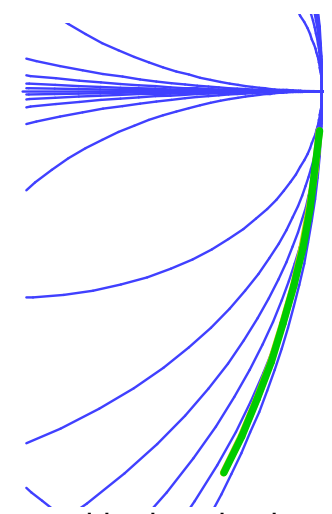

A close-up for delay near the open on smith chart is shown in figure depicted above.

\section{Step 3:}

An off-set short is measured using the IF calibration and the delay is calculated for each frequency spacing.

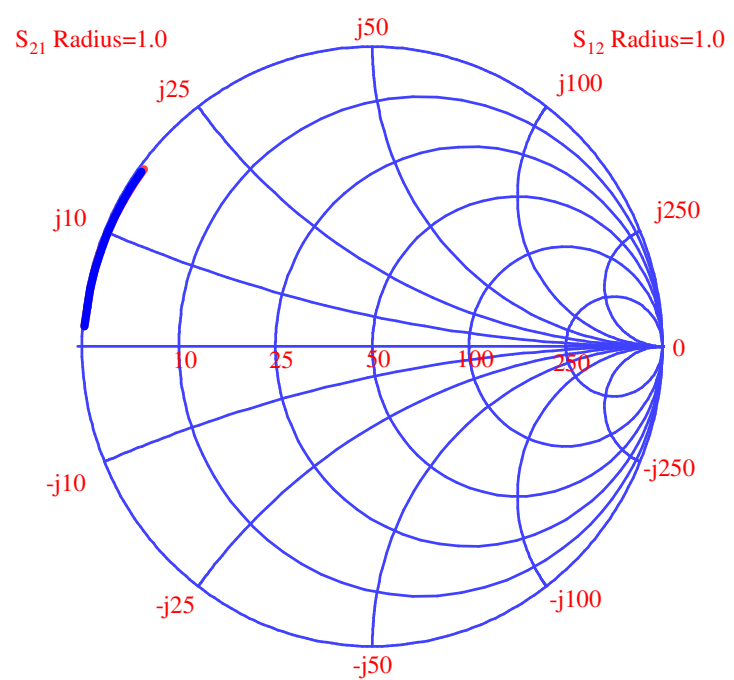

The measured S-parameters for both ports are listed in table B-2 and table B-3 which are very much same.

\begin{tabular}{|c|l|l|}
\hline \multicolumn{3}{|c|}{ Measured S11 } \\
\hline Frequency(MHz) & Magnitude & Phase \\
\hline 1 & 0.993958 & 176.129767 \\
\hline
\end{tabular}




\begin{tabular}{|c|l|l|}
\hline 2 & 0.991948 & 172.408392 \\
\hline 3 & 0.990433 & 168.733192 \\
\hline 4 & 0.989750 & 165.005774 \\
\hline 5 & 0.988915 & 161.324880 \\
\hline 6 & 0.987932 & 157.643689 \\
\hline 7 & 0.986946 & 153.972071 \\
\hline 8 & 0.985747 & 150.316109 \\
\hline 9 & 0.984504 & 146.687271 \\
\hline 10 & 0.983917 & 143.055001 \\
\hline
\end{tabular}

Table B-2: Measured S11 for short

\begin{tabular}{|c|l|l|}
\hline \multicolumn{3}{|c|}{ Measured S22 } \\
\hline Frequency $(\mathrm{MHz})$ & Magnitude & Phase \\
\hline 1 & 0.993803 & 176.121848 \\
\hline 2 & 0.991570 & 172.358148 \\
\hline 3 & 0.990119 & 168.848898 \\
\hline 4 & 0.989267 & 165.001684 \\
\hline 5 & 0.988124 & 161.338261 \\
\hline 6 & 0.987690 & 157.730461 \\
\hline 7 & 0.987248 & 154.161473 \\
\hline 8 & 0.986803 & 150.656734 \\
\hline 9 & 0.986369 & 147.206224 \\
\hline 10 & 0.986275 & 143.787029 \\
\hline
\end{tabular}

Table B-3: Measured S22 for short

The calculated delay based on the s-parameters listed in above both tables is approximately $10 \mathrm{~ns}$ which is twice of the previously calculated in case of thru.

\section{Step 4:}

Check the Beta file, it should be around 1 in magnitude and 0 in phase for all frequency list in order to achieve an excellent calibration.

\begin{tabular}{|c|l|l|}
\hline \multicolumn{3}{|c|}{ Beta File } \\
\hline Frequency $(\mathrm{MHz})$ & Magnitude & Phase \\
\hline 1 & 1.03388 & -0.0490795 \\
\hline 2 & 1.03066 & 0.0138316 \\
\hline 3 & 1.02828 & 0.0525044 \\
\hline 4 & 1.02549 & 0.0881534 \\
\hline 5 & 1.02122 & 0.119287 \\
\hline 6 & 1.01724 & 0.148774 \\
\hline 7 & 1.01239 & 0.177455 \\
\hline 8 & 1.00705 & 0.205179 \\
\hline 9 & 1.00145 & 0.232296 \\
\hline 10 & 0.994948 & 0.25914 \\
\hline
\end{tabular}

\section{Step 5:}

Table B-4: Beta file

Compare the error terms generated by the measurement system software with the actual attenuation or padding in the system. In case of IF, there is only $30 \mathrm{~dB}$ coupler is present in the source and load side which can be seen from the figure 1. However, the error terms obtained for transmission $\left(E_{10}\right)$ and reflection $\left(E_{01}\right)$ are found to be in agreement with the actual IF system attenuation. 

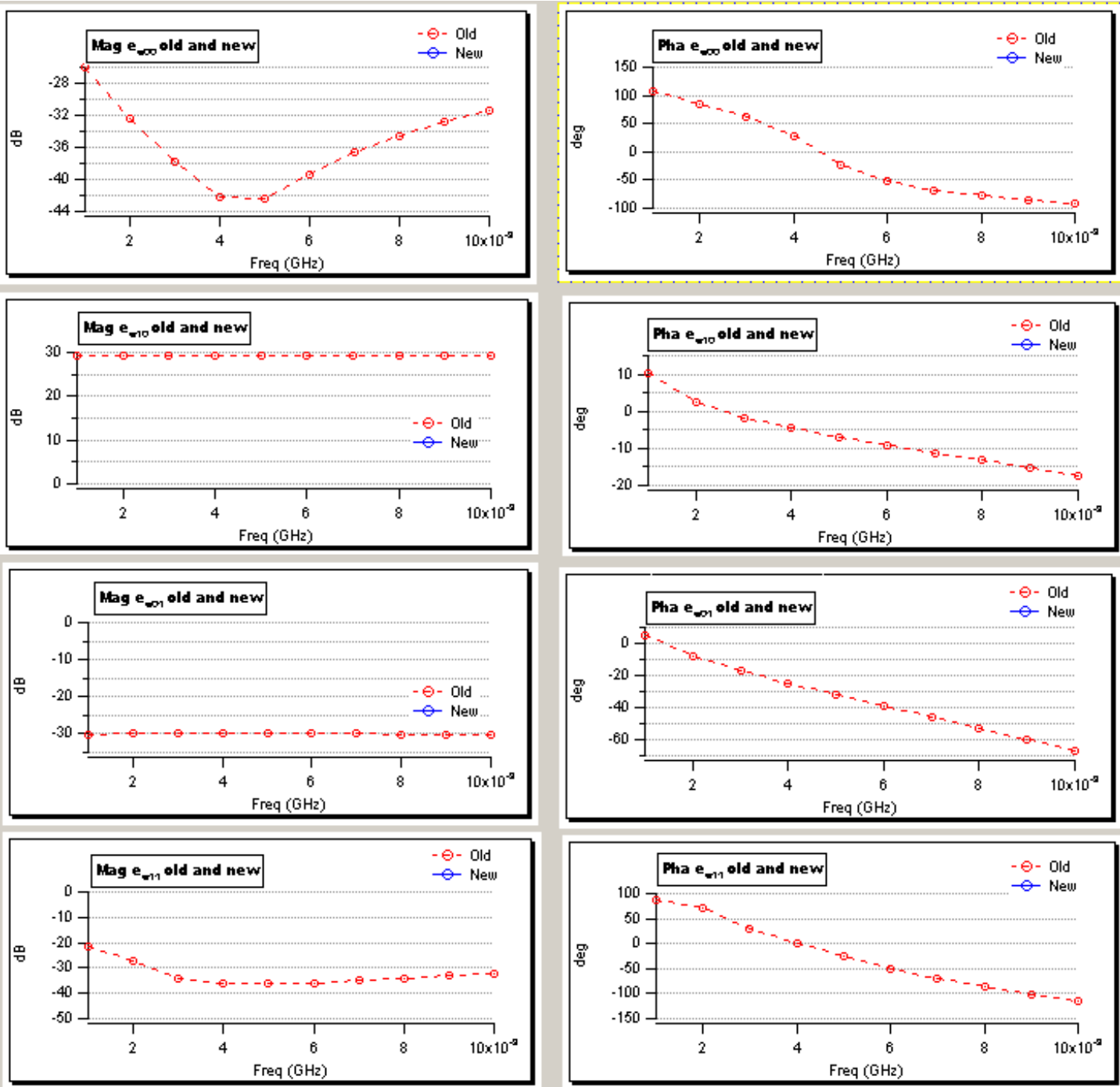

Figure B-3: Computed error terms on the input side
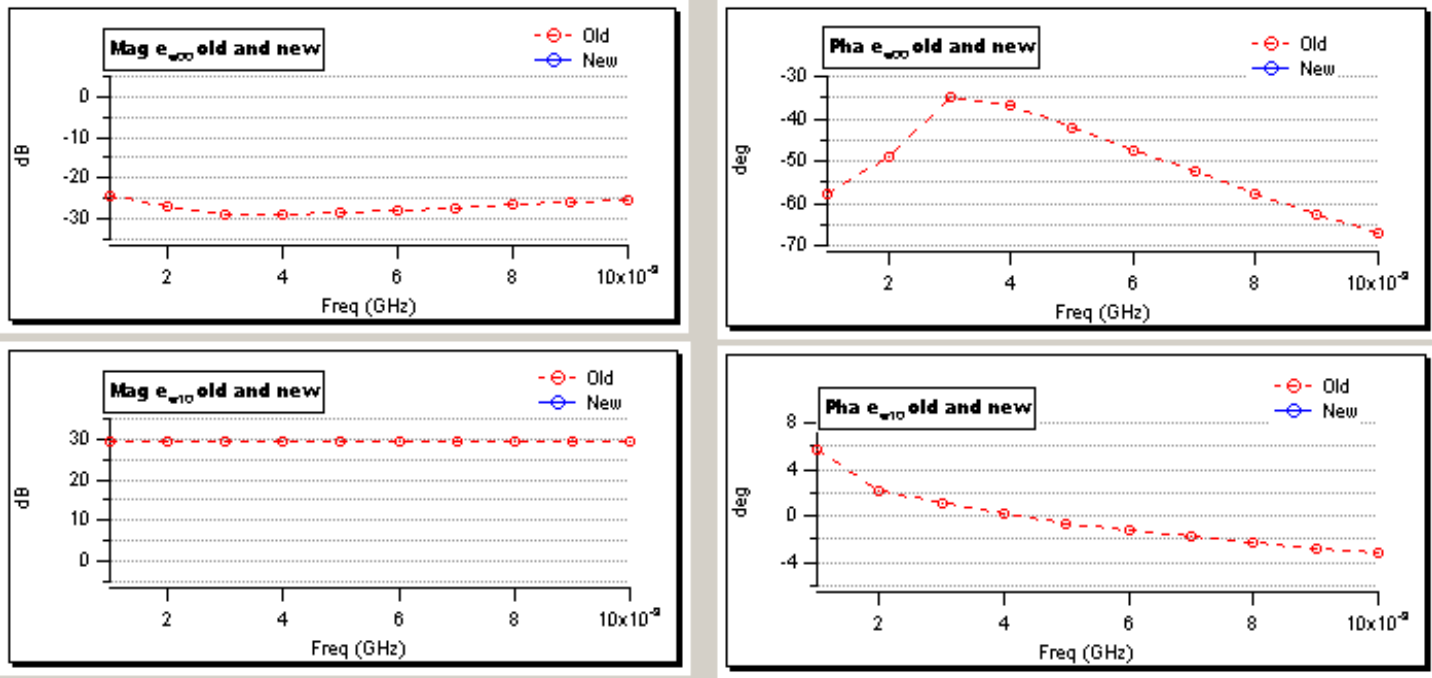

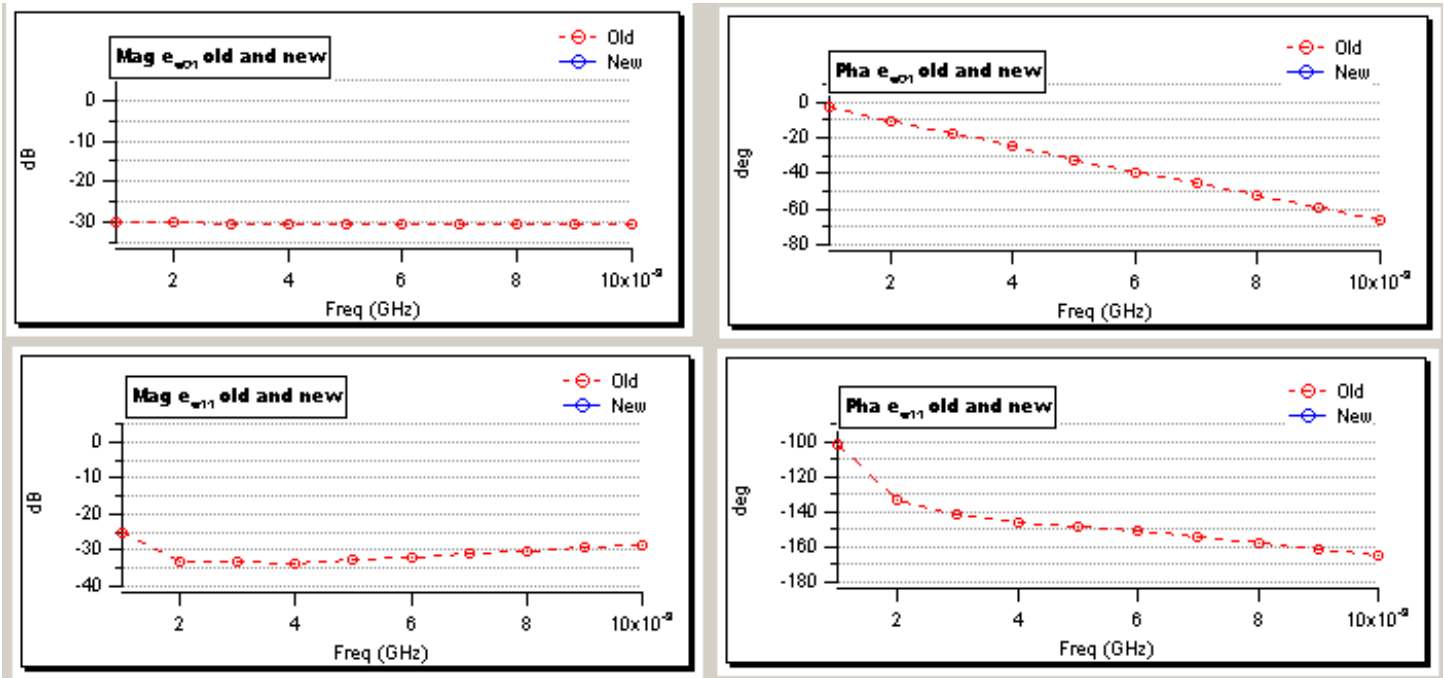

Figure B-3: Computed error terms on the output side

\section{Step 6:}

Finally, the calibration was loaded into the measurement software and performed the thru measurements. A $0 \mathrm{dBm}$ signal was injected at $1 \mathrm{MHz}$ and simple $50 \Omega$ line was used to calculate the power at the DUT plane (centre of the line was used as a reference). Moreover, an oscilloscope was used to measure the voltage for all the frequency spacing and then converted to the power using the formula giving in equation B-2.

Power $=\left(V_{p-p} / 2\right)^{2} / 2^{*} R$

The voltage measured at the centre of line with oscilloscope was $571 \mathrm{mV}$ and converted to average power.

Power $=\left(573^{*} 10^{-3} / 2\right) / 2^{*} 50=0.82 \mathrm{~mW}$

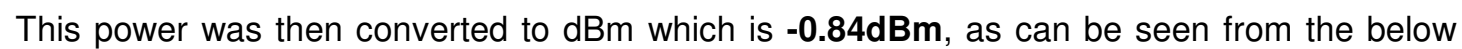

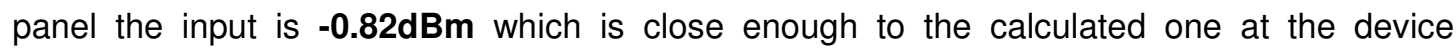
reference plane.

\begin{tabular}{|c|c|c|c|c|c|}
\hline \multicolumn{5}{|c|}{ Input Parameters } & \multirow[b]{2}{*}{ Impedance } \\
\hline & Input & Reflecteo & Load F & fl. Coef & \\
\hline IF & -0.827511 & -26.1404 & 0.0542446 & -78.6937 & 51.3617 \\
\hline Lower Tone & 4.93758 & -17.3737 & 0.0766361 & -160.199 & 43.3377 \\
\hline Fundamental & -42.4071 & -55.8863 & 0.211857 & -101.402 & 50.3143 \\
\hline Upper Tone & 4.81422 & -17.2663 & 0.0786995 & -162.228 & 43.0821 \\
\hline
\end{tabular}

Since a $50 \Omega$ line was measured so what power is at the input same was observed at the output. 


\section{APPENDIX-C}

\section{Tektronix Scope Setting}

Some control variations are required on the RF stimulus section for the multi-tone mode of operation. RF stimulus functionality of the panel allows the users to load and set the calibration parameters required to compute the number of windows for custom automated measurement of one complete modulation cycle.

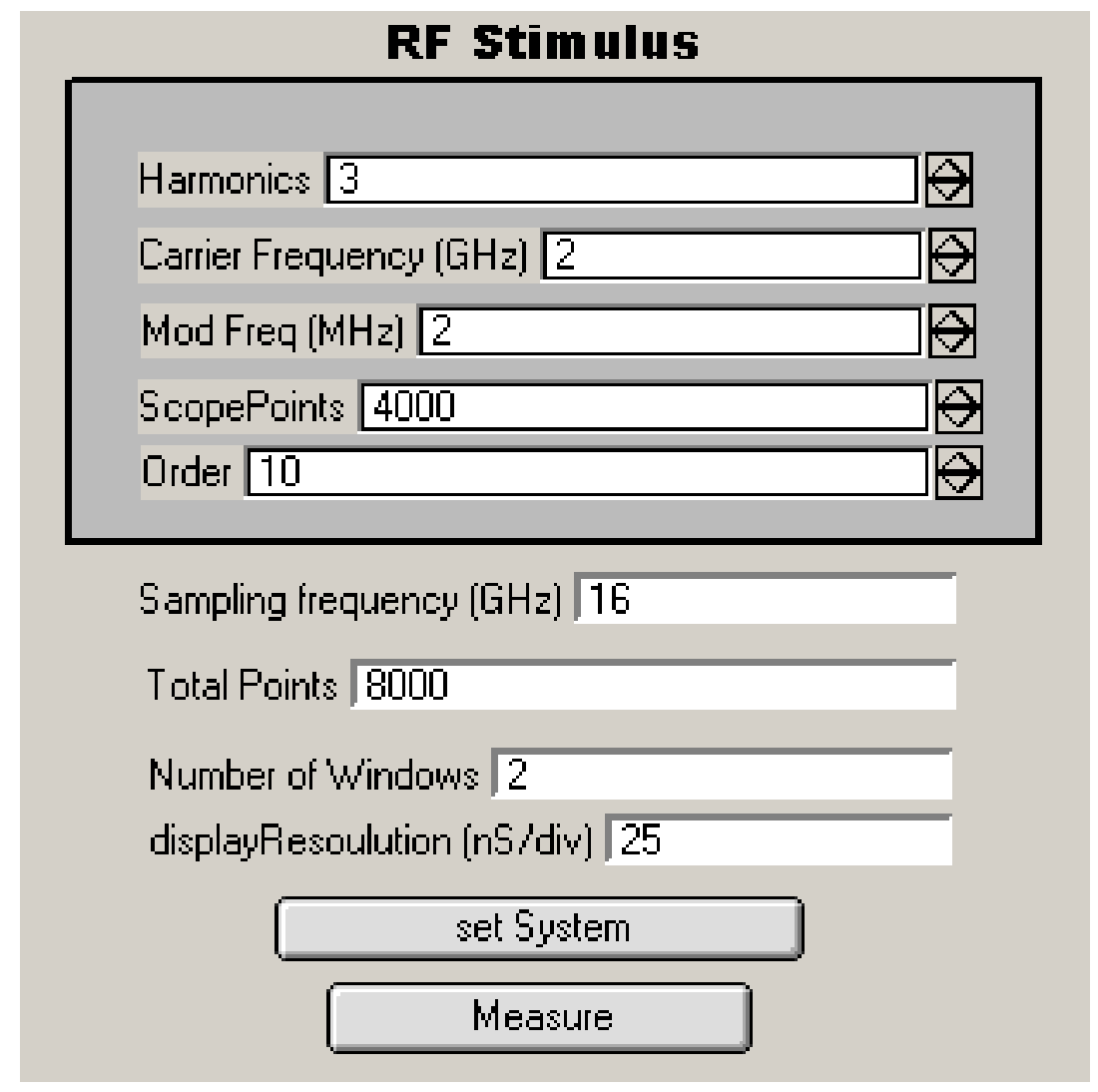

Figure C-1: RF Stimulus settings for multi-tone measurements

There are two main tabs on the panel as shown in Figure C-1 which are "Set System" and "Measure"

Set System sends the parameters values to Tektronix Scope Setting panel and sets the scope for modulated measurements

Measure is a common button switch with the button "Trigger a New measurement" on Tektronix Scope Setting panel depicted in Figure C-2. It sets the scope to measures the modulated time domain waveforms effectively.

The Tektronix scope panel allows setting the scope for the measurements of modulated signals. The measurement settings are determined in their respective tabs. 


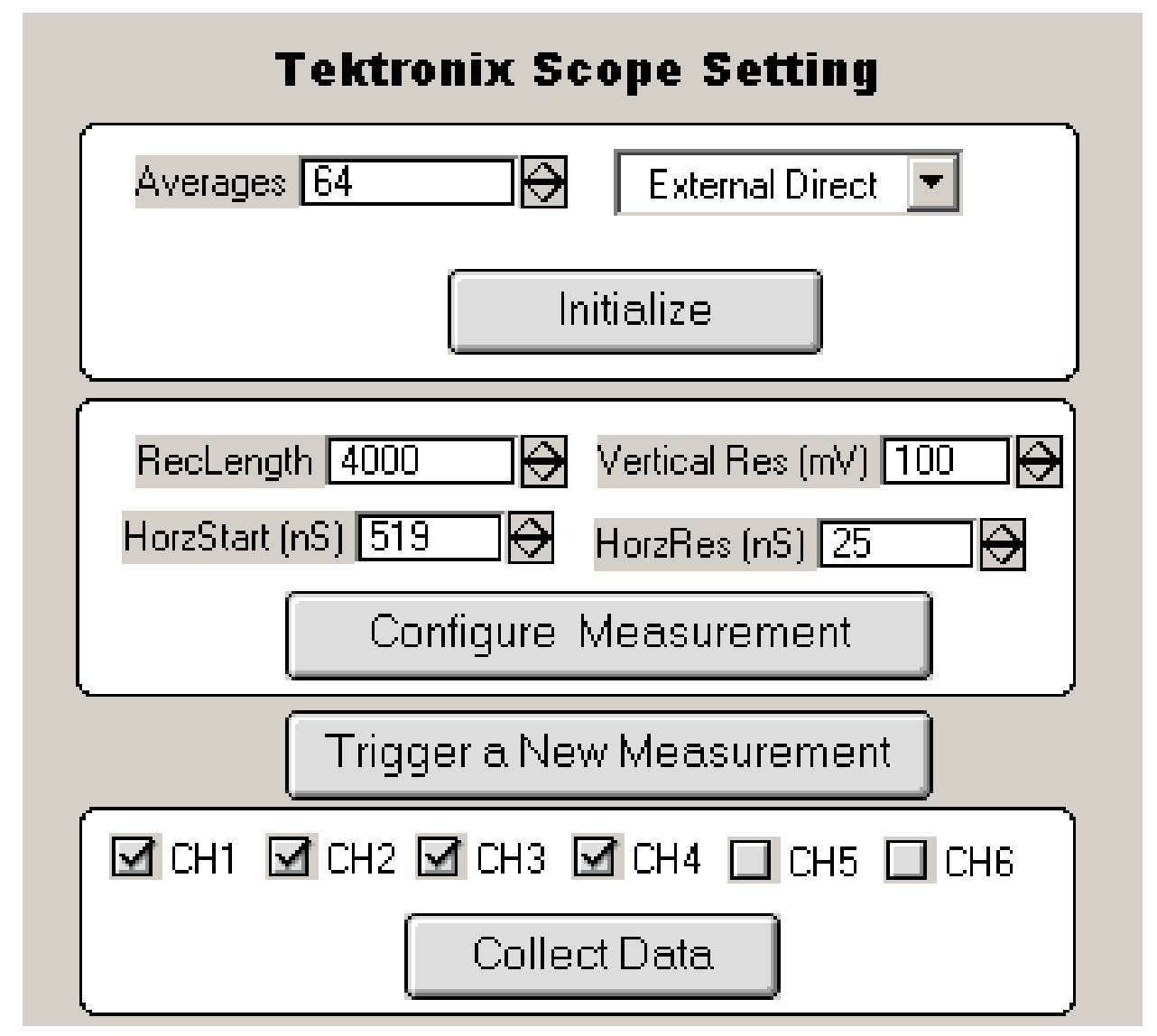

Figure C-2: The main interface panel for Tektronix scope setting

As shown in Figure C-2, there are four sections of functions that can be controlled via the interface. They are "Initialize", "Load Configure Measurement", "Trigger a New Measurement", and "Collect Data".

Initialize initializes the Tektronix scope by restoring the default settings and clear all the previously set variables.

Configure Measurement sets the averages, type of trigger, scope record length, vertical resolution and horizontal resolution of the scope which were computed from RF stimulus parameters.

Trigger a New Measurement triggers the scope for a measurement using the external high quality trigger clock.

Collect Data collects the raw data measured by the scope with different external parameters setting such as RF frequency, input power etc. 


\section{APPENDIX-D}

\section{Modulated Waveform Measurements}

Main Modulated Measurement panel provides the user interface to perform the modulated waveform measurement and display of the measured results in time and envelope domains. It also displays the measured power performance of the device under test (DUT).

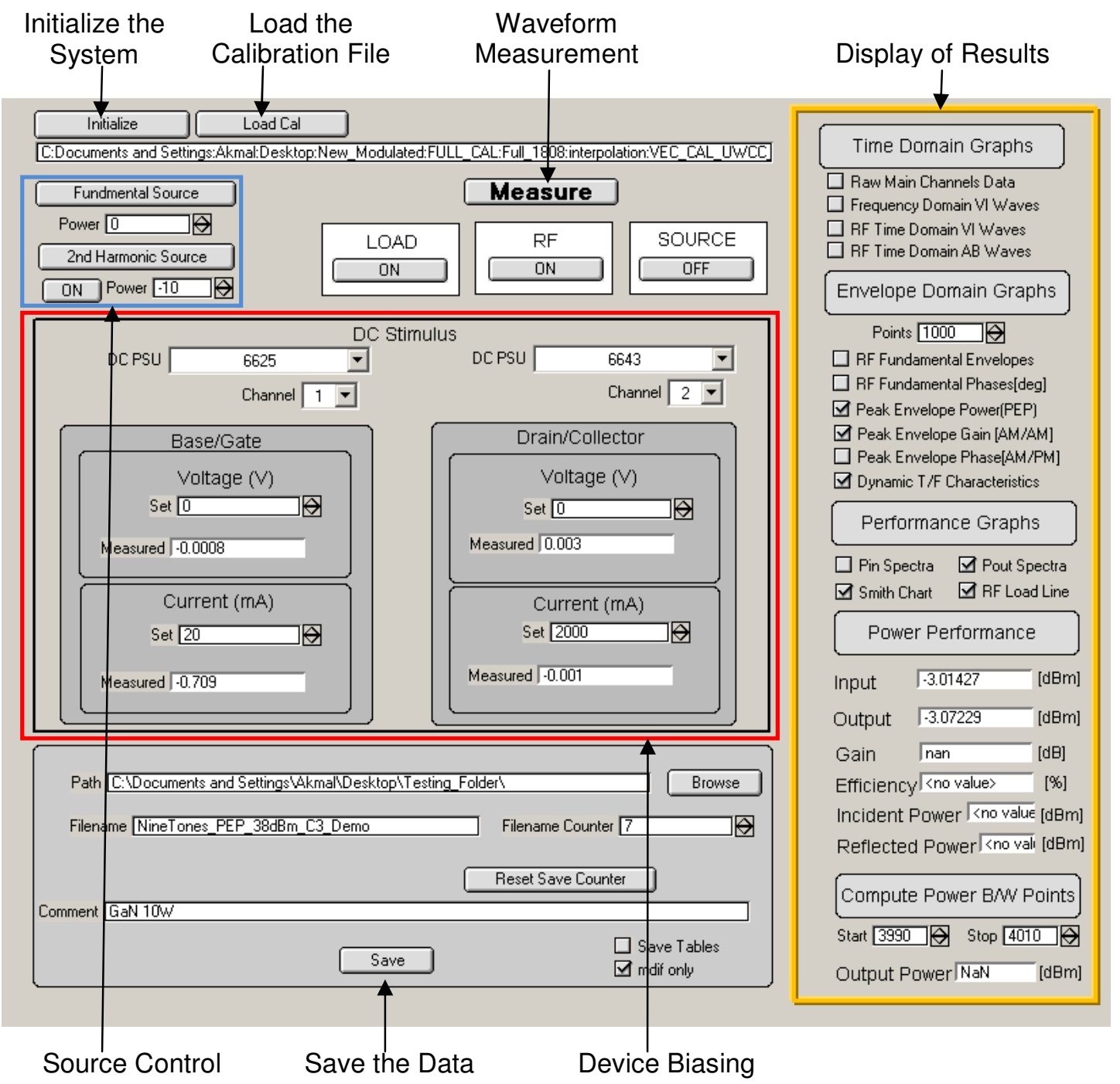

Figure D-1: The main user panel for measurement software

Initialize the System Enables IGOR to access the GBIP Bus and configures the measurement system instrumentation and switches. Control of the GPIB bus can also be achieved without an instrument and switch reset using SetupGPIB in the main menu.

Load the Calibration File opens the calibration location directory and loads the interpolated calibration file. 
Source Control changes the power and fundamental frequency in accordance with the panel depicted in Figure D-2. It downloads new values of the magnitude and phase to the source ESG. The RF ON/OFF button toggles the state for source as well as for $2^{\text {nd }}$ harmonic ESG.

Device Biasing Provides independent control of all the channels of the relevant DC power Supply. Allows biasing the device by changing the base voltage and drain voltage simultaneously and the measured data will be mathematically inserted into the RF waveforms.

Waveform Measurement performs a measurement at device reference plane and continuously measure the input or output voltage and current waveforms without switching ports. Measures the magnitude and phase of the travelling waveforms at the fundamental, second and third harmonic frequencies.

Save the Data determines the saved data location and allows the user to name the files.

Display of Results allows user to display a Smith chart and other graphs in time domain as well as in envelope domain.

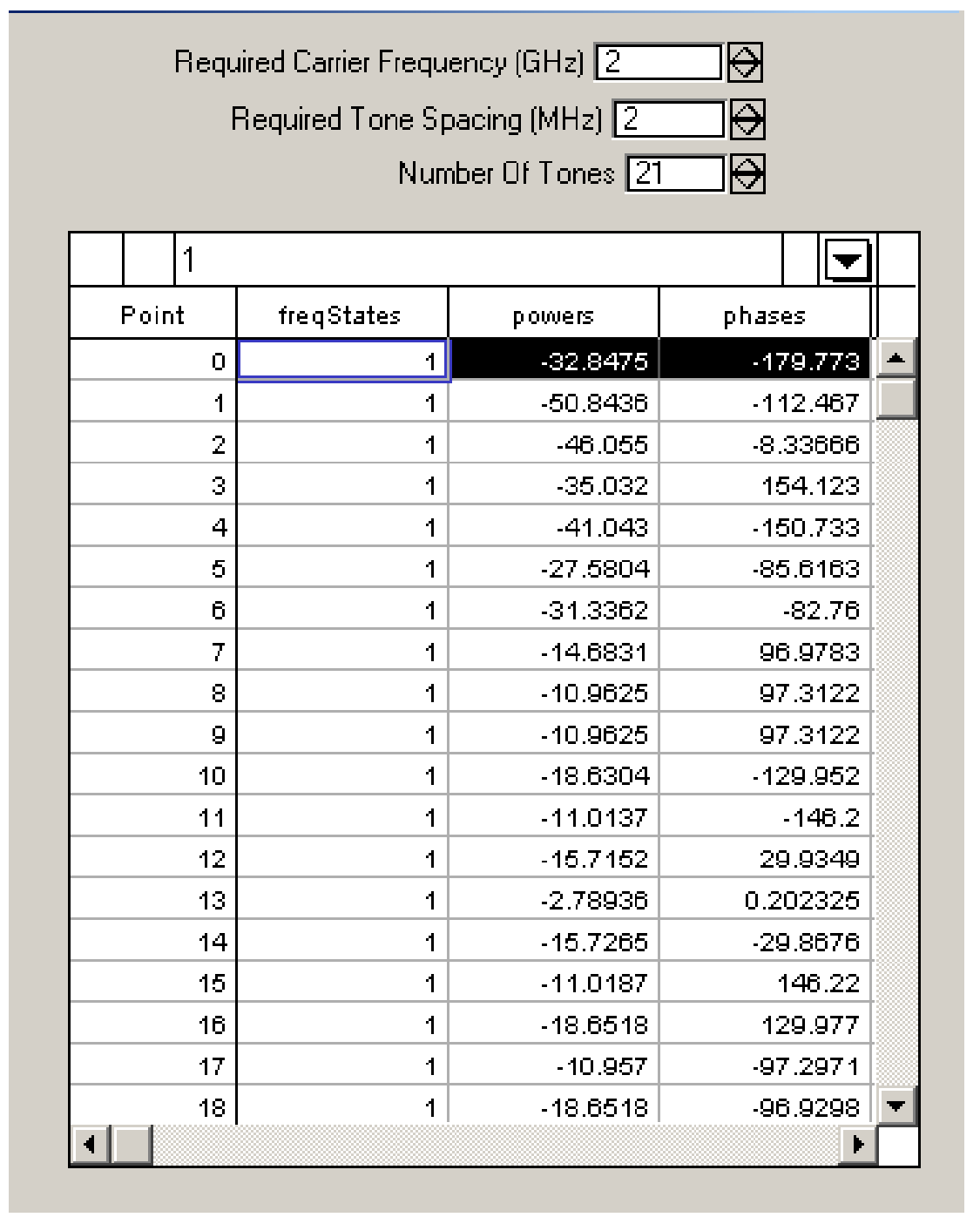

Figure D-2: The table to define the magnitude and phase of multi-tone signal to set the ESG 


\section{APPENDIX-E}

\section{Multi-Sine Stimuli}

\begin{tabular}{|c|c|c|}
\hline \multicolumn{3}{|c|}{ NINE TONE } \\
\hline No. & Amplitude & Phase \\
\hline 1 & -21.12471234 & -297.11 \\
\hline 2 & -37.84453698 & -115.4217 \\
\hline 3 & -33.69887126 & -18.21586 \\
\hline 4 & -14.02345689 & 214.568 \\
\hline 5 & -21.99012412 & -70.124 \\
\hline 6 & -19.04589687 & -125.745 \\
\hline 7 & -11.12366545 & -282.356 \\
\hline 8 & -22.63055897 & 145.389 \\
\hline 9 & -13.61243475 & 45.6235 \\
\hline
\end{tabular}

\begin{tabular}{|c|c|c|}
\hline No. & Amplitude & Phase \\
\hline 1 & -32.84750314 & -179.773 \\
\hline 2 & -50.84362168 & -112.467 \\
\hline 3 & -46.05498861 & -8.33666 \\
\hline 4 & -35.03202286 & 154.1229 \\
\hline 5 & -41.04299412 & -150.733 \\
\hline 6 & -27.58043687 & -85.6163 \\
\hline 7 & -31.33618015 & -82.76 \\
\hline 8 & -14.68307587 & 96.97827 \\
\hline 9 & -10.96253445 & 97.31224 \\
\hline 10 & -18.63043299 & -129.952 \\
\hline 11 & -11.01373613 & -146.2 \\
\hline 12 & -15.7151541 & 29.93485 \\
\hline 13 & -2.789364444 & 0.202325 \\
\hline 14 & -15.72651324 & -29.8676 \\
\hline 15 & -11.01874641 & 146.2201 \\
\hline 16 & -18.65182229 & 129.9766 \\
\hline 17 & -10.95701386 & -97.2971 \\
\hline
\end{tabular}




\begin{tabular}{|l|l|l|}
\hline 18 & -14.68251158 & -96.9298 \\
\hline 19 & -31.37801839 & 83.16438 \\
\hline 20 & -27.57500395 & 85.78428 \\
\hline 21 & -41.04183545 & 148.9179 \\
\hline 22 & -34.94699862 & -153.571 \\
\hline 23 & -46.73607638 & 8.974042 \\
\hline 24 & -50.39808355 & 111.384 \\
\hline
\end{tabular}




\section{APPENDIX-F}

\section{Complementary Cumulative Distribution Function (CCDF)}

The following code calculates the complementary cumulative distribution function and can be run as an m-file using Matlab.

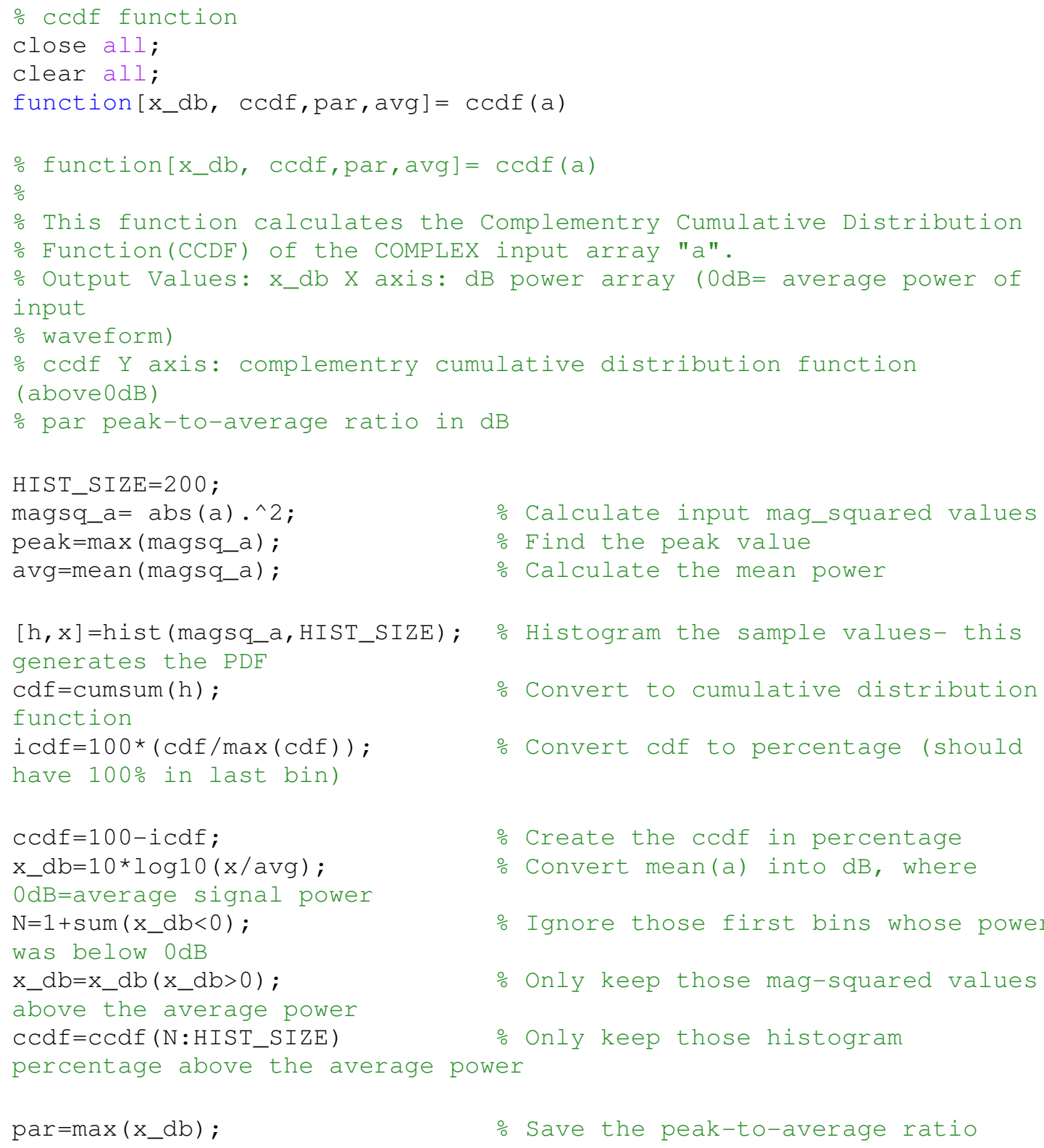




\section{APPENDIX-G}

\section{Tektronix AWG Control}

The detailed explanation of the options available in the Tektronix arbitrary waveform generator (AWG) software is listed on the panel.

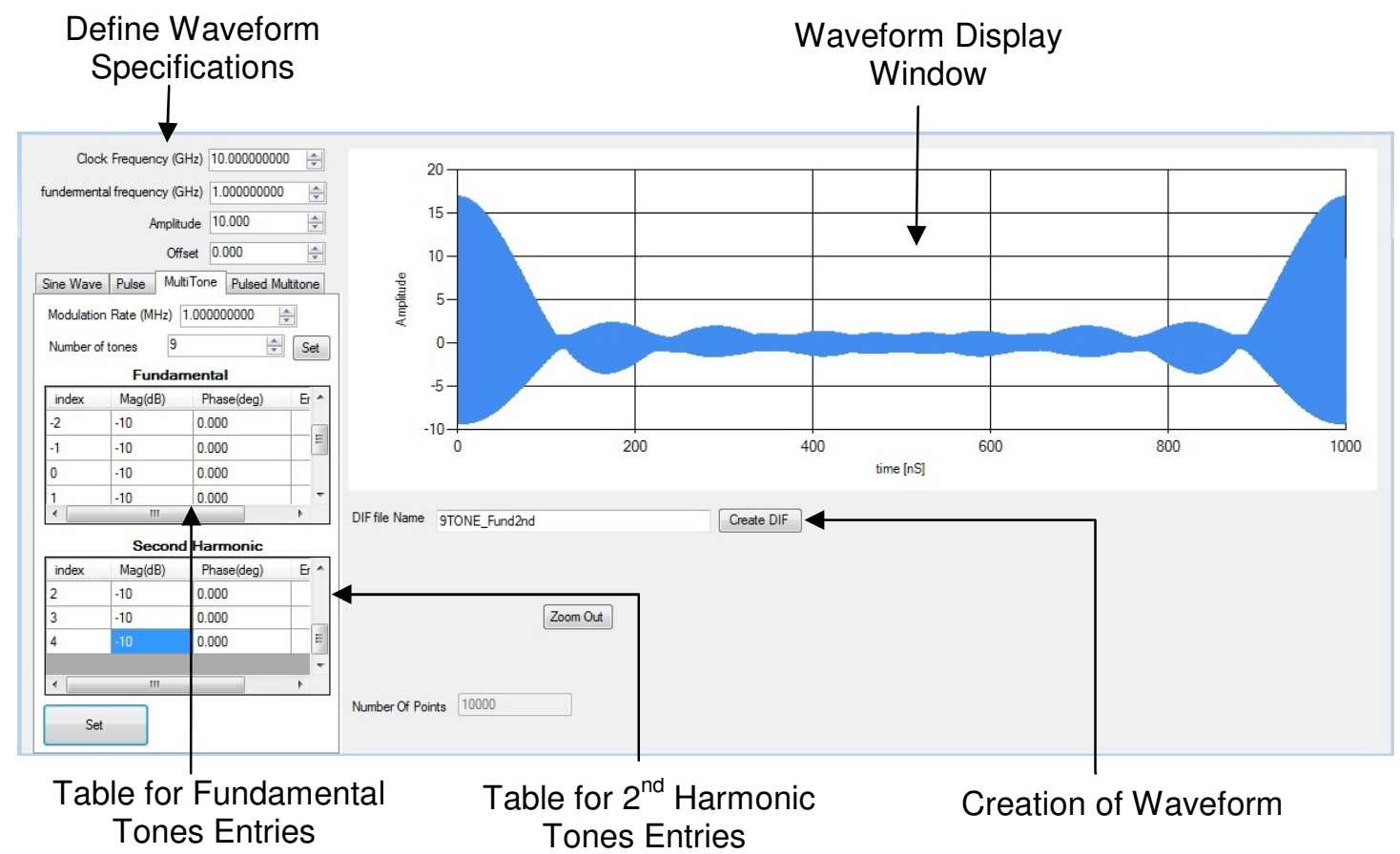

Figure G-1: The main user panel for AWG control software

Define Waveform Specifications allows setting the clock frequency and fundamental frequency of the waveform. The amplitude and offset of the waveform relative to zero crossing of the waveform can also be defined.

Waveform Display Window displays the waveform and for each edit in waveform magnitude and phase of the fundamental tones or $2^{\text {nd }}$ harmonic tones automatically updates the waveform.

Table for Fundamental Tones Entries defines the magnitude and phase of each individual tone at fundamental frequency.

Table for $2^{\text {nd }}$ Harmonic Tones Entries defines the magnitude and phase of each individual tone at $2^{\text {nd }}$ harmonic frequency.

Creation of Waveform creates the waveform with the defined values of each tone at fundamental and $2^{\text {nd }}$ harmonic frequencies and downloads to the AWG 


\section{APPENDIX-H}

\section{W, RF Power GaN HEMT}

\section{CFEEE상}

\section{CGH40010}

10 W, RF Power GaN HEMT

Cree's CGH40010 is an unmatched, gallium nitride (GaN) high electron mobility transistor (HEMT). The CGH40010, operating from a 28 volt rail, offers a general purpose, broadband solution to a variety of RF and microwave applications. GaN HEMTs offer high efficiency, high gain and wide bandwidth capabilities making the CGH40010 ideal for linear and compressed amplifier circuits. The transistor is available in both screw-down, flange and solderdown, pill packages.

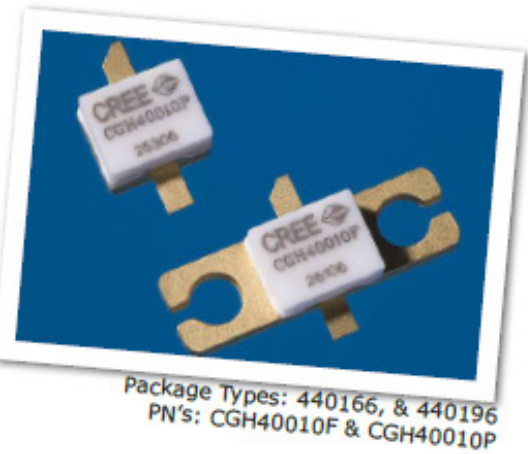

\section{FEATURES}

- Up to $6 \mathrm{GHz}$ Operation

- $16 \mathrm{~dB}$ Small Signal Gain at $2.0 \mathrm{GHz}$

- $14 \mathrm{~dB}$ Small Signal Gain at $4.0 \mathrm{GHz}$

- 13 W typical PAT $_{\text {SAT }}$

- 65 \% Efficiency at $P_{\text {SAT }}$

- 28 v Operation

\section{APPLICATIONS}

- 2-Way Private Radio

- Broadband Amplifiers

- Cellular Infrastructure

- Test Instrumentation

- Class A, AB, Linear amplifiers suitable for OFDM, W-CDMA, EDGE, CDMA waveforms 


\section{CFFE토승}

Absolute Maximum Ratings (not simultaneous) at $25^{\circ} \mathrm{C}$ Case Temperature

\begin{tabular}{|c|c|c|c|}
\hline Parameter & Symbol & Rating & Units \\
\hline Drain-Source Voltage & $v_{\mathrm{Das}}$ & 84 & Volts \\
\hline Gate-to-Source Voltage & $v_{c s}$ & $-10,+2$ & Volts \\
\hline Storage Temperature & $T_{\text {stac }}$ & $-65,+150$ & ${ }^{\circ} \mathrm{C}$ \\
\hline Operating Junction Temperature & $T_{3}$ & 225 & ${ }^{\circ} \mathrm{C}$ \\
\hline Maximum Forward Gate Current & $I_{\text {anax }}$ & 4.0 & $\mathrm{~mA}$ \\
\hline Soldering Temperature ${ }^{1}$ & $T_{s}$ & 245 & ${ }^{\circ} \mathrm{C}$ \\
\hline Screw Torque & $\tau$ & 60 & in-oz \\
\hline Thermal Resistance, Junction to Case & $R_{\infty e c}$ & 8.0 & ${ }^{\circ} \mathrm{C} / \mathrm{w}$ \\
\hline Case Operating Temperature.2. & $T_{c}$ & $-40,+150$ & ${ }^{\circ} \mathrm{C}$ \\
\hline
\end{tabular}

Note:

${ }^{1}$ Refer to the Application Note on soldering at www.cree.com/products/wireless_appnotes,asp

${ }^{2}$ Measured for the $\mathrm{CGH} 40010 \mathrm{~F}$ at $\mathrm{P}_{\text {DISS }}=14 \mathrm{~W}$.

${ }^{3}$ See also, the Power Dissipation De-rating Curve on Page 6.

Electrical Characteristics $\left(\mathrm{T}_{\mathrm{c}}=25^{\circ} \mathrm{C}\right)$

\begin{tabular}{|c|c|c|c|c|c|c|}
\hline Characteristics & Symbol & Min. & Typ. & Max. & Units & Conditions \\
\hline \multicolumn{7}{|l|}{ DC Characteristics ${ }^{1}$} \\
\hline Gate Threshold Voltage & $v_{\text {GSP(D) }}$ & -3.8 & -3.3 & -2.3 & $\mathrm{v}_{\mathrm{DC}}$ & $\mathrm{V}_{\mathrm{bos}}=10 \mathrm{~V}, \mathrm{I}_{\mathrm{o}}=3.6 \mathrm{~mA}$ \\
\hline Gate Quiescent Voltage & $v_{\text {canes }}$ & - & -3.0 & - & $\mathrm{v}_{\mathrm{DC}}$ & $V_{D S}=28 \mathrm{~V}, I_{0}=200 \mathrm{~mA}$ \\
\hline Saturated Drain Current & $\mathrm{I}_{\mathrm{ss}}$ & 2.9 & 3.5 & - & A & $V_{D s}=6.0 \mathrm{~V}, V_{G s}=2.0 \mathrm{~V}$ \\
\hline Drain-Source Breakdown Voltage & $v_{\mathrm{se}}$ & 120 & - & - & $\mathrm{v}_{\mathrm{DC}}$ & $V_{c G}=-8 \mathrm{~V}, I_{\mathrm{b}}=3.6 \mathrm{~mA}$ \\
\hline \multicolumn{7}{|c|}{ RF Characteristics ${ }^{2}\left(\mathrm{~T}_{\mathrm{c}}=25^{\circ} \mathrm{C}, \mathrm{F}_{\mathrm{q}}=3.7 \mathrm{GHz}\right.$ unless otherwise noted) } \\
\hline Small Signal Gain & $\mathrm{G}_{\mathrm{ss}}$ & 12.5 & 14.5 & - & $d B$ & $\mathrm{~V}_{D 0}=2 B \mathrm{~V}, \mathrm{I}_{\mathrm{DQ}}=200 \mathrm{~mA}$ \\
\hline Power Output ${ }^{2}$ & $P_{\text {ser }}$ & 10 & 12.5 & - & w & $v_{\infty O}=28 \mathrm{~V}, I_{D Q}=200 \mathrm{~mA}$ \\
\hline Drain Efficiency & $\pi$ & 55 & 65 & - & \% & $V_{D 0}=28 \mathrm{~V}, I_{D Q}=200 \mathrm{~mA}, P_{S A O}$ \\
\hline Output Mismatch Stress & VSWR & - & - & $10: 1$ & $\Psi$ & $\begin{array}{l}\text { No damage at all phase angles, } \\
\mathrm{V}_{\infty 0}=28 \mathrm{~V}, \mathrm{I}_{\mathrm{os}}=200 \mathrm{~mA}, \\
P_{\text {out }}=10 \mathrm{~W} \mathrm{CW}\end{array}$ \\
\hline \multicolumn{7}{|l|}{ Dynamic Characteristics } \\
\hline Input Capacitance & $c_{\text {cos }}$ & - & 4.5 & - & pF & $V_{o s}=28 \mathrm{~V}, V_{o f}=-8 \mathrm{~V}, f=1 \mathrm{MHz}$ \\
\hline Output Capacitance & $C_{s s}$ & - & 1.3 & - & pF & $v_{0 s}=28 \mathrm{~V}, v_{\mathrm{ct}}=-8 \mathrm{~V}, \mathrm{f}=1 \mathrm{MHz}$ \\
\hline Feedback Capacitance & $C_{c D}$ & - & 0.2 & - & pF & $v_{\mathrm{Ds}}=28 \mathrm{~V}, \mathrm{v}_{\mathrm{ef}}=-8 \mathrm{~V}, \mathrm{f}=1 \mathrm{MHz}$ \\
\hline
\end{tabular}

Notes:

${ }^{1}$ Measured on wafer prior to packaging.

2 Measured in CGH40010-TB.

${ }^{3} P_{\text {SAT }}$ is defined as $I_{G}=0.36 \mathrm{~mA}$.

4 Drain Efficiency $=\mathrm{P}_{\text {our }} / \mathrm{P}_{\mathrm{DC}}$

$$
\text { CGH40010 Rev } 3.1
$$




\section{CFFE토응}

Typical Performance

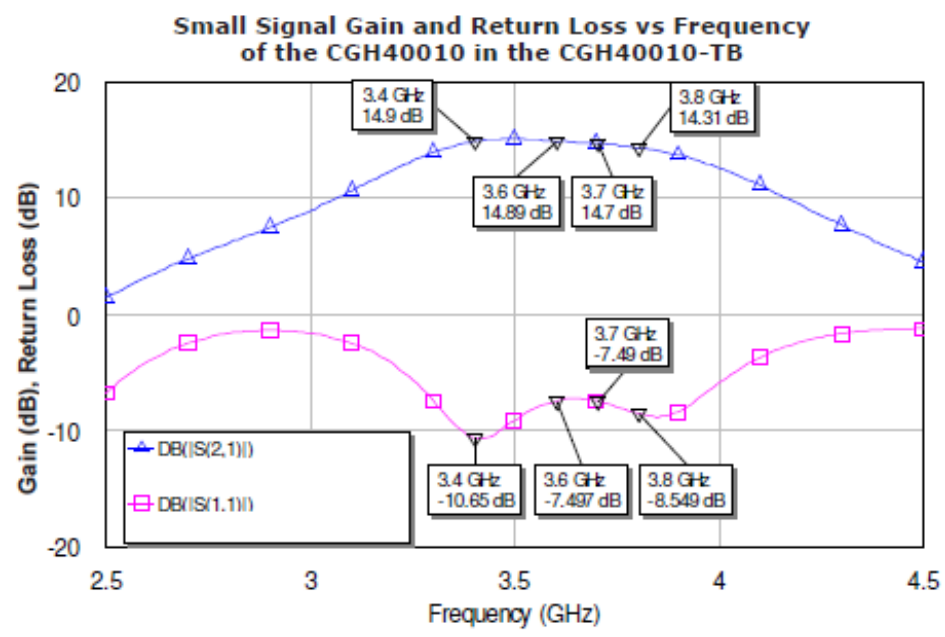

$P_{\text {SAT }}$ Gain, and Drain Efficiency vs Frequency of the CGH40010F in the CGH40010-TB $V_{D D}=28 \mathrm{~V}, I_{D Q}=200 \mathrm{~mA}$

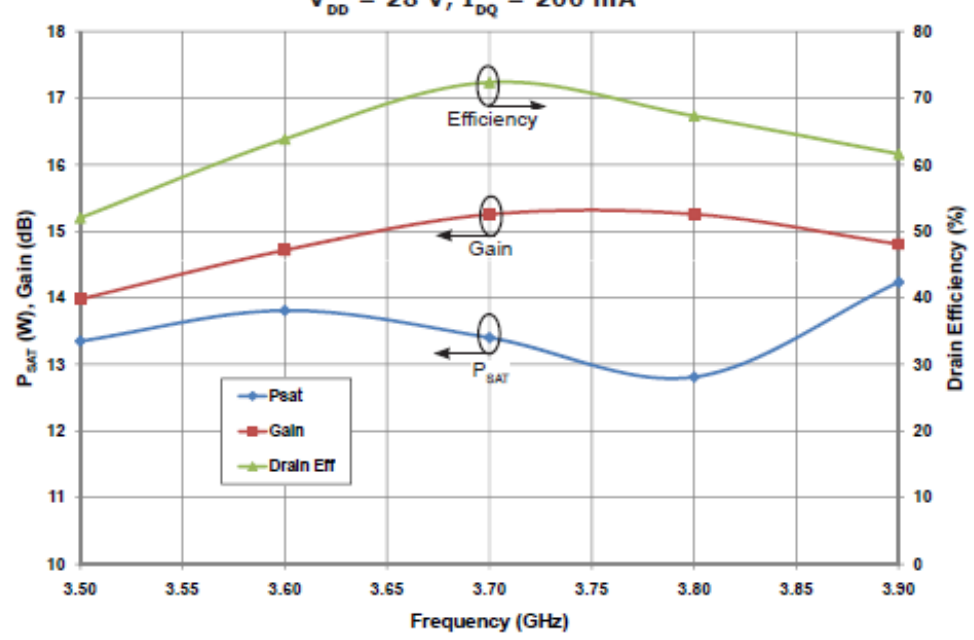




\section{다토도응}

Typical Performance

Swept CW Data of CGH40010F vs. Output Power with Source and Load Impedances Optimized for Drain Efficiency at $2.0 \mathrm{GHz}$

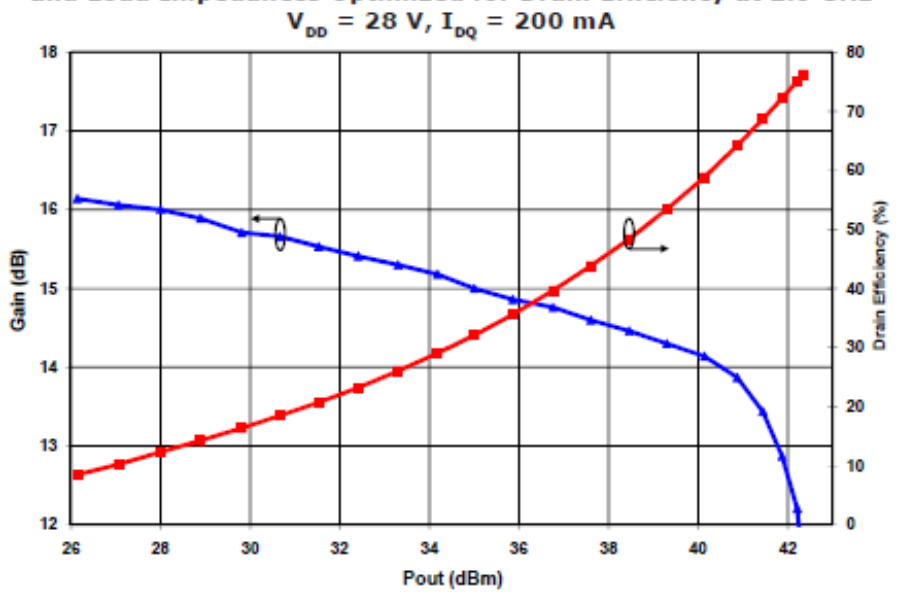

Swept CW Data of CGH40010F vs. Output Power with Source and Load Impedances Optimized for Drain Efficiency at $3.6 \mathrm{GHz}$ $\mathrm{V}_{\mathrm{DD}}=28 \mathrm{~V}, \mathrm{I}_{\mathrm{DQ}}=200 \mathrm{~mA}$

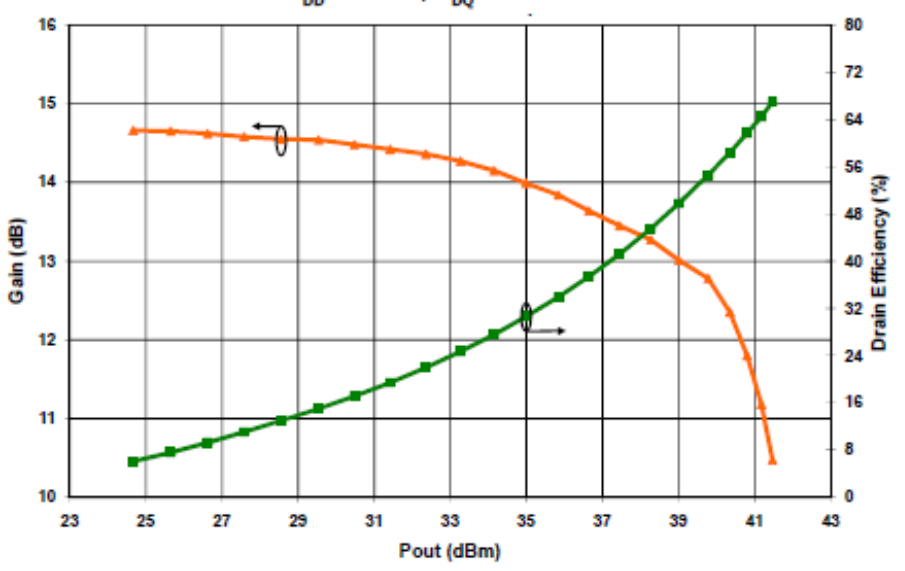




\section{CFFE토숭}

\section{Typical Performance}

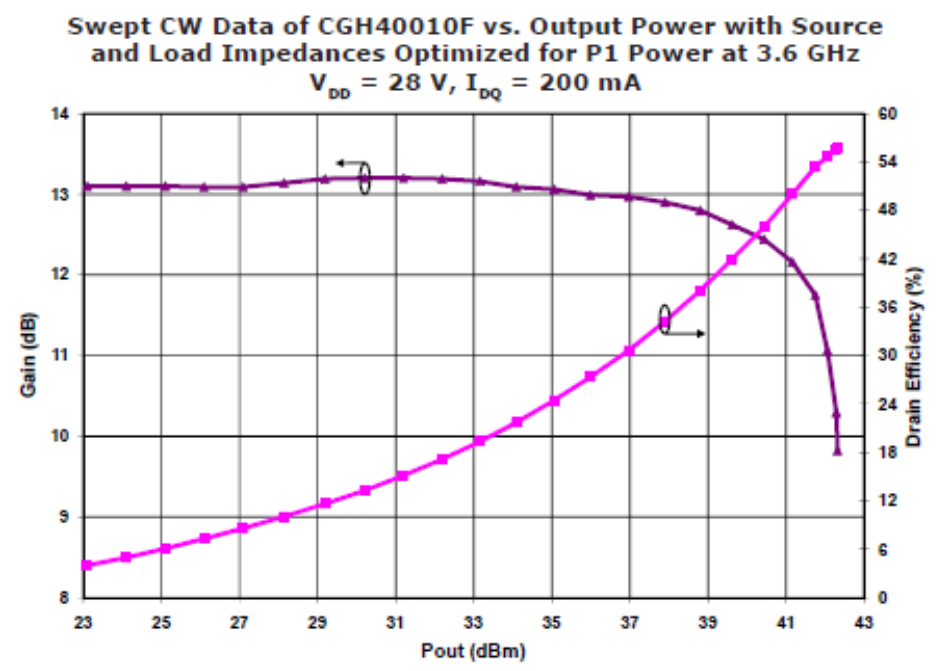

Simulated Maximum Available Gain and K Factor of the CGH40010F

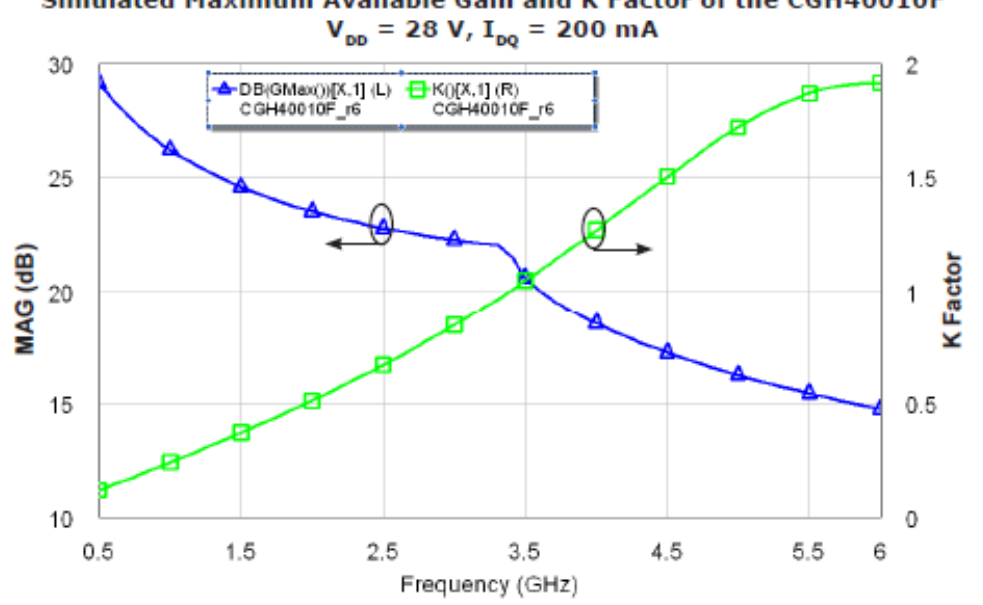

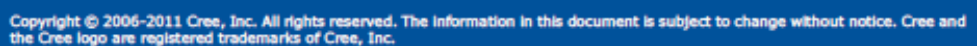




\section{CFE토탕}

Typical Noise Performance

Simulated Minimum Noise Figure and Noise Resistance vs Frequency of the CGH40010F $\mathrm{V}_{\mathrm{DD}}=28 \mathrm{~V}, \mathrm{I}_{\mathrm{DQ}}=100 \mathrm{~mA}$

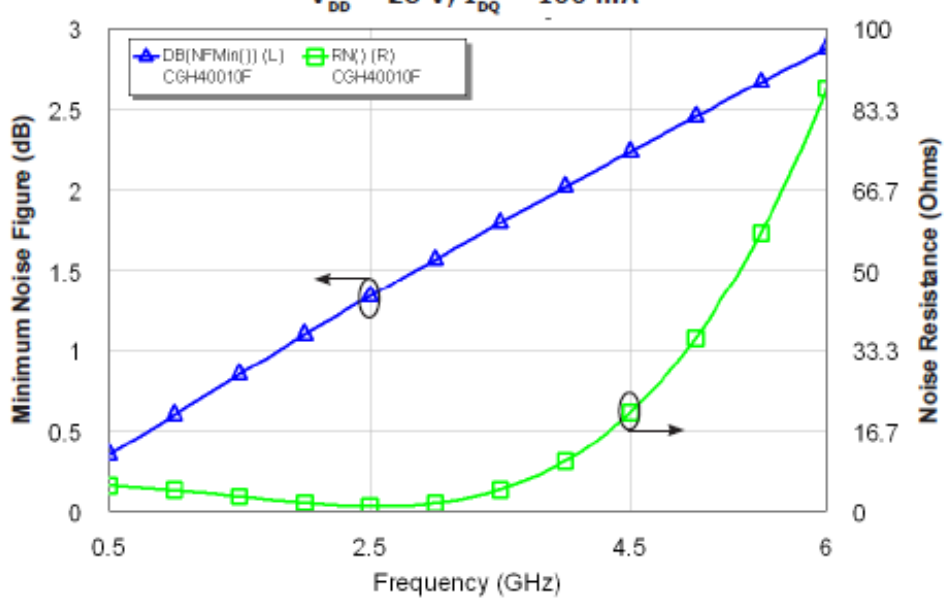

Electrostatic Discharge (ESD) Classifications

\begin{tabular}{|l|c|c|c|}
\hline Parameter & Symbol & Class & Test Methodology \\
\hline Human Body Model & HBM & $1 \mathrm{~A}>250 \mathrm{~V}$ & JEDEC JESD22 A114-D \\
\hline Charge Device Model & CDM & $1<200 \mathrm{~V}$ & JEDEC JESD22 C101-C \\
\hline
\end{tabular}




\section{CFFE토숭}

Source and Load Impedances

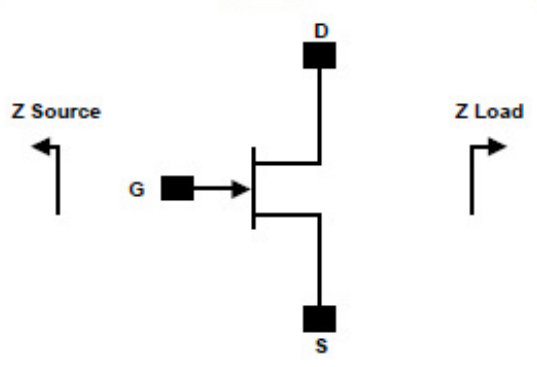

\begin{tabular}{|c|c|c|}
\hline Frequency (MHz) & Z Source & Z Load \\
\hline 500 & $20.2+j 16.18$ & $51.7+j 15.2$ \\
\hline 1000 & $8.38+j 9.46$ & $41.4+j 28.5$ \\
\hline 1500 & $7.37+j 0$ & $28.15+j 29$ \\
\hline 2500 & $3.19-j 4.76$ & $19+j 9.2$ \\
\hline 3500 & $3.18-j 13.3$ & $14.6+j 7.46$ \\
\hline
\end{tabular}

Note 1. $\mathrm{V}_{D O}=28 \mathrm{~V}, \mathrm{I}_{D Q}=200 \mathrm{~mA}$ in the 440166 package.

Note 2. Optimized for power, gain, $P_{\text {SaT }}$ and PAE.

Note 3 . When using this device at low frequency, series resistors

should be used to maintain amplifier stability.

CGH40010 Power Dissipation De-rating Curve

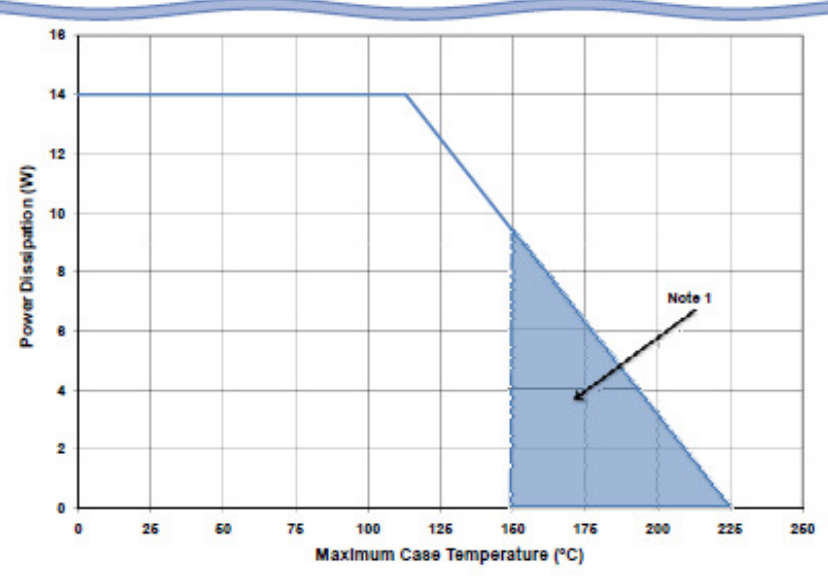

Note 1. Area exceeds Maximum Case Operating Temperature (See Page 2).

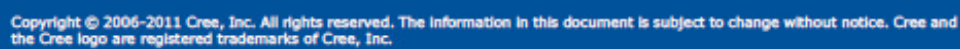




\section{CREEE}

CGH40010-TB Demonstration Amplifier Circuit Bill of Materials

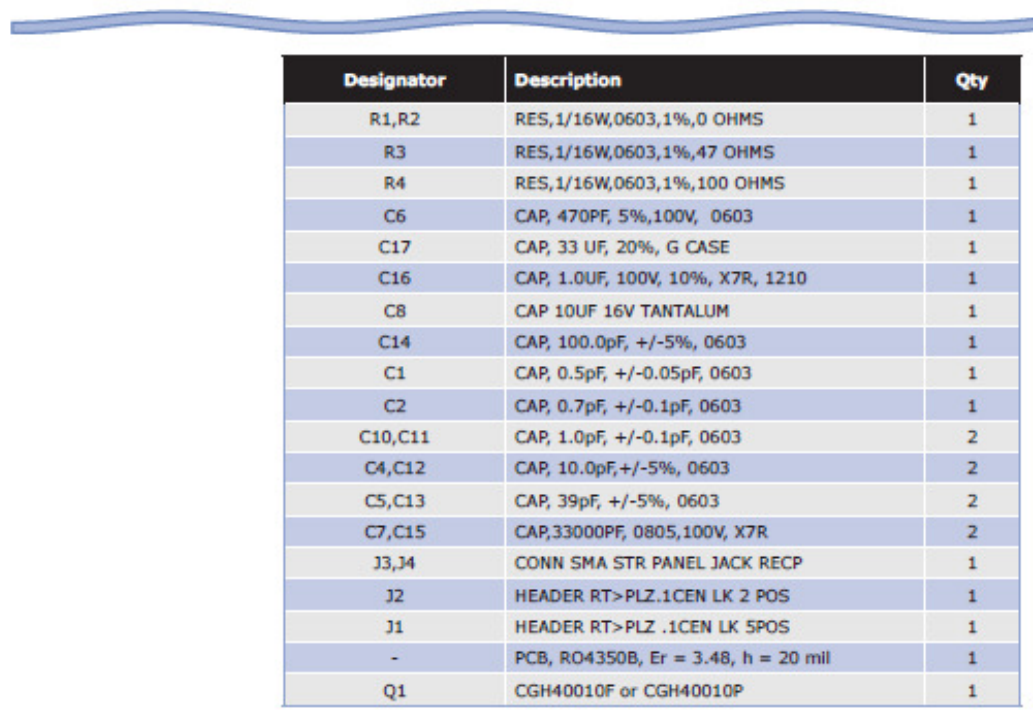

CGH40010-TB Demonstration Amplifier Circuit

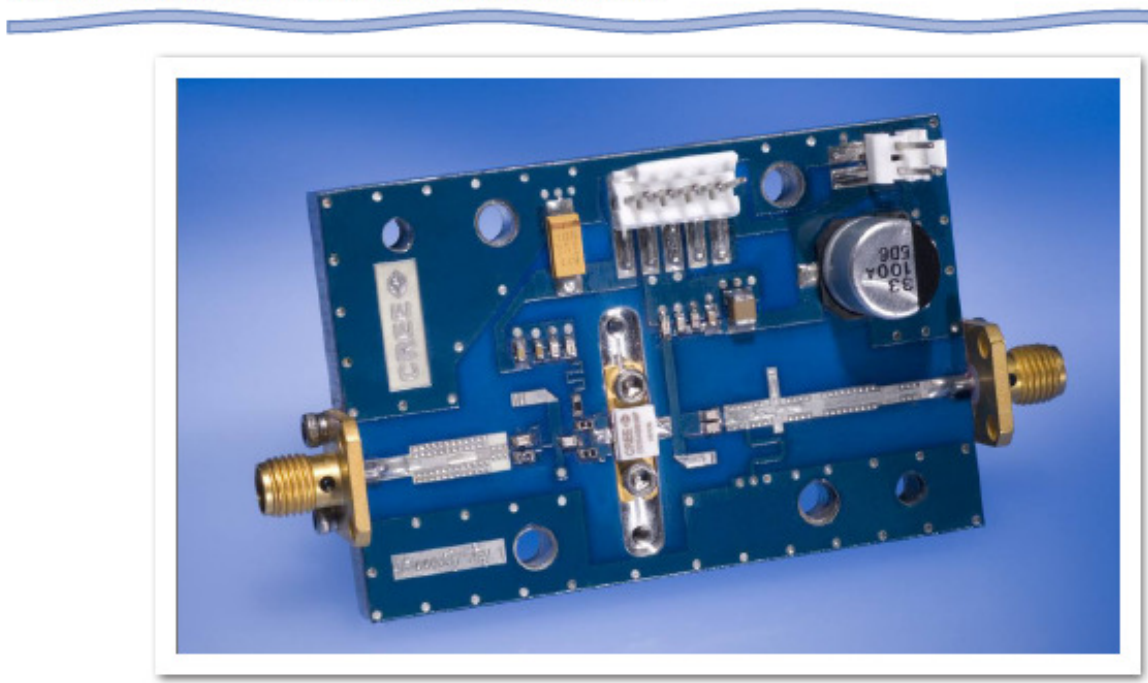

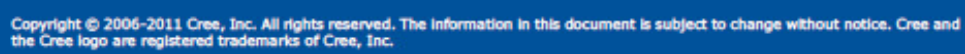




\section{CFFE토을}

CGH40010-TB Demonstration Amplifier Circuit Schematic

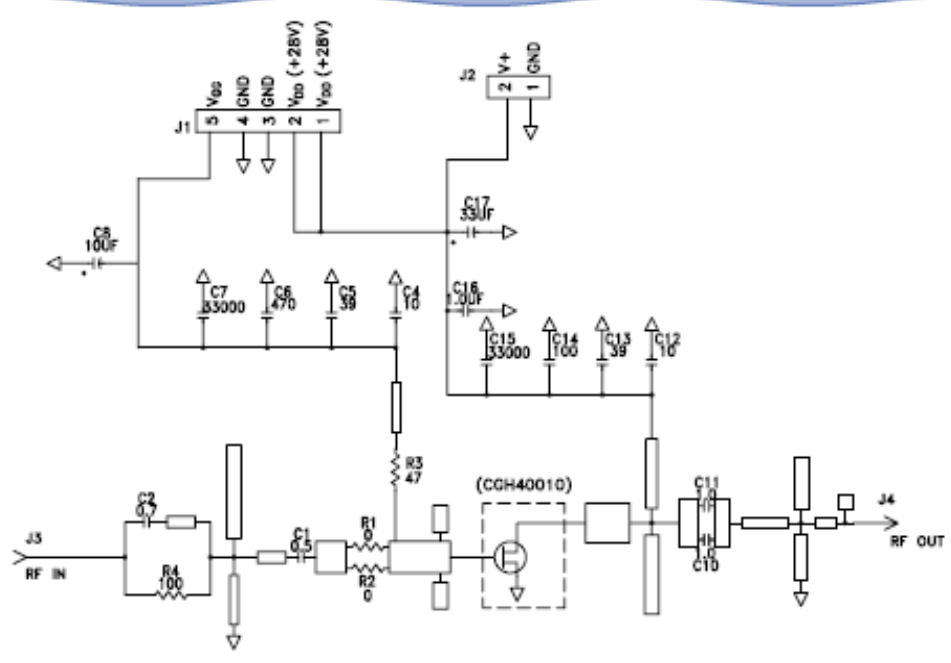

CGH40010-TB Demonstration Amplifier Circuit Outline

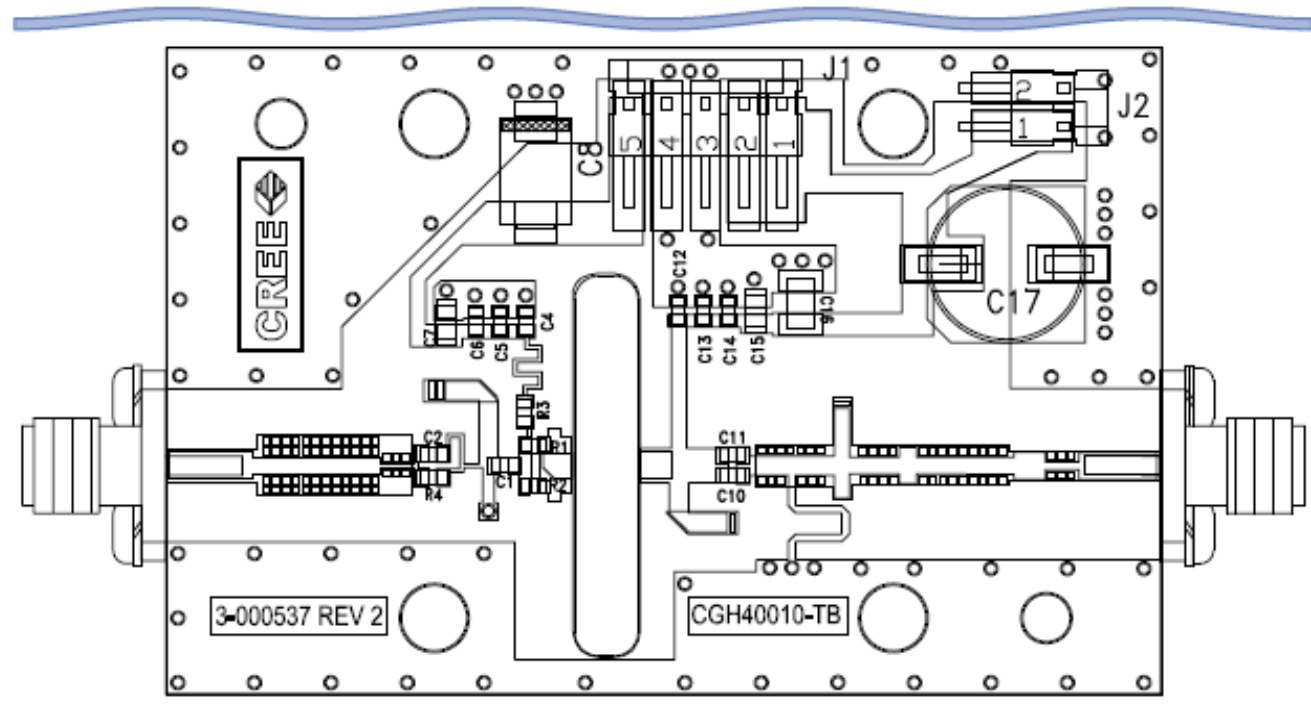




\section{CFFE토요}

Typical Package S-Parameters for CGH40010

(Small Signal, $\mathrm{V}_{\mathrm{DS}}=28 \mathrm{~V}, \mathrm{I}_{\mathrm{DQ}}=100 \mathrm{~mA}$, angle in degrees)

\begin{tabular}{|c|c|c|c|c|c|c|c|c|}
\hline Frequency & Mag S11 & Ang $\mathbf{S 1 1}$ & Mag $\$ 21$ & Ang $\$ 221$ & Mag S12 & Ang $\mathbf{5 1 2}$ & Mag 522 & Ang 522 \\
\hline $500 \mathrm{MHz}$ & 0.909 & -123.34 & 17.19 & 108.22 & 0.027 & 21.36 & 0.343 & -90.81 \\
\hline $600 \mathrm{MHz}$ & 0.902 & -133.06 & 14.86 & 101.82 & 0.028 & 15.60 & 0.329 & -98.65 \\
\hline $700 \mathrm{MHz}$ & 0.897 & -140.73 & 13.04 & 96.45 & 0.028 & 10.87 & 0.321 & -104.84 \\
\hline $800 \mathrm{MHz}$ & 0.894 & -146.96 & 11.58 & 91.78 & 0.029 & 6.84 & 0.317 & -109.84 \\
\hline $900 \mathrm{MHz}$ & 0.891 & -152.16 & 10.41 & 87.61 & 0.029 & 3.33 & 0.316 & -113.95 \\
\hline $1.0 \mathrm{GHz}$ & 0.890 & -156.60 & 9.43 & 83.82 & 0.029 & 0.19 & 0.318 & -117.42 \\
\hline $1.1 \mathrm{GHz}$ & 0.889 & -160.47 & 8.62 & 80.31 & 0.029 & -2.66 & 0.321 & -120.40 \\
\hline $1.2 \mathrm{GHz}$ & 0.888 & -163.90 & 7.93 & 77.02 & 0.029 & -5.28 & 0.326 & -123.02 \\
\hline $1.3 \mathrm{GHz}$ & 0.887 & -166.99 & 7.34 & 73.90 & 0.029 & -7.72 & 0.332 & -125.36 \\
\hline $1.4 \mathrm{GHz}$ & 0.887 & -169.80 & 6.82 & 70.92 & 0.029 & -10.01 & 0.338 & -127.51 \\
\hline $1.5 \mathrm{GHz}$ & 0.887 & -172.39 & 6.38 & 68.05 & 0.029 & -12.18 & 0.345 & -129.50 \\
\hline $1.6 \mathrm{GHz}$ & 0.887 & -174.80 & 5.98 & 65.28 & 0.028 & -14.24 & 0.353 & -131.37 \\
\hline $1.7 \mathrm{GHz}$ & 0.887 & -177.07 & 5.63 & 62.59 & 0.028 & -16.21 & 0.360 & -133.15 \\
\hline $1.8 \mathrm{GHz}$ & 0.887 & -179.22 & 5.32 & 59.97 & 0.028 & -18.09 & 0.369 & -134.87 \\
\hline $1.9 \mathrm{GHz}$ & 0.887 & 178.73 & 5.04 & 57.41 & 0.028 & -19.91 & 0.377 & -136.54 \\
\hline $2.0 \mathrm{GHz}$ & 0.888 & 176.76 & 4.78 & 54.89 & 0.027 & -21.66 & 0.385 & -138.17 \\
\hline $2.1 \mathrm{GHz}$ & 0.888 & 174.86 & 4.55 & 52.42 & 0.027 & -23.35 & 0.393 & -139.77 \\
\hline $2.2 \mathrm{GHz}$ & 0.888 & 173.02 & 4.34 & 49.99 & 0.027 & -24.98 & 0.402 & -141.34 \\
\hline $2.3 \mathrm{GHz}$ & 0.888 & 171.23 & 4.15 & 47.60 & 0.026 & -26.56 & 0.410 & -142.90 \\
\hline $2.4 \mathrm{GHz}$ & 0.889 & 169.48 & 3.97 & 45.24 & 0.026 & -28.08 & 0.418 & -144.45 \\
\hline $2.5 \mathrm{GHz}$ & 0.889 & 167.76 & 3.81 & 42.90 & 0.026 & -29.55 & 0.426 & -145.99 \\
\hline $2.6 \mathrm{GHz}$ & 0.890 & 166.07 & 3.66 & 40.59 & 0.025 & -30.98 & 0.434 & -147.53 \\
\hline $2.7 \mathrm{GHz}$ & 0.890 & 164.39 & 3.53 & 38.30 & 0.025 & -32.36 & 0.442 & -149.06 \\
\hline $2.8 \mathrm{GHz}$ & 0.890 & 162.74 & 3.40 & 36.03 & 0.025 & -33.69 & 0.450 & -150.59 \\
\hline $2.9 \mathrm{GHz}$ & 0.891 & 161.10 & 3.28 & 33.78 & 0.024 & -34.97 & 0.458 & -152.12 \\
\hline $3.0 \mathrm{GHz}$ & 0.891 & 159.46 & 3.17 & 31.55 & 0.024 & -36.20 & 0.465 & -153.65 \\
\hline $3.2 \mathrm{GHz}$ & 0.892 & 156.21 & 2.97 & 27.12 & 0.023 & -38.51 & 0.479 & -156.72 \\
\hline $3.4 \mathrm{GHz}$ & 0.893 & 152.96 & 2.79 & 22.73 & 0.022 & -40.63 & 0.493 & -159.80 \\
\hline $3.6 \mathrm{GHz}$ & 0.893 & 149.69 & 2.64 & 18.38 & 0.022 & -42.52 & 0.505 & -162.90 \\
\hline $3.8 \mathrm{GHz}$ & 0.894 & 146.38 & 2.50 & 14.05 & 0.021 & -44.17 & 0.517 & -166.03 \\
\hline $4.0 \mathrm{GHz}$ & 0.894 & 143.03 & 2.38 & 9.72 & 0.020 & -45.56 & 0.527 & -169.19 \\
\hline $4.2 \mathrm{GHz}$ & 0.894 & 139.61 & 2.28 & 5.40 & 0.019 & -46.67 & 0.537 & -172.39 \\
\hline $4.4 \mathrm{GHz}$ & 0.895 & 136.11 & 2.18 & 1.07 & 0.019 & -47.46 & 0.546 & -175.64 \\
\hline $4.6 \mathrm{GHz}$ & 0.895 & 132.53 & 2.09 & -3.29 & 0.018 & -47.90 & 0.554 & -178.95 \\
\hline $4.8 \mathrm{GHz}$ & 0.895 & 128.85 & 2.01 & -7.68 & 0.017 & -47.96 & 0.561 & 177.69 \\
\hline $5.0 \mathrm{GHz}$ & 0.895 & 125.06 & 1.94 & -12.10 & 0.017 & -47.61 & 0.568 & 174.25 \\
\hline $5.2 \mathrm{GHz}$ & 0.895 & 121.15 & 1.88 & -16.58 & 0.016 & -46.84 & 0.573 & 170.72 \\
\hline $5.4 \mathrm{GHz}$ & 0.895 & 117.11 & 1.82 & -21.12 & 0.016 & -45.67 & 0.578 & 167.10 \\
\hline $5.6 \mathrm{GHz}$ & 0.895 & 112.94 & 1.77 & -25.73 & 0.015 & -44.12 & 0.582 & 163.38 \\
\hline $5.8 \mathrm{GHz}$ & 0.895 & 108.62 & 1.72 & -30.42 & 0.015 & -42.30 & 0.586 & 159.54 \\
\hline $6.0 \mathrm{GHz}$ & 0.895 & 104.15 & 1.68 & -35.20 & 0.015 & -40.33 & 0.589 & 155.56 \\
\hline
\end{tabular}

Download this s-parameter file in ".s2p" format at httpi//www.cree.com/products/wireless_s-parameters,asp

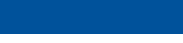




\section{CFEEFE}

Typical Package S-Parameters for CGH40010

(Small Signal, $\mathrm{V}_{\mathrm{DS}}=28 \mathrm{~V}, \mathrm{I}_{\mathrm{DQ}}=200 \mathrm{~mA}$, angle in degrees)

\begin{tabular}{|c|c|c|c|c|c|c|c|c|}
\hline Frequency & Mag S11 & Ang 511 & Mag 521 & Ang $\$ 21$ & Mag S12 & Ang $\mathbf{S 1 2}$ & Mag $\$ 22$ & Ang 522 \\
\hline $500 \mathrm{MHz}$ & 0.911 & -130.62 & 18.41 & 105.41 & 0.022 & 19.44 & 0.303 & -112.24 \\
\hline $600 \mathrm{MHz}$ & 0.906 & -139.65 & 15.80 & 99.47 & 0.023 & 14.31 & 0.299 & -119.83 \\
\hline $700 \mathrm{MHz}$ & 0.902 & -146.70 & 13.80 & 94.50 & 0.023 & 10.17 & 0.298 & -125.50 \\
\hline $800 \mathrm{MHz}$ & 0.899 & -152.41 & 12.22 & 90.19 & 0.023 & 6.68 & 0.299 & -129.85 \\
\hline $900 \mathrm{MHz}$ & 0.898 & -157.17 & 10.96 & 86.34 & 0.024 & 3.67 & 0.302 & -133.28 \\
\hline $1.0 \mathrm{GHz}$ & 0.896 & -161.24 & 9.92 & 82.82 & 0.024 & 0.99 & 0.305 & -136.05 \\
\hline $1.1 \mathrm{GHz}$ & 0.896 & -164.79 & 9.06 & 79.56 & 0.024 & -1.41 & 0.309 & -138.34 \\
\hline $1.2 \mathrm{GHz}$ & 0.895 & -167.95 & 8.33 & 76.49 & 0.024 & -3.62 & 0.314 & -140.30 \\
\hline $1.3 \mathrm{GHz}$ & 0.895 & -170.80 & 7.70 & 73.57 & 0.023 & -5.66 & 0.320 & -142.01 \\
\hline $1.4 \mathrm{GHz}$ & 0.894 & -173.41 & 7.17 & 70.78 & 0.023 & -7.56 & 0.326 & -143.54 \\
\hline $1.5 \mathrm{GHz}$ & 0.894 & -175.82 & 6.70 & 68.08 & 0.023 & -9.35 & 0.332 & -144.94 \\
\hline $1.6 \mathrm{GHz}$ & 0.894 & -178.09 & 6.28 & 65.47 & 0.023 & -11.05 & 0.338 & -146.24 \\
\hline $1.7 \mathrm{GHz}$ & 0.894 & 179.78 & 5.92 & 62.92 & 0.023 & -12.66 & 0.345 & -147.48 \\
\hline $1.8 \mathrm{GHz}$ & 0.894 & 177.75 & 5.59 & 60.43 & 0.023 & -14.19 & 0.352 & -148.68 \\
\hline $1.9 \mathrm{GHz}$ & 0.894 & 175.81 & 5.30 & 57.99 & 0.023 & -15.65 & 0.358 & -149.84 \\
\hline $2.0 \mathrm{GHz}$ & 0.894 & 173.94 & 5.04 & 55.59 & 0.022 & -17.05 & 0.365 & -150.99 \\
\hline $2.1 \mathrm{GHz}$ & 0.894 & 172.13 & 4.80 & 53.23 & 0.022 & -18.39 & 0.372 & -152.12 \\
\hline $2.2 \mathrm{GHz}$ & 0.894 & 170.37 & 4.58 & 50.91 & 0.022 & -19.67 & 0.379 & -153.26 \\
\hline $2.3 \mathrm{GHz}$ & 0.895 & 168.65 & 4.38 & 48.61 & 0.022 & -20.90 & 0.386 & -154.39 \\
\hline $2.4 \mathrm{GHz}$ & 0.895 & 166.96 & 4.20 & 46.33 & 0.021 & -22.08 & 0.393 & -155.54 \\
\hline $2.5 \mathrm{GHz}$ & 0.895 & 165.30 & 4.03 & 44.08 & 0.021 & -23.20 & 0.400 & -156.69 \\
\hline $2.6 \mathrm{GHz}$ & 0.895 & 163.66 & 3.88 & 41.84 & 0.021 & -24.27 & 0.407 & -157.85 \\
\hline $2.7 \mathrm{GHz}$ & 0.895 & 162.04 & 3.74 & 39.63 & 0.021 & -25.28 & 0.414 & -159.03 \\
\hline $2.8 \mathrm{GHz}$ & 0.895 & 160.43 & 3.60 & 37.43 & 0.020 & -26.25 & 0.420 & -160.22 \\
\hline $2.9 \mathrm{GHz}$ & 0.896 & 158.83 & 3.48 & 35.24 & 0.020 & -27.16 & 0.427 & -161.42 \\
\hline $3.0 \mathrm{GHz}$ & 0.896 & 157.24 & 3.37 & 33.06 & 0.020 & -28.02 & 0.433 & -162.64 \\
\hline $3.2 \mathrm{GHz}$ & 0.896 & 154.06 & 3.16 & 28.74 & 0.019 & -29.57 & 0.446 & -165.13 \\
\hline $3.4 \mathrm{GHz}$ & 0.896 & 150.87 & 2.98 & 24.44 & 0.019 & -30.88 & 0.457 & -167.69 \\
\hline $3.6 \mathrm{GHz}$ & 0.896 & 147.66 & 2.82 & 20.16 & 0.018 & -31.95 & 0.468 & -170.31 \\
\hline $3.8 \mathrm{GHz}$ & 0.897 & 144.41 & 2.68 & 15.89 & 0.018 & -32.76 & 0.478 & -173.00 \\
\hline $4.0 \mathrm{GHz}$ & 0.897 & 141.10 & 2.56 & 11.61 & 0.017 & -33.30 & 0.488 & -175.77 \\
\hline $4.2 \mathrm{GHz}$ & 0.897 & 137.72 & 2.45 & 7.33 & 0.017 & -33.55 & 0.497 & -178.61 \\
\hline $4.4 \mathrm{GHz}$ & 0.897 & 134.26 & 2.35 & 3.03 & 0.017 & -33.50 & 0.505 & 178.47 \\
\hline $4.6 \mathrm{GHz}$ & 0.897 & 130.71 & 2.26 & -1.31 & 0.016 & -33.18 & 0.512 & 175.46 \\
\hline $4.8 \mathrm{GHz}$ & 0.896 & 127.06 & 2.17 & -5.68 & 0.016 & -32.58 & 0.518 & 172.36 \\
\hline $5.0 \mathrm{GHz}$ & 0.896 & 123.30 & 2.10 & -10.09 & 0.016 & -31.74 & 0.524 & 169.16 \\
\hline $5.2 \mathrm{GHz}$ & 0.896 & 119.42 & 2.04 & -14.57 & 0.016 & -30.72 & 0.529 & 165.86 \\
\hline $5.4 \mathrm{GHz}$ & 0.896 & 115.41 & 1.98 & -19.10 & 0.016 & -29.60 & 0.534 & 162.44 \\
\hline $5.6 \mathrm{GHz}$ & 0.896 & 111.26 & 1.92 & -23.71 & 0.016 & -28.46 & 0.537 & 158.89 \\
\hline $5.8 \mathrm{GHz}$ & 0.895 & 106.97 & 1.87 & -28.40 & 0.017 & -27.41 & 0.540 & 155.20 \\
\hline $6.0 \mathrm{GHz}$ & 0.895 & 102.53 & 1.82 & -33.19 & 0.017 & -26.54 & 0.543 & 151.36 \\
\hline
\end{tabular}

Download this s-parameter file in ".s2p" format at http://www.cree.com/products/wireless_s-parameters,asp 


\section{CFFE탕}

Typical Package S-Parameters for CGH40010

(Small Signal, $\mathrm{V}_{\mathrm{DS}}=28 \mathrm{~V}, \mathrm{I}_{\mathrm{DQ}}=500 \mathrm{~mA}$, angle in degrees)

\begin{tabular}{|c|c|c|c|c|c|c|c|c|}
\hline Frequency & Mag S11 & Ang 511 & Mag $\$ 21$ & Ang $\$ 21$ & Mag $\$ 12$ & Ang $\$ 12$ & Mag $\$ 22$ & Ang 522 \\
\hline $500 \mathrm{MHz}$ & 0.914 & -135.02 & 18.58 & 103.70 & 0.020 & 18.36 & 0.300 & -126.80 \\
\hline $600 \mathrm{MHz}$ & 0.909 & -143.57 & 15.88 & 98.05 & 0.020 & 13.67 & 0.302 & -133.51 \\
\hline $700 \mathrm{MHz}$ & 0.906 & -150.23 & 13.83 & 93.33 & 0.021 & 9.90 & 0.304 & -138.40 \\
\hline $800 \mathrm{MHz}$ & 0.904 & -155.61 & 12.23 & 89.23 & 0.021 & 6.77 & 0.307 & -142.08 \\
\hline $900 \mathrm{MHz}$ & 0.903 & -160.09 & 10.95 & 85.56 & 0.021 & 4.08 & 0.311 & -144.94 \\
\hline $1.0 \mathrm{GHz}$ & 0.902 & -163.93 & 9.91 & 82.21 & 0.021 & 1.71 & 0.314 & -147.23 \\
\hline $1.1 \mathrm{GHz}$ & 0.901 & -167.29 & 9.04 & 79.09 & 0.021 & -0.41 & 0.319 & -149.10 \\
\hline $1.2 \mathrm{GHz}$ & 0.901 & -170.29 & 8.31 & 76.15 & 0.021 & -2.35 & 0.323 & -150.69 \\
\hline $1.3 \mathrm{GHz}$ & 0.900 & -173.00 & 7.69 & 73.35 & 0.021 & -4.12 & 0.328 & -152.07 \\
\hline $1.4 \mathrm{GHz}$ & 0.900 & -175.50 & 7.15 & 70.66 & 0.021 & -5.78 & 0.333 & -153.29 \\
\hline $1.5 \mathrm{GHz}$ & 0.900 & -177.81 & 6.69 & 68.07 & 0.021 & -7.32 & 0.338 & -154.41 \\
\hline $1.6 \mathrm{GHz}$ & 0.900 & -179.98 & 6.27 & 65.54 & 0.021 & -8.77 & 0.344 & -155.44 \\
\hline $1.7 \mathrm{GHz}$ & 0.900 & 177.96 & 5.91 & 63.08 & 0.020 & -10.15 & 0.349 & -156.43 \\
\hline $1.8 \mathrm{GHz}$ & 0.899 & 176.00 & 5.59 & 60.67 & 0.020 & -11.45 & 0.355 & -157.38 \\
\hline $1.9 \mathrm{GHz}$ & 0.899 & 174.12 & 5.30 & 58.30 & 0.020 & -12.68 & 0.361 & -158.30 \\
\hline $2.0 \mathrm{GHz}$ & 0.899 & 172.31 & 5.04 & 55.97 & 0.020 & -13.85 & 0.366 & -159.22 \\
\hline $2.1 \mathrm{GHz}$ & 0.899 & 170.54 & 4.80 & 53.67 & 0.020 & -14.96 & 0.372 & -160.14 \\
\hline $2.2 \mathrm{GHz}$ & 0.900 & 168.83 & 4.58 & 51.40 & 0.020 & -16.01 & 0.378 & -161.06 \\
\hline $2.3 \mathrm{GHz}$ & 0.900 & 167.15 & 4.39 & 49.16 & 0.019 & -17.01 & 0.384 & -161.99 \\
\hline $2.4 \mathrm{GHz}$ & 0.900 & 165.49 & 4.21 & 46.94 & 0.019 & -17.95 & 0.390 & -162.93 \\
\hline $2.5 \mathrm{GHz}$ & 0.900 & 163.87 & 4.04 & 44.73 & 0.019 & -18.85 & 0.396 & -163.88 \\
\hline $2.6 \mathrm{GHz}$ & 0.900 & 162.26 & 3.89 & 42.54 & 0.019 & -19.69 & 0.402 & -164.86 \\
\hline $2.7 \mathrm{GHz}$ & 0.900 & 160.66 & 3.75 & 40.37 & 0.019 & -20.48 & 0.407 & -165.85 \\
\hline $2.8 \mathrm{GHz}$ & 0.900 & 159.08 & 3.62 & 38.21 & 0.019 & -21.21 & 0.413 & -166.86 \\
\hline $2.9 \mathrm{GHz}$ & 0.900 & 157.51 & 3.50 & 36.05 & 0.018 & -21.89 & 0.418 & -167.89 \\
\hline $3.0 \mathrm{GHz}$ & 0.900 & 155.93 & 3.39 & 33.91 & 0.018 & -22.52 & 0.424 & -168.95 \\
\hline $3.2 \mathrm{GHz}$ & 0.900 & 152.79 & 3.18 & 29.65 & 0.018 & -23.61 & 0.435 & -171.12 \\
\hline $3.4 \mathrm{GHz}$ & 0.900 & 149.64 & 3.00 & 25.40 & 0.017 & -24.48 & 0.445 & -173.38 \\
\hline $3.6 \mathrm{GHz}$ & 0.900 & 146.45 & 2.85 & 21.17 & 0.017 & -25.11 & 0.454 & -175.73 \\
\hline $3.8 \mathrm{GHz}$ & 0.900 & 143.23 & 2.71 & 16.93 & 0.017 & -25.51 & 0.463 & -178.17 \\
\hline $4.0 \mathrm{GHz}$ & 0.900 & 139.94 & 2.58 & 12.69 & 0.017 & -25.67 & 0.471 & 179.30 \\
\hline $4.2 \mathrm{GHz}$ & 0.900 & 136.58 & 2.47 & 8.43 & 0.016 & -25.60 & 0.479 & 176.67 \\
\hline $4.4 \mathrm{GHz}$ & 0.899 & 133.14 & 2.38 & 4.15 & 0.016 & -25.32 & 0.486 & 173.94 \\
\hline $4.6 \mathrm{GHz}$ & 0.899 & 129.61 & 2.29 & -0.17 & 0.016 & -24.85 & 0.492 & 171.12 \\
\hline $4.8 \mathrm{GHz}$ & 0.899 & 125.97 & 2.21 & -4.53 & 0.016 & -24.24 & 0.498 & 168.18 \\
\hline $5.0 \mathrm{GHz}$ & 0.898 & 122.23 & 2.13 & -8.94 & 0.016 & -23.54 & 0.503 & 165.13 \\
\hline $5.2 \mathrm{GHz}$ & 0.898 & 118.36 & 2.07 & -13.41 & 0.016 & -22.80 & 0.507 & 161.96 \\
\hline $5.4 \mathrm{GHz}$ & 0.898 & 114.36 & 2.01 & -17.95 & 0.017 & -22.11 & 0.511 & 158.66 \\
\hline $5.6 \mathrm{GHz}$ & 0.897 & 110.22 & 1.95 & -22.56 & 0.017 & -21.54 & 0.514 & 155.22 \\
\hline $5.8 \mathrm{GHz}$ & 0.897 & 105.94 & 1.90 & -27.26 & 0.018 & -21.16 & 0.517 & 151.63 \\
\hline $6.0 \mathrm{GHz}$ & 0.897 & 101.51 & 1.86 & -32.04 & 0.019 & -21.04 & 0.519 & 147.87 \\
\hline
\end{tabular}

Download this s-parameter file in ".s2p" format at httpi//www.cree,com/products/wireless_s-parameters,asp. 


\section{CREEE}

Product Dimensions CGH40010F (Package Type - 440166)
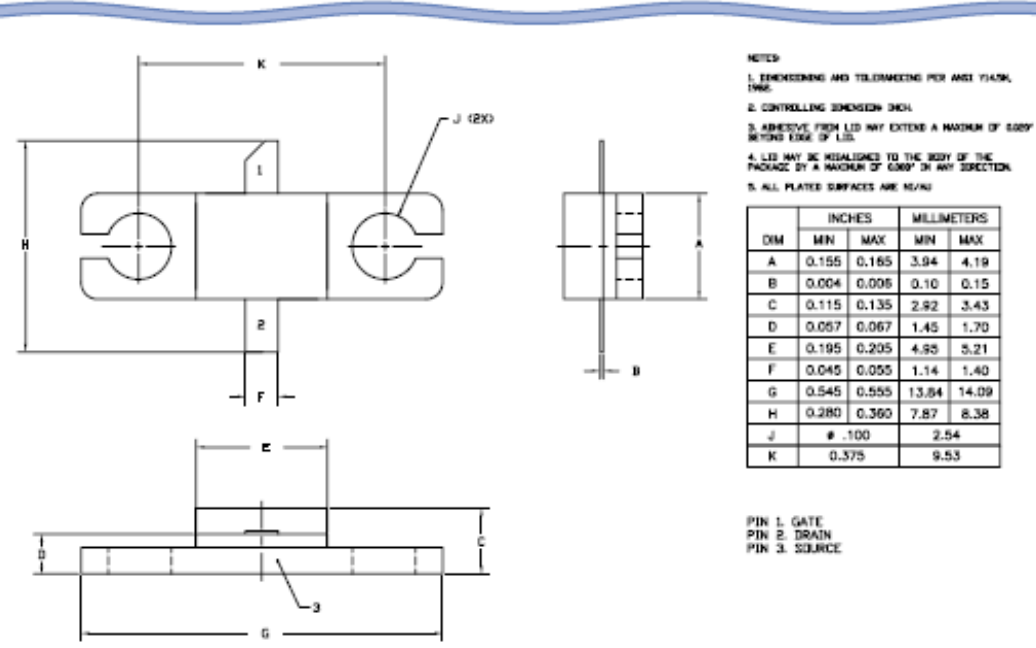

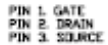

Product Dimensions CGH40010P (Package Type - 440196)
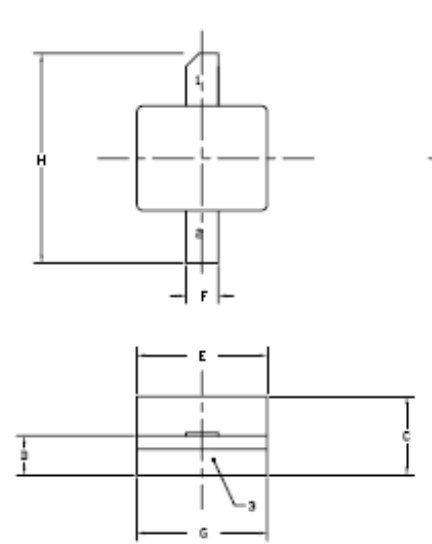

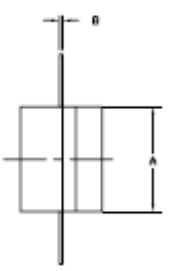

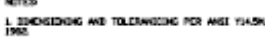

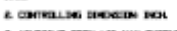

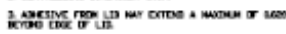

A

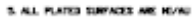

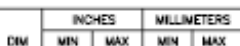

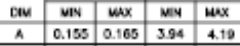

\begin{tabular}{|l|l|l|l|l|l|}
\hline A & 0.155 & 0.165 & 3.94 & 4.12 \\
\hline$\theta$ & 0.003 & 0.006 & 0.10 & 0.15 \\
\hline
\end{tabular}

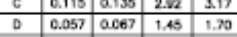

\begin{tabular}{|c|c|c|c|c|}
\hline $\mathrm{E}$ & 0.185 & 0.205 & 4.95 & 8.21 \\
\hline $\mathrm{F}$ & 0.015 & 0.055 & 1.14 & 1.40 \\
\hline
\end{tabular}

\begin{tabular}{|c|c|c|c|c|}
\hline $\mathrm{F}$ & 0.045 & 0.055 & 1.14 & 1.40 \\
\hline $\mathrm{G}$ & 0.185 & 0.205 & $\mathbf{4 . 9 5}$ & $\mathbf{5 2 1}$ \\
\hline
\end{tabular}

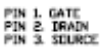




\section{다토도응}

\section{Disclaimer}

Specifications are subject to change without notice. Cree, Inc. believes the information contained within this data sheet to be accurate and reliable. However, no responsibility is assumed by Cree for any infringement of patents or other rights of third parties which may result from its use. No license is granted by implication or otherwise under any patent or patent rights of Cree. Cree makes no warranty, representation or guarantee regarding the suitability of its products for any particular purpose. "Typical" parameters are the average values expected by Cree in large quantities and are provided for information purposes only. These values can and do vary in different applications and actual performance can vary over time. All operating parameters should be validated by customer's technical experts for each application. Cree products are not designed, intended or authorized for use as components in applications intended for surgical implant into the body or to support or sustain life, in applications in which the failure of the Cree product could result in personal injury or death or in applications for planning, construction, maintenance or direct operation of a nuclear facility.

For more information, please contact:

Cree, Inc.

4600 Silicon Drive

Durham, NC 27703

www.cree.com/wireless

Ryan Baker

Marketing

Cree, RF Components

919.407.7816

Tom Dekker

Sales Director

Cree, RF Components

919.407.5639 


\section{RESEARCH PUBLICATIONS}




\section{An Enhanced Modulated Waveform Measurement System for the Robust Characterization of Microwave Devices under Modulated Excitation}

\author{
M. Akmal, J. Lees, S. Jiangtao, V. Carrubba, \\ Z. Yusoff, S. Woodington, J. Benedikt, P. J. Tasker \\ Centre for High Frequency Engineering \\ Cardiff University \\ Cardiff, United Kingdom \\ AkmalM1@Cardiff.ac.uk
}

\author{
S. Bensmida, K. Morris, M. Beach, J. McGeehan \\ Centre for Communications Research \\ University of Bristol \\ Bristol, United Kingdom \\ S.Bensmida@Bristol.ac.uk
}

\begin{abstract}
This paper presents a refined modulated waveform measurement system for the robust characterization of nonlinear microwave devices when driven by broadband multi-tone stimuli. This enhanced system has the ability to present specific, constant impedances, not only to a large number of baseband (IF) components, but also to signals located around the carrier and significant harmonic frequencies. Achieving such comprehensive impedance control across wide modulation bandwidths is critical in allowing the 'emulation' of new power amplifier modes and architectures, and the subsequent waveform characterization of devices operating in these complex and often dynamic impedance environments. The enhanced system is demonstrated through a number of applications: firstly the experimental investigation and baseband optimization of a $10 \mathrm{~W}$ GaN HEMT under ninetone excitation, and secondly, the emulation of a modulated Class-J impedance environment that interestingly highlights the presence of separate optimum baseband impedance conditions necessary for the reduction of individual IM products.
\end{abstract}

\section{INTRODUCTION}

The recent rapid development of mobile communications standards has resulted in a growing motivation to develop measurement systems capable of characterizing microwave power devices under realistic modulated stimuli. This is particularly relevant in the context of the highly dynamic RF envelopes that result from the use of modulation schemes such as multicarrier code division multiple access (CDMA) and orthogonal frequency division multiplexing (OFDM), where the coexistence of static and dynamic nonlinear distortion sources in microwave devices and circuits can severely compromise performance, not only in terms of general linearity, but also in terms of how easily such systems can be linearised. The minimization of effects that lead to such problematic distortions relies heavily on the accurate characterisation of the microwave power devices used.

It is now widely accepted that the linearity performance of a microwave device is effected by, not only the impedance environment presented at the fundamental and higher harmonic frequencies, but also at much lower baseband frequencies [1]-[3]. In reality, and in response to a simple two-tone excitation, the baseband spectrum constitutes not

This work has been carried out as part of an EPSRC grant EP/F033702/1. The authors would like to thank CREE for supplying the devices and supporting this activity. The authors further would like to thank Mesuro for providing access to Tektronix AWG. only the significant baseband components (IF1 and IF2) but also the higher baseband components (IF3, IF4), the presence of which can present a significant design challenge in achieving adequate broadband baseband impedance termination for high linearity. Previous work has highlighted the fact that the variation of baseband impedance over bandwidth is an important problem that needs further investigation to pave the way for the development of future high-efficiency, high-linearity and high-bandwidth communication systems [3]-[4].

The first part of this paper explains the significant measurement system enhancements performed to allow dynamic baseband impedance characterization under complex modulated excitations and quantifies the persistent influence of baseband impedance variation on ACPR performance. It is shown for instance that when a device is subjected to complex 9-tone excitation, a significant linearity improvement can be achieved by synthising specific baseband signals, characterised by specific nagative baseband impedances, as has already been shown for the two-tone case in [3].

The second part of this paper shows how it is possible to control all significant out-of-band frequency components, particularly at baseband, fundamental and $2^{\text {nd }}$ harmonic, through the significant modification of the measurement system RF architecture and enhancement of measurement dynamic range. These capabilities are then demonstrated through the active synthesis of the fundamental and second harmonic RF loads necessary to emulate a class-J impedance environment under modulated excitation. This new RF loadpull functionality has been achieved using a very-high bandwidth, dual channel arbitrary waveform generator - the Tektronix AWG7000. One motivation behind this analysis is to investigate the effectiveness of using the baseband linearisation for novel PAs where the fundamental and harmonic loads presented to the device can be highly reactive.

\section{The EnHANCED Modulated MEASUREMET SyStem}

Earlier work has generally focused completely on understanding the effects of baseband impedance variation over bandwidth, and the active load-pull systems used have been capable of presenting specific baseband impedances to the two most significant baseband components (IF1 and IF2), and measuring RF modulation envelopes generated as a result of 
simple modulated excitation [4]-[6]. Although effective, these systems are generally limited to two or three-tone excitations due to complexities in the triggering and averaging of complex modulated signals using high-speed sampling oscilloscopes.

\section{A. Improving Dynamic Range - Triggering and Averaging}

When measuring $\mathrm{CW}$ signals, the sampling oscilloscope is usually triggered from the input signal itself. This subsampling approach becomes impractical for multi-tone signals as each cycle of RF within the modulation envelope is no longer identical, and the waveform pattern no longer repeats on each rising edge of the RF waveform. In order to achieve high measurement speed and a useful dynamic range in the region of $60 \mathrm{~dB}$, it was critical that the enhanced modulated measurement system should be able to utilize the on-board averaging capability of the oscilloscope (Tektronix DSA8000).

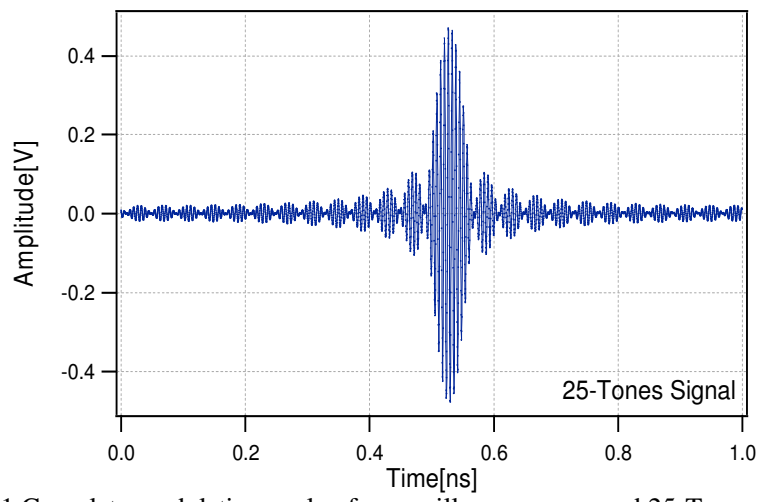

Fig. 1 Complete modulation cycle of on oscilloscope averaged 25-Tones signal at a carrier frequency of $2 \mathrm{GHz}$ with $500 \mathrm{KHz}$ tone separation.

When measuring complex modulated signals for the applications presented in this paper, it becomes critical to capture detailed features, both in the individual RF cycles, and the modulated RF envelopes, which can be difficult to achieve using sampling oscilloscopes where the number of horizontal points is limited. Folded and interleaved sub-sampling techniques have been developed and effectively compress the spectra of the captured waveforms, and reduce the number of RF cycles per cycle of modulation. Whilst these maintain the integrity of the captured signal [7], they were found to be problematic for capturing complex modulations.

The accurate triggering of the 4-channel sampling oscilloscope has been achieved here using an Agilent $120 \mathrm{MHz}$ arbitrary waveform generator (AWG) to generate high-quality, low-jitter repetitive trigger pulses at the modulation frequency of interest. When established correctly and synchronized with a multi-tone modulated source, complex modulated waveforms can be accurately captured, as depicted in Fig. 1.

\section{B. Multi-Tone Measurements}

In order to capture multi-tone signals using a standard sampling approach, the high-accuracy trigger was provided at the repetition rate of the modulated sequence. The DSA8000 has only 4000 measurement points making it impractical to capture, in one waveform, all of the relevant information present in a complex multi-sine modulation, so, a new technique was introduced that allowed the sequential capture of 'sections' of a complete modulation cycle, referred to here as "windowing". In this approach, the oscilloscope is caused to repeatedly trigger at a specific points within the modulation cycle, and by varying precisely the trigger delay and record length, it is possible to isolate and average specific parts of even complex modulation envelopes. Thus, it is possible to step through the modulated waveform, and accurately capture one complete modulation cycle in sufficient detail and accuracy for meaningful analysis.

Each captured, 4000 point window is typically averaged 500 times before being downloaded to a computer ready for assembly and analysis. The formulation given in (1) defines the number of windows $(\mathrm{W})$ required to capture one complete cycle of modulation.

$$
\mathrm{W}=\left(2 \cdot(\mathrm{H}+1) \cdot \mathrm{f}_{\mathrm{C}}\right) / P \cdot \mathrm{f}_{\mathrm{M}}
$$

In the above equation, $\mathrm{H}$ is the number of required harmonics, $f_{c}$ is carrier frequency, $f_{m}$ is modulation frequency and $P$ is number of points used, here limited to 4000 .

As well as improving dynamic range, this technique has allowed measurement time to be dramatically reduced - for example, it now takes less than 1 minute to completely capture a device's non-linear response (including baseband and five harmonics) to a $1 \mathrm{MHz}$ modulated $2 \mathrm{GHz}$ carrier. The frequency of the tones used to generate the modulation need to be considered carefully however to avoid waveform 'stitching' problems and subsequent spectral re-growth. To demonstrate the improved dynamic range of the enhanced measurement system, a thru measurement was conducted using a 9-tone stimulus with a notch created by progressively suppressing the central tone. For this case, and as can be seen from Fig. 2, the measurement system can measure this tone to approximately $50 \mathrm{dBc}$. From the same plot, the out-of-band dynamic range is closer to $60 \mathrm{~dB}$, and this is perfectly sufficient to be able to measure the relatively low distortion levels of interest here.

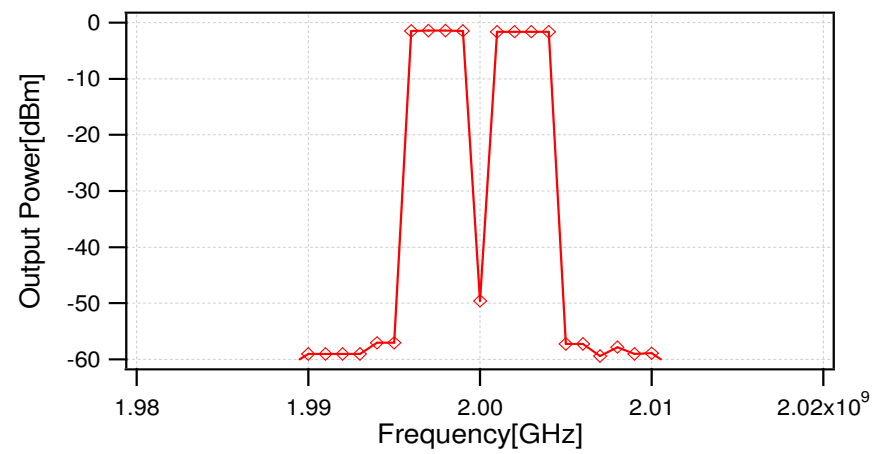

Fig. 2 Measured output spectrum for thru standard using 64 averages with the lowest spectral information.

A closer analysis of measurement system dynamic range for modulations using different numbers of tones, and using 64 averages is shown in Table-1. Increasing averaging beyond this has little effect on the in-band dynamic range as this is eventually limited by the leakage distortion properties of the modulated source. Increased averaging does however reduce the noise floor. One important observation to note is that when the phases of the tones used in the excitation are randomised, 
the dynamic range of measurement system is slightly reduced. For all measurements, the tone amplitudes were adjusted to ensure a constant peak envelope power.

TABLE I

Dynamic range of measurement system for different multi-tone excitations

\begin{tabular}{|c|c|c|c|c|}
\hline Fixed Amplitude & $\begin{array}{c}\text { 2-Tone } \\
(\mathbf{d B})\end{array}$ & $\begin{array}{c}\text { 5-Tone } \\
(\mathbf{d B})\end{array}$ & $\begin{array}{c}\text { 9-Tone } \\
(\mathbf{d B})\end{array}$ & $\begin{array}{c}\text { 25-Tone } \\
(\mathbf{d B})\end{array}$ \\
\hline Fixed Phase & 64 & 62 & 60 & 45 \\
\hline Randomised Phase & 61 & 59 & 56 & 39 \\
\hline
\end{tabular}

\section{Broadband Active RF and IF Load-pull}

Achieving broadband, baseband load emulation, required significant modification to the active load-pull architecture to account for the presence of higher baseband harmonics. This functionality was achieved in the time domain through the addition of a phase synchronised $80 \mathrm{MHz}$ arbitrary waveform generator (AWG). The generated waveforms comprise frequency components that are multiples of the baseband fundamental frequency, and by controlling the relative magnitude and phase of these, constant and specific baseband impedance scenarios can be presented to a device and maintained across a wide bandwidth. The resulting waveforms are fed directly to the output of device through a 200W baseband power amplifier, increasing the signal amplitude to the levels required for load-pull.

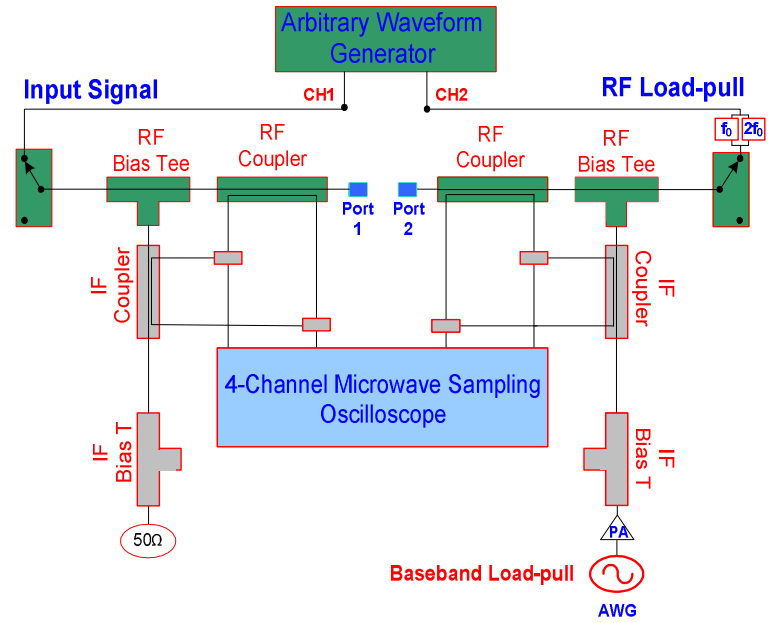

Fig. 3 Enhanced modulated waveform measurement system with the capability of active RF and IF load-pull.

The RF synthesizer used in the modulated waveform measurement system depicted in Fig. 3 is a two-channel Tektronix AWG7000 Arbitrary Waveform Generator, and its two independent yet coherent channels have been used here to synthesize both the modulated fundamental excitation and the complete modulated RF load-pull signal (comprising both fundamental band and harmonic band components) simultaneously, in the time domain. Through the addition of this instrument, the waveform measurement system is able to maintain independent and constant impedance control for each individual tone present across both IF and RF impedance environments, and over a wide modulation bandwidth. The enhanced modulated waveform measurement system depicted in Fig. 3 has been demonstrated in the first instance using wideband multi-sine stimuli to investigate the bandwidth dependent behavior of a CREE CGH40010 10W GaN HEMT device.

\section{MUlTi-Tone MEASUREMENTS AND INVESTIGATIONS}

The device was characterized at using a $1 \mathrm{MHz}$ modulated $2 \mathrm{GHz}$ carrier, and measurements were performed using a fully vector calibrated measurement system. The input stimulus comprised nine equally spaced tones of equal amplitude but with randomised phase. This was necessary to approximate a real wideband signal [8] with a peak-to-average power ratio (PAPR) of $9.54 \mathrm{~dB}$. The device was driven to deliver $39.5 \mathrm{dBm}$ peak envelope power (PEP) at approximately $1.5 \mathrm{~dB}$ compression, and active IF load-pull was used to present the constant baseband impedance to all the significant baseband components (IF1-IF5) whilst in the first instance, the RF was

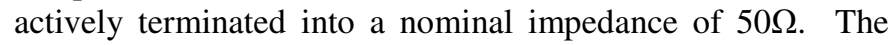
optimum IF impedance for the best linearity for this particular device, as has been identified using two-tone escitation in [3], lies outside the smith chart.

Fig. 4 illustrates a measurement where the broadband IF impedance was held constant for all baseband tones and swept over a measurement grid that includes the short circuit condition, and extends some way outside the Smith chart. For each of the measurement points, the bias and drive level was maintained constant. The optimum loads for $\mathrm{ACPR}_{\mathrm{L}}$ and $\mathrm{ACPR}_{\mathrm{H}}$ lie on the real axis and are almost identical in terms of their position and the degree of linearization they offer. The contours of $\mathrm{ACPR}_{\mathrm{L}}$ are plotted in Fig. 4 and show that at point $\mathrm{B}$, the performance is found to be $-43 \mathrm{dBc}$, which is approximately a $25 \mathrm{~dB}$ improvement over the classical short circuit case (point A).

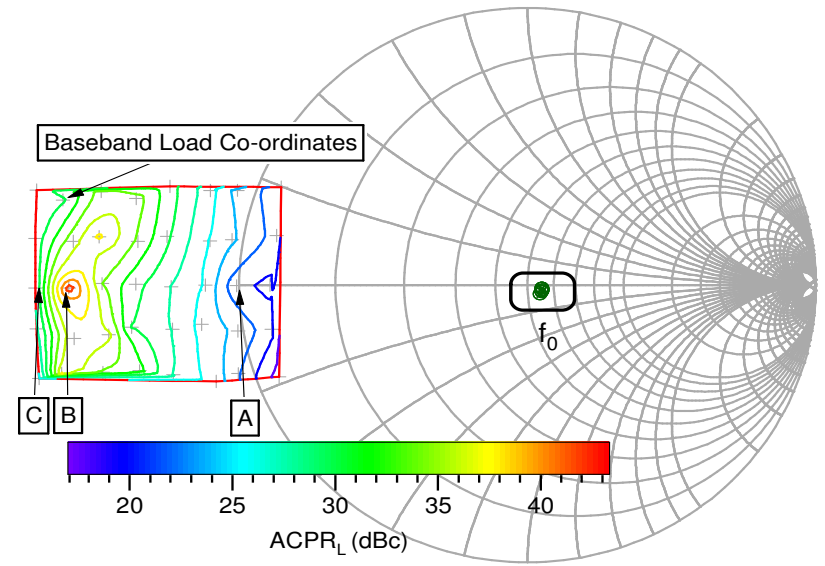

Fig. 4 Measured lower adjacent channel power linearity contours as a function of IF reflection coefficient $\left(\Gamma_{\mathrm{L}}\right)$.

The optimum load conditions for best linearity identified using this new sampling approach agree closely with the earlier documented results [3] on the same device, hence validating the approach. Fig. 5 depicts the baseband voltage and current waveforms required to present the device with the 
IF impedance of point B in Fig. 4. These relatively complex linearising waveforms are clearly in-phase due to the purely real negative nature of the baseband termination and contain five baseband harmonics.

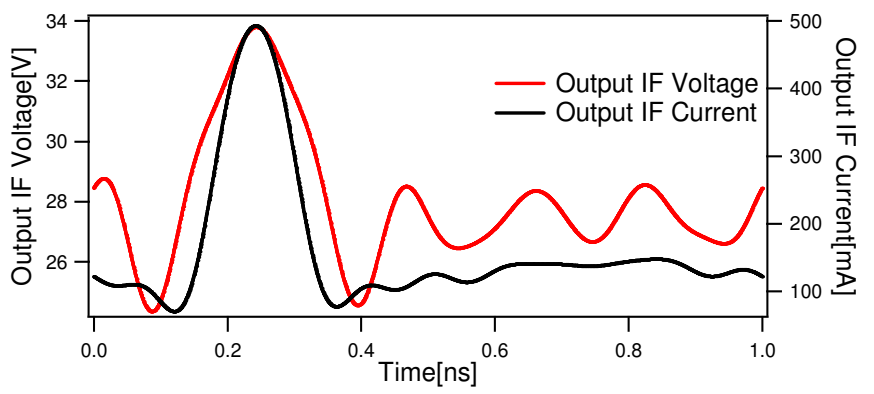

Fig. 5 Measured output baseband voltage and current at for best linearity at point $-\mathrm{B}$.

\section{EMULATION OF CLASS-J IMPEDANCE ENVIRONMENT}

To further demonstrate the enhanced broadband load-pull capabilities of the measurement system, a class-J mode was emulated by presenting established impedances [9] at the device package plane. The device was deep class-AB biased, and driven approximately $2 \mathrm{~dB}$ into compression with a twotone modulated excitation centred at $2 \mathrm{GHz}$ with a $4 \mathrm{MHz}$ tone spacing. An optimum reactive fundamental impedance was presented to all fundamental tones and a suitably phased reactive second harmonic impedance termination was presented to the tones around the $2^{\text {nd }}$ harmonic. The third harmonic components were terminated arbitrarily.

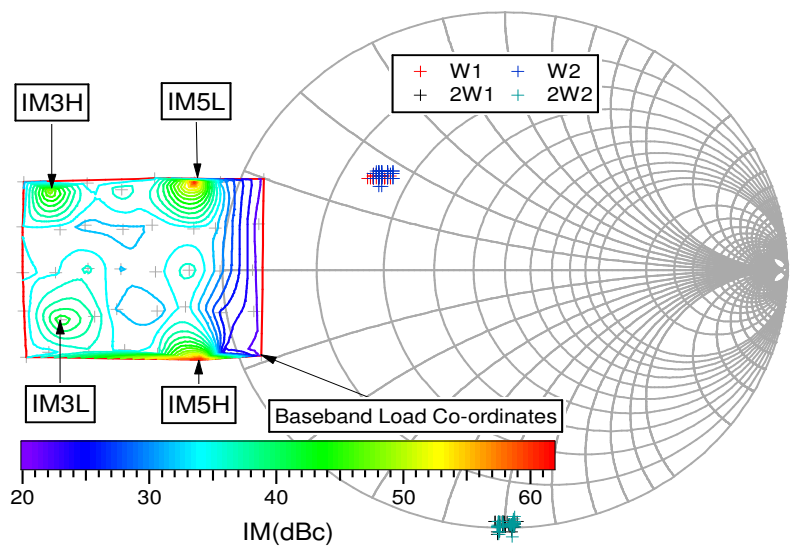

Fig. 6 Measured IM linearity contours as a function of IF reflection coefficient $\left(\Gamma_{\mathrm{L}}\right)$ for a class-J emulated RF impedance environment.

This analysis was specifically designed to investigate the effectiveness of baseband linearization techniques for novel PA modes and architectures, so as in the previous analysis, the impedance presented to all baseband tones was swept over a measurement around the short circuit condition. Selected inter-modulation distortion (IMD) contours are plotted in Fig. 6 , which in this case show an interesting result - different nonreal optima for different IMD terms. The ability of the system to maintain broadband baseband, fundamental and second harmonic loads is critical. This capability is evident in Fig.6 where the second and fundamental loads for all 40 baseband points are overlaid on the same smith chart - the variation and dispersion in these loads can be seen to be minimal and in terms of normalised Cartesian coordinates, this was measured to be in the region of $0.6 \%$ (1SD) for both fundamental and second harmonic loads.

\section{CONCLUSION}

An enhanced modulated waveform measurement system has been demonstrated that allows the emulation of novel PA modes and architectures, including for example class-J and envelope tracking respectively. Direct observation of the effects of baseband impedance variations is now possible over wide bandwidths, and under complex multi-sine excitations. Measurement speed and dynamic range have both been dramatically improved through the adoption of a new technique, here referred to as 'windowing', that allows the capture of a complete modulation cycle in a time efficient way and whilst employing the full capabilities of the sampling oscilloscope used.

The enhanced measurement system has been verified and validated through comparison with previous observations using earlier systems; relating to identification of optimal baseband impedances for linearity [3]. The true benefit of this enhanced system becomes apparent through the broadband emulation of a class-J mode of operation however, where initial results suggest that there exist separate optimum impedances for suppression of IM distortion products, and it is felt that this key observation may have significant implications for modern broadband PA design approaches.

\section{REFERENCES}

[1] Joel Vuolevi and Timo Rahkonen, "Distortion in RF Power Amplifiers," Norwood, MA: Artech House, 2003.

[2] J. Vuolevi, J. Manninen, T. Rahkonen, "Cancelling the memory effects in RF power amplifiers", in IEEE International Circuits and Systems Symposium, 2001, pp. 57-60.

[3] M. Akmal, et. al, "The Effect of Baseband Impedance Termination on the Linearity of GaN HEMTs", in Proc. of $40^{\text {th }}$ European Microwave Conference, September 2010, pages: 1046 -1049.

[4] A. Alghanim, J. Lees,T. Williams, J. Benedikt, P.J. Tasker, P "Using active IF load-pull to investigate electrical base-band induced memory effects in high-power LDMOS transistors," in Proc. Asia-Pacific Microwave Conference, 2007, 11-14 Dec. 2007 Page(s):1 - 4.

[5] M. Akmal, et. al, “ The Impact of Baseband Electrical Memory Effects on the Dynamic Transfer Characteristics of Microwave Power Transistors", in Proc. of $4^{\text {th }}$ International Nonlinear Microwave Monolithic Integrated Circuit(INMMIC), April 2010, pages: 148 - 151.

[6] J. Lees, et. al, "Demystifying Device Related Memory Effects Using Waveform Engineering and Envelope Domain Analysis", in Proc. $38^{\text {th }}$ European Microwave Conference, October 2008, pages:753-756.

[7] T. Williams, J. Benedikt, P. J. Tasker, "Fully Functional Real Time Non-Linear Device Characterization System Incorporating Active Load Control", in Proc. $36^{\text {th }}$ European Microwave Conference, October 2006, pages:1610-1613.

[8] N. B. de Carvalho, J. C. Pedro, "A comprehensive explanation of distortion sideband asymmetries," IEEE Trans. Microwave Theory Techniques, September 2002, vol. 50, pages. 2090-2101.

[9] P. Wright, J. Lees, P. J. Tasker, J. Benedikt, S.C. Cripps, “An efficient, linear, broadband class-J-mode PA realised using RF waveform engineering", in Proc. of IEEE MTT-S International, June 2009, pages: $653-656$. 


\title{
Linearity Enhancement of GaN HEMTs under Complex Modulated Excitation by Optimizing the Baseband Impedance Environment
}

\author{
M. Akmal ${ }^{\dagger}$, V. Carrubba ${ }^{\dagger}$, J. Lees ${ }^{\dagger}$, S. Bensmida ${ }^{*}$, J. Benedikt ${ }^{\dagger}$, K. Morris ${ }^{*}$, M. Beach ${ }^{*}$, J. McGeehan ${ }^{*}$, \\ P. J. Tasker ${ }^{\dagger}$
${ }^{\dagger}$ Centre for High Frequency Engineering, Cardiff University, The Parade, Cardiff, CF24 3AA, UK
Tel: +44 2920 875938, Email: AkmalM1@ Cardiff.ac.uk \\ *Centre for Communications Research, University of Bristol, Woodland Road, Bristol, BS8 1UB, UK
}

\begin{abstract}
This paper demonstrates how the linearity performance of a $10 \mathrm{~W}$ GaN HEMT can be dramatically improved by actively engineering the baseband impedance environment around the device. An important refinement to existing active load-pull measurement capability is proposed that allows the precise and independent control of all significant baseband and RF components that result from the amplification of a complex 9carrier multi-sine modulation. The synthesis of constant, modulation frequency independent negative baseband impedances, resulting in specific baseband voltage waveforms has delivered a 24dB improvement in ACPR compared to the classical baseband short case, even when the device is operating with RF components terminated into a non-optimal $50 \Omega \mathrm{RF}$ environment. This linearization concept is further investigated through the broadband emulation of a class-J impedance environment around a single device. Using this enhanced system and a two-tone modulated excitation, optimum baseband loads are identified that result in a $18.5 \mathrm{~dB}$ and $24 \mathrm{~dB}$ improvement in $\mathrm{IM}_{3}$ and $\mathrm{IM}_{5}$ inter-modulation products respectively, again relative to the case of a traditional IF short circuit. The significance of this last observation is that unlike the $50 \Omega$ case, the optimum class-J $\mathrm{IM}_{3}$ and $\mathrm{IM}_{5}$ baseband impedances disperse, becoming reactive and moving away from the real axis.
\end{abstract}

Index Terms - Active load-pull, memory effects, baseband, harmonics, power device.

\section{INTRODUCTION}

The continuing evolution of the wireless standards such as WiMAX and Long Term Evolution (LTE) is driving the need for power amplifiers (PAs) to be able to accommodate multiple modulation schemes at multiple frequencies, and also demands spectral efficiency to meet ever-increasing linearity requirements. These standards invariably impose higher peakto-average power ratios (PAR), which typically require increasing degrees of back-off to maintain good linearity, thus reducing the efficiency of the power amplifier considerably. As an example, the PAs used in LTE systems, employing orthogonal frequency division multiplexing (OFDM) need to accommodate scalable bandwidths ranging between $1.4 \mathrm{MHz}$ and $20 \mathrm{MHz}$, with PAR in the region of $10 \mathrm{~dB}$ [1].

In such wideband applications, any variation in baseband impedance over bandwidth can cause adverse effects in terms of device linearity performance where intermodulation distortion (IMD) levels can vary asymmetrically with signal bandwidth - these problems are generally termed memory effects [2]. In such case, filtering because it is too close in frequency to the desired signal cannot generally reduce the resulting IMD. Additionally, the reduction of IMD through simple pre-distortion linearization can become rather difficult when the side-bands become asymmetrical, since the simple pre-distorter assumes symmetrical IMD characteristics according to tone spacing. With the increasing signal bandwidth and PAR associated with modern wireless communication standards; it is frequently observed that predistortion linearization becomes very PA and modulation standard specific [3]. In addition, when PAs are driven into compression, pre-distortion algorithms have difficulty in capturing the true compression characteristics of the PA as the memory effects increase [3]. This highlights the fact that these bandwidth dependent baseband impedance effects on memory behavior are an important problem that needs further investigation. To further investigate such effects and their influence on the linearity performance of a device, a highpower modulated waveform measurement system has been developed and demonstrated in [4-8].

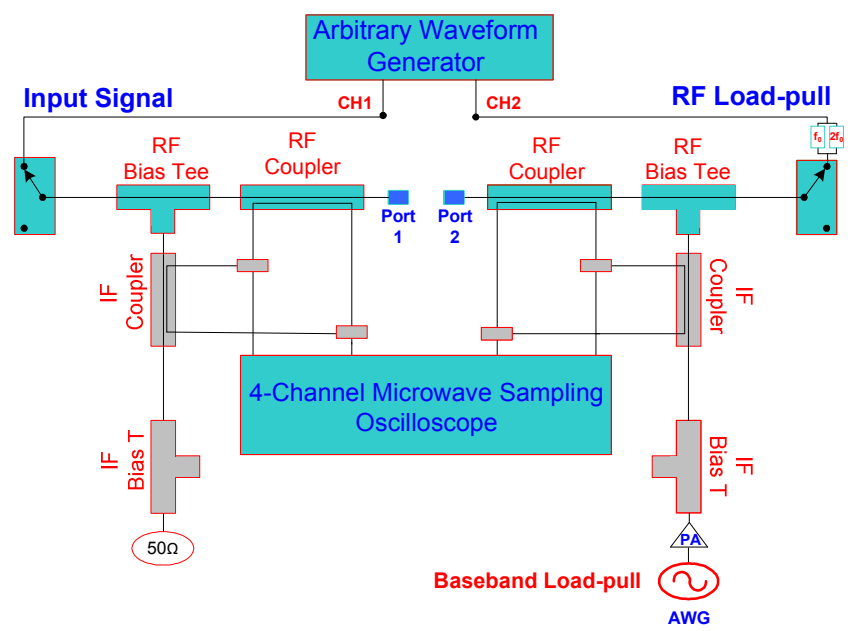

Fig. 1 Modulated waveform measurement system with the capability of active RF and IF load-pull.

The effects of varying the impedance presented to the two most significant baseband components; IF1 and IF2 that are generated as a result of 2-tone excitation has been shown in [4-7], where the device is driven at a relatively backed-off level, $1 \mathrm{~dB}$ below the $1 \mathrm{~dB}$ compression point. However, when 
the device is driven more deeply into compression with more complex excitations, significantly more mixing terms are generated, and in order to achieve a sufficiently broadband IF termination, significant modification of the load-pull measurement system has been required in order to accurately account for higher order baseband components. For this work, this impedance control is being achieved in the time domain using a single wide-band arbitrary waveform generator (AWG) to synthesise the necessray waveforms that result in a constant and specific IF impedance environment across a wide IF bandwidth. The RF synthesizer used in the modulated waveform measurement system depicted in Fig. 1 is a Tektronix AWG7000 Arbitrary Waveform Generator, and is used to synthesize both fundamental excitation and harmonic load-pull signals simultaneously, in the time domain. Using this instrument and its two independent yet coherent channels, the waveform measurement system is capable of maintaining independent and constant impedance control for each individual tone across both the IF and RF impedance environment, and over a wide modulation bandwidth. The approach adopted in this work is to actively engineer the baseband signal environment in order to achieve specific, modulation frequency independent impedances over a bandwidth of at least ten times the modulation bandwidth. The first part of this paper confirms and demonstrates that substantial linearity improvement can be achieved using this approach, when using a complex 9-tone excitation, as has already been shown for case of two-tone in [8]. As expected, for the $\mathrm{GaN}$ device considered, and for this degree of compression, the measured linearity significantly improves when specific negative baseband impedances are presented. The second part of this paper demonstrates the emulation of a modulated class-J PA, through the application of modulated RF active load-pull. This has been done to investigate the effectiveness of using this baseband linearising approach in improving PA linearity when the fundamental and reactive harmonic loads presented to the device become highly reactive. It is worth mentioning that although such 'negative' baseband impedances are non-realiseable using conventional, passive designs, this is not the case when considering active, baseband injection architectures such as Envelope Tracking (ET).

\section{LINEARITY MEASUREMENTS AND ANALYSIS}

All the measurements presented in this section are for a CREE CGH40010 discrete 10W GaN HEMT device, characterized at the centre frequency of $2 \mathrm{GHz}$, within a custom built $50 \Omega$ test fixture. This fixture was calibrated over a relatively wide $50 \mathrm{MHz}$ baseband bandwidth, and over 100 $\mathrm{MHz}$ RF bandwidths centered around the fundamental, second and third harmonics, with both baseband and RF calibrated reference planes established at the device's package plane. This allowed the accurate and absolute measurement of all the significant voltage and current spectra generated at the input and output of the device. Modulated measurements under nine tone stimuls at PAR of $9.54 \mathrm{~dB}$ were performed using a $2 \mathrm{MHz}$ modulation frequency, with the device class-AB biased. Respective drain and gate bias voltages of $28 \mathrm{~V}$ and $-2.05 \mathrm{~V}$ resulted in a quiescent drain current of $250 \mathrm{~mA}\left(\mathrm{I}_{\mathrm{DSQ}} \cong 5 \%\right.$ $\left.\mathrm{I}_{\mathrm{DSS}}\right)$. The device was driven into approximately $1.5 \mathrm{~dB}$ of compression whilst delivering a peak envelope power (PEP) of $41 \mathrm{dBm}$ with fundamental and harmonic components terminated into a nominal impedance of $50 \Omega$, at both input and the output. It should be noted that the broadband $50 \Omega$ load was used in this case to simplify the required measurement architecture. Although this is a non-optimal load condition, it was considered sufficiently representative for the linearity anaylysis presented here. Active IF load-pull was then used to synthesize a range of IF reflection coefficients in order to quantify the effects of the broadband baseband load impedance environment on the non-linear behavior of the DUT.

The optimum IF impedance for the best linearity, as has been identified in [8], lies some way outside the Smith chart. Fig. 2 illustrates a set of measurements where the broadband IF impedance was held constant for all baseband tones and swept over a measurement grid, including the short circuit condition. For each of the measurement points, the bias and drive level was maintained constant.

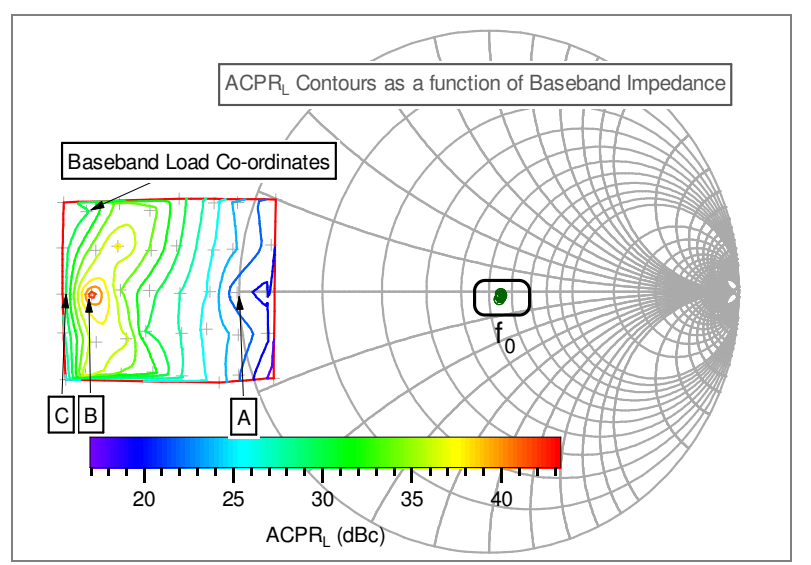

Fig. 2 Measured lower adjacent channel power linearity contours as a function of IF reflection coefficient $\left(\Gamma_{\mathrm{L}}\right)$.

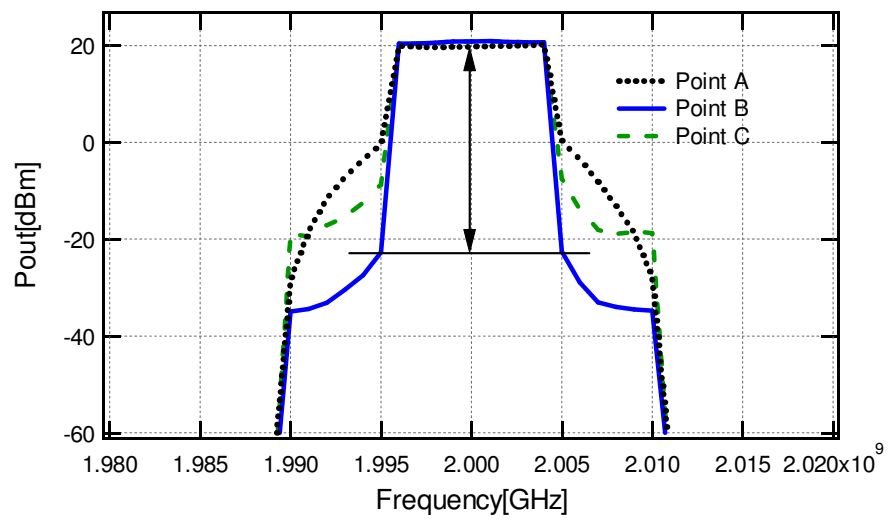

Fig. 3 Measured output power spectrum as a function of IF reflection coefficient $\left(\Gamma_{L}\right)$. 
The optimum loads for $\mathrm{ACPR}_{\mathrm{L}}$ and $\mathrm{ACPR}_{\mathrm{H}}$ lie on the real axis and are almost identical in terms of their position and the degree of linearization they offer. The contours of $\mathrm{ACPR}_{\mathrm{L}}$ are plotted in Fig 2 and show that at point-B, the linearity is found to be $-43 \mathrm{dBc}$, which is approximately a $25 \mathrm{~dB}$ improvement over the classical short circuit case (point-A). From the output power spectrum depicted in Fig. 3, it can be clearly seen that terminating all the significant IF components at point-B significantly improves the device's linearity over a $10 \mathrm{MHz}$ bandwidth, to a level of approximately $-43 \mathrm{dBc}$. This confirms that this method of baseband linearization is not only applicable to two-tone excitations, but can also apply to far more complex, multi-sine signals with high degrees of PAR.

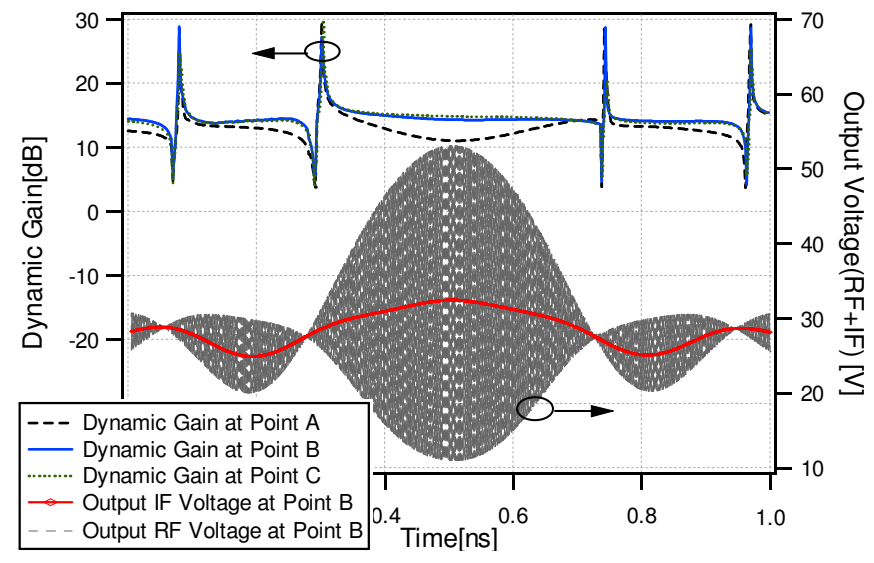

Fig. 4 Envelope domain [5] representation of gain as a function of IF reflection coefficient $\left(\Gamma_{\mathrm{L}}\right)$ at the indentified points, alongside RF and baseband voltage waveforms.

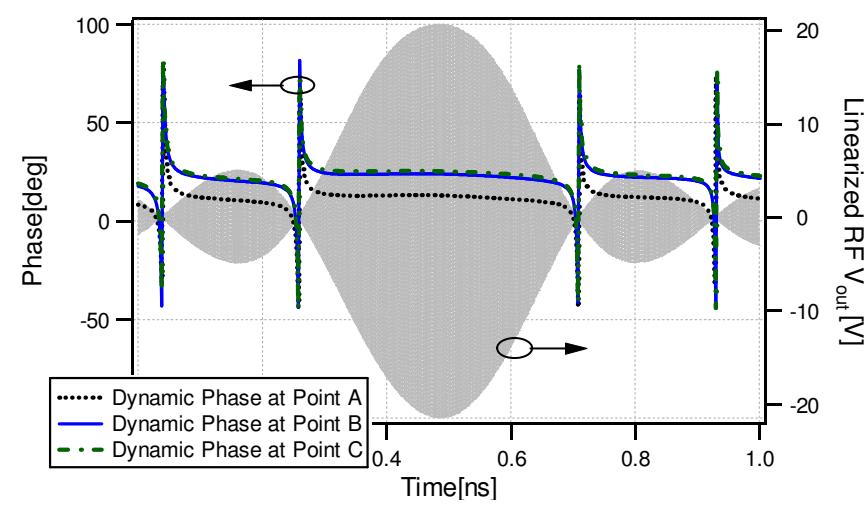

Fig. 5 Measured envelope phase $(\angle \mathrm{b} 2-\angle \mathrm{a} 1)$ as a function of IF reflection coefficient $\left(\Gamma_{\mathrm{L}}\right)$ at the indentified points with linearized output RF voltage at point-B inset.

Fig. 4 shows the dynamic changes in gain over the modulation cycle for the three cases of baseband load reflection co-efficient, identified by points A, B and C in Fig. 2. It is clear from this graph that the negative IF impedance presented at point-B results in an increased and almost constant gain and improved linearity. The effect of the baseband voltage at point-B in shaping and 'positioning' the output voltage waveform can clearly be seen. Setting the baseband load at a short circuit (Point-A) results in a significant depression in the dynamic gain envelope. Whilst point- $C$ delivers almost the same envelope gain as point $\mathrm{B}$, the linearity is reduced by approximately $13 \mathrm{~dB}$.

The phase shown in Fig.5 is defined here as the difference between measured $a_{1}$ and $b_{2}$ modulation envelopes, and its dependency on negative baseband impedance is shown in Fig. 5. It should be noted that the discontinuities observed in both the envelope gain and phase traces are measurement artifacts that occur due to the effect of linear delay as the magnitude of the modulation envelope approaching zero. Interestingly, there is a phase variation of approximately $18^{0}$ between point$\mathrm{A}$ and point-B, and this remains relatively constant over the entire period of the modulation cycle. The peak envelope efficiency is depicted in Fig. 6, and shows a maximum improvement of $8.1 \%$ for point-B compared to the short circuit case. This observation shows that presenting a negative impedances at point- $B$ increases the achievable peak output power and has thus enables a higher efficiency than is possible at point-A.

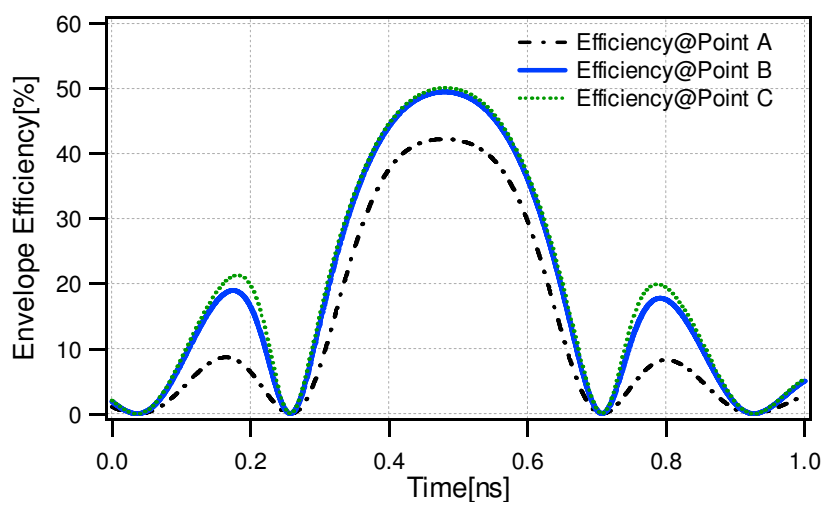

Fig. 6 Dynamic peak efficiency envelopes at an identified baseband loads.

\section{EMULATION OF CLASS-J MODE OF POWER AMPLIFIER}

In order to further investigate the suitability of this efficiency enhancing and linearizing technique under a realistic PA mode of operation, a class-J RF impedance environment was emulated. The device was biased in deep class- $\mathrm{AB}$, driven into approximately $2 \mathrm{~dB}$ of compression and excited using a $4 \mathrm{MHz}$ spaced two-tone modulated excitation centred at $2 \mathrm{GHz}$. With knowledge of the device's intrinsic and extrinsic parasitics, the required class-J impedances were emulated and presented to the package plane [9]. This involved maintaining constant, specific impedances for both fundamental tones as well as the tones $\left(2 \omega_{1}, 2 \omega_{2}\right)$ located around the $2^{\text {nd }}$ harmonic.

The measurement results documented in the previous section clearly indicated that significant linearity benefit can be gained by optimizing the baseband impedance environment, as opposed for example to simply presenting a short circuit. For this class-J investigation, the IF impedance 
presented to all significant baseband tones was swept over a similar measurement grid, again including the short circuit condition, and again extending outside the Smith chart. The IMD contours are plotted in Fig. 7, and similarly show that the optimum IF impedance for reduction of IMD resides outside the Smith chart. Importantly in this case however, the measurement shows that optimum baseband impedances are different for $\mathrm{IM} 3_{\mathrm{L}}, \mathrm{IM} 3_{\mathrm{H}}, \mathrm{IM} 5_{\mathrm{L}}$ and $\mathrm{IM} 5_{\mathrm{H}}$ and these are not, as was the case in the previous $50 \Omega$ measurement, co-located on the real axis. The optimum $\mathrm{IM} 3_{\mathrm{L}}$ and $\mathrm{IM} 3_{\mathrm{H}}$ baseband termination is found to be $-43.2 \mathrm{dBc}$ and $-45.5 \mathrm{dBc}$ respectively, and is approximately $18.5 \mathrm{dBc}$ better than the case where usual short circuit is provided to all the significant baseband components. But from an IM5 perspective, the optimum $\mathrm{IM} 5_{\mathrm{L}}$ and $\mathrm{IM} 5_{\mathrm{H}}$ showed the minimum distortion products which were found to be $-59.3 \mathrm{dBc}$ and $-61.1 \mathrm{dBc}$ respectively.

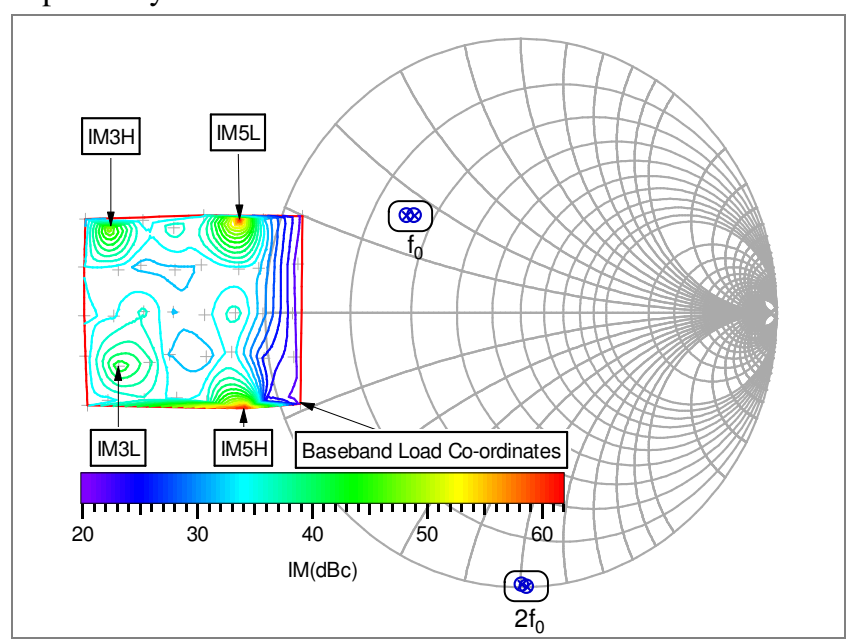

Fig. 7 Measured IM linearity contours as a function of IF reflection coefficient $\left(\Gamma_{\mathrm{L}}\right)$.

Fig. 8 shows the baseband voltage waveforms that result when the IF impedances for best IM3 low and high linearity are presented to the device. Although the magnitude and shape of both waveforms is similar, the phase is clearly different.

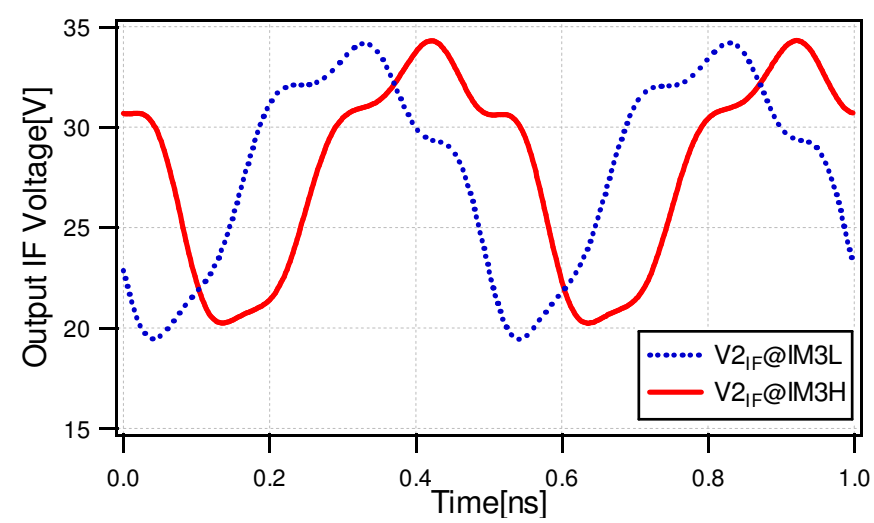

Fig. 8 Baseband voltages that resulted for the best IM3 linearity performance.

\section{Conclusion}

Linearity investigations and analysis of 10W GaN HEMT under complex multi-tone excitations have shown that the optimum impedance for best linearity lies some way outside the Smith chart. The results show that for a 9-tone stimulus, the presentation of specific baseband loads can deliver a linearity improvement of $24 \mathrm{~dB}$ and an efficiency improvement of $8.1 \%$, relative to the baseband short-circuit case.

To investigate the suitability of this baseband linearizing approach to real-world PA architectures, the measurement system was used to emulate a class-J impedance environment, and the results suggest that although linearity can be improved, the optimum impedances become dispersed and move away from the real axis. This important observation may have large implications for the linearization of emerging, broadband PA architectures, especially when these involve efficiency enhancing techniques such as envelope tracking.

\section{ACKNOWLEDGMENT}

This work has been carried out as part of EPSRC grant EP/F033702/1. The authors would also like to thank CREE for supporting this activity and supplying the devices; specifically Ray Pengelly and Mr. Simon Wood.

\section{REFERENCES}

[1] 3GPP Long Term Evolution specification, [Online]. Available: http://cp.literature.agilent.com/litweb/pdf/5989-8139EN.pdf

[2] Joel Vuolevi and Timo Rahkonen, "Distortion in RF Power Amplifiers," Norwood, MA: Artech House, 2003

[3] M. D. LeFevre, D. W. Runton, C. T. Burns, M. K. Mellor, "Digital Predistortion from an RF Perspective", 2010 IEEE Topical Symposium on Power Amplifiers for Wireless Communications, September 2010.

[4] A. Alghanim, J. Lees, T. Williams, J. Benedikt, P. Tasker, "Using active IF load-pull to investigate electrical base-band induced memory effects in high-power LDMOS transistors," in Proc. Asia-Pacific Microwave Conference, 2007, 11-14 Dec. 2007 Page(s): 1 - 4.

[5] J. Lees, et al, "Demystifying Device related Memory Effects using Waveform Engineering and Envelope Domain Analysis" in Proc. $38^{\text {th }}$ European Microwave Conference, October 2008, Page(s): 753 - 756

[6] M. Akmal, et al, " The Impact of Baseband Electrical Memory Effects on the Dynamic Transfer Characteristics of Microwave Power Transistors", in Proc. of $4^{\text {th }}$ International Nonlinear Microwave Monolithic Integrated Circuit(INMMIC), April 2010, pages: $148-151$.

[7] J. Lees, et al, "Waveform Engineering Applied to LinearEfficient PA Design", in Proc. of $11^{\text {th }}$ IEEE Wireless and Microwave Technology Conference (WAMICON), April 2010, pages: $1-5$.

[8] M. Akmal, et al, "The Effect of Baseband Impedance Termination on the Linearity of GaN HEMTs", in Proc. of $40^{\text {th }}$ European Microwave Conference (EuMC), September 2010, pages: $1046-1049$.

[9] P. Wright, J. Lees, P. J. Tasker, J. Benedikt, S.C. Cripps, “An efficient, linear, broadband class-J-mode PA realised using RF waveform engineering", in Proc. of IEEE MTT-S International, June 2009, pages: $653-656$. 


\title{
Minimization of Baseband Electrical Memory Effects in GaN HEMTs Using Active IF Load-pull
}

\author{
M. Akmal ${ }^{1}$, J. Lees ${ }^{1}$, V. Carrubba ${ }^{1}$, S. Bensmida ${ }^{2}$, S. Woodington ${ }^{1}$, J. Benedikt ${ }^{1}$, K. Morris ${ }^{2}$, M. Beach ${ }^{2}$, \\ J. McGeehan ${ }^{2}$ and P.J. Tasker ${ }^{1}$ \\ ${ }^{I}$ Cardiff School of Engineering, Cardiff University, The Parade, Cardiff, CF24 3AA, Wales, UK \\ ${ }^{2}$ Centre for Communications Research, University of Bristol, Woodland Rd, Bristol, BS8 1UB, UK \\ AkmalM1@Cardiff.ac.uk
}

\begin{abstract}
$A B S T R A C T$ - This paper presents a rigorous way to quantify the role played by higher baseband impedances in determining baseband electrical memory effects observed in power transistors under two-carrier excitation. These effects typically appear not only as asymmetrical distortion terms in the frequency domain, but also more reliably as a recognizeable hysteresis or looping in the dynamic transfer characteristics extracted from measured input voltage and output current envelopes of a power device. Investigations have been carried out using a commercially available $10 \mathrm{~W}$ GaN HEMT device characterised at $2 \mathrm{GHz}$ within a high-power modulated wavefor measurement system. Active IF loadpull has been employed to present specific baseband impedance environments, allowing the sensitivity of IMD symmetry to baseband impedance variations to be investigated.
\end{abstract}

Index Terms - Baseband, Gallium Nitride (GaN), active IF load-pull, hysteresis, memory effects, power amplifiers.

\section{INTRODUCTION}

Memory effects are recognised as a major obstacle in the design of highly linear power amplifiers for current and future wireless communication systems [1]. 'Memory' in this context is evident from variation in the magnitude and symmetry of intermodulation distortion (IMD) levels, as a function of signal bandwidth. These problematic effects can and do lead to significant difficulties and complexities in meeting the required power amplifier (PA) linearity requirements with standered linearization techniques often proving to be inadequate[1,2].

Electrical memory effects are generally caused by varying impedance across baseband (often significant) as well as RF modulation bandwidths. In classical PA design, the dependency of IMD on the envelope frequency is minimized by engineering baseband impedances to be as small as possible, for example less than $1 \Omega$ over a baseband frequency range significantly larger than the operational bandwidth [3]. In reality, this is difficult to achieve over increasingly large baseband impedance ranges, and the result can be drain bias modulation effects that contribute significantly to the PAs overall spectral distortion with increased magnitude and asymmetry in measured IMD[4].

Previous work clearly demonstrates the realistic scenario where high-power devices can have significant asymmetrical IMD behaviour, the consequence of which is that the modulated RF emerging from the output of the power device does not respond instantaneously to the applied excitation, leading to unwanted quasi-static memory effects. Intermodulation distortion suppression can be achieved by exploiting the second-order nonlinearity and the presence of second-order distortion signals at the second-harmonic or baseband (difference) frequencies. This has been achieved based on Volterra series and second-order signal injection [5,6]. Although quantitative measures have previously been proposed that attempt to capture the essence of memory effects [7], these tend to describe the causes of baseband electrical memory in terms of the impact only upon intermodulation product asymmetry [7] and leaving the effect on transfer characterstic hysteresis undemonstrated. Once examined, it is clear that this measured hysteresis, originating from the device's inherent nonlinearity, can be very sensitive to variations in baseband impedance, and this reinforces the importance of optimization of broad baseband impedance termination at the device output.

This paper focusses on demonstrating that baseband electrical memory effects have the most significant influence on intermodulation products and are the dominant cause of observed hysteresis in the measured dynamic transfer characteristics of a device. The techniques developed in $[8,9]$ are used to achieve the required broadband baseband impedance termination. Nevertheless, through the application and enhancement of an active IF load-pull arrangement, these baseband impedances have been controlled over a significant bandwidth; in this case at least eight times of modulating frequency.

\section{MODUlATED WAVEFORM MEASUREMENT SYSTEM}

To support the successful control of the out-of-band frequency components, particularly at baseband, a dedicated measurement setup has been developed and demonstrated in [8] to evaluate the device linearity performance under modulated excitations. In earlier work [9,10] only the impedance presented to the two most significant baseband components (IF1 and IF2) generated as a result of 2-tone excitation were controlled. This was achieved by combining two, phase coherent arbitrary waveform generators (AWGs) whilst the device was driven at a relatively backed-off level at $1 \mathrm{~dB}$ below the $1 \mathrm{~dB}$ compression point. However, when the device is driven more deeply into compression, significantly more distortion components are generated, and in order to achieve a sufficiently broad baseband termination, significant modification of the baseband load-pull measurement system was required in order to accurately control the higher baseband components. The integrated measurement 
architecture is shown in Fig. 1 and provides the ability to present independent impedances to all the baseband components that result from a multi-tone excitation. Enhancements performed as part of this work allow this now to be achieved in the time domain, using a single $80 \mathrm{MHz}$ arbitrary waveform generator (AWG) to synthesise the necessray waveforms to allow a constant and specific baseband impedance environment to be maintained across a wide bandwidth.

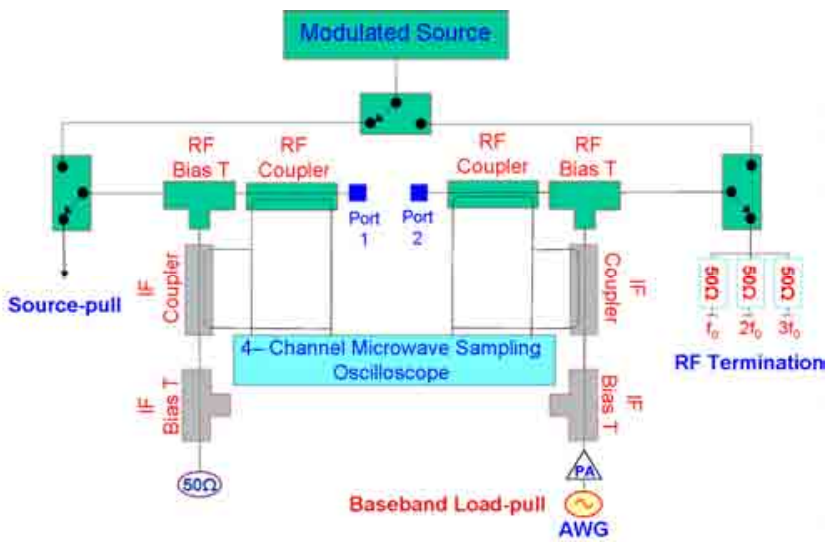

Fig. 1 Modulated waveform measurement system with broadband active IF load-pull.

\section{Modulated MEASUREMENTS AND ANALYSIS}

The system was calibrated using a custom $50 \Omega$ test fixture over a bandwidth of $50 \mathrm{MHz}$ at baseband, and over $180 \mathrm{MHz}$ RF bandwidths. This fully vector error corrected system can account for any errors introduced due to losses, mismatches and imperfect directivities in the system and allows for the measurement of the complete modulated voltage and current waveforms that exist at the DUT plane. In this set of measurements, RF fundamental and associated harmonics were terminated into a nominal impedance of $50 \Omega$, whilst the IF components were actively load-pulled. The investigations were performed on a $10 \mathrm{~W}$ GaN device biased in class- $\mathrm{AB}$ and characterized at a fundamental frequency of $2 \mathrm{GHz}$, for different values of tone spacing. Respective drain and gate bias voltages of $28 \mathrm{~V}$ and $-2.05 \mathrm{~V}$ resulted in a quiescent drain current of $250 \mathrm{~mA}\left(\mathrm{I}_{\mathrm{DSQ}} \cong 5 \% \mathrm{I}_{\mathrm{DSS}}\right)$. The device was driven to approximately $1.5 \mathrm{~dB}$ of compression resulting in $39.5 \mathrm{dBm}$ peak envelope power (PEP).

To understand the effects of baseband impedance, particularly the significant baseband components IF1 (twice the modulation frequency) and IF2 (four times the modulation frequency), the higher baseband components IF3 (six times the modulation frequency) and IF4 (eight times the modulation frequency) on memory effects and dynamic transfer characteristics, the baseband impedance environment was optimized. Generally, terminating baseband components into nominal $50 \Omega$ impedance will cause a ripple to appear on the DC drain voltage present at the device plane, which effectively results in additional modulation or re-modulation of the RF signal. Therefore, the IF active load-pull capability was employed as a means to control all of the baseband components. The magnitude of the synthesized reflection coefficient for all baseband components was set to unity whilst the phase was varied in the area of the short circuit at the edge of the Smith chart. Fig. 2 shows the variation of the four most significant baseband load impedances $\left(\Gamma_{\mathrm{IF}}\right)$ that result form a tone separation frequency of $2 \mathrm{MHz}$. It can be seen that $\Gamma_{\mathrm{IF}}=1 \angle 150^{\circ}$ clearly corresponds to an inductive baseband termination and $\Gamma_{\mathrm{IF}}=1 \angle 210^{\circ}$ corresponds to capacitive baseband termination.

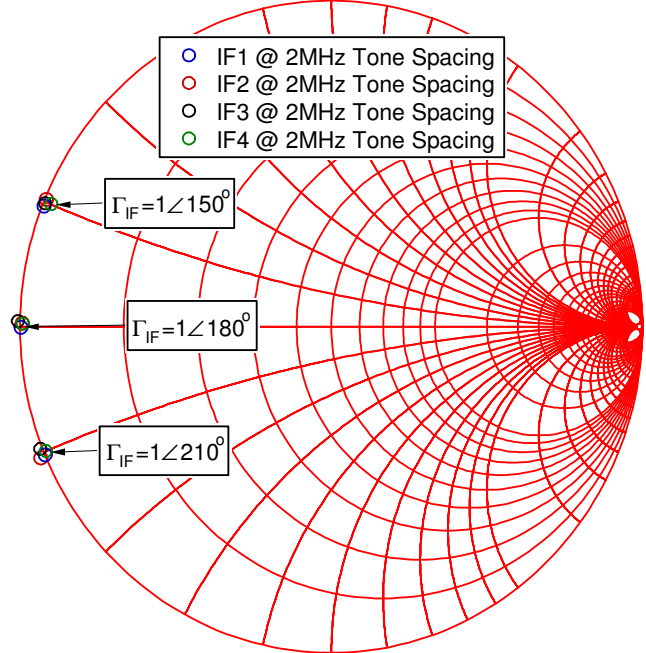

Fig. 2 Measured baseband impedances at $2 \mathrm{MHz}$ tone spacing for different baseband reflection coefficient $\left(\Gamma_{\mathrm{IF}}\right)$.

Working in the envelope domain, and using the measured RF voltage and current envelopes extracted from the measured magnitude and phase of all significant tones around the carrier, it is possible to begin to investigate the causes of envelope asymmetry.

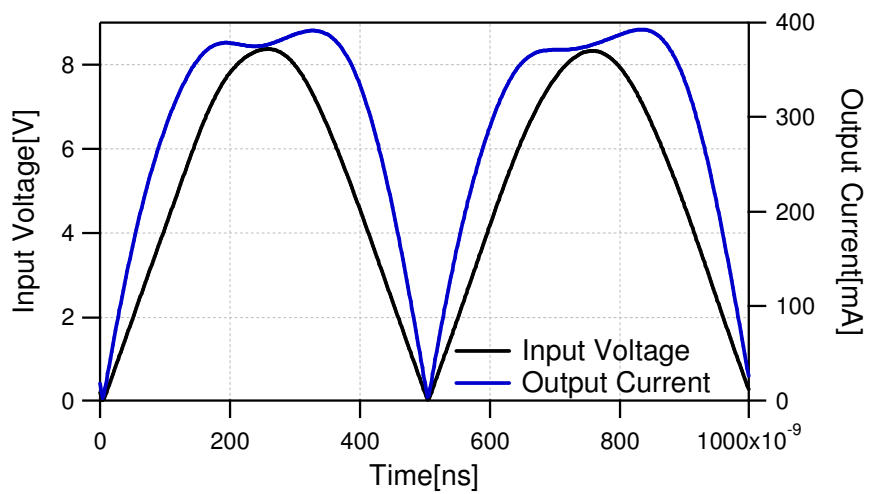

Fig. 3 Measured dynamic input voltage and output current envelopes for $2 \mathrm{MHz}$ tone spacing at $\Gamma_{\mathrm{IF}}=1 \angle 150^{\circ}$

Fig. 3, 4 and 5 show the input voltage and output current envelopes for each of the three cases of IF load shown in fig.2, and illustrate how terminating the baseband components with reactive impedances dramatically changes the shape of the output current envelopes and result in the different types of induced memory effects, observable in the dynamic transfer characteristics; it is this asymmetrical envelope shape that determines which IMD3 product $\left(2 \omega_{2^{-}}\right.$ $\left.\omega_{1}\right)$ or $\left(2 \omega_{1}-\omega_{2}\right)$ will have the greater magnitude. Explicitly, 
fig. 4 depicts the case where a short circuit impedance was maintained for all four of the baseband components considered, at a $2 \mathrm{MHz}$ tone-spacing, this results in symmetrical input voltage and output current RF envelopes.

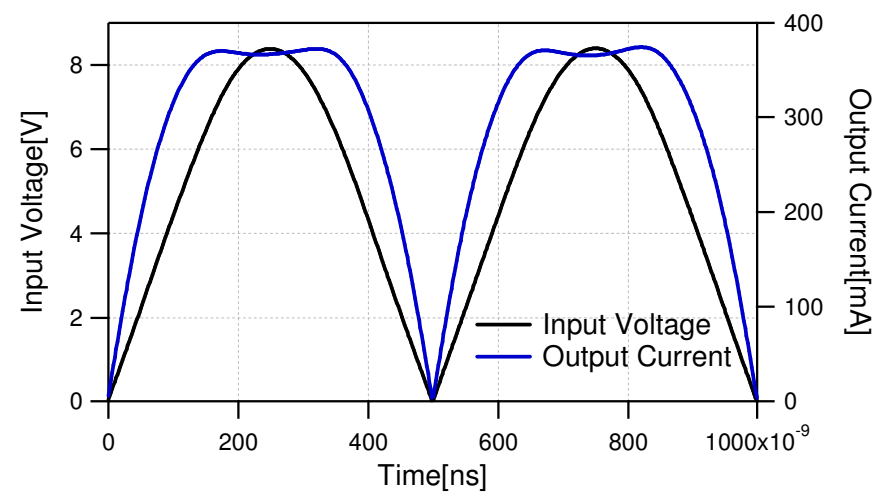

Fig. 4 Measured dynamic input voltage and output current envelopes for $2 \mathrm{MHz}$ tone spacing at $\Gamma_{\mathrm{IF}}=1 \angle 180^{\circ}$

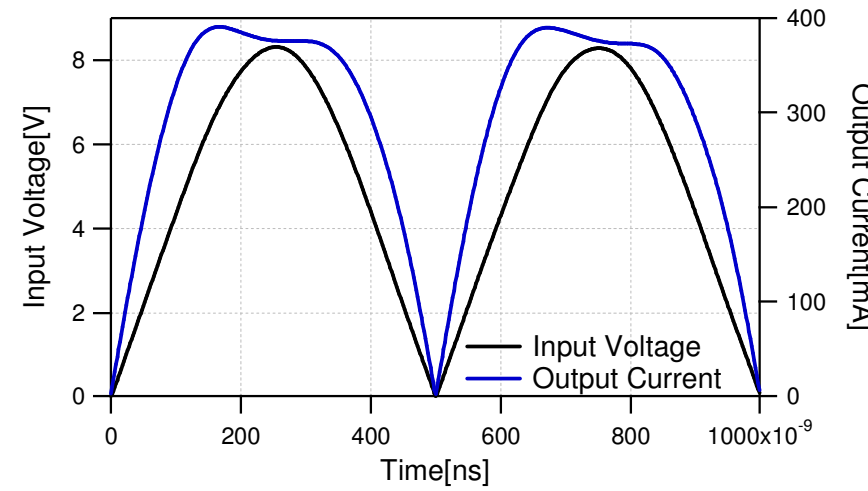

Fig. 5 Measured dynamic input voltage and output current envelopes for $2 \mathrm{MHz}$ tone spacing at $\Gamma_{\mathrm{IF}}=1 \angle 210^{\circ}$

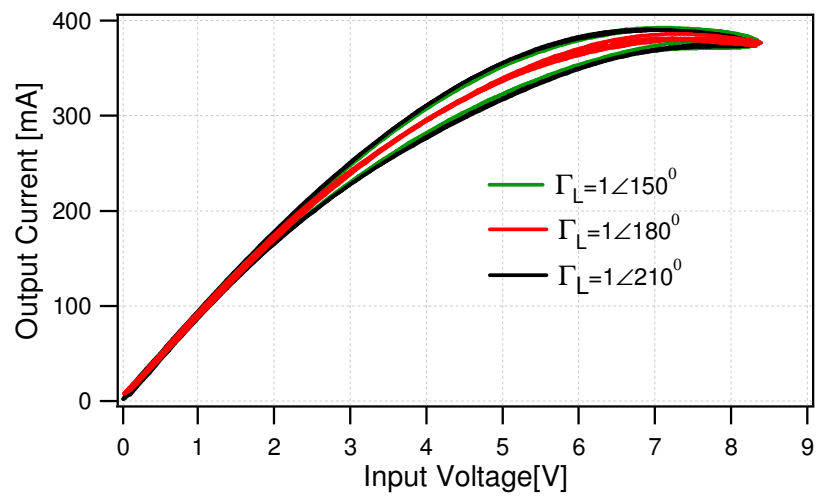

Fig. 6 Dynamic transfer characteristics at different baseband reflection coefficients for $2 \mathrm{MHz}$ tone spacing.

Fig. 6 presents the dynamic transfer characteristics obtained for the same three cases of $\Gamma_{\mathrm{IF}}$. In the cases where the baseband impedance presented to the transistor's output is no longer a short circuit, the presence of the inductive or capacitive reactance causes the baseband current and voltage waveforms to become phase shifted and as a consequence the output RF current envelope to become asymmetrical, appearing as a phenomenon of hysteresis in the $\mathrm{V}_{\mathrm{in}}-\mathrm{I}_{\text {out }}$ dynamic transfer characteristic. However, when short circuit impedance $\left(\Gamma_{\mathrm{IF}}=1 \angle 180^{\circ}\right)$ was maintained, then negligible hysteresis was observed. The asymmetrical current envelopes and hence hysteresis in the transfer characteristic can be explained by the fact that at higher drive levels, the RF dynamic load line begins to interact with the knee boundary region, and the degree and nature of the interaction becomes a strong function of the baseband impedance environment. In the case of baseband short circuit termination $\left(\Gamma_{\mathrm{IF}}=1 \angle 180^{\circ}\right)$, the IF load-line (achieved by plotting baseband current vs baseband voltage) remains completely vertical with no looping observed, as shown in fig.7. This particular behaviour of the IF load-line ensures that the RF load-line expands and contracts along the same trajectory, both in the linear region and when it interacts with the knee boundary. As a result, no hysteresis is observed in the dynamic transfer characteristics.

For the induced reactive baseband impedances conditions, there is a significant looping in the IF load-lines, both of which will cause an asymmetrical interaction with the knee boundary condition, as shown in fig.7. The impact of this interaction is clearly visible on either the rising edge or falling edge of each half of the modulation cycle. This behaviour causes the RF load-line to follow a different path in-to and then out-of the knee region. As a consequence asymmetrical output current envelopes emerge.

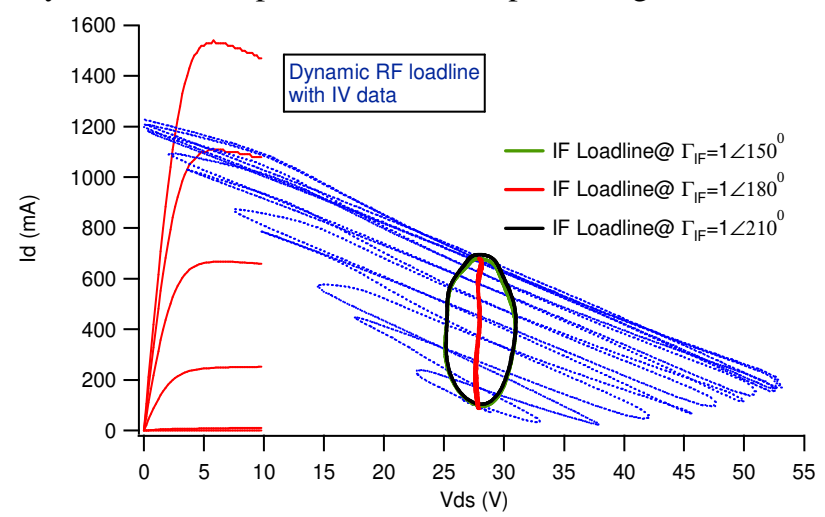

Fig. 7 Dynamic RF load-line and IF load-lines at different baseband reflection coefficients $\left(\Gamma_{\mathrm{IF}}\right)$.

\section{MinimiZATION OF BASEBAND ELECTRICAL MEMORY}

The electrical memory-effect originating from the baseband is not only a function of IF1 $\left(\omega_{2}-\omega_{1}\right)$, IF2 $\left(2 \omega_{2}-2 \omega_{1}\right)$ but also the higher baseband components (IF3 and IF4). In terms of suppression of baseband electrical memory effects, it is clear that all significant baseband frequencies need to be terminated with near short circuit impedance [11] - a common practice for envelope termination in order to achieve symmetrical IMD terms. This is indeed critical under realistic test signals which may have baseband components that extend from zero up to $100 \mathrm{MHz}$. The overall suppression of baseband components using active IF loadpull, can be used to emphasize the importance of broadband termination of the measurement setup when performing linearity analysis of an active device. The bandwidth of the active IF load-pull was limited to approximately $20 \mathrm{MHz}$ by the baseband power amplifier used to amplify the signal 
generated by the arbitrary waveform generator (AWG). This means that it becomes impossible to actively control the higher baseband components (IF3 and IF4) for tone separations above $5 \mathrm{MHz}$. This can be seen by the scattering of these components in the Smith chart plot of fig 8 .

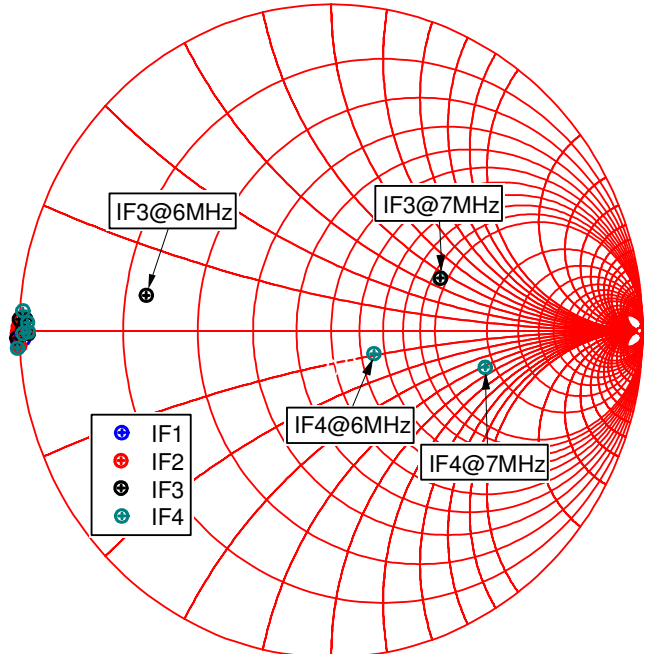

Fig. 8 Measured baseband impedances at different tone spacing after IF active load-pull.

Fig. 9 depicts the $\mathrm{IMD}_{3}$ and $\mathrm{IMD}_{5}$ behavior for different tone-spacing values ranging between $1 \mathrm{MHz}$ and $7 \mathrm{MHz}$, at a constant drive level. The behavior of the two output tones $\omega_{1}$ and $\omega_{2}$ is clearly observed to be almost independent of the tone-separation frequency. The reduction in $\mathrm{IMD}_{3}$ and $\mathrm{IMD}_{5}$ asymmetry confirms that if a frequency independent constant short circuit baseband impedance termination is utilized in the power amplifier drain bias network no modulation frequency sensitivity in IMD responses should be observed. The $\mathrm{IMD}_{3}$ and $\mathrm{IMD}_{5}$ responses are found to be modulation frequency independent only from $1 \mathrm{MHz}$ to $5 \mathrm{MHz}$.

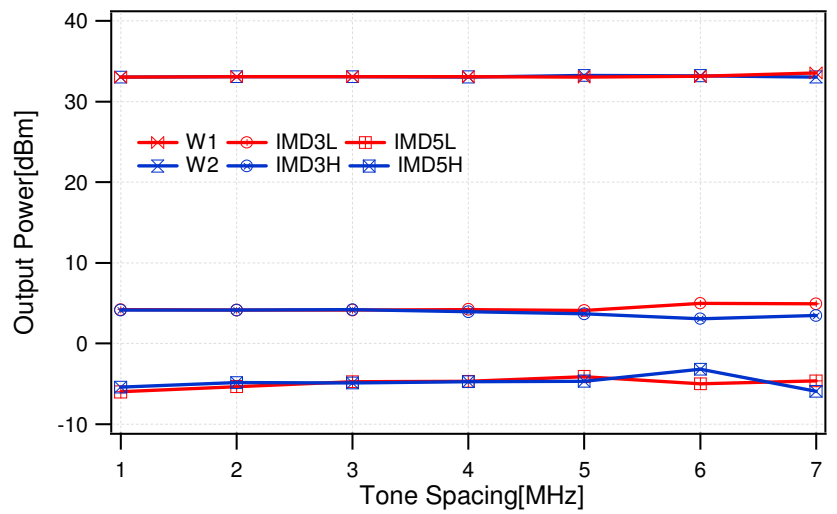

Fig. 9 Measured fundamental and IMD power for different values of tone spacing at a constant drive level of $1.5 \mathrm{~dB}$ compression point.

It is important to note that the variations in the magnitude of $\mathrm{IMD}_{3}$ and $\mathrm{IMD}_{5}$ components above $5 \mathrm{MHz}$ tone spacing are related to variation in base-band impedance, since it was difficult to present the short circuit impedance to the higher baseband components (IF3 and IF4) due to the bandwidth limitation of active IF load-pull power amplifier.

\section{CONCLUSION}

The suppression of electrical memory has been achieved by the synthesis and presentation of frequency invariant IF impedances to significant baseband components through active IF load-pull. The measurements show that hysteresis behavior in the dynamic transfer characteristics can be significantly improved by providing short circuit impedances to all significant baseband components.

The results also suggest however that IMD behavior in 10W GaN HEMT is not only dependent on the most significant IF component (IF1 and IF2), but is also sensitive to higher order IF components. In order to obtain the frequency independent response, the bandwidth over which the base-band impedances must be engineered should be extended to at least eight times the modulated bandwidth.

\section{ACKNOWLEDGEMENT}

This work has been carried out as part of EPSRC grant EP/F033702/1. The authors would also like to thank CREE for supporting this activity and supplying the devices; specifically Ray Pengelly and Mr. Simon Wood.

\section{REFERENCES}

[1] J. Vuolevi, J. Manninen, and T. Rahkonen, "Cancelling the memory effects in RF power amplifiers," in IEEE Int. Symp. Circuits and Systems, pp. 57-60, 2001.

[2] Joel Vuolevi and Timo Rahkonen, Distortion in RF Power Amplifiers, Norwood, MA: Artech House, 2003

[3] S. C. Cripps, RF power amplifiers for wireless communications, $2^{\text {nd }}$ edition. Norwood, MA: Artech House, 2006.

[4] N. Le Gallou, et. al, "Analysis of Low Frequency Memory and Influence on Solid State HPA Intermodulation Characteristics," IEEE MTT-S Int. Microwave Sym. Dig., pp. 979-982, May 2001.

[5] N. B. Carvalho and J. C. Pedro, "A Comprehensive Explanation of Distortion Sideband Asymmetries," IEEE Trans. on Microwave Theory and Tech., Vol. MTT-50, pp. $2090-2101$, Sep. 2002

[6] C. Fan and K. M. Cheng, "Theoretical and experimental study of amplifier linearization based on harmonic and baseband signal injection technique," IEEE Trans. Microwave Theory Tech., vol. 50, pp. 1801-1806, July 2002.

[7] João Paulo Martins, et. al, "A Metric for the Quantification of Memory Effects in Power Amplifiers", IEEE Trans. On Microwave Theory and Tech., vol. 54, no. 12, December 2006

[8] Alghanim, A.; Benedikt, J.; Tasker, P., "A measurement test-set for characterisation of high power LDMOS transistors including memory effects," High Frequency Postgraduate Student Colloquium, 2005 , vol., no.pp. 29- 32, 5-6 Sept. 2005.

[9] Alghanim, et. al. "Reduction of electrical base-band memory effects in high-power LDMOS DevicesUsing Optimum Termination for IMD3 and IMD5 using active load-pull”, in Proc., IEEE International Microwave Symposium, June 2008.

[10] J. Lees, et al, "Demystifying Device Related Memory Effects Using Waveform Engineering and Envelope Domain Analysis", in Proc. $38^{\text {th }}$ European Microwave Conference, October 2008.

[11] Peter B. Kenington, High Linearity RF Amplifier Design, Norwood, MA: Artech House, 2000. 


\title{
The Effect of Baseband Impedance Termination on the Linearity of GaN HEMTs
}

\author{
M. Akmal ${ }^{\dagger}$, J. Lees ${ }^{\dagger}$, S. Bensmida ${ }^{*}$, S. Woodington ${ }^{\dagger}$, V. Carrubba ${ }^{\dagger}$, S. Cripps ${ }^{\dagger}$, J. Benedikt ${ }^{\dagger}$, K. Morris ${ }^{*}$, M. Beach ${ }^{*}$, \\ J. McGeehan ${ }^{*}$ and P. J. Tasker ${ }^{\dagger}$ \\ ${ }^{\dagger}$ Cardiff School of Engineering, University of Cardiff, The Parade, Cardiff, CF24 3AA, Wales, UK \\ *Centre for Communications Research, University of B ristol, Woodland Rd, Bristol, BS8 1UB, UK \\ AkmalM1@Cardiff.ac.uk
}

\begin{abstract}
This paper demonstrates the significant effect of baseband impedance termination on the linearity performance of a $10 \mathrm{~W}$ GaN HEMT device driven to deliver a peak envelope power of approximately $40 \mathrm{dBm}$. The paper also proposes a further refinement to a state-of-art active IF load-pull measurement system to allow the precise independent control of all significant baseband components generated as a result of the multi-tone excitation used. The presentation of specific baseband impedances has delivered a $20 \mathrm{dBc}$ and $17 \mathrm{dBc}$ improvement in $\mathrm{IM}_{3}$ and $\mathrm{IM}_{5}$ inter-modulation products respectively, relative to the case of a classical, ideal short circuit. As expected for this device, this was achieved by emulating appropriate negative impedances lying outside of the Smith chart, and when this observation is considered alongside the Envelope Tracking PA architecture, this raises the interesting possibility of significantly improving PA linearity using the very mechanisms that are employed to improve PA efficiency.
\end{abstract}

\section{INTRODUCTION}

The advent of fourth generation (4G) wireless systems, namely Long Term Evolution (LTE) and mobile WiMAX, has significantly increased the demand on Power Amplifier (PA) linearity requirements. For example, the PAs used in LTE systems, employing orthogonal frequency division multiplexing (OFDM) for downlink need to accommodate scalable bandwidths ranging between $1.4 \mathrm{MHz}$ and $20 \mathrm{MHz}$ [1]. In such wideband applications, any variation in baseband impedance over bandwidth can cause adverse effects in terms of device linearity performance where intermodulation distortion (IMD) levels can vary asymmetrically with instantaneous signal bandwidth - these problems are generally termed memory effects [2], [3].

Such problematic effects can lead to significant difficulty in achieving PA performance that meets the required linearity specifications. Indeed, if the intermodulation distortion becomes asymmetrical, then this can lead to different digital pre-distortion compesation requirements for lower and upper sideband IMD signals, which itself can be problematic. As a result, some common linearization techniques can be rendered ineffective because their success relies upon constant and equal intermodulation levels over the signal bandwidth [3]. Thus, it is of prime importance to understand these anomalous baseband impedance variation effects. To further investigate such baseband impedance effects on the linearity performance of a device, two distinct measurement systems have been developed [4], [5] that can provide independent baseband impedance control. The general approach adopted in this work is to engineer actively the base-band impedance environment in order to achieve a modulation-frequencyindependent impedance environment over a bandwidth of at least eight times that of the modulation bandwidth.

In earlier work [5]-[7], only the impedance presented to the two most significant baseband components (IF1 and IF2) generated as a result of 2-tone excitation were controlled. This was achieved by combining two, phase coherent arbitrary waveform generators (AWGs) whilst the device was driven at a relatively backed-off level at $1 \mathrm{~dB}$ below the $1 \mathrm{~dB}$ compression point. However, when the device is driven more deeply into compression, significantly more mixing terms are generated, and in order to achieve a sufficiently broadband IF termination significant modification of the baseband load-pull measurement system was required in order to accurately account for higher baseband harmonics.

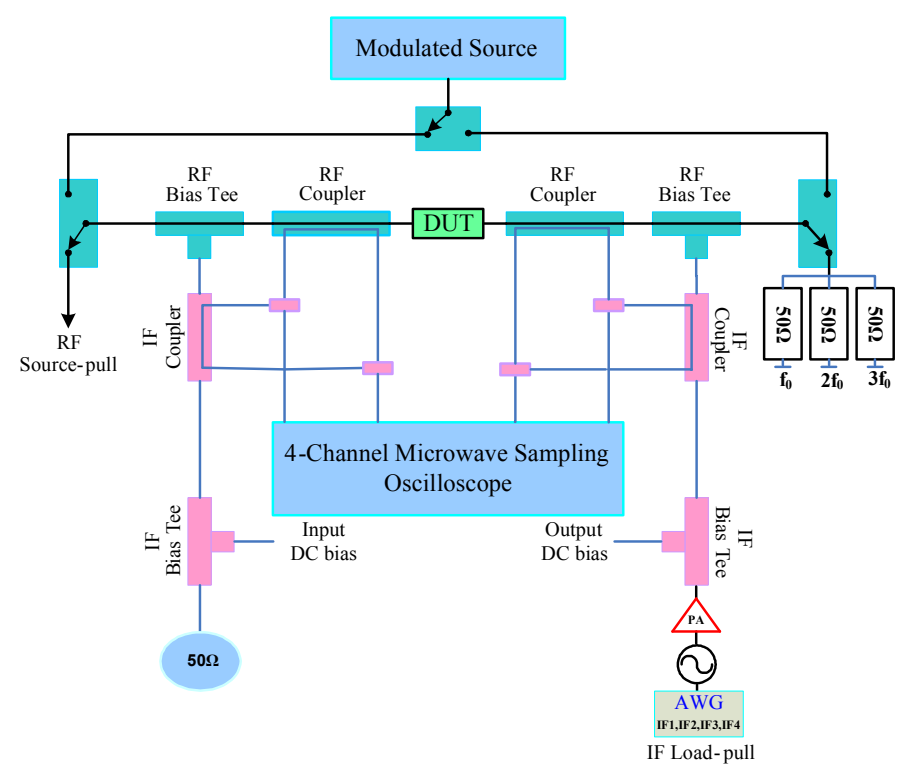

Fig. 1 Modulated waveform measurement system with active baseband load-pull. 
The integrated measurement architecture depicted in Fig. 1 provides the ability to present independent, baseband impedances to all the significant IF frequency components that result from a multi-tone excitation. This is now achieved in the time domain using a single arbitrary waveform generator (AWG) to synthesise the necessray waveforms to allow a constant and specific IF impedance environment to be maintained across a wide IF bandwidth.

The initial aim of this paper is to confirm and demonstrate that baseband electrical memory effects can be greatly reduced by terminating the baseband impedance into ideal short circuits: an impedance environment that would result from conventional design and the use of video bypass capacitors. The second part of the paper considers 'if' and 'how' this situation can be improved by considering alternative baseband impedance conditions. As expected, for the GaN device considered, and for this degree of compression, the measured linearity significantly improves when negative baseband impedances are presented. Although such impedances are non-realiseable using conventional, passive designs, this is not the case when active, baseband injection architectures such as Envelope Tracking (ET) are employed.

\section{LINEARITY INVESTIGATION AND ANALYSIS}

All the measurements presented in this section are for a CREE CGH40010 discrete 10W GaN HEMT device, characterised at the centre frequency of $2 \mathrm{GHz}$, within a custom $50 \Omega$ test fixture. This fixture was calibrated over a relatively wide $50 \mathrm{MHz}$ baseband bandwidth, and over 100 $\mathrm{MHz}$ RF bandwidths centred around fundamental, second and third harmonics, with both baseband and RF calibrated reference planes established at the device's package plane. This allowed the accurate and absolute measurement of all the significant voltage and current spectra generated at the input and output of the device.

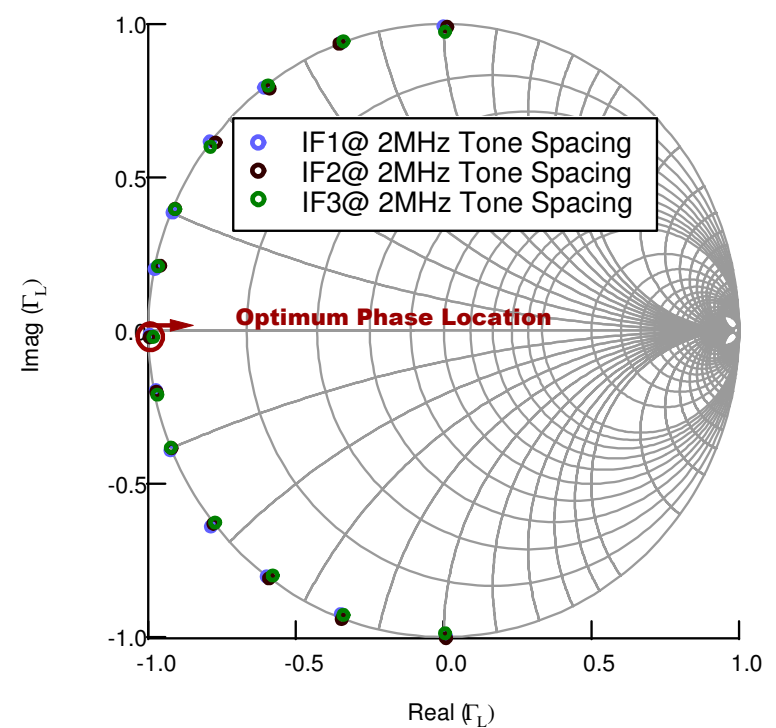

Fig. 2 Measured IF impedances at $2 \mathrm{MHz}$ tone spacing using IF active loadpull.
Two-tone measuremens were performed using a $2 \mathrm{MHz}$ tone spacing, with the device class-AB biased. Respective drain and gate bias voltages of $28 \mathrm{~V}$ and $-2.05 \mathrm{~V}$ resulted in a quiescent drain current of $250 \mathrm{~mA}\left(\mathrm{I}_{\mathrm{DSQ}} \cong 5 \% \mathrm{I}_{\mathrm{DSS}}\right)$. The device was driven into approximately $1.5 \mathrm{~dB}$ of compression whilst delivering $39.5 \mathrm{dBm}$ output peak envelope power (PEP) with fundamental and harmonic components terminated into a nominal impedance of $50 \Omega$, at both the input and the output.It should be noted that braodband $50 \Omega$ load is used to simplify the required measurement architecture. Although this is nonoptimal, but is considered sufficiently representative for the linearity anaylysis presented here. Active IF load-pull was then used to synthesise a range of IF reflection coefficients in order to quantify the effects of the low frequency, broad-band IF load impedance termination on the non-linear behaviour of the DUT.

Fig. 2 illustrates a measurement where the phase of the IF1, IF2 and IF3 loads were varied simultaneously, in steps of $15^{\circ}$ around the perimeter of the Smith chart, whilst keeping the magnitude of IF reflection co-efficient at unity. The results depicted in Fig. 3 clearly show that, as expected, there exists a strong dependence of IM3 and IM5 magnitude on the phase of the baseband impedance. The results explicitly identify an expected optimum phase in the region of $180^{\circ}$ for IF1, IF2 and IF3 loads, where IM3 and IM5 distortion products are minimised.

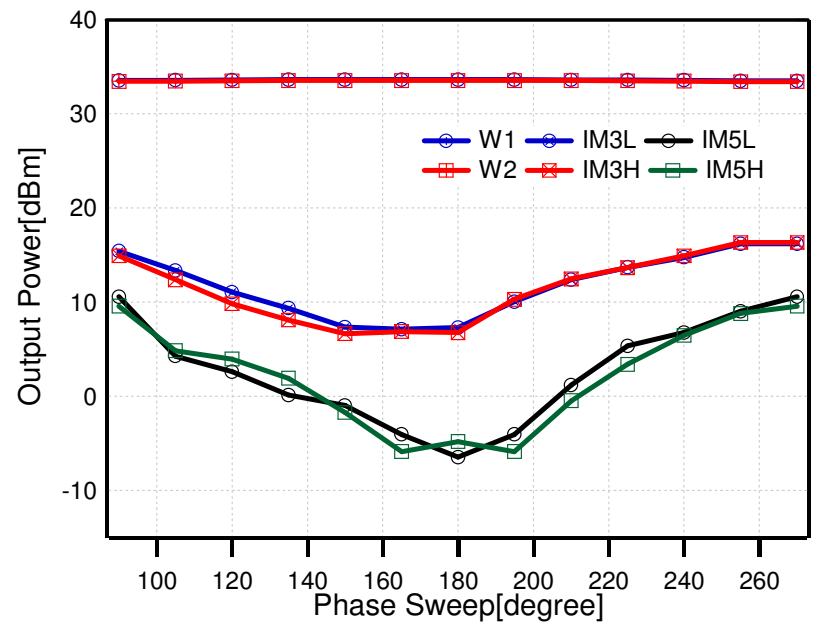

Fig. 3 Measured fundamental and IMD magnitudes at $2 \mathrm{MHz}$ Tone Spacing as a function of IF1, IF2 and IF3 phase.

The measured inter-modulation distortion products presented in Fig. 3 show that when a perfect short impedance $\left(\Gamma_{\mathrm{IF}}=1 \angle 180^{\circ}\right)$ is presented to the significant baseband components, the measured IM3 and IM5 magnitudes can be seen to be $-24 \mathrm{dBC}$ and $-38 \mathrm{dBC}$ respectively.

Active load-pull however has an important advantage in that it is able to seamlessly synthesise both positive impedances within the Smith chart, as well as negative impedances outside the Smith chart. So, in order to explore further the optimum baseband impedances for best linearity conditions, the broadband IF impedance was swept over a 
measurement grid, including the short circuit condition, and extending some way outside the Smith chart. IM3 ${ }_{\mathrm{L}}$ and IM5 $5_{\mathrm{L}}$ contours were then plotted and are shown in Fig. 4 and Fig. 5 respectively, and in both cases show a purely resistive negative optimum impedance. The optimum $\mathrm{IM} 3_{\mathrm{L}}$ performance (point B) is found to be $-43.5 \mathrm{dBc}$, and is approximately $19.5 \mathrm{dBc}$ better than the case where usual short circuit is provided to all the significant baseband components (point A).

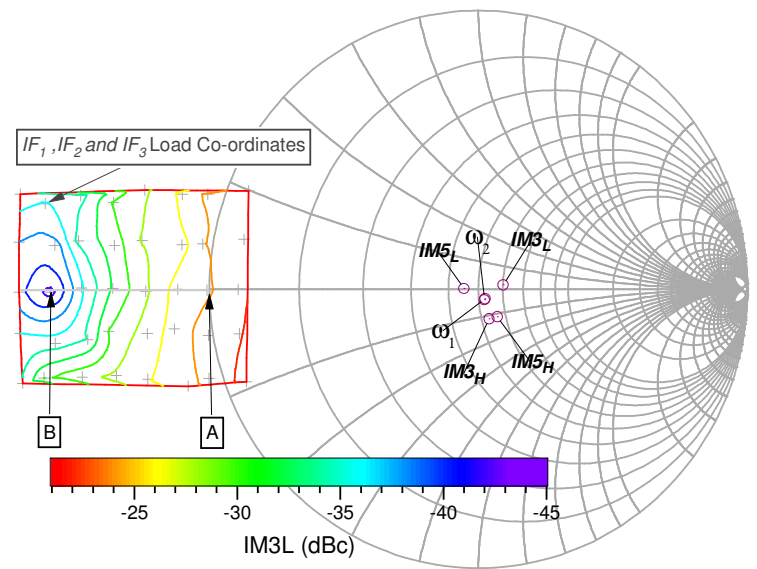

Fig. 4 Measured IM3L linearity contours as a function of IF1, IF2 and IF3 loads.

With regard to the $\mathrm{IM} 5_{\mathrm{L}}$ and $\mathrm{IM} 5_{\mathrm{H}}$, an improvement of $17 \mathrm{dBc}$ was achieved at an optimum termination (point $\mathrm{C}$ ) as compared to the short circuit case (point $\mathrm{A}$ ). As the contours for $\mathrm{IM} 3_{\mathrm{L}}$ and $\mathrm{IM} 3_{\mathrm{H}}$ were found to be almost identical, as was the case for $\mathrm{IM} 5_{\mathrm{L}}$ and $\mathrm{IM} 5_{\mathrm{H}}$, only $\mathrm{IM} 3_{\mathrm{L}}$ and $\mathrm{IM} 5_{\mathrm{L}}$ contours are presented here.

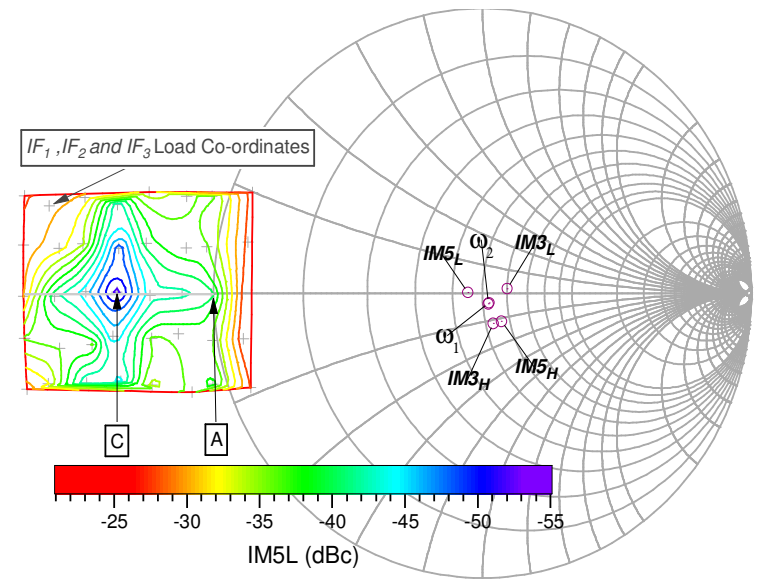

Fig. 5 Measured IM5L linearity contours as a function of IF1, IF2 and IF3 loads.

If we consider the behaviour of IM3 and IM5 components for the cases of IF loads only located along the real axis, it can be seen that with regard to Fig. 6, the real baseband impedances required to minimize IM3 and IM5 are different, and located at points $\mathrm{B}$ and $\mathrm{C}$ respectively. Establishing the broadband IF load at point B leads to an approximate $11 \mathrm{~dB}$ degradation in the established IM5 optimum. Conversely, fixing the IF load at point $\mathrm{C}$ results in an approximate $7 \mathrm{~dB}$ degradation from the established IM3 optimum. Having said this, it can be seen that adopting a broadband IF load impedance between points $\mathrm{B}$ and $\mathrm{C}$ offers a significant improvement in linearity when compared to the usual short circuit termination located at point-A.

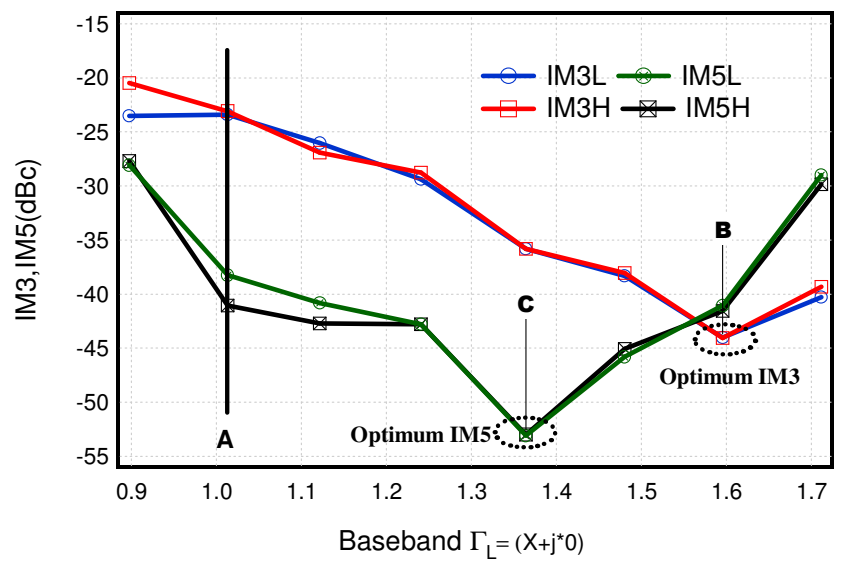

Fig. 6 Measured IM3 and IM5 linearity as a function of baseband reflection coefficient $\left(\Gamma_{\mathrm{L}}\right)$.

Fig. 7 shows the baseband voltage waveforms that result when the IF impedances for point $\mathrm{A}, \mathrm{B}$ and $\mathrm{C}$ are presented to the device.

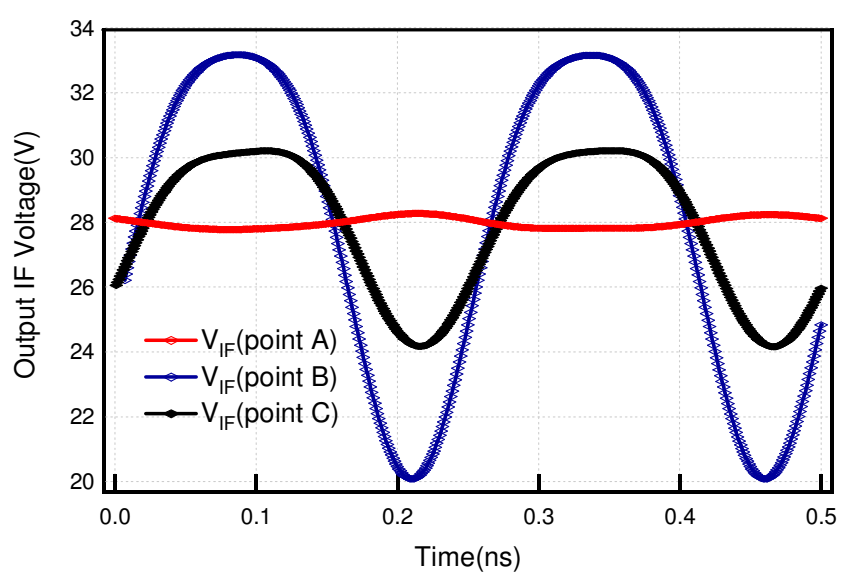

Fig. 7 Baseband voltage that result for the two cases of IF load impedances for minimum IM3 and IM5.

\section{OPTIMIZED DEVICE LINEARITY PERFORMANCE}

From the previous two-tone measurements, it was clear that for this device, the optimum baseband impedance for linearity resides outside the Smith chart. To investigate this observation further and specifically, to explore the possibility of linearization through the direct application of an ET voltage waveform, additional measurements were carried out for three different cases of DC drain voltage $\left(\mathrm{V}_{\mathrm{dc}}\right): 28 \mathrm{~V}, 24 \mathrm{~V}$ and $16 \mathrm{~V}$. 
The device was deep class-AB biased, and driven approximately $2.5 \mathrm{~dB}$ into compression with fundamental and harmonic components again terminated into a passive $50 \Omega$ load. Using a symmetrical 3-tone, 100\% AM excitation signal cantered at $2 \mathrm{GHz}$ with an envelope frequency of $2 \mathrm{MHz}$, a different IF load condition was used for each case of $\mathrm{V}_{\mathrm{dc}}$ : for the case of $\mathrm{V}_{\mathrm{dc}}=28 \mathrm{~V}$, an ideal short circuit termination was synthesised for each of the four significant baseband components, resulting in a near static supply rail. The slight 'ripple' that remained was associated with IF5, but this was very small and in the order of $200 \mathrm{mV}_{\mathrm{p}-\mathrm{p}}$, so was considered not to be of concern. For the second case of $\mathrm{V}_{\mathrm{dc}}=24 \mathrm{~V}$, a sufficiently negative resistance was presented to all four IF components to result in an $8 \mathrm{~V}_{\mathrm{p}-\mathrm{p}}$ baseband voltage being developed. For the third case of $\mathrm{V}_{\mathrm{dc}}=20 \mathrm{~V}$, the IF load was again adjusted such that a $16 \mathrm{~V}_{\mathrm{p} \text {-p }}$. baseband voltage waveform was developed.

Fig. 8 shows how in each case, the injected baseband signals track the RF input signal envelope and provide an 'ETlike' variable supply voltage to the device. It is also clear that for the three cases, the peak baseband voltage is the same at $28 \mathrm{~V}$, thus allowing meaningful comparison. For the case of $\mathrm{V}_{\mathrm{dc}}=28 \mathrm{~V}$, an average drain efficiency of $38.1 \%$ was achieved at a peak envelope power (PEP) of $39.72 \mathrm{dBm}$. For the case of $\mathrm{V}_{\mathrm{dc}}=24 \mathrm{~V}$, the average drain efficiency was $41.5 \%$ at a PEP of $40.3 \mathrm{dBm}$.

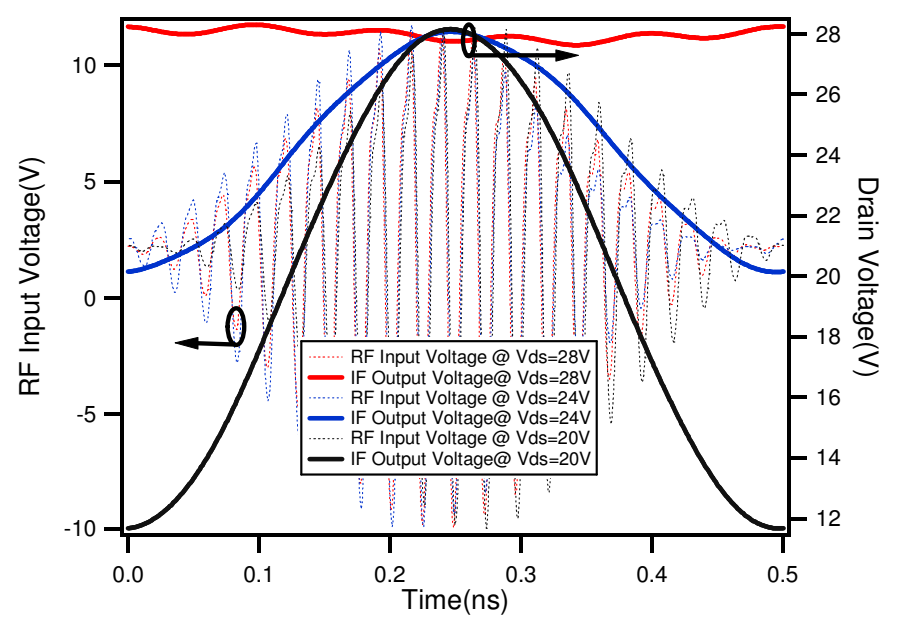

Fig. 8 Measured dynamic IF voltage envelops in-phase with input RF voltages

A slightly better performance in terms of efficiency and output power was achieved with $\mathrm{V}_{\mathrm{dc}}=20 \mathrm{~V}$. Therefore, applying a dynamic drain voltage has increased the achievable peak output power, and has thus enabled a higher efficiency than was possible with a fixed supply voltage of $28 \mathrm{~V}$. Linearity performance is summarized in Table-1, where it is shown that for the case of $\mathrm{V}_{\mathrm{dc}}=20 \mathrm{~V}$, IM3 and IM5 distortions are suppressed by $10 \mathrm{dBc}$ and $3 \mathrm{dBc}$ respectively compared to the static $\mathrm{V}_{\mathrm{dc}}=28 \mathrm{~V}$ case where a short circuit impedance was maintained for all four IF components. For the case where
$\mathrm{V}_{\mathrm{dc}}=24 \mathrm{~V}$, only a small improvement in IM3 distortions was observed along with an $8 \mathrm{dBc}$ improvement in IM5 distortion.

TABLE I

THREE TONES MEASURED LINEARITY RESULTS FOR THREE DISTINCT DRAIN VOLTAGES

\begin{tabular}{|c|c|c|c|c|c|c|c|}
\hline $\begin{array}{c}\text { Supply } \\
\text { Voltage(V) }\end{array}$ & $\begin{array}{c}\text { IM5L } \\
\mathrm{dBc}\end{array}$ & $\begin{array}{c}\text { IM3L } \\
\mathrm{dBc}\end{array}$ & $\begin{array}{c}\text { W1 } \\
\mathrm{dBm}\end{array}$ & $\begin{array}{c}\text { Wc } \\
\mathrm{dBm}\end{array}$ & $\begin{array}{c}\text { W2 } \\
\mathrm{dBm}\end{array}$ & $\begin{array}{c}\text { IM3H } \\
\mathrm{dBc}\end{array}$ & $\begin{array}{c}\text { IM5H } \\
\mathrm{dBc}\end{array}$ \\
\hline 28 & $\mathbf{- 3 5 . 6 8}$ & $\mathbf{- 1 2 . 8 3}$ & 27.85 & 35.69 & 28.02 & $\mathbf{- 1 3 . 1 2}$ & $\mathbf{- 3 6 . 4 7}$ \\
\hline 24 & $\mathbf{- 4 3 . 0 5}$ & $\mathbf{- 1 5 . 8 6}$ & 28.08 & 35.18 & 28.15 & $\mathbf{- 1 6 . 3 7}$ & $\mathbf{- 4 3 . 2 6}$ \\
\hline 20 & $\mathbf{- 3 8 . 9 5}$ & $\mathbf{- 2 2 . 8 1}$ & 28.39 & 34.39 & 28.49 & $\mathbf{- 2 2 . 5 7}$ & $\mathbf{- 3 9 . 4 1}$ \\
\hline
\end{tabular}

\section{CONCLUSION}

Linearity investigations of $10 \mathrm{~W}$ GaN HEMT under modulation excitations have shown that for the device considered, the optimum impedance for best linearity lies outside the Smith chart. Interestingly, the results also suggest that there exists separate optimum impedances for suppression of IM3 and IM5 distortion products.

Additionally, application focused measurements with nonoptimal load $(50 \Omega)$ have shown that efficiency as well as linearity can be improved at reduced drain supply voltages: for $\mathrm{V}_{\mathrm{dc}}=20 \mathrm{~V}$, the average drain efficiency is improved by approximately $5 \%$ together with an improvement of $10 \mathrm{dBc}$ in IM3 when compared to the static $V_{d c}$, where short circuit impedance was maintained for all four baseband components.

\section{ACKNOWLEDGMENT}

This work has been carried out as part of EPSRC grant EP/F033702/1. The authors would also like to thank CREE for supporting this activity and supplying the devices; specifically Ray Pengelly and Mr. Simon Wood.

\section{REFERENCES}

[1] 3GPP Long Term Evolution specification, [Online]. Available: http://cp.literature.agilent.com/litweb/pdf/5989-8139EN.pdf

[2] Joel Vuolevi and Timo Rahkonen, "Distortion in RF Power Amplifiers," Norwood, MA: Artech House, 2003

[3] J. Vuolevi, J. Manninen, and T. Rahkonen, "Cancelling the memory effects in RF power amplifiers," in IEEE Int. Symp. Circuits and Systems, pp. 57-60, 2001.

[4] Spirito, M., Pelk, M.J., van Rijs, F., Theeuwen, S.J.C.H., Hartskeerl, D. , de Vreede, L.C.N. , Active Harmonic Load-Pull for On-Wafer Out-of-Band Device Linearity Optimization," IEEE Trans. Microw. Theory Tech., vol. 54,no. 12, pp. 4225-4236, Dec. 2006.

[5] Alghanim, A.; Lees, J.; Williams, T.; Benedikt, J.; Tasker, P "Using active IF load-pull to investigate electrical base-band induced memory effects in high-power LDMOS transistors," in Proc. Asia-Pacific Microwave Conference, 2007, 11-14 Dec. 2007 Page(s):1 - 4.

[6] Alghanim, A;Less, J.; Williams, T.; Benedikt, J.; Tasker, P. "Reduction of electrical base-band memory effects in high-power LDMOS DevicesUsing Optimum Termination for IMD3 and IMD5 using active load-pull", in Proc., IEEE International Microwave Symposium, June 2008.

[7] J. Lees, et al, "Demystifying Device Related Memory Effects Using Waveform Engineering and Envelope Domain Analysis", in Proc. $38^{\text {th }}$ European Microwave Conference, October 2008. 


\title{
The Impact of Baseband Electrical Memory Effects on the Dynamic Transfer Characteristics of Microwave Power Transistors
}

\author{
M. Akmal ${ }^{\dagger}$, J. Lees ${ }^{\dagger}$, S. Bensmida ${ }^{*}$, S. Woodington ${ }^{\dagger}$, J. Benedikt ${ }^{\dagger}$, K. Morris ${ }^{*}$, M. Beach ${ }^{*}$, J. McGeehan*, \\ P. J. Tasker ${ }^{\dagger}$ \\ ${ }^{\dagger}$ Cardiff School of Engineering, University of Cardiff, The Parade, Cardiff, CF24 3AA, Wales, UK \\ *Centre for Communications Research, University of Bristol, Woodland Rd, Bristol, BS8 1UB, UK \\ Akmalm1@Cardiff.ac.uk
}

\begin{abstract}
The inter-modulation distortion products can vary both in terms of amplitude and asymmetry due to the effects of baseband and $2^{\text {nd }}$ harmonic impedance. This paper presents an investigation into the relationship between the IMD asymmetries caused by baseband impedance variation and the looping or hysteresis that can sometimes appear in the dynamic transfer characteristics of microwave power devices when subjected to modulated excitation. The investigation is carried out using a 2W GaN HFET bare die device characterized at $2.1 \mathrm{GHz}$, and using IF active load-pull to clarify the role of baseband impedance on observed hysteresis in the dynamic transfer characteristics. Analysis is performed using the envelope domain in order to more effectively reveal the DUT's sensitivity to impedance environments and specifically electrical baseband memory effects.
\end{abstract}

Index Terms - Envelope domain, GaN, hysteresis, IF active load-pull, inter-modulation, memory Effects.

\section{INTRODUCTION}

It is widely reported how electrical memory effects are caused by baseband, fundamental as well as $2^{\text {nd }}$ harmonic impedance variation as a function of modulation frequency [1] and can cause significant variation in the symmetry and magnitude of inter-modulation products generated within a signal bandwidth. This spectral asymmetry leads to, and in some cases can be considered a direct consequence of an asymmetrical output modulation envelope that results in an observed hysteresis in the measured dynamic input voltage output current transfer characteristics of the device - an effect often attributed to the presence of memory.

The third order inter-modulation (IMD3) products are of most interest, and for simple two-tone excitation comprise several contributors, some of which are mixed to IMD3 from different frequency bands. The interest here is mainly in the $2^{\text {nd }}$ order non-linearity that causes components to mix to IMD3 from the baseband and $2^{\text {nd }}$ harmonic band, and which can be affected by manipulating the impedances presented to these frequency bands [1]. Although the optimization of IMD3 terms is possible by controlling both the baseband and the second harmonic frequency components, in this work only load-pull of the low frequency baseband components is considered. In practical design, critical baseband impedances are usually determined by bias insertion networks, and typically extend from DC up to five times the modulation bandwidth. These impedances are notoriously difficult to maintain constant and as a consequence, are recognized as the main cause of bandwidth dependent memory effects observed in microwave power devices [1,2]. The first part of this paper focuses on probing the presence of nonlinear memory effects by examining both the input voltage and output current modulation envelopes and the resulting dynamic transfer characteristics obtained for modulated two-tone stimulus using various tone-spacings. The second part focuses on the use and application of active 'IF' or baseband load-pull measurements in order to control baseband impedances and to remove 'conventional' electrical memory. Finally, the effect of device inherent linear delay reported in [3] is removed to allow the residual hysteresis in the device's output characteristics to be analysed.

\section{Modulated MEASUREMENT SySTEM}

Until recently, there has been a lack of measurement systems that can support comprehensive non-linear device investigations under the modulated excitations. The majority of systems focus either upon the magnitude of the frequency spectra or upon in-band spectral components using instruments such as Vector Signal Analysers. Although these are extremely valuable approaches, they generally fail to capture all of the out-of-band information contained within both the base-band (IF) and around the harmonics that is essential for accurate envelope domain representation and analysis [3].

The developed measurement system is capable of handling IF and RF power levels in excess of $100 \mathrm{~W}$ which makes particularly relevant to the characterization of devices used in both mobile handset and base-station applications. The measurement system itself is shown in fig. 1 and consists of two main entities: the RF test-set and the IF test-set which are identical both in terms of component architecture and principle of operation. The architecture incorporates combined IF and RF capabilities allowing critically, the simultaneous collection of all four travelling waves at both IF and RF frequencies.

The simultaneous combining of coupled RF and IF components of the signal prior to the measurements is a key feature, and ensures phase coherence between the measured IF and RF components. The system is fully vector-error corrected, and can therefore account for any errors introduced due to losses, mismatches and imperfect directivities in the system, thus allowing for the measurement of the complete 
modulated voltage and current waveforms and impedances that exist at the DUT plane [2, 3]. The modulated measurement system shown in fig. 1 was used to perform the measurements where the significant baseband IF1 and IF2 components were actively suppressed. These 'active' IF loads were supplied via two phase coherent arbitrary waveform generators, which when amplified are capable of delivering $100 \mathrm{~W}$ over a bandwidth of $10 \mathrm{KHz}$ to $12 \mathrm{MHz}$.

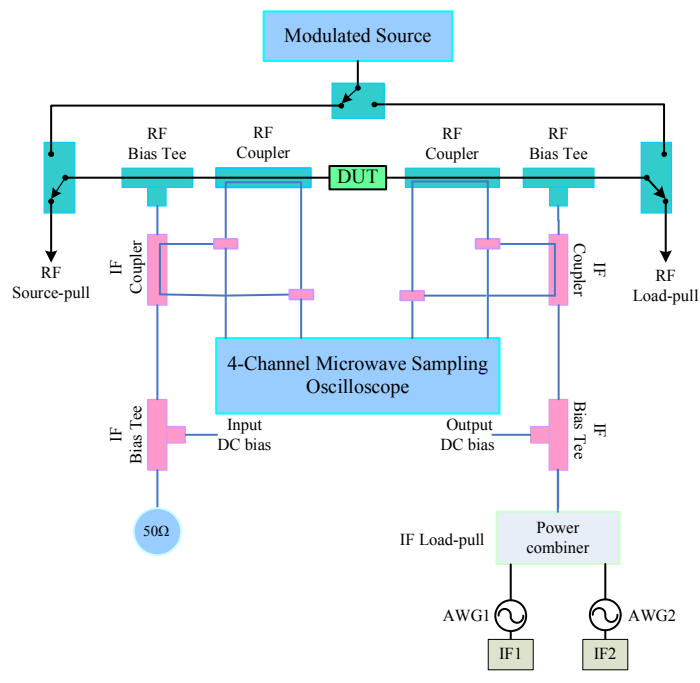

Fig. 1 Modulated waveform measurement system

\section{MEASUREMENT RESUlTS AND ANALYSIS}

The measurements presented here were carried out on a $2 \mathrm{~W}$ GaN HFET bare die supplied by CREE. For baseband electrical memory investigations, a two-tone stimulus signal with a centre frequency of $2.1 \mathrm{GHz}$ was used and the tonespacing was varied between 1 and $10 \mathrm{MHz}$. The device was biased at approximately $20 \%$ IDSS in Class-AB at a quiescent current of $130 \mathrm{~mA}$, and all significant RF signals were passively terminated into $50 \Omega$. The characteristics were measured dynamically and found to be strongly dependent on the separation frequency.

$$
\begin{aligned}
& A\left(\cos \omega_{1} t+\cos \omega_{2} t\right) \\
& =2 A \cos \left[\left(\frac{\omega_{1}-\omega_{2}}{2}\right) * t\right] \cos \left[\left(\frac{\omega_{1}+\omega_{2}}{2}\right) * t\right]
\end{aligned}
$$

Where the modulation frequency, $\omega_{\mathrm{m}}$, is given by

$$
\omega_{m}=\left(\omega_{1}-\omega_{2}\right) / 2
$$

To observe the dynamic behavior of the device whilst operating within a realistic operational environment, and in response to an applied modulated input stimulus signal, envelope domain analysis is used [3]. Fig 2 shows the fundamental current and voltage modulation envelopes for a $\mathrm{P}_{1 \mathrm{~dB}}-3 \mathrm{~dB}$ backed-off drive level, and clearly show that there is a significant phase delay between the input and output of the device at a tone spacing of $5 \mathrm{MHz}$, whereas at $1 \mathrm{MHz}$ tone spacing, negligible phase delay was observed . At this backedoff drive level, the output power spectrum illustrated the lowlevel of baseband and near absence of $2^{\text {nd }}$ harmonic distortion; a condition which unsurprisingly resulted in symmetrical IMD3 behaviour.

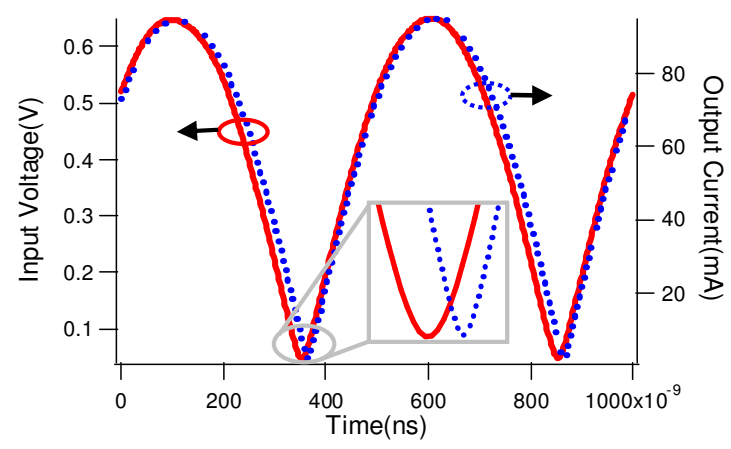

Fig. 2 Input voltage and output current envelopes at $5 \mathrm{MHz}$ tone spacing.

Fig. 3 shows a broadening in the dynamic transfer characteristic due to linear device delay as reported in [3]. It can be seen however that when the device is driven $1 \mathrm{~dB}$ into compression, the observed nonlinearities are much more significant and appear as a soft, asymmetrical clipping of the output current envelope, which significantly increases the $2^{\text {nd }}$ harmonic signal level and causes a significant baseband signal components to develop.

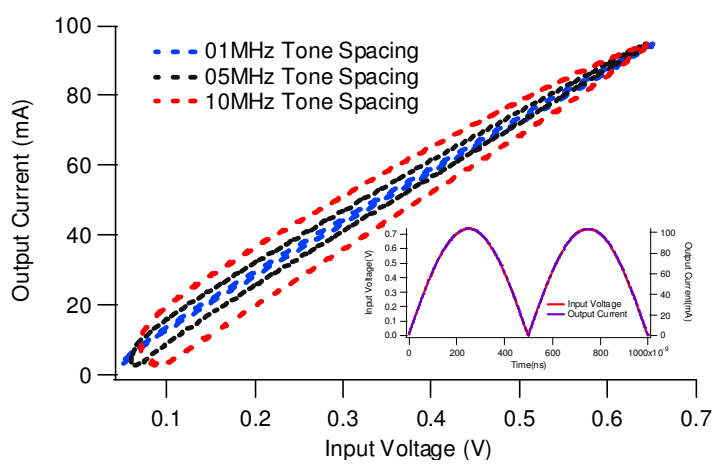

Fig. 3 Dynamic transfer characteristics at different tone spacings with input voltage and output current inset at $1 \mathrm{MHz}$ tone-spacing.

The asymmetrical envelops are shown in fig. 4 which are an explicit manifestation of presence of nonlinear memory effects at higher drive levels. The baseband signal generated from the device also includes higher order terms, and it is the asymmetric envelope shape that determines which $\mathrm{IMD}_{3}$ product $\left(2 \omega_{2}-\omega_{1}\right)$ or $\left(2 \omega_{1}-\omega_{2}\right)$ will have the greater magnitude. The presence of these asymmetrical spectral current components manifest themselves as recognizable electrical memory effects and can be clearly seen in the envelope domain as significant looping at the top-end of the input voltage / output current transfer characteristic. The hysteresis in the dynamic transfer characteristics worsen with the increasing tone spacing and can be seen explicitly from fig. 5 . 


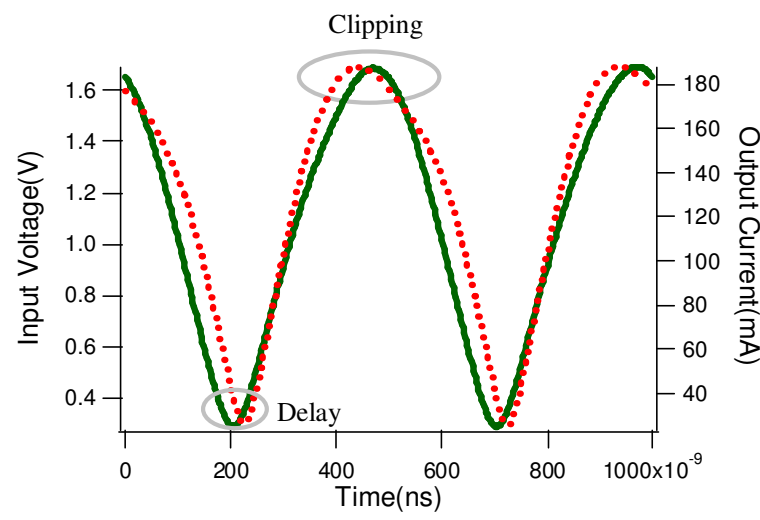

Fig. 4 Input voltage and output current envelops at $5 \mathrm{MHz}$ tone-spacing.

In this first case, the significant baseband frequency components are to be expected and are due to the fact that the significant baseband current components are termination into a relatively high broadband impedance of $50 \mathrm{Ohms}$. These are clearly the most significant contributor towards the observed electrical memory effects.

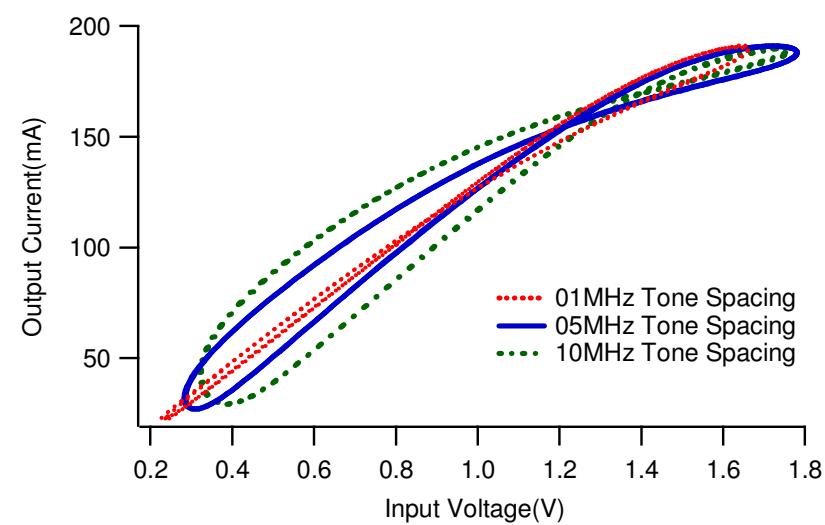

Fig. 5 Dynamic transfer characteristics at different tone spacings for compressed drive level.

\section{APPLICATION OF IF ACTIVE LOAD-PULL}

In a practical PA design, the first instinctive step is to nullify or cancel these unwanted baseband voltage components, preventing any contamination or 're-modulation' of the output bias supply. Therefore, the termination of these frequency components into short circuits is usually desirable and the normal course of action. For the measurements presented in this paper, active IF load-pull has been used as means of controlling the contribution of the significant baseband components (IF1 and IF2). The validity of this approach in maintaining a constant low-frequency impedance environment is reported in [2] where IF1, which is twice of the modulation frequency and IF2, which is four times the modulation frequency were actively load-pulled. In the work presented here, frequency independent IF1 and IF2 short circuit impedances were maintained for different tone separation and at a single drive level. The observed variation in impedanc is very small and can be seen from Fig. 6 to be less than 0.005 in magnitude and approximately 0.1 degrees in phase over the entire IF bandwidth.

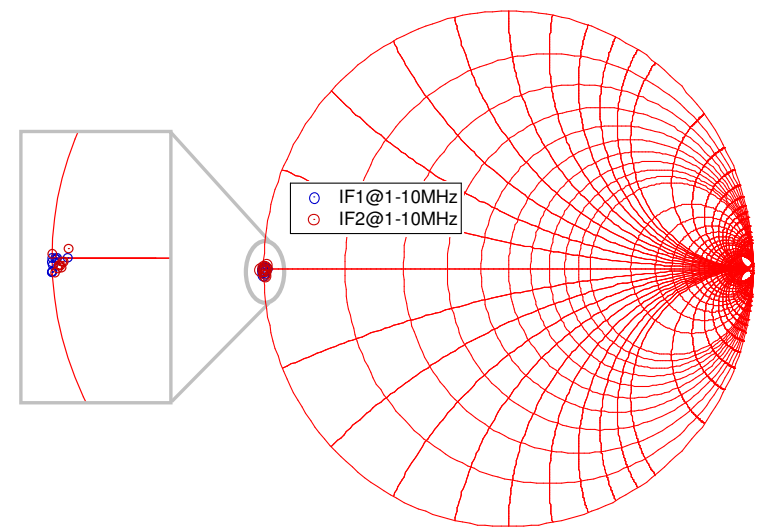

Fig .6 Measured $\mathrm{IF}_{1}$ and $\mathrm{IF}_{2}$ impedaces on Smith chart at different tonespacing.

The behaviour of the carrier tones (F1 and F2) is clearly independent of the tone spacing as shown in Fig. 7. It is also evident that a reduction in $\mathrm{IMD}_{3}$ components appears to have been possible by providing the IF short circuit impedance for frequency ranging from $1 \mathrm{MHz}$ to $10 \mathrm{MHz}$. This is due to the fact that the non-negligible baseband electrical memory effects are greatly reduced.

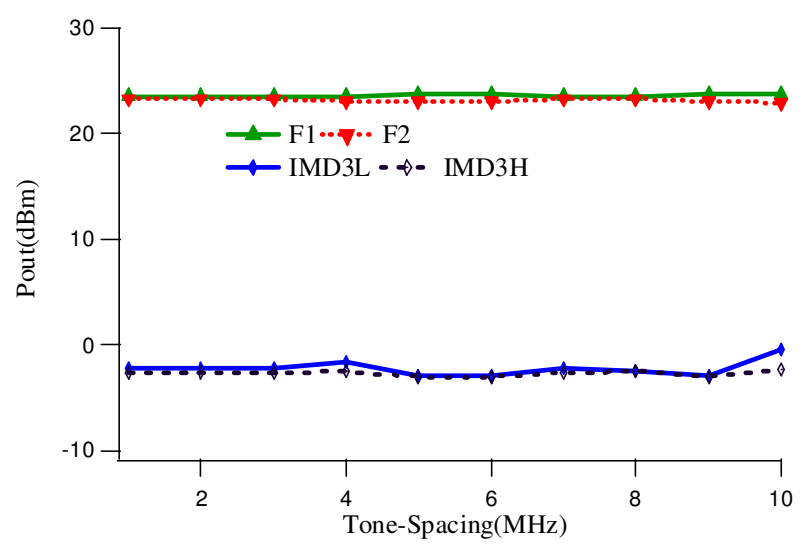

Fig. 7. Measured fundamental and IMD3 power for different tone-spacing at constant drive level of $1 \mathrm{~dB}$ compression point.

Measurements under such conditions show a notable reduction in $\mathrm{IMD}_{3}$ asymmetry and confirm the validity of IF active load-pull in maintaining a constant baseband impedance for the two significant baseband components IF1 and IF2 over the bandwidth of at least four times the modulation frequency. The baseband voltage components are greatly suppressed whilst maintaining the short-circuit baseband impedance for significant baseband components. Under such conditions, the IF load-line was almost completely vertical with only slight current variation present. This condition resulted in negligible looping in IF load-lines as the RF load-line follows the same trajectory in and out of the boundary conditions; consequently this reduced the hysteresis in the dynamic transfer characteristics. 


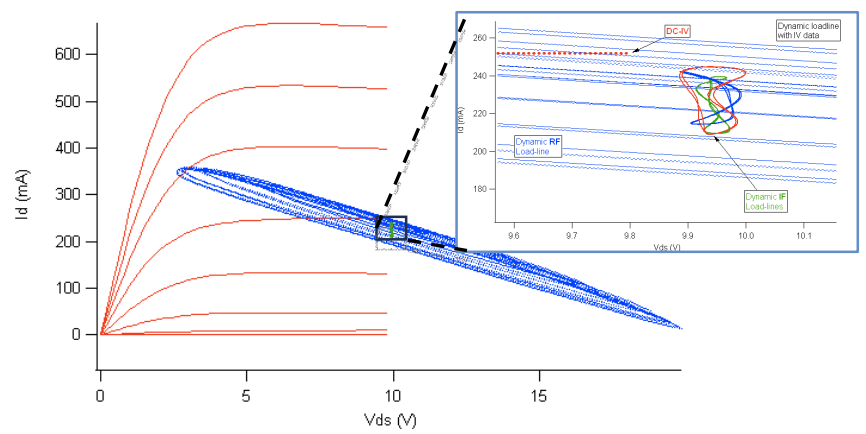

Fig. 8. Dynamic RF load-line with the IF loadline inset at different tone spacings.

\section{DElay AdJuSTMENT ESTIMATION}

The device's linear propagation delay has to be accurately computed so that the input and output envelopes can be time aligned. The delay is computed and applied to the output envelope, and fig. 9 shows that the envelopes can be perfectly aligned at their minima. There appears non-quasi static envelope distortion however even after terminating the significant baseband components into short circuits. This alignment changes the shape of the transfer characteristics such that the broadening at the bottom is diminished whilst the looping in the upper part of the characteristic remains.

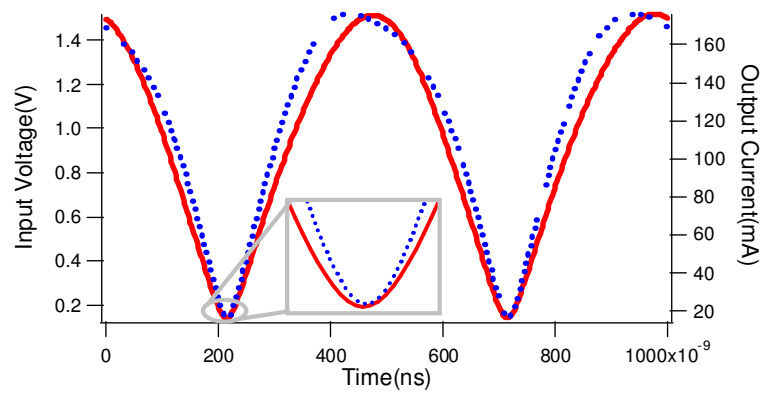

Fig. 9 Delay compensated output current envelope at 5MHz tone spacing.

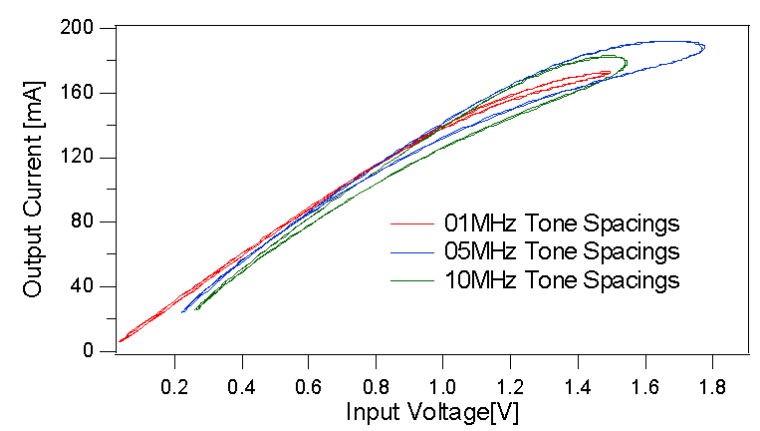

Fig. 10 Delay compensated dynamic transfer characteristics at different tone spacings for slghtly different drive level at $5 \mathrm{MHz}$ tone spacing.

Interestingly however, in the case of IF short circuit termination, it was observed that the approach of shorting these low-frequency voltage components is effective in influencing the IMD asymmetry, suggesting that the hysteresis in the dynamic transfer characteristics can also be minimized by terminating the significant baseband components IF1 and IF2 into short circuits. The residual spread remains in the dynamic transfer characteristics as can be seen from fig. 10 for certain bias conditions, and is most probably attributable to non-linear device delay or other sources of memory, specifically trapping effects, thermal effects [4][5], and in this case, is not due to electrical baseband memory effects.

\section{CONCLUSION}

In this paper, envelope domain analysis is performed for different drive levels and is used to investigate the effect of baseband electrical memory on envelope distortion and on the measured dynamic transfer characteristic.

At a backed-off drive level with the device operating linearly, symmetrical modulation envelopes are observed. When driven into compression, these envelopes become asymmetrical however. Using IF active load-pull to control the behaviour of the significant baseband components, $\mathrm{IMD}_{3}$ asymmetry can be significantly improved, an effect due to the suppression of baseband electrical memory. The causes of this 'electrical' effect may be understood by analysis of the IF dynamic load-lines - any significant looping in the IF load-line will result in different trajectories of the RF load-line through the static IV characteristic, for different parts of the modulation cycle. The suppressed baseband voltages translate to greatly reduced hysteresis for lower tone spacing, significant hysteresis remains for higher tone spacing.

\section{ACKNOWLEDGMENT}

This work has been carried out as part of EPSRC grant EP/F033702/1. The authors would also like to thank CREE for supporting this activity and supplying the devices; specifically Ray Pengelly and Mr. Simon Wood.

\section{REFERENCES}

[1] Joel Vuolevi and Timo Rahkonen, "Distortion in RF Power Amplifiers," Norwood, MA: Artech House, 2003.

[2] Alghanim, A.; Lees, J.; Williams, T.; Benedikt, J.; Tasker, P "Using active IF load-pull to investigate electrical base-band induced memory effects in high-power LDMOS transistors," in Proc. Asia-Pacific Microwave Conference, 2007, 11-14 Dec. 2007 Page(s): 1 - 4.

[3] J. Lees, et al, "Demystifying Device Related Memory Effects Using Waveform Engineering and Envelope Domain Analysis", in Proc. $38^{\text {th }}$ European Microwave Conference, October 2008.

[4] Le Gallou, N. Nebus, J.M. Ngoya, E. Buret, H., "Analysis of low frequency memory and influence on solid state HPA intermodulation characteristics", IEEE MTT-S Digest, Phoenix, vol.2, pp.979-982, May 2001,

[5] Ouarch, Z. Collantes, J.M. Teyssier, J.P. Quere, R., "Measurement based nonlinear electrothermal modeling of GaAs FETwith dynamical trapping effects", IEEE MTT-S Digest, Baltimore, vol.2, pp.599-602, Jun 1998. 


\title{
Waveform Engineering Applied to Linear-Efficient PA Design
}

\author{
J. Lees ${ }^{1}$, M. Akmal ${ }^{1}$, S. Bensmida ${ }^{2}$, S. Woodington ${ }^{1}$, S. Cripps ${ }^{1}$, J. Benedikt $^{1}$, K. Morris ${ }^{2}$, M. Beach ${ }^{2}$, J. McGeehan ${ }^{2}$ and \\ P. Tasker ${ }^{1}$ \\ ${ }^{1}$ Cardiff School of Engineering, University of Cardiff, the Parade, Cardiff, CF24 3AA, Wales, UK \\ ${ }^{2}$ Centre for Communications Research, University of Bristol, Woodland Rd, Bristol, BS8 1UB, UK \\ LeesJ 2 @cf.ac.uk
}

\begin{abstract}
Low-frequency, baseband effects in Power Amplifiers can become particularly important when steps are taken to improve operational efficiency through the application of 'envelope' based approaches such as Envelope Elimination and Restoration (EER) and Envelope Tracking (ET). The aim of this paper is to demonstrate how a broadband modulated time-domain measurement system, when used in combination with active IF load-pull, can be employed to gain valuable insight into the behaviour of high-frequency power transistors whilst operating under modulated conditions. Also considered is the interesting idea of how PA linearity can be improved using the very mechanisms that are employed to improve efficiency.
\end{abstract}

Index terms - Amplifier distortion, Amplifier linearity, Amplifier efficiency, Modulation, Power amplifier

\section{INTRODUCTION}

In order to achieve truly broadband, highly efficient linearization of increasingly complex PA architectures, baseband as well as RF effects must be carefully considered and taken into account [1]. This can be particularly important when working with complex, multiple-device architectures such as the Doherty PA, where successful design relies upon the precise and highly optimised interaction between two or more active devices, both in terms of both RF and baseband frequency components. One interesting approach presented in [2] exploits this interaction and connects the drain bias nodes of two similar PAs via a baseband processing circuit in such a way that the effects of baseband impedance on distortion are cancelled over a wide bandwidth.

The devices used within these types of structures and architectures may well be configured in different efficient or efficient-linear modes of operation such as inverse class-F or class-J, as well as using different types or versions of device. Such designs become challenging when steps are made to improve efficiency by the application of 'envelope' based approaches such as Envelope Elimination and Restoration (EER) and Envelope Tracking (ET). The latter has recently gained popularity and relies upon dynamically modifying the instantaneous DC applied to a device in response to a modulation envelope. Considered another way, these approaches directly modify the baseband impedance environment presented to a device through active baseband injection - an approach that through earlier work has already been shown to be able to significantly improve linearity [1][3].
It is worth considering therefore the idea of improving PA linearity using the very mechanisms that are employed to improve PA efficiency.

Whereas classical modulated analysis of active devices and power amplifiers usually involves observing the magnitude and symmetry of inter-modulation products as a function of varying tone-spacing excitation and drive [4][5], observing modulation envelopes directly - envelope domain analysis - offers an alternative approach to exposing problematic effects, and involves plotting the dynamic behaviour of key parameters such as gain, output power, efficiency and others in response to the modulation envelope. This representation can be highly intuitive and sometimes more representative of a device's behaviour whilst operating within a realistic environment. Analysis of time varying behaviour in the envelope domain, especially in comparison to $\mathrm{CW}$ equivalents, can often provide significant insight into a number of dynamic, non-linear processes including the presence or otherwise of PA or device related 'memory'[6].

\section{MOdUlated WAVEFORM MEASUREMENT SYSTEM}

Continuous Wave (CW) waveform measurement and engineering has established itself as a highly effective design solution for the development of power amplifiers [7][8][9]. However, modern modulation schemes such as CDMA and OFDM demand characterisation over extended instantaneous bandwidths of up to $100 \mathrm{MHz}$. In order to cater for these evolving requirements, existing $\mathrm{CW}$ waveform measurement and engineering capabilities have been extended into the modulated domain. This allows not only for the optimisation of traditional parameters such as output power and efficiency, but also the direct and simultaneous observation of linearity, memory and the sources of memory. Until recently, there has been a lack of measurement solutions that support comprehensive investigations into the full consequences of modulated excitation, with the majority of systems focusing either upon the magnitude of the frequency spectra using Spectrum Analysers, or upon magnitude and phase of in-band spectral components using Vector Signal Analysers (VSAs). Although these are both extremely valuable approaches, they are not able to completely describe the devices full operating state as they do not capture out-of-band information that exists at both the base-band (IF) and around the harmonics of the carrier 
frequency (RF). It is after all the vector summation of these individual components that results in the actual voltage and currents that develop at the nonlinear device's input and output terminals. The accurate capture of these waveforms is essential to develop accurate envelope domain representation and analysis. The approaches investigated in this paper have been made possible by the development of a modulated waveform measurement approach that allows the observation and control of all the frequency components (RF, IF $^{1}$ and DC) [10] produced by the device under test. The system architecture is capable of handling both low and high power IF and RF signals $(1 \mathrm{~mW}$ to $100 \mathrm{~W})$, which makes it relevant to the characterisation of devices used in both mobile handset and base-station applications. A simplified schematic of the measurement system itself is shown in Fig. 1

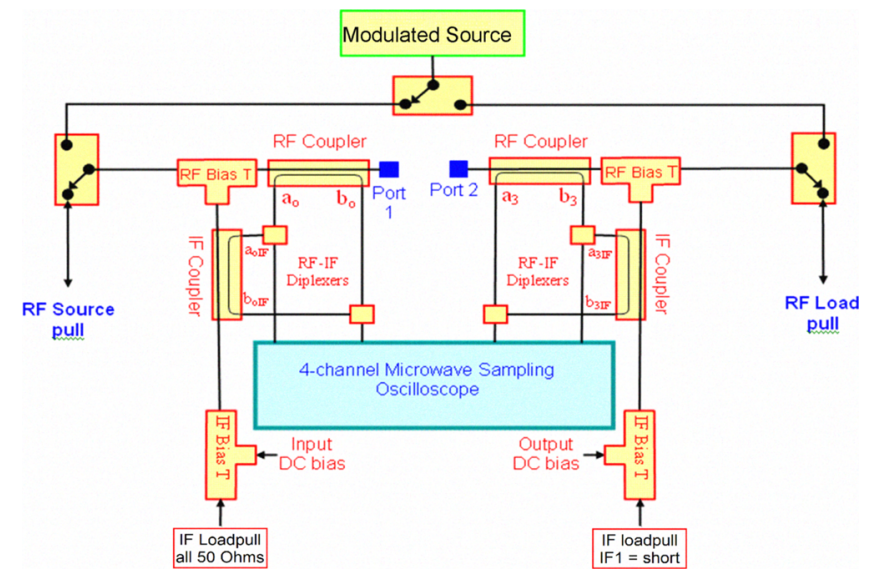

Fig. 1 Schematic of the modulated measurement system

The modulated measurement system consists of the RF testset and the IF test-set, which are identical in terms of both component architecture and principle of operation. This combined IF and RF architecture allows the collection of the four travelling waves both at IF $\left(a_{1}^{\mathrm{IF}}, b_{1}^{\mathrm{IF}}, a_{2}^{\mathrm{IF}}\right.$ and $\left.b_{2}^{\mathrm{IF}}\right)$ and RF ( $a_{1}^{R F}, b_{1}^{R F}, a_{2}^{R F}$ and $b_{2}^{R F}$ ) frequencies. Combination of the coupled RF and IF components of the signal prior to measurement by the broadband sampling oscilloscope is achieved using diplexers, and is a key feature that ensures phase coherence between measured IF and RF components. The system is fully vector-error corrected and can therefore account for any errors introduced due to losses, mismatches, imperfect directivities and delay in the system, thus allowing the measurement of the complete modulated voltage and current waveforms and impedances that are presented at the input and output of the DUT. For the measurements shown in this paper, the system was calibrated over a bandwidth, of $50 \mathrm{MHz}$ at baseband and around the fundamental and first three harmonic frequencies.

\footnotetext{
${ }^{1}$ Here, the term IF refers to the frequency components that exist in the baseband as a result of modulation. These may exist as a result of mixing, or be actively injected using active IF load-pull.
}

The specific focus of the measurement activities discussed in this paper was to investigate the impact of active baseband impedance conditions upon device linearity. For this reason, the fundamental and all harmonic frequency components were terminated into $50 \mathrm{Ohms}$. The IF load-pull architecture used here provided active impedance control of the most significant IF components; IF1 and IF2. This was achieved using an 80 $\mathrm{MHz}$ arbitrary waveform generator in combination with a highpower $(200 \mathrm{~W}), 1-10 \mathrm{MHz}$ power amplifier. All measurements were performed on a CREE CGH40010 10W GaN device within a custom $50 \mathrm{Ohm}$ test fixture. Calibrated reference planes were established at the package plane using a two-tier approach with error terms initially generated using a TRM coaxial calibration and then embedded with the extracted s-parameters of each half of the test fixture.

\section{APPLICATION OF THE MEASUREMENT SYSTEM}

Building upon previous work [7][12], the aim of this measurement activity was to explore in more detail the impact of baseband impedance upon linearity, and specifically 3rd order inter-modulation product magnitude and symmetry. Whereas previous work focussed primarily upon emulating passive baseband impedances inside the smith-chart, such as those that may be presented by an actual bias insertion network, this work was carried-out with an ET application very much in mind, where both positive (inside the Smith chart) and negative (outside the Smith chart) baseband impedances can be presented to the significant IF components.

The first investigation involved biasing a CREE CGH40010 $10 \mathrm{~W}$ GaN device in class $\mathrm{AB}(\mathrm{IDQ}=250 \mathrm{~mA})$, using a drain bias voltage of $16 \mathrm{~V}$ and exciting with a symmetrical 2-tone modulation centred at $2.1 \mathrm{GHz}$. A reduced value of $\mathrm{Vd}$ was used to represent a typical ET application, and the magnitude of the excitation limited to emulate a backed-off drive condition. In this way, the device remained largely linear with no significant load-line / knee boundary interaction occurring.

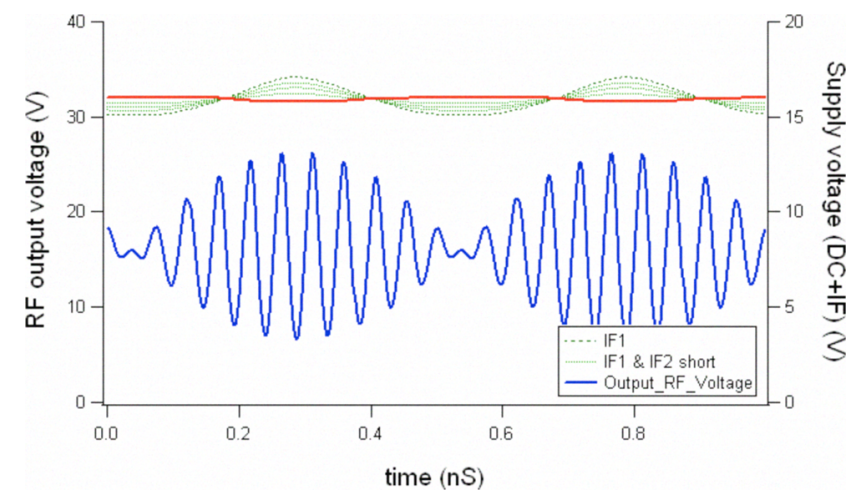

Fig. 2 measured modulated output voltage waveform and supply voltage.

Fig. 2 shows the measured voltage waveform present at the device plane ${ }^{2}$, where the 'backed-off' and linear operating condition is evident from the limited voltage peak-to-peak

\footnotetext{
2 Note that folded sampling approach used [5] allows individual cycles of RF to be visible inside the modulation envelope.
} 
swing between $8 \mathrm{~V}$ and $28 \mathrm{~V}$ - well away from the device's knee voltage at approx $4 \mathrm{~V}$.

IF1 and IF2 loads were then actively synthesised and swept together over the measurement grid shown in Fig. 3 and Fig. 4. The supply voltages comprising DC+IF, that correspond to the cases of IF1 and IF2 load along the real axis are shown as the upper traces in Fig. 2. These are plotted relative to the righthand axis, and are not to scale. The reference case is shown as a central, solid red line where both IF1 and IF2 are terminated into short circuits. The most negative IF1 load point results in approximately a $2 \mathrm{~V}$ p-p IF waveform established on a $16 \mathrm{~V}$ supply rail.

For each of the measurement points, the bias and drive level was maintained constant, and the IM3 behaviour measured. Due to the linear operation of the device and the dynamic range limitations of the system $(60 \mathrm{~dB})$, it was not possible to accurately measure the IM5 behaviour.

Contours of IM3 high and low are shown in Fig. 3 and Fig. 4 respectively, and indicate that interestingly, separate local optimum IF loads for linearity exist some way outside the Smith chart, and that for this device, a 15-20 dB improvement in IM3high magnitude exists for this load in comparison to the reference case where IF1 and IF2 are terminated into short circuits, as may be the case for example when using a conventional video bypass bias network arrangement. A slightly smaller improvement and different optimum load is observed for IM3-low.

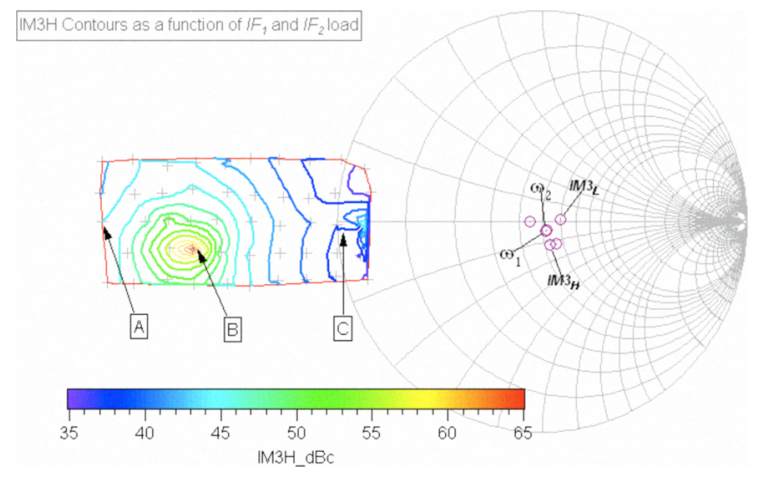

Fig. 3 IM3-high contours as a function of IF1 and IF2 load

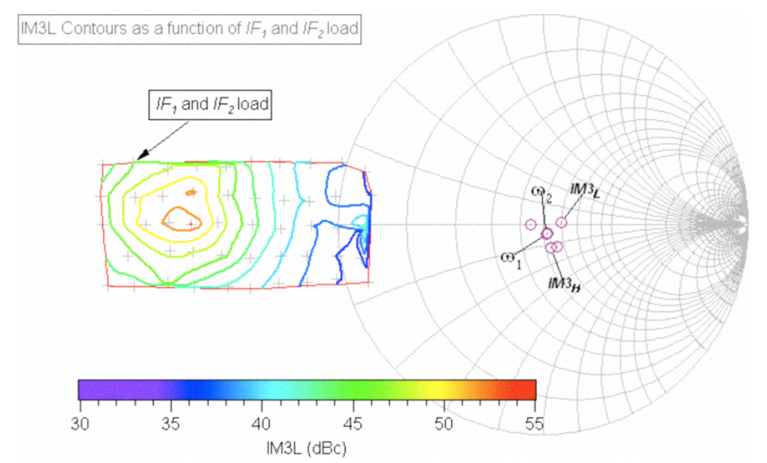

Fig. 4 IM3-low contours as a function of IF1 and IF2 load
Access to the envelope domain allows the dynamic analysis of other device parameters such as gain and drain efficiency as a function of the applied modulation envelope. Fig. 5 shows for example how the gain changes throughout the cycle of modulation for the three cases of IF1 \& IF2 load identified by points A, B and C in Fig. 4: point A - significant IF 'ET-like' component, point $\mathrm{B}$ - the identified optimum for minimum IM3L and point $C-$ a near IF short circuit.

It is clear from this graph that the negative IF impedance presented at point- $A$ results in the higher gain, yet the load at point-B offers improved linearity, at least in terms of IM3 behaviour. The load at Point- $\mathrm{C}$ causes significant distortion in the dynamic gain envelope, which is unsurprisingly reflected in the poor linearity performance.

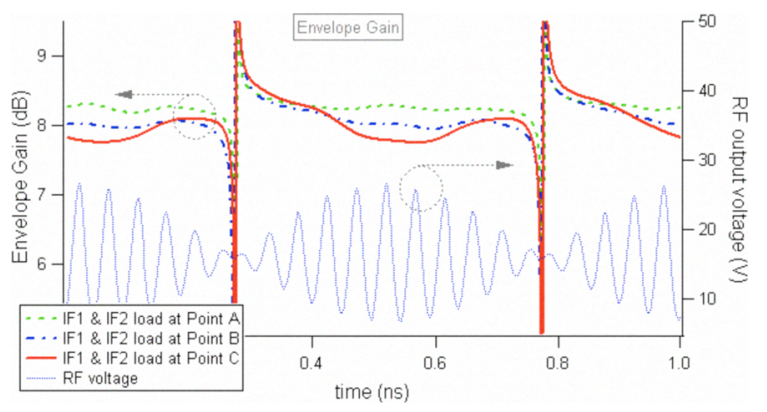

Fig. 5 Envelope gain profiles for IF1 and IF2 loads in positions A, B and C identified in on Fig. 4. RF voltage envelope is also shown for reference

\section{EXTENDING SYSTEM APPLICATION TO ENVELOPE TRACKING EMULATION}

The analysis in the previous section is interesting from a linearity perspective, yet it is limited in that it explores performance only in a backed-off condition. Synthesising negative IF impedances does allow us to understand however how this investigation can be extended from simple linearity investigations to full ET emulation. In this next measurement sequence, the same device has been excited, this time using a symmetrical 3-tone signal centred at $2.1 \mathrm{GHz}$, resulting in $100 \%$ AM modulation with an envelope frequency of $1 \mathrm{MHz}$. The Device was driven into approximately $2 \mathrm{~dB}$ of compression with fundamental and harmonic output components terminated into a passive $50 \mathrm{Ohm}$ load. At this frequency, the optimum output impedance for power is approximately $25+\mathrm{j} 10 \mathrm{Ohms}$, and although the device is not operating into the optimal fundamental load impedance, it is considered sufficiently representative for this analysis.

Three separate measurement cases were considered; case-1 (reference case): Vd=24V and IF1 and IF2 components actively terminated into short circuits, case-2: $\mathrm{Vd}=20 \mathrm{~V}$, injected IF $1=8 \mathrm{Vp}$-p and IF2 terminated into a short circuit, and finally case $3: \mathrm{Vd}=16 \mathrm{~V}$, injected $\mathrm{IF} 1=16 \mathrm{Vp}-\mathrm{p}$ and IF2 again terminated into a short circuit. 


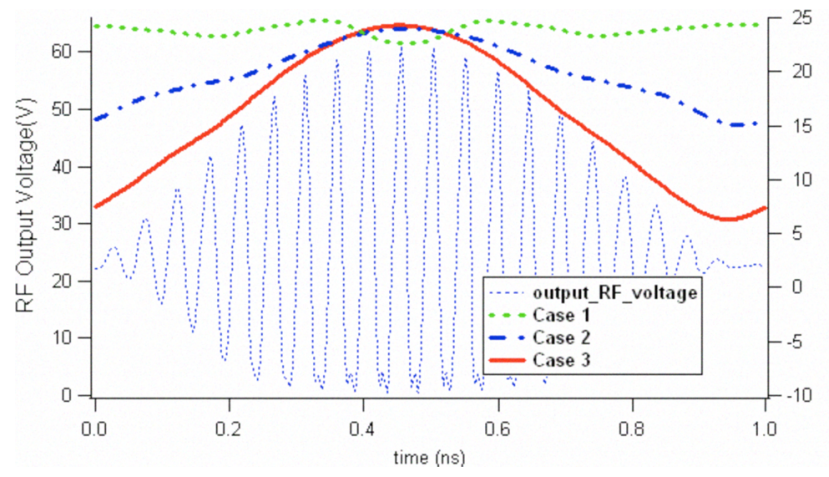

Fig. 6 measured supply voltages for the three cases of IF load, together with RF output voltage waveform - note that different scales are used.

These combinations of static DC supply and injected IF1 component ensure that the peak supply voltage remained as close as possible to a static $24 \mathrm{~V}$, as shown in Fig. 6 , thus allowing meaningful comparison between the three cases.

Plotting the measured (RF, IF \& DC) time-domain voltage vs. time-domain current yields a complete dynamic load-line which can be used to describe the trajectory of the modulated envelope relative to the device's DC characteristic. This intuitive view shows the actual voltage and current behaviour at the calibrated reference plane, and importantly, the nature of any interaction between the envelope and device boundary conditions. This is a familiar plot in $\mathrm{CW}$ waveform engineering yet adds an extra dimension to modulated analysis as it can show interesting effects such as self-bias, and in the case here of an emulated ET environment, the proximity of the load-line to the knee region. Fig. 7 below shows the full dynamic load-lines for the three cases considered, along with the IF load-line trajectories, which have been added by plotting and overlaying the baseband current vs. the baseband voltage waveforms. These clearly show the degree of baseband voltage (horizontal) and current (vertical) variation throughout the modulation cycle. The IF load-line itself can be considered as the component that 'pushes' or 'pulls' the dynamic RF load-line, in the case of electrical memory, asymmetrically into the knee region, and in the case of ET, into more efficient parts of the characteristic. A Perfect IF short would result in a straight, vertical IF load-line, and the shape of the IF load-line allows scope for baseband waveform engineering and optimisation for example of the proximity of dynamic RF load-line to knee boundary region.

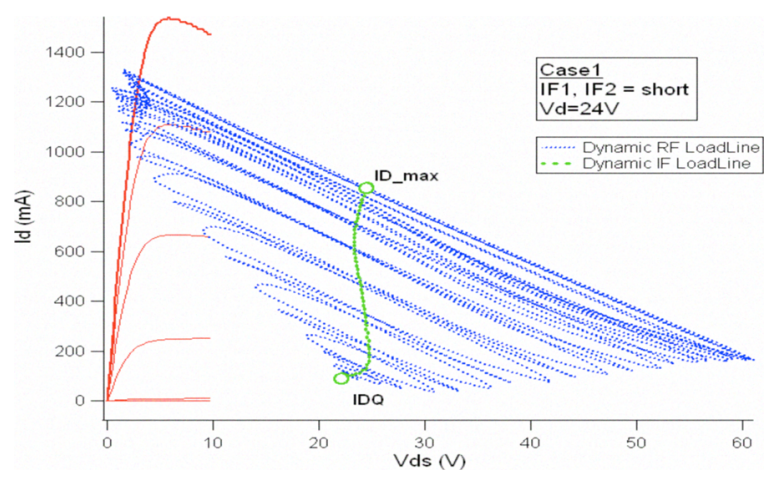

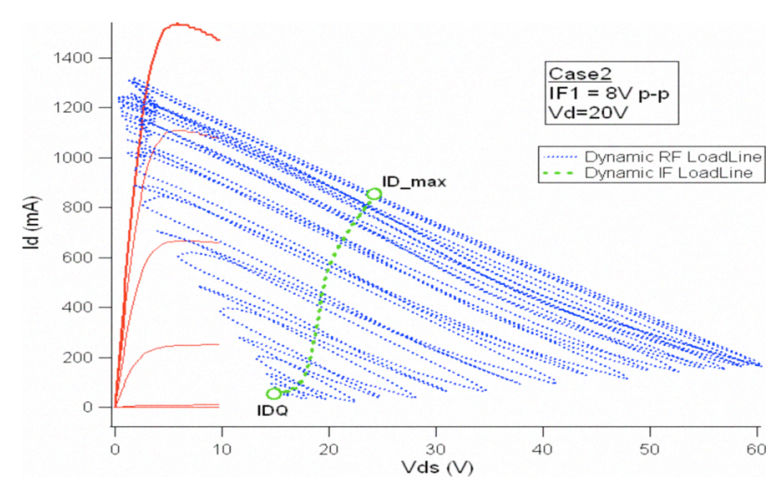

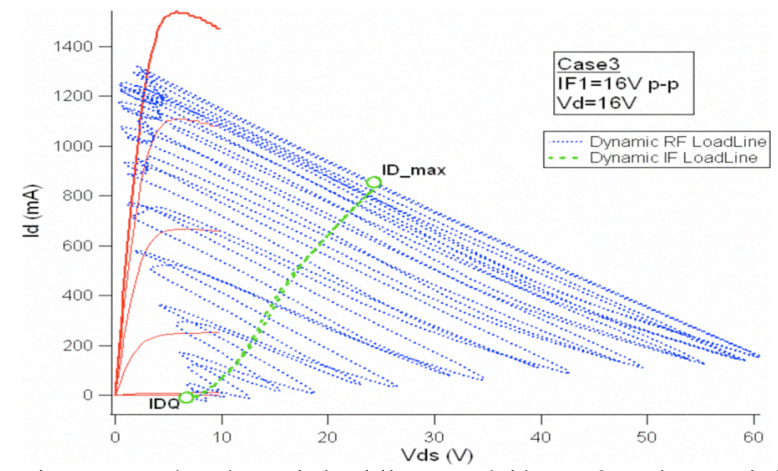

Fig. 7 RF and IF dynamic load-lines overlaid on DCIV characteristic for Case-3

Fig. 8 shows the extracted gain envelopes for the three cases, and how at the central point in the envelope where the DC supply voltage is the same at $24 \mathrm{~V}$, the gain is the same, albeit compressed. This plot also shows however that for other parts of the envelope, the dynamic gain can be very different. This effect is consistent with previous, $\mathrm{CW}$ based observations, where the gain for this device has been seen to reduce with reducing drain voltage.

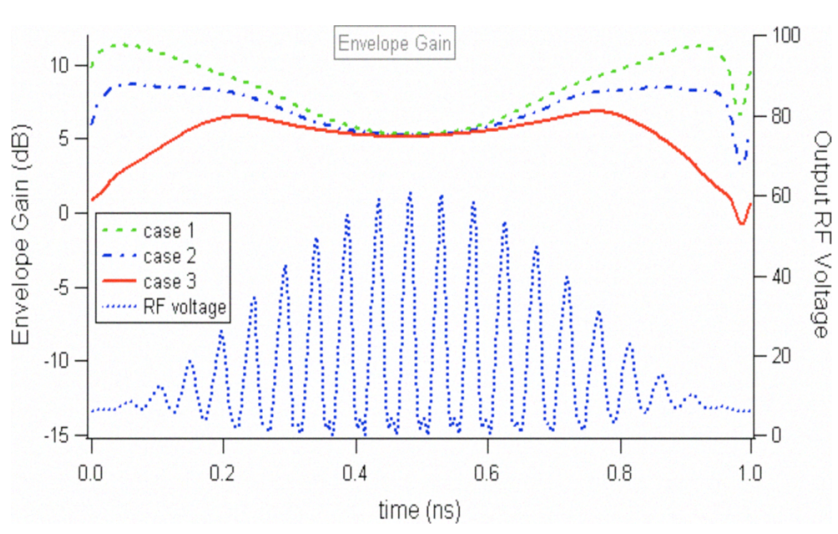

Fig. 8 Comparison of gain envelopes for the three cases

As with gain, it is possible to show the efficiency as a function of the envelope, and Fig. 9 shows a comparison of the three cases. Although it is clear from this graph that the applied ET does improve the average efficiency, it is also clear that the improvement is surprisingly small. In trying to explain this, it must be remembered that in each case, the device is driven 
fairly hard into compression, and no improvement can be expected in this region of the envelope. Although improvement is apparent around the envelope 'skirts', as mentioned, it maybe isn't as large as one would expect. This is again probably explained by the fact that for this device, the gain, output RF power and hence efficiency reduces with reducing drain voltage.

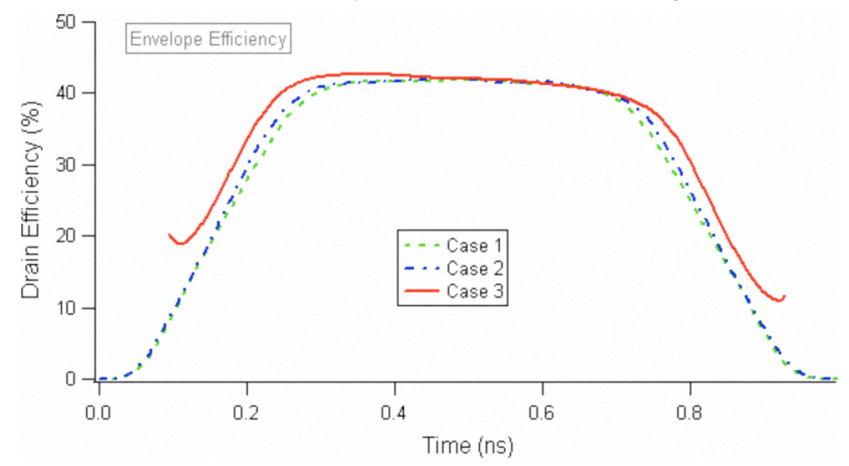

Fig. 9 Comparison of drain efficiency envelopes for the three cases

The linearity in terms of measured IM3 and IM5 mixing terms is shown in Table 1, which indicates that there is an approximate $10 \mathrm{~dB}$ improvement in IM3 for case 1 compared to the reference IF $1=I F 2=$ short case, and only a slight improvement in IM5 for case 2.

\begin{tabular}{|c|c|c|c|c|c|c|c|}
\hline case & $\begin{array}{c}\text { IM5I } \\
d B c\end{array}$ & $\begin{array}{c}\text { IM3I } \\
d B c\end{array}$ & $\begin{array}{c}\text { W1* } \\
d B m\end{array}$ & $\begin{array}{c}\text { WC } \\
d B m\end{array}$ & $\begin{array}{c}\text { W2 } \\
d B m\end{array}$ & $\begin{array}{c}\text { IM3h } \\
d B c\end{array}$ & $\begin{array}{c}\text { IM5h } \\
d B c\end{array}$ \\
\hline 1 & $\mathbf{3 1 . 9 3}$ & $\mathbf{2 3 . 6 2}$ & 27.42 & 33.57 & 27.46 & $\mathbf{2 4 . 4 3}$ & $\mathbf{3 1 . 2 3}$ \\
$\mathbf{2}$ & $\mathbf{4 7 . 4 6}$ & $\mathbf{1 6 . 3 2}$ & 27.13 & 34.29 & 27.22 & $\mathbf{1 6 . 5 3}$ & $\mathbf{4 3 . 5 7}$ \\
3 & $\mathbf{4 1 . 5 7}$ & $\mathbf{1 2 . 7 7}$ & 27.04 & 34.95 & 27.10 & $\mathbf{1 2 . 9 4}$ & $\mathbf{4 1 . 0 2}$ \\
\hline
\end{tabular}

Table 1 - linearity for the three cases

\section{CONCLUSIONS}

The aim of this paper has been to demonstrate that a broadband modulated time-domain measurement system, when used in combination with active IF load-pull, can be employed to gain valuable insight into the behaviour of high-frequency power transistors when operating under realistic conditions. With the increasingly popular application of ET in mind, the specific objective has been to question the impact of both positive and negative baseband impedance upon measured linearity, and to examine the idea of how PA linearity can be improved using the very mechanisms that are employed to improve efficiency.

In the case of a 'backed-off' device, contours of IM3-high and IM3-low indicate separate local optimum IF loads that lie some way outside the Smith chart, and for this particular device, a 15$20 \mathrm{~dB}$ improvement in IM3-high magnitude is observed in comparison to the reference case, where IF1 and IF2 are terminated into short circuits.

Whereas dynamic RF load-lines are a familiar plot in CW waveform engineering, the inclusion of baseband voltage and current to these plots adds an extra dimension that shows interesting and useful modulated behaviour, such as self-bias, and in this case of an emulated ET environment, the proximity of the load-line to the knee region, which may of course be optimised. For the ET analysis, where significant a significant IF component is injected, it is shown how plotting the envelope efficiency and gain can be useful in understanding device behaviour. In this case, it is clear from these time domain plots that there is an overall reduction in gain and hence output power with the application of an envelope tracking voltage - an effect that is largely explained by the fact that for this device, the gain reduces with reducing drain bias voltage.

\section{ACKNOWLEDGMENTS}

This work has been carried out as part of EPSRC grant EP/F033702/1. The authors would also like to thank CREE for supporting this activity and supplying the devices; specifically Ray Pengelly, Dr. Chris Harris and Mr. Simon Wood.

\section{REFERENCES}

[1] S. Cripps, "RF Power Amplifiers for Communications", Second Edition, Artech House, ISBN 1-59693-018-7

[2] Richards, A. Morris, K.A. McGeehan, J.P. "Removing the effects of baseband impedance on distortion in FET amplifiers" Microwaves, Antennas and Propagation, IEE Proceedings Volume: 153, Issue: 5 Oct.2006 page(s):401-406.

[3] Alghanim, A.; Lees, J.; Williams, T.; Benedikt, J.; Tasker, P.J., "Sensitivity of electrical baseband memory effects to higher-order IF components for high-power LDMOS power amplifiers" in Electronics Letters, Volume 44, Issue 5, 2008 pp. 358 - 359, Feb. 28

[4] N.B. Carvalho, J.C. Pedro, "Two-Tone IMD Asymmetry in Microwave Power Amplifiers" IEEE MTT-S Int. Microwave Symp. Dig., Vol. 1, 2000 pp. 445-448

[5] Joel H. K. Vuolevi, Timo Rahkonen, Jani P. Manninen, "Measurement technique for characterizing memory effects in RF power amplifiers," in IEEE Trans. MTT, vol. 49, no.9, pp. 1383-1389, Aug. 2001.

[6] Lees, J.; Williams, T.; Woodington, S.; McGovern, P.; Cripps, S.; Benedikt, J.; Tasker, P.; 38th European Microwave Conference, 2008. 2731 Oct. 2008 Page(s): 753 - 756.

[7] Alghanim, A.; Benedikt, J.; Tasker, P., "A measurement test-set for characterisation of high power LDMOS transistors including memory effects," High Frequency Postgraduate Student Colloquium, 2005 , vol., no.pp. 29- 32, 5-6 Sept. 2005.

[8] Benedikt, J.; Gaddi,R.; Tasker, P.J.; Goss, M.; "High-power time-domain measurement system with active harmonic load-pull for high-efficiency base-station amplifier design", IEEE Transactions on Microwave Theory and Techniques, Vol. 48, Issue 12, Dec. 2000, pp. 2617-2624.

[9] Brinkhoff, J.; Parker, A.E., "Effect of baseband impedance on FET intermodulation," Microwave Theory and Techniques, IEEE Transactions on, vol.51, no.3, pp. 1045-1051, Mar 2003

[10] T.V.Williams, "A Large-Signal Multi-Tone Time Domain Waveform Measurement System With Broadband Active Impedance Control" Ph.D. thesis, Cardiff University, December 2007.

[11] A. E. Parker and J. G. Rathmell, "Bias and frequency dependence of FET characteristics," in IEEE Trans. Microwave Theory Tech., vol. 51, pp. 588-592, Feb. 2003

[12] D.J. WilLiams, 1. Leckey, P.J. Tasker "A Study of the Effect of Envelope Impedance on intermodulation asymmetry using a two-tone Time Domain Measurement System" IEEE MTT-S Int. Microwave S p p . Dig., Vol. 3, 2002 pp. $1841-1844$ 


\title{
The Benefit of GaN Characteristics over LDMOS for Linearity Improvement using Drain Modulation in Power Amplifier System
}

\author{
Z. Yusoff, M. Akmal, V. Carrubba, J. Lees, J. Benedikt, P.J. Tasker and S.C. Cripps \\ School of Engineering, Cardiff University, \\ Cardiff, CF24 3AA, United Kingdom. \\ yusoffz@cardiff.ac.uk
}

\begin{abstract}
This paper reports that significant linearity improvement can be obtained in gallium nitride $(\mathrm{GaN}) \mathrm{RF}$ power amplifiers (RFPAs) in comparison to laterally diffused metal oxide semiconductor (LDMOS) RFPAs through the use of a modulated drain supply. It is shown that the gain characteristic of a GaN RFPA has significant variation with the drain bias voltage and this results in a $10-20 \mathrm{~dB}$ reduction in intermodulation (IM) levels. The LDMOS RFPA was measured and the result showed that the gain of LDMOS did not change substantially with drain bias voltage. As a consequence, when the LDMOS RFPA is measured using modulated drain bias, the IM levels showed only a much smaller improvement. These results appear to indicate that GaN devices have an important advantage over LDMOS in linear RFPA applications.
\end{abstract}

Keywords-component; Gallium nitride (GaN), Linearity, Power amplifiers, Intermodulation.

\section{INTRODUCTION}

In today's advanced wireless communication technology, such as WCDMA and Long Term Evolution (LTE), linearity performance has been an important aspect in designing the RF power amplifiers (RFPAs). Future applications such as mobile TV and multimedia streaming will also require high spectral efficiency and linear output power. Currently, the expected adjacent channel interference requirement for both standards is about $-30 \mathrm{dBc}$ to assure that two adjacent frequency operators to operates without any interference [12]. There are three methods of power amplifier linearization mentioned in [3] which are categorized by the way the RFPA's input signal, output signal or bias signal is being modified. The most popular method is by modifying the input signal (e.g. pre-distortion), secondly by modifying the output signal (e.g. feed forward) and the least popular is by modifying the amplifier characteristics dynamically (e.g. adaptive bias). This paper explores the last method by investigating the gain characteristic of GaN HEMT and SiLDMOS RFPAs at different drain bias voltages and measuring the linearity performances of the RFPAs by supplying modulated signal to the drain bias.

RFPAs based on LDMOS technology have been widely used in to the communication industry. The short channel length of the LDMOS structure provides a high current handling capability and low doping on drain side of this FET contributes to high blocking voltage [4]; this combination is a desired property for high power RFPAs. The low cost of LDMOS technology has resulted in it becoming the technology of choice for RFPA. However, as the LDMOS device operation reaches its limit, GaN technology represents the future for microwave power amplifiers. As compared to LDMOS technology, GaN is a wide band gap material thus it offers high breakdown voltage, higher carrier mobility and high temperature applications [5]. The high power density property of $\mathrm{GaN}$ contributes to reduced parasitic capacitance thus offering wider bandwidth in RFPA broadband matching design as compared to LDMOS. GaN also offers higher operating frequency because LDMOS performance degrades when operating frequency is greater than $3 \mathrm{GHz}$ [6]. All of these advantages towards high power broadband RFPA design have therefore attracted a large amount of recent attention, both in research and industry.

In this paper, GaN RFPA linearity characteristics are compared to corresponding LDMOS RFPA performance. It is shown that the GaN RFPA gain characteristic has possibility for linearity improvement using a modulated drain supply voltage. An earlier investigation of linearity on the same $10 \mathrm{~W}$ GaN device using an advanced active IF load pull measurement setup has been presented in [7] where IM3 and IM5 distortions were observed to improve significantly by emulating appropriate negative baseband impedance terminations. Such negative impedance terminations can be implemented in practice by using a suitably modulated supply voltage.

\section{GAIN CHARACTERISTIC OF GAN AND LDMOS PA}

A 10W GaN Class J RFPA that was reported in [8] and a 20W LDMOS Class AB RFPA were used in this measurement. GaN HEMT is a depletion mode device, therefore, the $10 \mathrm{~W}$ GaN Class J PA is biased with negative voltage at the gate of the RFPA. Both RFPAs are however biased in deep Class $\mathrm{AB}$ region. In the $\mathrm{CW}$ measurement at 2 $\mathrm{GHz}$, the RF power was swept at different drain bias voltage from $16 \mathrm{~V}$ to $28 \mathrm{~V}$. The output power was measured and the gain in $\mathrm{dBs}$ versus output power was plotted. The measurement of GaN RFPA showed that the gain varied significantly from $8 \mathrm{~dB}$ to $12 \mathrm{~dB}$ as shown in Figure 1 . At drain voltage of $16 \mathrm{~V}$, the gain measured was linearly around $8 \mathrm{~dB}$ but compresses as the peak output level was reached. The behavior of gain at different drain bias voltage was 
almost similar but at each higher drain bias voltage, the gain increased by about $0.5 \mathrm{~dB}$.

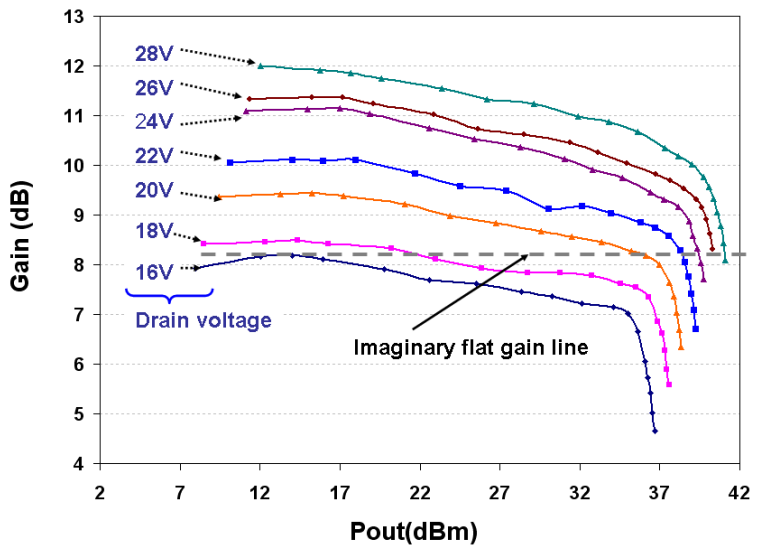

Figure 1. GaN PA gain characteristic as drain voltage was varied

In order to show the gain variation clearly, the gain in decibel scale was then plotted at a linear level of output power, $30 \mathrm{dBm}$. The logarithmic gain was observed to change approximately linearly with the drain bias voltage as shown in Figure 2. As observed in Figure 1, the rate of change of this linear gain in decibel in Figure 2 is about $0.5 \mathrm{~dB}$ per Volt.

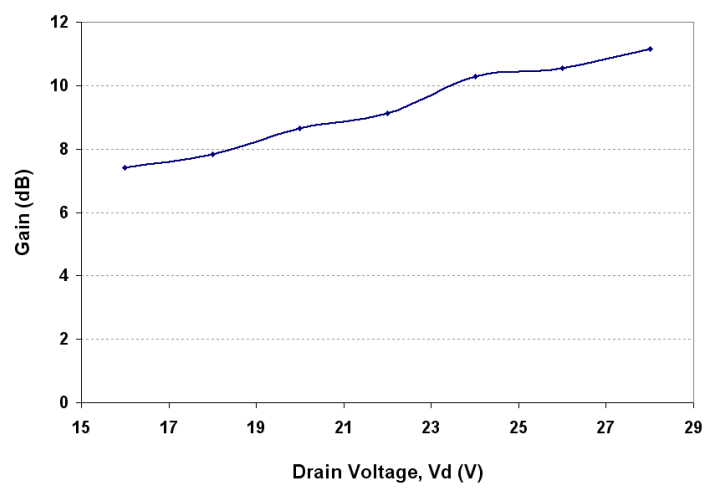

Figure 2. GaN PA gain characteristic at $30 \mathrm{dBm}$ output power

CW measurement was also done on the 20W LDMOS Class AB RFPA. The same RF power was swept at drain bias voltage from $16 \mathrm{~V}$ to $28 \mathrm{~V}$. The output power was measured and the again the logarithmic gain was plotted against the output power. This measurement result is presented in Figure 3 . The figure shows that the gain was linear but compressed towards peak output level. The gain behavior of LDMOS RFPA was almost similar for all drain bias voltages. However, it is observed that the gain varied much less significantly with drain bias voltage in comparison to the GaN measurements. In order to see clearly the insignificant rate of change in the logarithmic gain versus drain bias voltage, the LDMOS gain was plotted at a linear level of output power, $30 \mathrm{dBm}$. As shown in Figure 4, the gain was seen to be almost constant with the drain bias voltage.

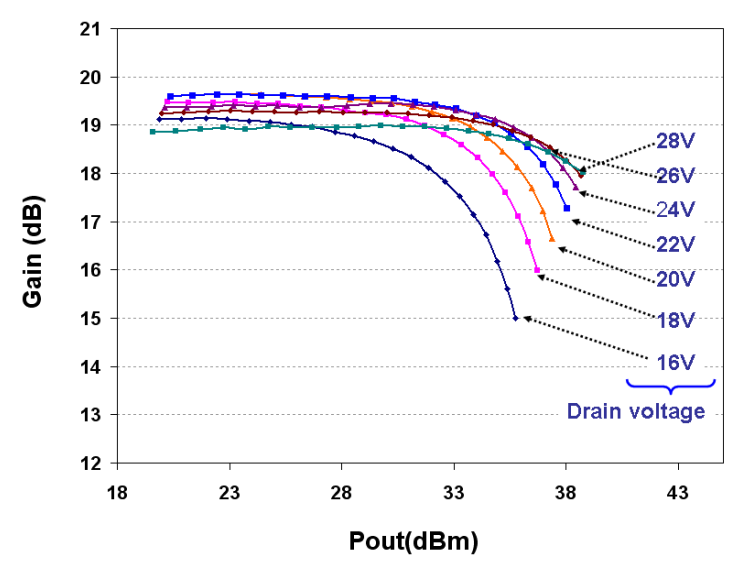

Figure 3. LDMOS gain characteristic as drain voltage was varied.

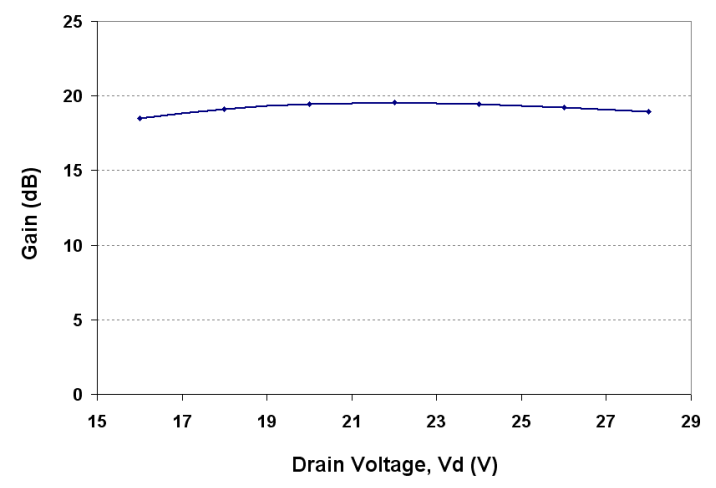

Figure 4. LDMOS PA gain characteristic at $30 \mathrm{dBm}$ output power

The measurement results above show that the GaN RFPA has an expansive gain characteristic with increasing drain voltage. Theoretically, when the device reaches the peak power level the device compressive behavior can be linearized by the expansive gain characteristic if the drain bias voltage is suitably increased. In order to visualize the linearizing effect, an imaginary linear line is drawn in Figure 1. From this figure, as we increase a suitable drain bias voltage in the compressive area at the peak power level, a flat gain can be achieved. As we improve a linear gain at its peak power level, the linearity of the RFPA will be observed as well. We have called such drain voltage adjustment "Auxiliary Envelope Tracking (AET)", inasmuch as the voltage tracking is primarily intended to linearize the PA, rather than improve its efficiency.

\section{IMD MEASUREMENT FOR GAN AND LDMOS PA}

For intermodulation distortion (IMD) investigation, the GaN RFPA and LDMOS were measured on two experimental setups. The first experimental setup was used to measure the IMD of the RFPA when the RFPAs were biased by a fixed drain supply as shown in Figure 5 (a). This IMD measurement using fixed drain supply is needed to make comparison to RFPA when biased with modulated signal. The second experimental setup was used to measure the IMD when the RFPAs were biased with a modulated drain supply 
voltage as shown in Figure 5(b). In the first setup, the 2carrier input signal at $2 \mathrm{GHz}$ with frequency separation of $1 \mathrm{MHz}$ was generated by two signal generators. The input signal is then amplified before the input signal was fed into the gate of RFPA. The drain of the RFPA was biased from a fixed supply.

In this second experimental setup, there were three signal generators used for this measurement. Two signal generators generated a 2-carrier input signal for the RFPA at $2 \mathrm{GHz}$ with frequency separation of $1 \mathrm{MHz}$. The input signal was amplified by a driver amplifier before inserted to the gate of RFPA. The drain bias of the RFPA was provided with the sinusoidal signal at the 2-carrier signal envelope frequency of $1 \mathrm{MHz}$ by the third signal generator. This $1 \mathrm{MHz}$ sinusoidal signal was then amplified by an amplifier that we termed Envelope Amplifier and the DC component is inserted through a diplexer. The combined signal that we called AET signal was then fed to the RFPA drain supply, which of course has to have all bias decoupling components disconnected.

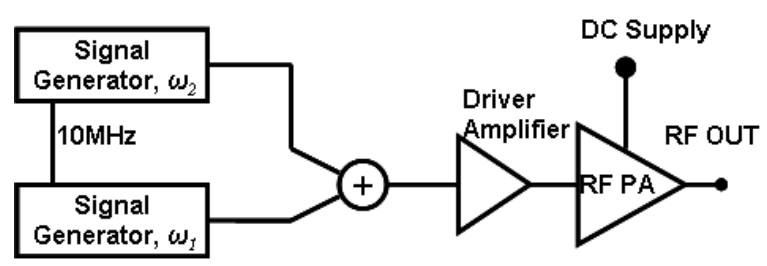

(a)

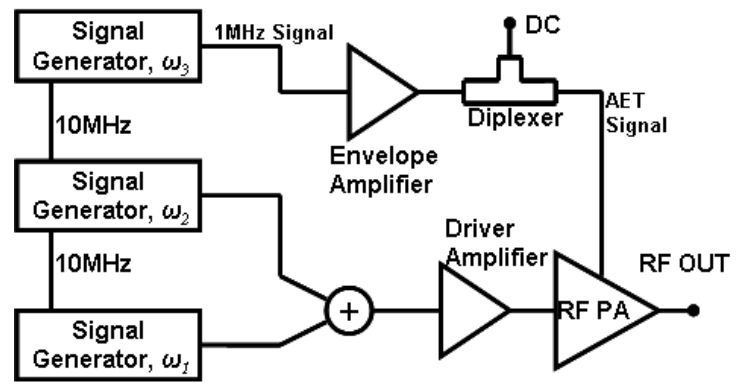

(b)

Figure 5. Experimental setup for intermodulation distortion measurement

(a) Fixed drain supply (b) Modulated drain supply

In the 2-carrier signal measurement for GaN RFPA, RF power was swept and the third-order intermodulation (IM3) and average output power were measured. For the GaN RFPA, on the first experimental setup for fixed drain bias measurement, the GaN RFPA was measured at fixed drain bias of $37 \mathrm{~V}$. On the second experimental setup; the peak voltage that was provided to the drain bias, (that is the sum of the fixed and sinusoidal components) was $37 \mathrm{~V}$. The values were chosen to make a very fair comparison between the fixed drain supply and the drain modulated bias results measured on the modulated drain supply setup. For the LDMOS RFPA, the fixed drain bias voltage on the first setup was $28 \mathrm{~V}$ and the peak voltage of the combined fixed and sinusoidal signal that was provided to the drain bias on the second setup was $28 \mathrm{~V}$.

For both fixed and drain modulated bias measurements, the IM3 performance were plotted. In Figure 6, the IM3 performance of the GaN RFPA when biased with fixed drain supply showed that the IM3 value of lower than $-30 \mathrm{dBc}$ for average output power lower than about $36 \mathrm{dBm}$. A 'null' effect was observed around average output power of $31 \mathrm{dBm}$ but greater than $36 \mathrm{dBm}$ average output power, the IM3 level was worsening. The IM3 performance of GaN RFPA under modulated drain supply however showed that the IM3 level was below $-30 \mathrm{dBc}$ even at its maximum average output signal. This is a promising performance for WCDMA and LTE technology that require high linearity. At the maximum measured output power level, the IM3 performance under modulated drain supply as compared to fixed drain supply was improved from $-22 \mathrm{dBc}$ to $-33 \mathrm{dBc}$, and an IM3 improvement between $10 \mathrm{~dB}$ and just over $20 \mathrm{~dB}$ was observed over a $3 \mathrm{~dB}$ power backoff $(\mathrm{PBO})$ range, and significant

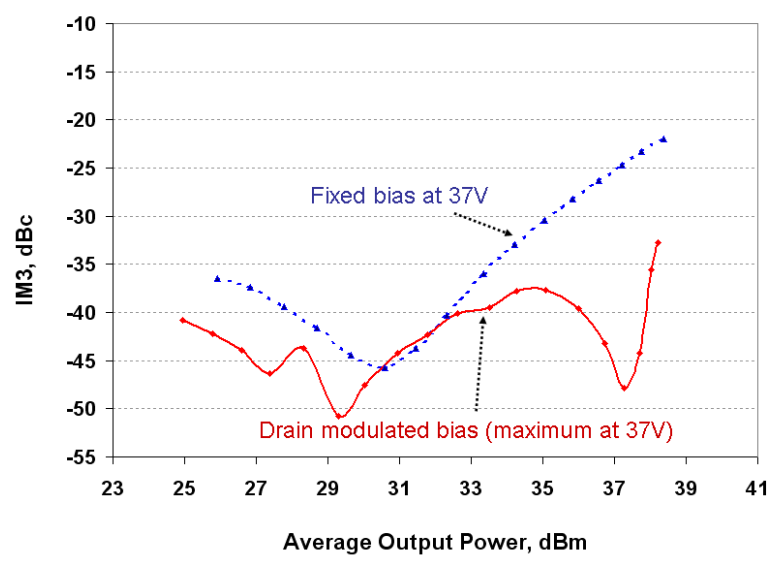

Figure 6. IM3 for GaN PA at fixed and drain modulated bias

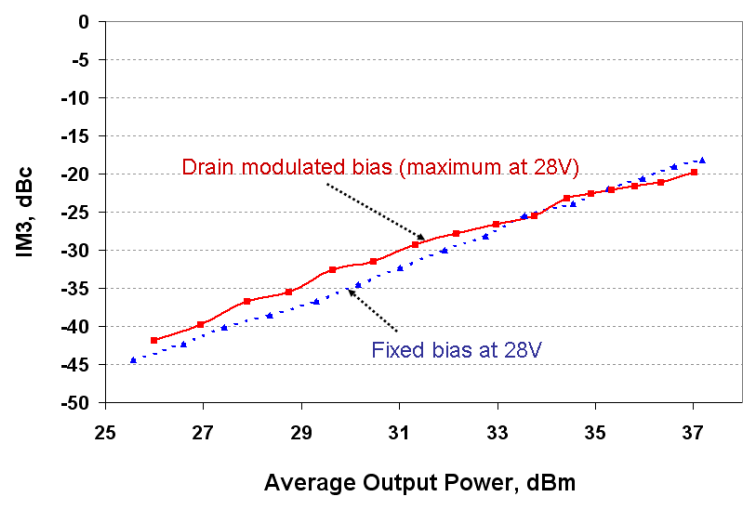

Figure 7. IM3 for LDMOS PA at fixed and drain modulated bias 
In Figure 7, the LDMOS performance in IM3 is presented and it is observed that the LDMOS, as expected from the gain measurements, did not show substantial IM3 improvement. The IM3 performance of LDMOS RFPA for fixed drain supply was less than $-30 \mathrm{dBc}$ for average output power greater than $33 \mathrm{dBm}$. The IM3 performance of this LDMOS RFPA under modulated drain supply showed a slight decline for average output power lower than $33 \mathrm{dBm}$ but showed a slight but insignificant improvement for average output power greater than $33 \mathrm{dBm}$.

\section{CONCLUSIONS}

It has been shown that the gain variation of $\mathrm{GaN}$ device has a potential to contribute substantial linearity improvement using drain modulation, an effect which is absent in an LDMOS device. The improvement in linearity using the AET system showed that the GaN RFPA can be useful for modern communication systems that require the RFPA to be optimized at highest power rating while maintaining the linearity and spectrum purity of the signal. Future work on this research program is to investigate the gain characteristic on higher power GaN devices and at different bias point, and also to explore the possibility of this drain modulation method to improve efficiency.

\section{ACKNOWLEDGMENT}

This work has been carried out as part of EPSRC grant EP/F033702/1. The authors would also like to thank CREE for supporting this activity and supplying the devices and
Yayasan Telekom Malaysia, Kuala Lumpur for $\mathrm{PhD}$ student sponsorship.

\section{REFERENCES}

[1] H. Lilja, H. Mattila," WCDMA Power Amplifier Requirements and Efficiency Optimization Criteria", 1999 IEEE MTT-S International Microwave Symposium Digest, Anaheim, USA, vol.4, pages 1943-1846.

[2] Y. Li, R. Zhu, D. Prikhodko, Y. Tkachenko, “ LTE Power Amplifier Module Design: Challenges and Trends", 2010 10th IEEE International Conference on Solid-State and Integrated Circuit Technology (ICSICT), Shanghai, China, pages 192195.

[3] S. Cripps, "RF Power Amplifier for Wireless Communications", Norwood, MA: Artech House, Apr. 1999.

[4] Bart Van Zeghbroeck, " Principles of Semiconductor Devices", http://ece-www.colorado.edu/ bart/book/

[5] "LDMOS", Microwave 101 Website, http://www.microwaves101.com/encyclopedia/LDMOS.cfm

[6] "GaN Essentials- Application Notes", AN009 - AN 013, Nitronex Corporation, http:// www.nitronex.com/ganessentials .html

[7] M.Akmal,J. Lees, S. Bensmida, S. Woodington, V. Carrubba, S. Cripps, J. Benedikt, K. Morris, M. Beach, J. McGeehan, P.Tasker, "The Effect of Baseband Impedance Termination on the Linearity of GaN HEMTs," 40th European Microwave Conference, 2010.

[8] P. Wright, J. Lees, P.J. Tasker, J. Benedikt, S.C. Cripps, “An Efficient, Linear, Broadband Class-J-Mode PA Realised Using RF Waveform Engineering", IEEE/MTT-S International Microwave Symposium Digest, 7-12 June 2009, pp. 653-656 


\title{
Investigation and Analysis into Device Optimization for Attaining Efficiencies In-Excess of 90\% When Accounting for Higher Harmonics
}

\author{
A. L. Clarke, M. Akmal, J. Lees, P. J. Tasker, J. Benedikt \\ Cardiff School of Engineering, Cardiff University, Cardiff, CF24 3AA, United Kingdom \\ Tel: +44 2920 875938, Email: ClarkeA4@ cardiff.ac.uk
}

\begin{abstract}
A rigorous, systematic, measurement-founded approach is presented that enables the design of highly efficient power amplifiers. The identified process allows the designer to quickly identify the parameters necessary for completion of a design whilst ascertaining their flexibility and impact on performance degradation. The investigation continues to consider the impact of the higher harmonics and gate bias as design tools on the performance of the design and proposes a strategy that utilizes their positive effect as well as considering the subsequent impact on device scaling. This was carried out on GaAs pHEMT devices from commercial processes that obtained measured peak efficiencies of $90.1 \%$ at $P_{1 d B}$ in a class-B bias.
\end{abstract}

Index Terms - Power amplifiers, microwave measurements, load-pull, class-F, inverse class-F.

\section{INTRODUCTION}

In recent years, the focus of the wireless communication industry has shifted towards energy conservation due to environmental and consumer demands. This is especially true of microwave power amplifier (PA) design for the mobile market, where minimizing DC power consumption is critical. In order to achieve this, harmonically-engineered amplifiers, such as class-F [1][2], inverse class-F [3][4] and class-J [5][6], are of increasing interest.

The class- $F$ and inverse class- $F$ designs in particular provide excellent performance in terms of output power and efficiency whilst operating at $1 \mathrm{~dB}$ compression levels. By providing higher peak efficiencies they lend themselves to extended high efficiency topologies such as in envelope tracking (ET) or the Doherty PA. Various prior publications have utilized a developed measurement-based strategy [4][7][8], but were unable to investigate more deeply into the dependencies on higher harmonics and their impact on efficiency due to a lack in measurement speed and time; this will be addressed here.

This paper moves away from the CAD and simulation environment to a purely measurement based strategy for the design of these highly-efficient modes of operation, that has the capability of including analysis for the optimization of higher harmonic content as well as assessing other simple design tools available, other than load termination. The measurements for this investigation were carried out using an envelope load-pull based system [9] at a fundamental frequency of $900 \mathrm{MHz}$. This system allows the necessary waveforms to be captured in addition to the ability of rapidly establishing fundamental and harmonic impedances - a key requirement for this type of exhaustive, in-depth investigation. Analyses carried out at these frequencies provide the PA designer with critical information on the capabilities of the device and its relative performance, whilst narrowing down the number of measurements and investigation time required for designs at higher frequencies. Devices used throughout this paper were from the TriQuint TQPED GaAs Foundry process, specifically $6 \times 50 \mu \mathrm{m}$ depletion mode pHEMTs.

\section{BACKGROUND AND THEORETICAL ANALYSIS}

The overall efficiency can be described by the product of the fundamental RF waveform efficiencies, $\eta_{\text {voltage }}$ and $\eta_{\text {current }}$, and the physical limitations imposed on the waveform extensions by the excitation boundaries, $\eta_{\text {boundary }}$. It is known that bandlimiting, e.g. limiting the positive effect of higher harmonic content, in a class-F or inverse class-F design will yield less than the theoretical $100 \%$ drain efficiencies through the production of non-ideal waveforms [10].

The boundary efficiency, described by (1), for the devices used in this investigation for class- $F$ at $V_{D}=6 \mathrm{~V}$ and inverse class- $\mathrm{F}$ at $\mathrm{V}_{\mathrm{D}}=4 \mathrm{~V}$ were 0.95 and 0.925 respectively.

$$
\eta_{\text {boundary }}=\left(1-\frac{V_{\min }}{V_{D}}\right) \cdot\left(1-\frac{I_{\min }}{I_{D}}\right) .
$$

Therefore, the overall theoretical efficiencies for class-F operation are $80.1 \%$ with 3 harmonics utilized, $90.1 \%$ with 5 harmonics, and $95 \%$ with ideal waveform shaping.

\section{ClASS-F INVESTIGATION AND ANALYSIS}

\section{A. Optimization Strategy}

Following a class-F design procedure similar to [7], the starting point is to establish the optimum input bias at a suitable power level that will ensure a null in the oddharmonic current content. To investigate this, the device input bias was swept for conduction angles, $\alpha_{\mathrm{c}}$, of $90^{\circ} \leq \alpha_{\mathrm{c}} \leq 270^{\circ}$, over a range of input power levels, whilst maintaining constant active harmonic loads, in order to engineer the appropriate current magnitude spectrum for the class-F mode. Provision of this type of design information has not been previously viable due to the volume of measurements required and the speed of 

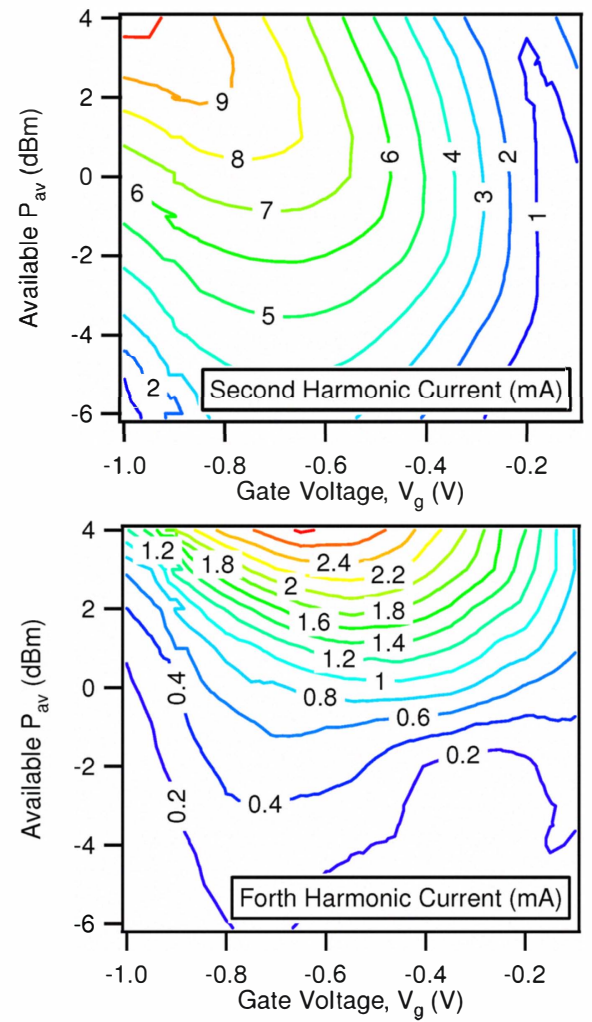
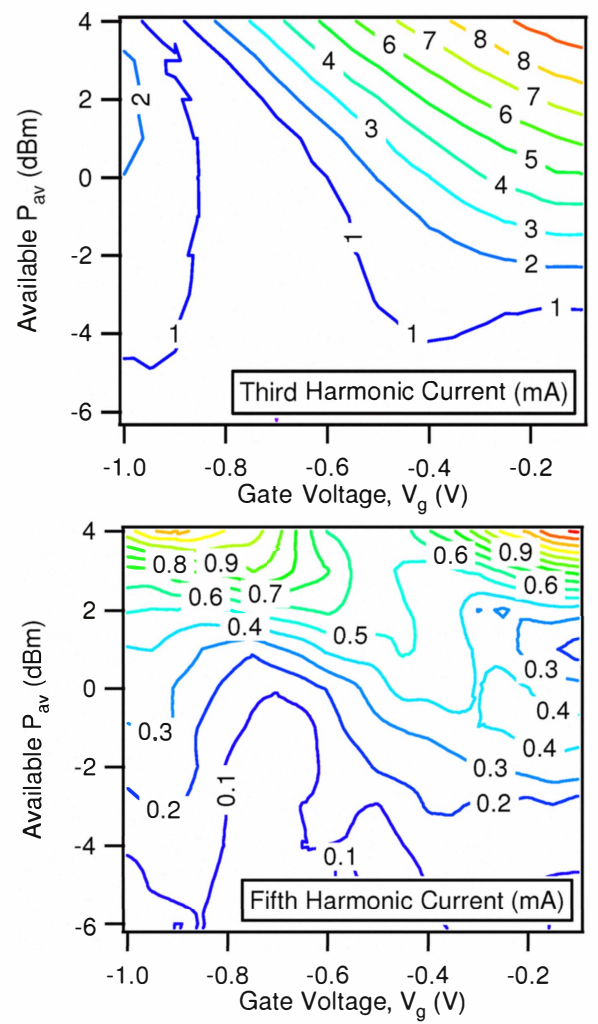

Fig. 1. Measured second, third, forth and fifth harrnonic current magnitudes as a function of gate bias and drive power, at $V_{D}=6 \mathrm{~V}$.

previous measurement systems. Fig. 1 displays the resultant information in contour form.

Through direct device measurement, it is clear that harmonic current levels are dependant on both the drive level as well as input bias. Using this analysis, the PA designer has a continuum of bias and drive levels available to achieve their goal; in the case of class-F, nulling the third harmonic current, whilst only considering the first 2 harmonics, $2 \mathrm{f}_{0}$ and $3 \mathrm{f}_{0}$. Although, it is important to note that the archetypal class-F state, of square voltages and half-sinusoid currents, only occurs when enough harmonic content is generated to ensure waveform shaping. Fig. 1 indicates that this is only at higher input drive levels when the current waveform would be compressed [2], therefore it would be prudent to select the gate bias level based on these power levels. Here a gate voltage, $\mathrm{V}_{\mathrm{G}}$, was chosen to be $-0.85 \mathrm{~V}$, close to pinch-off, for a third harmonic minimum in mild compression.

The methodology employed here does not try to force the device into the class-F state solely through the given harmonic states, but tries to 'relax' it into the condition through the correct biasing of the device.

To ensure that the voltage waveform is a full sinusoid, the second harmonic is required to be terminated into a short. This is from the fact that the natural starting point for class-F is a class-B state, where all harmonics are ideally shorted.

The classic class-F procedure involves terminating the third harmonic into an open circuit, although it has been shown that the resulting efficiency is relatively insensitive to the magnitude of the load, so long as the third harmonic resistance $\left(\mathrm{Z}_{3 \mathrm{fO}}\right)$ is at least 10 times greater than that of the fundamental [2]. Although this does not take into account the effect of the output capacitance, $C_{d s}$, which offsets the harmonic impedance by the susceptance, $B$, at that frequency. By experimentally sweeping the third harmonic, the required phase was shown to be $\arg \left(\mathrm{Z}_{3 \mathrm{fO}}\right)=15^{\circ}$.

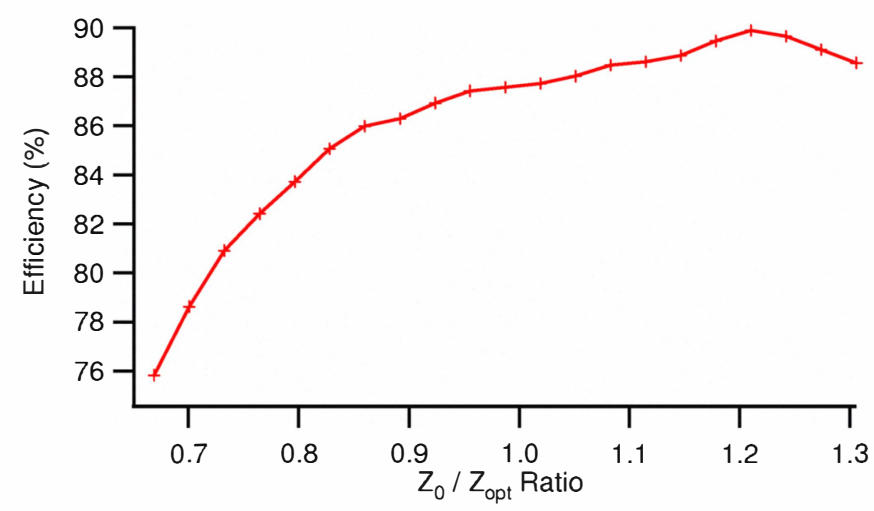

Fig. 2. Measured efficiency as a function of $\left|Z_{\mathrm{f} 0}\right|:\left|Z_{\text {opt }}\right|$ ratio for constant output power.

With the natural increase in RF voltage at the third harmonic, due to the high impedance presented, it is required that the fundamental RF voltage also increase to maintain the required $\mathrm{f}_{0} / 3 \mathrm{f}_{0}$ voltage ratio. Usual practice is to increase the impedance seen by the fundamental; the increase being a 


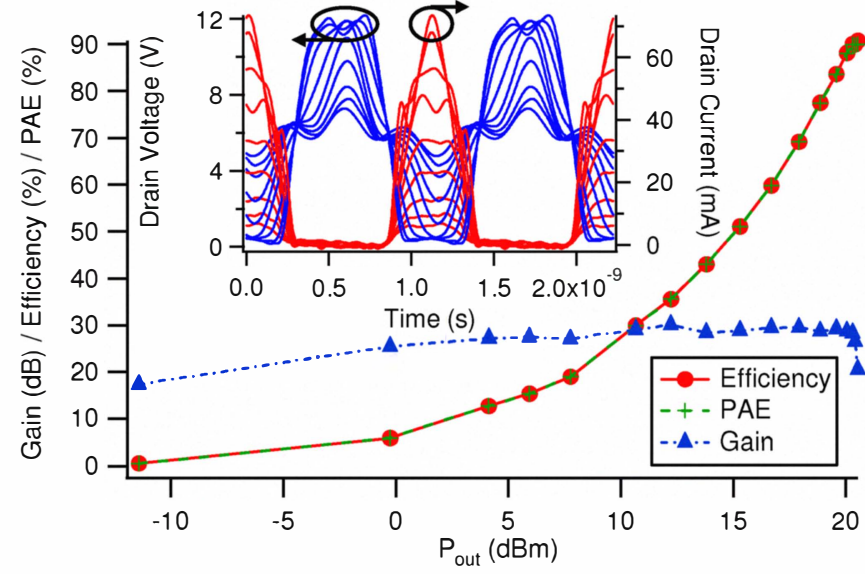

Fig. 3. Measured class-F power sweep with recorded drain voltage and current waveforms at the current-generator plane.

factor of $4 / \pi$ for fully harmonic terminated class-F. A design that only considers waveform engineering to the third harmonic requires a smaller increase in $\mathrm{Z}_{\mathrm{f} 0}$ of 1.154 [11]. Although this is an assumption based on the complete absence and non-interaction of higher harmonics, which in reality cannot be achieved easily. Therefore it cannot be assumed in the design routine that an increase of $1.154 \cdot \mathrm{Z}_{\mathrm{opt}}$ is the optimum termination for a realistic class-F operation. In order to explore this, the fundamental load was swept along the contour of constant susceptance, corresponding to the effect of $C_{d s}$, whilst maintaining a constant output power. Fig. 2 plots the variation in efficiency as the ratio of $\left|Z_{\mathrm{f} 0}\right|$ to $\left|Z_{\mathrm{opt}}\right|$ is increased, and indicates that the optimum fundamental impedance environment is $1.21 \cdot \mathrm{Z}_{\mathrm{opt}}=210 \Omega$. This is a marked increase over what theory predicts for a 3 harmonic situation.

\section{B. Results}

The result of this optimization is achieved drain efficiencies of $90.1 \%$ at $\mathrm{P}_{1 \mathrm{~dB}}$ and $90.3 \%$ at $\mathrm{P}_{3 \mathrm{~dB}}$. Fig. 3 displays a power sweep for the device in the established class-F mode, along with select de-embedded waveforms. As can be seen in the final power sweep, a very high efficiency for this device has been obtained, greater than the theory predicts for a three harmonic emulation. The RF voltage and current waveforms are very close to those expected, with only a small amount of voltage clipping apparent in the final current waveforms.

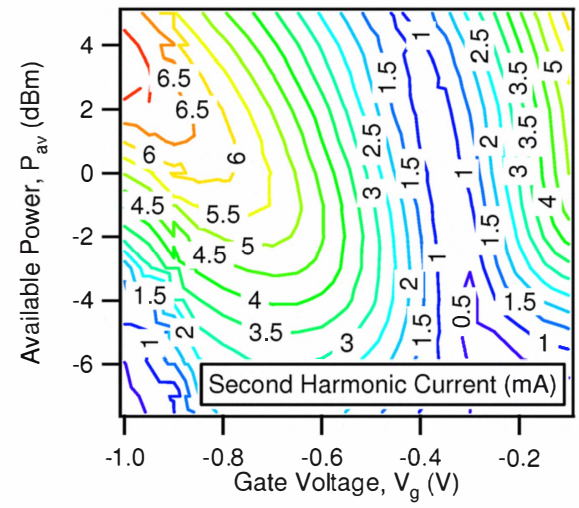

\section{INVERSE CLASS-F INVESTIGATION AND ANALYSIS}

\section{A. Optimization Strategy}

A similar procedure to the class-F design was adopted for an inverse class-F emulation. This mode produces output voltage peaking in the order of $\pi \cdot \mathrm{V}_{\mathrm{DC}}$; knowing that the voltage breakdown of the device is in the region of $12 \mathrm{~V}$ it is necessary to reduce the drain voltage to $4 \mathrm{~V}$, which will also help facilitate higher efficiencies [8].

Again, as a starting point, the gate bias was swept as well as the input power; the harmonic currents being shown in Fig. 4 as contours. Considering that the third harmonic current was nulled in the class-F approach, aiding its half-rectification, it is logical that in the inverse class-F case the $2^{\text {nd }}$ harmonic current be minimized. Thus relaxing it into the squaring of the current waveform with only odd harmonic content; inherently around the class-A bias point. The inverse class-F mode builds upon the inverted class- $\mathrm{B}$ mode, where all the harmonic impedances are set to open, producing an initial inverted, half-rectified voltage waveform and a sinusoid current, also having a class-A bias point. Fig. 4 suggests that the optimal bias condition is $\mathrm{V}_{\mathrm{G}}=-0.4 \mathrm{~V}$ for the higher-end power levels. The harmonic impedance environments were set as required, $Z_{2 \mathrm{fo}}$ to an open and $Z_{3 \mathrm{fo}}$ to a short with the second harmonic requiring the optimization for the $C_{d s}$ offset. Again, the fundamental load was optimized along the contour of constant susceptance.

\section{B. Results}

Fig. 5 displays the power sweep of the inverse class-F emulation with select de-embedded waveforms inset. It can be seen that at $1 \mathrm{~dB}$ of compression a drain efficiency of $81.0 \%$ was achieved, $82.3 \%$ at $\mathrm{P}_{3 \mathrm{~dB}}$, near predicted values. Although the RF waveforms are not as ideal, with significant peaking in the voltage waveform and a deep notch forming in the current.

\section{COMPARATIVE ANALYSIS OF RESUlTS}

On first comparison of the final results, it is particularly interesting that the class-F emulation is by far the better performer both in terms of drain efficiency and output power, with 9 percentage-points increase over the inverse class- $F$ efficiency at an excellent $90.1 \%$ at $\mathrm{P}_{1 \mathrm{~dB}}$.

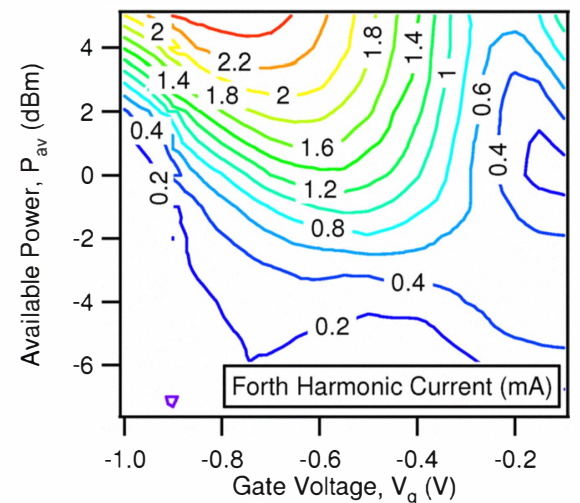

Fig. 4. Second and forth harmonic current magnitudes as a function of gate bias and drive power, at $V_{D}=4 \mathrm{~V}$. 


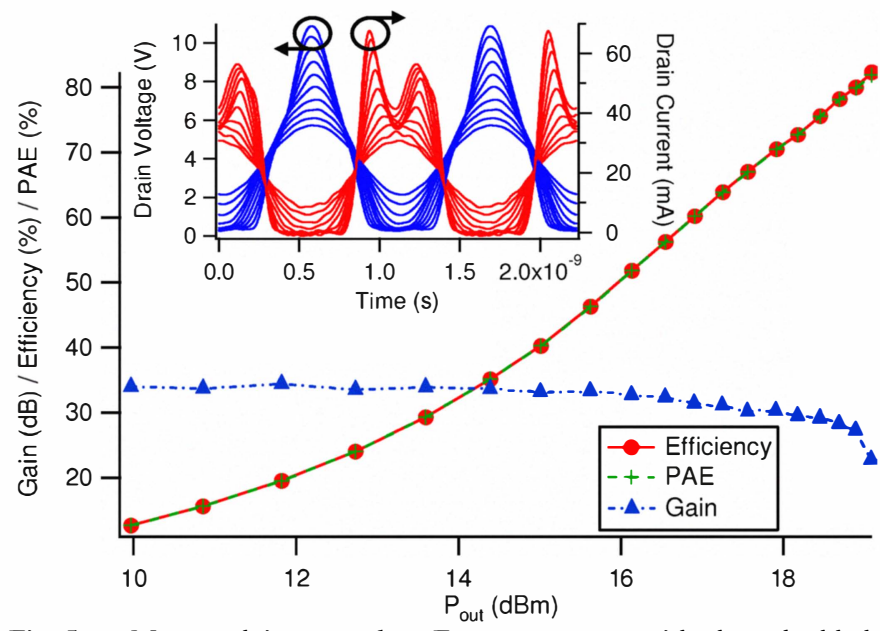

Fig. 5. Measured inverse class-F power sweep with de-embedded output voltage and current waveforms inset.

According to the band-limited theory, it is not possible to operate these devices with greater efficiency than $80.1 \%$ in class-F with an engineered 3 harmonic content, yet here this was managed. Viewing the $4^{\text {th }}$ and $5^{\text {th }}$ harmonics, it was found that they were terminated into $\mathrm{Z}_{4 \mathrm{f} 0}=29.4-\mathrm{j} 34.8 \Omega$ and $Z_{5 f 0}=95+j 35 \Omega$. When considering that these GaAs devices are low-power, requiring a high impedance termination at the fundamental (in the order of $210 \Omega$ ) these upper harmonic impedances appear relatively low. As the class-F starting condition is from a class- $\mathrm{B}$ mode, where all harmonic content is ideally shorted, the small upper harmonic impedances can be seen to be beneficial to a class-F design. Now, given that the inverse class-F design has an inverted-class- $\mathrm{B}$ starting point, where the harmonic content is desired to be terminated into open circuits, the relatively small upper harmonic impedances have a negative impact in terms of efficiency.

The correct shaping of the squared current waveform through bias control is more important in the inverse class-F mode, as seen, due to the fact that greater control of the higher harmonics is required to shape the waveform without distortion, as load engineering plays little part in this. By examining the harmonic content in Fig. 4, it can be seen that whilst trying to achieve a second harmonic null, a significant forth harmonic exists, degrading the quality of the squared current waveform. The minimum of the forth component existing nearer a gate voltage of $\mathrm{V}_{\mathrm{G}}=-0.2 \mathrm{~V}$. The solution to increased performance could lie in a trade-off in favor of minimizing the higher, forth harmonic with a bias greater than the optimum class-A, which was seen to be necessary in [4].

\section{CONCLUSION}

The emulated class-F and inverse class- $F$ modes on the same GaAs pHEMT device has shown how the higher harmonics can play a large part in the achieved overall performance.

This becomes important when considering the mode of operation necessary for a specific device to obtain efficiencies in excess of $85 \%$. For low-power devices with a high $\mathrm{Z}_{\mathrm{opt}}$, it is sensible to focus on a class-F design where the higher harmonics have relatively more freedom to be terminated into lower impedances. The reverse is true for high-power devices which have a low $\mathrm{Z}_{\mathrm{opt}}$; this has been seen with a class-F design in [7] and an inverse class-F in [4]. Device scaling would also be affected by this observation, so where a class-F design would provide increased efficiency for the lower power, this performance does not necessarily scale up with higher power, where inverse class-F terminations would be more beneficial.

This conclusion has been reached through a measurement based approach that culminated in a design with a measured drain efficiency of $90.1 \%$ in $1 \mathrm{~dB}$ compression, which to the authors' knowledge is the highest efficiency reported without requiring the trade-off of a class- $\mathrm{C}$ bias.

\section{ACKNOWLEDGEMENT}

The authors wish to thank Cobham Defense \& Space and EPSRC for funding the research. As well as thanking Tony St Denis and Paul Litzenberg from TriQuint Semiconductor for their support with this project.

\section{REFERENCES}

[1] F. H. Raab, "Maximum Efficiency and Output of Class-F Power Amplifier," IEEE Trans. Microwave Theory \& Tech., Vol. 49, No. 6, Jun. 2001, pp. 1162-1166.

[2] S. C. Cripps, $R F$ Power Amplifiers for Wireless Communications, 2nd Ed., Norwood, MA: Artech House, 2006.

[3] S. Goto, et al, "Effect of Bias Condition and Input Harmonic Termination on High Efficiency Inverse Class-F Amplifiers," in Proc. IEEE EuMC., Vol. 3, Sept. 2001, pp. 113-116.

[4] P. Wright, A. Sheikh, C. Roff, P. J. Tasker, and J. Benedikt, "Highly Efficient Operation Modes in GaN Power Transistors Delivering Upwards of $81 \%$ Efficiency and $12 \mathrm{~W}$ Output Power," IEEE MTT-S Int. Dig., Jun. 2008, pp. 1147-1150.

[5] P. Wright, J. Lees, P. J. Tasker, J. Benedikt, and S. C. Cripps, "An Efficient, Linear, Broadband Class-J-Mode PA Realised Using RF Waveform Engineering," IEEE MTT-S Int. Dig., Jun. 2009, pp. 653-656.

[6] S. C. Cripps, P. J. Tasker, A. L. Clarke, J. Lees and J. Benedikt "On the Continuity of High Efficiency Modes in Linear RF Power Amplifiers," IEEE Microwave and Wireless Components Letters, Vol. 19, No. 10, Oct. 2009, pp. 665-667.

[7] C. Roff, J. Benedikt and P. J. Tasker, "Design Approach for Realization of Very High Efficiency Power Amplifiers," IEEE MTT-S Int. Dig., June 2007, pp. 143-146.

[8] A. Sheikh et al. "Peak Class F and Inverse Class F Drain Efficiencies Using Si LDMOS in a Limited Bandwidth Design," IEEE Microwave and Wireless Components Letters, Vol. 19, No. 7, Jul. 2009, pp. 473-475.

[9] M. S. Hashmi, A. L. Clarke, S. P. Woodington, J. Lees, J. Benedikt, and P. J. Tasker, "Electronic multi-harmonic load-pull system for experimentally driven power amplifier design optimization," IEEE MTT-S Int. Dig, Jun. 2009, pp. 1549-1552.

[10]F. H. Raab, "Class-F Amplifiers with Maximally Flat waveforms," IEEE Trans. Microwave Theory \& Tech., Vol. 45, No. 11, Nov. 1997, pp. 2007-2012.

[11] A. Mallet et al., "A Design Method for High Efficiency Class F HBT Amplifiers," IEEE MTT-S Int., Vol. 2, 1996, pp. 855-858. 


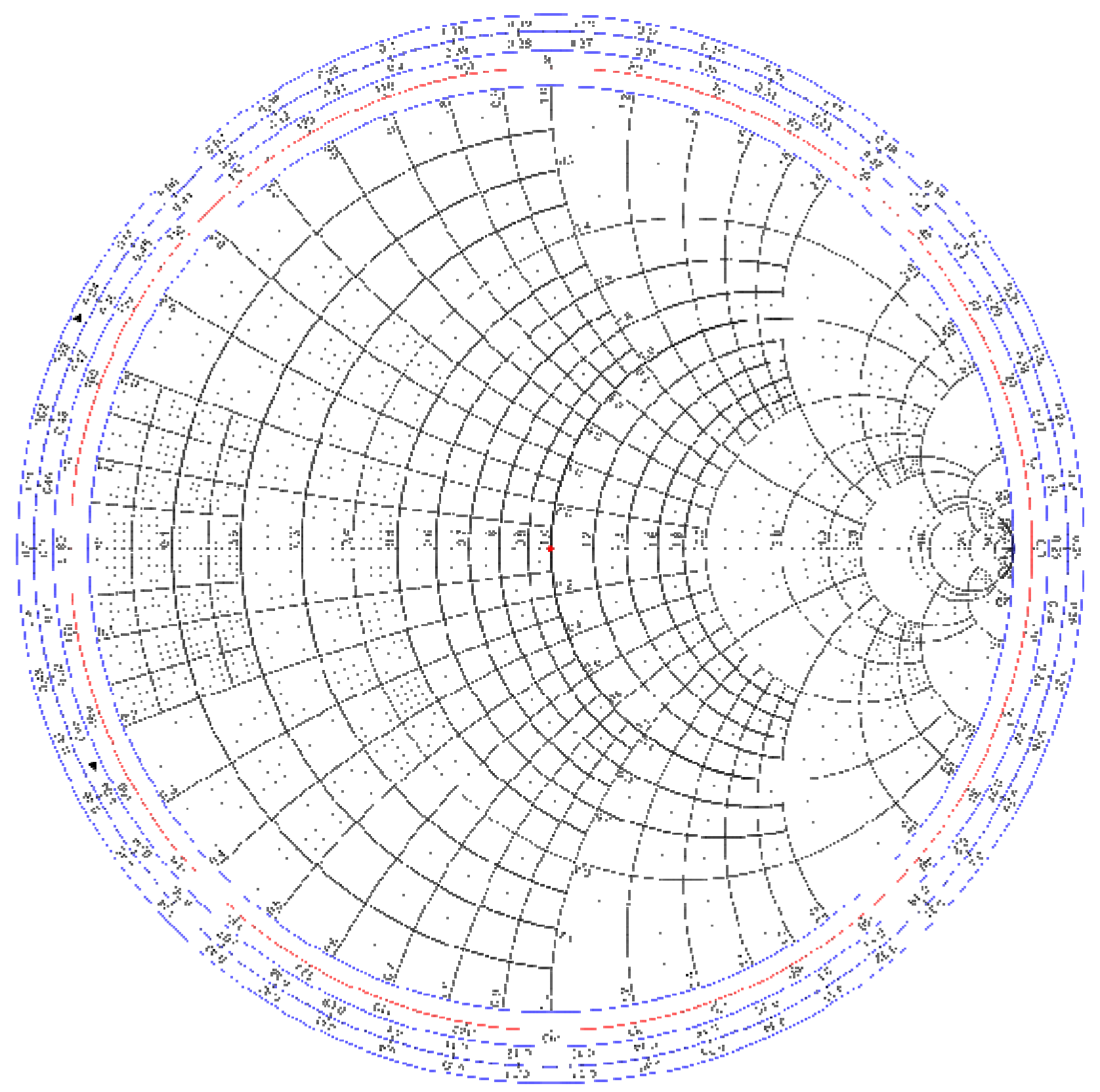

